\title{
Erdflechten und ihre Gesellschaften in Nordhessen mit besonderer Berücksichtigung der morphologischen und genetischen Variabilität bei Cladonia furcata (Hudson) Schrader
}

\author{
Dissertation \\ zur Erlangung des Doktorgrades \\ der Mathematisch-Naturwissenschaftlichen Fakultäten \\ der Georg-August-Universität zu Göttingen
}

vorgelegt von

Bettina Günzl

aus Tübingen

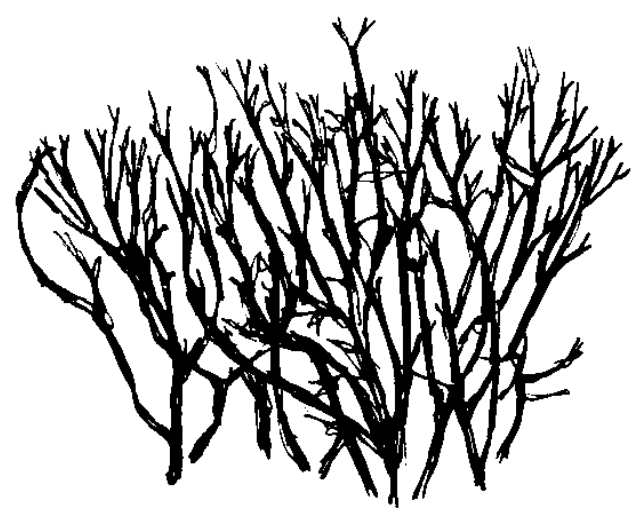

Göttingen 2003 
D 7

Referent: Prof. Dr. H. Dierschke

Korreferent: Prof. Dr. E. Bergmeier

Tag der mündlichen Prüfung: 22.01.2004 


\section{Inhaltsverzeichnis}

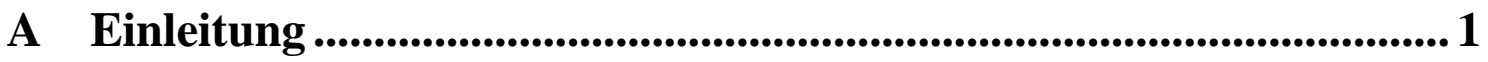

1 Erdflechtenvegetation............................................................................................ 1

2 Variabilität bei Cladonien ......................................................................................2

3 Zusammenfassung der Arbeitsziele........................................................................

B Das Untersuchungsgebiet ............................................................................ 5

1 Lage der Flächen und naturräumliche Zuordnung...........................................5

2 Geologie und Böden .........................................................................................

3 Klima .......................................................................................................................11

4 Historische und aktuelle Nutzung der Flächen ...............................................13

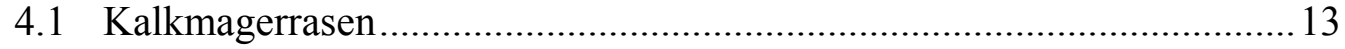

4.2 Zwergstrauchheiden ..........................................................................14

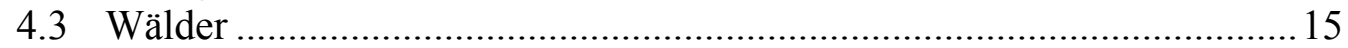

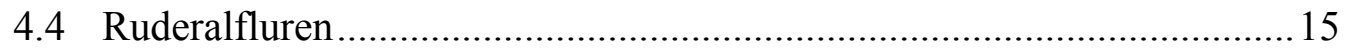

C Charakterisierung der Art Cladonia furcata ..................................... 16

D Übersicht der angewandten Methoden ........................................... 19

E Vegetationskundliche und floristische Untersuchungen ................ 20

1 Methoden .............................................................................................................20

1.1 Auswahl und Verteilung der Aufnahmeflächen .......................................20

1.2 Vegetationsaufnahmen und Bodenanalysen.............................................21

1.3 Nomenklatur der Arten und Vegetationseinheiten .....................................22

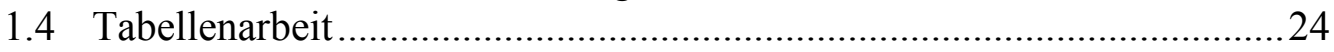

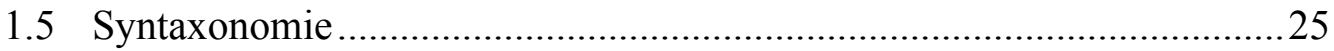

2 Die Erdflechtengesellschaften ......................................................................27

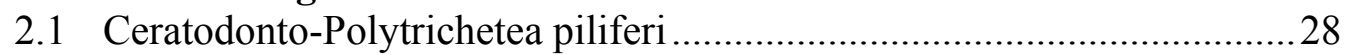

2.1.1 Baeomycion rosei....................................................................

2.1.1.1 Dibaeetum baeomycetis ...................................................33

2.1.1.2 Lecideetum uliginosae ....................................................... 35

2.1.1.3 Pycnothelio-Cladonietum cervicornis und Cladonia cervicornis-Baeomycion rosei-Fragmentgesellschaft....36

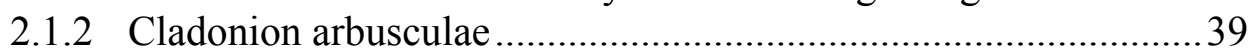

2.1.2.1 Cladonia bacillaris-Gesellschaft .....................................39

2.1.2.2 Cladonietum mitis ........................................................... 43

2.1.2.3 Cladonia furcata-Cladonion arbusculae- und Cladonia gracilis-Cladonion arbusculae-Fragmentgesellschaft ....46

2.1.2.4 Cladonietum foliaceae ...................................................4 48

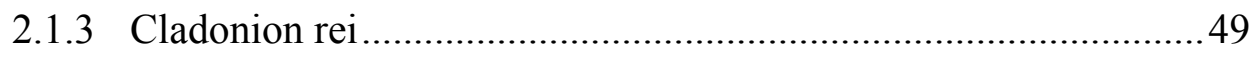

2.1.3.1 Cladonia floerkeana-Cladonion rei-

Fragmentgesellschaft ……………………………......52

2.1.3.2 Cladonietum rei............................................................53

2.1.3.3 Cladonia subulata-Cladonion rei-Fragmentgesellschaft 54

2.1.4 Standorte und Dynamik im Überblick ..............................................56 


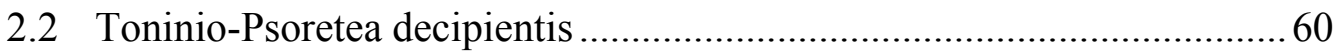

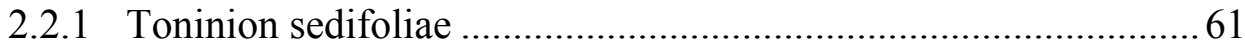

2.2.1.1 Toninia sedifolia-Toninion-Initialgesellschaft..............64 64

2.2.1.2 Toninio-Psoretum decipientis ....................................6 65

2.2.1.3 Cladonia symphycarpa-Toninion-

Fragmentgesellschaft ................................................ 70

2.2.1.4 Peltigera rufescens-Toninion-Fragmentgesellschaft...... 72

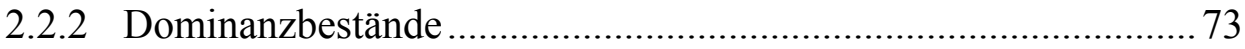

2.2.3 Standorte und Dynamik im Überblick ....................................... 74

3 Die Vergesellschaftung von Cladonia furcata ..........................................77

3.1 Cladonia furcata ssp. furcata .............................................................. 77

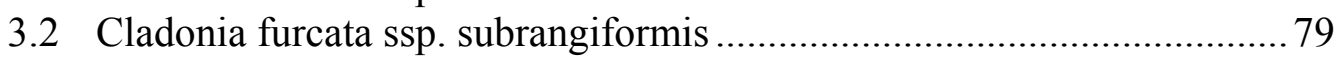

4 Einbindung der Flechtengesellschaften in die Phanerogamenvegetation . 81

5 Fruchtkörperbildung.....................................................................................82 82

6 Gefährdung der Arten und Gesellschaften sowie Schutzmaßnahmen ......89

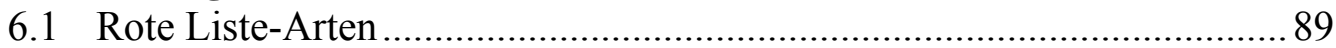

6.2 Bestandssituation der Flechtengesellschaften ..................................... 91

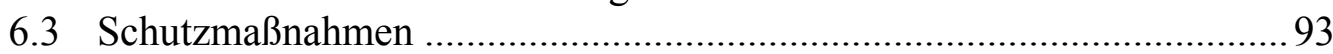

6.3.1 Erdflechten in Kalkmagerrasen und auf Felsen .......................... 94

6.3.2 Erdflechten in Zwergstrauchheiden ..........................................95

6.3.3 Erdflechten in Steinbrüchen........................................................99

6.3.4 Erdflechten in Wäldern ......................................................... 97

7 Zusammenfassung ............................................................................98

F Chemismus ausgewählter Cladonia-Arten ....................................99

1 Methodik zur Identifizierung der Flechtenstoffe..........................................99

2 Ergebnisse der Dünnschichtchromatographie .................................................100

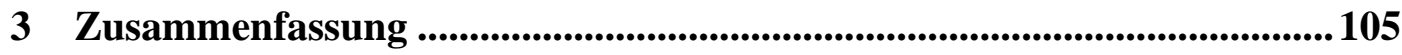

\section{G Morphologische und anatomische Variabilität von}

Cladonia furcata ..............................................................................106

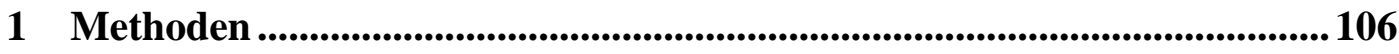

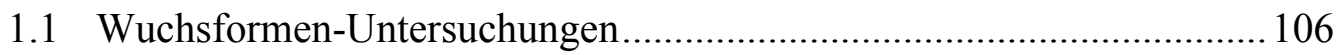

1.1.1 Probenahme und untersuchte Merkmale................................. 106

1.1.2 Auswertung und Darstellung der Ergebnisse............................ 109

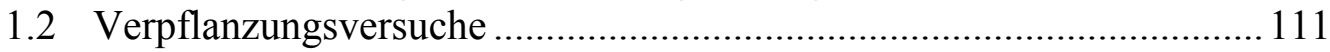

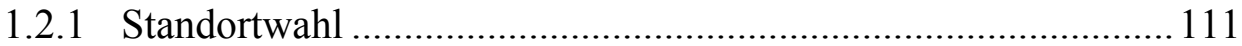

1.2.2 Auswahl der Flechten-Polster und Bearbeitung der Podetien .... 112

1.2.3 Mikroklima-Messungen .......................................................... 113

2 Einführung: Varietäten und Formen....................................................... 114

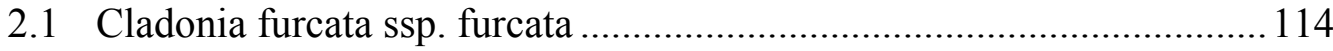

2.1.1 Die Varietät racemosa (= furcata) .......................................... 114

2.1.2 Die Varietät pinnata .............................................................. 115

2.1.3 Die Varietät palamaea............................................................. 115

2.1.4 Die Formen der Varietäten........................................................ 116

2.2 Cladonia furcata ssp. subrangiformis ................................................. 120

2.3 Ein kurzer Vergleich mit der Variabilität anderer Cladonia-Arten........ 121 
3 Morphologie und morphologische Variabilität in Nordhessen ................122

3.1 Allgemeine Beschreibung der Proben ................................................... 122

3.2 Abhängigkeiten der morphologischen Merkmale von den

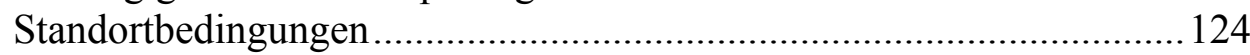

3.3 Abhängigkeiten und Korrelationen der morphologischen Merkmale

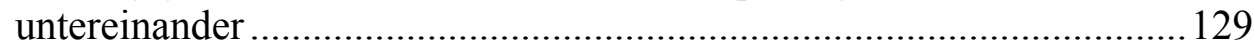

3.4 Rolle äußerer und innerer Faktoren bei der Merkmalsausbildung ......... 132

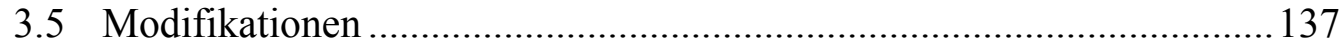

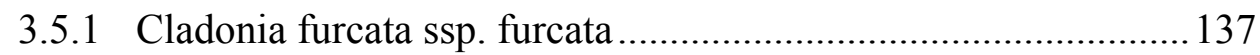

3.5.2 Cladonia furcata ssp. subrangiformis.................................... 139

4 Anatomische Merkmale..............................................................................140

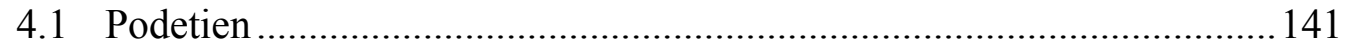

4.2 Beschreibung der einzelnen Podetienquerschnitte ................................ 143

4.3 Schuppen am Podetium (= Phylloide) und Grundschuppen .................. 145

5 Verpflanzungsversuche ........................................................................... 148

5.1 Das Makroklima im Versuchszeitraum ............................................. 148

5.2 Das Mikroklima an den Erdflechtenstandorten.................................... 150

5.3 Morphologische Veränderungen der Thalli und Zuwachsraten .............. 156

5.3.1 Die Morphologie der Polster am Versuchsende ....................... 156

5.3.2 Zuwachsraten und Neubildung von Verzweigungen ................... 157

5.3.3 Veränderungen der Podetienbündel .......................................... 160

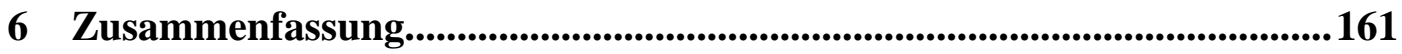

\section{H Genetische Variabilität der Bionten von Cladonia furcata} und Cladonia gracilis ............................................................................ 162

1 Molekularbiologische Methoden ..........................................................162

1.1 Auswahl und Herkunft der Proben, DNA-Extraktion ............................162

1.2 Polymerase-Chain-Reaktion (PCR), Gelelektrophorese und DNA-

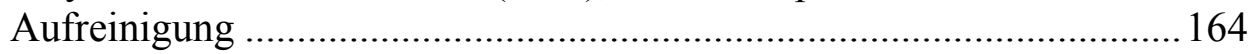

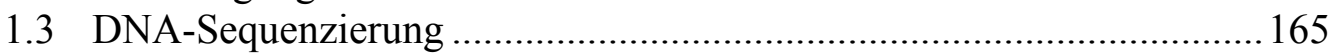

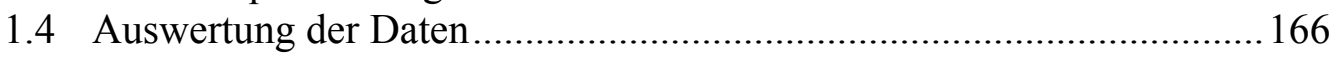

2 Ergebnisse der ITS rDNA-Sequenzierung...........................................167

2.1 ITS rDNA-Sequenzen der Mycobionten ................................................. 167

2.1.1 Polymorphismen der sequenzierten ITS-Bereiche..................... 167

2.1.2 Korrelation der ITS-Varianten mit systematischer Einstufung, Chemismus, Morphologie und Standortbedingungen................. 171

2.2 ITS rDNA-Sequenzen der Photobionten .............................................. 172

2.2.1 Polymorphismen der sequenzierten ITS-Bereiche.................... 172

2.2.2 Vergleich mit Photobionten-Sequenzen aus GenBank .............. 172

2.2.3 Korrelation der ITS-Varianten mit systematischer Einstufung, Chemismus, Morphologie und Standortbedingungen.................. 175

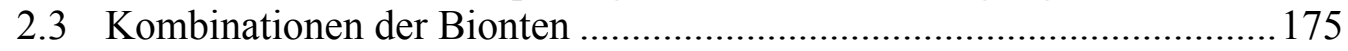

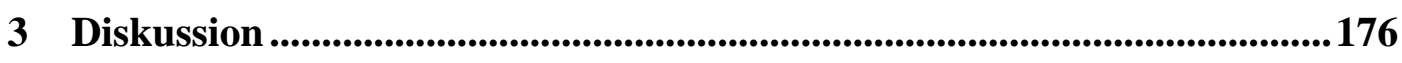

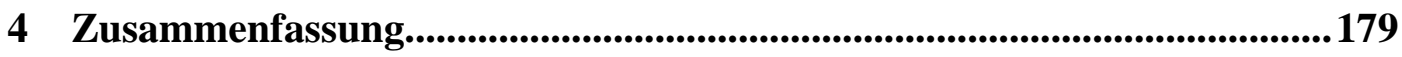


I Zusammenfassende Diskussion zu Cladonia furcata.......................181

1 Ursachen der morphologischen Erscheinungsformen der Subspezies

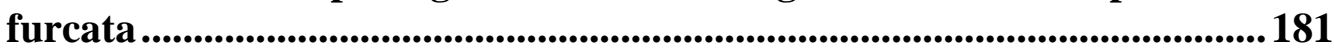

1.1 Auswirkungen des Mikroklimas ...................................................... 181

1.2 Gestaltveränderungen bei fortschreitender Podetienentwicklung .......... 186

1.3 Modifikatorische Varianten oder phänologische Stadien ..................... 187

2 Zur Artberechtigung von Cladonia subrangiformis ................................ 188

2.1 Morphologische und chemische Merkmale ....................................... 188

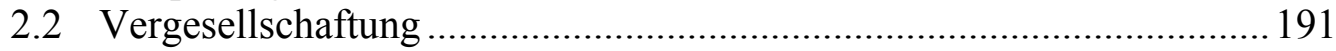

2.3 ITS rDNA-Sequenzen der Mycobionten ............................................... 191

2.4 ,subrangiformis“ - eine edaphisch bedingte Modifikation innerhalb der Cladonia furcata? ......................................................................... 192

J Gesamtzusammenfassung...................................................................193

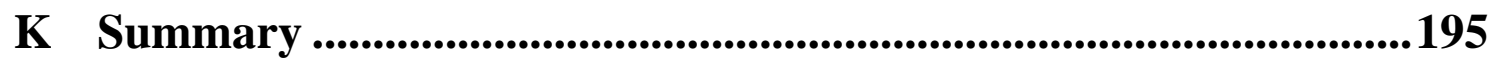

L Literaturverzeichnis ......................................................................197

M Tabellen- und Abbildungsverzeichnis .........................................213

N Anhang 1: Abkürzungen, Arten- und Belegeliste, Tabellen .........219

O Anhang 2: Bildtafeln ..................................................................233

P Anhang 3: Vegetationstabellen....................................................242 


\section{A Einleitung}

\section{Erdflechtenvegetation}

Nordhessen stellt ein Gebiet mit hoher standörtlicher Vielfalt und einem bemerkenswerten Reichtum an seltenen und schützenswerten Biotopen dar. Stellvertretend hierfür seien die gut erhaltenen Zwergstrauchheiden sowie die Magerrasen basenreicher Standorte genannt. Die Vielfalt unterschiedlichster Biotope bietet Lebensraum für zahlreiche Pflanzen und Tiere, unter anderem auch für viele auf Erde siedelnde Flechten, die als konkurrenzschwache Doppelorganismen ganz spezielle Ansprüche an ihre Habitate stellen. Trotz des Reichtums an Erdflechten ist bis in die heutige Zeit relativ wenig über epigäische Flechten und ihre Vergesellschaftung in Nordhessen publiziert worden. Neben einigen rein lichenologischen Veröffentlichungen wurden die Flechten gelegentlich bei der Bearbeitung der Phanerogamenvegetation mit erfaßt (z.B. Bultmann 1993, Schmidt 2000). Die Arbeit von KüMmERLing (1991) ist eine der wenigen ausführlichen flechtenkundlichen Publikationen aus dem Gebiet, beschränkt sich aber auf den Hohen Meißner und sein Vorland. Vegetationsaufnahmen der Flechtengesellschaften liegen aus dem gesamten nordhessischen Raum nur in geringer Zahl vor; so berücksichtigt beispielsweise die Arbeit von PAUS (1997) einige Erdflechtenvorkommen im Meißner-Gebiet und im Waldecker Upland. In den von FOLLMANN veröffentlichten Artikeln aus den 1970er und 1980er Jahren finden sich zwar Angaben zur Flechtenvegetation, diese Schriften behandeln jedoch nur wenige, ausgewählte Flechtenarten bzw. -gesellschaften (z.B. FOLLMANN 1974/1981/1983). Eine umfassende Bearbeitung der Erdflechtengesellschaften in Nordhessen stand also bisher aus. Es bedurfte zudem einer Überprüfung, ob die vor zwanzig bis dreißig Jahren aufgenommenen Gesellschaften heute noch im selben Maße vorhanden sind wie damals, nicht zuletzt auch deshalb, weil viele Flechtenarten und -gesellschaften aktuell in ihrem Bestand gefährdet sind (s. z.B. WIRTH et al. 1996). Umfassende Kenntnisse über die derzeitigen Vorkommen sind zu ihrem Schutz dringend notwendig. Ein Ziel der vorliegenden Arbeit ist es, hierfür notwendige Daten zu liefern und gleichzeitig einen Beitrag zur Kenntnis des Inventars an Flechtengesellschaften in Deutschland zu leisten. Die Erfassung und Dokumentation der Gesellschaften erfolgt anhand von Vegetationsaufnahmen. Auf Grundlage dieser Daten werden die aktuelle Gefährdungssituation der Erdflechten-Gesellschaften in Nordhessen beurteilt sowie Schutzmöglichkeiten aufgezeigt. Punktrasterkarten geben Auskunft über derzeitige Vorkommen der Bestände. Die Ergebnisse des ersten, vegetationskundlichen Teils stellen zudem die Grundlage für alle weiteren Untersuchungen der vorliegenden Arbeit dar. 


\section{Variabilität bei Cladonien}

Die Gattung Cladonia spielt innerhalb der Erdflechtenvegetation eine bedeutende Rolle. Ihre Arten sind maßgeblich am Aufbau zahlreicher Gesellschaften beteiligt. Bemerkenswert ist weiterhin, $d a ß$ in dieser Gattung etliche morphologisch ausgesprochen variable, epigäische Arten vorkommen. Cladonia furcata zählt hierzu und weist eine beeindruckende Formenvielfalt auf. Die ausgeprägte morphologische Variabilität von Cl.furcata offenbart sich beispielsweise bei Betrachtung der floristischen Arbeiten von ANDERS (1928), SANDSTEDE (1922/1931) oder ERICHSEN (1957). Ihre wechselnde Gestalt führte zur Beschreibung zahlreicher Varietäten und Formen, die besonders zahlreich in den Werken dieser drei Autoren zu finden sind. Viel zu wenig wurde dagegen, nicht nur bei Cl. furcata, der Frage nachgegangen, welches die Ursachen der Vielfalt an morphologischen Erscheinungsformen sind, wie schon Schade (1964) bemerkte. Als einer der wenigen hat sich SEMBDNER (1957/58) dieser Frage angenommen und die Standorteinflüsse auf die morphologische und anatomische Ausgestaltung bei Cl. chlorophaea (Syn. Cl. pyxidata ssp. chlorophaea) und Cl. coniocraea untersucht. Die vorliegende Arbeit befaßt sich mit den Ursachen der morphologischen Variabilität bei Cl. furcata und geht dabei über die bisherigen, eher beschreibenden Arbeiten (z.B. SCHADE 1964) hinaus. Anhand statistisch abgesicherter Daten werden Aussagen über die Abhängigkeit der verschiedenen morphologischen Merkmale von Standortfaktoren bzw. vom Entwicklungszustand der Cladonie gemacht. Ergänzend wird mit einem Verpflanzungsversuch die Anpassungsfähigkeit der Art an sich verändernde Umgebungsbedingungen sowie der Einfluß der Standortfaktoren auf den Podetienzuwachs bestimmt.

Variabilität kann nicht nur die äußerliche Gestalt einer Flechte, sondern auch ihre Inhaltsstoffe (Flechtensäuren) betreffen. Ebenfalls von großem Interesse ist daher die Frage, ob ein Zusammenhang zwischen der Morphologie und den Flechtensäuren, d.h. verschiedenen Chemotypen einer Art besteht. CHRISTENSEN (1987) untersuchte die morphologische und chemische Variabilität beim Cladonia macilenta/bacillarisAggregat und stellte fest, daß das Auftreten von Thamnolsäure nicht mit irgendwelchen morphologischen Mustern korreliert. Mit dem Zusammenhang zwischen morphologischen und chemischen Merkmalen bei Cl. [pyxidata ssp.] chlorophaea bzw. dem Cl. chlorophaea-Komplex befaßten sich AHTI (1966) und DePriest (1993). Während AHTI abschließend bemerkt, die „chemical species“ innerhalb des Cl. chlorophaea-Komplexes besäßen einige spezifische morphologische Merkmale, kommt DEPRIEST zu einem ähnlichen Ergebnis wie CHRISTENSEN: Es können zwar morphologische Tendenzen für die Chemotypen ausgemacht werden, die Morphologie kann aber nicht verwendet werden, um auf den Chemotyp zu schließen. Innerhalb von Cl. furcata sind ebenfalls verschiedene Chemotypen bekannt (s. z.B. BARENDREGT et al. 1982 und PAUS 1997). Die Unterteilung der Art in zwei Unterarten erfolgt u.a. aufgrund des Vorhandenseins oder Fehlens der Flechtensäure Atranorin (s. WIRTH 1995). Aus diesem Grunde wird in der vorliegenden Untersuchung auch der Chemismus von $\mathrm{Cl}$. furcata im Untersuchungsgebiet betrachtet und nach Chemotypen sowie Korrelationen 
derselben mit morphologischen und, wie nachfolgend aufgeführt, molekularen Merkmalen gesucht. Die dünnschichtchromatographischen Untersuchungen leisten darüber hinaus einen Beitrag zur Kenntnis und Verbreitung der Chemotypen von weiteren, ebenfalls bearbeiteten Arten der Gattung Cladonia.

Der letzte Abschnitt dieser Arbeit widmet sich der Variabilität von Cladonia furcata s.1 auf molekularer Ebene. Umfangreiche Untersuchung zur genetischen Variabilität einer Flechtenart auf der Basis von DNA-Sequenzen publizierten erstmals ZOLLER et al. (1999). Sie stellten bei der Blattflechte Lobaria pulmonaria eine Variabilität innerhalb des ITS 1 der ribosomalen DNA fest. Die ITS rDNA-Sequenzierung wird bei Flechten erst seit wenigen Jahren, vorwiegend für systematische Zwecke angewandt (z.B. STENROOS et al. 2002). In der vorliegenden Arbeit tauchen beide Zielsetzungen auf. Zum einen soll mit Hilfe dieser Methode nach intraspezifischer Variabilität bei Cl. furcata gesucht werden, von der, ebenso wie von anderen Cladonien, bislang nur wenige ITS-Sequenzen bekannt sind (PIERCEY-Normore \& DePriest 2001, Stenroos et al. 2002). Zum anderen tragen die molekularbiologischen Untersuchungen zur Klärung der Frage bei, ob es gerechtfertigt ist, Cl. [furcata ssp.] subrangiformis in den Rang einer eigenen Art zu heben. Dies ist in einigen floristischen Arbeiten der Fall (s. Kap. G2.2). Es werden hierbei sowohl die Mycobionten, als auch die Photobionten untersucht. Um mögliche Zusammenhänge zwischen molekularen und morphologischen Merkmalen aufzudecken, wurden Thalli verschiedener Gestalttypen ausgewählt. Zum Vergleich wurden solche Exemplare von Cl.gracilis ebenfalls bearbeitet, die morphologisch der Cl. furcata ähnlich sehen. Die Flechtenthalli entstammen dabei nicht weit voneinander entfernt liegenden Regionen (wie z.B. bei STENROOS et al. 2002) sondern einem einzigen, mitteleuropäischen Untersuchungsraum und belegen somit die Variabilität innerhalb einer geographisch wenig ausgedehnten Region.

\section{Zusammenfassung der Arbeitsziele}

Anhand der in Kap. A1 und A2 aufgeführten Themen lassen sich folgende Fragestellungen formulieren, die jeweils in getrennten Kapiteln behandelt werden (s. Abb. 1):

1. Welche Erdflechtengesellschaften sind aktuell im Untersuchungsgebiet vorhanden? Wie ist ihre Bestandssituation und was kann zu ihrem Schutz getan werden? (Kapitel $\mathrm{E}$ )

- Bestandsaufnahme und Dokumentation der Erdflechtenvegetation in Nordhessen

- Die Vergesellschaftung der Strauchflechte Cladonia furcata

- Darstellung der aktuellen Gefährdungssituation der Arten und Gesellschaften sowie Erarbeiten von Vorschlägen geeigneter Schutzmaßnahmen.

- Klärung der Frage, ob sich bei einigen ausgewählten Erdflechten Abhängigkeiten bei der Fruchtkörperbildung von der Gesellschaft bzw. vom Standort erkennen lassen.

2. Welche Chemotypen treten bei Cladonia furcata und weiteren Cladonien im Untersuchungsgebiet auf? (Kapitel F) 
3. Wie variabel sind verschiedene morphologische (und anatomische) Merkmale bei Cladonia furcata ssp. furcata und wodurch werden sie hauptsächlich beeinflußt? (Kapitel G)

4. Wie paßt sich Cladonia furcata ssp. furcata an sich verändernde Standortbedingungen an (Verpflanzungsversuch)? (Kapitel G)

5. Die genetische Variabilität der Bionten von Cladonia furcata und Cl. gracilis (Kapitel H):

- Vergleich der ITS rDNA-Sequenzen beider Unterarten von Cl. furcata: Zeigen die Sequenzen Unterschiede, die gegen eine Einstufung als Unterarten und für eine Abtrennung als eigene Arten sprechen?

- Korrelieren die morphologischen Erscheinungsformen bzw. eventuell auftretende Chemotypen mit molekularen Merkmalen?

- $\quad$ Sind bei Cl. furcata standortabhängige ITS-Varianten nachweisbar?

- Welche Photobionten treten als Symbionten in den beiden Cladonia-Arten auf?

- Zeigen sich bei der Photobionten-Wahl Abhängigkeiten von der Mycobionten-ITS-Variante, vom Standort oder von der Morphologie?

Die verschiedenen Teile der Arbeit bieten letztlich die Möglichkeit, in einer zusammenfassenden Diskussion (Kap. I) die Untergliederung von Cladonia furcata sowie die Artberechtigung von Cl. furcata ssp. subrangiformis von verschiedenen Seiten zu beleuchten. Diskussionen, die sich nur mit einem Themenbereich befassen, also etwa mit den Gesellschaften oder der genetischen Variabilität, werden in den jeweiligen Kapiteln aufgeführt.

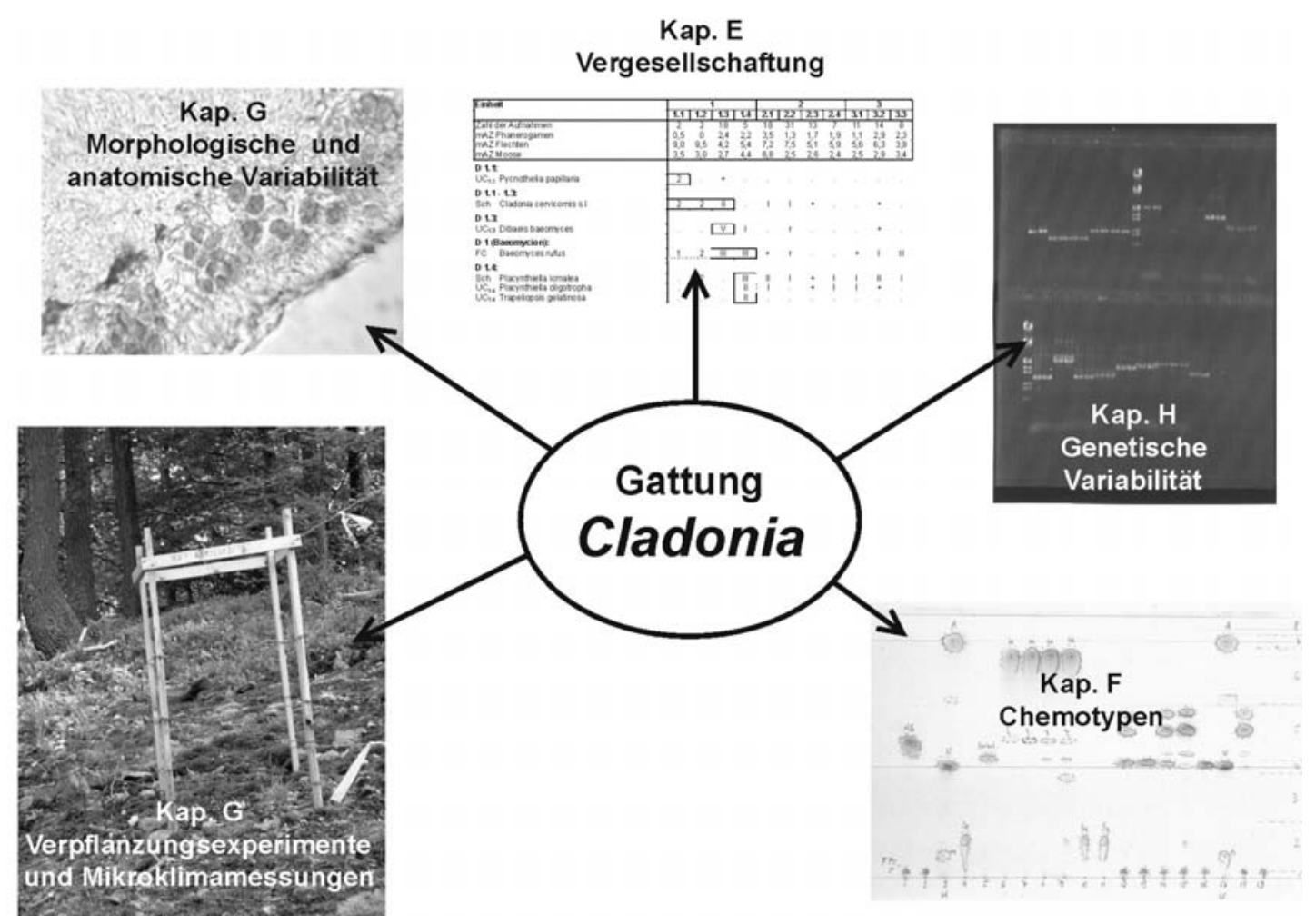

Abb. 1: Schematische Darstellung der Gliederung der Arbeit. 


\section{B Das Untersuchungsgebiet}

\section{Lage der Flächen und naturräumliche Zuordnung}

Die untersuchten Aufnahmegebiete liegen im nordhessischen Regierungsbezirk Kassel. Das Untersuchungsgebiet (UG) erstreckt sich über dessen 5 nördliche Landkreise. Dies sind die Landkreise Waldeck-Frankenberg und Kassel, der Schwalm-Eder-Kreis, der Werra-Meißner-Kreis sowie der Landkreis Hersfeld-Rotenburg (Abb. 2 und

Abb. 3). Der Regierungsbezirk Kassel umfaßt zahlreiche Naturräumliche Haupteinheiten (s. KLAUSING 1988), von denen im Folgenden nur die für die vorliegenden Untersuchungen interessanten, da an Erdflechtenvorkommen besonders reichen, genannt werden sollen:

332 Ostsauerländer Gebirgsrand

333 Hochsauerland

342 Habichtswälder Bergland

343 Westhessische Senke

344 Kellerwald
357 Fulda-Werra-Bergland

358 Unteres Werraland

361 Oberwälder Land

370 Solling, Bramwald und Reinhardswald

Bearbeitungsschwerpunkte liegen im Fulda-Werra-Bergland, im Unteren Werraland, im Kellerwald (Edersee-Gebiet) sowie im Hochsauerland (Waldecker Upland). Im Rahmen einer lichenologischen Exkursion der Bryologisch-Lichenologischen AG Mitteleuropa (BLAM) im Frühjahr 2001 konnte zudem das Gladenbacher Bergland (naturräumliche Einheit 320) besucht und in die Untersuchungen mit aufgenommen werden.

Innerhalb dieser Naturräume wurden zahlreiche Orte besichtigt; an insgesamt 70 fanden sich für die Untersuchungen geeignete Erdflechtenbestände (s. Tab. 57 im Anhang 1). Die häufigsten Biotoptypen, in denen dort Erdflechten auftreten, sind Kalkmagerrasen, Zwergstrauchheiden, Wälder trockenwarmer, kalkarmer Standorte sowie Ruderalfluren, deren aktuelle und historische Nutzung in Kap. B4 beschrieben wird. 


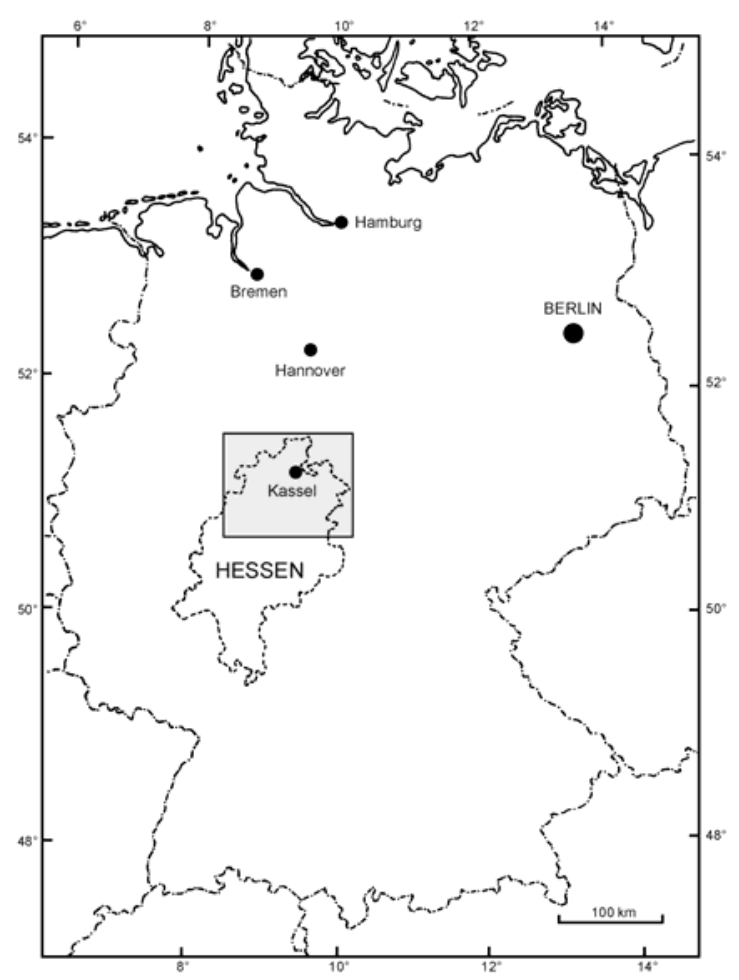

Abb. 2: Lage des Untersuchungsgebietes.

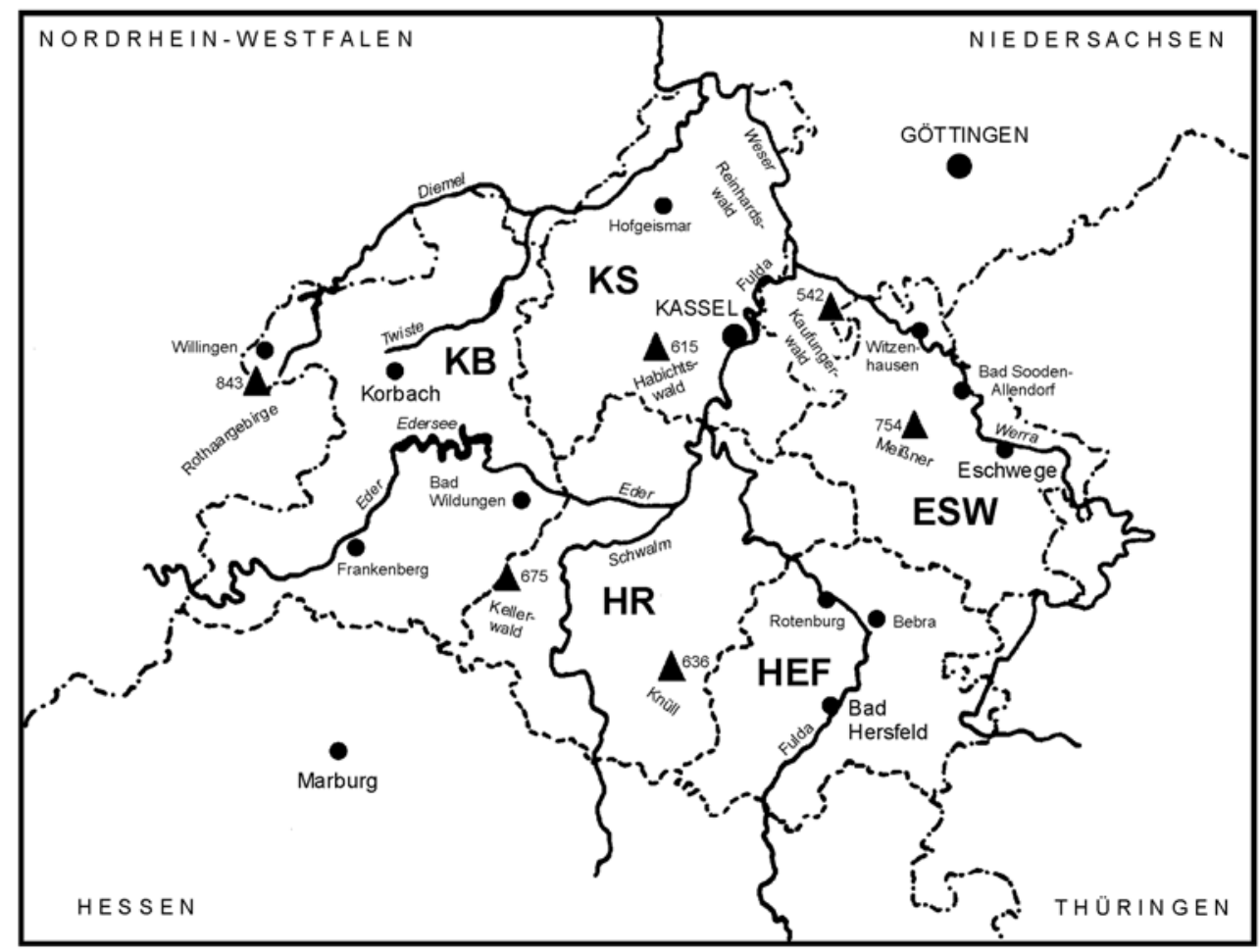

Abb. 3: Übersichtskarte des Untersuchungsgebietes mit den bearbeiteten Landkreisen: KS = Landkreis Kassel, ESW = Werra-Meißner-Kreis, HEF = Landkreis Hersfeld-Rotenburg, HR = SchwalmEder-Kreis, $K B=$ Landkreis Waldeck-Frankenberg. 


\section{Geologie und Böden}

Die Angaben zur Geologie des Untersuchungsgebietes sind, soweit nicht anders vermerkt, aus den Geologischen Übersichtskarten 1:200000 Bundesrepublik Deutschland, CC 5518 (Blatt Fulda) und CC 4718 (Blatt Kassel) entnommen.

Die Geologie des Untersuchungsgebietes ist ausgesprochen vielfältig (s. Abb. 4). Die erdgeschichtlich ältesten Gesteine an den bearbeiteten Erdflechten-Standorten stammen aus dem Erdaltertum (Paläozoikum). Sie treten vor allem im südwestlichen Teil Nordhessens an der Oberfläche zutage, und zwar in den nordöstlichen Ausläufern des Rheinischen Schiefergebirges, welches den Kellerwald und das Waldecker Upland mit einschließt (DIEDERICH 1991). Während das Rheinische Schiefergebirge insgesamt hauptsächlich von devonischen Gesteinen aufgebaut wird, dominieren in dem zum Untersuchungsgebiet gehörenden Teil Tonschiefer und Grauwacken des Unterkarbon, auch bezeichnet als Kulmtonschiefer und Kulmgrauwacke (LOTZ 1995). Diese sind beispielsweise an den Steilhängen des Edersees, im Battenfelder Driescher und in Teilen des Waldecker Uplands anzutreffen. Westlich von Bad Wildungen sowie in anderen Bereichen des Waldecker Uplands (z.B. bei Usseln) kommen dagegen devonische Gesteine (Tonschiefer etc.) vor (s. auch KRAUSSE 1971).

Im Nordosten des Untersuchungsgebietes treten im Unterwerra-Sattel (s.u.) ebenfalls paläozoische Gesteine auf. Es handelt sich dabei vor allem um devonische Grauwacken (Werra-Grauwacke, s. RITZKOWSKI 1978). Darüber hinaus ist auch das DiabasVorkommen am Bilstein im Höllental bei Albungen im Devon entstanden.

Der Unterwerra-Sattel zwischen Witzenhausen und Eschwege stellt eine ZechsteinAufwölbung dar. Der Zechstein liegt dort direkt auf devonischen Gesteinen des Grundgebirges, die stellenweise an die Oberfläche treten (HENNINGSEN \& KATZUNG 1998). Beispiele für dortige Lokalitäten auf Grauwacke sind die Traubeneichenwälder am Roßkopf und am Klepperberg sowie im Dohlsbachtal. Etwas südlich von Witzenhausen befindet sich im Zechstein-Gebiet das mächtigste Vorkommen an Alabaster in Deutschland. Er wird im Steinbruch bei Hundelshausen abgebaut (LOTZ 1995). Dieses Gebiet am Gottesberg wurde im Rahmen der vorliegenden Untersuchungen ebenso bearbeitet wie weitere Lokalitäten auf Zechstein im Unterwerra-Sattel, da sie reich an Erdflechten sind. Stratigraphisch läßt sich der Zechstein nach RichTER-BERNBURG (1955) in 4 salinare Serien (Z 1 - Z 4) unterteilen. Auf Werra-Anhydrit (Werra-Serie Z 1), der oberflächlich als Gips vorliegt, finden sich Erdflechten z.B. im NSG ,Jestädter Weinberg“ oder im NSG „Kripplöcher und Hielöcher" bei Frankershausen; in beiden Gebieten siedeln Flechten auch über Dolomit. Die Erdfälle der Kripp- und Hielöcher sind auf die Lösung des Gipses zurückzuführen (s. RITZKOWSKI 1978). Felsen aus dem verwitterungsresistenteren Dolomit ragen an vielen Stellen aus den Magerrasen auf. Die größte Verbreitung im Zechstein-Ausstrich des Unterwerra-Sattels besitzt der Hauptdolomit (Stassfurt-Serie Z 2). Er ist dort zugleich der wichtigste Klippenbildner. Die Flechtenvorkommen in den Steinbrüchen 
„Steinröllchen“ bei Oberhone siedeln auf Standorten mit Hauptanhydrit (Leine-Serie Z 3, s. RITZKOWSKI 1978).

Weiterhin finden sich Gesteine des Zechstein in dem südlich des Unterwerra-Sattels gelegenen Richelsdorfer Gebirge, das unter den Zechsteinschichten auch Rotliegendes aufweist (HENNINGSEN \& KATZung 1998). Die Zechstein-Aufwölbung des Richelsdorfer Gebirges quert zwischen Heinebach und Rotenburg an der Fulda deren Tal (LOTZ 1995). In zahlreichen Steinbrüchen wurden und werden dort die Gipsvorkommen abgebaut. Im ehemaligen Gips-Steinbruch bei Heinebach sind einige Vegetationsaufnahmen dieser Arbeit entstanden. Nicht zuletzt kommen auch am Ostrand des Rheinischen Schiefergebirges Gesteine des Zechstein vor, wo das Devon und Unterkarbon von einem schmalen Saum Zechstein-zeitlicher Schichten bedeckt wird (HENNINGSEN \& KATZUNG 1998).

Größtenteils wird der geologische Untergrund des Untersuchungsgebietes jedoch nicht von paläozoischen, sondern von Trias-Gesteinen gebildet. Vor allem der Buntsandstein nimmt große Flächen ein und formt Höhenzüge wie den Reinhardswald und den südlich davon gelegenen Kaufunger Wald. Das nordhessische Buntsandsteingebiet grenzt im Westen an das Rheinische Schiefergebirge und erstreckt sich bis an die östliche Landesgrenze. Es wird nur durch die Niederhessische Senke unterbrochen. Das osthessische Buntsandsteingebiet reicht im Süden von Vorderrhön, Hoher Rhön, FuldaBergland einschließlich Knüll-Hochland, Richelsdorfer Gebirge, Ringgau und Sontraer Hügelland bis zum Meißner und dem unteren Werratal (DIEDERICH 1991). Größtenteils ist es der Mittlere Buntsandstein, der an der Oberfläche ansteht und sowohl den Reinhardswald als auch den Kaufunger Wald aufbaut. Als Beispiele für ErdflechtenStandorte auf Mittlerem Buntsandstein seien der Grenzstreifen bei der Burg Hanstein, die Heide „Steinbülz“ bei Hombressen (am Westrand des Reinhardswaldes), die Termenei bei Wilhelmshausen (südlicher Reinhardswald) und die Flächen im Niestetal (Kaufunger Wald) genannt. Auch am Osthang des Hohen Meißners steht der Mittlere Buntsandstein an.

Gesteine der Muschelkalkzeit treten im Untersuchungsgebiet flächenhaft, z.B. im Ringgau und an der Diemel auf (s. RITZKOWsKI 1978 u. Geologische Übersichtskarten 1:200 000 CC 5518/CC 4718). Außerdem durchziehen drei tektonische Gräben mit Muschelkalkgesteinen das Untersuchungsgebiet. Der Altmorschen-Lichtenauer Graben zieht von Witzenhausen nach Süd-Südwest. Weitere Gräben mit Muschelkalkresten verlaufen zum einen in west-nordwestlicher Richtung über Kassel und zum anderen von Warburg an der Diemel in südlicher Richtung (LOTZ 1995). In der Regel steht als Ausgangsgestein auf den untersuchten Muschelkalk-Flächen der Untere Muschelkalk an (Bsp. Schäferburg (Graburg), NSG „Bühlchen bei Weißenbach“, Sommerberg und Papenbreite bei Sielen, Dörnberg, NSG „Hohekopf bei Epterode“, NSG „Festberg bei Philippinenthal“"). Ausnahmen mit Oberem Muschelkalk stellen der Heiligenberg (NSG „Meißner“) und der Eisenberg bei Walburg dar.

Zwischen Alsfeld im Süden und Kassel im Norden erstreckt sich die bis zu 18 km breite Niederhessische Senke. Geologisch handelt es sich um einen Senkungsraum, in dem oberflächennah meist tertiäre Sedimente oder tertiäre Basalte anstehen (DIEDERICH 
1991). Sie zählt zu einer Kette von einzelnen Senken, die mit Tertiären Lockersedimenten gefüllt sind und sich vom nordöstlichen Ende des Oberrhein-Grabens durch Hessen und Südniedersachsen bis etwa an den Westrand des Harzes erstrecken. Die sandig-tonigen Schichten in vielen dieser Tertiärbecken enthalten Braunkohle-Flöze (HENNINGSEN \& KATZUNG 1998). So auch in der ehemaligen Zeche Glimmerode, in der bis etwa Ende der 60er Jahre des letzten Jahrhunderts untertage Bergbau betrieben wurde (s. BROSIUS 1959, BAIER mündl.). Die obereozänen bis mitteloligozänen Sedimentfolgen der Glimmeröder Tertiärmulde schlossen nach BROSIUS (1959) 5 Braunkohleflöze ein, die z.T. in Abbau standen. Heute bestimmt ein See das Bild der Mulde. Die Erdflechten siedeln an den Seeufern und an abgerutschten Hängen sowohl über sandigen als auch über tonigen Sedimenten, stellenweise treten auch Braunkohlehaltige Substrate an der Oberfläche auf. Das stärkste Flöz in Hessen hatte jedoch der Hohe Meißner. Es war $50 \mathrm{~m}$ mächtig und ist rund 400 Jahre lang abgebaut worden. Die Vorräte sind nun fast erschöpft (LOTZ 1995). Zurück bleiben großflächige Abraumhalden, beispielsweise an der Kalbe, die ihrerseits wiederum Flechtenstandorte darstellen können.

Während der Tertiärzeit waren in der Niederhessischen Senke, wie auch im Vogelsberg und in der Rhön, Vulkane aktiv. Die vulkanischen Basalte sind widerstandsfähiger gegen Erosion und Abtragung als die sie umgebenden Sande und Tone. Die Reste der Vulkane blieben daher bis heute erhalten und prägen die Landschaft der Niederhessischen Senke (s. auch HLUG 2001). Beispiele solcher Basaltvorkommen im Untersuchungsgebiet sind der Habichtswald mit dem angrenzenden Dörnberg und den Helfensteinen sowie die Gudensberger Basaltkuppen.

An der Kreuzung zweier Gräben drangen im Tertiär auch die Basaltmassen des Hohen Meißners empor. Sie waren ursprünglich in eine tertiäre Senke geflossen. Durch die Verwitterung der tertiären Tone und Sande wurde später der Basalt herauspräpariert, so daß hier eine Reliefumkehr stattgefunden hat. Die Gesteine am Westrand des Meißners sanken an einer Verwerfung um etwa $160 \mathrm{~m}$ gegenüber dem Meißner-Plateau ab. Die oben erwähnten beachtlichen Braunkohle-Vorkommen lagerten unter dem bis zu $90 \mathrm{~m}$ mächtigen Basalt (LoTz 1995). Die Klimabedingungen des Pleistozäns führten zu einer intensiven Hangschuttbildung, es entstanden Blockmeere, Blockwülste und Schuttdecken mit wechselndem Basaltanteil (MöLlER 1989). Diese Blockhalden, z.B. an der $\underline{\text { Petersruh }}$ oder Kalbe, stellen heute Lebensraum für viele seltene Flechtenarten dar.

Die Blaue Kuppe bei Eschwege ist im Tertiär im Zusammenhang mit der in NW-SORichtung durch den Ringau verlaufenden Fortsetzung des Kasseler Grabens entstanden. Sie ist ein im Buntsandstein stecken gebliebener Basaltpfropf aus alkalibasaltischem Gestein (LOTZ 1995 und Geolog. Übersichtskarte). 


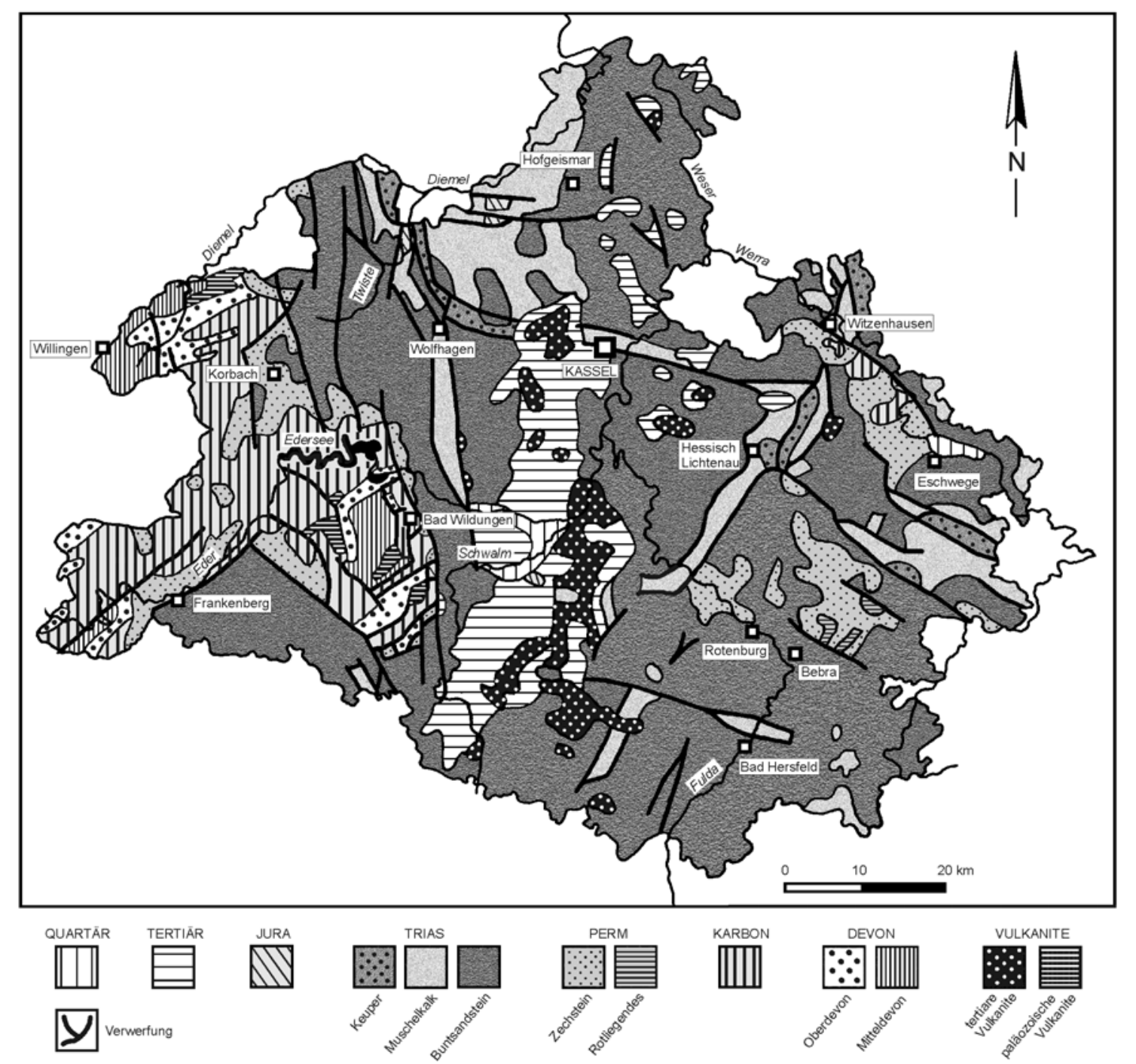

Abb. 4: Geologische Übersichtskarte von Nordhessen (Quelle: Landschaftsrahmenplan Nordhessen 2000 unter: http://www.rp-kassel.de/themen/naturschutz/lrp2000/index.htm. Ausschnittsbearbeitung durch RP Kassel auf Grundlage von: HESS. LANDESAMT FÜR BODENFORSCHUNG (1989) GÜK 1:300 000 vereinf. in 1:2 000 000).

Die kleinräumige Vielfalt der Böden an Erdflechtenstandorten ist vor allem durch Gesteins- und Reliefunterschiede bedingt. So treten in steileren Kuppen- und Hanglagen neben Fels-Rohböden, über silikatischem Festgestein auch Bodengesellschaften aus Rankern und sauren Braunerden auf, während auf carbonat- und gipshaltigen Gesteinen Rendzinen mit wenig oder nicht versauerten Braunerden vergesellschaftet sind (KUNTZE et al. 1994). Bei den Böden an den Erdflechtenstandorten handelt es sich im Untersuchungsgebiet vielerorts um Rohböden (Syroseme), vor allem über Gips, Dolomit und Muschelkalk sowie um Rendzinen bzw. Ranker. Sofern andere Bodentypen auftreten, z.B. Braunerden bzw. podsolige Braunerden, ist nicht selten aus unterschiedlichen Gründen der Ah-Horizont teilweise oder überwiegend abgetragen. In einigen wenigen Heidegebieten ist dies stellenweise eine Folge des Abplaggens. Weiterhin sind in diesem Zusammenhang Humusansammlungen in den Gesteinsspalten der Blockhalden zu nennen, die ebenfalls von Erdflechten besiedelt werden. 


\section{$3 \quad$ Klima}

Im Untersuchungsgebiet zeigen sich starke Unterschiede bezüglich des mittleren Jahresniederschlages (s. Abb. 5 und Abb. 6). Die höchsten Niederschlagsmengen mit bis über $1200 \mathrm{~mm}$ weist das Waldecker Upland bei Willingen und Usseln auf. Ebenfalls regenreich ist der Hohe Meißner mit über $950 \mathrm{~mm}$, der Kaufunger Wald mit bis über $900 \mathrm{~mm}$ sowie Reinhardswald, Knüllgebirge, Wildunger Bergland und Kellerwald mit bis über $850 \mathrm{~mm}$. Die an der Westgrenze des Untersuchungsgebietes hohen Niederschläge sinken Richtung Korbach bereits auf unter $700 \mathrm{~mm}$ ab. Den geringsten mittleren Jahresniederschlag von unter $600 \mathrm{~mm}$ weisen die Niederhessische Senke, die Region um Eschwege sowie das Gebiet um Volkmarsen an der Diemel auf. Auch im Edersee-Gebiet und im Fuldatal oberhalb Rotenburg werden durchschnittlich nicht mehr als $650 \mathrm{~mm}$ Jahresniederschlag gemessen. Das Niederschlagsmaximum liegt im Juni/Juli, das Niederschlagsminimum im Februar. Eine Ausnahme liefert die Station Kahler Asten im Rothaargebirge (s. Abb. 5). Dort wird das Niederschlagsmaximum im Dezember und Januar, das Minimum im Mai erreicht (DT. WeTTERDIENST IN DER USZONE 1950, MÜHR 2002/2003).
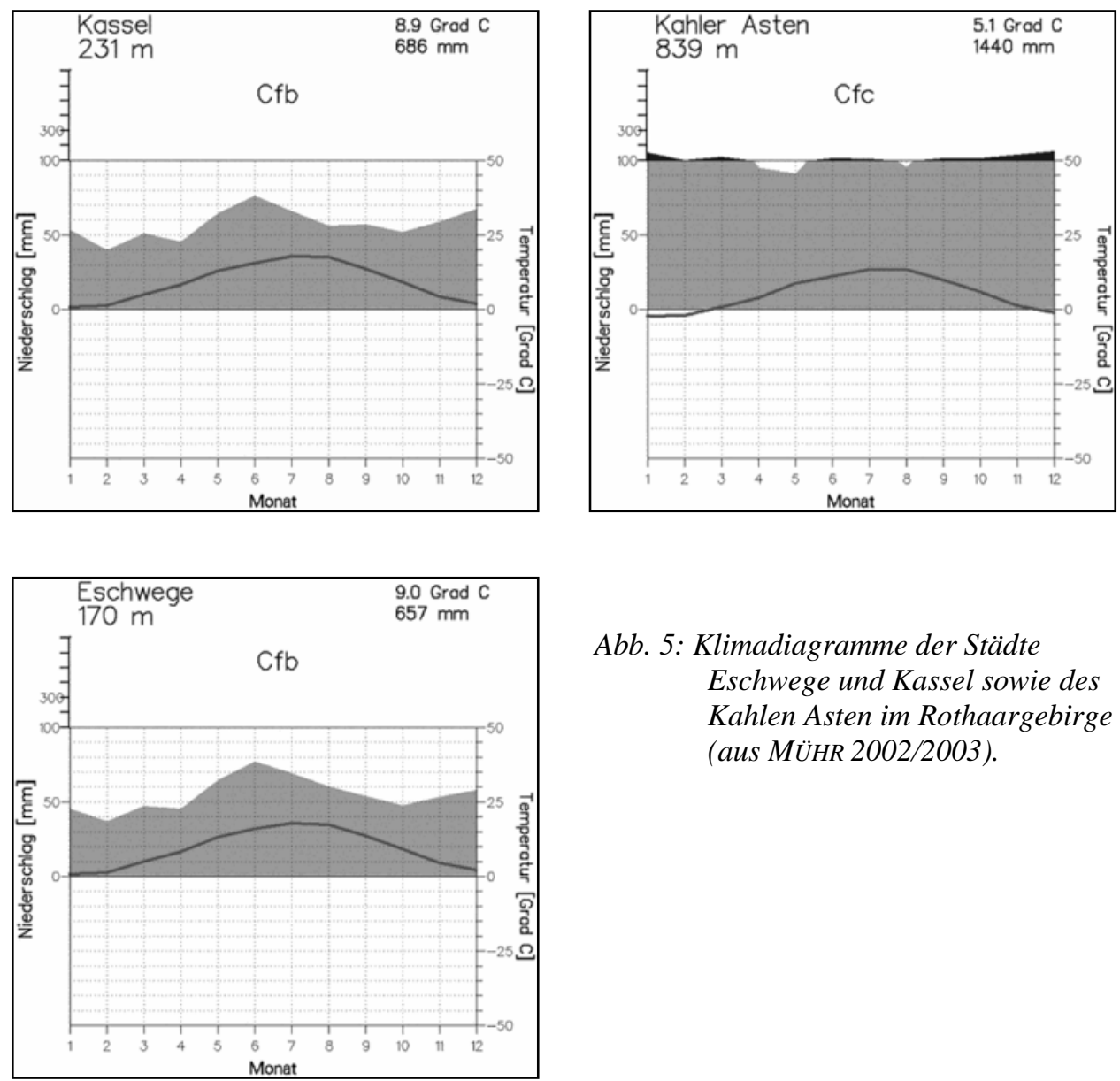

Abb. 5: Klimadiagramme der Städte Eschwege und Kassel sowie des Kahlen Asten im Rothaargebirge (aus MÜHR 2002/2003). 
Der Einfluß der Berglagen auf das Umland wird besonders im Vorland des Hohen Meißners deutlich. Dort zeigen sich je nach Lage der Flächen auffallende klimatische Unterschiede. Im westlichen Meißner-Vorland treten aufgrund der vorherrschenden Westwetterlagen und der größeren Meereshöhe 800 bis $900 \mathrm{~mm}$ Jahresniederschlag und eine Jahresmittel-Temperatur von $6-8^{\circ} \mathrm{C}$ auf (kühleres, niederschlagsreicheres Berglandklima). Im östlichen Vorland, im Regenschatten des Hohen Meißners, fallen dagegen nur 550 bis $750 \mathrm{~mm}$ Jahresniederschlag; die dortige Jahresmittel-Temperatur beträgt $7-9{ }^{\circ} \mathrm{C}$, es herrscht ein trocken-warmes Hügelklima (BRÜHL \& HEGER 1989, DT. WetTERDIENST IN DER US-ZONE 1950, HeINTZE 1966). Die mittlere Jahresschwankung der Lufttemperatur liegt im Untersuchungsgebiet bei 16,0 bis 17,5 ${ }^{\circ} \mathrm{C}$. Den niedrigsten Wert weisen die kühlen Berglagen des Waldecker Uplandes mit lediglich $15,5^{\circ} \mathrm{C}$ Jahresschwankung auf (DT. WeTtERDIENST IN DER US-ZONE 1950).

Die Hauptwindrichtungen in Nordhessen sind über das Jahr betrachtet Südwest und Nordwest. Im Juni herrschen an den Stationen Mengeringhausen und Kassel NordwestWinde vor, im Dezember überwiegen Winde aus Südwesten bzw. Süden (DT. WETTERDIENST IN DER US-ZONE 1950).

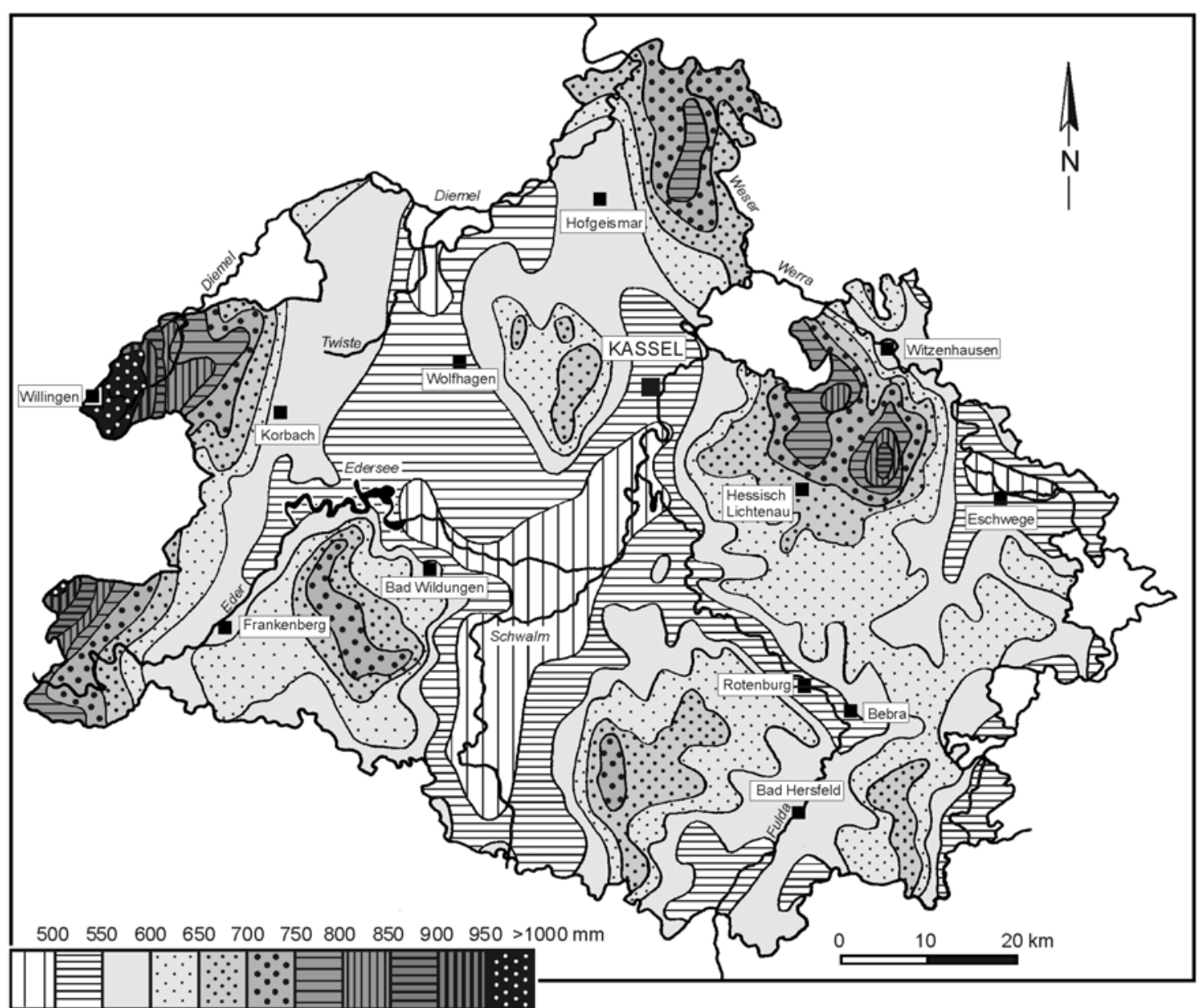

Abb. 6: Mittlere Niederschlagshöhen im Regierungsbezirk Kassel (1931-1960). (Quelle: Landschaftsrahmenplan Nordhessen 2000 unter: http://www.rp-kassel.de/ themen/naturschutz/lrp2000/index.htm. Ausschnittsbearbeitung durch RP Kassel auf Grundlage von: KALB \& VENT-SCHMIDT (1981)). 
Von großer Bedeutung für die Kryptogamen sind, neben dem Makroklima, auch die jeweiligen mikroklimatischen Verhältnisse. Besonders die Grenz- und Extremwerte sowie der zeitliche Verlauf des Mikroklimas während des Tages spielen eine große Rolle, z.B. wann der Thallus austrocknet oder welche Thallustemperaturen bei einem noch feuchten Thallus auftreten (ScHÖLLER 1991). Bei der Beschreibung der Vegetationstypen (Kap. E2) werden daher mikroklimatische Faktoren wie Feuchtigkeits- und Lichtverhältnisse jeweils kurz erwähnt. Darüber hinaus wurden an exemplarisch ausgewählten Standorten Mikroklima-Messungen durchgeführt (s. Kap. G5.2).

\section{Historische und aktuelle Nutzung der Flächen}

\subsection{Kalkmagerrasen}

Die Kalkmagerrasen des Untersuchungsgebietes sind hauptsächlich seit dem Mittelalter als Schafweiden entstanden. Die Schafzucht in Hessen erreichte ihren Höchststand in der zweiten Hälfte des 18ten Jahrhunderts, die beweideten Flächen hatten in diesem Zeitraum daher ihre größte Ausdehnung. Mit dem Verfall des Wollpreises ging die Schäferei danach immer weiter zurück (WILKE 1996). Den Schilderungen von PFUHL (1935) zufolge fand jedoch beispielsweise im Gebiet Östliches Westfalen und Lippe, nahe der Trendelburger Kalkmagerrasen, noch in den 1930er Jahren Schafhaltung in größeren Betrieben in bedeutendem Umfang statt. Im Gebiet um Frankershausen blieb diese Bewirtschaftungsweise ebenso wie am Dörnberg, am Festberg bei Philippinenthal und in der Region Ellingerode-Rückerode bis nach dem Zweiten Weltkrieg erhalten, wurde dann aber in den 1950er Jahren mehr und mehr aufgegeben (GLAVAC et al. 1979, Von DER Heide 1984, LeNARduZZi 1999a/b, Auge 2000, Nitsche \& NitsChe 2003). HAKES (1987) gibt für die Halbtrockenrasen in der Umgebung von Kassel als Periode zunehmender Extensivierung den Zeitraum zwischen 1950 und 1960 an. Der Schafbestand erreichte in Hessen 1965 seinen Tiefststand (WILKE 1996). Als Folge der Nutzungsaufgabe bzw. der nur noch sporadischen Beweidung fielen zahlreiche Magerrasen brach. Gehölze konnten in die Flächen einwandern, die ursprüngliche Magerrasenvegetation wurde zunehmend verdrängt (HAKES 1987). Als weitere Nutzungsform der Kalkmagerrasen nennt BRUELHEIDE (1989/1991) für einige Flächen im westlichen Meißner-Vorland die Mahd mit der Sense (bis etwa 1940). Am Jestädter Weinberg wurde bis 1840 Weinbau betrieben (HochHUT 1952 zitiert in BRUELHEIDE 1989).

Seit den 1990er Jahren werden einige Flächen aus Gründen des Naturschutzes wieder regelmäßig mit Schafen beweidet, z.B. der Dörnberg bei Zierenberg (NITSCHE \& BULTMANN 1995), der Festberg bei Philippinenthal, der Warmberg bei Zwergen (NiTSCHE \& NiTSCHE 2003), die Magerrasen im Meißner-Vorland mit den beiden NSG „Bühlchen bei Weißenbach“ bzw. „Kripplöcher und Hielöcher“ (LENARDUZzI 1999a/b) sowie das NSG „Kalkmagerrasen bei Roßbach” (AUGE 2000). Auch der Sommerberg und die Papenbreite an der Diemel bei Trendelburg werden mit Schafen beweidet (BultManN 1993). Darüber hinaus finden stellenweise weitere Pflegemaßnahmen statt. 
So wird beispielsweise vor bzw. parallel zur Wiedereinführung der Hütehaltung im Meißner-Vorland eine Entbuschung durchgeführt (LENARDUZZI 1999a/b), ebenso am Dörnberg bei Zierenberg (NiTsChe \& BULTMANN 1995), am Warmberg bei Zwergen und am Festberg bei Philippinenthal, wo stellenweise auch gemulcht wurde (NITSCHE \& NiTSCHE 2003). Im NSG „Kalkmagerrasen bei Roßbach” dienen seit 1989 neben Beweidung und Entbuschung auch Mahd, Abbrennen und das Entfernen von Kiefern dem Erhalt der Flächen (AugE 2000). Über die Nutzung der Kalkmagerrasen im Untersuchungsgebiet berichtet auch GÜNZL (2001b).

\subsection{Zwergstrauchheiden}

Zwergstrauchheiden sind vorwiegend anthropogenen Ursprungs und haben früher größere Flächen eingenommen. Auf den Heideflächen Nordhessens wurde durch Rechen oder Mähen, seltener auch durch Abplaggen Stallstreu gewonnen. Heideflächen dienten zudem als Schafweiden (BonNEMANN 1984, Nitsche 1995). Die Hochheiden der Kahlen Pön bzw. des Ettelsberges im Waldecker Upland verdanken ihre Erhaltung beispielsweise der Plaggenwirtschaft (ENGELHARD 1971). Die Nutzung der Besenheide durch Abplaggen wurde jedoch bereits in den 1950er Jahren aufgegeben. Durch die Nutzungsaufgabe bzw. -änderung sind die Zwergstrauchheiden allgemein stark zurückgegangen und heute in Nordhessen nur noch auf Restflächen, meist kleinflächig, vorhanden. Viele dieser Flächen sind verbuscht oder haben sich auf Teilflächen zum Vorwald entwickelt (NITSCHE 1995). In einigen nordhessischen Heidegebieten wurden während der letzten Jahre zumindest kleine Flächen maschinell oder in traditioneller Weise von Hand abgeplaggt (NITSCHE 1995, WELTICKE mündl.), beispielsweise am Osterkopf bei Usseln und auf der Kahlen Pön.

Einige Heideflächen werden mit Schafen beweidet. In der Termenei bei Wilhelmshausen, einem ehemaligen Truppenübungsplatz bzw. ehemaligen Brandflächen auf Mittlerem Buntsandstein, werden hierfür Moorschnucken eingesetzt. Ebenso in der kleinen Heide „Steinbülz“ bei Hombressen. Auf der Kahlen Pön übernehmen diese Aufgabe Heidschnucken. Am Osterkopf bei Usseln werden seit 1994 nur die stärker vergrasten Bereiche mit Heidschnucken beweidet, während sich die eigentliche Hochheide weitgehend auf natürliche Weise (durch Windaushagerung) erhält. Als einzige Pflegemaßnahme wird lediglich von Zeit zu Zeit eine Gehölzentnahme durchgeführt. Gehölzrücknahme findet auch in anderen Heiden, z.B. der Termenei, dem „Steinbülz“ bei Hombressen oder dem Battenfelder Driescher statt (Nitsche 1995, Nitsche \& Nitsche 2003). Der Battenfelder Driescher, ein Biotopkomplex u.a. aus Calluna-Heide und Borstgrasrasen, wird ebenfalls mit Schafen beweidet, allerdings nicht wie in einigen anderen Gebieten (Osterkopf etc.) in Hute-, sondern in Koppelhaltung. Mit Schafen beweidet wird auch das NSG „Wacholderheiden bei Niederlemp“ (BERGMEIER 1987). Als weitere, bisher noch nicht genannte Pflegemaßnahme wird nach NiTSCHE (1995) im Battenfelder Driescher ebenso wie in der Termenei die Heide zurückgeschnitten.

Am Ettelsberg bei Willingen wird, wie in kaum einer anderen Heidefläche Nordhessens, eine neuartige Heidenutzung sichtbar, nämlich als Erholungsgebiet. Die mit 
Heidschnucken beweideten Zwergstrauchheiden werden von Ski- und Sesselliftanlagen, Spazierwegen und Skipisten durchzogen. Einer weniger erfreulichen Nutzung unterlag der ehemalige Grenzstreifen bei der Burg Hanstein; die Flächen neben dem Kolonenweg wurden zu DDR-Zeiten mit Herbiziden vegetationsfrei gehalten. In den Jahren nach der Wende waren diese stellenweise noch mit lückigen, flechtenreichen Calluna-Heiden überwachsen, die nun jedoch zunehmend vergrasen und verbuschen. Einige in der vorliegenden Arbeit dokumentierte Flechtenvorkommen dürften daher schon bald von konkurrenzstärkeren Phanerogamen verdrängt und somit verschwunden sein.

\subsection{Wälder}

Die Traubeneichenwälder am Roßkopf wurden bis etwa 1920 als Eichenschälwälder zur Gewinnung von Gerberlohe für die Gerbereibetriebe in Eschwege genutzt (BAIER mündl.). Die jungen Eichenstämme wurden zu diesem Zweck abgehauen und entrindet (KollmanN 1993). Niederwälder im Gebiet Waldeck dienten verschiedenen Nutzungszwecken: zur Brennholzversorgung, zur Kohlholzgewinnung (landesherrliche Forste) oder als Eichenschälwälder (ENGELHARD 1971). Die Niederwaldwirtschaft führte aufgrund der relativ kurzen Umtriebszeiten (vgl. Pотт 1985) und der Verringerung des Rotbuchenanteils zu einer Auflichtung der Wälder. An Steilhängen konnten Bodenerosionen die Folge starker Nutzung sein (vgl. POTT 1981). Die Veränderungen im Baumartengefüge wirkten sich auf den Wärme- und Wasserhaushalt aus (MANZ 1993) und ermöglichten so die Ansiedlung lichtliebender, trockenheitsertragender Arten.

\subsection{Ruderalfluren}

An Ruderalstandorten sind hauptsächlich Steinbrüche zu nennen, in denen Gips, Dolomit (für den Feldwegebau) bzw. Grauwacke abgebaut wurde. Alle untersuchten Steinbrüche sind heute nicht mehr in Betrieb. Die Flächen bleiben sich selbst überlassen. Allerdings werden sie leider vielerorts als Grillplatz genutzt oder als Müllkippe mißbraucht. Die Basaltvorkommen der Blauen Kuppe bei Eschwege haben bis 1910 ebenfalls als Steinbruch gedient (LOTZ 1995), heute besiedeln jedoch Trockenrasen die ehemaligen Abbauflächen. Desweiteren finden sich Erdflechtenvorkommen im Untersuchungsgebiet innerhalb von Ruderalfluren auf ehemaligen Bergbauflächen. Einige Informationen zu diesen Flächen und zum Bergbau in Nordhessen gibt das Kap. B2. 


\section{Charakterisierung der Art Cladonia furcata}

Cladonia furcata (Hudson) Schrader zählt zu den bereits seit 1762 bekannten Flechtenarten (SCHADE 1964). Schon früh tauchte sie auch in der Literatur des deutschsprachigen Raumes auf. Den Erstnachweis für Mecklenburg-Vorpommern erbrachte beispielsweise BRÜCKNER (1803), der sie als Lichen furcatus bezeichnete. Cl. furcata ist heute auch unter den Trivialnamen „Vielgestaltige Becherflechte“ (KREMER \& MUHLE 1991), „Many-forked clad“, „forking clad“, „fork cladonia“ oder „fork lichen“ (GOWARD 1999) bekannt.

\section{a) Systematische Stellung}

Aнті (2000) unterteilt die Gattung Cladonia in 7 Sektionen. Eine Unterteilung, die im wesentlichen auf morphologischen und chemischen Merkmalen beruht. Cl. furcata wird in die Sektion Ascyphiferae sensu Ahti gestellt. STENRoos et al. (2002) unterstützen diese Gliederung in Sektionen nicht. In ihrer Phylogenie, aufgestellt auf Basis molekularer, morphologischer und chemischer Daten, wird die Gattung Cladonia in 3 sogenannte Subdivisions unterteilt. Der überwiegende Teil der Sektion Ascyphiferae, inclusive der Typusart Cl. furcata, zählt zur Subdivision II, Supergroup Cladonia. In diese Subdivision II wird auch die Subgroup Graciles mit der provisorischen Typusart Cl. gracilis gestellt, welche bei den im Rahmen der vorliegenden Arbeit durchgeführten Untersuchungen ebenfalls eine Rolle spielt.

Nach WiRTh (1995) und Scholz (2000) wird Cladonia furcata in die beiden Unterarten Cl. furcata (Hudson) Schrader ssp. furcata und ssp. subrangiformis (Sandst.) Abbayes unterteilt. Dieser Unterteilung schließe ich mich zunächst an, möchte sie jedoch im Anschluß an die Ergebnis-Darstellungen diskutieren. Einen Überblick über andere taxonomische Einstufungen der Sippe subrangiformis gibt Kap. G2.2.

Die gelegentlich als var. scabriuscula (s. FINK 1904 und HenNiPMAN 1978) zu Cladonia furcata gestellte Cl. scabriuscula (Delise) Nyl. wird als eigene Art gewertet (s. WIRTH 1995) und im Rahmen dieser Untersuchungen nicht mit bearbeitet.

\section{b) Morphologie}

Bei Cladonia furcata handelt es sich um eine Strauchflechte mit aufrechten bis niederliegenden, stets becherlosen Podetien mit offenen Achseln. Beide Unterarten haben weiterhin folgende Gemeinsamkeiten (s. WIRTH 1995): sie sind nicht sorediös, haben braune Apothecien, sind glatt berindet und der Aspekt des Lagers wird in der Regel von den Podetien bestimmt. Eine ausführliche Beschreibung der Morphologie von Cl. furcata findet sich in Kap. G2 und G3 sowie in ANDERS (1928), HILlmanN \& GrummanN (1957), SAndstede (1931) und Schade (1964/66). Tab. 1 zeigt eine Gegenüberstellung der Merkmale, in denen sich beide Unterarten unterscheiden können. 
Tab. 1: Merkmale der beiden Unterarten von Cladonia furcata (nach WIRTH 1995)

\begin{tabular}{l|l}
\hline Cladonia furcata ssp. furcata & Cladonia furcata ssp. subrangiformis \\
\hline $\begin{array}{l}\text { grundständiges kleinblättriges Lager oft vorhanden } \\
\text { Podetien ohne Schuppen bis dicht schuppig }\end{array}$ & $\begin{array}{l}\text { Grundschuppen gewöhnlich verschwindend } \\
\text { Podetien spärlich beschuppt } \\
\text { Podetien gewöhnlich +/- aufrecht }\end{array}$ \\
$\begin{array}{l}\text { Podetien in manchen (oft reich fruchtenden) Formen } \\
\text { stellenweise längs aufgeschlitzt und dann rinnig niederliegend bis aufsteigend } \\
\text { Penig bis stark verzweigt (oft dichotom), schlank }\end{array}$ & $\begin{array}{l}\text { aufbrechenden Beulen } \\
\text { relativ kräftig und locker verzweigt }\end{array}$ \\
Podetien grauweiß bis graugrünlich bis dunkelbraun. & Podetien meist dunkelbraun bis olivbraun \\
Äste meist gabelig, oft auch ziemlich spitzwinklig abgehend. & Äste zugespitzt, oft fast rechtwinklig abgehend \\
K- oder grünlich bis bräunlich, selten gelblich, & K+ gelb bis K-, Atranorin, FPC, (+/- Bourgeansäure). \\
P+ rot, FPC. Ohne oder sehr selten mit Atranorin. & P+ gelborange bis rot \\
verbreitete und häufige, variable Flechte & ziemlich selten \\
\hline
\end{tabular}

\section{c) Ökologie}

Cladonia furcata ssp. furcata besitzt eine sehr weite ökologische Amplitude und kommt auf Silikatfels ebenso vor wie auf Baumstümpfen oder (steinigem) Erdboden. Sie gedeiht auf offenem Erdboden, zwischen anderen Cladonia-Arten und Moosen, auf mit Moosen und anderen Flechten bewachsenem Fels, selten auch auf Stubben oder in Halbtrockenrasen. In niederen, felsarmen Lagen siedelt sie besonders an Waldrändern und in lichten Wäldern (v.a. Eichen-Buchen-Wälder, Kiefern-Wälder), auf steinigen, sandigen und sandig-lehmigen Böden, seltener auch in Kalktrockenrasen. In höheren Lagen kommt sie besonders in Zwergstrauchheiden, an felsigen Abhängen und in moosreichen Wäldern vor. Ihren Schwerpunkt hat sie auf mäßig bis ziemlich sauren Substraten. Sie gilt als skiophytisch bis sehr photophytisch und mesophytisch bis xerophytisch. Die Art zeigt eine weite Verbreitung von der Ebene bis in den Montanbereich, auch in intensiv genutzten Gebieten. Sie ist auf schattigen Standorten genauso konkurrenzkräftig wie in der Sonne, wenn auch mit unterschiedlicher Morphologie (SCHÖLleR 1991, WIRTH 1995, vgl. CANTERS 1988). Cl. furcata ssp. furcata kann sich in trockenen Rasen gelegentlich auch gegenüber Höheren Pflanzen behaupten (s. POELT 1969).

Cladonia furcata ssp. subrangiformis siedelt v.a. in Kalktrockenrasen, kommt aber auf humusarmen, basen- oder kalkreichen, seltener sauren Sanden ebenfalls vor. Sie bevorzugt trockene, sommerwarme, lichtreiche Habitate (PAUS 1997, WIRTH 1995, s. auch FISCHER 2003).

\section{d) Verbreitung weltweit}

Die Subspezies furcata zeigt in Europa ein Vorkommen vom borealen Nadelwaldgebiet bis in den Mittelmeerraum (WIRTH 1995). Die Art ist nach LITTERSKI (1999) in der gesamten Nordhemisphäre weit verbreitet und reicht dort von der arktischen bis zur meridionalen Florenzone (vgl. z.B. THOMSON 1967, HenNiPMAN 1978, AHTI \& LAI 1979, Nimis 1993, SANTESSON 1993, EgEA 1996, GowARD 1999, SupPAN et al. 2000, und Checklisten bei FEUERER 2003). Darüber hinaus kommt sie auch in den tropischsubtropischen Zonen (s. Checklisten bei FEUERER 2003) sowie in der australen und antarktischen Florenzone vor (DoIGDE 1950, GALlOWAY 1985, ARCHER 1992, STENROOS \& AHTI 1992 und Checklisten bei FEUERER 2003). 
Die Subspezies subrangiformis ist in Europa von der mitteleuropäischen Zone bis ins Mittelmeergebiet verbreitet (WIRTH 1995). Sie kommt von Südost-England bis Südwest-Asien (LITTERSKI 1999, vgl. auch HAWKSWORTH 1969, HENNIPMAN 1978, PURVIS \& JAMES 1992) sowie in Südeuropa (BURGAZ \& AHTI 1992) und Nordafrika vor (EGEA 1996). Cladonia furcata ssp. subrangiformis tritt in Nordeuropa (vgl. z.B. SANTESSON 1993) ebenso auf wie in Osteuropa, dort werden Vorkommen z.B. aus der ehem. Tschechoslowakei, aus Ungarn, aus Slowenien und aus Estland beschrieben (CERnohorsky 1950, PišUt \& WAgner 1973, Verseghy 1975, Suppan et al. 2000, vgl. auch FEUERER 2003). EvANS (1954) führt die Art für Nordamerika auf - dies wird jedoch von Aнті (1962) widerlegt. Er schreibt, die entsprechenden Proben seien eher Cl. furcata ssp. furcata zuzuordnen. In der aktuellen Flora von GOWARD (1999) über die Flechten British Columbias taucht Cl. subrangiformis bzw. Cl.furcata ssp. subrangiformis nicht auf.

\section{e) Verbreitung in Deutschland}

Cladonia furcata ist in ganz Deutschland verbreitet. Die ssp. furcata wird in ScHOLZ (2000) aus den Bundesländern Mecklenburg-Vorpommern, Niedersachsen, RheinlandPfalz, Saarland, Hessen, Thüringen, Sachsen-Anhalt, Sachsen und Baden-Württemberg gemeldet, die ssp. subrangiformis aus Mecklenburg-Vorpommern, Niedersachsen, Rheinland-Pfalz, Saarland, Hessen, Thüringen, Sachsen-Anhalt, Brandenburg, Sachsen, Baden-Württemberg und Bayern. Den Erstnachweis der ssp. subrangiformis für das Untersuchungsgebiet (Hessisches Bergland) publizierte KÜMMERLING (1991). Erwähnt wird die Unterart jedoch schon bei BEUG (1963-1989) in der unpublizierten Artenliste des Jahres 1963. 


\section{D Übersicht der angewandten Methoden}

Zur Dokumentation der Erdflechtengesellschaften des Untersuchungsgebietes wurden Vegetationsaufnahmen nach der Methode von BRAUn-BLANQUET (s. z.B. DieRsCHKE 1994) angefertigt. Zur Anwendung kam eine modifizierte Abundanz-Dominanz-Skala nach WIRTH (1972). Darüber hinaus erhielt jeder Aufnahmebogen Angaben zur Ausdehnung der Lager und zur Fruchtkörperbildung. Die an jedem der Standorte entnommenen Bodenproben dienten der anschließenden Bestimmung von pH-Werten und Leitfähigkeiten. Die Vegetationsaufnahmen wurden nach floristisch-soziologischen Kriterien geordnet (s. Kap. E1).

Kritische Flechten-Belege aus den Aufnahmeflächen wurden mit Hilfe der Dünnschichtchromatographie auf charakteristische Inhaltsstoffe hin untersucht (Methode nach CULBERSON \& AMMANN 1979), ebenso alle im Untersuchungsgebiet gesammelten Cladonia furcata-Belege (s. Kap. F1).

Um die Variabilität von Cladonia furcata ssp. furcata anhand möglichst vieler Merkmale zu erfassen und Abhängigkeiten der Merkmale aufzuzeigen, wurden insgesamt $65 \mathrm{Cl}$. furcata-Polster gesammelt und an ihnen jeweils 16 Merkmale protokolliert. $\mathrm{Zu}$ jedem gesammelten Polster wurde darüber hinaus eine Kurzbeschreibung des Standorts angefertigt (s. Kap. G1.1).

Im Rahmen der Verpflanzungsversuche erfolgte eine Messung der Zuwachsraten der Podetien sowie eine Beobachtung der morphologischen Veränderungen. An den Standorten der ausgepflanzten Polster wurden mit einem eigens für Flechten konstruierten tragbaren Meßgerät (s. SCHUSTER et al. 1982, 2002) MikroklimaMessungen durchgeführt. Zusätzlich wurden die Evaporation, die Windgeschwindigkeit und die relative Luftfeuchte bestimmt (s. Kap. G1.2).

Bei den abschließenden molekularbiologischen Untersuchungen handelt es sich um die Sequenzierung der ITS rDNA von Mycobiont und Photobiont (s. Kap. H1). 


\section{E Vegetationskundliche und floristische Untersuchungen}

\section{Methoden}

\subsection{Auswahl und Verteilung der Aufnahmeflächen}

Die Zielsetzung lautete, das gesamte Spektrum an Erdflechten-Gesellschaften in Nordhessen zu erfassen und dabei möglichst viele bezüglich Topographie und Geologie unterschiedliche Gegenden des Untersuchungsgebietes abzudecken. Die Datenerhebung erfolgte in den Jahren 1999 bis 2002. Den Geländearbeiten ging eine Auswertung der vorhandenen Literatur über Flechtenvorkommen in Nordhessen voran. Ergänzend wurden aus den topographischen Karten (TK 25, TK 50) potentielle Flechtenwuchsorte (Steinbrüche, Heideflächen, Magerrasen, Felsen etc.) herausgesucht sowie Ortskundige über Flechtenvorkommen befragt. An erster Stelle bei der Suche nach Flechtenwuchsorten im Gelände wurden die in der Literatur aufgeführten Stellen berücksichtigt (z.B. FOLLMANN \& MALECKI 1973, FOLLMANN 1974/1979/1981/1983, BRUELHEIDE 1991/1992, KÜMMERLING 1991, BULTMANN 1993). Leider erwiesen sich etliche dieser Fundortangaben als heute nicht mehr zutreffend. Des weiteren wurden Auflistungen von Naturschutzgebieten (z.B. LEICHT 1988/1990, ANONYMUS 1994, Hessisches Ministerium FÜR LANDESENTWICKLUNG, WOHNEN, LANDWIRTSCHAFT, Forsten UND NATURSCHUTZ 1994) sowie Berichte über interessante Biotoptypen (Nieschalk \& Nieschalk 1983a/b, Nitsche 1995, Nitsche \& Bultmann 1995) in Bezug auf mögliche Flechtenwuchsorte ausgewertet. Unter den potentiell geeigneten Aufnahmegebieten mußte eine Auswahl getroffen werden, da es aus zeitlichen Gründen nicht realisierbar war, alle ausgesuchten Orte zu überprüfen. Vegetationsaufnahmen wurden an 63 der insgesamt 70 bearbeiteten Gebiete mit gut entwickeltem Flechtenbewuchs angefertigt.

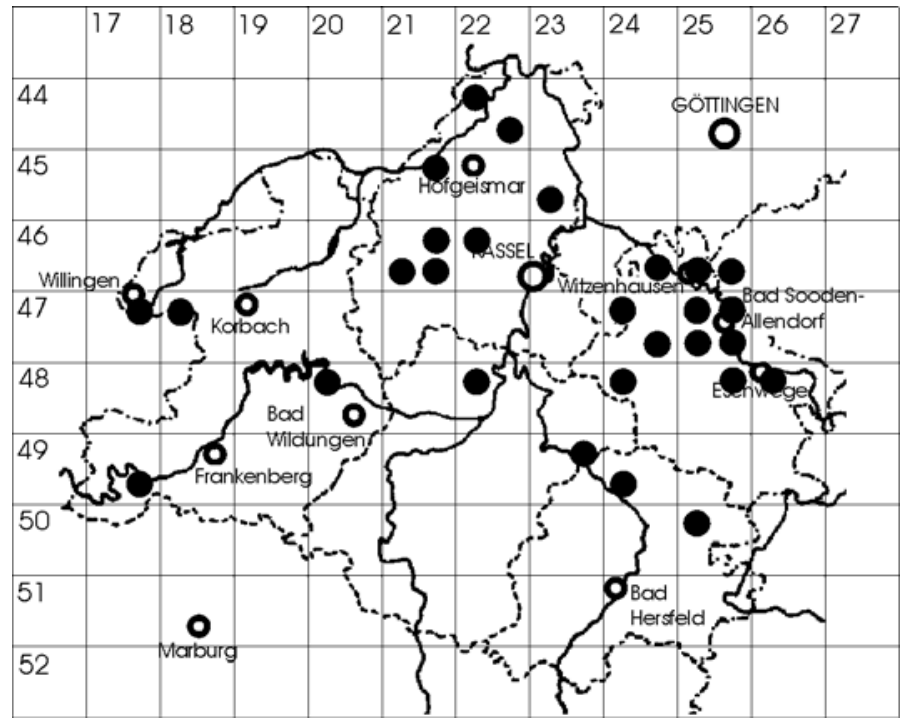

Abb. 7: Lage der Aufnahmegebiete. 
Die Verteilung der bearbeiteten Aufnahmeflächen im Untersuchungsgebiet auf Quadrantenbasis der TK 25 zeigt Abb. 7. Zur Beschreibung aller bearbeiteten Orte siehe Tab. 57 im Anhang 1.

\subsection{Vegetationsaufnahmen und Bodenanalysen}

\section{a) Vegetationsaufnahme}

Die pflanzensoziologischen Aufnahmen wurden nach der Methode von Braun-Blanquet (s. DIERSCHKE 1994) durchgeführt. Die Flächenauswahl erfolgte nach Kriterien der floristischen Homogenität. Die Größe der Aufnahmenflächen beträgt in der Regel $30 \times 30 \mathrm{~cm}^{2}$. Sofern eine quadratische Fläche nicht möglich war, wurden Rechtecke gleicher Flächengröße (9 bis $10 \mathrm{dm}^{2}$ ) gewählt. Bei der Erfassung der Artmächtigkeiten fand die von PAUS (1997) modifizierte Abundanz-Dominanz-Skala nach WIRTH (1972) Anwendung:

$\begin{array}{lll}\mathrm{r} & \text { 1-2 Lager/Ansiedlungen, auch außerhalb nur sporadisch } & \begin{array}{l}\text { Deckung in \% } \\ <1\end{array} \\ + & \text { bis 5 Lager/Ansiedlungen } & <1 \\ 1 & \text { bis 20 Lager/Ansiedlungen } & <5 \\ 2 \mathrm{~m} & \text { über 20 Lager/Ansiedlungen } & <5 \\ 2 \mathrm{a} & \text { Anzahl der Lager/Ansiedlungen beliebig } & 5-12,5 \\ \text { 2b } & \text { Anzahl der Lager/Ansiedlungen beliebig } & 12,5-25 \\ 3 & \text { Anzahl der Lager/Ansiedlungen beliebig } & 25-50 \\ 4 & \text { Anzahl der Lager/Ansiedlungen beliebig } & 50-75 \\ 5 & \text { Anzahl der Lager/Ansiedlungen beliebig } & 75-100\end{array}$

Zusätzlich zu den Artmächtigkeiten wurden Angaben zur Ausdehnung der Lager (s. Soziabilität bei WILMANNS 1962) und zur Fruchtkörperbildung notiert:

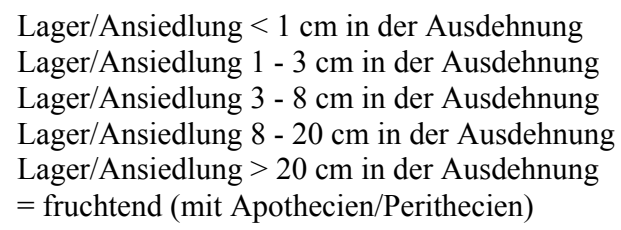

Für jede Vegetationsaufnahme wurden darüber hinaus folgende Angaben zur Vegetation sowie zu Wuchs- und Standort erhoben (die Erläuterung der Abkürzungen von Geologie und Fundorten finden sich im Anhang 1):

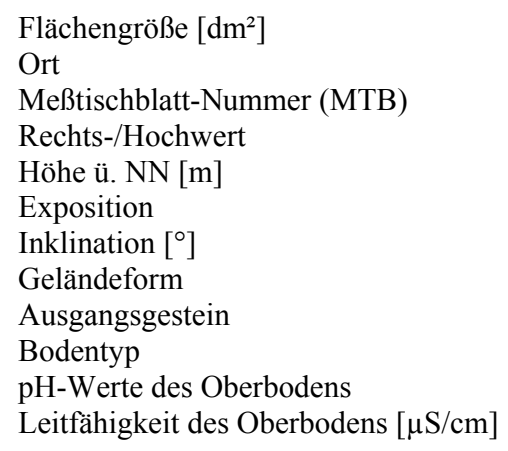

Skelettanteil in oberster Bodenschicht

Mikroklima

Größe des Gesamtbestandes

umgebende Phytozönose

Nutzung

Störeinflüsse

Deckung der Schichten [\%]

Deckung offener Boden [\%]

Deckung Steine [\%]

Deckung Streu [\%]

Wuchshöhen [cm]

Es wurden insgesamt 203 Vegetationsaufnahmen erstellt. 


\section{b) Bodenanalysen}

Zur Charakterisierung der Standorte wurden an jeweils 3 verschiedenen Stellen in unmittelbarer Nähe der Aufnahmeflächen Proben der obersten Bodenschicht (bis max. $5 \mathrm{~cm}$ Tiefe) entnommen, vermischt und bei Zimmertemperatur getrocknet. Die Bodenentnahme erfolgte in der Regel nicht innerhalb der Aufnahmeflächen, um die ohnehin oft nur noch sehr sporadisch auftretenden Erdflechtenbestände nicht unnötig zu schädigen. Für die Messung der pH-Werte und elektrischen Leitfähigkeit wurde eine wässrige Suspension aus Boden und demineralisiertem Wasser im Verhältnis 1:2 angesetzt. 24 Stunden später erfolgte die Messung der $\mathrm{pH}\left(\mathrm{H}_{2} \mathrm{O}\right)$ - und Leitfähigkeitswerte. Nach der anschließenden Zugabe von einer Spatelspitze $\mathrm{KCl}$ und etwa 30 minütiger Wartezeit konnte der $\mathrm{pH}(\mathrm{KCl})$-Wert ermittelt werden. Für die Messungen wurden Einstabmeßelektroden verwendet. Da die elektrische Leitfähigkeit als indirektes Maß für die in der Bodenlösung gelösten Nährstoffe dienen sollte, wurde die korrigierte, d.h. die um den Anteil der $\mathrm{H}^{+}$-Ionen verringerte Leitfähigkeit errechnet. Die Korrektur wurde bei allen $\mathrm{pH}\left(\mathrm{H}_{2} \mathrm{O}\right)$-Werten $\leq 5$ vorgenommen, da der Einfluß der $\mathrm{H}^{+}$-Ionen auf die elektrolytische Leitfähigkeit bei $\mathrm{pH}$-Werten $>5,5$ nur noch unbedeutend, bei $\mathrm{pH}-$ Werten $<4,5$ dagegen ganz erheblich ist (BAUMANN 2000). Die Berechnung erfolgte in Anlehnung an SJÖRS (1950) und HöLZER (1977/1984) (s. auch BAUMANN 2000).

\subsection{Nomenklatur der Arten und Vegetationseinheiten}

Die Nomenklatur der Flechtenarten richtet sich nach SchOlz (2000), die der Moose nach KOPERSKI et al. (2000) und die der Phanerogamen nach WISSKIRCHEN \& HAEUPLER (1998), mit Ausnahme von Taraxacum officinale, das nach EHRENDORFER (1973) als Taraxacum officinale agg. aufgeführt wird. Angaben zu den Charakterarten sind Paus (1997) sowie FollmanN (1974) und DRehwald (1993) entnommen. Die Nomenklatur der Flechtengesellschaften richtet sich im wesentlichen nach WIRTH (1995) sowie nach DREHWALd (1993) und PAUs (1997). Ergänzend zu Charakter- und Differentialarten werden in Anlehnung an PAUs (1997) Arten, die schwerpunktmäßig in einer der Vegetationseinheiten auftreten, jedoch weite soziologische Amplituden haben und auch auf andere syntaxonomische Einheiten übergreifen, als Schwerpunktarten (Sch) bezeichnet. Unter dem Begriff Diagnostische Arten werden Charakter- und Differentialarten zusammengefasst. Die Charakteristische Artenverbindung (CAV) beinhaltet neben den Diagnostischen Arten alle Begleiter mit einer Stetigkeit von über 40 \% (Klasse III und höher) (DIERSCHKE 1994).

In der vorliegenden Untersuchung wird der Begriff „Bunte-Erdflechten-Gesellschaft” in Anlehnung an REIMERS (1940a) als Bezeichnung der Assoziation Toninio-Psoretum decipientis und nicht des Verbandes Toninion verwendet.

\section{Bestimmungskritische Sippen:}

Bryum-Arten wurden nur selten mit Kapseln und in gutem Entwicklungszustand angetroffen. Eine genaue Bestimmung war daher in der Regel nicht möglich. Die 
Sippen werden als Bryum spec. zusammengefaßt. Darin dürften zumindest Bryum capillare agg. und Bryum caespiticium enthalten sein.

Cephaloziella: Diese schwer bestimmbare Gruppe wird nicht weiter unterteilt, sondern als Cephaloziella spec. in den Tabellen aufgeführt.

Cladonia arbuscula: Die Thalli wurden nur stichprobenhaft auf ihren Chemismus hin untersucht (s. Kap. F). In den Vegetationstabellen wird die Art nicht weiter in Subspezies unterteilt, sondern Cladonia arbuscula benannt.

Cladonia cervicornis wird in den Tabellen als Cl. cervicornis bezeichnet, da sich viele Proben weder der Subspezies cervicornis noch der Subspezies verticillata eindeutig zuordnen lassen, sondern Übergangscharakter zeigen.

Cladonia furcata: Alle Proben von Cladonia furcata wurden mit Hilfe der Dünnschichtchromatographie überprüft (s. Kap. F) und in die beiden Unterarten furcata und subrangiformis aufgetrennt.

Cladonia gracilis: Die Unterarten werden nicht unterschieden, da einige Proben nicht bis zur Subspezies bestimmt werden konnten.

Cladonia macilenta: Die Proben lassen sich, bis auf wenige Ausnahmen, einer der beiden Unterarten zuordnen und werden daher getrennt als ssp. floerkeana bzw. ssp. macilenta aufgeführt. In Gesellschaftsnamen werden sie verkürzt mit ihren Synonymen als Cladonia bacillaris (Syn. Cladonia macilenta ssp. macilenta) und Cladonia floerkeana bezeichnet (s. SCHOLZ 2000).

Cladonia pyxidata: Da die Chemosippen von Cladonia pyxidata unterschiedliche Standortansprüche aufweisen und nach PAUS (1997) Trennarten von Gesellschaften sein können, werden sie im Aufnahmematerial unterschieden. $\mathrm{Zu}$ diesem Zweck wurden innerhalb der Aufnahmeflächen an mehreren Stellen Proben genommen und anschließend im Labor dünnschichtchromatographisch untersucht (s. Kap. F). Sofern makroskopisch erkennbare Unterschiede auftraten (Thallusfärbung, Becherdurchmesser, Fruchtkörperbildung, Körnung der Soredien/Granulae etc.), konnten die Proben bereits im Gelände getrennt werden. In den anderen Fällen wurden an mehreren Stellen der Fläche Thallusproben gesammelt und anschließend auf Einheitlichkeit hinsichtlich des Chemismus geprüft. Um übermäßig lange Namen in Text und Tabellen zu umgehen, werden die Chemosippen innerhalb von Cladonia pyxidata ssp. grayi nach WIRTH (1995) folgendermaßen benannt:

Chemosippe cryptochlorophaea $=$ Cladonia cryptochlorophaea

Chemosippe grayi s.str. = Cladonia grayi s.str.

Chemosippe merochlorophaea $=$ Cladonia merochlorophaea

Chemosippe novochlorophaea $=$ Cladonia novochlorophaea

Collema-Arten werden in den Vegetationstabellen als Collema spec. zusammengefaßt, da ein Großteil der Proben so schlecht entwickelt ist, daß sie sich nicht bis auf Artebene bestimmen lassen. Im Gebiet nachgewiesen werden konnten Collema crispum, Collema tenax und Collema cf. auriforme (s. Artenliste im Anhang 1). 
Hypnum cupressiforme s.l.: Innerhalb der Klasse Ceratodonto-Polytrichetea ergaben sich Schwierigkeiten beim Bestimmen der oft mangelhaft entwickelten HypnumBelege. Diese werden daher, der Nomenklatur von FrAHM \& FREY (1992) folgend, in den Tabellen als Hypnum cupressiforme s.l. vereinigt. Deutlich besser entwickelt und eindeutig der var. lacunosum (KOPERSKI et al. 2000) zuzuordnen, ist Hypnum cupressiforme auf den basenreichen Standorten, innerhalb der Klasse Toninio-Psoretea.

Leptogium: Die in den Aufnahmeflächen vorkommenden Thalli der Gattung Leptogium sind größtenteils für eine Bestimmung auf Artniveau ungenügend entwickelt. Es scheint sich bei den Proben um L. lichenoides und L. schraderi zu handeln, welche nach KÜMMERLING (1991) beide im Untersuchungsgebiet vorkommen. In den Tabellen werden sie als Leptogium cf. lichenoides bzw. cf. schraderi bezeichnet.

Racomitrium: Die Benennung erfolgt nach FRAHM \& FREY (1992) als Racomitrium heterostichum agg. und Racomitrium canescens s.l.

Trichostomum spec. und Weissia spec.: Wie bei vielen Proben der kleinwüchsigen Moose basenreicher Standorte, können auch hier nicht alle eindeutig bestimmt werden. Es ist daher nicht möglich, beide Gattungen eindeutig voneinander zu trennen, weshalb sie in den Vegetationstabellen als Trichostomum et Weissia spec. vereinigt werden.

Einige Flechten-Belege wurden im Herbar der Universität Göttingen bzw. im Herbar der Verfasserin hinterlegt. Diese sind in der Belegeliste im Anhang 1 aufgeführt.

\subsection{Tabellenarbeit}

Die Vegetationsaufnahmen wurden mit Hilfe des Computerprogrammes TABWIN Version 3.3 (PEPPLER 1999) nach floristisch-soziologischen Kriterien geordnet. Die Ausdehnung der Lager und die Fruchtkörperbildung (falls vorhanden) sind in den Tabellen als Exponenten dargestellt. Die Stetigkeitsklassen richten sich nach BERGMEIER et al. (1990). Folgende Abkürzungen werden in den Vegetationstabellen verwendet:

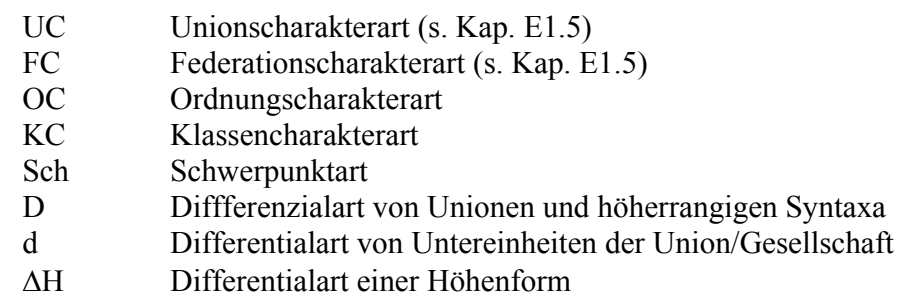

$\mathrm{Zu}$ den Abkürzungen in den Tabellenköpfen siehe Abkürzungsverzeichnis im Anhang 1.

Die Vorkommen der Gesellschaften werden in Punktrasterkarten dargestellt. Die Eintragung der Fundorte erfolgt auf Quadrantenbasis der TK 25. 
Da Cladonia furcata bei den weiteren Untersuchungen der vorliegenden Arbeit eine zentrale Rolle spielt, wurden die beiden Unterarten der Sippe (ssp. furcata und ssp. subrangiformis) in den Vegetationstabellen jeweils markiert. Ihre Vergesellschaftung wird in einem eigenen Kapitel besprochen (Kap. E3).

\subsection{Syntaxonomie}

Im allgemeinen werden terrestrische Flechten und Moose bei der Vegetationsanalyse und Klassifikation von Gefäßpflanzengesellschaften nach BRAUN-BLANQUET (1964) als Bestandteile der Moosschicht erfaßt und in das Phytozönosensystem integriert. Kryptogamenbestände auf ökologisch deutlich abweichenden Mikrostandorten wie beispielsweise Felsblöcken, Totholz oder Stammbasen werden dagegen oft als eigenständige Gesellschaften betrachtet (BARKMAN 1958, WIRTH 1972). Vor allem skandinavische Bearbeiter betonten, daß derartige Gemeinschaften nicht als Assoziationen bezeichnet werden dürfen, da die gleiche Kategorie nicht für den Teil und das Ganze verwendet werden kann. In der Folge wurden daher „Mikrogemeinschaften“ mehr und mehr im Synusialsystem beschrieben (SCHUHWERK 1986).

Floristisch klar abgrenzbare Kryptogamenbestände existieren auch auf terrestrischen Standorten, sind dort allerdings in noch stärkerem Maße von den durch die umgebende Gefäßpflanzenvegetation geschaffenen Standortbedingungen abhängig. Da diese Erdflechtengesellschaften also mehr oder weniger abhängige Untereinheiten von Phytozönosen darstellen, werden sie von PAUS (1997) als Synusien aufgefaßt, nach ihrer floristischen Zusammensetzung typisiert und im Synusialsystem eingeordnet. Unter Synusien werden nach WILMANNS (1998: 17) ökologisch und morphologisch einander nahestehende und unter annähernd gleichen kleinstandörtlichen Bedingungen lebende Artengruppen verstanden.

Die Syntaxa erhalten im Synusialsystem z.T. eine andere Nomenklatur: Die Union als Grundeinheit entspricht der Assoziation, die Federation dem Verband. Die Syntaxa Ordnung und Klasse werden nach WILMANNS (1966) ebenso übernommen wie die in der Terminologie gebräuchlichen Endungen (-etum, -ion, -etalia, -etea). BARKMAN (1973) benutzt für die höheren Einheiten dagegen die Begriffe Ordulus und Classicula.

Entscheidend für die Einordnung in das Synusialsystem ist der Grad der Abhängigkeit der Kryptogamengemeinschaft von der Makrophytenvegetation. Nach SCHUHWERK (1986) werden Kryptogamengemeinschaften dann als Assoziationen in das Phytozönosensystem eingeordnet, wenn sie ökologisch von der sie umgebenden Makrophytenvegetation unabhängig sind. Abhängige Vereine, die nur Teile komplexer Phytozönosen sind, werden dagegen im Synusialsystem behandelt. In der Praxis ist die Abhängigkeit einer Kryptogamengesellschaft nicht immer eindeutig feststellbar, zudem kann ein und dieselbe Kryptogamengemeinschaft in verschiedenen Makrogesellschaften auftreten und in diesen unterschiedlich starke Abhängigkeit zeigen.

Im nordhessischen Raum treten sowohl Gesellschaften auf, die als weitgehend unabhängig betrachtet werden können (z.B. die „Bunte-Erdflechten-Gesellschaft“ auf 
Felsköpfen) als auch solche, die offensichtlich eine stärkere Abhängigkeit von der umgebenden Phytozönose aufweisen und als „Teil des Ganzen“ verstanden werden sollten (z.B. Flecken von Rentierflechten in Zwergstrauchheiden). Das Synusialsystem bietet die Möglichkeit, beide Kategorien vergleichen zu können (SCHUHWERK 1986); daher werden in der vorliegenden Arbeit die Begriffe des Synusialssystems in Anlehnung an WiLMANNS (1966) bzw. SCHUHWERK (1986) verwendet, um sprachlich deutlich $\mathrm{zu}$ machen, daß viele der Kryptogamengesellschaften kleine Lebensgemeinschaften innerhalb einer Makrogesellschaft darstellen. Sofern im weiteren der Begriff „Gesellschaft“ verwendet wird, ist er als Synonym zu der Bezeichnung „Synusie“ zu sehen.

Unter Fragmentgesellschaft (FG) werden in Anlehnung an BRUN-HoOL (1966) Vegetationstypen verstanden, die gegenüber der Assoziation an Diagnostischen Arten verarmt sind. Die Einheiten werden nach der stetesten Art bzw. einer der stetesten Arten und dem niedrigstmöglichen Syntaxon benannt (z.B. Cladonia symphycarpa-ToninionFG).

Der Zielsetzung der vorliegenden Arbeit folgend, wurden nur solche Aufnahmeflächen ausgewählt, in denen Flechten dominieren oder zumindest eine bedeutende Rolle am Aufbau der Gesellschaft haben. Zumeist treten jedoch darüber hinaus auch Gefäßpflanzen, Moose oder Algen auf. Diese wurden bei den Vegetationsaufnahmen mit erfaßt und in die Vegetationsanalyse einbezogen. Bei der syntaxonomischen Bewertung sind Flechten und Moose gleichberechtigt. Die Phanerogamen, deren Auftreten aufgrund der geringen Größe der Aufnahmeflächen stark zufallsbedingt ist, werden lediglich als Strukturelemente betrachtet und bei der Klassifikation nicht berücksichtigt. Von der relativ aufwendigen Bestimmung der Grün- bzw. Blaualgen mußte im Rahmen dieser Untersuchungen aus zeitlichen Gründen, mit Ausnahme einiger Stichproben, abgesehen werden. 


\section{Die Erdflechtengesellschaften}

Die Erdflechten-Bestände des Untersuchungsgebietes gehören zwei Klassen an: den Toninio-Psoretea decipientis und den Ceratodonto-Polytrichetea piliferi. Beide lassen sich durch zahlreiche Arten klar voneinander trennen, wobei die stärkeren Differentialarten auf Seite der Toninio-Psoretea zu finden sind, während die Klasse Ceratodonto-Polytrichetea mit Cladonia macilenta ssp. floerkeana nur eine gute Differentialart aufweist (s. Tab. 2). Cl. subulata, Cl. gracilis, Polytrichum piliferum und Trapeliopsis granulosa sind Beispiele für weitere Differentialarten dieser Klasse, erreichen jedoch nur eine Stetigkeit von maximal $40 \%$.

Tab. 2: Diagnostische Arten der Vegetationsklassen

$\mathrm{C}=$ Charakterart innerhalb der Klasse; Exponent $=$ Spanne der Deckungsgrade

\begin{tabular}{|c|c|c|c|}
\hline \multicolumn{2}{|c|}{ Klasse } & $\begin{array}{l}\text { Cerato- } \\
\text { donto- } \\
\text { Polytri- } \\
\text { chetea }\end{array}$ & $\begin{array}{l}\text { Toninio- } \\
\text { Psoretea }\end{array}$ \\
\hline \multicolumn{2}{|c|}{ Zahl der Aufnahmen } & 129 & 74 \\
\hline \multicolumn{4}{|c|}{ D Ceratodonto-Polytrichetea piliferi: } \\
\hline $\mathrm{C}$ & Cladonia macilenta ssp. floerkeana & $\operatorname{III}^{r-2 b}$ & . \\
\hline $\mathrm{C}$ & Cladonia subulata & $I I^{r-4}$ & . \\
\hline $\mathrm{C}$ & Cladonia gracilis & $I I^{r-3}$ & . \\
\hline $\mathrm{C}$ & Polytrichum piliferum & $I I^{r-4}$ & . \\
\hline $\mathrm{C}$ & Cladonia coccifera & $I I^{r-2 a}$ & . \\
\hline \multirow[t]{7}{*}{$\mathrm{C}$} & Trapeliopsis granulosa & $I I^{r-5}$ & . \\
\hline & Cladonia merochlorophaea & $I I^{r-4}$ & . \\
\hline & Cladonia macilenta ssp. macilenta & $I I^{r-3}$ & . \\
\hline & Hypnum cupressiforme s.1. & $I I^{r-4}$ & . \\
\hline & Pohlia nutans & $I I^{r-2 b}$ & . \\
\hline & Dicranum scoparium & $I I^{r-3}$ & $r^{3}$ \\
\hline & Cladonia fimbriata & $I I^{r-5}$ & $r^{+}$ \\
\hline $\mathrm{C}$ & Cladonia pyxidata ssp. chlorophaea & $I I^{r-3}$ & $r^{+}$ \\
\hline $\mathrm{C}$ & Cladonia furcata ssp. furcata & $I I^{r-5}$ & $r^{+}$ \\
\hline \multicolumn{4}{|c|}{ D Toninio-Psoretea decipientis: } \\
\hline & Ceratodon purpureus & $I I^{r-3}$ & $I V^{r-3}$ \\
\hline & Cyanophyta div. spec. & $++^{+-3}$ & $I V^{r-4}$ \\
\hline & Ditrichum flexicaule & . & $I V^{r-3}$ \\
\hline & Hypnum cupressiforme var. lacunosum & . & $\mathrm{IV}^{\mathrm{r}-4}$ \\
\hline & Peltigera rufescens & . & $\operatorname{III} I^{r-5}$ \\
\hline $\mathrm{C}$ & Fulgensia bracteata & . & $\operatorname{III}^{r-4}$ \\
\hline $\mathrm{C}$ & Cladonia symphycarpa & . & $\operatorname{III}^{r-3}$ \\
\hline $\mathrm{C}$ & Toninia sedifolia & . & $\operatorname{III}^{r-3}$ \\
\hline $\mathrm{C}$ & Cladonia pyxidata ssp. pocillum & . & $\operatorname{III} I^{r-5}$ \\
\hline \multirow[t]{3}{*}{$\mathrm{C}$} & Barbula convoluta & . & $\operatorname{III}^{+-3}$ \\
\hline & Collema spec. & . & $\operatorname{III} I^{r-2 b}$ \\
\hline & Tortella inclinata & . & $\mathrm{II}^{+-2 b}$ \\
\hline $\mathrm{C}$ & Placidium squamulosum & . & $I I^{r-2 a}$ \\
\hline $\mathrm{C}$ & Psora decipiens & . & $I I^{r-2 a}$ \\
\hline \multirow[t]{3}{*}{$\mathrm{C}$} & Fulgensia fulgens & . & $\mathrm{II}^{+-3}$ \\
\hline & Leptogium cf. lichenoides & . & $I I^{r-3}$ \\
\hline & Aloina spec. & . & $I I^{r-2 m}$ \\
\hline \multirow[t]{4}{*}{$\mathrm{C}$} & Cladonia furcata ssp. subrangiformis & . & $I I^{r-3}$ \\
\hline & Thuidium abietinum & . & $I I^{r-2 m}$ \\
\hline & Bryum spec. & $r^{+}$ & $I I^{r-2 m}$ \\
\hline & Cladonia rangiformis & $++^{r-3}$ & $I I^{r-3}$ \\
\hline
\end{tabular}

$\mathrm{Zu}$ den guten Kenn- bzw. Trennarten der Toninio-Psoretea gehören unter anderem die Moose Ditrichum flexicaule und Hypnum cupressiforme var. lacunosum sowie die Flechten Peltigera rufescens, Fulgensia bracteata, Cladonia symphycarpa, Toninia sedifolia und Cl. pyxidata ssp. pocillum. 
Innerhalb der Sippe Cladonia furcata zählt die Subspezies furcata zu den Diagnostischen Arten der Ceratodonto-Polytrichetea, die Subspezies subrangiformis aber zur Charakteristischen Artenverbindung der Toninio-Psoretea.

Phanerogamen können unterschiedlich großen Anteil an der Gesamtdeckung der Bestände haben. In der Regel treten nur vereinzelte, oft kümmerlich entwickelte Exemplare derjenigen Pflanzen auf, welche auch Bestandteil der umgebenden Phytozönose sind. Beispielsweise einzelne Halme von Deschampsia flexuosa oder Festuca ovina agg. oder einzelne Pflänzchen z.B. von Calluna vulgaris. Lediglich in einigen Fragmentgesellschaften oder Dominanzbeständen auf basenreichen Substraten spielen Phanerogamen eine bedeutendere Rolle und sind dann stellenweise gut entwickelt. Aber auch an diesen Wuchsorten gedeihen meist nur vereinzelt stehende Kräuter und Gräser.

\subsection{Ceratodonto-Polytrichetea piliferi}

(Tab. 4)

Die Klasse schließt alle Moos- und Flechtengesellschaften saurer, meist trockener Böden an lichtreichen Standorten ein. Sie gliedert sich in die zwei Ordnungen Peltigeretalia und Polytrichetalia piliferi (DREHWALD 1993). Erstere umfaßt Flechten-, die zweite Moosgesellschaften, daher ist nur die Ordnung Peltigeretalia für die vorliegenden Untersuchungen von Interesse. Nach DREHWALD wird sie in zwei Federationen, Baeomycion rosei und Cladonion sylvaticae (Syn. Cladonion arbusculae), unterteilt, welche PAUS (1997) noch um die Federation Cladonion rei ergänzt. Im Untersuchungsgebiet konnten Gesellschaften aller 3 Federationen nachgewiesen werden (s. Tab. 3 und Tab. 4).

Tab. 3: Syntaxonomische Übersicht der Gesellschaften innerhalb der Klasse CeratodontoPolytrichetea piliferi

\begin{tabular}{|c|c|c|}
\hline \multicolumn{3}{|c|}{ Ceratodonto-Polytrichetea piliferi } \\
\hline \multicolumn{3}{|c|}{ Ordnung } \\
\hline \multicolumn{3}{|c|}{ Peltigeretalia } \\
\hline Federation & Federation & Federation \\
\hline Baeomycion rosei & Cladonion arbusculae & Cladonion rei \\
\hline Union, Gesellschaft & Union, Gesellschaft & Union, Gesellschaft \\
\hline $\begin{array}{c}\text { Pycnothelio-Cladonietum cervicornis } \\
\text { Cladonia floerkeana- } \\
\text { Cladonion rei-FG }\end{array}$ & Cladonia bacillaris-Ges. & Cladonietum rei \\
\hline Dibaeetum baeomycetis & Cladonietum mitis & Cladonia furcata- und \\
& $\begin{array}{c}\text { Cladonia subulata-Cladonion rei-FG } \\
\text { Cladonia gracilis-Cladonion arb.-FG }\end{array}$ & \\
\hline Lecideetum uliginosae & Cladonietum foliaceae & \\
\hline
\end{tabular}


Tab. 4: Übersichtstabelle Ceratodonto-Polytrichetea piliferi

Einheit 1: Baeomycion rosei

1.1 Pycnothelio-Cladonietum cervicornis

1.2 Cladonia cervicornis-Baeomycion-Fragmentgesellschaft

1.3 Dibaeetum baeomycetis

1.4 Lecideetum uliginosae

Einheit 2: Cladonion arbusculae

2.1 Cladonia bacillaris-Gesellschaft

2.2 Cladonietum mitis

2.3 Cladonia furcata- und Cladonia gracilis-Cladonion arbusculae-Fragmentgesellschaft

2.4 Cladonietum foliaceae

Einheit 3: Cladonion rei

3.1 Cladonia floerkeana-Cladonion rei-Fragmentgesellschaft

3.2 Cladonietum rei

3.3 Cladonia subulata-Cladonion rei-Fragmentgesellschaft

\begin{tabular}{|l|l|}
\hline Einheit & \\
\cline { 2 - 3 } & Zahl der Aufnahmen \\
mAZ Phanerogamen & \\
mAZ Flechten & \\
mAZ Moose & \\
\hline
\end{tabular}

\begin{tabular}{|ccc|c|c|c|c|c|c|c|c|c|}
\hline \multicolumn{5}{|c|}{1} & \multicolumn{5}{c|}{2} & \multicolumn{3}{c|}{3} \\
\hline 1.1 & 1.2 & 1.3 & 1.4 & 2.1 & 2.2 & 2.3 & 2.4 & 3.1 & 3.2 & 3.3 \\
2 & 2 & 18 & 5 & 18 & 31 & 13 & 7 & 11 & 14 & 8 \\
0,5 & 0 & 2,4 & 2,2 & 3,5 & 1,3 & 1,7 & 1,9 & 1,1 & 2,9 & 2,3 \\
9,0 & 9,5 & 4,2 & 5,4 & 7,2 & 7,5 & 5,1 & 5,9 & 5,6 & 6,3 & 3,9 \\
3,5 & 3,0 & 2,7 & 4,4 & 6,8 & 2,5 & 2,6 & 2,4 & 2,5 & 2,9 & 3,4 \\
\hline
\end{tabular}

D 1.1:

$\mathrm{UC}_{1.1}$ Pycnothelia papillaria

D 1.1 - 1.3:

Sch Cladonia cervicornis

D 1.3:

$\mathrm{UC}_{1.3}$ Dibaeis baeomyces

D 1 (Baeomycion):

FC Baeomyces rufus

D 1.4:

Sch Placynthiella icmalea

$\mathrm{UC}_{1.4}$ Placynthiella oligotropha

$\mathrm{UC}_{1.4}$ Trapeliopsis gelatinosa

D 2.1:

Cladonia grayi s.str.

D 2.2:

$\mathrm{FC}_{2} \quad$ Cladonia gracilis

$\mathrm{UC}_{2.2}$ Cladonia arbuscula

$\mathrm{UC}_{2.2}$ Cladonia portentosa

$\mathrm{FC}_{2} \quad$ Cladonia uncialis

D $2.2+2.3$ :

$\mathrm{FC}_{2} \quad$ Cladonia rangiferina

D 2.2 - 2.4:

$\mathrm{FC}_{2} \quad$ Cetraria aculeata

OC Cladonia furcata ssp. furcata

D 2.4:

KC Polytrichum piliferum

Cladonia rangiformis

$\mathrm{UC}_{2.4}$ Cladonia foliacea

D $2.4+3$ :

Ceratodon purpureus

\section{3 (Cladonion rei):}

Cladonia fimbriata

D $3.2+3.3$ :

$\mathrm{UC}_{3.2}$ Cladonia pyxidata ssp. chlorophaea

$\mathrm{UC}_{3.2}$ Cladonia humilis

D 3.2:

$\mathrm{UC}_{3.2}$ Cladonia rei

Atrichum undulatum

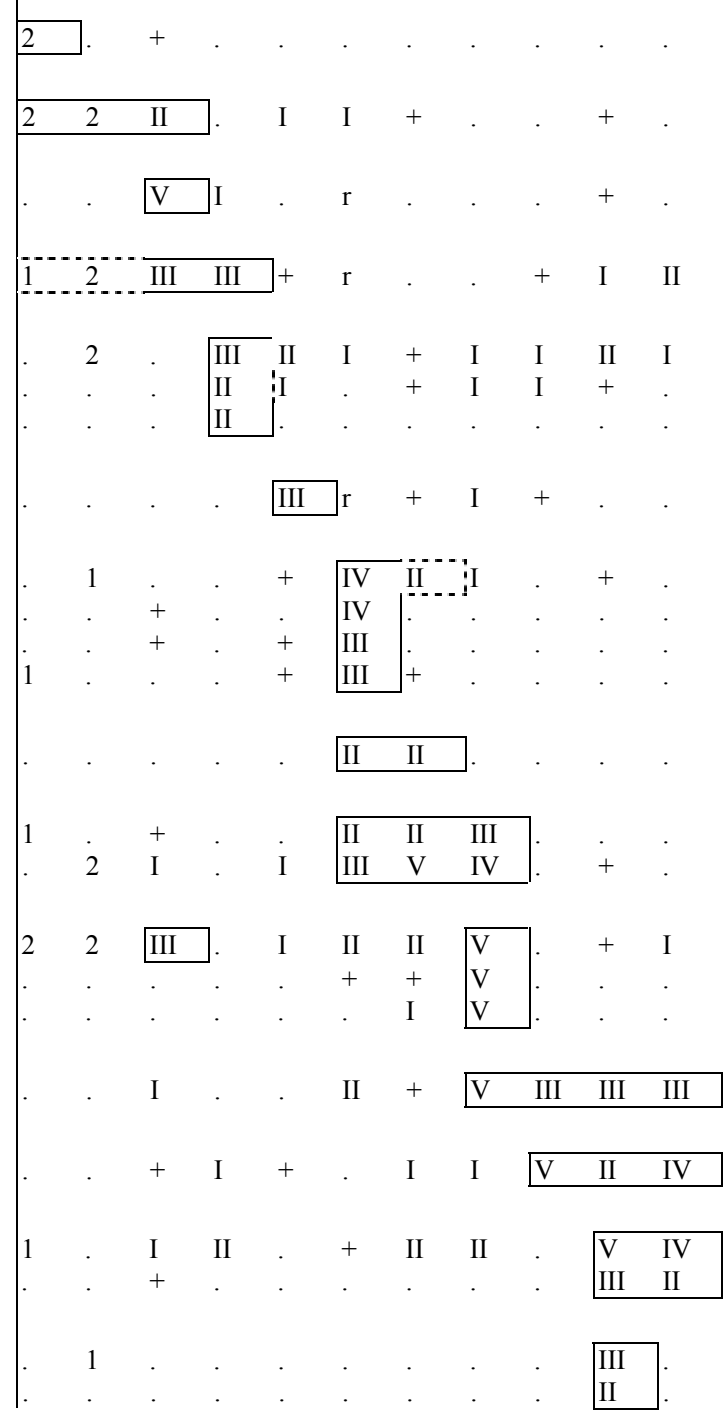


Forts. Tab. 4: Übersichtstabelle Ceratodonto-Polytrichetea piliferi

\begin{tabular}{|c|c|c|c|c|c|c|c|c|c|c|c|c|}
\hline \multirow{2}{*}{\multicolumn{2}{|c|}{ Einheit }} & \multicolumn{4}{|c|}{1} & \multicolumn{4}{|c|}{2} & \multicolumn{3}{|c|}{3} \\
\hline & & 1.1 & 1.2 & 1.3 & 1.4 & 2.1 & 2.2 & 2.3 & 2.4 & 3.1 & 3.2 & 3.3 \\
\hline \multicolumn{13}{|c|}{ D 2 + 3: } \\
\hline \multirow[t]{2}{*}{$\mathrm{OC}$} & Trapeliopsis granulosa & 1 & 2 & . & . & II & II & II & . & IV & $\mathrm{I}$ & \\
\hline & Cladonia merochlorophaea & 1 & 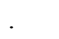 & + & II & $\mathrm{V}$ & III & II & & IV & III & . \\
\hline \multirow[t]{2}{*}{$\mathrm{OC}$} & Cladonia macilenta ssp. floerkeana & 2 & 2 & II & I & $\mathrm{V}$ & III & $\mathrm{I}$ & I & V & III & . \\
\hline & Cladonia macilenta ssp. macilenta & 1 & . & . & II & $\mathrm{V}$ & I & . & I & III & IV & 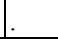 \\
\hline $\mathrm{OC}$ & Cladonia subulata & . & 1 & I & . & IV & $\mathrm{I}$ & I & I & IV & IV & $\mathrm{V}$ \\
\hline \multicolumn{13}{|c|}{ OC Peltigeretalia: } \\
\hline $\mathrm{OC}$ & Cladonia coccifera & 2 & 2 & I & . & II & II & II & II & . & I & . \\
\hline $\mathrm{FC}_{2}$ & Cladonia ramulosa & . & 1 & . & . & I & + & . & . & . & II & . \\
\hline $\mathrm{FC}_{2}$ & Cladonia phyllophora & . & . & . & . & . & + & I & I & . & . & $\mathrm{I}$ \\
\hline & Cetraria islandica & . & . & + & . & . & + & + & . & . & . & . \\
\hline $\mathrm{UC}_{1.4}$ & Placynthiella uliginosa & . & . & + & I & + & . & . & . & + & . & . \\
\hline $\mathrm{UC}_{2.2}$ & Cladonia ciliata & . & . & . & . & . & I & . & . & . & . & . \\
\hline $\mathrm{OC}$ & Peltigera didactyla & . & . & . & . & . & $\mathrm{r}$ & . & . & . & . & $\mathrm{I}$ \\
\hline $\mathrm{FC}_{2}$ & Cladonia crispata & . & . & . & . & . & $\mathrm{r}$ & . & . & . & . & . \\
\hline Begle & iter Kryptogamen: & & & & & & & & & & & \\
\hline & Cephaloziella spec. & 2 & 2 & II & III & II & II & I & III & I & III & III \\
\hline & Hypnum cupressiforme s.l. & . & 1 & I & II & II & II & IV & $\mathrm{I}$ & I & III & $\mathrm{I}$ \\
\hline & Chlorophyta div. spec. & 1 & . & IV & III & III & $\mathrm{r}$ & II & . & III & I & II \\
\hline & Pohlia nutans & 1 & . & I & III & III & II & I & . & II & + & III \\
\hline & Dicranum scoparium & 1 & 1 & I & II & II & II & II & I & + & + & . \\
\hline & Polytrichum formosum & . & . & I & . & II & I & + & . & II & II & $\mathrm{I}$ \\
\hline & Cladonia glauca & 1 & . & . & . & II & II & II & II & . & . & . \\
\hline & Cladonia cryptochlorophaea & 1 & . & I & II & II & + & . & . & . & I & . \\
\hline & Pleurozium schreberi & . & . & + & . & . & II & + & . & I & $\mathrm{I}$ & $\mathrm{I}$ \\
\hline & Cladonia squamosa & . & 1 & . & I & I & II & I & . & . & + & . \\
\hline & Cladonia novochlorophaea & . & . & + & I & I & $\mathrm{I}$ & I & . & + & . & . \\
\hline & Campylopus introflexus & . & . & . & I & II & I & + & . & II & . & . \\
\hline & Cladonia spec. & . & . & II & . & $\mathrm{I}$ & $\mathrm{r}$ & I & I & I & + & . \\
\hline & Cyanophyta div. spec. & 1 & . & I & . & . & + & . & I & + & + & II \\
\hline & Cladonia coccifera/deformis agg. & . & . & I & . & I & + & . & I & . & + & . \\
\hline & Cladonia coniocraea & . & . & + & . & I & + & . & . & + & + & I \\
\hline & Polytrichum juniperinum & . & . & + & . & I & $\mathrm{r}$ & I & . & . & + & $\mathrm{I}$ \\
\hline & Dicranaceae spec. & . & . & $\mathrm{I}$ & I & I & . & . & . & II & . & . \\
\hline & Cladonia pyxidata ssp. pyxidata & . & . & + & . & . & + & . & I & . & + & $\mathrm{I}$ \\
\hline & Ditrichum heteromallum & . & . & II & I & . & . & . & . & . & . & . \\
\hline & diverse Algen & . & . & . & I & I & $\mathrm{r}$ & . & . & . & . & . \\
\hline & Dicranella heteromalla & 1 & . & + & II & + & . & . & . & . & . & . \\
\hline & Rhytidiadelphus squarrosus & . & . & + & . & . & $\mathrm{r}$ & + & . & . & . & $\mathrm{I}$ \\
\hline & Cladonia cf. cornuta & . & . & + & . & . & . & . & . & . & I & $\mathrm{I}$ \\
\hline & Buxbaumia aphylla & 1 & . & . & . & + & $\mathrm{r}$ & . & . & + & . & . \\
\hline & Musci spec. & . & . & I & . & + & . & . & . & . & + & . \\
\hline & Racomitrium lanuginosum & . & . & . & . & . & + & + & . & . & . & $\mathrm{I}$ \\
\hline & Racomitrium heterostichum agg. & . & . & . & . & . & $\mathrm{r}$ & + & . & . & . & $\mathrm{I}$ \\
\hline & Lepraria rigidula & 1 & . & . & I & . & . & + & . & . & . & . \\
\hline & Lepraria lobificans & . & . & + & I & + & . & . & . & . & . & . \\
\hline & Bryophyta spec. & . & . & + & . & + & . & . & . & . & + & . \\
\hline & Diplophyllum albicans & . & . & . & II & . & . & . & . & . & . & . \\
\hline & Cladonia digitata & . & . & . & II & . & . & . & . & . & . & . \\
\hline & Bryum spec. & . & . & . & . & . & . & . & . & . & + & II \\
\hline Pha & erogamen: & & & & & & & & & & & \\
\hline & Deschampsia flexuosa & 1 & 1 & II & II & II & III & II & . & III & II & $\mathrm{I}$ \\
\hline & Festuca ovina agg. & . & . & III & I & I & II & II & II & + & II & . \\
\hline & Calluna vulgaris & . & . & IV & III & II & $r$ & . & . & + & II & II \\
\hline & Agrostis capillaris & . & . & II & . & . & . & . & . & . & II & $\mathrm{I}$ \\
\hline & Hieracium pilosella & . & . & + & . & . & I & I & . & . & + & . \\
\hline & Hieracium spec. & . & . & $\mathrm{I}$ & . & . & $\mathrm{r}$ & . & . & + & + & III \\
\hline & Rumex acetosella & . & . & . & . & . & $\mathrm{r}$ & I & II & . & I & . \\
\hline & Vaccinium myrtillus & . & . & I & . & + & . & + & . & + & + & . \\
\hline & Festuca rubra agg. & . & . & . & . & . & . & . & . & . & I & II \\
\hline & Nardus stricta & . & . & . & II & I & . & . & . & . & . & . \\
\hline & Carex spec. & . & . & + & . & + & $\mathrm{r}$ & . & . & . & . & $\mathrm{I}$ \\
\hline & Scleranthus annuus agg. & . & . & . & . & . & . & + & II & . & . & . \\
\hline & Achillea millefolium & . & . & . & . & . & $\mathrm{r}$ & . & . & . & + & I \\
\hline & Galium saxatile & . & . & + & . & . & . & + & . & . & + & . \\
\hline & Hieracium lachenalii & $\cdot$ & . & + & I & . & . & . & . & . & + & . \\
\hline & Holcus lanatus & . & . & . & . & . & . & . & . & + & + & $\mathrm{I}$ \\
\hline
\end{tabular}




\section{Ergänzung zu Tab. 4}

Außerdem mit Stetigkeit $<$ II und in weniger als 3 Einheiten:

Agrostis stolonifera 10:+; Andreaea rupestris 11:I; Arthrorhaphis citrinella 3:I; Betula pendula juv. 10:I; Brachythecium rutabulum 3:+; Brachythecium spec. 10:+; Calliergonella cuspidata 10:+; Campanula rotundifolia 3:+; Campylopus flexuosus 5:+, 11:I; Carex ornithopoda 5:+; Carex pilulifera 5:+; Centaurea jacea 11:I; Cerastium semidecandrum 7:+; Cladonia caespiticia 9:I; Cladonia pleurota 5:I; Cladonia polydactyla 5:I; Cladonia pyxidata 3:+; Cladonia pyxidata ssp. grayi 7:+; Cladonia scabriuscula 3:+, 7:+; Cladonia strepsilis 6:r; Dianthus carthusianorum 8:I; Dicranodontium denudatum 5:+; Dicranum montanum 5:I; Dicranum polysetum 5:+; Ditrichum spec. 5:+, 11:I; Epilobium spec. 11:I; Erophila verna 8:I; Galium glaucum 8:I; Helictotrichon pratense 7:+; Hepaticae spec. 3:+; Hypericum hirsutum 10:+; Hypochaeris radicata 6:r, 10:+; Juncus effusus 11:I; Jungermannia gracillima 3:I; Jungermannia spec. 3:+; Leontodon autumnalis 10:+; Lepidozia reptans 4:I; Lepraria spec. 8:I; Leucobryum glaucum 5:+; Linum catharticum 10:+; Lophocolea cf. heterophylla 7:+; Lophocolea heterophylla 4:I, 11:I; Lophoziaceae spec. 9:+; Luzula campestris 11:I; Luzula campestris agg. 3:+, 7:+; Luzula spec. 6:r; Melampyrum arvense 6:r; Micarea leprosula 6:r; Micarea peliocarpa 10:+; Neofuscelia verruculifera 8:I; Picea abies juv. 5:I; Pimpinella saxifraga 10:+; Pinus sylvestris juv. 3:+; Plagiothecium laetum 4:I; Polytrichum commune 7:+,10:+; Populus tremula juv. 11:I; Potentilla anserina 10:+; Potentilla erecta 3:+, 4:I; Pottiaceae spec. 5:+, 6:r; Psilolechia lucida 5:+; Ptilidium ciliare 5:+; Quercus petraea juv. 6:r; Quercus spec. 7:+; Racomitrium canescens s.1. 6:r; Rumex acetosa 6:+; Scleropodium purum 10:I, 11:I; Sedum rupestre agg. 8:I; Silene spec. 7:+; Tetraphis pellucida 4:I; Thymus praecox 8:I; Trapeliopsis pseudogranulosa 3:+, 5:+; Trifolium arvense 8:I; Trifolium spec. 7:+; Vaccinium vitis-idaea 9:+; Veronica spec. 10:+; Veronica verna agg. 4:I, 8:I; Viola arvensis 7:+; Weissia spec. 5:+.

\subsubsection{Baeomycion rosei}

(Tab. 5; Tab. 6 im Anhang 3)

Das Baeomycion rosei vereinigt acidophytische Erdkrusten-Vereine auf Sand- und Rohhumusböden von der Niederung bis in die alpine Region (KLEMENT 1955). Die raschwüchsigen Erdkrusten der Federation können extreme Standortbedingungen gut meistern und für andere Kryptogamen eine besiedelbare Unterlage schaffen (SCHÖLLER 1991). In meinem nordhessischen Aufnahmematerial lassen sich innerhalb der Federation 3 Unionen und eine Fragmentgesellschaft unterscheiden.

\section{Tab. 5: Stetigkeitstabelle Baeomycion rosei}

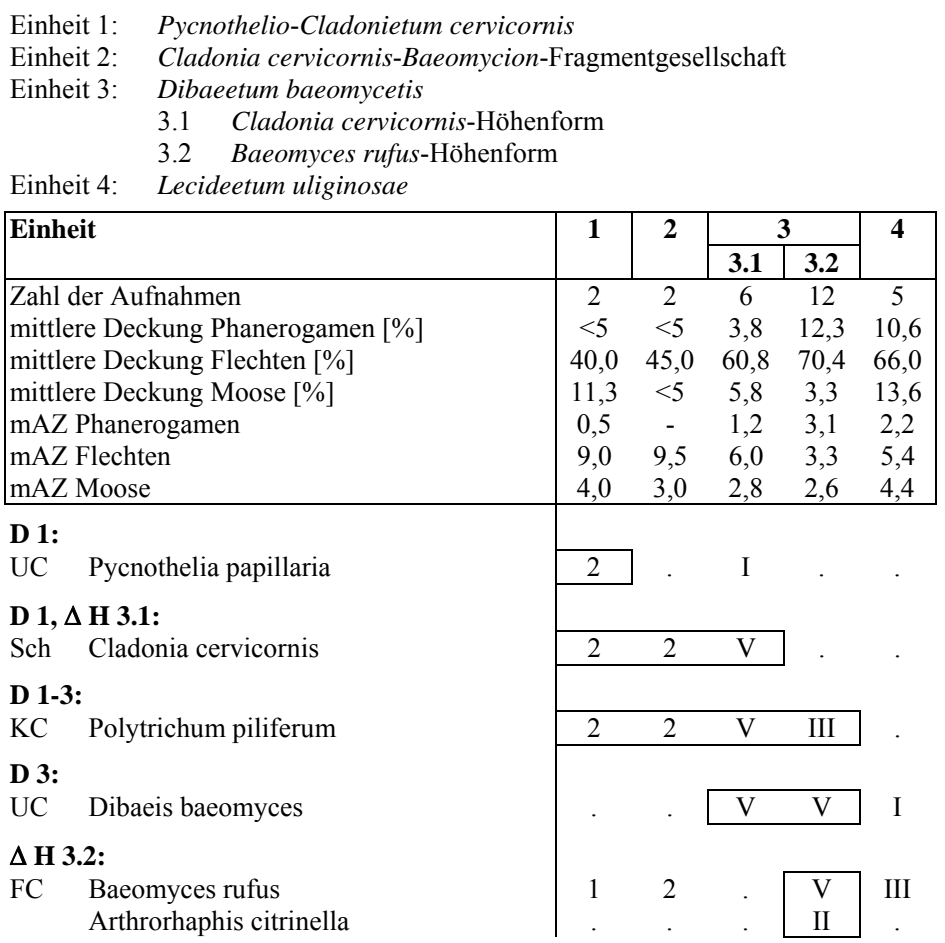


Forts. Tab. 5: Stetigkeitstabelle Baeomycion rosei

\begin{tabular}{|c|c|c|c|c|c|c|}
\hline \multirow{2}{*}{\multicolumn{2}{|c|}{ Einheit }} & \multirow[t]{2}{*}{1} & \multirow[t]{2}{*}{2} & \multicolumn{2}{|c|}{3} & \multirow[t]{2}{*}{4} \\
\hline & & & & 3.1 & 3.2 & \\
\hline \multicolumn{7}{|l|}{ D 4: } \\
\hline Sch & Placynthiella icmalea & . & 2 & . & . & III \\
\hline $\mathrm{UC}$ & Placynthiella oligotropha & . & . & . & . & II \\
\hline & Cladonia macilenta ssp. macilenta & 1 & . & . & . & II \\
\hline & Trapeliopsis gelatinosa & . & . & . & . & II \\
\hline $\mathrm{UC}$ & Placynthiella uliginosa & . & . & . & + & I \\
\hline \multicolumn{7}{|c|}{ OC Peltigeretalia: } \\
\hline & Cladonia furcata ssp. furcata & . & 2 & II & . & . \\
\hline & Cladonia coccifera & 2 & 2 & II & . & . \\
\hline & Cladonia macilenta ssp. floerkeana & 2 & 2 & II & II & I \\
\hline & Cladonia subulata & . & 1 & $\mathrm{I}$ & I & . \\
\hline & Trapeliopsis granulosa & 1 & 2 & . & . & . \\
\hline & Cetraria aculeata & 1 & . & $\mathrm{I}$ & . & . \\
\hline & Cladonia ramulosa & . & 1 & . & . & . \\
\hline & Cladonia rei & . & 1 & . & . & . \\
\hline & Cladonia arbuscula & . & . & $\mathrm{I}$ & . & . \\
\hline & Cladonia portentosa & . & . & $\mathrm{I}$ & . & . \\
\hline & Cladonia humilis & . & . & . & + & . \\
\hline & Cetraria islandica & . & . & $\mathrm{I}$ & . & . \\
\hline & Cladonia gracilis & . & 1 & . & . & . \\
\hline & Cladonia uncialis & 1 & . & . & . & . \\
\hline \multicolumn{7}{|c|}{ Begleiter Kryptogamen: } \\
\hline & Chlorophyta div. spec. & 1 & . & IV & IV & III \\
\hline & Cephaloziella spec. & 2 & 2 & II & II & III \\
\hline & Hypnum cupressiforme s.l. & . & 1 & I & I & II \\
\hline & Cladonia cryptochlorophaea & 1 & . & II & + & II \\
\hline & Pohlia nutans & 1 & . & II & + & III \\
\hline & Dicranum scoparium & 1 & 1 & II & . & II \\
\hline & Cladonia pyxidata ssp. chlorophaea & 1 & . & . & I & II \\
\hline & Cladonia merochlorophaea & 1 & . & $\mathrm{I}$ & . & II \\
\hline & Ditrichum heteromallum & . & . & . & II & I \\
\hline & Cladonia spec. & . & . & III & + & . \\
\hline & Cladonia coccifera/deformis agg. & . & . & III & . & . \\
\hline & Dicranella heteromalla & 1 & . & I & . & II \\
\hline & Cyanophyta div. spec. & 1 & . & II & . & . \\
\hline & Polytrichum formosum & . & . & . & II & . \\
\hline & Ceratodon purpureus & . & . & . & II & . \\
\hline & Cladonia digitata & . & . & . & . & II \\
\hline & Diplophyllum albicans & . & . & - & . & II \\
\hline & Dicranella cf. heteromalla & . & . & II & . & . \\
\hline \multicolumn{7}{|c|}{ Phanerogamen: } \\
\hline & Deschampsia flexuosa & 1 & 1 & III & II & II \\
\hline & Festuca ovina agg. & . & . & $\mathrm{I}$ & III & I \\
\hline & Calluna vulgaris & . & . & I & $\mathrm{V}$ & III \\
\hline & Agrostis capillaris & . & . & . & II & . \\
\hline & Nardus stricta & . & . & . & . & II \\
\hline
\end{tabular}

Außerdem mit Stetigkeit < II und in weniger als 3 Einheiten:

Brachythecium rutabulum 4:+; Bryophyta spec. 4:+; Buxbaumia aphylla 1:1; Campanula rotundifolia 4:+; Campylopus introflexus 5:I; Carex spec. 4:+; Cladonia cf. cornuta 4:+; Cladonia coniocraea 4:+; Cladonia fimbriata 4:+, 5:I; Cladonia glauca 1:1; Cladonia novochlorophaea 4:+, 5:I; Cladonia pyxidata 3:I; Cladonia pyxidata ssp. pyxidata 3:I; Cladonia scabriuscula 4:+; Cladonia squamosa 2:1, 5:I; Dicranaceae spec. 5:I; diverse Algen 5:I; Galium saxatile 4:+; Hepaticae spec. 3:I; Hieracium lachenalii 4:+, 5:I; Hieracium pilosella 3:I; Hieracium spec. 4:I; Jungermannia gracillima 4:I; Jungermannia spec. 4:+; Lepidozia reptans 5:I; Lepraria lobificans 3:I, 5:I; Lepraria rigidula 1:1, 5:I; Lophocolea heterophylla 5:I; Luzula campestris agg. 4:+; Musci spec. 4:I; Pinus sylvestris juv. 3:I; Plagiothecium laetum 5:I; Pleurozium schreberi 3:I; Polytrichum juniperinum 4:+; Potentilla erecta 4:+, 5:I; Rhytidiadelphus squarrosus 4:+; Tetraphis pellucida 5:I; Trapeliopsis pseudogranulosa 3:I; Vaccinium myrtillus 4:I; Veronica verna agg. 5:I. 


\subsubsection{Dibaeetum baeomycetis}

(Tab. 5; Tab. 6 (Einheit 3) im Anhang 3; Taf. 1.1 im Anhang 2)

Syn.: Baeomyces roseus-Bestand (BIERMANN et al. 1994), Baeomyces-Facies $($ Sommer 1972) $=$ Synonym für Baeomyces rufus-dominierte Bestände ohne Dibaeis baeomyces.

\section{a) Floristische Zusammensetzung, Physiognomie und Struktur}

Das Dibaeetum baeomycetis grenzt sich durch Dibaeis baeomyces und durch das kleinwüchsige, für sonnige Standorte typische Moos Polytrichum piliferum gegen das Lecideetum uliginosae ab. Dibaeis ist die einzige Charakterart der Gesellschaft.

Das Dibaeetum baeomycetis kommt im Untersuchungsgebiet in Höhenlagen zwischen 300 und $780 \mathrm{~m}$ ü. NN vor. Es können zwei Höhenformen unterschieden werden. Die Cladonia cervicornis-Höhenform (Einheit 3.1) ist in tieferen Lagen, in 300$400 \mathrm{~m}$ ü. NN anzutreffen. In höheren Lagen, zwischen (410) 660 und $780 \mathrm{~m}$ ü. NN tritt dagegen die Baeomyces rufus-Höhenform (Einheit 3.2) auf. Beide Höhenformen werden jeweils durch die namengebende Art differenziert. Ordnungscharakterarten der Peltigeretalia kommen nur in geringer Stetigkeit vor, sind aber in der Cladonia cervicornis-Höhenform etwas häufiger vertreten.

Das Dibaeetum baeomycetis ist eine artenarme Flechtengesellschaft. Die mittlere Artenzahl der Flechten beträgt 6,0 in der Cladonia- und 3,3 in der BaeomycesHöhenform. An der mittleren Gesamtdeckung von $74 \%$ in der Cladonia-Höhenform und $83 \%$ in der Baeomyces-Höhenform haben die Flechten mit 61 bzw. $70 \%$ hohen Anteil. Kümmerlich entwickelte Phanerogamen, wie etwa Calluna vulgaris, dringen meist nur randlich in die relativ geschlossenen Bestände ein und auch Moose spielen nur eine untergeordnete Rolle. Grünalgen können dagegen in einigen Fällen 15 bis 30 \% Deckung aufweisen.

Physiognomisch handelt es sich um Krustenflechten-dominierte Bestände, in denen Becher- und Strauchflechten allenfalls geringe Deckungsgrade erreichen. Die dominante Art kann dabei sowohl Dibaeis baeomyces als auch Baeomyces rufus sein. Reinbestände von Baeomyces rufus sind im Untersuchungsgebiet gelegentlich an beschatteten Erdanrissen von Wegböschungen über saurem Ausgangsgestein zu finden, allerdings in der Regel nur kleinflächig ausgebildet. PAUS (1997) bezeichnet derartige Bestände als Baeomyces rufus-Baeomycion rosei-Fragmentgesellschaft. Aufnahme 22 in Tab. 6 entspricht dieser Gesellschaft, da es sich aber um die einzige derartige Aufnahme aus dem Untersuchungsgebiet handelt, wurde sie im vorliegenden Fall der Baeomyces-Höhenform des Dibaeetum angegliedert.

Am Orenberg bei Willingen tritt als floristische Besonderheit Arthrorhaphis citrinella in der Baeomyces rufus-Höhenform auf. Arthrorhaphis parasitiert anfangs häufig auf Baeomyces-Arten (WIRTH 1995). Sie wurde im Untersuchungsgebiet nur an dieser einen Lokalität gefunden und wird in der Roten Liste von Hessen (WIRTH et al. 1996) mit Gefährdungsgrad 2 eingestuft (s. Kap. E6.1). 


\section{b) Standorte und Vorkommen im UG}

Das Dibaeetum baeomycetis ist in Nordhessen wenig verbreitet. Nur an wenigen Wuchsorten, z.B. in den Hochheiden des Waldecker Uplandes (MTB 4717/18), treten gut entwickelte Bestände auf. Die Union wurde im Untersuchungsgebiet mit insgesamt 18 Aufnahmen in 10 Teilgebieten erfaßt. Die Baeomyces-Höhenform ist in den Hochheiden am besten entwickelt. Es handelt sich dabei um Bereiche, die vor etwa 20 Jahren abgeplaggt wurden (WELTICKE mündl.) und nun ein Mosaik von größeren und kleineren

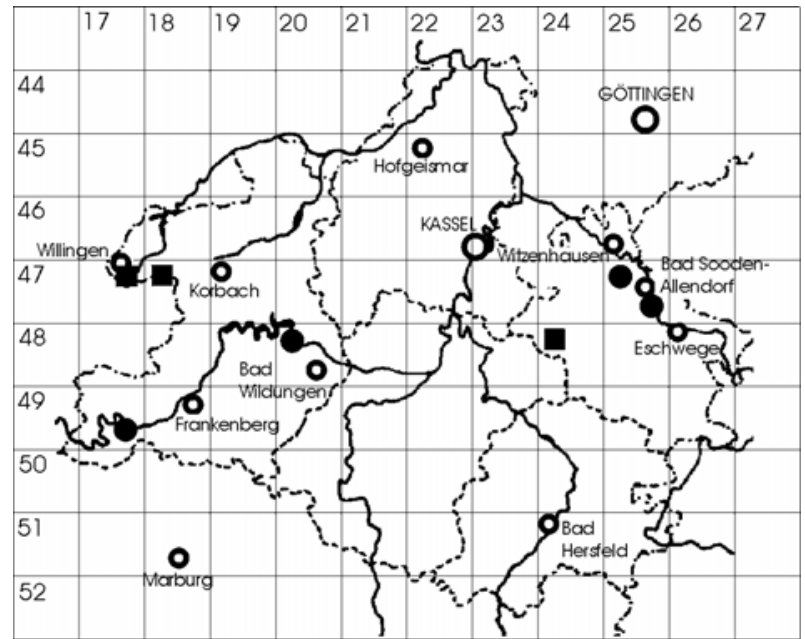

Abb. 8: Verbreitung des Dibaeetum baeomycetis im Untersuchungsgebiet: Cladonia-Höhenform; Baeomyces-Höhenform. Flecken mit Flechtenbewuchs sowie Calluna-Beständen darstellen. Weiter kommt das Dibaeetum an Wegrändern (z.B. am Ettelsberg bei Willingen) oder Erdanrissen von Wegböschungen vor, also auf etwas verdichteten Böden (s. auch BIERMANN et al. 1994). Die Größe der Bestände variiert erheblich: sie kann nur wenige Quadratdezimeter betragen, in abgeplaggten Heideflächen jedoch auch bei mehr als $10 \mathrm{~m}^{2}$ liegen. Das Abplaggen der Heide wirkt sich demnach ausgesprochen günstig auf diese Union aus.

Im Gegensatz zu Dibaeis baeomyces kommt Baeomyces rufus auch an schattig-feuchten Stellen vor. Die Art gedeiht optimal bei ausreichender Substrat- und/oder hoher Luftfeuchte, meist an ziemlich lichtarmen Standorten (SCHÖLLER 1991). Wohl aus diesen Gründen gibt ScHÖLLER für den Taunus an, die Art trete fast nie im Baeomycion rosei auf. In den niederschlagsreichen Berglagen Nordhessens sind die Standortbedingungen für Baeomyces rufus jedoch so günstig, daß sich sogar eine Baeomyces rufus-Höhenform ausbilden kann. Rötliche Eisenkonkretionen im Boden weisen an dem am tiefsten gelegenen Baeomyces-Standort in der Zeche Glimmerode (MTB 4824) auf wechselfeuchte Bedingungen hin, wie sie auch BIERMANN et al. (1994) für Baeomyces-Dominanzbestände angeben. Die Cladonia cervicornis-Höhenform wurde überwiegend in Traubeneichenwäldern am Edersee (MTB 4820) bzw. im Gebiet des Hohen Meißners (MTB 4725) gefunden. Aufgrund der trockeneren Standortverhältnisse bleibt Baeomyces rufus in diesen Wäldern auf wenige Stellen, auf substratfeuchte Erdanrisse an Wegrändern beschränkt. Beide Höhenformen stehen somit auch für eine unterschiedlich gute Feuchtigkeitsversorgung der jeweiligen Standorte.

Das Dibaeetum baeomycetis ist als Pioniergesellschaft einzustufen und kann bei fortschreitender Vegetationsentwicklung von Phanerogamen oder, sofern die Standortbedingungen für Flechtenbewuchs günstig bleiben, von Gesellschaften des Cladonion arbusculae (s. Kap. E2.1.2) abgelöst werden. Das Pycnothelio-Cladonietum cervicornis (s. Kap. E2.1.1.3) als Folgegesellschaft dürfte in Nordhessen nur an 
wenigen Standorten in Traubeneichenwäldern eine Rolle spielen. Das Dibaeetum baeomycetis siedelt meist auf skelettreichen Böden über Grauwacke bzw. Tonschiefer. Die Böden haben allenfalls eine dünne Streuauflage. Die $\mathrm{pH}(\mathrm{KCl})$-Werte liegen zwischen 3,4 und 4,3 (s. Tab. 11). Die Standorte sind überwiegend sonnig bzw. werden nur gelegentlich beschattet.

\subsubsection{Lecideetum uliginosae}

(Tab. 5; Tab. 6 (Einheit 4) im Anhang 3)

Syn.: Lecidea uliginosa-Gesellschaft (TOBLER \& MATTICK 1938), Lecidea granulosa-uliginosa-Assoziation (LANGERFELDT 1939), Baeomycetum rufi Duvigneaud 1939 p.p., Biatoretum uliginosae Langerfeldt ex. Klement 1947, Placynthiella uliginosa-Verein (DANIELS et al. 1993).

\section{a) Floristische Zusammensetzung, Physiognomie und Struktur}

Die Trennartengruppe dieser Gesellschaft wird gebildet aus Placynthiella icmalea, P. oligotropha, P. uliginosa, Trapeliopsis gelatinosa und Cladonia macilenta ssp. macilenta. Placynthiella oligotropha und P. uliginosa sind als Charakterarten einzustufen (s. PAUS 1997), im vorliegenden Aufnahmematerial jedoch ziemlich selten. Im Untersuchungsgebiet wesentlich häufiger ist die verwandte Art Placynthiella icmalea, die zwar in den anderen Federationen der Peltigeretalia ebenfalls auftritt, ihren Schwerpunkt aber im Lecideetum uliginosae hat.

Bezüglich der Dominanzverhältnisse erweisen sich die Bestände als recht uneinheitlich. Für das Untersuchungsgebiet sind Dominanzbestände von Placynthiella oligotropha, Trapeliopsis gelatinosa und Placynthiella icmalea durch Aufnahmen belegt. Letztere sind mit den ranglosen Placynthiella icmalea-Beständen bei BIERMANN et al. (1994) gleichzusetzen, welche im Gegensatz zu Baeomyces- bzw. Dibaeis-Beständen Böden mit höherem Humusgehalt bevorzugen sollen. Dem Trapeliopsis gelatinosaDominanzbestand (Tab. 6, Aufn. 27) fehlen die Kennarten des Lecideetum, es sind jedoch auch keine Charakterarten vorhanden, die eine Einordnung in eine der anderen Unionen zuließen. Aufgrund der physiognomischen Ähnlichkeit wird der Bestand daher dem Lecideetum angeschlossen.

Das Lecideetum ist geprägt von den dunkelbraun bis braungrün gefärbten Krusten der Placynthiella-Arten, in die gelegentlich Becherflechten und einige meist kleinwüchsige Moose eingestreut sind. Die mittlere Artenzahl der Flechten liegt bei 5,4, die der Moose bei 4,4 und die der Phanerogamen lediglich bei 2,2. Die dominierende Artengruppe sind die Flechten, die im Mittel mit $66 \%$ den bei weitem größten Anteil an der Gesamtdeckung von durchschnittlich $86 \%$ haben. 


\section{b) Standorte und Vorkommen im UG}

Das Lecideetum uliginosae konnte in Nordhessen nur durch 5 Aufnahmen an 3 Wuchsorten belegt werden und ist daher $\mathrm{zu}$ den seltenen Flechtengesellschaften zu zählen. Die Vegetationsaufnahmen stammen überwiegend aus Buntsandsteingebieten, wo die Union bei guter Entwicklung mehrere Quadratmeter bedecken kann. Die zugehörigen, sauren Böden weisen $\mathrm{pH}(\mathrm{KCl})$-Werte von 3,2 bis 3,8 auf. Die elektrolytische Leitfähigkeit des Bodens ist gering (Median $85 \mu \mathrm{S} / \mathrm{cm}$ ), liegt jedoch

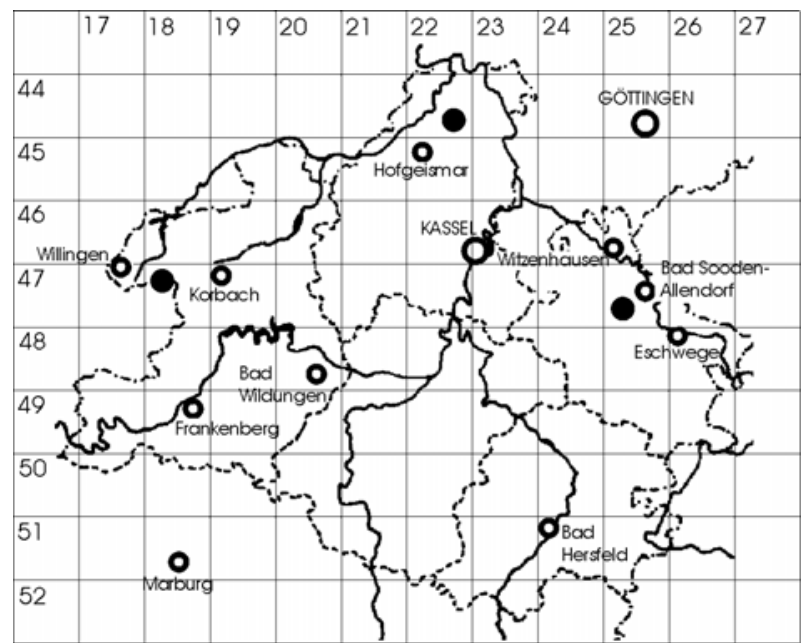

Abb. 9: Verbreitung des Lecideetum uliginosae im Untersuchungsgebiet. deutlich über den Werten der anderen Baeomycion-Gesellschaften im Untersuchungsgebiet (s. Tab. 11). Die Standorte sind bezüglich der Feuchtigkeitsverhältnisse als frisch einzustufen und sonnig bis halbschattig. Die Union tritt an offenen Stellen innerhalb von Calluna vulgarisBeständen auf oder besiedelt Erdanrisse, beispielsweise an Wegböschungen in bodensauren Buchenwäldern (Luzulo-Fagetum). Wie das Dibaeetum baeomycetis hat auch das Lecideetum Pioniercharakter. Anhand der wenigen Aufnahmen lassen sich allerdings kaum Aussagen über die weitere Vegetationsentwicklung machen; an den Wegböschungen dürfte die Gesellschaft jedoch zumindest so lange Bestand haben, bis entweder neue Erdbewegungen den Flechtenbewuchs zerstören oder Phanerogamen die konkurrenzschwachen Flechten überwachsen. Nach SOMMER (1972) ist die Gesellschaft resistenter gegenüber Tritteinwirkungen als Baeomyces-Bestände und kann in Heideflächen im Sukzessionsverlauf in ein Cladonietum mitis übergehen.

\subsubsection{Pycnothelio-Cladonietum cervicornis und Cladonia cervicornis- Baeomycion rosei-Fragmentgesellschaft}

(Tab. 5; Tab. 6 (Einheiten 1+2) im Anhang 3)

Syn.: Cladonia cervicornis-Assoziation (LANGERFELDT 1939)

\section{a) Floristische Zusammensetzung, Physiognomie und Struktur}

Das Pycnothelio-Cladonietum cervicornis ist im Untersuchungsgebiet nur schlecht repräsentiert. Lediglich in zwei erfaßten Beständen kommt die bei PAUS (1997) als Charakterart der Gesellschaft angegebene Pycnothelia papillaria vor. Sie ist im Untersuchungsgebiet generell sehr selten, es konnten nur 3 Fundorte notiert werden (s. Artenliste im Anhang 1). Die zweite bei PAUs (1997) aufgeführte Unionscharakterart Cladonia strepsilis hat in Nordhessen ebenfalls nur wenige Vorkommen, sie wurde im Rahmen der vorliegenden Untersuchungen nur ein einziges Mal, allerdings in einem Cladonion arbusculae-Bestand, entdeckt. 
Bei Einheit 2 handelt es sich um eine artenarme Form der Gesellschaft, nur gekennzeichnet durch das Auftreten von Cladonia cervicornis, die jedoch nicht als Unionscharakterart, sondern lediglich als Schwerpunktart des Pycnothelio-Cladonietum cervicornis gewertet werden kann (PAUS 1997). Aufgrund des Fehlens beider Unionscharakterarten und des Auftretens der Federationscharakterart Baeomyces rufus werden diese beiden Bestände als Cladonia cervicornis-Baeomycion roseiFragmentgesellschaft eingestuft.

Sowohl im Pycnothelio-Cladonietum cervicornis als auch in der Cladonia cervicornisBaeomycion rosei-Fragmentgesellschaft spielen Ordnungscharakterarten der Peltigeretalia eine gewisse Rolle, z.B. Cladonia coccifera s.str. und Cl. macilenta ssp. floerkeana. Sie zeigen, ebenso wie Cl. subulata und Trapeliopsis granulosa, die Nähe zu Gesellschaften des Cladonion arbusculae an, als deren Vorstufe das PycnothelioCladonietum cervicornis auf manchen Standorten gelten kann (s. BIERMANN et al. 1994). Die wenigen Bestände im Untersuchungsgebiet siedeln jedoch an Hängen in Traubeneichenwäldern und stellen dort vermutlich Dauerstadien der Flechtenbesiedlung dar, da auf diesen Standorten eine Entwicklung hin zum Cladonion arbusculae aufgrund der Instabilität des Substrates nicht zu erwarten ist.

Die Bestände sind sehr lückig, auf größerer Fläche zeigt sich offener Boden sowie das verwitterte Ausgangsgestein. Cladonien bestimmen mit ihren Primärthalli das Erscheinungsbild. Podetien werden, zumindest bei Cl. cervicornis, selten ausgebildet. In den wenigen Fällen, in denen bei Cl. cervicornis Podetien auftreten, lassen diese erkennen, daß es sich um die Subspezies verticillata handeln muß. Krustenflechten spielen nur eine untergeordnete Rolle. Mit durchschnittlich 9,3 Flechtenarten sind beide Gesellschaften artenreicher als die anderen Baeomycion-Gesellschaften des Untersuchungsgebietes. Als eine floristische Besonderheit der Bestände ist das Koboldmoos Buxbaumia aphylla zu nennen.

\section{b) Standorte und Vorkommen im UG}

Außer dem oben genannten Faktor der Substratinstabilität sind die Standorte gekennzeichnet durch skelettreiche Böden über Grauwacke bzw. Tonschiefer, deren $\mathrm{pH}(\mathrm{KCl})-$ Wert bei 3,3 bis 4,2 liegt und die nur geringe Wasserhaltekapazität besitzen. Die Streuauflage ist gering bis fehlend. Durch die Kronen der umstehenden, schlechtwüchsigen Eichen gelangt reichlich Sonnenlicht auf den Boden. Dieser Umstand ermöglicht photophilen Arten wie Cladonia cervicornis ssp. verticillata und Pycnothelia papillaria ein

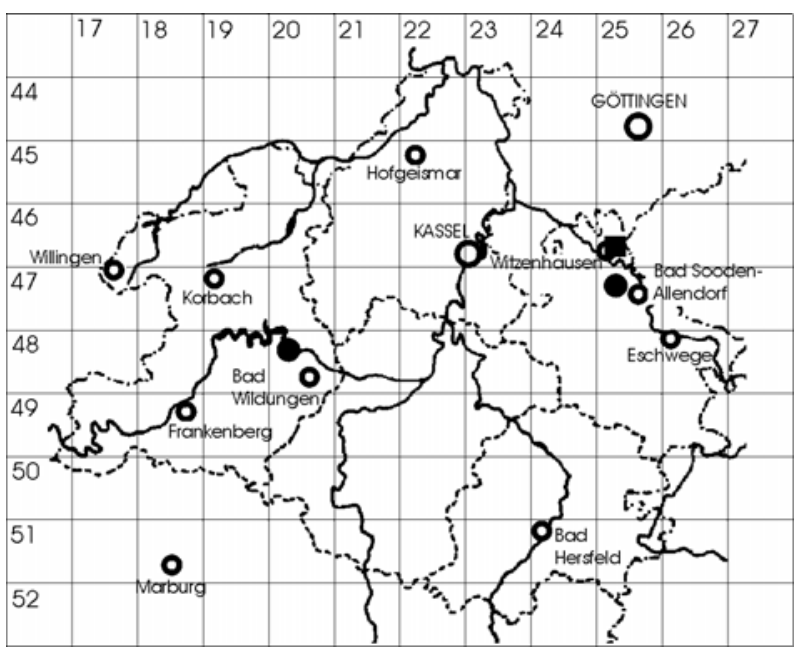

Abb. 10: Verbreitung des Pycnothelio-Cladonietum cervicornis und der Cladonia cervicornisBaeomycion-FG im Untersuchungsgebiet. 
Vorkommen innerhalb des Waldes. Auch wenn die einzelnen Flecken mit Flechtenbewuchs auf dem Waldboden oft sehr klein sind, so können zusammengenommen an den Hängen doch an die $20 \mathrm{~m}^{2}$ große Vorkommen auftreten. Forstwirtschaftlich sind diese ehemaligen Niederwälder im Meißner- und Ederseegebiet (MTB 4725 und 4820), nicht zuletzt wegen der Steilhanglage, heute uninteressant. Eine Beeinträchtigung der Flechtenbestände durch die Forstwirtschaft besteht daher nicht.

\section{Anmerkung:}

Die nordhessischen Bestände sind in etwa den artenarmen Dominanzbeständen von Cl. cervicornis ssp. verticillata (PAUS 1997) gleichzusetzen, welche vor allem an trockenen, stärker exponierten Standorten entstehen sollen. Generell ist das Pycnothelio-Cladonietum cervicornis in Nordwestdeutschland allerdings in erster Linie kennzeichnende Synusie des Genisto-Callunetum. Sie ist typisch für binnenländische Zwergstrauchheiden in subatlantisch getönten Tieflandregionen sowie niederschlagsreichen montanen Lagen, wo sie bevorzugt an Stellen siedelt, an denen der Boden länger feucht bleibt (PAUS 1997). Als Pioniergesellschaft soll die Union für frühe Aufbauphasen der Heiden charakteristisch sein (BIERMANN et al. 1994), konnte jedoch in keiner der untersuchten nordhessischen Heiden nachgewiesen werden. 


\subsubsection{Cladonion arbusculae}

(Tab. 7; Tab. 8 im Anhang 3)

Syn.: Cladonion silvaticae Klement 1950

Die Federation faßt azidiphile Strauchflechten-Gesellschaften auf sterilen Sand- und Rohhumusböden oder auch auf trockenen, zersetzten Stubben zusammen (KLEMENT 1955). Sie ist im Gebiet mit 4 Gesellschaften vertreten.

\subsubsection{Cladonia bacillaris-Gesellschaft}

(Tab. 7; Tab. 8 (Einheit 1) im Anhang 3; Taf. 1.2 im Anhang 2)

Syn.: Cladonia bacillaris-Assoziation (KRIEGER 1937), Cladonia bacillaris-Cladonia glauca-Gesellschaft (TOBLER \& MATTICK 1938), Cladonietum glaucae Daniels 1983, Lecidea-Cladonia floerkeana-Mikrogesellschaft (DANIELS et al. 1987), Placynthiella uliginosa-Cladonia glauca-Verein (DANIELS et al. 1993), Cladonia bacillaris-Mikrogesellschaft (PAUS 1997).

\section{a) Floristische Zusammensetzung, Physiognomie und Struktur}

Trennart der Gesellschaft ist Cladonia grayi s.str. Zur Abtrennung gegen andere Cladonion arbusculae-Gesellschaften kann auch die Gruppe der Grünalgen herangezogen werden. Das Fehlen von Charakterarten läßt eine Einordnung lediglich als ranglose Gesellschaft zu (s. PAUS 1997). Federationscharakterarten (Cladonia uncialis, Cl. portentosa und Cl. ramulosa) sind wenig vertreten und erreichen nur geringe Stetigkeiten, ebenso die Ordnungscharakterarten Cl. coccifera s.str. und Trapeliopsis granulosa. Cl. subulata (OC) tritt dagegen mit hoher Stetigkeit in den Beständen auf.

Weitere Arten, die ihren Schwerpunkt in der Gesellschaft haben und zur Charakteristischen Artenverbindung gehören sind Cl. macilenta ssp. floerkeana (OC) und Cl. macilenta ssp. macilenta sowie Cl. merochlorophaea. Auf eine Unterteilung in Typische Ausbildung und Ausbildung mit Cl. glauca, wie dies PAUS (1997) vorgenommen hat, wurde bei den nordhessischen Beständen verzichtet. Cl.glauca kommt in einem Teil der Aufnahmen vor. Cl. novochlorophaea, von PAUS als zweite Trennart der Cladonia glauca-Ausbildung genannt, ist aber im gesamten Untersuchungsgebiet und somit auch in der Cladonia bacillaris-Gesellschaft zu selten, um zur Differenzierung von Einheiten herangezogen werden zu können.

Stift- und becherförmige, sorediöse Cladonien sind bestandsbildend, darunter vor allem die auffallenden rotfrüchtigen Unterarten von Cl. macilenta. Häufig treten diese Arten in gleichen Anteilen auf, jedoch finden sich im vorliegenden Aufnahmematerial auch Dominanzbestände von Cl. merochlorophaea und Cl. subulata. Strauchflechten sind nur sehr sporadisch eingestreut. Die mittleren Artenzahlen verteilen sich wie folgt: Flechten 7,2, Moose 6,9 und Phanerogamen 3,5. Phanerogamen können bis $40 \%$ Deckung erreichen (Mittelwert $18 \%$ ). Moose kommen mit einer mittleren Deckung von $17 \%$ vor. Algen treten gelegentlich auf, jedoch meist nur in geringer Deckung. Die Bestände sind unterschiedlich lückig, der Anteil an offenem Boden liegt zwischen 4 und $45 \%$. 
Tab. 7: Stetigkeitstabelle Cladonion arbusculae

Einheit 1: Cladonia bacillaris-Gesellschaft

Einheit 2: Cladonietum mitis
2.1 cladonietosum merochlorophaeae
2.2 typicum
2.2.1 Typische Variante
2.2.2 Cladonia rangiferina-Variante

Einheit 3: Cladonia furcata-Cladonion arbusculae-FG und

Cladonia gracilis-Cladonion arbusculae-FG

Einheit 4: Cladonietum foliaceae

\begin{tabular}{|c|c|c|c|c|c|c|}
\hline \multirow{3}{*}{ Einheit } & \multirow[t]{3}{*}{1} & \multicolumn{3}{|c|}{2} & \multirow[t]{3}{*}{3} & \multirow[t]{3}{*}{4} \\
\hline & & \multirow{2}{*}{2.1} & \multicolumn{2}{|c|}{2.2} & & \\
\hline & & & 2.2 .1 & 2.2 .2 & & \\
\hline \multirow{7}{*}{\begin{tabular}{|l|} 
Zahl der Aufnahmen \\
mittlere Deckung Phanerogamen [\%] \\
mittlere Deckung Flechten [\%] \\
mittlere Deckung Moose [\%] \\
mAZ Phanerogamen \\
mAZ Flechten \\
mAZ Moose \\
\end{tabular}} & 18 & 14 & 5 & 12 & 13 & 7 \\
\hline & 8,2 & 11,6 & 13,0 & 1,2 & 7,2 & 4,6 \\
\hline & 50,0 & 60,4 & 72,0 & 63,8 & 54,2 & 50,7 \\
\hline & 11,5 & 12,6 & 11,6 & 23,8 & 22,5 & 26,1 \\
\hline & 3,5 & 1,2 & 2,2 & 1,0 & 1,7 & 1,9 \\
\hline & 7,2 & 9,6 & 5,0 & 6,0 & 5,1 & 5,9 \\
\hline & 6,9 & 3,1 & 1,6 & 2,2 & 2,6 & 2,4 \\
\hline \multirow{3}{*}{ D 1: } & & & & & & \\
\hline & III & + & & . & + & $\mathrm{I}$ \\
\hline & III & + & . & . & II & . \\
\hline \multicolumn{7}{|l|}{ D 1, d 2.1: } \\
\hline Cladonia macilenta ssp. macilenta & $\mathrm{V}$ & III & & . & . & I \\
\hline OC Cladonia subulata & IV & II & . & 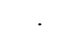 & I & I \\
\hline Pohlia nutans & III & II & & I & I & . \\
\hline OC Trapeliopsis granulosa & II & III & . & + & II & 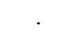 \\
\hline Campylopus introflexus & II & II & & & + & \\
\hline Cladonia macilenta ssp. floerkeana & $\mathrm{V}$ & IV & II & II & I & I \\
\hline Cladonia merochlorophaea & $\mathrm{V}$ & IV & II & II & II & . \\
\hline \multicolumn{7}{|l|}{ D 2: } \\
\hline Cladonia gracilis & + & IV & IV & IV & III & I \\
\hline Cladonia uncialis & + & III & IV & II & + & . \\
\hline UC Cladonia arbuscula & . & IV & III & III & . & . \\
\hline UC Cladonia portentosa & + & II & III & III & . & . \\
\hline \multicolumn{7}{|l|}{ d 2.1 + 2.2.1: } \\
\hline Pleurozium schreberi & . & II & III & . & + & . \\
\hline \multicolumn{7}{|l|}{ d 2.1: } \\
\hline UC Cladonia ciliata & & III- & & II & & \\
\hline OC Cladonia coccifera & II & IV & & I & II & II \\
\hline \multicolumn{7}{|l|}{ d 2.1, D 4: } \\
\hline KC Polytrichum piliferum & I & IV & I & . & II & $\mathrm{V}$ \\
\hline Ceratodon purpureus & . & III & & . & + & $\mathrm{V}$ \\
\hline \multicolumn{7}{|l|}{ D 4: } \\
\hline Cladonia rangiformis & . & . & . & I & + & $\mathrm{V}$ \\
\hline UC Cladonia foliacea & . & . & & . & I & $\mathrm{V}$ \\
\hline \multicolumn{7}{|l|}{ d 2.2.2, D 3 + 4: } \\
\hline OC Cladonia furcata ssp. furcata & I & II & I & IV & $\mathrm{V}$ & IV \\
\hline d 2.2.2, D 3: & & & & & & \\
\hline Hypnum cupressiforme s.l. & II & + & & $\mathrm{V}$ & IV & I \\
\hline
\end{tabular}


Forts. Tab. 7: Stetigkeitstabelle Cladonion arbusculae

\begin{tabular}{|c|c|c|c|c|c|c|c|}
\hline \multirow{3}{*}{\multicolumn{2}{|c|}{ Einheit }} & \multirow[t]{3}{*}{1} & \multicolumn{3}{|c|}{2} & \multirow[t]{3}{*}{3} & \multirow[t]{3}{*}{4} \\
\hline & & & \multirow[t]{2}{*}{2.1} & \multicolumn{2}{|c|}{2.2} & & \\
\hline & & & & 2.2 .1 & 2.2 .2 & & \\
\hline \multicolumn{8}{|c|}{ d 2.2.2: } \\
\hline & Cladonia rangiferina & . & + & . & IV & II & . \\
\hline & Cladonia squamosa & I & + & I & III & I & . \\
\hline & Polytrichum formosum & II & . & . & III & + & . \\
\hline & Dicranum scoparium & II & I & . & III & II & $\mathrm{I}$ \\
\hline \multicolumn{8}{|c|}{ FC*, OC: } \\
\hline$*$ & Cetraria aculeata & . & III & II & . & II & III \\
\hline \multirow[t]{2}{*}{$*$} & Cladonia phyllophora & . & I & . & + & $\mathrm{I}$ & I \\
\hline & Placynthiella oligotropha & I & . & . & . & + & I \\
\hline$*$ & Cladonia ramulosa & $\mathrm{I}$ & + & . & + & . & . \\
\hline \multirow[t]{6}{*}{$*$} & Cladonia crispata & . & . & . & + & . & . \\
\hline & Dibaeis baeomyces & . & + & . & . & . & . \\
\hline & Baeomyces rufus & + & + & . & . & . & . \\
\hline & Peltigera didactyla & . & + & . & . & . & . \\
\hline & Placynthiella uliginosa & + & . & . & . & . & . \\
\hline & Cetraria islandica & . & I & I & . & + & . \\
\hline \multicolumn{8}{|c|}{ Begleiter Kryptogamen: } \\
\hline & Cladonia glauca & II & III & . & I & II & II \\
\hline & Cephaloziella spec. & II & II & II & . & $\mathrm{I}$ & III \\
\hline & Placynthiella icmalea & II & II & I & + & + & I \\
\hline & Cladonia novochlorophaea & I & II & . & $\mathrm{I}$ & I & . \\
\hline & Cladonia pyxidata ssp. chlorophaea & . & I & I & . & II & II \\
\hline & Cladonia cervicornis & $\mathrm{I}$ & II & . & + & + & . \\
\hline & Cladonia cryptochlorophaea & II & I & . & . & . & . \\
\hline & Cladonia spec. & $\mathrm{I}$ & + & . & . & $\mathrm{I}$ & $\mathrm{I}$ \\
\hline & Cladonia coccifera/deformis agg. & $\mathrm{I}$ & + & . & + & . & I \\
\hline & Cladonia fimbriata & + & . & . & . & $\mathrm{I}$ & I \\
\hline & Polytrichum juniperinum & I & . & I & . & I & . \\
\hline \multicolumn{8}{|c|}{ Phanerogamen: } \\
\hline & Deschampsia flexuosa & II & I & II & IV & II & . \\
\hline & Festuca ovina agg. & I & III & III & . & II & II \\
\hline & Hieracium pilosella & $\cdot$ & II & I & . & I & . \\
\hline & Calluna vulgaris & II & . & I & . & ${ }^{\circ}$ & . \\
\hline & Rumex acetosella & . & . & I & . & I & II \\
\hline & Scleranthus annuus agg. & . & . & . & . & + & II \\
\hline
\end{tabular}

Außerdem mit Stetigkeit < II und in weniger als 3 Einheiten:

Achillea millefolium 3:I; Bryophyta spec. 1:+; Buxbaumia aphylla 1:+, 2:+; Campylopus flexuosus 1:+; Carex ornithopoda 1:+; Carex pilulifera 1:+; Carex spec. 1:+, 2:+; Cerastium semidecandrum 5:+; Cladonia coniocraea 1:I, 4:I; Cladonia pleurota 1:I; Cladonia polydactyla $1: I$; Cladonia pyxidata ssp. grayi $5:+$; Cladonia pyxidata ssp. pyxidata 2:I, 6:I; Cladonia scabriuscula 5:+; Cladonia strepsilis 2:+; Cyanophyta div. spec. 2:I, 6:I; Dianthus carthusianorum 6:I; Dicranaceae spec. 1:+; Dicranella cf. heteromalla 1:+; Dicranella heteromalla 1:+; Dicranodontium denudatum 1:+; Dicranum montanum 1:I; Dicranum polysetum 1:+; Dicranum scoparium 4:+; Ditrichum spec. 1:+; diverse Algen 1:I, 2:+; Erophila verna 6:I; Galium glaucum 6:I; Galium saxatile 5:+; Helictotrichon pratense 5:+; Hieracium spec.3:I; Hypochaeris radicata 2:+; Lepraria lobificans 1:+; Lepraria rigidula 5:+; Lepraria spec. 6:I; Leucobryum glaucum 1:+; Lophocolea cf. heterophylla 5:+; Luzula campestris agg. 5:+; Luzula spec. 3:I; Melampyrum arvense 4:+; Micarea leprosula 2:+; Musci spec. 1:+; Nardus stricta 1:I; Neofuscelia verruculifera 6:I; Picea abies juv. 1:I; Polytrichum commune 5:+; Pottiaceae spec. 1:+,3:I; Psilolechia lucida 1:+; Ptilidium ciliare 1:+; Quercus petraea juv. 4:+; Quercus spec. 5:+; Racomitrium canescens s.1. 2:+; Racomitrium heterostichum agg. 2:+, 5:+; Racomitrium lanuginosum 2:I, 5:+; Rhytidiadelphus squarrosus 2:+, 5:+; Rumex acetosa 2:I; Sedum rupestre agg. 6:I; Silene spec. 5:+; Thymus praecox 6:I; Trapeliopsis pseudogranulosa 1:+; Trifolium arvense 6:I; Trifolium spec. 5:+; Vaccinium myrtillus 1:+, 5:+; Veronica verna agg. 6:I; Viola arvensis 5:+; Weissia spec. 1:+. 


\section{b) Standorte und Vorkommen im UG}

Die Cladonia bacillaris-Gesellschaft wurde für das Untersuchungsgebiet durch 18 Aufnahmen belegt und an 8 Wuchsorten angetroffen. Viele der am Aufbau der Gesellschaft beteiligten Arten sind im Untersuchungsgebiet weit häufiger, treten jedoch meist nur als vereinzelte Ansiedlungen und nicht innerhalb größerer Flechtenbestände auf. Die Gesellschaft besiedelt schwach bis stark geneigte Flächen unterschiedlichster Expositionen vorwiegend in Buntsandsteingebieten. Überwiegend treten dort skelettarme,

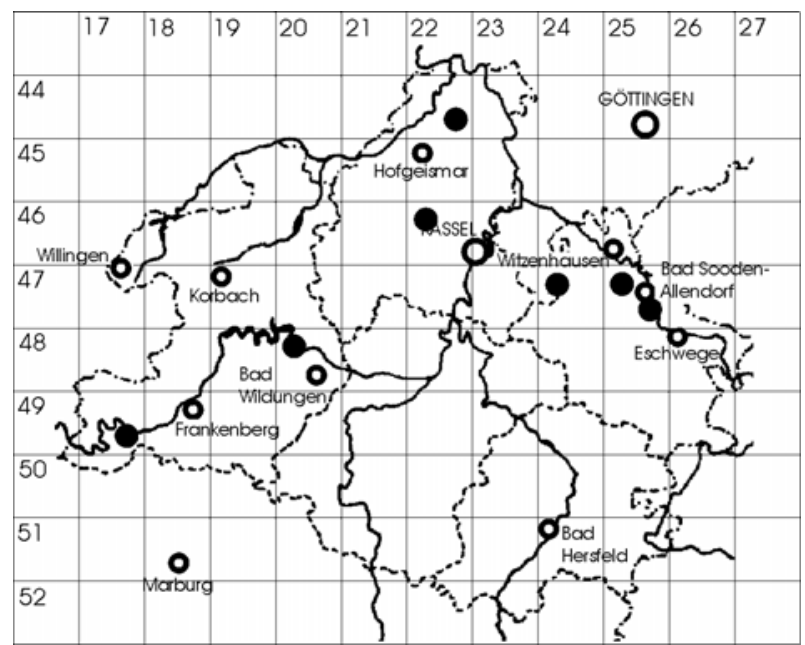

Abb. 11: Verbreitung der Cladonia bacillarisGesellschaft im Untersuchungsgebiet. saure Böden auf, deren $\mathrm{pH}(\mathrm{KCl})$-Wert zwischen 2,9 und 4,0 liegt. Die Streubedeckung variiert erheblich (5 bis $60 \%$ ). Ebenso stark unterscheiden sich die elektrolytischen Leitfähigkeitswerte der Bodenproben (s. Tab. 11). Einige der Bestände siedeln auf einer Rohhumusauflage. Das Mikroklima an den Standorten kann als sonnig bis leicht beschattet und meist trocken bezeichnet werden.

Die Gesellschaft bedeckt immer nur kleine Flächen. Jedoch kann die Summe aller Flächen an einem Wuchsort bis zu $10 \mathrm{~m}^{2}$ betragen. Als umgebende Phytozönosen sind Zwergstrauchheiden (z.B. Battenfelder Driescher, MTB 4917), Fichtenforste (z.B. Niestetal, MTB 4724) und Traubeneichenwälder (z.B. Ederseegebiet, MTB 4820) zu nennen. Bevorzugte Standorte innerhalb der Wälder sind Erdanrisse an Wegen und Pfaden oder sonstige Abbruchkanten z.B. an Steinbrüchen. Nach PAUS (1997) nehmen derartige Bestände im Sukzessionsverlauf eine intermediäre Stellung zwischen den Pioniergesellschaften des Baeomycion rosei und dem Cladonietum mitis als Endstadium der Flechtenentwicklung ein. Alle Sukzessionsstadien dieser Abfolge lassen sich beispielsweise in der Heidelandschaft „Battenfelder Driescher" beobachten. An Wegböschungen und Steinbruchkanten dürfte die Entwicklung aufgrund der Substratinstabilität dagegen wohl kaum über das Stadium der Cladonia bacillarisGesellschaft hinausgehen.

\section{Syntaxonomische Anmerkung:}

Paus (1997) verwendet den Namen Cladonia bacillaris-Mikrogesellschaft. Unter dem Begriff „Mikrogesellschaft“ werden nach BARKMAN (1968/1973) jedoch floristisch eigenständige Teilbestände mit mehreren Schichten ( $\leftrightarrow$ Synusie), als strukturelles Element verstanden, die auf einem Kleinstandort innerhalb einer größeren Pflanzengesellschaft siedeln. „Mikrogesellschaft“ nach BARKMAN bezieht sich also nicht syntaxonomisch wie bei PAUS auf das Fehlen von Charakterarten. Aus diesem Grunde wird in der vorliegenden Arbeit die Bezeichnung „Gesellschaft“ verwendet.

Das Cladonietum bacillaris Krieger 1937 wird bei DREHWALD (1993) innerhalb des Verbandes Cladonion coniocraeae in die Klasse Cladonio-Lepidozietea reptantis Ježek \& Vondráček 1962 
eingeordnet. Als Charakterarten gelten Cladonia glauca und Cl. macilenta ssp. floerkeana. Die Gesellschaft siedelt auf morschem Holz, Rohhumus und humusreichen Sandböden. Sie läßt sich in zwei Subassoziationen unterteilen. Die Subassoziation polytrichetosum piliferi bevorzugt humusreiche Sandböden, die Subassoziation typicum kommt auf morschem Holz und Rohhumus vor. Beide Subassoziationen entsprechen in ihrer Artenzusammensetzung teilweise der Cladonia bacillarisGesellschaft, enthalten jedoch darüber hinaus eine Reihe von Flechtenarten, die auch auf morschem Holz häufig anzutreffen sind, z.B. Cladonia digitata, Cl. coniocraea, Trapeliopsis flexuosa etc.

\subsubsection{Cladonietum mitis}

(Tab. 7; Tab. 8 (Einheit 2) im Anhang 3; Taf. 1.3 im Anhang 2)

Syn.: Cladonietum Preis 1937, Cladonia bacillaris-Assoziation (KRIEGER 1937 p.p.), Cladonia furcata-Assoziation (KrIEgER 1937), Cladonia mitis-Assoziation (LANGERFELDT 1939), Cladonia squamosa-Assoziation (KAISER 1926) p.p., Cladonia uncialis-Assoziation (KRIEGER 1937) p.p., Cladonia glauca-Cladina mitis-Gesellschaft (MAGNUSSON 1982), Cladonia portentosa-Gesellschaft (MAGnusson 1982), Cladonietum portentosae prov. (SLOOF et al. 1986), Cladina portentosa-Verein (DANIELS et al. 1993), Cladinetum mitis (Krieg.) Follm. (in FolLMANN 1981).

\section{a) Floristische Zusammensetzung, Physiognomie und Struktur}

Die Trennartengruppe der Union setzt sich zusammen aus Cladonia arbuscula (UC), Cl. portentosa (UC) sowie den Federationscharakterarten Cl. gracilis und Cl. uncialis. Die Union läßt sich in zwei Subunionen gliedern (s. auch PAUS 1997). Das Cladonietum mitis cladonietosum merochlorophaea (Einheit 2.1) wird durch die Arten Cl. ciliata (UC), Cl. coccifera s.str. (OC), Polytrichum piliferum (KC) und Ceratodon purpureus differenziert. Als weitere Trennartengruppe ist die von Cl. macilenta ssp. macilenta zu nennen, zu der unter anderem auch Cl. subulata (OC) und Trapeliopsis granulosa (OC) gehören. Cl. macilenta ssp. floerkeana und Cl. merochlorophaea zeigen höhere Stetigkeiten als in der zweiten Subunion des Cladonietum mitis. Diese Arten stellen vermutlich Relikte der Cladonia bacillaris-Gesellschaft als vorhergehendem Sukzessionsstadium dar (PAUS 1997). Die Nähe der Subunion zur Cladonia bacillaris-Gesellschaft wird vor allem durch die Aufnahmen 19 bis 21 in Tab. 8 verdeutlicht, in denen Trapeliopsis granulosa dominiert, die jedoch auch Unionscharakterarten des Cladonietum mitis aufweisen. Trapeliopsis zeigt sich in diesen Beständen außerdem reich fruchtend. Aspektbestimmend in der Subunion cladonietosum merochlorophaea sind weniger die sogenannten Rentierflechten, als vielmehr die konkurrenzschwächeren stift- und becherförmigen Arten.

Das Cladonietum mitis typicum (Einheit 2.2; Taf. 1.3) tritt in zwei Varianten auf. Die Typische Variante (Einheit 2.2.1) umfaßt relativ artenarme Strauchflechtenbestände, in denen jeweils eine der Arten der CAV dominant ist. Diesen Beständen lassen sich die Aufnahmen 36 und 37 in Tab. 8 angliedern, die keine Unionscharakterarten des Cladonietum mitis aufweisen, als besonders artenarme Ausprägung aber dennoch in diese Einheit gestellt wurden. Korrekterweise müßten sie jedoch, jeweils nach der dominierenden Art benannt, als Cladonia uncialis- bzw. Cetraria aculeata-Cladonion arbusculae-Fragmentgesellschaft bezeichnet werden. Die Cladonia rangiferinaVariante (Einheit 2.2.2) wird durch die Differentialarten Cl. furcata ssp. furcata (OC), Hypnum cupressiforme s.l., Cl. rangiferina (FC), Cl. squamosa sowie das Moos Polytrichum formosum von der Typischen Variante abgegrenzt. Sie ist die 
charakteristische Ausbildung der Bestände in den Traubeneichenwäldern des Untersuchungsgebietes.

Mit durchschnittlich 9,6 Flechtenarten ist das Cladonietum mitis cladonietosum merochlorophaeae artenreicher als die beiden Varianten der Typischen Subunion (mittlere Artenzahl 5,0 bzw. 6,0). Phanerogamen und Moose kommen in allen Einheiten nur mit wenigen Arten vor. Auch die Cladonia rangiferina-Variante des Cladonietum mitis typicum ist nicht reich an Moosarten, es sind dort nur wenige, kräftige Moose, wie z.B. Polytrichum formosum oder Hypnum cupressiforme s.1., die hochstet und mit teilweise recht hohen Deckungsgraden auftreten.

In den dicht geschlossenen Beständen des Cladonietum mitis erreichen Phanerogamen und Kryptogamen eine Gesamtdeckung von bis $\mathrm{zu} 100 \%$. Nur in der Subunion cladonietosum merochlorophaeae sind bisweilen Steine an der Bodenoberfläche sichtbar. Die höchste Flechtendeckung (durchschnittlich rund 70 \%) zeigt die Typische Variante des Cladonietum mitis typicum, in den anderen Einheiten liegt sie etwas darunter. Algen treten gelegentlich in der Subunion cladonietosum merochlorophaeae auf. Dagegen ist die Streuauflage dort geringer als im Cladonietum mitis typicum. Typischerweise haben die Moose bezüglich der Deckung am Aufbau der Cladonia rangiferina-Variante höheren Anteil als in den anderen Untereinheiten, während die Phanerogamen dort nur eine geringe Rolle spielen.

\section{b) Standorte und Vorkommen im UG}

Das Cladonietum mitis ist im Aufnahmematerial mit 31 Aufnahmen vertreten, welche jedoch nur aus 8 verschiedenen Teilgebieten aus 6 MTB der TK 25 stammen. Die Union tritt im Untersuchungsgebiet in Traubeneichenwäldern und Zwergstrauchheiden über Tonschiefer oder Grauwacke, seltener auch über Basalt auf. Sie besiedelt schwach geneigte, die Cladonia rangiferinaVariante auch etwas steilere Flächen aller Expositionen. Bei den $\mathrm{pH}$ Werten zeigen sich nur geringe Unterschiede zwischen den Untereinheiten des Cladonietum mitis.

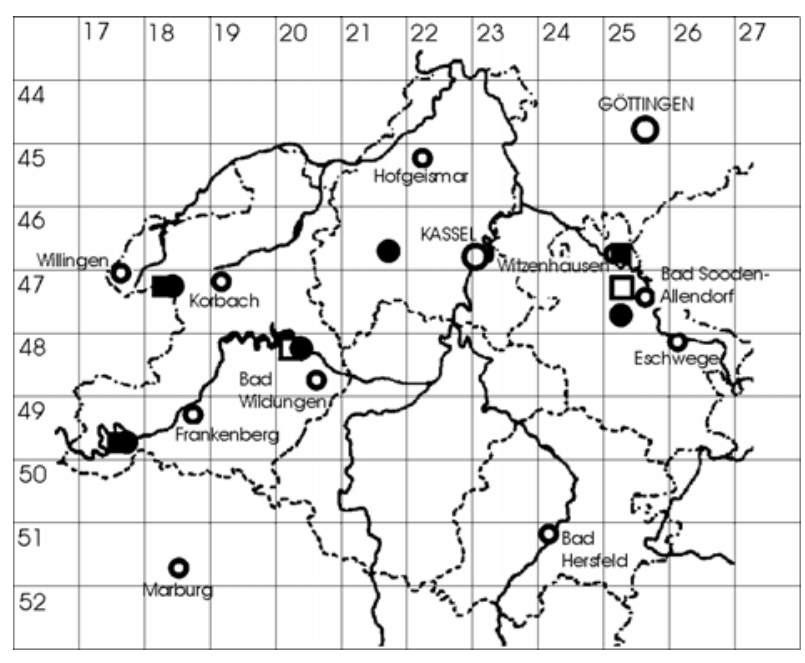

Abb. 12: Verbreitung des Cladonietum mitis im Untersuchungsgebiet: Cladonietum mitis cladonietosum; Cladonietum mitis typicum, Typische Variante; Cladonietum mitis typicum, Cladonia-Variante.

Der Median des $\mathrm{pH}(\mathrm{KCl})$-Wertes beträgt für die Cladonia rangiferina-Variante 3,4 und für die Subunion cladonietosum merochlorophaeae 3,9. Die Typische Variante des Cladonietum mitis typicum nimmt mit einem Median des $\mathrm{pH}(\mathrm{KCl})-W e r t e s$ von 3,7 eine Mittelstellung ein. 
Das Cladonietum mitis cladonietosum merochlorophaeae siedelt an sonnigen und trockenen Standorten, bevorzugt in Zwergstrauchheiden, seltener in Wäldern. Es erreicht 1 bis $20 \mathrm{~m}^{2}$ Ausdehnung.

Die Typische Variante des Cladonietum mitis typicum kommt an mäßig bis stärker beschatteten, eher frischen Stellen vor und bildet dort kleine, nur selten knapp $10 \mathrm{~m}^{2}$ groß werdende Flecken. Die Vorkommen der Cladonia rangiferina-Variante in den Traubeneichenwäldern gedeihen an zeitweilig beschatteten bis schattigen Standorten. Diese Variante kann in Nordhessen mancherorts (z.B. an der Mühlecke am Edersee, MTB 4820) beträchtliche Ausmaße von mehr als $50 \mathrm{~m}^{2}$ erreichen. Mit der namengebenden Art Cl. rangiferina und mit Cl. squamosa sind für diese Variante zwei hygrisch anspruchsvollere Arten kennzeichnend. Die Bestände entsprechen in etwa dem Cladina-Polytrichum formosum-Bestand von SCHÖLLER (1991). Er wählt diesen Namen für die Bodenvegetation im Schatten der Gehölze. Typisch sei eine relative Artenarmut und ein hoher Anteil an Moosen. Als charakteristische Arten nennt er Cl. arbuscula, Cl. rangiferina, Cl. squamosa, Polytrichum formosum, Dicranum scoparium sowie Pleurozium schreberi. Cl. arbuscula und Cl. rangiferina sind SCHÖLLER zufolge hygrisch etwas anspruchsvoller als $\mathrm{Cl}$. portentosa und $\mathrm{Cl}$. ciliata. Sie können daher an derartigen Schattenstandorten dominant werden. Die Ubiquistin Cl. furcata ssp. furcata, Trennart der nordhessischen Bestände, tritt im Schatten der Gehölze ebenfalls mit hohen Stetigkeiten auf.

\section{Syntaxonomische Anmerkung:}

KLEMENT (1955) unterteilt die Gesellschaft in ein Cladonietum mitis atlanticum im Einflußbereich des ozeanischen Klimas (also vornehmlich im nordwestdeutschen Tiefland sowie auf den Nordseeinseln) und in ein Cladonietum mitis continentale in Gebieten hoher Kontinentalität. Er bemerkt weiterhin, Überschneidungen würden zu den Seltenheiten zählen. Ein solches Überschneidungsgebiet könnte jedoch laut FolLmanN (1981) in Nordhessen liegen, wo er im Cladonietum mitis damals noch sowohl Cladonia rei als Differentialart der Subassoziation (Subunion) atlanticum als auch Cladonia cariosa als Differentialart der kontinental verbreiteten Subassoziation (Subunion) nachweisen konnte. Aktuell wurde Cladonia cariosa weder im Rahmen der vorliegenden Untersuchungen gefunden, noch wird sie in der floristischen Arbeit von KÜMMERLING (1991) genannt.

Moosreiche Untereinheiten wie die Cladonia rangiferina-Variante des Cladonietum mitis typicum finden sich auch bei PAUS (1997) und BIERMANN et al. (1994). Sowohl im Cladonietum mitis hypnetosum (PAUS 1997) als auch in den sogenannten „Moosreichen Abbaustadien des Cladonietum mitis” (BIERMANN et al. 1994) zählt jedoch, anders als bei der Cladonia rangiferina-Variante, Pleurozium schreberi zu den Trennarten. Beide von PAUS bzw. BIERMANN et al. beschriebenen Vegetationseinheiten sind Gesellschaften der Heiden, in denen Pleurozium schreberi als typischer Bestandteil der Moosschicht auftritt. In den Traubeneichenwäldern Nordhessens spielt diese Moosart dagegen in der Moosschicht keine Rolle.

Aufgrund dieser Unterschiede bezüglich Standort und Trennartengruppe wurde daher in der vorliegenden Arbeit eine Einordnung des nordhessischen Aufnahmematerials in die Subunion hypnetosum abgelehnt. Statt dessen wird mit der Cladonia rangiferina-Variante eine Untereinheit aufgestellt, die nach einer für die Standortverhältnisse typischen Art benannt ist. 


\subsubsection{Cladonia furcata-Cladonion arbusculae- und Cladonia gracilis-Cladonion arbusculae-Fragmentgesellschaft}

(Tab. 7; Tab. 8 (Einheit 3) im Anhang 3)

\section{a) Floristische Zusammensetzung, Physiognomie und Struktur}

Im gesamten Untersuchungsgebiet finden sich auf unterschiedlichsten Standorten Dominanzbestände von Cl. furcata ssp. furcata. Die Dominanz von Cl. furcata ssp. furcata kann unterschiedlich stark ausgeprägt sein. Zu Einheit 3 wurden außerdem zwei Vegetationsaufnahmen (Aufnahmen $50+51$ in Tab. 8) von Blockhalden gestellt, auf denen Cl. gracilis-geprägte Bestände auftreten. Diese Cladonia gracilis-Cladonion arbusculae-Fragmentgesellschaft unterscheidet sich nur in der dominierenden Art von der Cladonia furcata-Cladonion arbusculae-Fragmentgesellschaft. Die Fragmentgesellschaften umfassen Bestände mit bis zu 13 Flechtenarten ebenso wie Bestände, in denen neben $\mathrm{Cl}$. furcata ssp. furcata keine weitere Flechte auftritt. Die mittlere Artenzahl der Flechten liegt bei 5,1. Es gibt artenreichere Bestände mit Charakterarten des Cladonion arbusculae. Selten treten mit Cl. fimbriata und Cl. pyxidata ssp. chlorophaea auch Diagnostische Arten des Cladonion rei auf, so daß die Bestände der Cladonia furcata-Cladonion arbusculae-Fragmentgesellschaft teils mehr zur einen, teils mehr zur anderen Federation tendieren. Alle Bestände sind negativ gekennzeichnet durch das nahezu vollständige Fehlen der für das Cladonietum mitis typischen Artengruppe um Cl. arbuscula und Cl. portentosa. Die zweite hochstete Art neben Cl. furcata ssp. furcata ist Hypnum cupressiforme s.l. Als Charakterarten der Federation treten Cl. rangiferina, Cl. uncialis und Cetraria aculeata mit geringer Stetigkeit auf.

Geprägt wird das Erscheinungsbild der Fragmentgesellschaft durch die strauchförmigen, kriechend bis aufrecht wachsenden Thalli von Cl. furcata ssp. furcata bzw. zierlichen Podetien von Cl. gracilis. Stift- und becherförmige Arten sind oft nur bei genauem Hinsehen auffindbar. Die Gesamtdeckung liegt im Mittel in diesen dicht geschlossenen Beständen bei $81 \%$. Davon entfallen durchschnittlich auf die Phanerogamen $28 \%$, die Flechten $54 \%$ und die Moose $32 \%$, während die Algen mit weniger als $5 \%$ kaum eine Rolle spielen.

Die Cladonia furcata-Cladonion arbusculae-Fragmentgesellschaft kann nur kleinflächig entwickelt sein $\left(\sim 0,5 \mathrm{~m}^{2}\right)$ oder auch Flächen von bis zu $30 \mathrm{~m}^{2}$ bedecken! Die Bestände mit Cl. gracilis beschränken sich auf einzelne Felsblöcke in Blockhalden. 


\section{b) Standorte und Vorkommen im UG}

Im Untersuchungsgebiet wurde die Fragmentgesellschaft von $\mathrm{Cl}$. gracilis in 2 Aufnahmen an 2 Wuchsorten, die Fragmentgesellschaft von $\mathrm{Cl}$. furcata ssp. furcata in 11 Aufnahmen an 9 Wuchsorten erfaßt. Die Cladonia furcata-Fragmentgesellschaft siedelt über unterschiedlichsten kalkfreien Ausgangsgesteinen in tieferen und höheren Lagen, von 260 bis $740 \mathrm{~m}$ ü. NN. Die meist flachgründigen Böden haben eine fehlende bis nur geringe Streuauflage. Es wurden Bestände sowohl auf Rohböden (Syrosem) als auch auf Ranker, auf skelettreichen

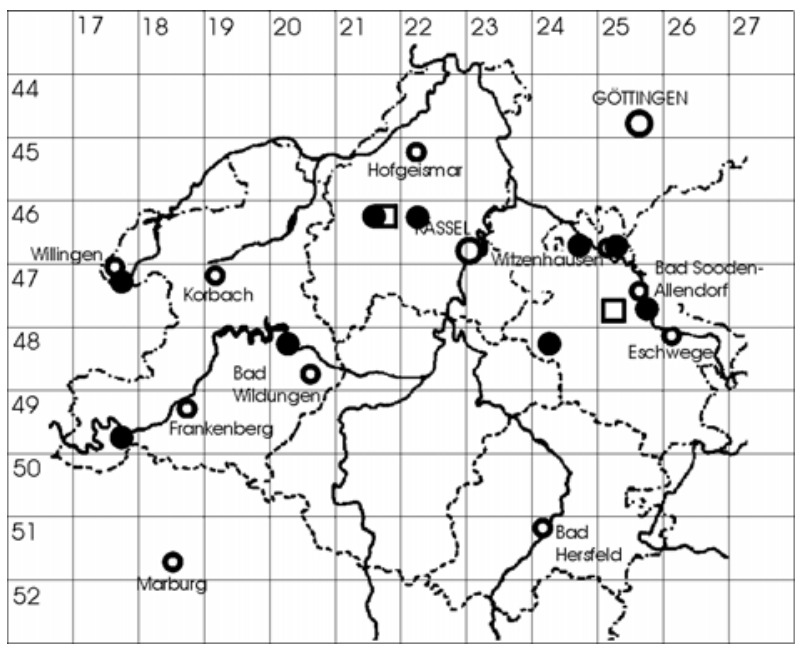

Abb. 13: Verbreitung der Cladonia furcata- und der Cladonia gracilis-Cladonion arbusculaeFG im Untersuchungsgebiet. oder skelettarmen Böden angetroffen. Ebenso unterschiedlich wie die Böden sind auch Exposition und Inklination der Flächen sowie die mikroklimatischen Verhältnisse, die von sonnig bis schattig und von trocken bis frisch reichen. Einheitliche Standortbedingungen lassen sich für diese Fragmentgesellschaft also nicht ausmachen. Es müssen andere Faktoren bei der Ausbildung derartiger Bestände eine Rolle spielen (s.u.). Die stellvertretend für weitere derartige Vorkommen in Nordhessen stehenden Aufnahmen der Cladonia gracilis-Fragmentgesellschaft stammen von Humusansammlungen auf Basalt-Blockhalden.

Die Median-Werte des $\mathrm{pH}(\mathrm{KCl})$ und der elektrolytischen Leitfähigkeit der Fragmentgesellschaften unterscheiden sich nicht nennenswert von denen der anderen, oben beschriebenen Cladonion arbusculae-Gesellschaften (s. Tab. 11).

\section{Anmerkung zur Dominanz von Cladonia furcata ssp. furcata:}

Im Abschnitt „Floristische Zusammensetzung” wurde erläutert, daß die Cladonia furcata-Cladonion arbusculae-Fragmentgesellschaft Beziehungen sowohl zum Cladonion arbusculae als auch zum Cladonion rei aufweist. PAUs (1997) bezeichnet die artenärmsten Bestände in ihrer Arbeit ranglos als Cladonia furcata-Dominanzbestände und stellt sie neben die Cladonia furcata-Cladonion reiFragmentgesellschaft. Diese ist an Diagnostischen Arten ebenfalls deutlich verarmt. Ihre Zuordnung zur Federation Cladonion rei ist aber floristisch noch erkennbar. Darüber hinaus weist PAUS jedoch ebenfalls darauf hin, daß die Standortverhältnisse teilweise auch auf andere Flechtengesellschaften als Vorstadien der Cladonia furcata-Dominanzbestände schließen lassen, z.B. auf das Cladonietum mitis. Die Cladonia furcata-Cladonion rei-Fragmentgesellschaft ist ihrer Meinung nach als degradierte Restgesellschaft des Cladonietum rei aufzufassen. Als Grund für die zunehmende Dominanz von $\mathrm{Cl}$. furcata ssp. furcata nennt sie die fortschreitende Vergrasung der Bestände. Cl. furcata ssp. furcata sei daher als Degenerationszeiger zu werten.

Im allgemeinen ist $C l$. furcata ssp. furcata vor allem an stärker anthropogen beeinflußten Standorten häufig. Die zur Cl. furcata-Gruppe gehörenden Sippen dominieren bei Störeinflüssen aufgrund ihrer hohen Regenerationskraft und sind zudem relativ schnellwüchsig (PAUs 1997). Auch das Mikroklima hat 
einen Einfluß auf die Dominanz solcher Arten. Unter mikroklimatisch monotoneren Bedingungen wird das Wechselspiel verschiedener Arten zugunsten solcher konkurrenzkräftiger Arten entschieden (SCHÖLLER 1991).

\subsubsection{Cladonietum foliaceae}

(Tab. 7; Tab. 8 (Einheit 4) im Anhang 3; Taf. 1.4 im Anhang 2)

Syn.: Cornicularia tenuissima-Assoziation (KRIEGER 1937), Cornicularia tenuissima-Gesellschaft (ToBLER \& MATTICK 1938), Cladonietum alcicornis Klement 1953.

\section{a) Floristische Zusammensetzung, Physiognomie und Struktur}

Zur CAV zählen Cladonia foliacea (UC), Cl. rangiformis (D), Ceratodon purpureus (D), Polytrichum piliferum (KC), Cl. furcata ssp. furcata (OC) und Cetraria aculeata (FC). Das vorliegende Aufnahmematerial läßt sich weder der von PAUS (1997) genannten Subunion cladonietosum subrangiformis noch der Subunion cladonietosum furcatae zuordnen, da Arten beider Trennartengruppen hochstet in den Beständen des Untersuchungsgebietes auftreten (Cl. furcata ssp. furcata, Cl. rangiformis, Ceratodon purpureus). Somit nimmt die Ausbildung des Cladonietum foliaceae in Nordhessen eine Mittelstellung zwischen den beiden nordwestdeutschen Subunionen ein. Während PAUS (1997) Cl. rangiformis lediglich als Trennart der Union einstuft, bezeichnet sie SCHÖLLER (1991) als Charakterart des Cladonietum foliaceae.

An der hohen mittleren Gesamtdeckung von $82 \%$ haben die Flechten einen Anteil von $51 \%$. Dominante Art ist Cl. foliacea (Syn. Cl. alcicornis), deren Grundschuppen besonders bei Trockenheit auffallen, wenn sie eingerollt sind und ihre weißen Unterseiten sichtbar werden. Es können aber auch Cl. rangiformis oder Cl. furcata ssp. furcata aspektbestimmend sein. Eine Untersuchung der Cl. furcata-Proben mittels Dünnschichtchromatographie ergab, daß im Gegensatz zu den nordwestdeutschen, von PAUS (1997) bearbeiteten Beständen, die nordhessischen nur die Subspezies furcata, nicht jedoch die Subspezies subrangiformis enthalten.

\section{b) Standorte und Vorkommen im UG}

Das Cladonietum foliaceae zählt zu den Besonderheiten der Flechtenvegetation in Nordhessen. Es konnten daher nur 7 Aufnahmen dieser Union, an 3 Wuchsorten angefertigt werden. Die Union siedelt im Untersuchungsgebiet vorwiegend auf Ranker über den vulkanischen Gesteinen Basalt und Diabas. Der Median der $\mathrm{pH}(\mathrm{KCl})$ Werte im Ah-Horizont liegt mit 4,2 deutlich über den Werten der anderen Cladonion arbusculae-Gesellschaften. Die Standorte sind sonnig, trocken, süd- bis westlich exponiert und von

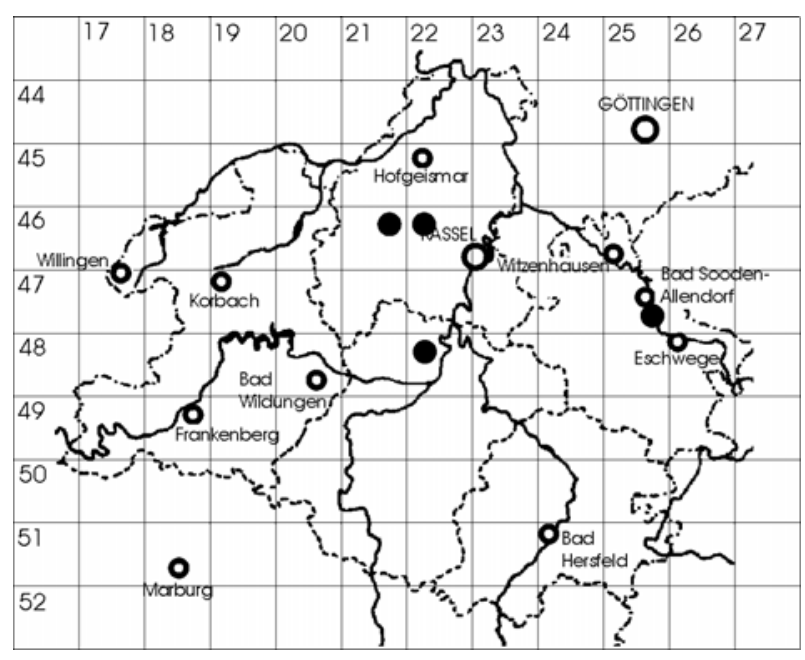

Abb. 14: Verbreitung des Cladonietum foliaceae im Untersuchungsgebiet. 
eher geringer Neigung und daher optimal für die wärmeliebende Art Cl. foliacea und die xerophytische Strauchflechte Cl. rangiformis.

Während die Gesellschaft in Nordwestdeutschland nach PAUS (1997) eine charakteristische Dünengesellschaft darstellt, bleibt sie in Nordhessen auf solche Gebiete beschränkt, an denen die Vulkanite Basalt und Diabas an die Oberfläche treten. Besonders gut ausgeprägte Bestände finden sich daher z.B. auf Basalt am Dörnberg bei Zierenberg (MTB 4621/22) und auf Diabas am Bilstein bei Albungen (MTB 4725). Auf diesen Felsstandorten dürfte die Gesellschaft dauerhaften Bestand haben, sofern, gegebenenfalls durch Pflegemaßnahmen, die Felsbereiche ihren offenen Charakter behalten und eine Verbuschung verhindert wird.

Das Cladonietum foliaceae tritt innerhalb der Felsgrus- und Felsbandgesellschaften der Sedo-Scleranthetea (z.B. im Polytricho-Allietum montani, s. BERND 1998) sowie innerhalb von Halbtrockenrasen des Verbandes Mesobromion auf und kann dort, alle Einzelvorkommen aufsummiert, mehr als $10 \mathrm{~m}^{2}$ Gesamtgröße aufweisen.

\section{Anmerkungen:}

Das Cladonietum foliaceae zeigt nach KLEMENT (1955) eine optimale Entwicklung bei viel größerem Artenreichtum in den vorgeschobenen Steppeninseln des Böhmischen Raumes. Die Vorkommen in Deutschland stellen demnach lediglich verarmte Bestände dar. Im Untersuchungsgebiet liegt die mAZ der Flechten bei 5,9. Mit Cladonia foliacea, Cl. coccifera, Cl. gracilis, Cl. phyllophora, Cetraria aculeata und Placythiella oligotropha sind im eigenen nordhessischen Aufnahmematerial allerdings 6 in Hessen gefährdete Arten zu verzeichnen (vgl. Tab. 27 S. 91 und WIRTH et al. 1996).

\subsubsection{Cladonion rei}

(Tab. 9; Tab. 10 im Anhang 3)

Für die Erdflechtengesellschaften gestörter Standorte schlägt PAUS (1997) die Aufstellung der Federation Cladonion rei vor, als dritte Federation innerhalb der Ordnung Peltigeretalia. Charakterarten des Cladonion rei, welche auch in Nordhessen auftreten sind Cladonia rei, Cl. pyxidata ssp. chlorophaea, Cl. humilis und Peltigera didactyla. Als Trennarten gelten Cl. fimbriata und Ceratodon purpureus.

Die Aufnahmen des Cladonion rei stammen schwerpunktmäßig aus dem Gebiet um den Hohen Meißner, das insgesamt sehr reich an Flechtenvorkommen ist. Möglicherweise ist diese Federation auch in anderen Teilen des Untersuchungsgebietes häufiger, als dies das Aufnahmematerial nahelegt. Es konnten jedoch aus zeitlichen Gründen nicht alle Meßtischblätter der TK 25 von Nordhessen auf Ruderalstandorte, also potentielle Wuchsorte dieser Gesellschaften abgesucht werden. 
Tab. 9: Stetigkeitstabelle Cladonion rei

Einheit 1: Cladonia floerkeana-Cladonion rei-Fragmentgesellschaft

Einheit 2: Cladonietum rei

$$
2.1 \text { Cladonia floerkeana-Variante }
$$

2.2 Typische Variante

Einheit 3: Cladonia subulata-Cladonion rei-Fragmentgesellschaft

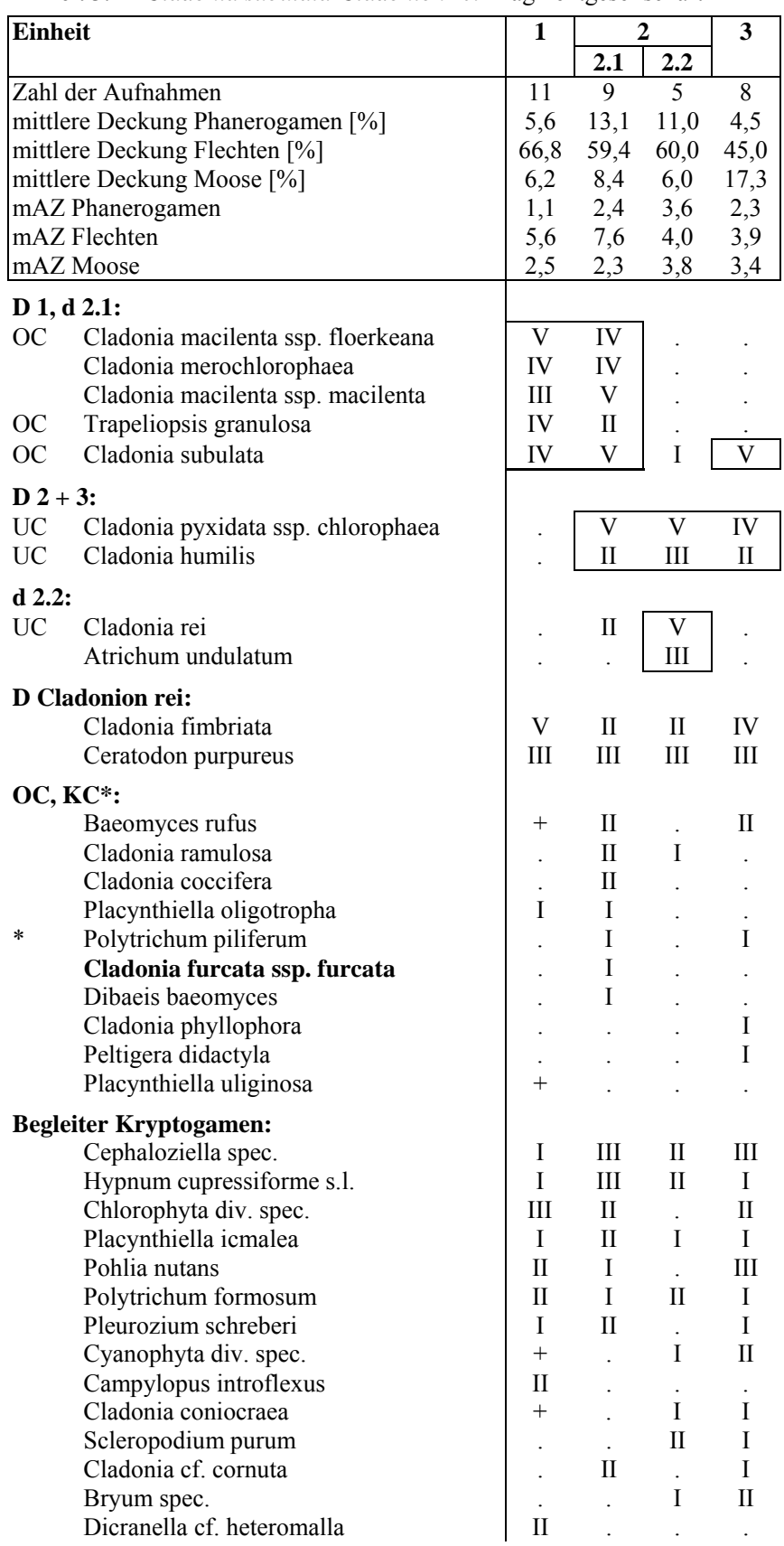


Forts. Tab. 9: Stetigkeitstabelle Cladonion rei

\begin{tabular}{|c|c|c|c|c|}
\hline \multirow[t]{2}{*}{ Einheit } & \multirow[t]{2}{*}{1} & \multicolumn{2}{|c|}{2} & \multirow[t]{2}{*}{3} \\
\hline & & 2.1 & 2.2 & \\
\hline \multicolumn{5}{|l|}{ Phanerogamen: } \\
\hline Deschampsia flexuosa & III & II & II & I \\
\hline Calluna vulgaris & + & III & . & II \\
\hline Hieracium spec. & + & 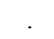 & I & III \\
\hline Agrostis capillaris & & II & II & I \\
\hline Festuca ovina agg. & + & III & . & $\cdot$ \\
\hline Festuca rubra agg. & . & . & II & II \\
\hline Holcus lanatus & + & & $\mathrm{I}$ & I \\
\hline Betula pendula juv. & . & . & II & . \\
\hline Rumex acetosella & 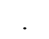 & II & . & . \\
\hline
\end{tabular}

\section{Außerdem mit Stetigkeit < II und in weniger als 3 Einheiten:}

Achillea millefolium 3:I, 4:I; Agrostis stolonifera 3:I; Andreaea rupestris 4:I; Brachythecium spec. 3:I; Bryophyta spec. 2:I; Buxbaumia aphylla 1:+; Calliergonella cuspidata 3:I; Campylopus flexuosus 4:I; Carex spec. 4:I; Centaurea jacea 4:I; Cladonia caespiticia 1:I; Cladonia cervicornis 2:I; Cladonia coccifera/deformis agg. 2:I; Cladonia cryptochlorophaea 2:I, 3:I; Cladonia gracilis 2:I; Cladonia grayi s.str. 1:+; Cladonia novochlorophaea 1:+; Cladonia pyxidata ssp. pyxidata 2:I, 4:I; Cladonia spec. 1:I, 2:I; Cladonia squamosa 2:I; Dicranum scoparium 1:+, 3:I; Ditrichum spec. 4:I; Epilobium spec. 4:I; Galium saxatile 3:I; Hieracium lachenalii 2:I; Hieracium pilosella 2:I; Hypericum hirsutum 3:I; Hypochaeris radicata 2:I; Juncus effusus 4:I; Leontodon autumnalis 2:I; Linum catharticum 3:I; Lophocolea heterophylla 4:I; Lophoziaceae spec. 1:+; Luzula campestris 4:I; Micarea peliocarpa 2:I; Musci spec. 3:I; Pimpinella saxifraga 2:I; Polytrichum commune 2:I; Polytrichum juniperinum 2:I, 4:I; Populus tremula juv. 4:I; Potentilla anserina 3:I; Racomitrium heterostichum agg. 4:I; Racomitrium lanuginosum 4:I; Rhytidiadelphus squarrosus 4:I; Vaccinium myrtillus 1:+, 3:I; Vaccinium vitis-idaea 1:+; Veronica spec. 3:I. 


\subsubsection{Cladonia floerkeana-Cladonion rei-Fragmentgesellschaft}

(Tab. 9; Tab. 10 (Einheit 1) im Anhang 3; Taf. 1.5 im Anhang 2)

Syn.: „Übergang zum Cladonion arbusculae“ (PAUs 1997).

\section{a) Floristische Zusammensetzung, Physiognomie und Struktur}

Die Fragmentgesellschaft stellt einen Übergang zwischen dem Cladonietum rei und der Cladonia bacillaris-Gesellschaft dar. Als Trennart der Federation tritt Cl. fimbriata hochstet auf, etwas weniger häufig auch Ceratodon purpureus. Zur CAV zählt darüber hinaus die Artengruppe um Cl. macilenta ssp. floerkeana und Cl. merochlorophaea; eine Gruppe von Flechten also, die einen ihrer Schwerpunkte auch in der Cladonia bacillaris-Gesellschaft haben. Negativ differenziert wird die Fragmentgesellschaft durch das Fehlen der für das Cladonietum rei charakteristischen Arten.

Geprägt wird das Erscheinungsbild durch sorediöse stift- und becherförmige Arten, so daß diese Fragmentgesellschaft optisch nur schwer von der Cladonia bacillarisGesellschaft zu unterscheiden ist. An der Gesamtdeckung von im Mittel $75 \%$ (max. $95 \%$ !) haben die Flechten mit durchschnittlich $67 \%$ (max. $90 \%$ !) einen beträchtlichen Anteil. Phanerogamen und Moose sind nur ausnahmsweise, Algen nie von Bedeutung beim Aufbau der Gesellschaft. Die mittlere Artenzahl der Flechten beträgt 5,6, die der Moose 2,5 und die der Phanerogamen nur 1,1.

\section{b) Standorte und Vorkommen im UG}

Die Cladonia floerkeana-Cladonion rei-Fragmentgesellschaft konnte an 8 Wuchsorten insgesamt 11 mal aufgenommen werden. Sie siedelt in verschiedenen Höhenlagen, Hangneigungen und Expositionen. Als Ausgangsgestein ist vor allem der Mittlere Buntsandstein zu nennen. Verglichen mit anderen, nachfolgend beschriebenen Gesellschaften des Cladonion rei sind die $\mathrm{pH}(\mathrm{KCl})$-Werte der Böden relativ niedrig (Median 3,6), ebenso die elektrolytische Leitfähigkeit (Median $46 \mu \mathrm{S} / \mathrm{cm}$ ). Die

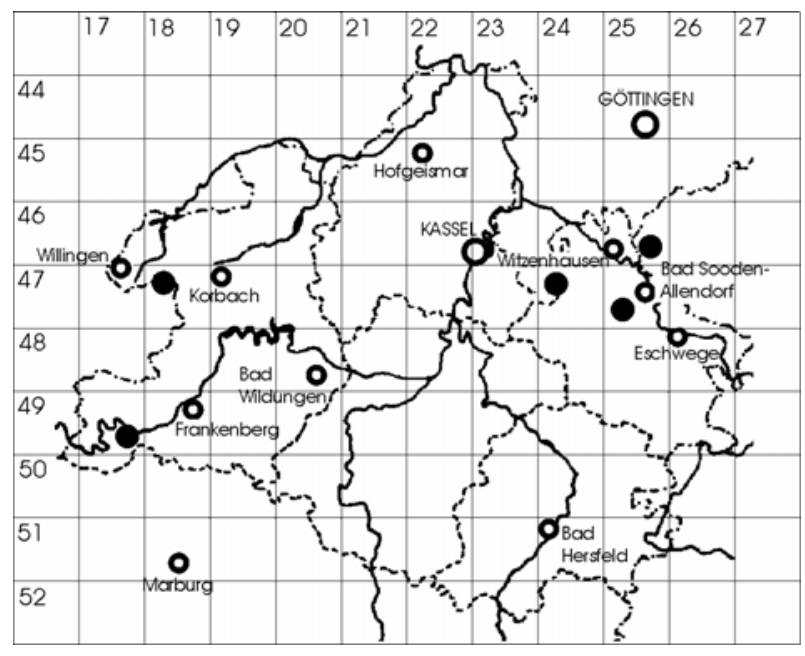

Abb. 15: Verbreitung der Cladonia floerkeanaCladonion rei-FG im Untersuchungsgebiet.

Streuauflage kann fehlen oder auch beträchtliche Ausmaße haben. Der Skelettanteil der Böden ist eher gering.

Das Mikroklima dieser Erdflechtenstandorte zeichnet sich durch lange Sonneneinstrahlung oder allenfalls zeitweise Beschattung aus. Die Böden sind trocken bis frisch. Die Gesellschaft tritt innerhalb von Zwergstrauchheiden, bodensauren Laubwäldern mit Eiche und Rotbuche oder Fichtenforsten auf. Die Standorte entsprechen denen der Cladonia bacillaris-Gesellschaft (Erdanrisse an Wegen etc.). 
Ebenso wie diese dürfte sie ein intermediäres Sukzessionsstadium darstellen und wird, wie mancherorts schon zu beobachten, im Untersuchungsgebiet wohl in der Regel von Phanerogamengesellschaften abgelöst.

\subsubsection{Cladonietum rei}

(Tab. 9; Tab. 10 (Einheit 2) im Anhang 3; Taf. 1.6 im Anhang 2)

\section{a) Floristische Zusammensetzung, Physiognomie und Struktur}

Als Charakterarten der Union treten zusätzlich zu den Differentialarten der Federation noch Cladonia pyxidata ssp. chlorophaea, Cl. humilis und Cl. rei auf. Es lassen sich zwei Varianten unterscheiden.

Die Cladonia floerkeana-Variante (Einheit 2.1) wird abgegrenzt durch die Gruppe von Cl. macilenta welche zugleich auch Differentialartengruppe der im vorigen Kapitel beschriebenen Cladonia floerkeana-Cladonion rei-Fragmentgesellschaft ist. Aufgrund dieses Übergangscharakters ist die mittlere Artenzahl der Flechten hier mit 7,6 Arten relativ hoch. Der Typischen Variante (Einheit 2.2) fehlen dagegen diese Arten, es sind im Mittel nur 4,0 Flechtenarten zu verzeichnen. In der Typischen Variante tritt das Moos Atrichum undulatum in Erscheinung, und Cl. rei kommt, im Gegensatz zur Cladonia floerkeana-Variante, hochstet vor.

Einzelne Aufnahmen weisen relativ viele Phanerogamenarten auf, die im Ausnahmefall bis zu $45 \%$ Deckung erreichen. An der Gesamtdeckung von durchschnittlich $76 \%$ in beiden Varianten sind jedoch hauptsächlich die Flechten mit 59 bzw. 60 \% beteiligt. Die Bestände können relativ geschlossen oder auch sehr lückig sein (bis zu $50 \%$ offener Boden).

\section{b) Standorte und Vorkommen im UG}

Das Cladonietum rei ist im Aufnahmematerial mit 14 Aufnahmen vertreten. Im Untersuchungsgebiet wurde die Union an 9 Wuchsorten angetroffen. Anthropogene Störstellen treten zwar zahlreich auf, Flechtenbestände des Cladonietum rei sind dort offensichtlich jedoch nur ausnahmsweise $\mathrm{zu}$ finden. Die Gesellschaft siedelt überwiegend an Ruderalstandorten wie Erdanrissen, Böschungen und auf Bergbauflächen, an denen sich wenig bis keine Streu angesammelt hat. Die Standorte sind sonnig bis etwas beschattet, meist

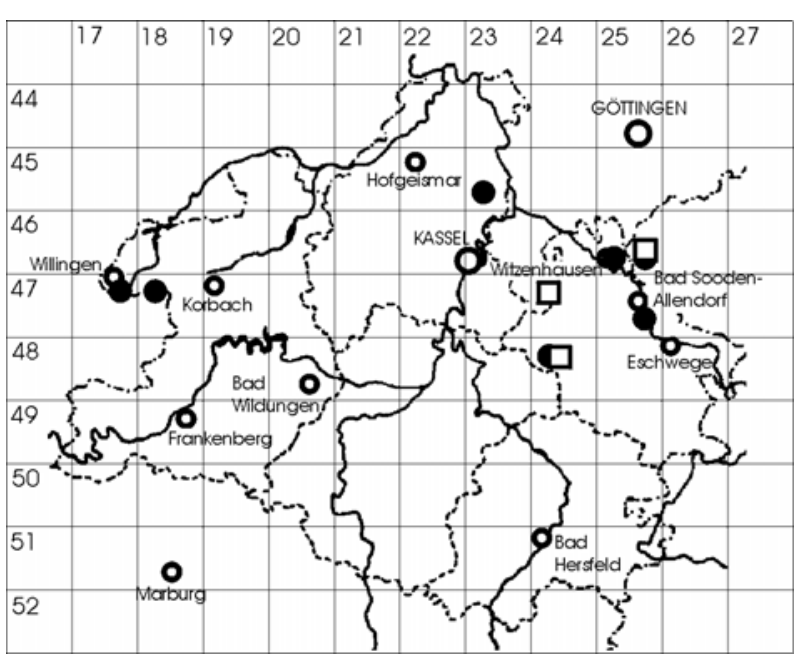

Abb. 16: Verbreitung des Cladonion rei im Untersuchungsgebiet: Cl. floerkeanaVariante; Typische Variante. frisch oder trocken, seltener wechselfeucht. Exposition und Inklination der Flächen sind 
uneinheitlich. Als Ausgangsgestein treten neben Grauwacke, Tonschiefer und Mittlerem Buntsandstein auch tertiäre Sedimente auf. Bei diesen, in der ehemaligen Zeche Glimmerode (MTB 4824) anstehenden Sedimenten handelt es sich um Sande mit mehr oder weniger deutlicher Beimischung von Braunkohle.

Bezüglich der $\mathrm{pH}(\mathrm{KCl})-W e r t e$ zeigen sich große Unterschiede zwischen den beiden Varianten des Cladonietum rei. Die zur Cladonia bacillaris-Gesellschaft vermittelnde Variante weist einen Medianwert von 3,5 auf, während die Typische Variante einen Wert von 5,6 hat! Die Leitfähigkeitswerte schwanken innerhalb der Untereinheiten erheblich und sind an den Standorten mit Braunkohlebeimischung besonders hoch.

Das Cladonietum rei tritt als Flechtengemeinschaft innerhalb verschiedenster Phytozönosen auf, sofern sich dort geeignete Störstellen in der Vegetation befinden. Als angrenzende und teilweise überschirmende Phanerogamenvegetation wurden in Nordhessen Zwergstrauchheiden, lichte Buchen- oder Eichenwälder sowie Pioniergehölzvegetation mit Hängebirke, Grünerle oder Zitterpappel notiert. Es ist anzunehmen, daß die Gesellschaft nur vorübergehend bestehen kann und nach einiger Zeit von der die Störstellen überwachsenden Phanerogamenvegetation verdrängt wird.

\section{Anmerkung zum Vorkommen von Cladonia rei in Nordhessen:}

In seiner Schrift aus dem Jahre 1981 weist FoLLMANN Cl. rei erstmalig für das Hessische Bergland nach. Als Wuchsorte gibt er offene Kulturstandorte wie Bahndämme, Trockenrasen oder Wegränder sowie Kalkfelsen mit geringfügiger Humusauflage an. Besonders gut besetzte Fundorte seien einige gut drainierte Sohlen seit längerem aufgelassener Basaltbrüche.

Im Rahmen der eigenen Geländearbeiten wurde Cl. rei nur an 6 Orten nachgewiesen (s. Artenliste im Anhang 1), lediglich an 2 Stellen erreichen die Thalli Ausdehnungen von immerhin etwa $5 \mathrm{~cm}$. Einer der von FOLLMANN (1981) aufgeführten „,besonders gut besetzten” Standorte - der Hangarstein - wurde ebenfalls aufgesucht. Der Bereich des dortigen ehemaligen Steinbruchs ist inzwischen aber bis an das Ufer des Gewässers bewaldet und $C l$. rei nicht mehr auffindbar.

\subsubsection{Cladonia subulata-Cladonion rei-Fragmentgesellschaft}

(Tab. 9; Tab. 10 (Einheit 3) im Anhang 3)

\section{a) Floristische Zusammensetzung, Physiognomie und Struktur}

Diese Fragmentgesellschaft wird durch das Vorherrschen von Cladonia subulata geprägt. An Diagnostischen Arten des Cladonion rei treten Cl. fimbriata (D), Ceratodon purpureus (D), Cl. pyxidata ssp. chlorophaea (FC) und Cl. humilis (FC) auf; letztere beiden deuten die Nähe der Fragmentgesellschaft zum Cladonietum rei an.

Die artenarmen Bestände dieser Fragmentgesellschaft zeigen sich bezüglich der Deckungsverhältnisse sehr uneinheitlich. Die Gesamtdeckung schwankt zwischen 10 und $90 \%$ (MW 66\%), gleiches gilt für die Deckung der Flechten, die ebenfalls zwischen 10 und $90 \%$ liegt (MW 45\%). Die Aufnahmeflächen können also sehr lückig, mit hohem Anteil an offenem Boden oder Gestein, oder ziemlich geschlossen sein. Mancherorts nehmen die filzigen Watten fädiger Grünalgen große Teile der 
Bodenoberfläche ein und prägen dann, neben den immer auffallenden sorediösen becher- oder stiftförmigen Flechten, das Erscheinungsbild. Für die Algenkrusten in der Zeche Glimmerode sind die Gattungen Trentepohlia, Gloeocapsa, Oscillatoria, Mesotaenium (cf.) und die oft dominierende Jochalge Zygogonium ericetorum charakteristisch (HOPPERT unpubl.). Sofern Höhere Pflanzen in die Flechtenrasen eindringen, handelt es sich dabei oft um Gräser.

\section{b) Standorte und Vorkommen im UG}

Die Fragmentgesellschaft wird für das Untersuchungsgebiet mit 8 Aufnahmen von 5 Lokalitäten belegt. Besiedelt werden unterschiedlich exponierte und geneigte Stellen an sonnigen bis halbschattigen, frischen bis trockenen Standorten. Der Median des $\mathrm{pH}(\mathrm{KCl})$-Wertes des Substrates liegt mit 5,1 im schwach sauren Bereich. Die Ausgangsgesteine entsprechen denen an Cladonietum rei-Standorten, jedoch wurde die Cladonia subulata-Cladonion reiFragmentgesellschaft zusätzlich auch

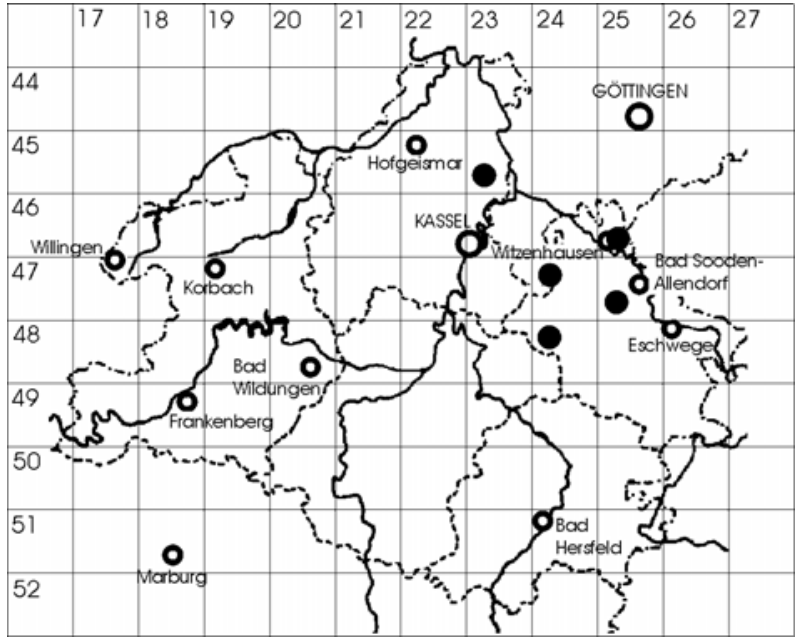

Abb. 17: Verbreitung der Cladonia subulataCladonion rei-FG im Untersuchungsgebiet. auf Basalt und in einem Falle sogar auf stark verwitterter Dachpappe angetroffen. Die Gesellschaft tritt im Untersuchungsgebiet an Stellen mit offenem Boden innerhalb von Calluna vulgaris-, Deschampsia flexuosa- oder Festuca ovina agg.-Beständen auf bzw. siedelt an Standorten mit angrenzenden Pioniergehölzen wie Hängebirke, Zitterpappel oder Kiefer. Sie zählt zur Gruppe der intermediären Sukzessionsstadien und wird mit fortschreitender Vegetationsentwicklung an den meisten Wuchsorten wohl von der sich schließenden Phanerogamenvegetation verdrängt.

\section{Anmerkung:}

Cladonia subulata-Dominanzen treten auch in der Cladonia bacillaris-Gesellschaft (Kap. E2.1.2.1) auf. PAUS (1997) hält sie häufig für zufallsbedingt als Folge der Erstansiedlung. PAUS et al. (1993) bezeichnen Cl. subulata als eine Art mit weiter soziologischer Spanne und einem Schwerpunkt in der Cladonia bacillaris-Gesellschaft. Dies bestätigen auch die vorliegenden Daten aus Nordhessen. 


\subsubsection{Standorte und Dynamik im Überblick}

Geeignete Standorte für Gesellschaften der Klasse Ceratodonto-Polytrichetea zeichnen sich durch relativ extreme Bedingungen (Trockenheit, Substratinstabilität, geringe Bodenbildung) aus, die den Phanerogamen eine Ansiedlung erschweren oder unmöglich machen. Die fehlende Konkurrenz durch Höhere Pflanzen ist sicherlich einer der Hauptgründe, warum sich Flechtengesellschaften ausbilden können. Das Ausgangsgestein ist insofern von Bedeutung, als daß die Ceratodonto-PolytricheteaGesellschaften auf Standorte mit Böden über mehr oder weniger sauren Gesteinen beschränkt sind.

Nur in Ausnahmefällen gedeihen die Flechtenbestände im Schatten, meist werden sie wenigstens zeitweise besonnt. Oft müssen die Erdflechten Trockenheit ertragen (z.B. an den Standorten des Cladonietum foliaceae). Besser wasserversorgte, frische Böden bevorzugen dagegen beispielsweise die Gesellschaftsbildner des Dibaeetum baeomycetis. Andere Faktoren wie Skelettanteil der Böden, Exposition und Inklination scheinen eine relativ geringe Rolle $\mathrm{zu}$ spielen und sind innerhalb einer Vegetationseinheit oft recht uneinheitlich. Eine eindeutige Abhängigkeit von der Höhe zeigen nur die beiden Höhenformen des Dibaeetum baeomycetis. Der Medianwert von 595 m ü. NN für das Lecideetum uliginosae deutet zwar auch eine Bevorzugung höherer Lagen an, die wenigen Werte streuen allerdings stark. Alle anderen Vegetationseinheiten kommen in verschiedenen Höhenlagen vor.

\section{a) Bodenökologischer Vergleich}

$\mathrm{pH}(\mathrm{KCl})$-Werte (Abb. 18, Tab. 11):

Das Cladonietum foliaceae (Nr. 2.4), das Cladonietum rei (Nr. 3.2) und die Cladonia subulata-Cladonion rei-Fragmentgesellschaft (Nr. 3.3) kommen tendenziell auf etwas weniger sauren Böden vor. Auffallend hohe Medianwerte bei beiden $\mathrm{pH}-$ Werten zeigt die Cladonia subulata-Cladonion rei-Fragmentgesellschaft. Das Cladonietum foliaceae weist einen höheren Median auf, als die anderen Cladonion arbusculae-Gesellschaften und liegt etwa im Bereich des Cladonietum rei. Unter den Pioniergesellschaften zeigt das Dibaeetum baeomycetis (Nr. 1.3) deutlich höhere Werte als das Lecideetum uliginosae (Nr. 1.4).

Die Cladonia bacillaris-Gesellschaft (Nr. 2.1) gedeiht auf Böden mit niedrigeren $\mathrm{pH}(\mathrm{KCl})$-Werten als die möglichen Folgegesellschaften Cladonietum mitis (Nr. 2.2) und andere Cladonion arbusculae-Gesellschaften. Der Median liegt etwa im Bereich des Wertes der nahestehenden Cladonia floerkeana-Cladonion rei-Fragmentgesellschaft (Nr. 3.1). 


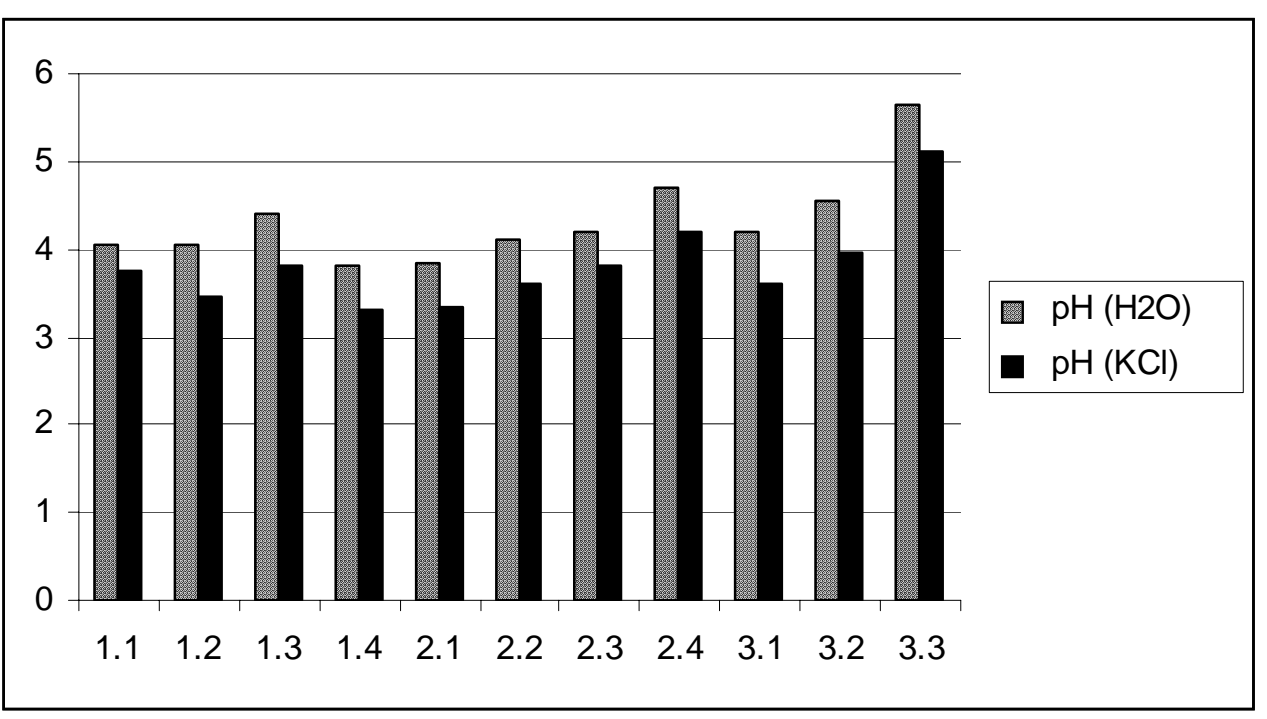

Abb. 18: Ceratodonto-Polytrichetea piliferi: Medianwerte der $\mathrm{pH}$-Werte (Nummerierung der Einheiten s. Tab. 4, S. 29).

Elektrolytische Leitfähigkeit (Abb. 19, Tab. 11):

Alle Vegetationseinheiten haben einen relativ niedrigen Median der Leitfähigkeit, d.h. die Gesellschaften siedeln auf Standorten mit elektrolytarmem Bodenwasser bzw. mit mittleren Elektrolytgehalten.

Das Dibaeetum baeomycetis (Nr. 1.3) weist einen deutlich geringeren Median auf, als die anderen Gesellschaften der Klasse. Nur die beiden Bestände des PycnothelioCladonietum (Nr. 1.1) zeigen einen ähnlich niedrigen Wert. Besonders deutlich ist der Unterschied zwischen Dibaeetum und Lecideetum uliginosae (Nr. 1.4), das als zweite Pioniergesellschaft der Federation Baeomycion rosei Böden mit etwas höherer elektrolytischer Leitfähigkeit besiedelt.

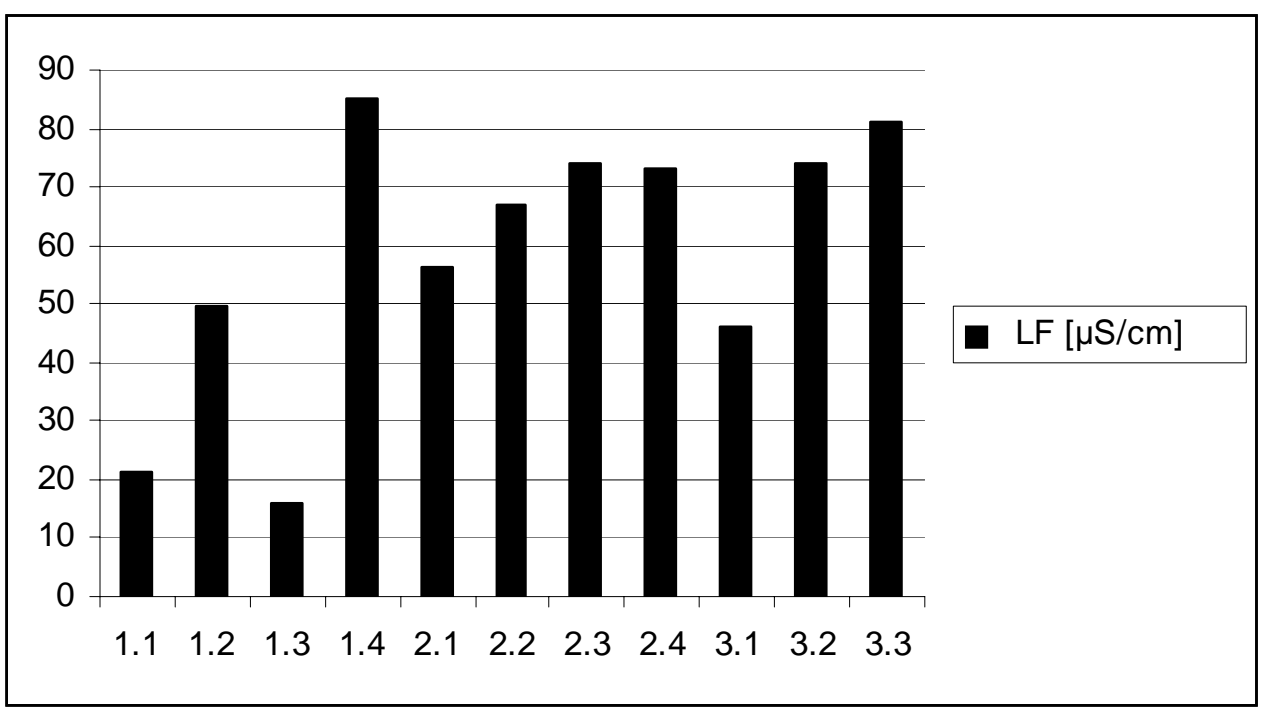

Abb. 19: Ceratodonto-Polytrichetea piliferi: Medianwerte der elektrolytischen Leitfähigkeit (Nummerierung der Einheiten s. Tab. 4, S. 29). 
Die Gesellschaften des Cladonion arbusculae (Nr. 2.1 bis 2.4) erweisen sich bezüglich der Medianwerte als recht einheitlich. Die Böden an den Standorten der Cl. bacillarisGesellschaft (Nr. 2.1) haben den niedrigsten Wert innerhalb der Federation. Die nahestehende Cl. floerkeana-Cladonion rei-Fragmentgesellschaft (Nr.3.1) den geringsten Wert aller Einheiten des Cladonion rei (Nr. 3.1 bis 3.3).

Tab. 11: Ceratodonto-Polytrichetea piliferi: pH-Werte und elektrolytische Leitfähigkeiten der Böden

\begin{tabular}{|c|c|c|c|c|c|c|c|c|c|}
\hline Vegetationseinheit & 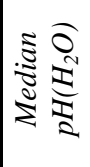 & 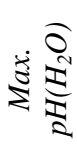 & 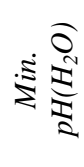 & 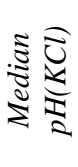 & 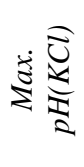 & : & 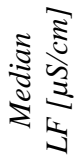 & 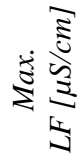 & 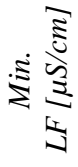 \\
\hline Pycnothelio-Cladoniet & 4,1 & 4,2 & 3,9 & 3,5 & 4,2 & 3,3 & 40 & 53 & 10 \\
\hline Dibaeetum baeomycetis, Cladonia cervicornis-HF & 4,4 & 4,7 & 4,2 & 3,8 & 4,0 & 3,6 & 31 & 49 & 16 \\
\hline Dibaeetum baeomycetis, Baeomyces rufus-HF & 4,4 & 4,8 & 4,1 & 3,9 & 4,3 & 3,4 & 10 & 41 & 0 \\
\hline Lecideetum uliginosae & 3,8 & 4,0 & 3,8 & 3,3 & 3,8 & 3,2 & 85 & 396 & 35 \\
\hline Cl. bacillaris-Gesellschaft & 3,9 & 4,5 & 3,6 & 3,4 & 4,0 & 2,9 & 57 & 630 & 29 \\
\hline Cladonietum mitis cladonietos & 4,3 & 4,7 & 4,0 & 3,9 & 4,6 & 3,6 & 55 & 89 & 21 \\
\hline Cladoniet & 4,1 & 4,2 & 3,6 & 3,7 & 3,8 & 3,3 & 53 & 87 & 30 \\
\hline ina-Var & 3 & 4,2 & 3, & 3 & 3,6 & 3,0 & 93 & 113 & 43 \\
\hline Cl. f & 4 & 4,8 & 3,9 & 3 & 4,2 & 3,4 & 74 & 136 & 33 \\
\hline liaceae & 4 & 4,9 & 4,5 & 4 & 4,3 & 4,0 & 73 & 146 & 29 \\
\hline Cl. floerkeana-Cladonion & 4,2 & 4,8 & 3,7 & 3,6 & 4,3 & 2,9 & 46 & 94 & 23 \\
\hline Cladonietum rei, $\mathrm{Cl}$. floerkeana-Vari & 4,0 & 4,8 & 3,3 & 3,5 & 4,3 & 2,9 & 59 & 372 & 10 \\
\hline Cladonietum rei, Typische Variante & 6,0 & 6,5 & 4,7 & 5,6 & 6,0 & 3,8 & 102 & 356 & 34 \\
\hline Cl. subulata-Cladonion rei-FG & 5,7 & 6,3 & 4,5 & 5,1 & 6,2 & 4,0 & 81 & 156 & 40 \\
\hline
\end{tabular}

\section{b) Dynamik}

$\mathrm{Zu}$ den Pioniergesellschaften zählen das Dibaeetum baeomycetis und das Lecideetum uliginosae. Als mögliche Folgegesellschaften dieser Einheiten sind nach PAUS (1997) beispielsweise das Pycnothelio-Cladonietum cervicornis, die Cladonia bacillarisGesellschaft, bei ungünstiger Entwicklung aber auch Phanerogamenbestände denkbar. In Nordhessen ist das Pycnothelio-Cladonietum allerdings, wie bereits erwähnt, nur sehr sporadisch anzutreffen, so daß Gesellschaften des Cladonion arbusculae als Folgegesellschaften wahrscheinlicher sind. Endstadium der Flechtenentwicklung ist das Cladonietum mitis, das entweder über die Cladonia bacillaris-Gesellschaft oder direkt aus den Pioniergesellschaften entstehen kann.

Abb. 20 zeigt eine Vegetationsabfolge an einem Wegrand innerhalb der Traubeneichenwälder am Roßkopf. Hier sind unterschiedliche Sukzessionsstadien nebeneinander entwickelt. Ein Fortschreiten der Entwicklung ist v.a. bei den Pionierund Intermediärstadien an diesem Standort zunächst nicht zu erwarten. An der steilen Wegböschung entstehen durch Abrutschen des Bodenmaterials ständig neue Pionierstandorte, die vor allem Dibaeis baeomyces als Substrat dienen. Darüber siedeln im Traufbereich der Eichen krustige Arten wie Trapeliopsis granulosa und einige offensichtlich lichtliebende Cladonien wie Cladonia uncialis und Cl. crispata. Unter den Bäumen werden die Flechtenbestände überwiegend nur noch von Cl. portentosa und Cl. gracilis gebildet. $\mathrm{Da}$ sich die Baumschicht unter den gegebenen Standortbedingungen in absehbarer Zeit kaum verändern wird, können auch diese Arten am Roßkopf dauerhaft existieren. Die Transektflächen unter den Eichen sind dem Cladonietum mitis typicum zuzuordnen, einige Flächen im Traufbereich mit Arten wie 
Placynthiella uliginosa und Trapeliopsis granulosa zählen dagegen eher zum Cladonietum mitis cladonietosum merochlorophaeae. Im Übergangsbereich beider Subunionen wechseln im vorliegenden Transekt kleinräumig Bestände der Subunion typicum mit solchen der Subunion cladonietosum ab.

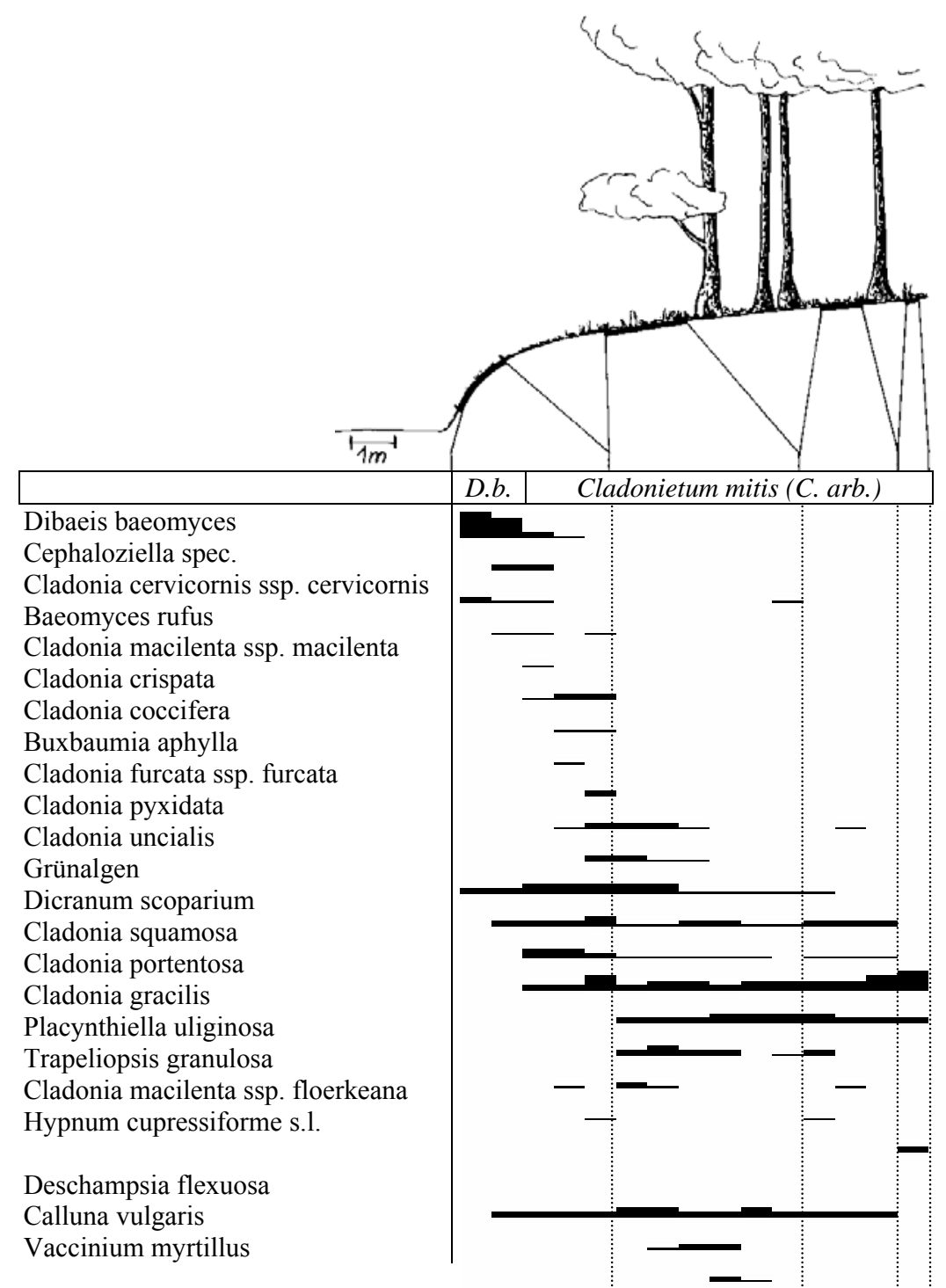

Abb. 20: Transekt an einem Wegrand innerhalb der Eichenwälder am Roßkopf.

Die Balken geben den Wuchsbereich und die Artmächtigkeit der Arten an.

Zugrunde liegt die siebenteilige Braun-Blanquet-Skala (s. DIERSCHKE 1994: 160).

Je nach Höhe der Balken entsprechen diese der Artmächtigkeit r/+, 1, 2, 3 oder 4.

D.b. = Dibaeetum baeomycetis (Baeomycion rosei),

C. arb. = Cladonion arbusculae.

Vermutlich sind alle Erdflechtenbestände im Untersuchungsgebiet, die von Cl. macilenta und/oder Cl. subulata beherrscht werden, als intermediäre Stadien anzusehen (z.B. auch die Cladonia floerkeana-Cladonion rei-Fragmentgesellschaft). Oft werden schon Pionier- und intermediäre Gesellschaften von Phanerogamenbeständen abgelöst. Als Vorstadien von Cladonia furcata-dominierten Beständen kommen sowohl Gesellschaften des Cladonion arbusculae als auch des Cladonion rei in Frage (PAUS 1997). 
Das Cladonietum foliaceae der nordhessischen Vulkanitfelsen ist eine Initialgesellschaft, die auf den extrem trockenen Standorten dauerhaften Bestand haben kann, sofern eine Verbuschung die Union nicht verdrängt.

Das Cladonietum rei auf Störstellen dürfte im allgemeinen wohl von sich ausdehnenden Phanerogamengesellschaften abgelöst werden. Initialstadien der Union sind nach PAUS (1997) gekennzeichnet durch das Auftreten von Placynthiella-Arten.

\subsection{Toninio-Psoretea decipientis}

\section{(Tab. 14)}

Syn.: Psoretea decipientis Mattick ex Follmann 1974 = Barbuletea unguiculatae Mohan 1978 p.p.; Psoretalia decipientis Mattick ex Follmann 1974 = Toninietalia Hadač 1948 prov.; Toninion sedifoliae Hadac 1948 = Toninion coeruleonigricantis Reimers 1951.

In der Klasse Toninio-Psoretea decipientis werden Kryptogamengesellschaften trockener bis frischer, kalk- bis basenreicher Böden zusammengefaßt. Ihre Klassencharakterarten sind nach DREHWALD (1993): Barbula unguiculata, B. convoluta, Didymodon fallax und Riccia sorocarpa. Bis auf Riccia treten diese Arten auch in den Beständen des Untersuchungsgebietes auf. Innerhalb der Klasse wird die Ordnung Psoretalia decipientis, welche die flechtenreichen Gesellschaften vereint, den moosreichen Gesellschaften der Ordnung Barbuletalia unguiculatae gegenübergestellt. Alle Gesellschaften der Psoretalia decipientis besiedeln kalk- oder zumindest basenreiche, längerfristig stark austrocknende Böden (DREHWALD 1993).

Tab. 12: Syntaxonomische Übersicht der Gesellschaften innerhalb der Klasse Toninio-Psoretea decipientis

\begin{tabular}{|c|c|}
\hline \multicolumn{2}{|l|}{ Klasse } \\
\hline \multicolumn{2}{|l|}{ Psoretea decipientis } \\
\hline \multicolumn{2}{|l|}{ Ordnung } \\
\hline \multicolumn{2}{|l|}{ Psoretalia decipientis } \\
\hline Federation & \multirow{7}{*}{$\begin{array}{c}\text { diverse } \\
\text { Dominanzbestände }\end{array}$} \\
\hline Toninion sedifoliae & \\
\hline Union, Gesellschaft & \\
\hline Toninia sedifolia-Toninion-Initialgesellschaft & \\
\hline Toninio-Psoretum decipientis & \\
\hline Cladonia symphycarpa-Toninion sedifoliae-FG & \\
\hline Peltigera rufescens-Toninion sedifoliae-FG & \\
\hline
\end{tabular}

Die Angaben bezüglich der Einstufung der auftretenden Flechtenarten als Charakterarten sind in der Literatur sehr uneinheitlich. Tab. 13 faßt die Aussagen sechs verschiedener Autoren zusammen. Jeweils fettgedruckt sind die für die vorliegende Untersuchung übernommenen Einstufungen. 
Tab. 13: Autorenangaben zu Kennarten innerhalb der Klasse Toninio-Psoretea decipientis

\begin{tabular}{l|llllll}
\hline & $\begin{array}{l}\text { Drehwald } \\
(1993)\end{array}$ & $\begin{array}{l}\text { Wirth } \\
(1995)\end{array}$ & $\begin{array}{l}\text { Follmann } \\
(1974)\end{array}$ & $\begin{array}{l}\text { Klement } \\
(1955)\end{array}$ & $\begin{array}{l}\text { Marstaller } \\
(1971)\end{array}$ & $\begin{array}{l}\text { Wilmanns } \\
(1959)\end{array}$ \\
\hline Buellia epigaea & - & - & AC & AC & - & AC \\
Cetraria aculeata & - & - & OC/KC & $*$ & - & - \\
Cladonia furcata ssp. subrangiformis & - & VC & - & $*$ & - & - \\
Cladonia pyxidata ssp. pocillum & - & - & OC/KC & $*$ & - & $*$ \\
Cladonia rangiformis & - & - & OC/KC & $*$ & - & $*$ \\
Cladonia symphycarpa & VC & - & VC & VC & VC & VC \\
Endocarpon pusillum & - & - & AC & AC & VC & AC \\
Fulgensia bracteata & AC & - & AC & VC & AClokal & - \\
Fulgensia fulgens & AC & AC & AC & AC & AClokal & AC \\
Peltigera rufescens & - & - & OC/KC & $*$ & - & $*$ \\
Placidium squamulosum & VC & - & - & VC & VC & VC \\
Psora decipiens & VC & VC & OC/KC & VC & AClokal & VC \\
Squamarina cartilaginea & VC & - & AC & AC & AClokal & AC \\
Squamarina lentigera & VC & VC & AC & AC & AClokal & AC \\
Toninia sedifolia & VC & VC & VC & VC & VC & VC \\
\hline
\end{tabular}

* Ordnungs- bzw. Klassencharakterart der Epigaeetalia bzw. -etea.

\subsubsection{Toninion sedifoliae}

Das Toninion sedifoliae ist die einzige Federation innerhalb der Ordnung Psoretalia.

\section{a) Syntaxonomische Anmerkungen}

Bei Klement (1955) wird der Verband Toninion coeruleonigricantis (Syn. Toninion sedifoliae) zur Ordnung Epigaeetalia (Syn. Peltigeretalia Klem. 1950) und in die Klasse Epigaeetea lichenosa Klem. gestellt. Als Verbandscharakterarten führt er u.a Psora decipiens, Toninia sedifolia (Syn. T. coeruleonigricans) und Placidium squamulosum (Syn. Dermatocarpon hepaticum) auf. Ordnungscharakterarten der Epigaeetalia sind nach KLEMENT (1955) u.a. Cladonia pyxidata ssp. pocillum, Cetraria aculeata (Syn. Cornicularia aculeata), Peltigera rufescens, Cl. rangiformis, Cl. subrangiformis (Syn. Cl. furcata ssp. subrangiformis) und Cl. furcata palamaea. FOLLMANN (1974) scheint diese Einstufung der Ordnungscharakterarten (mit Ausnahme von Cl. furcata ssp. subrangiformis) übernommen zu haben, obwohl er den Verband Toninion in eine andere Ordnung, die Psoretalia decipientis (Klasse Psoretea decipientis) stellt. Zumindest ein Teil der hier aufgeführten Arten können nach heutigem Wissensstand nicht als Ordnungscharakterarten der Psoretalia gelten. Cl. rangiformis beispielsweise tritt auch in Cladonion arbusculae-Gesellschaften hochstet auf (s. Kap. E2.1.2.4), gleiches gilt für Cetraria aculeata.

\section{b) Anmerkung zu den Standorten und Standortbedingungen}

Die gemessenen $\mathrm{pH}-$ Werte weisen nur geringe Unterschiede zwischen den Vegetationseinheiten der Toninio-Psoretea auf. Bei der elektrolytischen Leitfähigkeit zeigen sich deutliche Unterschiede zwischen den Böden über Zechsteingips und denen über Zechsteindolomit bzw. Muschelkalk. Da jedoch keine der nachfolgend beschriebenen Vegetationseinheiten auf ein Ausgangsgestein beschränkt ist, schwanken dementsprechend die Leitfähigkeitswerte innerhalb der Aufnahmegruppen sehr stark. Beiden Meßgrößen kommt also keine differenzierende Bedeutung zu. Tab. 19 (S. 76) 
faßt die Ergebnisse der Bodenanalysen zusammen. Aufgrund der geringen Aussagekraft dieser Meßwerte wird bei der weiteren Besprechung der Vegetationseinheiten auf die $\mathrm{pH}$ - und Leitfähigkeitswerte nicht näher eingegangen.

\section{Tab. 14: Übersichtstabelle Toninio-Psoretea decipientis}

Einheit 1: Toninia sedifolia-Toninion-Initialgesellschaft

Einheit 2: $\quad$ Toninio-Psoretum decipientis

Einheit 3: Cladonia symphycarpa-Toninion-Fragmentgesellschaft

Einheit 4: Peltigera rufescens-Toninion-Fragmentgesellschaft

Einheit 5: Dominanzbestände

\begin{tabular}{|c|c|c|c|c|c|c|}
\hline \multicolumn{2}{|c|}{ Einheit } & 1 & 2 & 3 & 4 & 5 \\
\hline \multicolumn{2}{|c|}{ Zahl der Aufnahmen } & 3 & 41 & 11 & 8 & 11 \\
\hline \multicolumn{2}{|c|}{ mAZ Phanerogamen } & 5,0 & 4,1 & 6,2 & 8,4 & 10,9 \\
\hline \multicolumn{2}{|c|}{ mAZ Flechten } & 2,0 & 6,7 & 6,4 & 3,3 & 1,8 \\
\hline \multicolumn{2}{|c|}{ mAZ Moose } & 3,3 & 6,0 & 6,6 & 5,4 & 3,5 \\
\hline \multicolumn{2}{|c|}{ D 2: } & & & & & \\
\hline $\mathrm{UC}$ & Fulgensia fulgens & . & III & . & . & . \\
\hline $\mathrm{UC}$ & Fulgensia bracteata & . & IV & . & . & . \\
\hline $\mathrm{UC}$ & Endocarpon pusillum & 1 & II & + & . & . \\
\hline $\mathrm{UC}$ & Squamarina cartilaginea & . & II & $\cdot$ & . & . \\
\hline \multicolumn{7}{|c|}{ D $2+3:$} \\
\hline $\mathrm{FC}$ & Cladonia symphycarpa & . & III & $\mathrm{V}$ & I & . \\
\hline \multirow[t]{2}{*}{$\mathrm{FC}$} & Toninia sedifolia & 3 & III & IV & . & . \\
\hline & Tortella inclinata & . & III & IV & I & . \\
\hline FC & Psora decipiens & . & III & II & . & . \\
\hline $\mathrm{FC}$ & Placidium squamulosum & . & III & II & . & . \\
\hline \multicolumn{7}{|c|}{ D 3: } \\
\hline & Encalypta streptocarpa & . & I & III & . & . \\
\hline & Leptogium cf. lichenoides & . & I & IV & II & . \\
\hline \multicolumn{7}{|c|}{ D $3+4$ : } \\
\hline & Tortella tortuosa & . & $\mathrm{r}$ & II & II & . \\
\hline \multicolumn{7}{|c|}{ D 2-4: } \\
\hline & Peltigera rufescens & . & IV & IV & $\mathrm{V}$ & . \\
\hline & Cyanophyta div. spec. & 3 & IV & IV & IV & . \\
\hline & Ceratodon purpureus & 2 & $\mathrm{~V}$ & IV & III & . \\
\hline $\mathrm{OC}$ & Cladonia pyxidata ssp. pocillum & . & IV & IV & II & + \\
\hline $\mathrm{KC}$ & Barbula convoluta & 1 & III & II & II & + \\
\hline \multicolumn{7}{|c|}{ D 2, 3, 4, 5: } \\
\hline & Ditrichum flexicaule & 1 & IV & $\mathrm{V}$ & III & II \\
\hline & Hypnum cupressiforme var. lacunosum & . & III & $\mathrm{V}$ & III & V \\
\hline \multirow[t]{5}{*}{ FC } & Cladonia furcata ssp. subrangiformis & & II & IV & III & III \\
\hline & Cladonia rangiformis & . & + & II & III & III \\
\hline & Homalothecium lutescens & . & . & I & III & II \\
\hline & Fissidens dubius & . & + & I & II & III \\
\hline & Ctenidium molluscum & . & $\mathrm{r}$ & + & . & III \\
\hline \multicolumn{7}{|c|}{$\mathrm{UC}_{2}, \mathrm{KC}^{*}:$} \\
\hline & Barbula unguiculata & . & $\mathrm{r}$ & . & I & + \\
\hline & Squamarina lentigera & . & I & . & . & . \\
\hline$*$ & Didymodon fallax & . & . & . & I & . \\
\hline & Buellia epigaea & . & . & + & . & . \\
\hline \multicolumn{7}{|c|}{ Begleiter Kryptogamen: } \\
\hline & Collema spec. & 2 & III & II & I & + \\
\hline & Bryum spec. & 1 & II & II & II & II \\
\hline & Thuidium abietinum & 1 & I & I & II & II \\
\hline & Aloina spec. & 2 & II & I & . & . \\
\hline & Pseudocrossidium revolutum & . & II & I & II & . \\
\hline & Encalypta vulgaris & . & II & . & I & + \\
\hline & Trichostomum crispulum & 1 & II & + & I & . \\
\hline
\end{tabular}


Forts. Tab. 14: Übersichtstabelle Toninio-Psoretea decipientis

\begin{tabular}{|c|c|c|c|c|c|}
\hline Einheit & 1 & 2 & 3 & 4 & 5 \\
\hline \multicolumn{6}{|l|}{ Forts. Begleiter Kryptogamen: } \\
\hline Cephaloziella spec. & . & II & II & I & . \\
\hline Pseudocrossidium hornschuchianum & 1 & I & + & I & . \\
\hline Leptogium cf. schraderi & . & I & + & I & . \\
\hline Chlorophyta div. spec. & 1 & I & . & II & . \\
\hline Didymodon rigidulus & . & I & + & . & . \\
\hline Cetraria aculeata & . & $\mathrm{r}$ & I & $\mathrm{I}$ & I \\
\hline Tortula ruralis & . & + & + & $\mathrm{I}$ & . \\
\hline Cladonia furcata ssp. furcata & & & + & & \\
\hline \multicolumn{6}{|l|}{ Phanerogamen: } \\
\hline Thymus pulegioides & 3 & III & IV & IV & III \\
\hline Festuca ovina agg. & 2 & II & III & IV & IV \\
\hline Koeleria pyramidata & 1 & II & III & IV & III \\
\hline Potentilla tabernaemontani & 2 & II & IV & III & III \\
\hline Bromus erectus & 1 & II & III & II & II \\
\hline Sanguisorba minor & 1 & II & III & III & III \\
\hline Hieracium pilosella & 2 & $\mathrm{r}$ & I & III & IV \\
\hline Arenaria serpyllifolia & 1 & I & I & III & I \\
\hline Sedum sexangulare & . & I & II & II & + \\
\hline Euphorbia cyparissias & 1 & $\mathrm{r}$ & III & II & II \\
\hline Scabiosa columbaria & . & + & . & $\mathrm{I}$ & III \\
\hline Carex caryophyllea & . & + & + & I & III \\
\hline Pimpinella saxifraga & . & + & + & II & II \\
\hline Lotus corniculatus & . & $\mathrm{r}$ & + & II & II \\
\hline Briza media & . & $\mathrm{r}$ & . & $\mathrm{I}$ & II \\
\hline Linum catharticum & . & + & I & I & + \\
\hline Carex flacca & . & $\mathrm{r}$ & + & I & II \\
\hline Plantago lanceolata & . & + & + & I & II \\
\hline Leontodon hispidus & . & + & + & . & I \\
\hline Medicago lupulina & 1 & $\mathrm{r}$ & . & . & II \\
\hline Helictotrichon pratense & . & $\mathrm{r}$ & I & II & . \\
\hline Carlina vulgaris & . & $\mathrm{r}$ & . & II & + \\
\hline Poa compressa & . & + & . & II & . \\
\hline Campanula rotundifolia & . & + & + & I & . \\
\hline Anthyllis vulneraria & . & . & + & $\mathrm{I}$ & II \\
\hline Acinos arvensis & . & $\mathrm{r}$ & + & II & . \\
\hline Erophila verna & . & $\mathrm{r}$ & I & . & + \\
\hline Arabis hirsuta & . & . & + & $\mathrm{I}$ & + \\
\hline Fragaria viridis & . & . & . & . & II \\
\hline Trifolium campestre & . & . & . & II & . \\
\hline
\end{tabular}

Außerdem mit Stetigkeit $<$ II und in weniger als 3 Einheiten:

Achillea millefolium 2:r, 5:I; Agrostis stolonifera 2:r; Asplenium ruta-muraria 3:+; Bacidia bagliettoana 3:+; Barbula spec. 2:+, 3:+; Brachypodium pinnatum 2:r, 4:I; Brachythecium albicans 2:r; Bryophyta spec. 3:+; Carex ornithopoda 2:+; Centaurea jacea 4:I; Centaurea scabiosa 5:I; Cerastium pumilum agg. 2:r, 5:+; Cirsium acaule 5:+; Cirsium spec. 5:+; Cladonia ciliata 5:+; Cladonia fimbriata 5:+; Cladonia pyxidata ssp. chlorophaea $3:+$; Cladonia ramulosa 5:+; Cladonia rei 2:r; Convolvulus arvensis 5:+; Dactylis glomerata 5:I; Daucus carota 5:+; Dianthus carthusianorum 4:I; Dicranum scoparium 5:+; Didymodon acutus 2:r; Didymodon cf. fallax 2:r; Didymodon tophaceus 2:r; Didymodon vinealis 2:r; Diploschistes muscorum 2:r, 3:I; Distichum capillaceum 2:r; Euphrasia officinalis 5:+; Eurhynchium hians 4:I; Festuca rubra agg. 5:+; Fissidens spec. 3:+; Fissidens taxifolius 5:+; Galium mollugo 5:+; Galium pumilum 3:+, 5:I; Galium verum 2:r, 5:+; Gentianella germanica 5:+; Gyroweisia tenuis 4:I; Hepaticae spec. 2:r; Hieracium spec. 5:+; Hippocrepis comosa 2:r, 4:I; Hypericum perforatum 2:r; Knautia arvensis 3:+; Leucanthemum vulgare 4:I; Lichenes spec. 2:+, 3:+; Medicago falcata 5:+; Musci spec. 3:+; Mycobilimbia sabuletorum 2:r, 4:I; Mycobilimbia et Bacidia spec. 2:r; Ononis spinosa agg. 5:+; Peltigera didactyla 5:+; Phascum curvicolle 2:r; Pinus sylvestris 5:+; Placidium pilosellum 2:+; Plagiomnium rostratum 5:+; Plantago media 2:+; Poa pratensis 5:+; Poa trivialis 2:r, 5:+; Poaceae spec. 3:+, 5:+; Pottia trunctata 2:r; Pottiaceae spec. 2:+, 3:+; Primula veris 5:+; Prunus avium juv. 5:+; Racomitrium canescens 4:I; Sarcosagium campestre 2:r; Sedum acre 2:r; Silene vulgaris 4:I, 5:+; Taraxacum officinale agg. 3:+; Trichostomum et Weissia spec. 2:+, 3:+; Viola hirta 5:+; Weissia controversa 4:I. 


\subsubsection{Toninia sedifolia-Toninion-Initialgesellschaft}

(Tab. 14; Tab. 15 (Einheit 1) im Anhang 3; Taf. 1.7 im Anhang 2)

\section{a) Floristische Zusammensetzung, Physiognomie und Struktur}

Nach FollmanN (1974) und KLEMENT (1955) setzt die Gesellschaftsentwicklung des Toninio-Psoretum (s. Kap. E2.2.1.2) mit Verbandscharakterarten wie Placidium squamulosum (Syn. Dermatocarpon hepaticum), Psora decipiens und Toninia sedifolia (Syn. T. coeruleonigricans) ein. Beispiele solcher Initialstadien auf Zechsteindolomit stellen die Aufnahmen 1 bis 3 der Tab. 15 dar. Es dominieren diverse Blaualgen, die zu den Pionieren bei der Besiedlung neuer Standorte zählen und als schwarze Kruste das verwitterte Gesteinsmaterial überziehen. In den Blaualgen-reichen mikrobiotischen Krusten treten die Gattungen Nostoc, Scytonema, Gloeocystis, Gloeocapsa, Oscillatoria und Chroococcus sowie vereinzelt auch die Jochalge Zygogonium auf (HOPPERT unpubl.). Stellenweise ist Toninia sedifolia die einzige Flechtenart, deren aufgeblasene Schuppen sich eingestreut in diesen Krusten finden. Weiterhin können Collema-Arten, das Pioniermoos Ceratodon purpureus (s. NeBEL \& PHILIPPI 2000) sowie weitere kleinwüchsige Moose auftreten. Die mittlere Artenzahl beträgt für die Flechten nur 2,0, für die Moose 3,3 und für die Phanerogamen 5,0. Unionscharakterarten des ToninioPsoretum haben sich in den Beständen noch nicht eingestellt, daher erfolgt die Einordnung der Bestände als Toninia sedifolia-Toninion-Initialgesellschaft. Die Gesamtdeckung liegt zwischen 35 und $85 \%$, wobei den größten Anteil daran die Cyanophyceen mit bis zu $70 \%$ Deckung haben. Flechten mit $5 \%$ und Moose mit weniger als $5 \%$ Deckung spielen in diesen Stadien lediglich eine geringe Rolle. Einige wenige Phanerogamen wie Thymus pulegioides sind in der Lage, aus dem angrenzenden Gentiano-Koelerietum in die Initialgesellschaft einzudringen. Sofern die Algenkrusten nicht große Bereiche bedecken, können die Bestände mit bis zu 55 \% offenem Boden recht lückig sein.

\section{b) Standorte und Vorkommen im UG}

3 Aufnahmen können dieser Initialgesellschaft zugeordnet werden. Die Bodenbildung ist noch wenig fortgeschritten. Das Festgestein wird lediglich von einer Schicht verwitterten Dolomits und einem humusarmen, gelblichgrauen Ai-Horizont bedeckt. Die Bestände siedeln auf diesem skelettreichen Substrat an den mitunter ziemlich steilen Wänden zweier ehemaliger Dolomit-Steinbrüche und können eine Gesamtgröße von 1 bis

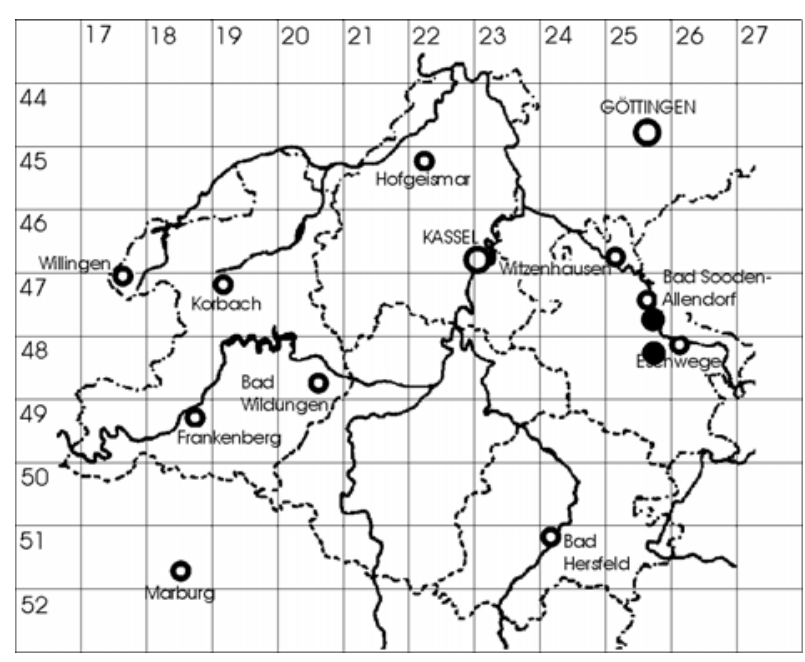

Abb. 21: Verbreitung der Toninia sedifolia-ToninionInitialgesellschaft im Untersuchungsgebiet. 
$5 \mathrm{~m}^{2}$ erreichen. Die Standorte sind sonnig und trocken.

\subsubsection{Toninio-Psoretum decipientis}

(„Bunte-Erdflechten-Gesellschaft“; Tab. 16; Tab. 17 im Anhang 3; Taf. 1.8 im Anhang 2)

Syn.: Psoretum decipientis Frey 1922, Toninia caeruleonigricans-Assoziation (KAISER 1926), Fulgensietum fulgentis Gams 1927, Thalloedema coeruleonigricans-Lecidea decipiens-Assoziation (STODIEK 1937) (ThalloedemaLecideetum decipientis Stodiek 1937), Bunte Flechtengesellschaft (REIMERS 1940a), Toninia caeruleonigricans-Psora decipiens-Assoziation (SuZA 1937), Mikroassoziation von Toninia caeruleonigricans (SUZA 1937), Cetraria aculeataAssoziation (KAISER 1926) p.p., Fulgensietum continentale Reimers 1951.

\section{a) Floristische Zusammensetzung, Physiognomie und Struktur}

Die Union wird durch Fulgensia bracteata, F. fulgens und Squamarina cartilaginea differenziert. Auch Endocarpon pusillum kann zumindest als schwache Differentialart herangezogen werden (s. Tab. 14). Innerhalb des Toninio-Psoretum decipientis werden im Untersuchungsgebiet zwei Subunionen unterschieden: die Typische Subunion (Einheit 1) und die Subunion fulgensietosum bracteatae (Einheit 2). Deren Unterschiede in der Artenzusammensetzung sind zumindest teilweise standortbedingt. Die beiden Fulgensia-Arten zeigen zwar nicht generell unterschiedliche Vorkommen (es gibt sowohl über Gips als auch über Dolomit Bestände in denen beide Arten auftreten) jedoch wurde Fulgensia bracteata in Nordhessen nie an MuschelkalkStandorten angetroffen. Aus diesem Grund kann sie als Trennart der Subunion fulgensietosum bracteatae gelten und stellt im Untersuchungsgebiet zudem eine lokale Unionscharakterart dar. Fulgensia fulgens, die zumindest sporadisch auf allen basenreichen Substraten in Nordhessen auftritt, wird den Literaturangaben folgend als Unionscharakterart angesehen (s. DreHWALd 1993, KLEMENT 1955, WiRTH 1995). Sie zeigt allerdings einen Schwerpunkt ihres Vorkommens in der Typischen Subunion. Eine weitere Trennart der Fulgensia bracteata-Subunion gegen die Typische Subunion ist Endocarpon pusillum.

Die Aufnahmen 1 bis 9 in Tab. 17 können in Anlehnung an DrEHWALD (1993) der Cladonia-Variante der Typischen Subunion zugeordnet werden. Differentialarten dieser Variante sind Cladonia furcata ssp. subrangiformis, Leptogium cf. schraderi und Thuidium abietinum. Die mittlere Artenzahlen in der Typischen Subunion betragen 7,7 bei den Flechten, 6,1 bei den Moosen und 6,7 bei den Phanerogamen.

\section{Varianten der Fulgensia bracteata-Subunion:}

Das Toninio-Psoretum decipientis fulgensietosum bracteatae läßt sich in zwei Varianten unterteilen:

Die relativ artenreiche Cladonia symphycarpa-Variante (Einheit 2.1) ist gekennzeichnet durch Cladonia symphycarpa, Psora decipiens und Placidium squamulosum sowie die kräftigeren Moose Hypnum cupressiforme var. lacunosum und Ditrichum flexicaule. Sie läßt sich im Untersuchungsgebiet in zwei Subvarianten untergliedern: 
Tab. 16: Stetigkeitstabelle Toninio-Psoretum decipientis

Einheit 1: Toninio-Psoretum decipientis typicum, Cladonia-Variante

Einheit 2: Toninio-Psoretum decipientis fulgensietosum bracteatae

$2.1 \quad$ Cladonia symphycarpa-Variante

2.1.1 Squamarina cartilaginea-Subvariante

2.1.2 Cephaloziella-Subvariante

2.2 Artenarme Variante

\begin{tabular}{|c|c|c|c|c|c|}
\hline \multirow{3}{*}{\multicolumn{2}{|c|}{ Einheit }} & \multirow[t]{3}{*}{1} & \multicolumn{3}{|c|}{2} \\
\hline & & & \multicolumn{2}{|c|}{2.1} & \multirow[t]{2}{*}{2.2} \\
\hline & & & 2.1 .1 & 2.1 .2 & \\
\hline \multirow{7}{*}{\multicolumn{2}{|c|}{$\begin{array}{l}\text { Zahl der Aufnahmen } \\
\text { mittlere Deckung Phanerogamen [\%] } \\
\text { mittlere Deckung Flechten [\%] } \\
\text { mittlere Deckung Moose [\%] } \\
\text { mAZ Phanerogamen } \\
\text { mAZ Flechten } \\
\text { mAZ Moose } \\
\end{array}$}} & 9 & 17 & 6 & 9 \\
\hline & & 24,4 & 8,8 & 7,2 & 2,1 \\
\hline & & 22,8 & 29,1 & 16,7 & 37,2 \\
\hline & & 24,4 & 32,4 & 23,8 & 20,0 \\
\hline & & 6,7 & 4,0 & 4,7 & 1,6 \\
\hline & & 7,7 & 8,1 & 6,3 & 3,2 \\
\hline & & 6,1 & 6,5 & 5,5 & 5,4 \\
\hline \multirow{2}{*}{\multicolumn{2}{|c|}{ Fulgensia fulgens }} & & & & \\
\hline & & $\mathrm{V}$ & I & I & III \\
\hline \multicolumn{2}{|c|}{ d Variante: } & & & & \\
\hline & Cladonia furcata ssp. subrangiformis & III & I & I & . \\
\hline & Leptogium cf. schraderi & III & I & . & . \\
\hline & Thuidium abietinum & III & $\mathrm{I}$ & II & . \\
\hline \multicolumn{2}{|l|}{ d 2: } & & & & \\
\hline $\mathrm{UC}$ & Fulgensia bracteata & . & $\mathrm{V}$ & $\mathrm{V}$ & $\mathrm{V}$ \\
\hline & Endocarpon pusillum & . & II & III & II \\
\hline \multicolumn{2}{|c|}{ d 1, d 2.1: } & & & & \\
\hline $\mathrm{FC}$ & Cladonia symphycarpa & III & IV & IV & . \\
\hline $\mathrm{FC}$ & Psora decipiens & III & IV & $\mathrm{V}$ & . \\
\hline & Placidium squamulosum & $\mathrm{V}$ & III & $\mathrm{V}$ & . \\
\hline & Ditrichum flexicaule & $\mathrm{V}$ & $\mathrm{V}$ & IV & I \\
\hline & Hypnum cupressiforme var. lacunosum & III & IV & $\mathrm{V}$ & I \\
\hline \multicolumn{2}{|c|}{ d 1, d 2.1.1: } & & & & \\
\hline & Peltigera rufescens & $\mathrm{V}$ & $\mathrm{V}$ & I & I \\
\hline & Tortella inclinata & III & IV & I & . \\
\hline & Encalypta streptocarpa & II & II & . & . \\
\hline & Leptogium cf. lichenoides & II & II & . & . \\
\hline \multicolumn{2}{|c|}{ d 2.1.1: } & & & & \\
\hline & Encalypta vulgaris & I & III & . & . \\
\hline UC & Squamarina cartilaginea & . & III & . & . \\
\hline & Squamarina lentigera & . & II & . & . \\
\hline & Placidium pilosellum & . & II & . & . \\
\hline \multicolumn{6}{|c|}{ d 2.1.2, d 2.2: } \\
\hline & Cephaloziella spec. & I & + & III & III \\
\hline & Trichostomum et Weissia spec. & II & I & $\mathrm{V}$ & II \\
\hline \multicolumn{6}{|c|}{ d 2.2: } \\
\hline & Aloina spec. & II & + & I & IV \\
\hline & Bryum spec. & II & II & I & IV \\
\hline UC & Barbula unguiculata & . & . & . & II \\
\hline \multicolumn{6}{|c|}{ FC, OC, KC: } \\
\hline & Toninia sedifolia & IV & III & III & II \\
\hline & Cladonia pyxidata ssp. pocillum & III & IV & $\mathrm{V}$ & III \\
\hline & Barbula convoluta & II & III & III & IV \\
\hline
\end{tabular}


Forts. Tab. 16: Stetigkeitstabelle Toninio-Psoretum decipientis

\begin{tabular}{|c|c|c|c|c|}
\hline \multirow[t]{3}{*}{ Einheit } & \multirow[t]{3}{*}{1} & \multicolumn{3}{|c|}{2} \\
\hline & & \multicolumn{2}{|c|}{2.1} & \multirow[t]{2}{*}{2.2} \\
\hline & & 2.1.1 & 2.1 .2 & \\
\hline \multicolumn{5}{|l|}{ Begleiter Kryptogamen: } \\
\hline Ceratodon purpureus & $\mathrm{V}$ & IV & V & IV \\
\hline Cyanophyta div. spec. & $\mathrm{V}$ & IV & II & V \\
\hline Collema spec. & IV & III & II & II \\
\hline Pseudocrossidium revolutum & . & II & I & III \\
\hline Pseudocrossidium hornschuchianum & . & I & I & II \\
\hline Didymodon rigidulus & . & I & I & II \\
\hline Chlorophyta div. spec. & . & II & I & . \\
\hline Cladonia rangiformis & II & I & . & . \\
\hline Barbula spec. & I & + & II & . \\
\hline Fissidens dubius & II & + & . & . \\
\hline Pottiaceae spec. & II & . & . & . \\
\hline Brachythecium albicans & . & . & II & . \\
\hline Cetraria aculeata & II & . & . & . \\
\hline \multicolumn{5}{|l|}{ Phanerogamen: } \\
\hline Thymus pulegioides & V & III & IV & . \\
\hline Festuca ovina agg. & IV & III & I & I \\
\hline Bromus erectus & III & III & . & . \\
\hline Koeleria pyramidata & II & II & IV & II \\
\hline Potentilla tabernaemontani & IV & I & I & . \\
\hline Sanguisorba minor & II & II & . & II \\
\hline Sedum sexangulare & II & II & II & . \\
\hline Arenaria serpyllifolia & . & I & I & I \\
\hline Scabiosa columbaria & II & + & . & . \\
\hline Poa compressa & I & + & I & . \\
\hline Carex ornithopoda & II & . & . & . \\
\hline Plantago media & II & + & . & . \\
\hline Plantago lanceolata & II & . & . & . \\
\hline Pimpinella saxifraga & II & . & . & . \\
\hline Lotus corniculatus & II & . & . & . \\
\hline Helictotrichon pratense & II & . & . & . \\
\hline Briza media & II & . & . & . \\
\hline
\end{tabular}

Außerdem mit Stetigkeit < II und in weniger als 3 Einheiten:

Achillea millefolium 1:I; Acinos arvensis 2:+; Agrostis stolonifera 3:I; Brachypodium pinnatum 3:I, 4:I; Campanula rotundifolia 1:I, 2:I; Carex caryophyllea 2:I; Carex flacca 2:+; Carlina vulgaris 2:I; Cerastium pumilum agg. 2:+; Cladonia rei 4:I; Ctenidium molluscum 1:I, 2:+; Didymodon acutus 2:+; Didymodon cf. fallax 4:I; Didymodon tophaceus 2:+; Didymodon vinealis 2:+, 4:I; Diploschistes muscorum 1:I; Distichum capillaceum 2:+; Erophila verna 2:+; Euphorbia cyparissias 1:I, 3:I; Galium verum 2:+, 3:I; Hepaticae spec. 3:I; Hieracium pilosella 2:+, 4:I; Hippocrepis comosa 1:I; Hypericum perforatum 4:I; Leontodon hispidus 2:I, 3:I; Lichenes spec. 2:I; Linum catharticum 2:I, 3:I; Medicago lupulina 1:I, 3:I; Mycobilimbia sabuletorum 4:I; Mycobilimbia et Bacidia spec. 2:I; Phascum curvicolle 2:+, 4:I; Poa trivialis 4:I; Pottia trunctata 4:I; Sarcosagium campestre 3:I, 4:I; Sedum acre 2:I; Tortella tortuosa 1:I; Tortula ruralis 2:I, 3:I. 
$\mathrm{Zu}$ den Diagnostischen Arten der Subvariante von Squamarina cartilaginea (Einheit 2.1.1) zählen neben Peltigera rufescens unter anderem Squamarina cartilaginea, Squamarina lentigera, Encalypta vulgaris und die seltene Art Placidium piliferum. Die Bestände dieser Variante zählen aufgrund ihres Artenreichtums zu den wertvollsten Erdflechtengesellschaften auf basenreichen Standorten. Die CephaloziellaSubvariante (Einheit 2.1.2) ist negativ durch das Fehlen sowohl der Peltigera rufescens- als auch der Squamarina cartilaginea-Gruppe gekennzeichnet. In ihr kommen als Trennarten dagegen kleinwüchsige Moose wie etwa die CephaloziellaArten vor. Es treten im Mittel 8,1 Flechtenarten in der Squamarina-Subvariante und 6,3 in der Cephaloziella-Subvariante auf. Bei den Moosarten betragen die Werte der mittleren Artenzahl 6,5 bzw. 5,5, bei den Phanerogamen 4,0 bzw 4,7.

Eine weitere Gruppe kleinwüchsiger Moose, die Gattung Aloina, ist typisch für die Artenarme Variante (Einheit 2.2) der Fulgensia bracteata-Subunion. Sie ist darüber hinaus nur negativ gekennzeichnet. Außer der Peltigera rufescens- und der Squamarina cartilaginea-Gruppe fehlen ihr auch die Arten der Gruppe von Cladonia symphycarpa. Die mittlere Artenzahl der Flechten liegt daher mit 3,2 Arten deutlich unter den Werten der anderen Einheiten. Auch die mittlere Artenzahlen der Moose $(5,4)$ und der Phanerogamen $(1,6)$ sind geringer.

Obwohl einige Vertreter der sogenannten „Bunten Erdflechten” nicht vorkommen (z.B. Squamarina cartilaginea und Psora decipiens), sind diese Bestände, bedingt durch die Dominanz der leuchtend gelben Fulgensia-Arten und ihrer bisweilen großen Ausdehnung, optisch sehr eindrucksvoll. Nicht eindeutig beantworten läßt sich die Frage, ob derartige Bestände im Untersuchungsgebiet generell Initialstadien der Flechtenbesiedlung auf Gips darstellen, analog den der Toninia sedifolia-ToninionInitialgesellschaft zugeordneten Vorkommen über Dolomit. Auf Gipsfelsen scheint schon von Beginn der Besiedlung an Fulgensia bracteata eine wesentliche Rolle zu spielen. An einigen Standorten siedelt die Art jedenfalls, oft ohne weitere Begleitarten, bereits auf wenig verwittertem Gips, an Stellen, wo noch keine nennenswerte Bodenbildung stattgefunden hat. Vielleicht hängt das Fehlen einiger Bunter Erdflechten jedoch auch mit einer stärkeren Trittbelastung der z.T. als Freizeit- und Grillgelände genutzten Standorte zusammen (s. GÜNZL 2001b).

\section{Physiognomie:}

Das Toninio-Psoretum decipientis wird von Krustenflechten und kleinwüchsigen Moosen (vor allem Pottiaceen) geprägt. Strauchartig wachsende Cladonien sind, sofern überhaupt vorhanden, in der Regel nur kümmerlich entwickelt. Nur die blättrigen Grundschuppen von Cladonia symphycarpa oder die Loben der Blattflechte Peltigera rufescens können gelegentlich das Erscheinungsbild der Bestände mitbestimmen, besonders in der Squamarina cartilaginea-Subvariante der Cladonia symphycarpaVariante. Die normalerweise kräftigeren Moose Hypnum cupressiforme var. lacunosum und Ditrichum flexicaule neigen in der Bunten-Erdflechten-Gesellschaft zum Kümmerwuchs. 
Die Gesamtdeckung schwankt in allen Untereinheiten erheblich. Sie ist im Mittel in der Typischen Subvariante der Cladonia symphycarpa-Variante mit $43 \%$ am geringsten und in der Artenarmen Variante mit $71 \%$ am höchsten. Phanerogamen, die aus den umgebenden Festuco-Brometea-Beständen (meist handelt es sich um das GentianoKoelerietum) eindringen, bedecken maximal $35 \%$ der Flächen, können aber auch vollständig fehlen. Die Deckung der Flechten zeigt erheblich Unterschiede selbst innerhalb der Untereinheiten. So reicht sie beispielsweise innerhalb der Squamarina cartilaginea-Subvariante der Cladonia symphycarpa-Variante von 5 bis 65 \%. Im Mittel ist sie in der Cephaloziella-Subvariante der Cladonia symphycarpa-Variante mit $17 \%$ am geringsten und in der Artenarmen Variante mit 37\% am höchsten. Eine ähnlich breite Spanne zeigt die Moosdeckung, die allerdings im Mittel in allen Einheiten bei rund 20 bis $30 \%$ liegt. Algen haben keinen oder aber auch hohen Anteil am Aufbau der Gesellschaft. In der Artenarmen Variante bedecken sie im Ausnahmefall $45 \%$ der Fläche, sonst maximal $30 \%$. Einige der aufgenommenen Bestände sind extrem lückig, auf bis zu $60 \%$ der Fläche treten dann Steine und offener Boden zutage. Eine Streubedeckung spielt mit $<5 \%$ keine Rolle.

\section{b) Standorte und Vorkommen im UG}

Das Toninio-Psoretum decipientis konnte im Untersuchungsgebiet durch 41 Aufnahmen belegt und an 12 Wuchsorten angetroffen werden. Ein Verbreitungszentrum dieser Union liegt im östlichen bzw. südöstlichen Meißner-Vorland (MTB 4725/4826). Das Toninio-Psoretum decipientis typicum besiedelt überwiegend Standorte auf Unterem Muschelkalk, seltener auf Zechsteindolomit. Das Toninio-Psoretum decipientis fulgensietosum bracteatae kommt außer auf Zechsteindolomit auch auf Zechsteingips vor. Die Artenarme Variante ist in ihrem Auftreten sogar

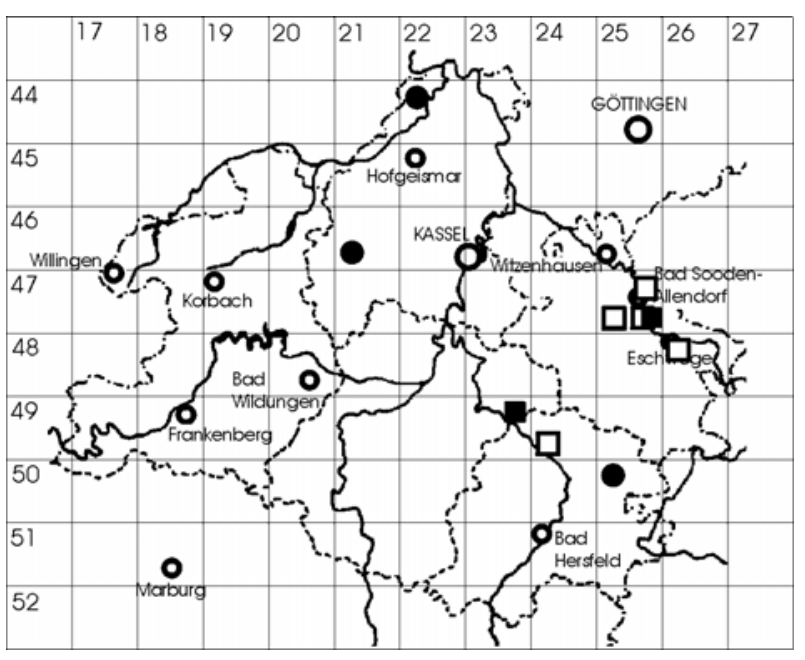

Abb. 22: Verbreitung des Toninio-Psoretum decipientis im Untersuchungsgebiet.

T.-P. typicum;

T.-P. fulgensietosum, Cl.-Variante;

T.-P. fulgensietosum, Artenarme Variante.

ausschließlich auf Gips beschränkt. Die Bodenentwicklung ist in der Regel wenig fortgeschritten, die Böden sind daher meist als Syroseme einzustufen. Rendzinen kommen vor allem bei der Typischen Subunion sowie bei der Cladonia symphycarpaVariante der Subunion fulgensietosum bracteatae vor.

Die überwiegend sonnigen und trockenen Standorte sind schwach bis mäßig geneigt, das Substrat weist einen hohen bis sehr hohen Skelettanteil auf. Etliche der Vorkommen liegen auf Pfaden innerhalb von Schafweiden. Der Tritt der Weidetiere scheint ihnen, solange er nicht zu mächtig wird, nicht zu schaden. Die Schafe halten die Magerrasen offen und schaffen neue Ansiedlungsmöglichkeiten für Erdflechten, die mancherorts 
durch Vergrasen und Verbuschen der Enzian-Schillergras-Rasen (GentianoKoelerietum) verdrängt werden. Weitere Standorte der Gesellschaft sind Felsköpfe und aufgelassene Gips-Steinbrüche. In letzteren werden sie durch Aktivitäten des Menschen geschädigt. Einen detaillierteren Überblick über Vorkommen und Gefährdungsursachen des Toninio-Psoretum decipientis in Nordhessen gibt GüNZL (2001b).

\section{Syntaxonomische Anmerkung:}

Die Subunion fulgensietosum bracteatae stellt die „Gipsausbildung” (im weiteren Sinne), die Typische Subunion eher die „Kalkausbildung” dar. Diese Bezeichnungen verwendet beispielsweise BoRNKAMM (1958) für seine Untergliederung der Bestände des südwestlichen Harzvorlandes. Aufgrund des vollständigen Fehlens von Fulgensia bracteata auf Muschelkalkstandorten in Nordhessen scheint jedoch eine Untergliederung der Union in Subunionen als standörtliche Einheiten, wie dies DREHWALD (1993) anhand des Aufnahmematerials von BORNKAMM vorgenommen hat, sinnvoller.

\subsubsection{Cladonia symphycarpa-Toninion-Fragmentgesellschaft}

(Tab. 14; Tab. 15 (Einheit 2) im Anhang 3)

\section{a) Floristische Zusammensetzung, Physiognomie und Struktur}

Die Fragmentgesellschaft steht der artenreichen Cladonia symphycarpa-Variante des Toninio-Psoretum decipientis fulgensietosum bracteatae nahe. Sie wird negativ gekennzeichnet durch das Fehlen der Diagnostischen Arten des Toninio-Psoretum (Fulgensia fulgens, F. bracteata, Endocarpon pusillum, Squamarina cartilaginea). Einige Federationscharakterarten gehören dagegen zur CAV: Psora decipiens, Placidium squamulosum, Toninia sedifolia und Cl. symphycarpa. Höhere Stetigkeit als im Toninio-Psoretum erreichen Encalypta streptocarpa, Tortella tortuosa und Leptogium cf. lichenoides. Hohen Anteil am Aufbau der Bestände haben darüber hinaus die relativ kräftigen Moose Ditrichum flexicaule und Hypnum cupressiforme var. lacunosum sowie Cl. furcata ssp. subrangiformis. Letztere tritt in der Cladonia symphycarpa-Toninion-Fragmentgesellschaft häufiger auf als in der BuntenErdflechten-Gesellschaft.

MARStaller (1971) deutet seine Cladonia-Variante des Toninio-Psoretum, in der in zunehmendem Maße Strauchflechten in die Bestände eindringen, als Terminalphase der Gesellschaft. Die Cladonia symphycarpa-Toninion-Fragmentgesellschaft könnte demnach als Folgestadium ehemaliger Toninio-Psoretum-Bestände interpretiert werden. Aufgrund der relativ hohen Artenzahl (mittlere Artenzahl 6,4 bei den Flechten, 6,6 bei den Moosen, 6,3 bei den Phanerogamen) und dem Vorkommen einiger Rote Liste-Arten sind diese Bestände aus Naturschutzsicht ebenso wertvoll wie die eigentliche BunteErdflechten-Gesellschaft (s. Kap. E6).

Physiognomisch handelt es sich bei der Cladonia symphycarpa-ToninionFragmentgesellschaft um Bestände, die zwar oft von strauchigen oder blättrigen Arten geprägt werden, in denen die Krustenflechten jedoch ebenfalls noch eine bedeutende Rolle spielen. Vereinzelt treten Cladonia-Arten dominant auf. Die Gesamtdeckung der Bestände liegt zwischen 40 und $80 \%$, wovon 10 bis $50 \%$ auf die Flechten entfallen. 
Phanerogamen und Moose weisen maximal $40 \%$ Deckung auf, Algen maximal $20 \%$. Mit bis zu $60 \%$ Anteil an offenem Boden können die Bestände sehr lückig sein. Vergleichbar mit den Untereinheiten des Toninio-Psoretum erweisen sich auch die Bestände der Cladonia symphycarpa-Toninion-Fragmentgesellschaft bezüglich der Deckungswerte als sehr uneinheitlich.

Die Gesamtvorkommen innerhalb eines Gebietes bedecken oft weniger als $5 \mathrm{~m}^{2}$. Eine Ausnahme stellen die rund $40 \mathrm{~m}^{2}$ großen Bestände im NSG „Bühlchen bei Weißenbach“ dar, dem zugleich einzigen Wuchsort, an dem Cladonia symphycarpa mit Fruchtkörpern angetroffen wurde.

\section{b) Standorte und Vorkommen im UG}

Am Bühlchen und an 7 weiteren Lokalitäten konnten insgesamt 11 Vegetationsaufnahmen dieser Fragmentgesellschaft angefertigt werden. Sie tritt innerhalb des GentianoKoelerietum der Kalkmagerrasen über Muschelkalk und Zechsteindolomit auf, besonders im MTB 4725. Die Flächen sind in der Regel wenig bis mäßig geneigt, sonnig bzw. allenfalls gelegentlich beschattet und werden zum Teil von Schafen beweidet. Die Böden, Rendzinen oder Syroseme, sind trocken oder wechseltrocken und

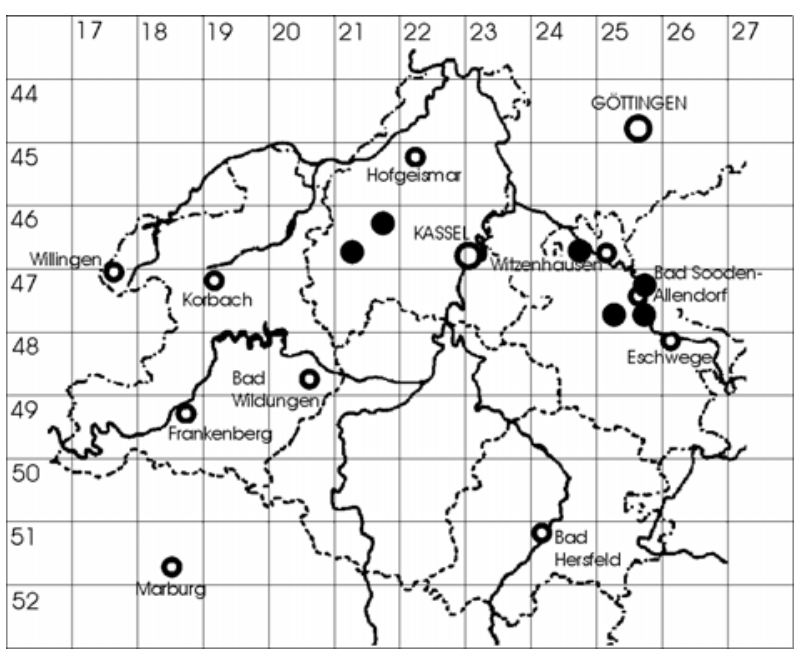

Abb. 23: Verbreitung der Cladonia symphycarpaToninion-FG im Untersuchungsgebiet. sehr skelettreich.

\section{Syntaxonomische Anmerkung:}

Die Cladonia symphycarpa-Toninion-Fragmentgesellschaft kann anhand der Aussagen von DREHWALD (1993) als Übergangsstadium zwischen Toninio-Psoretum und Cladonietum symphycarpae interpretiert werden. Das Cladonietum symphycarpae bezeichnet DREHWALD als eine Pioniergesellschaft trockener Kalkböden. Es könne jedoch gelegentlich auch durch Feinerdeansammlung aus dem Toninio-Psoretum decipientis hervorgehen und Übergänge zu diesem bilden. Die Abgrenzung des Cladonietum symphycarpae vom Toninio-Psoretum ist daher oft unklar und in der Literatur nicht immer eindeutig nachvollziehbar, da eine große Zahl von Arten beiden Assoziationen gemeinsam ist und die nach DREHWALD (1993) einzige Charakterart der Union, Cl. symphycarpa, auch im Toninio-Psoretum decipientis hochstet vorkommt (s. Tab. 14). MARSTALLER (1971: 38/45) beschreibt das Cladonietum symphycarpae als Bestände, in denen "die Bunten Erdflechten nur noch eine unbedeutende Rolle spielen und die Cladonia-Arten völlig zur Herrschaft gelangt sind" und deren Physiognomie ganz von Cl. symphycarpa beherrscht wird. Dieser Beschreibung nach dürfte nur eine einzige Aufnahme (Aufn. 4, Tab. 15) aus dem nordhessischen Raum dem Cladonietum symphycarpae zugeordnet werden, da nur in dieser Cl. symphycarpa dominiert. Aus den oben erläuterten Gründen der unklaren Abgrenzung dieser Union wird im vorliegenden Fall jedoch auf die Ausweisung einer eigenen Union Cladonietum symphycarpae verzichtet und die Aufnahme aus dem NSG „Bühlchen bei Weißenbach“ ebenso wie das floristisch ähnliche Aufnahmematerial der Cladonia symphycarpa-Toninion-Fragmentgesellschaft 
zugeordnet. PAUS (1997) nahm Bestände ähnlicher Artenzusammensetzung, die sie dem Cladonietum symphycarpae zuordnet, unter anderem im nordwestlichen Vorland des Hohen Meißners auf, also nicht weit entfernt von den Cladonia symphycarpa-reichen Flechtenvorkommen am Bühlchen.

\subsubsection{Peltigera rufescens-Toninion-Fragmentgesellschaft}

(Tab. 14; Tab. 15 (Einheit 3) im Anhang 3)

\section{a) Floristische Zusammensetzung, Physiognomie und Struktur}

Obwohl floristisch der Cladonia symphycarpa-Toninion-Fragmentgesellschaft noch ähnlich, fehlen dieser Fragmentgesellschaft jedoch zusätzlich einige Federationscharakterarten des Toninion fast vollständig (Cl. symphycarpa, Psora decipiens, Placidium squamulosum). Zur CAV zählen, neben der dominanten Art Peltigera rufescens, die Moose Ceratodon purpureus, Tortella tortuosa, Ditrichum flexicaule und Hypnum cupressiforme var. lacunosum, welche sich, ebenso wie Cl. pyxidata ssp. pocillum, Cl. furcata ssp. subrangiformis (FC) und Cl. rangiformis in den bisweilen kleinen Lücken zwischen den blättrigen Lagern von Peltigera anzusiedeln vermögen. Weitere Besiedler dieser Lücken sind diverse Blaualgen (zu denen auch die Photobionten von Peltigera zu rechnen sind). Die mittlere Artenzahl der Flechten liegt mit nur 3,3 Arten deutlich unter dem Wert der Cladonia symphycarpaToninion-Fragmentgesellschaft. Bei den Moosen (mittlere Artenzahl 5,4) und Phanerogamen (mittlere Artenzahl 8,4) weichen die Werte dagegen weniger ab.

Unter den Flechten dominiert die eng an das Substrat anliegende, fast krustig erscheinende Blattflechte Peltigera rufescens. Die Ansiedlungen von Peltigera erreichen stellenweise einen Durchmesser von mehr als $8 \mathrm{~cm}$ und sind damit deutlich größer als in der Cladonia symphycarpa-Toninion-Fragmentgesellschaft und im Toninio-Psoretum decipientis, in denen Peltigera rufescens ebenfalls mit hoher Stetigkeit auftritt. Allein Peltigera kann bis zu 80 \% Deckung erreichen. Gewöhnlich liegt jedoch die Deckung der Flechten bei 15 bis $55 \%$. Moose, Algen und Phanerogamen treten wiederum in stark wechselnder Deckung auf. Letztere können bei fortschreitender Vegetationsentwicklung die Flechten überwuchern und verdrängen. Die Bestände wirken insgesamt weniger lückig als die der Cladonia symphycarpa-ToninionFragmentgesellschaft. 


\section{b) Standorte und Vorkommen im UG}

8 Aufnahmen von 5 Wuchsorten belegen diese Vegetationseinheit für das Untersuchungsgebiet. Die Bestände liegen umgeben von oder angrenzend an Magerrasen des Gentiano-Koelerietum hauptsächlich im Meißner-Gebiet. Typische Standorte sind sonnige, trockene (bzw. wechseltrockene), flach geneigte bis ebene Flächen innerhalb von Kalkmagerrasen oder an Wegrändern bzw. am Grund aufgelassener Steinbrüche. Ausgangsgestein ist Zechsteindolomit oder Muschelkalk.

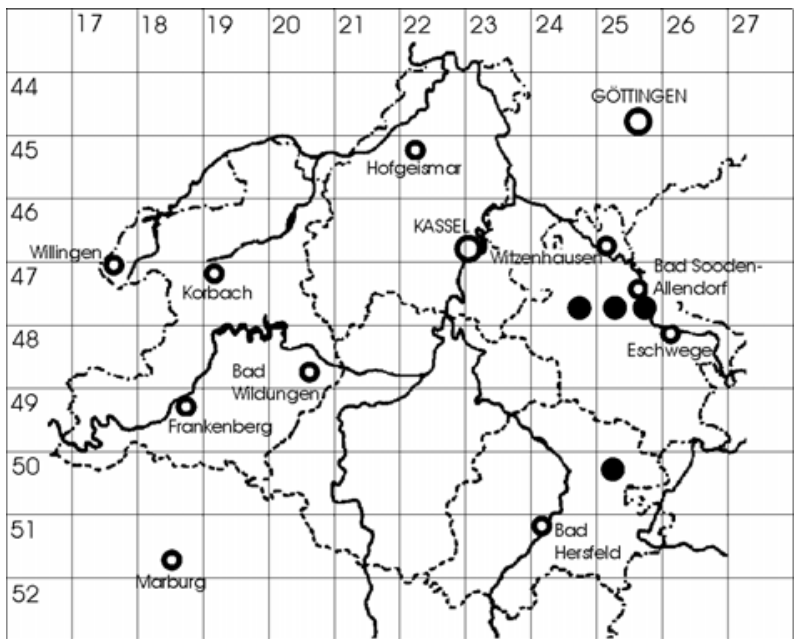

Abb. 24: Verbreitung der Peltigera rufescensToninion-FG im Untersuchungsgebiet.

Die Böden wurden als Syrosem bzw.

Rendzina angesprochen und sind sehr skelettreich. Die Bestände bedecken nie mehr als $5 \mathrm{~m}^{2}$ an einem Wuchsort.

\subsubsection{Dominanzbestände}

(Tab. 14; Tab. 15 (Einheit 4) im Anhang 3)

\section{a) Floristische Zusammensetzung, Physiognomie und Struktur}

Die Einengung des Siedlungsraumes durch eine Ausbreitung der Trockenrasenpflanzen oder das Dominieren einzelner kräftiger Strauchflechten führt zum Rückgang konkurrenzschwacher Erdflechten. Es fehlen daher den Dominanzbeständen die typischen Bunten Erdflechten vollständig, nur noch kräftige, höherwüchsige Kryptogamen können sich behaupten. Typischerweise treten daher nun auch vermehrt größere Moose (Ctenidium molluscum, Fissidens dubius, Homalothecium lutescens) auf. Die dominierenden Flechtenarten der Bestände sind Cladonia furcata ssp. subrangiformis und Cl. rangiformis, stellenweise kann auch Cetraria aculeata Dominanzbestände innerhalb des Gentiano-Koelerietum bilden. Die Ansiedlungen beider Cladonia-Arten werden in diesen Dominanzbeständen größer als in anderen Vegetationseinheiten der Klasse Toninio-Psoretea. Das typische Bild solcher Dominanzen zeigt sich als ein Gemisch aus den charakteristischen Phanerogamen der Trockenrasen (mit bis zu 75\% Deckung), einigen Moosen und dazwischen Flechtenpolstern, die 10 bis $40 \%$ Deckung erreichen. Die Bestände wirken recht geschlossen, lediglich an wenigen Stellen ist der Boden oder das verwitterte Gestein deutlich erkennbar.

Cladonia pyxidata ssp. pocillum-Dominanzbestände treten gelegentlich in Kalkmagerrasen auf, finden aber in der Regel wegen ihrer Eintönigkeit und Artenarmut keine Beachtung. Der aufgeführte Bestand (Aufn. 33, Tab. 15) soll hier jedoch der 
Vollständigkeit halber bei der Beschreibung der aktuell in Nordhessen vorhandenen Erdflechtengesellschaften basenreicher Standorte erwähnt werden. Physiognomisch weicht er von den anderen Dominanzbeständen ab, da sich zwischen den fast krustigen Lagern von Cladonia pyxidata ssp. pocillum kaum andere Kryptogamen oder Phanerogamen behaupten können.

Die Artenarmut aller Dominanzbestände zeigt sich bei den mittleren Artenzahlen von Flechten und Moosen. Den durchschnittlich nur 1,8 Flechten- und 3,5 Moosarten stehen 10,9 Arten der Phanerogamen gegenüber.

\section{b) Standorte und Vorkommen im UG}

Dominanzbestände wurden an mehreren Standorten vegetationskundlich erfaßt (11 Aufnahmen, 8 Wuchsorte). Jedoch sind noch etliche weitere, nicht bearbeitete Vorkommen im Untersuchungsgebiet vorhanden.

Dominanzbestände von Cladonia furcata ssp. subrangiformis und Cl. rangiformis siedeln in verschiedenen Expositionen und Neigungen, an sonnigen, trockenen Stellen in Magerrasen über Muschelkalk, Zechsteingips, Zechsteindolomit und

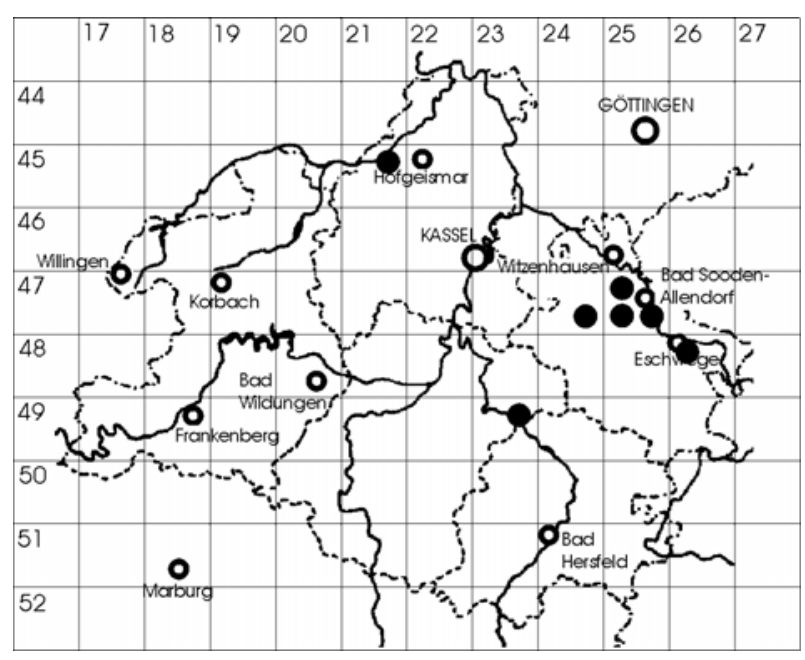

Abb. 25: Lage der bearbeiteten Dominanzbestände im Untersuchungsgebiet.

Basalt. Die Bodenentwicklung ist etwas weiter fortgeschritten, die Rendzinen sind meist weniger skelettreich als an den Standorten der anderen Toninio-Psoretea-Gesellschaften. Größere Ausdehnung (bis $10 \mathrm{~m}^{2}$ ) erreichen die Bestände nur in den Kalkmagerrasen, die von Schafen beweidet werden, da nur sie ausreichend Lücken für Flechtenbewuchs aufweisen. Ein Aufhören der traditionellen Nutzungsweise führt zum Verschwinden der Flechten durch Verbuschung und Vergrasung der Flächen.

\subsubsection{Standorte und Dynamik im Überblick}

Wie einleitend in Kap. E2.2.1 erwähnt, haben die Meßwerte von $\mathrm{pH}$ und elektrolytischer Leitfähigkeit keine differenzierende Bedeutung für die Einheiten der Klasse Toninio-Psoretea decipientis. Die $\mathrm{pH}\left(\mathrm{H}_{2} \mathrm{O}\right)$ - und $\mathrm{pH}(\mathrm{KCl})$-Werte unterscheiden sich mit Ausnahme der Probe von einem Basaltstandort kaum. Bei der elektrolytischen Leitfähigkeit fallen die außergewöhnlich hohen Werte der Gipsböden auf, die rund fünfmal so hoch sind wie die Werte der Böden über Muschelkalk. Noch etwas geringere Medianwerte weisen die Bodenlösungen von Dolomit- bzw. Basaltstandorten auf (s. Tab. 18). 
Tab. 18: Medianwerte von $\mathrm{pH}$ und elektrolytischer Leitfähigkeit in Abhängigkeit vom Ausgangsgestein

\begin{tabular}{l|llll}
\hline Gestein & $n$ & $p H\left(\mathrm{H}_{2} \mathrm{O}\right)$ & $p H(\mathrm{KCl})$ & $\mathrm{LF}[\mu \mathrm{S} / \mathrm{cm}]$ \\
\hline $\mathrm{b}$ & 1 & 6,1 & 5,4 & 122 \\
$\mathrm{mo}$ & 5 & 7,4 & 7,2 & 275 \\
$\mathrm{mu}$ & 13 & 7,3 & 7,1 & 301 \\
$\mathrm{zd}$ & 33 & 7,4 & 7,3 & 192 \\
$\mathrm{zg}$ & 15 & 7,2 & 7,1 & 1441 \\
sug & 7 & 7,2 & 7,0 & 1378 \\
\hline
\end{tabular}

Die Gesellschaften der Klasse Toninio-Psoretea decipientis siedeln überwiegend an sonnigen und trocken Stellen über basenreichem Ausgangsgestein. Gelegentlich können umgebende Phanerogamen die Flechten etwas beschatten. Selten treten die Kryptogamenbestände an wechseltrockenen Standorten auf (z.B. einige Bestände der Cladonia symphycarpa-Toninion-Fragmentgesellschaft). Die Böden, Syroseme und Rendzinen, sind im allgemeinen skelettreich und sehr flachgründig. Die Tatsache, daß sich die untersuchten Standorte bezüglich Boden und Klima kaum unterscheiden, deutet darauf hin, daß der entscheidende Faktor für das Auftreten oder Fehlen der verschiedenen Vegetationseinheiten der Grad der Nutzung der Flächen und die damit verbundene Entwicklung der Magerrasen ist.

Toninio-Psoretea-Bestände lassen sich im Untersuchungsgebiet vor allem in Vegetationslücken von Kalkmagerrasen (Gentiano-Koelerietum) sowie in aufgelassenen Gips- und Dolomit-Steinbrüchen finden. Entscheidend für ihr Vorkommen ist wiederum hauptsächlich die fehlende Konkurrenz durch Phanerogamen. Magerrasen, die infolge fehlender oder ungenügender Nutzung vergrasen, verstauden oder verbuschen stellen keine Lebensräume für Kryptogamengesellschaften mehr dar. Detailliertere Angaben zu Gefährdungsursachen und Schutzmaßnahmen gibt Kap. E6 und GÜNZL (2001a+b).

\section{Dynamik:}

Die Primärsukzession auf neu entstandenen Felsstandorten beginnt auf Dolomit offensichtlich mit der Toninia sedifolia-Initialgesellschaft, auf Gips mit Rein- oder Dominanzbeständen von Fulgensia bracteata (Artenarme Variante des ToninioPsoretum decipientis fulgensietosum bracteatae).

Neu entstehende Lücken innerhalb der Magerrasen, also Standorte, an denen die Bodenbildung teilweise schon bis zur Rendzina fortgeschritten ist, werden von den Gesellschaftsbildnern der „Bunten-Erdflechten-Gesellschaft“ (Toninio-Psoretum decipientis) besiedelt. Bei der Vegetationsentwicklung vom Initialstadium der Flechtenbesiedlung zum flechtenreichen Kalkmagerrasen (Gentiano-Koelerietum) treten kleinwüchsige bzw. krustige Arten, darunter die typischen Arten der BuntenErdflechten-Gesellschaft mehr und mehr zurück, während kräftigere Moos- und Flechtenarten zunehmen. In der Cladonia symphycarpa-Toninion-Fragmentgesellschaft gewinnen bereits die wuchskräftigeren Arten Hypnum cupressiforme var. lacunosum, 
Cladonia furcata ssp. subrangiformis und Cl. rangiformis an Bedeutung. Im Extremfall können diese Arten Dominanzbestände bilden.

Die Peltigera rufescens-Toninion-Fragmentgesellschaft scheint sich an offenen, jedoch zumindest gelegentlich durch ablaufendes oder sich am Grund von Steinbrüchen ansammelndes Wasser gekennzeichnete Standorte zu besiedeln und wird vermutlich im weiteren Sukzessionsverlauf durch Phanerogamengesellschaften, z.B. das GentianoKoelerietum abgelöst.

Tab. 19: Toninio-Psoretea decipientis: pH-Werte und elektrolytische Leitfähigkeiten der Böden

\begin{tabular}{|c|c|c|c|c|c|c|c|c|c|}
\hline & 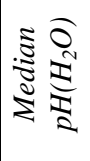 & 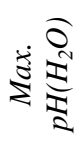 & 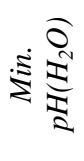 & 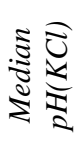 & 泀这 & 这这 & 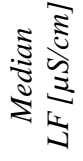 & 胥 & 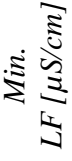 \\
\hline Toninia sedifolia-Toninion-Initialgesellschaft & 7,7 & 7,8 & 7,6 & 7,3 & 7,5 & 7,3 & 157 & 192 & 109 \\
\hline Toninio-Psoretum decipientis (Gesamt) & 7,3 & 7,8 & 7,0 & 7,1 & 7,4 & 6,9 & 359 & 1609 & 172 \\
\hline Toninio-Psoretum typicum, Cladonia-Variante & 7,3 & 7,3 & 7,2 & 7,1 & 7,2 & 7,1 & 301 & 362 & 258 \\
\hline $\begin{array}{l}\text { Toninio-Psoretum fulgensietosum, Cladonia-Var., } \\
\text { Squamarina-Subvariante }\end{array}$ & 7,3 & 7,8 & 7,0 & 7,3 & 7,4 & 7,0 & 192 & 1566 & 172 \\
\hline $\begin{array}{l}\text { Toninio-Psoretum fulgensietosum, Cladonia-Var., } \\
\text { Cephaloziella-Subvariante }\end{array}$ & 7,2 & 7,6 & 7,0 & 7,1 & 7,3 & 7,0 & 1376 & 1596 & 203 \\
\hline Toninio-Psoretum fulgensietosum, Artenarme Var. & 7,2 & 7,8 & 7,1 & 7,1 & 7,4 & 6,9 & 1500 & 1609 & 359 \\
\hline Cladonia symphycarpa-Toninion-FG & 7,5 & 7,8 & 7,2 & 7,2 & 7,5 & 7,0 & 197 & 395 & 161 \\
\hline Peltigera rufescens-Toninion-FG & 7,5 & 7,6 & 6,3 & 7,3 & 7,5 & 6,6 & 198 & 306 & 128 \\
\hline Dominanzbestände & 7,3 & 7,4 & 6,1 & 7,1 & 7,3 & 5,4 & 315 & 1609 & 122 \\
\hline
\end{tabular}




\section{Die Vergesellschaftung von Cladonia furcata}

Cladonia furcata steht im Mittelpunkt der weiteren Untersuchungen dieser Arbeit, daher wird in diesem Kapitel speziell auf die Vergesellschaftung ihrer beiden Unterarten in Nordhessen eingegangen. Die Ergebnisse werden mit entsprechenden Angaben für Nordwestdeutschland (PAUS 1997) verglichen.

\subsection{Cladonia furcata ssp. furcata}

Cladonia furcata ssp. furcata wurde im Untersuchungsgebiet in insgesamt 39 Vegetationsaufnahmen erfaßt. Hohe Stetigkeit erreicht die Subspezies in der Cladonia rangiferina-Variante des Cladonietum mitis typicum, in der Cladonia furcata-Cladonion arbusculae-Fragmentgesellschaft sowie im Cladonietum foliaceae. Mit geringer Stetigkeit tritt sie außerdem in 6 weiteren Vegetationseinheiten der Klasse CeratodontoPolytrichetea, jedoch nur in einer Einheit der Klasse Toninio-Psoretea auf. Im Untersuchungsgebiet ist Cl. furcata ssp. furcata daher eine schwache Trennart der Ceratodonto-Polytrichetea. Nach PAUS (1997) wird die Unterart als Ordnungscharakterart gewertet. In Nordhessen liegt der Schwerpunkt ihres Vorkommens in der Federation Cladonion arbusculae.

Tab. 20: Stetigkeiten, Ausdehnung der Lager und Deckungsgrade von Cladonia furcata ssp. furcata ( $n$ = Anzahl Aufnahmen, in denen Cl. furcata ssp. furcata auftritt.

Ausdehnung der Lager s. Kap. E1.2, S. 21)

\begin{tabular}{l|llll}
\hline Vegetationseinheit & $\begin{array}{l}\text { Stetig- } \\
\text { keit }\end{array}$ & $\begin{array}{l}\text { Ausdehnung } \\
\text { der Lager: } \\
\text { Min.,Max., } \\
\text { Median }\end{array}$ & $\begin{array}{l}\text { Deckungsgrad: } \\
\text { Minimum, } \\
\text { Maximum }\end{array}$ \\
\hline Pycnothelio-Cladonietum + Cladonia cervicornis-FG & 2 & 2 & $3,3,3$ & $1,2 \mathrm{a}$ \\
Dibaeetum baeomycetis, Cladonia-Höhenform & II & 2 & $1,1,1$ & r,1 \\
Dibaeetum baeomycetis, Baeomyces-Höhenform & - & - & - & - \\
Lecideetum uliginosae & - & - & - & - \\
Cl. bacillaris-Gesellschaft & I & 2 & $1,2,2$ &,+ 1 \\
Cladonietum mitis cladonietosum merochlorophaea & II & 5 & $1,3,1$ &,$+ 2 \mathrm{a}$ \\
Cladonietum mitis typicum, Typische Variante & I & 1 & 1 & + \\
Cladonietum mitis typicum, Cl. rangiferina-Variante & IV & 9 & $1,5,3$ &,+ 4 \\
Cl. furcata-Cladonion arbusculae-FG & V & 11 & $2,5,4$ & $2 \mathrm{a}, 5$ \\
Cl. gracilis-Cladonion arbusculae-FG & - & - & - & - \\
Cladonietum foliaceae & IV & 5 & $1,3,2$ & 1,3 \\
Cl. floerkeana-Cladonion rei-FG & - & - & - & - \\
Cladonietum rei, Cl. floerkeana-Variante & I & 1 & 1 & 1 \\
Cladonietum rei, Typische Variante & - & - & - & - \\
Cl. subulata-Cladonion rei-FG & - & - & - & - \\
Toninia sedifolia-Toninion-Initialgesellschaft & - & - & - & - \\
Toninio-Psoretum decipientis & - & - & - & - \\
Cl. symphycarpa-Toninion-FG & + & 1 & 1 & + \\
Peltigera rufescens-Toninion-FG & - & - & - & - \\
Dominanzbestände & - & - & - & - \\
\hline
\end{tabular}

In der Cladonia furcata-Cladonion arbusculae-Fragmentgesellschaft und in der Cladonia rangiferina-Variante des Cladonietum mitis typicum erreicht die Subspezies die größte Ausdehnung der Polster. Die Ansiedlungen werden dort bis über $20 \mathrm{~cm}$ groß. Bis zu $8 \mathrm{~cm}$ Polsterdurchmesser kann sie z.B. im Cladonietum mitis cladonietosum 
merochlorophaeae und im Cladonietum foliaceae aufweisen. In den meisten anderen Einheiten bleiben die Lager kleiner als $3 \mathrm{~cm}$ bzw. sogar kleiner als $1 \mathrm{~cm}$, d.h. es treten nur einzelne Podetien und keine Polster auf.

\section{a) Vergleich mit Nordwestdeutschland (Tab. 21)}

Bei einem Vergleich der eigenen Vegetationsaufnahmen aus Nordhessen mit solchen aus Nordwestdeutschland (PAUS 1997) zeigen sich Übereinstimmungen bezüglich der hohen Stetigkeiten im Cladonietum mitis typicum und im Cladonietum foliaceae. Innerhalb des Cladonietum mitis typicum stellt PAUS allerdings eine eigene Ausbildung mit Cladonia furcata ssp. furcata auf, während in der vorliegenden Untersuchung die Cl. furcata-reichen Bestände der Cladonia rangiferina-Variante zugeordnet werden. Eine Subunion cladonietosum furcatae des Cladonietum foliaceae, in der die Subspezies furcata bei PAUS mit hoher Stetigkeit auftritt, kann im nordhessischen Aufnahmematerial nicht abgetrennt werden.

Tab. 21: Stetigkeiten von Cladonia furcata ssp. furcata: Vergleich der Daten aus Nordhessen und Nordwestdeutschland

\begin{tabular}{|c|c|c|c|}
\hline \multicolumn{2}{|l|}{ Nordhessen (eigene Daten) } & \multicolumn{2}{|c|}{ Nordwestdeutschland (PAUS 1997) } \\
\hline Vegetationseinheit & $\begin{array}{l}\text { Stetig- } \\
\text { keit }\end{array}$ & $\begin{array}{l}\text { Stetig- } \\
\text { keit }\end{array}$ & Vegetationseinheit \\
\hline Pycnothelio-Cladonietum $/ \mathrm{Cl}$. cervicornis-FG & 2 & + & Pycnothelio-Cladonietum, Terminalphase \\
\hline Dibaeetum, Cladonia cervicornis-Höhenform & II & + & $\begin{array}{l}---- \\
\text { Dibaeetum baeomycetis, typische Ausbildung }\end{array}$ \\
\hline Lecideetum uliginosae & - & $\begin{array}{l}+ \\
\mathrm{r}\end{array}$ & $\begin{array}{l}\text { Lecideetum uliginosae typicum } \\
\text { Lecideetum uliginosae cladonietosum portentosae }\end{array}$ \\
\hline Cladonia bacillaris-Gesellschaft & I & $\begin{array}{l}+ \\
\mathrm{I} \\
\mathrm{r} \\
\mathrm{I}\end{array}$ & $\begin{array}{l}\text { Cl. bacillaris-MG, typische Ausb., jüngere Entw.phasen } \\
\text { Cl. bacillaris-MG, typische Ausb., fortgeschr. Phasen } \\
\text { Cl. bacillaris-MG, Ausb. mit Cl. glauca, jüngere Phasen } \\
\text { Cl. bacillaris-MG, Ausb. Cl. glauca., fortgeschr. Phasen }\end{array}$ \\
\hline $\begin{array}{l}\text { Cladonietum mitis cladonietosum merochl. } \\
\text { Cladonietum mitis typicum, Typische Variante } \\
\text { Cl. mitis typicum, Cl. rangiferina-Variante } \\
\text {---- } \\
\text {---- }\end{array}$ & $\begin{array}{l}\text { I } \\
\text { IV }\end{array}$ & $\begin{array}{l}\mathrm{r} \\
\mathrm{r} \\
\mathrm{V} \\
+ \\
\mathrm{V} \\
+ \\
\end{array}$ & $\begin{array}{l}\text { Cladonietum mitis cladonietosum merochl., typ. Ausb. } \\
\text { Cl. mitis cladonietosum merochl., Ausb. mit Cl. glauca } \\
\text { Cl. mitis cladonietosum merochl., Ausb. mit Cl. furcata } \\
\text { Cladonietum mitis typicum, typische Ausbildung } \\
\text {---- } \\
\text { Cladonietum mitis typicum, Ausbildung mit } \mathrm{Cl} \text {. furcata } \\
\text { Cladonietum mitis hypnetosum jutlandicae }\end{array}$ \\
\hline Cladonia furcata-Cladonion arbusculae-FG & $\mathrm{V}$ & & ----- \\
\hline $\begin{array}{c}---- \\
\end{array}$ & & $\mathrm{I}$ & Dominanzbestände von Cladonia uncialis \\
\hline Cladonietum foliaceae & IV & $\begin{array}{l}\text { IV } \\
\text { IV }\end{array}$ & $\begin{array}{l}\text { Cladonietum foliaceae cladonietosum furcatae } \\
\mathrm{Cl} \text {. foliaceae, Übergang zu cladonietosum furcatae }\end{array}$ \\
\hline----- & & II & Cetraria aculeata-Cladonion arbusculae-Fragmentges. \\
\hline $\begin{array}{l}\text { Cladonietum rei, Cl. floerkeana-Variante } \\
\text { Cladonietum rei, Typische Variante } \\
\text {---- }\end{array}$ & $\begin{array}{l}\mathrm{I} \\
-\end{array}$ & + & $\begin{array}{l}---- \\
\text { Cladonietum rei, typische Ausbildung } \\
\text { Cladonietum rei, Ausbildung mit Peltigera didactyla }\end{array}$ \\
\hline $\begin{array}{ll}---- \\
----- \\
----\end{array}$ & & $\begin{array}{l}\text { I } \\
\text { V } \\
\text { V } \\
\text { II }\end{array}$ & $\begin{array}{l}\text { Cladonia humilis-Cladonion rei-Fragmentgesellschaft } \\
\text { Cladonia furcata-Cladonion rei-Fragmentgesellschaft } \\
\text { Cladonia furcata-Dominanzbestände } \\
\text { Übergang Cladonietum rei zu Cladonion arbusculae }\end{array}$ \\
\hline
\end{tabular}

In Nordwestdeutschland erreicht die Unterart zusätzlich eine hohe Stetigkeit in einer gleichnamigen Ausbildung des Cladonietum mitis cladonietosum merochlorophaeae. In Nordhessen kommt sie innerhalb dieser Subunion nur mit Stetigkeit II vor; eine eigene Ausbildung läßt sich nicht abtrennen. Ein weiterer Unterschied zwischen beiden 
Gebieten zeigt sich innerhalb der Federation Cladonion rei. PAUS nennt eine Cladonia furcata-Cladonion rei-Fragmentgesellschaft, die sich aus dem nordhessischen Aufnahmematerial nicht ergab. Sie stellt auch die Cladonia furcata-Dominanzbestände in die Nähe des Cladonion rei, weist aber auf andere Vorstadien dieser Dominanzen, etwa das Cladonietum mitis hin. Letzteres zeigt wiederum verwandtschaftliche Beziehungen zu der in der vorliegenden Untersuchung beschriebenen Cladonia furcataCladonion arbusculae-Fragmentgesellschaft.

\section{b) Vergleich mit weiteren Literaturangaben}

Nach der Ansicht von KLEMENT (1955) ist die Varietät racemosa von Cladonia furcata als Federationscharakterart des Cladonion silvaticae (Syn. Cladonion arbusculae) zu betrachten. Das stete Auftreten der Art innerhalb dieser Federation hat wohl auch zu der Bezeichnung Cladonia furcata-Assoziation Krieger 1937 als Synonym des Cladonietum mitis geführt. Durch eine Dominanz von Cl. furcata zeichnen sich nach KLEMENT (1955) gelegentlich Ausbildungsformen des Cladonietum mitis aus, die schwach geneigte Hangflächen oder Waldränder besiedeln. Diese Ausbildungsform sei ziemlich windhart und kennzeichne gut Aushagerungsgebiete.

Hochstet tritt im Cladonietum alcicornis (Syn. Cladonietum foliacea) laut KLEMENT (1955) die Varietät Cladonia furcata-palamaea (s. Kap. G2.1.3) auf; er wertet sie als Charakterart der Gesellschaft. Weitere Angaben zur Verbreitung von Cl. furcata in Nordostdeutschland finden sich in Doll (1996); als Begleiter tritt die Art dort in der sogenannten Cladonia cariosa-Gesellschaft, der Cladonia zopfii-Gesellschaft und der Cladonia scabriuscula-Gesellschaft auf.

Bezüglich des Vorkommens von Cladonia furcata ssp. furcata in Gesellschaften basenreicher Standorte finden sich überraschende Angaben bei KLEMENT (1955): Die Varietät Cladonia furcata-palamaea (s. Kap. G2.1.3) erreicht in seinem Aufnahmematerial Stetigkeit IV im Fulgensietum fulgentis (Syn. Toninio-Psoretum) und Stetigkeit III im Cladonietum symphycarpiae (Syn. Cladonietum symphycarpae). Beide Angaben erscheinen mir ungewöhnlich hoch und sind mit Sicherheit nicht repräsentativ für den mitteleuropäischen Raum! FOLLMANN (1974) wertete 30 Aufnahmen aus dem Nordhessischen Bergland aus und führt die Varietät palamaea mit Stetigkeit II auf.

\subsection{Cladonia furcata ssp. subrangiformis}

Die Subspezies subrangiformis wurde im Untersuchungsgebiet in 26 Aufnahmeflächen nachgewiesen und tritt darin stets nur steril auf. Ihr Vorkommen beschränkt sich ausschließlich auf Vegetationseinheiten innerhalb der Klasse Toninio-Psoretea decipientis, zu deren Trennarten sie im Untersuchungsgebiet zählt (s. Tab. 2). Hohe Stetigkeiten erreicht sie im Toninio-Psoretum typicum, in der Cladonia symphycarpaToninion-Fragmentgesellschaft, in der Peltigera rufescens-Toninion-FG sowie in den Dominanzbeständen, deren Erscheinungsbild sie mit bis zu $20 \mathrm{~cm}$ großen Lagern prägen kann. Im allgemeinen sind ihre Lager jedoch wesentlich unauffälliger. Meist sind nur einzelne, unregelmäßig verzweigte Podetien zu finden (s. Tab. 22). 
Tab. 22: Stetigkeiten, Ausdehnung der Lager und Deckungsgrade von Cladonia furcata ssp. subrangiformis ( $n=$ Anzahl Aufnahmen, in denen Cladonia furcata ssp. subrangiformis auftritt. Ausdehnung der Lager s. Kap. E1.2, S. 21)

\begin{tabular}{|c|c|c|c|c|}
\hline Vegetationseinheit & Stetigkeit & $n$ & $\begin{array}{l}\text { Ausdehnung } \\
\text { der Lager: } \\
\text { Min.,Max., } \\
\text { Median }\end{array}$ & $\begin{array}{l}\text { Deckungsgrad: } \\
\text { Minimum, } \\
\text { Maximum }\end{array}$ \\
\hline Toninia sedifolia-Toninion-Initialgesellschaft & - & - & - & - \\
\hline Toninio-Psoretum typicum, Cladonia-Variante & III & 5 & $1,2,1$ & $\mathrm{r}, 1$ \\
\hline $\begin{array}{l}\text { Ton.-Psoretum fulgensietosum, Cl. symphycarpa-Variante, } \\
\text { Subvariante von Squamarina cartilaginea }\end{array}$ & $\mathrm{I}$ & 3 & $1,1,1$ & $\mathrm{r},+$ \\
\hline $\begin{array}{l}\text { Ton.-Psoretum fulgensietosum, Cl. symphycarpa-Variante, } \\
\text { Cephaloziella-Subvariante }\end{array}$ & $\mathrm{I}$ & 1 & 1 & + \\
\hline Ton.-Psoretum fulgensietosum, Artenarme Variante & - & - & - & - \\
\hline Cl. symphycarpa-Toninion-FG & IV & 7 & $1,2,1$ & $\mathrm{r}, 1$ \\
\hline Peltigera rufescens-Toninion-FG & III & 4 & $1,2,2$ &,$+ 2 \mathrm{a}$ \\
\hline Dominanzbestände & III & 6 & $2,4,3$ &,+ 3 \\
\hline
\end{tabular}

Tab. 23: Stetigkeiten von Cladonia furcata ssp. subrangiformis: Vergleich der Daten aus Nordhessen und Nordwestdeutschland

\begin{tabular}{|c|c|c|c|}
\hline \multicolumn{2}{|l|}{ Nordhessen (eigene Daten) } & \multicolumn{2}{|c|}{ Nordwestdeutschland (PAUS 1997) } \\
\hline Vegetationseinheit & $\begin{array}{l}\begin{array}{l}\text { Stetig- } \\
\text { keit }\end{array} \\
\end{array}$ & \begin{tabular}{|l|}
$\begin{array}{l}\text { Stetig- } \\
\text { keit }\end{array}$ \\
\end{tabular} & Vegetationseinheit \\
\hline $\begin{array}{l}---- \\
----\end{array}$ & & $\begin{array}{l}\mathrm{r} \\
\mathrm{r}\end{array}$ & $\begin{array}{l}\text { Cladonietum zopfii, Optimalphase } \\
\text { Cladonietum zopfii, Terminalphase }\end{array}$ \\
\hline Cladonia bacillaris-Gesellschaft & - & I & $\begin{array}{l}\text { Cl. bacillaris-Mikrogesellschaft, Ausbildung mit } \\
\text { Cl. glauca, jüngere Entwicklungsphasen } \\
\text { Cl. bacillaris-Mikrogesellschaft, Ausbildung mit } \\
\text { Cl. glauca, fortgeschrittene Entwicklungsphasen } \\
\end{array}$ \\
\hline $\begin{array}{l}\text { Cladonietum mitis cladonietosum } \\
\text { Cladonietum mitis typicum, Typ. Var. }\end{array}$ & - & $\begin{array}{l}\mathrm{r} \\
+ \\
+\end{array}$ & $\begin{array}{l}\text { Cladonietum mitis cladonietosum, Typ. Ausbildung } \\
\text { - “-, Ausbildung mit Cl. glauca } \\
\text { Cladonietum mitis typicum, Typische Ausbildung }\end{array}$ \\
\hline Cladonietum foliaceae & - & $\begin{array}{l}\text { IV } \\
\text { III } \\
\text { V }\end{array}$ & $\begin{array}{l}\text { Cladonietum foliaceae cladonietosum subrangiformis, } \\
\text { Typische Variante } \\
\text { - “-, Var. von Cl. novochlor., Ausb. mit Cl. ciliata } \\
\text { - “-, Var. von Cl. novochloroph., Typ. Ausbildung }\end{array}$ \\
\hline Toninio-Psoretum decipientis & II & & ----- \\
\hline Cl. symphycarpa-Toninion-FG & IV & & \begin{tabular}{|l|l}
---- \\
\end{tabular} \\
\hline----- & & III & Cladonietum symphycarpae \\
\hline Peltigera rufescens-Toninion-FG & III & & $\begin{array}{cc}---- \\
\end{array}$ \\
\hline----- & & III & Cladonia pocillum-Toninion-Fragmentgesellschaft \\
\hline----- & & - & Mycobilimbia microcarpa-Toninion-Fragmentges. \\
\hline Dominanzbestände & III & & $\begin{array}{ll}---- \\
--1\end{array}$ \\
\hline
\end{tabular}

PAus (1997) konnte Cl. furcata ssp. subrangiformis auch in einigen Vegetationseinheiten der Klasse Ceratodonto-Polytrichetea nachweisen. Nur im Cladonietum foliaceae erreicht die Subspezies jedoch nennenswerte Stetigkeit und zwar innerhalb der Subunion cladonietosum subrangiformis auf basenreichen Sanden (s. Tab. 23). Der relative Basenreichtum verbindet diese Gesellschaft mit den Gesellschaften der Klasse Toninio-Psoretea, innerhalb derer Cl. furcata ssp. subrangiformis in Nordwestdeutschland im Cladonietum symphycarpae und der Cladonia pocillumToninion-Fragmentgesellschaft auftritt (allerdings wurden von PAUS nur wenige Bestände dieser Klasse und darunter kein Toninio-Psoretum bearbeitet). KLEMENT (1955) zählt Cladonia [furcata ssp.] subrangiformis zu den Ordnungscharakterarten der von ihm aufgestellten Ordnung Epigaeetalia (Syn. Peltigeretalia). In seiner Publikation führt er sie mit geringer Stetigkeit innerhalb des Fulgensietum fulgentis (Syn. ToninioPsoretum) auf; in Nordhessen ist sie in dieser Gesellschaft etwas steter vertreten. 
Erstaunlicherweise kommt im Aufnahmematerial von FolLMANN (1974) die Unterart überhaupt nicht vor, wohingegen BORNKAMM (1958) sie etliche Male in Beständen seiner „Kalkausbildung“ fand. Somit zeigen sich Parallelen zwischen den Ergebnissen von BORNKAMM und dem Vorkommen von Cl. furcata ssp. subrangiformis als Trennart der Cladonia-Variante des Toninio-Psoretum typicum in Nordhessen.

\section{Einbindung der Flechtengesellschaften in die Phanerogamenvegetation}

Erdflechtengesellschaften treten im Untersuchungsgebiet innerhalb verschiedenster Phanerogamengesellschaften auf. Allerdings sind sie nicht immer von gut entwickelten Phanerogamenbeständen umgeben. Im Extremfall sind an den Wuchsorten sogar nur einzelne Exemplare Höherer Pflanzen vorhanden. Jedoch lassen sich für das Untersuchungsgebiet einige typische Erdflechten-reiche Vegetationstypen nennen.

Die Vegetation der Magerrasen auf basenreichen Standorten ist größtenteils dem Gentiano-Koelerietum (vgl. BRUELHEIDE 1991) zuzuordnen. Als Kryptogamenschicht innerhalb dieser Gesellschaft siedeln beispielsweise Dominanzbestände von Cladonia furcata ssp. subrangiformis, in größeren Vegetationslücken die Cladonia symphycarpaToninion-Fragmentgesellschaft. Die Bunte-Erdflechten-Gesellschaft (Toninio-Psoretum decipientis) ist zwar ebenfalls innerhalb der Kalkmagerrasen anzutreffen, nutzt jedoch überwiegend Sonderstandorte wie Felsköpfe oder Wegränder und ist daher in noch stärkerem Maße von der umgebenden Phanerogamenvegetation unabhängig wie die Cladonia symphycarpa-Toninion-Fragmentgesellschaft. Die Bunte-ErdflechtenGesellschaft besiedelt zudem mancherorts Flächen in aufgelassenen Steinbrüchen, wo die Phanerogamenvegetation oft so spärlich entwickelt ist, daß eine Einordnung lediglich als Brometalia- bzw. Festuco-Brometea-Bestände erfolgen kann. Auch die Toninia sedifolia-Toninion-Initialgesellschaft sowie gelegentlich die Peltigera rufescens-Toninion-Fragmentgesellschaft wurden in aufgelassenen Steinbrüchen angetroffen. Erstere nutzt die Steinbruchwände, letztere die ebenen Flächen am Grund. Beide werden u.U. von Kalkmagerrasen-Fragmenten umgeben, sind aber von diesen nicht abhängig.

Auf Basalt, z.B. am Dörnberg und am Maderstein treten Felsfluren der Klasse SedoScleranthetea (Syn. Koelerio-Corynephoretea) auf (s. BERnd 1998). Das Cladonietum foliaceae wächst in Lücken solcher Felsfluren, deren Arten sporadisch in die Flechtenbestände eindringen. Auf Diabas, am Bilstein bei Albungen werden die Flechtenbestände dieser Union von Festuca pallens umgeben.

Dominierende Art der Zwergstrauchheiden ist die Besenheide (Calluna vulgaris). Auch Vaccinium myrtillus und V. vitis-idaea können größere Flächen einnehmen, so z.B. im Waldecker Upland. Stellenweise treten vergraste Heidebestände auf. Ausführliche Beschreibungen der Zwergstrauchheiden in Nordhessen finden sich in NIESCHALK \& Nieschalk (1983a/b), Nitsche (1995) und Nitsche \& Bultmann (1995). Innerhalb der Zwergstrauchheiden sind verschiedene Erdflechtengesellschaften anzutreffen. Das Dibaeetum baeomycetis, welches gerne abgeplaggte Flächen besiedelt, wird nur 
randlich von den Zwergsträuchern beschattet und verschwindet sofern sich diese ausbreiten. Dagegen kann das Cladonietum mitis dauerhaften Charakter haben und als Teil der Zwergstrauchheide angesehen werden, in die es fleckenweise eingestreut ist. Aber auch in diesem Falle durchmischen sich Kryptogamen und Phanerogamen wenig. Als Kryptogamenschicht unterhalb der Calluna-Sträucher wurden stellenweise z.B. ausgedehnte Polster von Cladonia furcata ssp. furcata festgestellt.

Die bearbeiteten Traubeneichenwälder der steilen Hänge auf Grauwacke und Tonschiefer dürften dem Hainsimsen-Traubeneichenwald (Luzulo-Quercetum petraea) zuzuordnen sein (BAUFELD 1991, SCHÄFER 1998). BAUFELD (1991) beschreibt diese Gesellschaft beispielsweise von den Steilhängen an Roßkopf, Klepperberg und Sengelharth. Sowohl an den Ederseehängen als auch im Roßkopf-Gebiet treten außerdem lichte Bestände des Hainsimsen-Buchenwaldes (Luzulo-Fagetum) auf (BAUfEld 1991, ScHÄFER 1998). Das Pycnothelio-Cladonietum bzw. die Cladonia cervicornis-Baeomycion-Fragmentgesellschaft sind in Nordhessen typische Gesellschaften solcher Eichenwälder. Die Cladonia rangiferina-Variante des Cladonietum mitis typicum stellt sich ebenfalls nur unter den mikroklimatischen Bedingungen innerhalb der Eichenwälder ein. Die Beschattung durch die Bäume spielt hierbei eine große Rolle.

Bleiben zuletzt noch die Flechtenbestände der ehemaligen Bergbauflächen, beispielsweise das Cladonietum rei. Erdflechten derartiger Flächen siedeln in Vegetationslücken bzw. auf Pionierstandorten, an denen Höhere Pflanzen noch nicht Fuß fassen können. Nur gelegentlich durchmischen sich Kryptogamen und Phanerogamen. Ist das der Fall, so spricht dies für ein fortgeschrittenes Stadium der Sukzession, in dem die Flechten durch Höhere Pflanzen verdrängt werden. In unmittelbarer Umgebung der Flechtenbestände treten z.B. Festuca ovina agg. sowie Pioniergehölze (Betula pendula, Alnus alnobetula und Populus tremula) auf.

\section{$5 \quad$ Fruchtkörperbildung}

Im folgenden wird der Frage nachgegangen, ob Diagnostische Arten einer Gesellschaft auf den zugehörigen Standorten nicht nur verhältnismäßig häufig sind, sondern auch günstige Entwicklungsmöglichkeiten vorfinden. Kümmerliche Exemplare zeichnen sich bei manchen Flechten (z.B. bei Cladonia macilenta) oft durch fehlende Fruchtkörper aus. Das Auftreten oder Fehlen fruchtender Stadien, vorausgesetzt solche gehören zum normalen Entwicklungszyklus einer Art, kann also Auskunft darüber geben, ob die Bedingungen am Standort eine optimale Entwicklung ermöglichen. Nachfolgend wird die Fruchtkörperbildung einiger exemplarisch ausgewählter Arten besprochen, die größtenteils diagnostischen Wert haben. In den Tab. 59 bis Tab. 60 im Anhang 1 werden die Ergebnisse für weitere Arten aufgeführt, sofern diese in mindestens 15 Vegetationsaufnahmen auftreten und zumindest gelegentlich Fruchtkörper ausbilden. 
Die Auswertung erfolgte unter zwei Fragestellungen:

1. Weisen die fruchtenden Exemplare bezüglich der Stetigkeiten den selben/die selben Verbreitungsschwerpunkt(e) auf wie die Art insgesamt (s. Tab. 24)?

2. In welcher Vegetationseinheit finden sich prozentual die meisten fruchtenden Thalli gemessen an der Gesamtzahl aller gefundenen Thalli mit Fruchtkörperbildung? (s. Tab. 25)

Ergänzend dazu enthält Tab. 26 Angaben darüber, in wieviel Prozent der Aufnahmen einer Vegetationseinheit die entsprechende Art auch Fruchtkörper ausgebildet hatte. Dieser Tabelle läßt sich also beispielsweise entnehmen, in welchen Einheiten eine in der Regel fruchtende Art nur in Kümmerexemplaren ohne Fruchtkörper auftritt.

Hinweise auf fruchtende Stadien (dargestellt als Exponenten) enthalten auch die Vegetationstabellen (Tab. 6, Tab. 8, Tab. 10, Tab. 15, Tab. 17) im Anhang 3.

\section{a) Diagnostische Arten innerhalb des Baeomycion rosei}

\section{Dibaeis baeomyces:}

Sowohl bei den Stetigkeiten der fruchtenden Thalli als auch bei der Gesamtstetigkeit der Art läßt sich ein eindeutiger Schwerpunkt im Dibaeetum baeomycetis feststellen (s. Tab. 24). Es wurden in der Hälfte aller Aufnahmen der Cladonia-Höhenform und in einem Drittel der Aufnahmen der Baeomyces-Höhenform Apothecien beobachtet (s. Tab. 26). In den anderen 3 Vegetationseinheiten, außerhalb des Dibaeetum baeomycetis, zeigt Dibaeis keine Apothecienbildung. Sie tritt dort jedoch auch nur mit geringer Stetigkeit auf. Insgesamt waren die Lager nur an 7 von 20 Fundstellen fertil. Gut entwickelte Vorkommen wachsen in den höheren Lagen Nordhessens, beispielsweise am Osterkopf bei Usseln. Günstige Bedingungen findet Dibaeis im Untersuchungsgebiet demnach offensichtlich nur an den dortigen Standorten des Dibaeetum, als deren Kennart sie gilt (s. Kap. E2.1.1.1). Nach SchÖLLER (1991) hängt die Sterilität bei Dibaeis mit ungünstigen Standortbedingungen wie ungenügender Feuchtigkeitsversorgung oder Kälte zusammen. Die Lebensdauer der Fruchtkörper von Dibaeis scheint auf gut ein Jahr beschränkt zu sein (JAHNS \& OTT 1982).

Baeomyces rufus:

Als Federationscharakterart des Baeomycion liegen die Verbreitungsschwerpunkte in der Baeomyces-Höhenform des Dibaeetum baeomycetis sowie im Lecideetum uliginosae. Fruchtende Lager treten hochstet jedoch nur in der Baeomyces-Höhenform auf (s. Tab. 24). Der prozentual höchste Anteil an fruchtenden Lagern stammt ebenfalls aus dieser Vegetationseinheit. In den anderen Einheiten wurden nur 8 bzw. $15 \%$ der insgesamt notierten fruchtenden Ansiedlungen gefunden (s. Tab. 25). Als optimale Standorte für die Entwicklung der Apothecien von Baeomyces rufus erweisen sich die Zwergstrauchheiden der niederschlagsreichen, höheren Lagen des Untersuchungsgebietes. Die Art liebt JAHNS \& OTT (1982) zufolge wohl einen relativ dunklen und feuchten Standort, jedoch scheint nach meinen Beobachtungen mehr die ausreichende Feuchtigkeitsversorgung entscheidend zu sein als die Lichtverhältnisse. Die Flechte 
zählt zu den kurzlebigen Arten. Thallus und Apothecien von Baeomyces leben nur etwa 2 Jahre (JAHNS \& OTT 1982). Die Art paßt sich in ihrem Lebenszyklus, wie auch Dibaeis baeomyces, dem temporären Auftreten von Pionierstandorten an. Die sterilen Thalli von Baeomyces rufus tragen im Untersuchungsgebiet fast stets sogenannte Schizidien, die als Diasporen fungieren und somit auf ungeschlechtlichem Weg eine Vermehrung und Verbreitung der Thalli gewährleisten.

\section{b) Diagnostische Arten innerhalb der Peltigeretalia bzw. des Cladonion arbusculae}

Cladonia furcata ssp. furcata:

Die Art wird als Ordnungscharakterart der Peltigeretalia angesehen und weist die höchsten Stetigkeiten in der Cladonia rangiferina-Variante des Cladonietum mitis typicum, in der Cladonia furcata-Cladonion arbusculae-Fragmentgesellschaft und im Cladonietum foliaceae auf (s. Tab. 24, vgl. Kap. E3.1). Insgesamt ließen die Polster nur in 13 Aufnahmeflächen eine Fruchtkörperbildung erkennen, und zwar in den 4 Vegetationseinheiten Cladonietum mitis cladonietosum merochlorophaeae, Cladonia rangiferina-Variante des Cladonietum mitis typicum, Cladonia furcata-Cladonion arbusculae-Fragmentgesellschaft und Cladonietum foliaceae. Während in der Cladonia rangiferina-Variante des Cladonietum mitis $67 \%$ der Aufnahmen mit Cl. furcata ssp. furcata fruchtende Thalli enthielten, ist das beispielsweise im Cladonietum foliaceae nur in $20 \%$ der Fall (s. Tab. 26), obwohl die Art dort hohe Stetigkeit erreicht. Äußere und innere Einflüsse auf die Fruchtkörperbildung bei Cl. furcata ssp. furcata werden in Kap. G3 ausführlich besprochen und in Kap. I1 diskutiert.

\section{Cladonia merochlorophaea:}

Die Chemosippe merochlorophaea besitzt Verbreitungsschwerpunkte in der Cladonia bacillaris-Gesellschaft, dem Cladonietum mitis cladonietosum merochlorophaeae, der Cl. floerkeana-Cladonion rei-Fragmentgesellschaft sowie in der Cl. floerkeana-Variante des Cladonietum rei. Sie tritt also bevorzugt in Gesellschaften auf, die zu den intermediären Sukzessionsstadien zählen und von sorediösen Arten dominiert werden. Ein ähnliches Bild zeigen die Stetigkeiten der fruchtenden Exemplare, die jedoch v.a. in der Cl. bacillaris-Gesellschaft hochstet auftreten (s. Tab. 24). Fast die Hälfte aller Nennungen fruchtender Thalli stammen aus dieser Gesellschaft (s. Tab. 25). Eine interessante Beobachtung bei Cl. merochlorophaea ist, daß sie in $75 \%$ der Bestände der Cl. bacillaris-Gesellschaft, in denen sie auftritt, Fruchtkörper ausbildet, während in den anderen Cladonion arbusculae-Gesellschaften nur in höchstens $40 \%$ aller Bestände mit Cl. merochlorophaea eine Apothecienbildung notiert werden konnte (s. Tab. 26). Auch die maximalen Durchmesser der Ansiedlungen dieser Chemosippe sind in der Cl. bacillaris-Gesellschaft größer als in den anderen, nahestehenden Gesellschaften (s. Tab. 8 im Anhang 3). Insgesamt wies etwa jedes zweite Vorkommen fertile Podetien auf. 


\section{c) Diagnostische Arten innerhalb des Cladonion rei}

\section{Cladonia fimbriata:}

Die Trennart des Cladonion rei taucht schwerpunktmäßig in den beiden Fragmentgesellschaften des Cladonion rei auf. Jedoch kommen nur in der $\mathrm{Cl}$. floerkeana-Cladonion rei-Fragmentgesellschaft auch fruchtende Exemplare in höherer Stetigkeit vor (s. Tab. 24). Dementsprechend stammt der prozentual höchste Anteil aller Nennungen von Apothecien aus dieser Gesellschaft (s. Tab. 25). Außerhalb der Federation Cladonion rei wurde die Art nie fruchtend angetroffen. Insgesamt zeigten sich nur in 11 Aufnahmeflächen fruchtende Podetien.

Cladonia pyxidata ssp. chlorophaea:

Die Art ist typisch für das Cladonietum rei (s. Kap. E2.1.3.2), tritt hochstet aber auch in der Cl. subulata-Cladonion rei-Fragmentgesellschaft auf. Das gleiche Bild zeigt sich bei Betrachtung der fruchtenden Vorkommen, jedoch kommen fertile Podetien mit deutlich höherer Stetigkeit im Cladonietum rei vor (s. Tab. 24). Rund ein Drittel aller Nennungen von Apothecienvorkommen stammen aus Flächen der Cl. floerkeanaVariante, etwa ein Viertel aus Flächen der Typischen Variante (s. Tab. 25). Insgesamt waren aber nur knapp die Hälfte aller gefundenen Ansiedlungen fertil. Außerhalb der Federation Cladonion rei wurde sie 14 mal registriert, davon jedoch nur viermal fertil (=29\%). Unter den 19 Vorkommen im Cladonion rei zeigten dagegen immerhin 13 Ansiedlungen $(=68 \%$ ) Apothecien.

JAHNS \& OTT (1982) konnten nachweisen, daß bei Cl. pyxidata ssp. chlorophaea der Entwicklungsablauf von der Ansiedlung bis zum fruchtenden Podetium nur wenige Jahre in Anspruch nimmt. Eine Tatsache, die sicher bei der Besiedlung der nur über einen begrenzten Zeitraum für Flechtenbewuchs ausreichend offenen, anthropogenen Störstellen (wie sie dem Cladonion rei als Lebensraum dienen) von Vorteil ist. Die Apothecien bei Cl. pyxidata ssp. chlorophaea zeigen einen Entwicklungsverlauf, der in seiner Geschwindigkeit und Vollständigkeit durch äußere Faktoren geregelt und synchronisiert wird. Unter extremeren mikroklimatischen Bedingungen entwickelt sich die Art schlechter (JAHNS \& OTT 1982). Wie Cl. fimbriata gedeiht die Art offensichtlich vor allem im Cladonion rei optimal. Gekennzeichnet sind die dazugehörigen Standorte durch Lichtreichtum, häufige Trockenperioden und Böden, deren pH-Werte oftmals im schwach sauren Bereich liegen (s. auch Kap. E2.1.3). 


\section{d) Diagnostische Arten innerhalb des Toninion sedifoliae}

\section{Psora decipiens:}

Die Art ist ein typischer Vertreter der „Bunten Erdflechten“ (FC Toninion sedifoliae). Jedoch kommen fertile Psora-Schuppen hochstet nur in Beständen der Typischen Subunion sowie in der Squamarina-Subvariante innerhalb der Subunion fulgensietosum vor (s. Tab. 24). Diesen Einheiten entstammen ein Drittel bzw. knapp die Hälfte aller Nennungen fruchtender Lager (s. Tab. 25). Sterile Thalli wurden relativ oft in der Cephaloziella-Subvariante innerhalb des Toninio-Psoretum fulgensietosum entdeckt (s. Tab. 26). Eventuell ist die hohe Trittbelastung mancher Standorte für das Ausbleiben fruchtender Stadien mitverantwortlich (s. GüNZL 2001b).

Cladonia furcata ssp. subrangiformis:

Die Sippe tritt in den Aufnahmeflächen nur steril auf. Lediglich außerhalb der Aufnahmeflächen wurde sie vereinzelt mit braunen Fruchtkörpern beobachtet.

\section{e) Hochstete Begleitart innerhalb der Klasse Toninio-Psoretea}

\section{Peltigera rufescens:}

Apothecien sind bei Peltigera rufescens generell mäßig häufig (WIRTH \& DÜLL 2000) bzw. nach Angaben von VITIKAINEN (1994) „fairly common“. Im Untersuchungsgebiet zeigten sich aber an keinem Wuchsort Fruchtkörper. Die Art ist ihren Zeigerwerten (WIRTH 1991) nach zu urteilen, eine typische Flechte trockener, heller, basenreicher und nährstoffarmer Standorte. Obwohl sie also die relativ extremen Bedingungen an den Toninion-Standorten gut meistern kann, erreichen ihre Thalli in vielen Aufnahmeflächen nur eine Ausdehnung der Stufe 1 oder 2 (s. Tab. 15 und Tab. 17 im Anhang 3), d.h. maximal $3 \mathrm{~cm}$ Durchmesser. Diese Lager sind eventuell zu klein, um Apothecien zu bilden, da Flechtenthalli eine gewisse Größe erreicht haben müssen, ehe Fruchtkörper entstehen können (JAHNS \& FREY 1982). Bei Peltigera canina sehen JAHNS \& FREY (1982) einen Grund für diese Mindestgröße darin, daß die Fruchtkörperentwicklung das weitere Thalluswachstum hemmt. Jedoch kann bei Peltigera, im Gegensatz zu Cladonia furcata, dem Fruchten erneutes Wachstum und erneute Fruchtkörperbildung folgen. Es lassen sich daher an ein und demselben Lager u.U. ältere und jüngere Apothecien gleichzeitig beobachten (JAHNS \& FREY 1982). In einigen Beständen v.a. der Peltigera rufescens-Toninion-Fragmentgesellschaft erreichen die Lager von Peltigera rufescens bis zu $20 \mathrm{~cm}$ in der Ausdehnung (d.h. Stufe 3 und 4, s. Tab. 15 und Tab. 17 im Anhang 3). Diese Thalli sollten eine für die Fruchtkörperbildung ausreichende Größe erreicht haben. Da jedoch auch an den großen Lagern nie Apothecien beobachtet wurden, dürften die ungünstigen Standortbedingungen (v.a. die Trockenheit) für das Ausbleiben der Apothecienbildung im Untersuchungsgebiet wesentlich mit verantwortlich sein. Es ist bekannt, daß klimatische Verhältnisse bei der nahe verwandten Art Peltigera canina einen Einfluß auf die Fruchtkörperbildung haben. Das Erscheinen der ersten Primordien wird bei 
dieser Blattflechte durch günstiges Wetter, und zwar im Frühherbst ausgelöst (JAHNS \& FREY 1982).

\section{f) Schlußfolgerung}

Anhand der Fruchtkörperbildung kann keine Aussage darüber getroffen werden, ob eine Art in der betreffenden Einheit diagnostischen Wert hat. Cladonia merochlorophaea beispielsweise eignet sich als Trennart für mehrere Einheiten, fruchtet aber nur in einer sehr häufig. Fruchtkörper weisen in der Regel auf besonders günstige Standortbedingungen für die jeweilige Art hin. Jedoch können bei Cl. merochlorophaea, wie auch bei anderen besprochenen Flechten (z.B. Cl. furcata ssp. furcata), die sterilen Thalli ebenfalls sehr vital sein. Cl. furcata ssp. subrangiformis ist ein Beispiel für eine Art, die innerhalb der Aufnahmeflächen kein einziges Mal fruchtend angetroffen wurde - trotzdem sind ihre mitunter sehr kräftigen sterilen Thalli kennzeichnend für Bestände der Federation Toninion sedifoliae.

Tab. 24: Stetigkeiten fruchtender Thalli und Gesamtstetigkeiten ausgewählter Arten

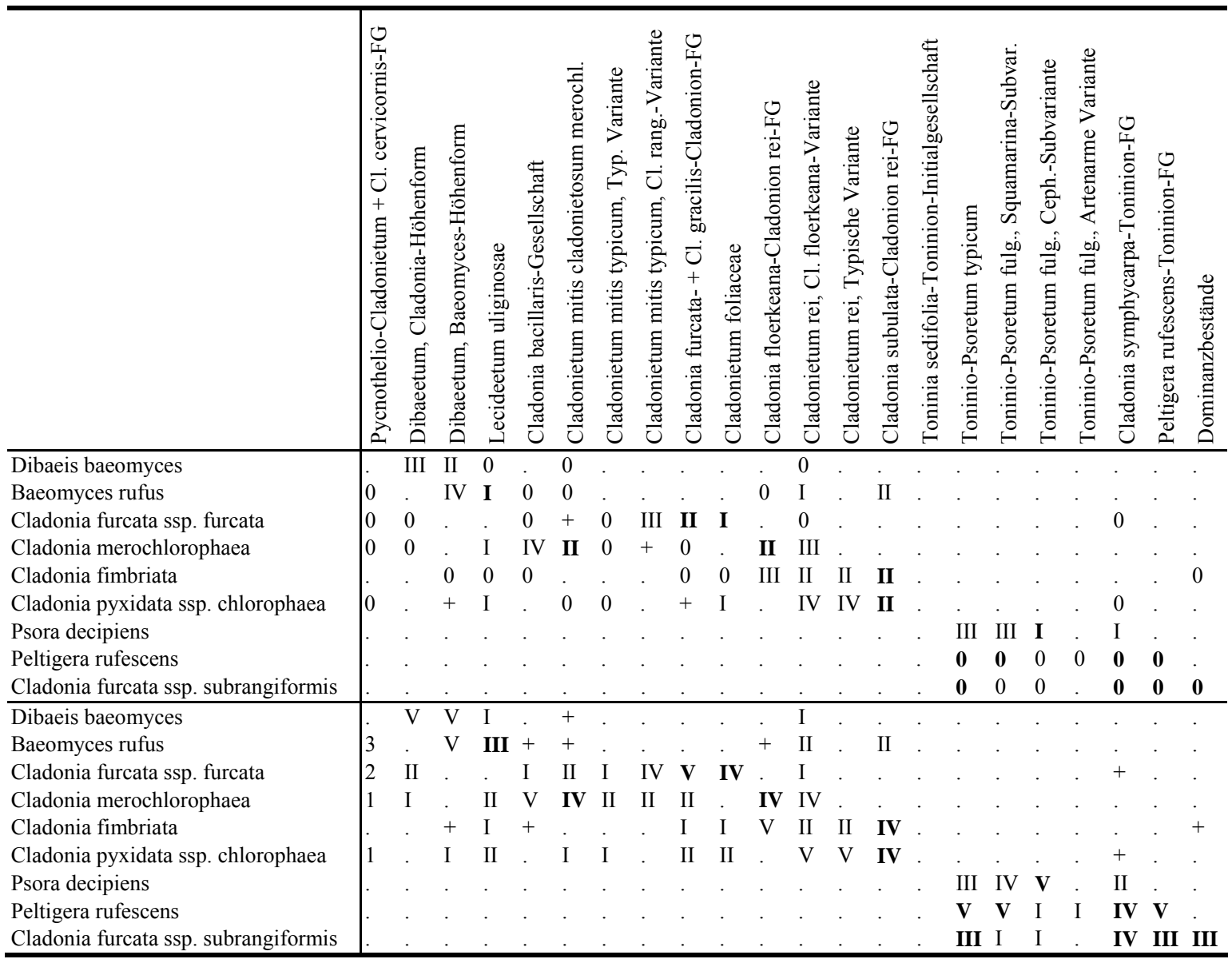

Oben: Stetigkeiten der fruchtenden Thalli. Unten: Gesamtstetigkeiten. Fett gedruckt sind besonders deutliche Stetigkeitsunterschiede zwischen der Gesamtstetigkeit und der Stetigkeit fruchtender Thalli. 
Tab. 25: Prozentualer Anteil der fruchtenden Thalli ausgewählter Arten

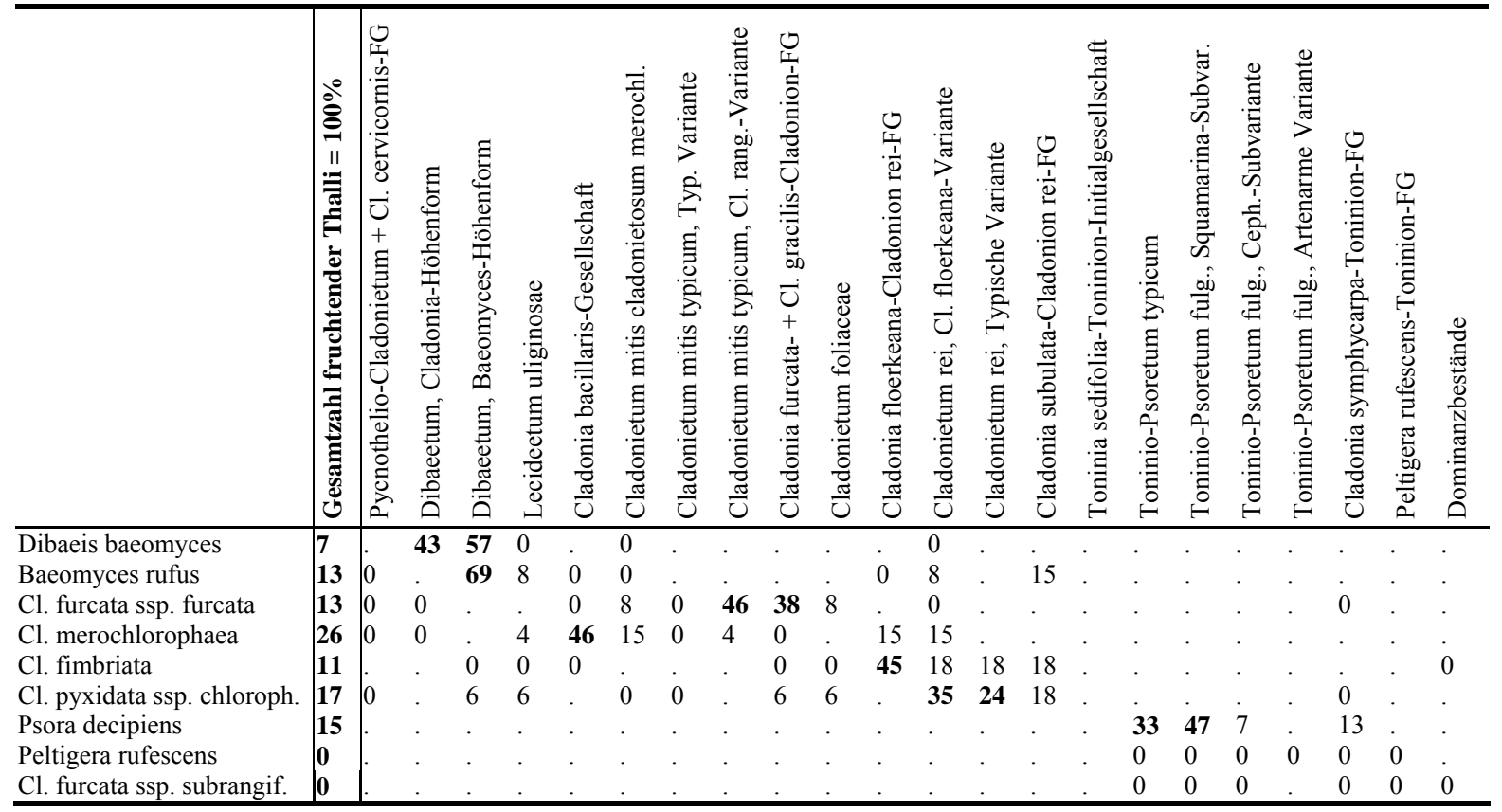

Tab. 26: Anteil fruchtender Thalli an den absoluten Stetigkeiten

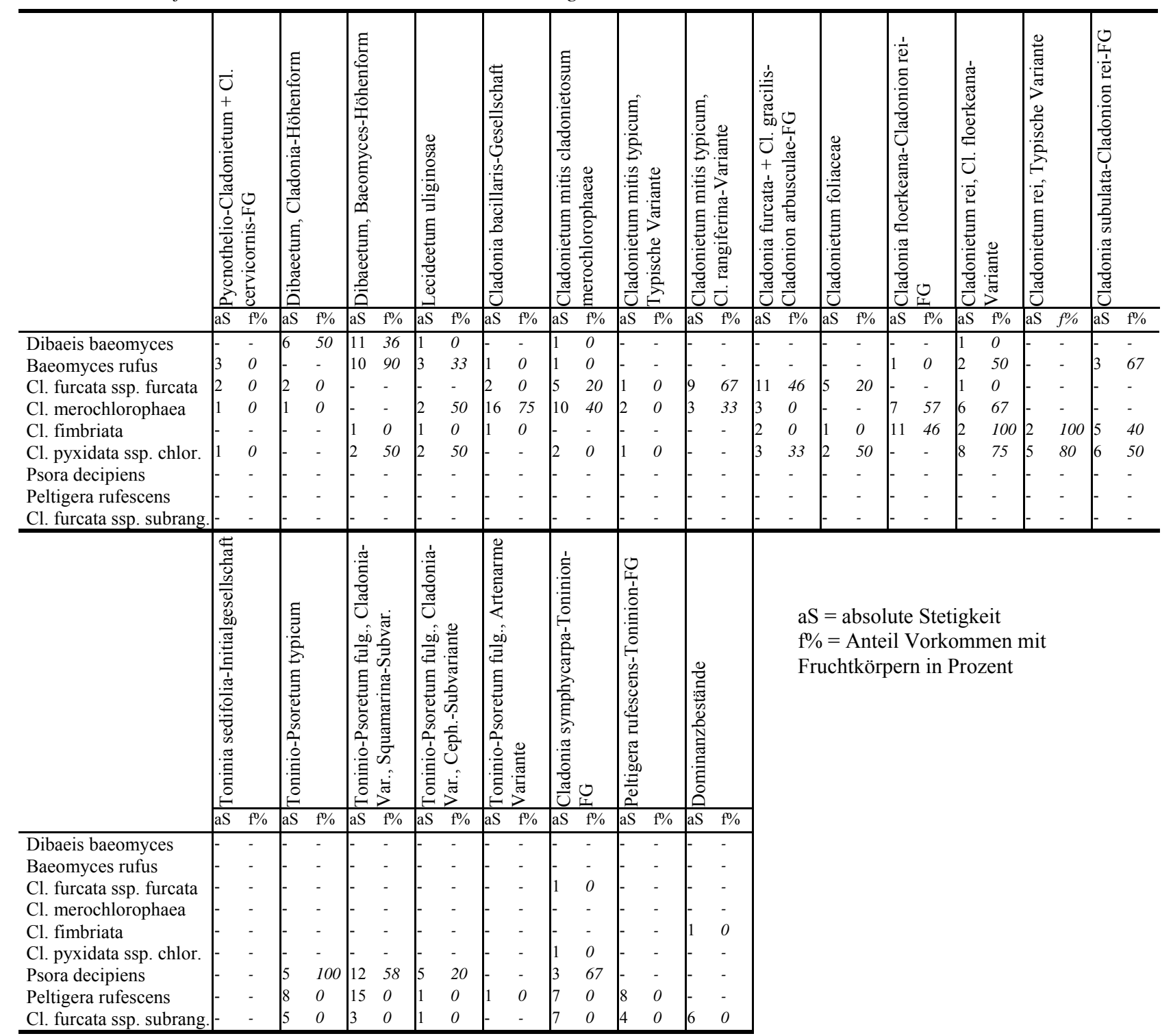




\section{Gefährdung der Arten und Gesellschaften sowie Schutzmaßnahmen}

\subsection{Rote Liste-Arten}

Im Untersuchungsgebiet wurden insgesamt 88 epigäische Flechtenarten bzw. Unterarten nachgewiesen, von denen über die Hälfte auf der Roten Liste von Deutschland bzw. von Hessen stehen (vgl. WIRTH et al. 1996). Neben typischen Bodenbewohnern zählen zu den gefundenen Arten auch zwei gewöhnlich epiphytisch wachsende Flechten, Hypogymnia physodes und Pseudevernia furfuracea, die nur ausnahmsweise auf Gesteinsbrocken oder Erdboden übergehen sowie 3 Arten (Cladonia coniocraea, Cl. digitata und Cl. polydactyla), die in der Regel eher auf morschem Holz, der Stammbasis von Bäumen oder Rohhumus anzutreffen sind als auf Erdboden.

\section{a) Rote Liste Deutschland (s. Tab. 27)}

39 der im Rahmen der vorliegenden Arbeit gefundenen Arten bzw. Unterarten werden in Gefährdungskategorien eingestuft. Dies entspricht einem Anteil von $44 \%$ an der Gesamtartenzahl. Davon gelten $3(=3 \%)$ als ,vom Aussterben bedroht“, 13 (=15\%) als „stark gefährdet" und $23(=26 \%)$ als ,gefährdet“". Bei weiteren 4 Arten wird eine Gefährdung angenommen, bei 2 Arten reicht die Datenlage für eine Bewertung nicht aus.

b) Rote Liste Hessen (s. Tab. 27)

46 Arten/Unterarten des Untersuchungsgebietes sind in Gefährdungskategorien eingestuft. Dies entspricht einem Anteil von $52 \%$ an der Gesamtartenzahl. Davon gelten $10(=11 \%)$ als ,vom Aussterben bedroht“, 15 (=17\%) als ,stark gefährdet" und $21(=24 \%)$ als ,gefährdet“. Bei 3 Arten wird eine Gefährdung angenommen, bei weiteren 3 Arten reicht die Datenlage für eine Bewertung nicht aus. Eine Art erhält den Vermerk ,extrem selten“.

\section{c) Vom Aussterben bedrohte Arten}

Bei den meisten der in Hessen vom Aussterben bedrohten Arten handelt es sich um Vertreter der Bunten Erdflechten-Gesellschaft (s. Kap. E2.2.1.2):

Buellia epigaea, eigentlich Charakterart dieser Gesellschaft, wurde nur in den Kalkmagerrasen bei Roßbach gefunden. Ein Vergleich mit KüMMERLING (1991) und BRUELHEIDE (1991) zeigt, daß die Art im Vergleich zu Anfang der 1990er Jahre zurückgegangen ist. Gleiches gilt auch für die beiden Fulgensia-Arten des Untersuchungsgebietes. KÜMMERLING (1991) konnte diese Krustenflechten noch an Wuchsorten nachweisen, an denen die Arten heute nicht mehr vorkommen. Mit Squamarina cartilaginea und Squamarina lentigera weisen zwei weitere Vertreter der Bunten Erdflechten-Gesellschaft den Gefährdungsgrad 1 auf. Beide kamen in früheren Jahren nach BEUG (1963-1989) und KÜMMERLING (1991) ebenfalls an mehr Lokalitäten 
vor als dies heute der Fall ist. Squamarina lentigera konnte im Jahre 2001 nur noch auf einem Dolomitfelsen am Ortsrand von Hergershausen nachgewiesen werden. Nach FOLLMANN (1974) scheint sie Anfang der 1970er Jahre am Aufbau der Bunten Erdflechten-Gesellschaft in Nordhessen beteiligt und demnach häufiger gewesen zu sein. Auch Psora decipiens ist wohl im Rückgang begriffen, wie ein Vergleich der heutigen Fundorte mit älterem Datenmaterial (KÜMMERLING 1991) zeigt. REIMERS (1951) bezeichnet diese Art als die empfindlichste der „Charakterflechten“ der Bunten Erdflechten-Gesellschaft, die in den Degenerationsstadien der Gesellschaft offensichtlich zuerst verschwindet.

Der Fund von Placidium pilosellum am Wenkeberg bei Frankershausen stellt den Erstnachweis der Art in Hessen dar (GÜNZL 2001b). Neben den deutlich bewimperten Thalli dieser Art treten am Wenkeberg auch die unbewimperten Schuppen von Placidium squamulosum auf. Letztere ist die deutlich häufigere der beiden PlacidiumArten, bleibt jedoch ebenfalls auf wenige basenreiche Standorte im Untersuchungsgebiet beschränkt. Nur an zwei Lokalitäten in Nordhessen wurde Solorina saccata entdeckt, sie siedelt dort auf beschatteten Zechstein-Felsen.

In diesem Zusammenhang ist auch Sarcosagium campestre zu nennen. In der Roten Liste Hessen wird der Art zwar kein Gefährdungsstatus zugeordnet, sie wird aber als „extrem selten“ eingestuft. Den Erstnachweis für Hessen erbrachte KÜMMERLING (1991) aus dem Meißner-Vorland. Im Rahmen der vorliegenden Untersuchungen wurde Sarcosagium im gesamten Untersuchungsgebiet nur an zwei Lokalitäten, jeweils in Gips-Steinbrüchen, gefunden. Detaillierte Angaben zur Verbreitung der genannten Arten basenreicher Standorte in Nordhessen finden sich bei GÜNZL (2001b).

Mit Cladonia crispata und Pycnothelia papillaria gehören außerdem zwei Erdflechten bodensaurer Standorte zu den vom Aussterben bedrohten Arten. Pycnothelia kommt in den Traubeneichenwäldern am Edersee und im Meißner-Vorland vor und auch Cladonia crispata zeigte sich im Meißner-Vorland, und zwar an den RoßkopfStandorten, am besten entwickelt.

Eine bryologische Besonderheit des Untersuchungsgebietes ist das Koboldmoos Buxbaumia aphylla, das in der Roten Liste Deutschlands mit Gefährdungsgrad 2 eingestuft wird. Für Hessen liegen keine Angaben zum Gefährdungsgrad vor. Es ist aber davon auszugehen, daß dieses kleinwüchsige Moos landesweit zu den seltenen Arten zählt. Aufgrund seiner geringen Größe wird es wohl oft übersehen und aus diesem Grund hier mit aufgeführt. Im Rahmen der vorliegenden Untersuchungen wurde es im Battenfelder Driescher (MTB/Q 4917/4) und in den Traubeneichenwäldern im MeißnerVorland angetroffen (MTB/Q 4725/1). 
Tab. 27: Rote Liste-Arten der Flechten

\begin{tabular}{|c|c|c|c|c|c|}
\hline & $B R D$ & $\mathrm{He}$ & & $B R D$ & $\mathrm{He}$ \\
\hline Arthrorhaphis citrinella & 3 & 2 & Cladonia scabriuscula & $\mathrm{G}$ & $\mathrm{G}$ \\
\hline Bacidia bagliettoana & 3 & 2 & Cladonia strepsilis & 3 & 2 \\
\hline Buellia epigaea & 1 & 1 & Cladonia symphycarpa & 3 & 3 \\
\hline Cetraria aculeata & 3 & 2 & Cladonia uncialis & 3 & $\mathrm{G} / 3^{1}$ \\
\hline Cetraria islandica & 3 & 2 & Collema cf.auriforme & $*$ & 3 \\
\hline Cetraria muricata & 3 & 2 & Dibaeis baeomyces & 2 & 2 \\
\hline Cladonia arbuscula & 3 & - & Diploschistes muscorum & 3 & 3 \\
\hline Cladonia arbuscula ssp. mitis & - & 3 & Endocarpon pusillum & 2 & 2 \\
\hline Cladonia arbuscula ssp. squarrosa & - & 3 & Fulgensia bracteata & 2 & 1 \\
\hline Cladonia cervicornis & 3 & - & Fulgensia fulgens & 2 & 1 \\
\hline Cladonia cervicornis ssp. cervicornis & - & 2 & Lepraria rigidula (cf.) & $*$ & $\mathrm{D}$ \\
\hline Cladonia cervicornis ssp. verticillata & - & 2 & Leptogium schraderi (cf.) & $\mathrm{D}$ & $\mathrm{G}$ \\
\hline Cladonia ciliata & 3 & 3 & Leptogium lichenoides & $\mathrm{G}$ & 3 \\
\hline Cladonia coccifera & $*$ & 3 & Micarea leprosula & $*$ & 3 \\
\hline Cladonia cornuta (cf.) & 2 & 2 & Micarea peliocarpa & 2 & 3 \\
\hline Cladonia crispata & 2 & 1 & Peltigera rufescens & 3 & 3 \\
\hline Cladonia foliacea & 3 & 2 & Placidium pilosellum & 1 & - \\
\hline Cladonia furcata ssp. subrangiformis & 3 & 3 & Placidium squamulosum & 3 & 1 \\
\hline Cladonia gracilis & 3 & 3 & Placynthiella oligotropha & 3 & 3 \\
\hline Cladonia humilis & $*$ & $\mathrm{D}$ & Placynthiella uliginosa & $\mathrm{D}$ & $\mathrm{G}$ \\
\hline Cladonia phyllophora & 3 & 3 & Psora decipiens & 2 & 1 \\
\hline Cladonia pleurota & $*$ & 3 & Pycnothelia papillaria & 2 & 1 \\
\hline Cladonia polydactyla & $*$ & 3 & Sarcosagium campestre & $\mathrm{G}$ & $\mathrm{R}$ \\
\hline Cladonia portentosa & 3 & 3 & Solorina saccata & 2 & 1 \\
\hline Cladonia pyxidata ssp. grayi & - & $\mathrm{D}$ & Squamarina cartilaginea & 2 & 1 \\
\hline Cladonia pyxidata ssp. pocillum & - & 3 & Squamarina lentigera & 1 & 1 \\
\hline Cladonia ramulosa & 3 & 3 & Toninia sedifolia & 3 & 2 \\
\hline Cladonia rangiferina & 2 & 2 & Trapeliopsis gelatinosa & 2 & 2 \\
\hline Cladonia rangiformis & 3 & $*$ & & & \\
\hline
\end{tabular}

Kategorien nach WIRTH et al. (1996): 1 = vom Aussterben bedroht; 2 = stark gefährdet;

3 = gefährdet; * = nicht als gefährdet angesehen; $\mathrm{G}=$ Gefährdung anzunehmen;

$\mathrm{D}=$ Daten mangelhaft; $\mathrm{R}=$ extrem selten; ${ }^{1} \mathrm{ssp}$. biuncialis/ssp. uncialis

\subsection{Bestandssituation der Flechtengesellschaften}

In Kap. E2 wird bei der Besprechung der einzelnen Gesellschaften teilweise bereits auf deren Bestandssituation eingegangen. Nachfolgend soll jedoch als Grundlage für die Besprechung der Schutzmaßnahmen in Kap. E6.3 nochmals ein zusammenfassender Überblick über die Häufigkeit und Schutzwürdigkeit der Flechtengesellschaften im Untersuchungsgebiet gegeben werden (Tab. 28). Die Einschätzung der Bestandssituation erfolgt auf der Datengrundlage der eigenen Vegetationsaufnahmen.

Da gut entwickelte Erdflechtenbestände in unserer Landschaft allgemein nicht häufig auftreten, sollten sie generell als schützenswert betrachtet werden, auch dann, wenn nur häufige Arten an ihrem Aufbau beteiligt sind. Einige Gesellschaften bzw. ihre Gesellschaftsbildner sind allerdings nicht nur im Untersuchungsgebiet, sondern auch in anderen Gebieten Deutschlands so selten, daß jedes ihrer Vorkommen unbedingt zu erhalten und zu schützen ist. 
Tab. 28: Bestandssituation der Erdflechtengesellschaften in Nordhessen

\begin{tabular}{|c|c|}
\hline Flechtengesellschaft & Bestandssituation \\
\hline Dibaeetum baeomycetis & Selten und nur an weni \\
\hline Lecideetum uliginosae & $\begin{array}{l}\text { Schutzwürdig auch aufgrund der Rote Liste-Arten. } \\
\text { Nur an wenigen Standorten erfaßt möglicherweise jedoch im }\end{array}$ \\
\hline & $\begin{array}{l}\text { Gebiet häufiger. Gelegentlich treten Rote Liste-Arten auf, } \\
\text { daher sollten die Bestände geschützt werden. }\end{array}$ \\
\hline Pycnothelio & Selten und schützenswert, besonders Bestände mit Pycnothelia. \\
\hline Cl. ce & \\
\hline Cl. bacillaris-Gesellschaft & $\begin{array}{l}\text { Wohl relativ verbreitet. Artenreiche Bestände sind jedoch } \\
\text { selten und daher schützenswert. }\end{array}$ \\
\hline Cladonietum & $\begin{array}{l}\text { Selten und meist reich an Rote Liste-Arten, daher sind alle } \\
\text { Bestände unbedingt zu schützen. }\end{array}$ \\
\hline Cl. furcata-Cladoni & Wohl häufiger, als durch Aufnahmematerial belegt. \\
\hline Cl. gracilis-Clado & Selten \\
\hline Cladon & Sehr s \\
\hline Cl. floerkeana-Cladonion rei-Fragmentgesellschaft & $\begin{array}{l}\text { Bestandssituation unklar, aber wohl häufiger, als durch } \\
\text { Aufnahmematerial belegt. }\end{array}$ \\
\hline Cladonietum rei & $\begin{array}{l}\text { Wohl häufiger, als durch Aufnahmematerial belegt, da } \\
\text { anthropogene Standorte unvollständig erfaßt. }\end{array}$ \\
\hline Cl. subulata-Cladonion rei-Fragmentgesellschaft & $\begin{array}{l}\text { Bestandssituation unklar, aber wohl häufiger, als durch } \\
\text { Aufnahmematerial belegt. }\end{array}$ \\
\hline Toninia sedi & Selten und schutzwürdig. \\
\hline Toninio-Psoretum decipier & $\begin{array}{l}\text { Selten und mit mehreren Rote Liste-Arten, daher sind alle } \\
\text { Vorkommen unbedingt zu schützen. }\end{array}$ \\
\hline Cl. symphycarpa-Toninion-Fragmentgesellsch & $\begin{array}{l}\text { Selten und mit mehreren Rote Liste-Arten, daher alle } \\
\text { Vorkommen unbedingt schützenswert. }\end{array}$ \\
\hline Peltigera rufescens-Toninion & Selten und aufgrund der Rote Liste-Arten schützenswert. \\
\hline
\end{tabular}

Keine der Flechtengesellschaften der Klasse Ceratodonto-Polytrichetea piliferi kann für Nordhessen als häufig eingestuft werden. Gut entwickelte und auf größerer Fläche ausgebildete Bestände, auch wenn sie von häufigen Arten aufgebaut werden, sind ziemlich selten. Relativ weit verbreitet über das Untersuchungsgebiet sind die Cladonia bacillaris-Gesellschaft, die Cladonia furcata-Cladonion arbusculae-Fragmentgesellschaft sowie das Cladonietum mitis, welches zugleich diejenige Vegetationseinheit in Nordhessen ist, die mit teilweise mehr als $50 \mathrm{~m}^{2}$ die größten Flächen einnimmt. Im Gegensatz dazu wurden andere Vegetationseinheiten nur in kleinen Teilen des Untersuchungsgebietes, die Baeomyces-Höhenform des Dibaeetum baeomycetis z.B. nur im nordwestlichsten Bereich angetroffen.

Alle Gesellschaften der Klasse Toninio-Psoretea decipientis gehören $\mathrm{zu}$ den Besonderheiten des Untersuchungsgebietes und werden von seltenen Arten aufgebaut. Daher sind alle derartigen Bestände schutzwürdig, auch die artenarmen Ausbildungen.

Abb. 20 läßt sich entnehmen, wieviele Erdflechtengesellschaften in welchem Meßtischblatt der TK 25 vorkommen. In die Auswertung einbezogen sind alle Gesellschaften mit Ausnahme der Dominanzbestände, da diese nicht immer vegetationskundlich erfaßt wurden. Ein Zentrum besonders hoher Gesellschaftsdiversität liegt im Gebiet des Hohen Meißners und seines östlichen Vorlandes. Besonders das MTB 4725 zeichnet sich durch eine außergewöhnlich hohe Zahl an Erdflechtengesellschaften aus. Dies ist zum einen bedingt durch die geologische Vielfalt dieses Gebietes und zum anderen durch eine hohe Zahl gut erhaltener, für Flechten geeigneter Biotope, wie etwa den Kalkmagerrasen bei Frankershausen. Die schönsten Flechtenbestände liegen innerhalb dieses Meßtischblattes in 
Naturschutzgebieten, z.B. im NSG „Kripplöcher und Hielöcher“, im NSG „Bühlchen bei Weißenbach“" und im NSG „Meißner“. Unterschiedliches Ausgangsgestein sowie Fels- und Magerstandorte sind beispielsweise auch für das MTB 4621 kennzeichnend. In den dort gelegenen Naturschutzgebieten „Festberg bei Philippinenthal“, „Burghasunger Berg“ und „Dörnberg“ finden sich ebenfalls bemerkenswerte Flechtenvorkommen. Allerdings haben die Flechten auf den Basaltköpfen des Burghasunger Berges durch Beweidung im Jahr 2002 schwere Trittschäden erlitten (trotzdem wird Beweidung, wie in Kap. E6.3.1 erläutert, generell als positiv angesehen). Auch in geologisch einheitlicheren Gegenden können mehrere Gesellschaften nebeneinander auftreten, so z.B. in den teilweise unter Naturschutz stehenden Hochheiden des Waldecker Uplands (MTB 4717/18), im NSG „Battenfelder Driescher“ (MTB 4917) oder in den Eichenwäldern am Edersee (MTB 4820). Diese Gebiete sind besonders reich an Erdflechtengesellschaften der Ceratodonto-Polytrichetea piliferi.

Eine geringe Zahl an Gesellschaften ist nicht immer gleichbedeutend mit einer schlechten Ausstattung an Flechtenbeständen, wie anhand der MTB 4825 und 4923/24 deutlich wird. Für alle diese MTB wurde zwar nur das Toninio-Psoretum decipientis belegt, welches jedoch in den genannten Regionen ungewöhnlich gut und flächendeckend entwickelt ist. Wuchsorte sind aufgelassene Steinbrüche. Über Maßnahmen zum Schutz dieser Bestände gibt Kap. E6.3.3 Auskunft.

Der mittlere Teil Nordhessens ist verhältnismäßig arm an Flechtenstandorten wie Magerrasen oder Felsen, dementsprechend zeigen sich Lücken in der Karte zur Gesellschaftsdiversität.

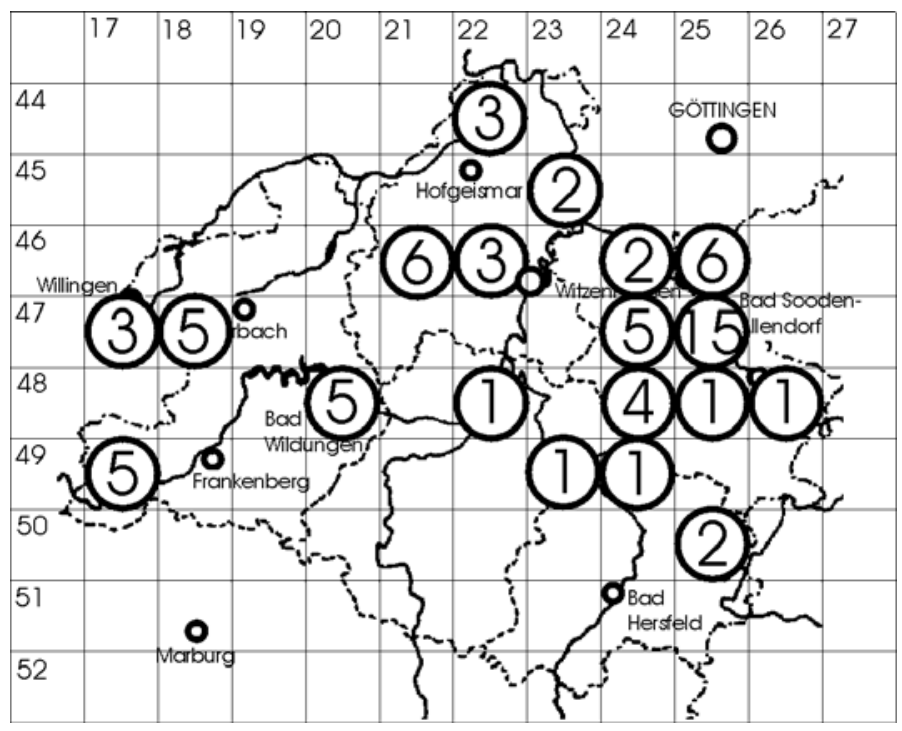

Abb. 26: Gesellschaftsdiversität im Untersuchungsgebiet. Angegeben ist die Zahl der Erdflechtengesellschaften innerhalb der einzelnen MTB.

\subsection{Schutzmaßnahmen}

Flechtenreiche Biotope verdienen generell besondere Beachtung, um gefährdete Arten und Lebensgemeinschaften zu schützen. Mit Hilfe sogenannter Indikatorarten können wertvolle Biotope mit weiteren gefährdeten Flechtenarten ermittelt werden (WIRTH 2002). Rentierflechten (Cladonia arbuscula, Cl. rangiferina) und das Isländisch Moos 
(Cetraria islandica) sowie die an offenen Stellen siedelnden Arten Dibaeis baeomyces und Pycnothelia papillaria weisen beispielsweise auf schützenswerte Magerrasen hin. Darüber hinaus zeigen diese Arten, mit Ausnahme von Cetraria islandica, in Nordhessen auch Traubeneichenwälder von besonderem Wert an. Die auffallenden Bunten Erdflechten (Fulgensia spec., Psora decipiens, Toninia sedifolia) sowie Placidium squamulosum gelten als Indikatoren für wertvolle Kalkmagerrasen (WIRTH 2002), deren Erhalt nicht nur für den Flechtenartenschutz von großer Bedeutung ist. Nachfolgend werden Maßnahmen zum Erhalt solcher flechtenreicher Biotope aufgeführt.

\subsubsection{Erdflechten in Kalkmagerrasen und auf Felsen}

Im Untersuchungsgebiet stellt die Aufgabe der traditionellen Nutzungsweisen und der damit verbundene Rückgang offener Bodenstellen infolge von Sukzessionsprozessen momentan die größte Gefahr für die Flechten der Magerrasen dar. Auch eine Nutzungsintensivierung oder Aufforstungsmaßnahmen haben bereits zum Verlust vieler Erdflechtenstandorte in Magerrasen beigetragen (vgl. NitSCHE \& BULTMANN 1995).

Für das Gedeihen der Bunten-Erdflechten-Gesellschaft ist das Vorhandensein offener Stellen zwingend erforderlich, welche beispielsweise durch eine gesteuerte Beweidung mit Schafen geschaffen werden (s. BöHLE \& HALFMANN 1992). Auch MARSTALLER (1968) unterstreicht den günstigen Einfluß einer Beweidung auf diese Flechtengesellschaft. Bei längerem Ausbleiben der Beweidung wird die BunteErdflechten-Gesellschaft dagegen von Moosen und Blütenpflanzen überwachsen. Nur wenige Arten, wie beispielsweise Cladonia pyxidata ssp. pocillum, können dieser Entwicklung noch einige Zeit standhalten. Ein positives Beispiel zur Erhaltung von Magerrasen ist das vom Forstamt Bad Sooden-Allendorf und dem Naturpark Meißner erarbeitete Beweidungskonzept, das in Zusammenarbeit mit einem Schäfereibetrieb, Verbänden und Institutionen auf dem Meißner und in seinem Vorland umgesetzt wird. $\mathrm{Zu}$ dem auf diese Weise entstandenen Biotopverbund zählen einige der schönsten flechtenreichen Kalkmagerrasen des Untersuchungsgebietes (z.B. das NSG „Kripplöcher und Hielöcher“ und das NSG „Bühlchen bei Weißenbach“), die in der traditionellen stationären Hütehaltung bewirtschaftet werden (LENARDUZZI 1999a/b). Die krustigen Erdflechten sind erstaunlich trittfest (s. auch FOLLMANN 1974) und scheinen die Schafbeweidung und die damit verbundene Trittbelastung gut $\mathrm{zu}$ überstehen. Ausführlicher über Gefährdung und Schutz der Arten basenreicher Standorte im Untersuchungsgebiet berichtet GÜNZL (2001b).

Eine extrem starke Trittbelastung durch Spaziergänger bzw. Kletterer, z.B. auf den Gipsfelsen des Wenkebergs bei Frankershausen, schadet dagegen dem Flechtenbewuchs, da beim Klettern auf den Felsen der verwitterte Gips samt den darauf siedelnden Flechtenlager abgeschürft wird. Einige weitere Flechtenwuchsorte auf Felsen innerhalb der Magerrasenflächen gingen durch fortschreitende Sukzession und die damit verbundene Verbuschung (z.B. NSG „Jestädter Weinberg“) oder durch Wiederbewaldung (Dolomitfelsen bei Hollstein) verloren. Zum Schutz der Flechten wäre es daher vorteilhaft, die Trittbelastung auf den Gipsfelsen zu reduzieren sowie das 
Verbuschen der Felsen und ihrer Umgebung zu verhindern. Dies gilt im Übrigen nicht nur für Felsen aus Gips und Muschelkalk, sondern für alle Felsen bzw. Felswände, die von Flechten besiedelt werden. Auch das Cladonietum foliaceae ist im Untersuchungsgebiet beispielsweise auf sonnige, offene Felsen aus Diabas oder Basalt angewiesen.

\subsubsection{Erdflechten in Zwergstrauchheiden}

Für die in Zwergstrauchheiden basenarmer Standorte wachsenden Erdflechten sind offenen Stellen innerhalb der Phanerogamenvegetation ebenfalls unerläßlich. In älteren geschlossenen Heiden gehen Flechtengemeinschaften stark zurück und werden von Moosen wie Pleurozium schreberi, Dicranum scoparium und Hypnum jutlandicum verdrängt (MÜLLER et al. 1997).

Heidegesellschaften lassen sich in der Regel nur durch gezielte Pflegemaßnahmen erhalten, die sich an den ehemaligen Nutzungsformen orientieren. Die Sukzession bei Aufgabe der Nutzung verläuft in Richtung Wald, der ursprünglich die meisten Heidestandorte bedeckte. Der Sukzessionsdruck ist je nach Standort unterschiedlich stark ausgeprägt. So können z.B. die Hochheiden im Upland an den windexponierten Bergkuppen mittelfristig ohne Pflege gedeihen, sofern im weiteren Umkreis durch Landnutzung die Gehölze zurückgedrängt werden. Zur Aushagerung trägt der Wind bei, der auf den exponierten Kuppen die Streu wegbläst (NITSCHE 1995). Calluna vulgaris als typische Art der Heiden kommt in Nordhessen auf fast allen geologischen Formationen vor. Der niedrige Nährstoffgehalt der Standorte ermöglicht es der Besenheide, sich gegen Konkurrenzarten durchzusetzen. Daher ist der Nährstoffentzug durch sinnvoll gesteuerte Mahd oder Beweidung für die Heide die wichtigste Pflegemaßnahme (NITSCHE 1995).

Wie in Kap. E2.1.1.1 bereits dargestellt, wirkt sich in den Zwergstrauchheiden das Abplaggen förderlich auf das Ansiedeln von Pionier-Flechtengesellschaften wie dem Dibaeetum baeomycetis aus (s. auch BIERMANN et al. 1994). Dies läßt sich in den Hochheiden des Waldecker Uplands (Kahle Pön und Osterkopf bei Usseln) besonders gut beobachten. Bereiche, an denen offenen Stellen und Arten wie Dibaeis baeomyces oder Pycnothelia papillaria bereits vorkommen, sollten als Tabuzonen bei der weiteren Heidepflege gelten (s. BIERMANN et al. 1994). Vom kleinräumigen Abplaggen profitieren nach PAUS (1997) auch fortgeschrittenere Stadien der Flechtensukzession, die Cladonia bacillaris-Gesellschaft und das Cladonietum mitis. Das Abplaggen ist besonders in der Degenerationsphase der Heide (nach mehr als rund $16 \mathrm{~J}$. Bestandsentwicklung) zur Verjüngung der Flächen sinnvoll (EIGNER 1991). Aus Kostengründen ist es heute aber nicht mehr großflächig durchführbar. Kostengünstiger ist der Schnitt der Besenheide, z.B. mit Hilfe eines Kreiselmähers (NITSCHE 1995, s. a. BIERMANN et al. 1994).

Pionierstadien sind im NSG „Battenfelder Driescher“, eventuell aufgrund des Mangels an geeigneten offenen Bodenstellen, eher selten. Das Cladonietum mitis ist dort dagegen bemerkenswert gut entwickelt und wird durch die stattfindende Beweidung sicherlich profitieren. Eine Schafbeweidung kann auch in den Magerrasen und Heiden 
bodensaurer Standorte für die Erdflechtenflora von großem Nutzen sein, da sie Lücken in den geschlossenen Vegetationsdecken (z.B. aus Calluna vulgaris) entstehen läßt und auf diese Weise neue, geeignete Lebensräume für Flechten schafft. STOKER \& DE SMIDT (1985) stellten eine Erhöhung der Artenzahl und der Deckung der Flechten auf beweideten Heideflächen fest. Damit neu entstandene Störstellen jedoch besiedelt werden können, müssen in der Nähe Flechtenbestände vorhanden sein, von denen aus eine Neubesiedlung erfolgen kann. Dies ist im NSG „Battenfelder Driescher“ beispielsweise der Fall. Die dort durchgeführte Schafbeweidung ist daher prinzipiell als positiv für den Erhalt der Flechtenvegetation $\mathrm{zu}$ bewerten. Jedoch scheint im Battenfelder Driescher die Trittbelastung durch die Schafe auf manchen mit Rentierflechten bewachsenen Kuppen sehr hoch, vielleicht auch zu hoch zu sein, mit der Folge, daß die Flechtenthalli vollständig zertreten werden. Die Beweidung sollte sich daher zunächst auf Flächen mit geschlossener Calluna-Bedeckung konzentrieren, Flächen mit Rentierflechtenbewuchs dagegen zumindest solange geschont werden, bis weitere potentielle Flechtenstandorte geschaffen wurden. BIERMANN et al. (1994) schreiben zum Thema Beweidung, daß beim Einsatz von Schafen die optimale Weidedichte (1-3 Tiere pro ha nach BEYER 1968 bzw. TOEPFER 1971) zu beachten ist, da besonders junge Heide empfindlich auf Überweidung reagiert. Die Schafe sollen zudem möglichst nicht über Nacht auf der Heide bleiben. Schafbeweidung (ebenso wie Mahd) erweist sich besonders in der Aufbau- und Optimalphase der Heideentwicklung als sinnvoll (EIGNER 1991).

Als weitere Pflegemaßnahme zur Heideverjüngung nennen MüLlER et al. (1997) das Abbrennen, dessen Auswirkungen ich in den nordhessischen Heideflächen jedoch nie selbst beobachten konnte. Flechten gelten als feuergefährdete Organismen, allerdings zeigte sich auf den Dauerflächen von MüLLER et al. (1997), daß nach einem Brand viele angekohlte Grundschuppen zurückbleiben, aus denen sich innerhalb eines Jahres bereits wieder Fruchtkörper entwickeln können. Bedeutsam ist, daß mit der Zerstörung einer dichten Phanerogamenschicht auch Flechtenstandorte neu geschaffen werden können. Wichtig für den Einsatz von Brand als Pflegemaßnahme ist der Erhalt flechtenreicher Populationen in der Umgebung gebrannter Flächen, aus denen eine Einwanderung in gebrannte Bereiche erfolgen kann. Bleiben auf Brandflächen Teile des Flechtenlagers erhalten, so entwickeln sich aus diesen Thallusfragmenten sogenannte Regenerationsthalli. Diese wiederum können fruchtende Podetien ausbilden. Bei einer vollständigen Zerstörung der Flechtenthalli und Diasporen müssen die Flechten entweder durch Sporen, Bruchstücke oder Soredien neu einwandern. Flechtenbruchstücke können nur kurze Distanzen zurücklegen, Sporen aus Fruchtkörpern längere Distanzen. Am leichtesten kann eine Wiederbesiedlung durch Soredien geschehen. So findet man häufig auf gebrannten Heiden sorediöse Arten wie Cladonia pleurota oder Cl. subulata. Auch Pionierarten wie Placynthiella uliginosa können sich nach einem Brand schnell wieder etablieren, da sie, dicht mit dem Substrat verbunden, auch extreme Trockenheit während der Pionierphase nach Brand tolerieren oder den Brand sogar überdauern können. Arten wie Cladonia portentosa, die sich über Bruchstücke verbreiten, sind bei totaler Zerstörung der Flechtenthalli erst später zu erwarten. Entscheidend für die gute Entwicklung der Bodenflechten auf Pionier- und 
Brandstandorten sind die anfänglich fehlende Selektion durch Lichtkonkurrenz der erst später in der Sukzession dominierenden Höheren Pflanzen (MüLLER et al. 1997). Generell sind jedoch vor dem Brennen die positiven Aspekte mit eventuellen negativen Folgen für andere Lebewesen (z.B. Insekten) abzuwägen.

Um Heideflächen als Mosaik vieler verschiedener Kleinlebensräume zu erhalten bzw. zu entwickeln, wäre es generell in den Heiden sinnvoll, nicht alle Flächen in gleichem Maße durch Tritt und Beweidung zu beeinflussen. Die wenigen Standorte, an denen aktuell in Heiden Pionier- und Folgegesellschaften vorkommen, sollten unbedingt vor Zerstörung jeglicher Art bewahrt werden. Dies bedeutet z.B. auch, Wegränder und sonstige Erdanrisse vor dem Befahren mit Arbeitsmaschinen, vor übermäßigem Tritt sowie vor der Zerstörung z.B. durch das Ablagern von Baumstämmen zu schützen.

\subsubsection{Erdflechten in Steinbrüchen}

Aufgelassene Steinbrüche stellen als vom Menschen geschaffene Felsbiotope eine wichtige Ergänzung der natürlichen Wuchsorte der Erdflechten dar. Besonders die Gips- und Dolomit-Steinbrüche sind mitunter reich an seltenen Arten, aber auch in den Grauwacke-Steinbrüchen des Untersuchungsgebietes lassen sich zahlreiche Erdflechten finden. Leider sind einige kleinere Steinbrüche im Zuge fortschreitender Sukzession stark zugewachsen und als Flechtenwuchsort somit untauglich geworden. Andere, noch wenig verbuschte Gips-Steinbrüche, z.B. das Steinröllchen bei Oberhone, werden als Freizeitgelände genutzt, in welchem die Besucher zwar durch ihren Tritt die Flächen offen halten, leider aber auch Feuerstellen und vor allem reichlich Müll zurücklassen. Im Extremfall werden die Steinbrüche gar als Müllkippen mißbraucht, beispielsweise um Gartenabfälle oder Sperrmüll zu entsorgen. Zum Schutz der Arten ist es daher notwendig, dafür zu sorgen, daß die Steinbrüche nicht verfüllt oder durch Freizeitbetrieb übermäßig stark beansprucht, sondern als wertvolle Felsstandorte und somit Lebensraum, nicht nur für Flechten, betrachtet und erhalten werden.

\subsubsection{Erdflechten in Wäldern}

Erdflechtenreiche Wälder treten in Nordhessen, sieht man von den Flechtenvorkommen an Wegrändern und auf Schneisen innerhalb des Waldes ab, im Meißner-Vorland und am Edersee auf. Es handelt sich um ehemalige Eichen-Niederwälder an Steilhängen und Kuppen über Grauwacke und Tonschiefer. Die Wälder sind aufgrund ihrer ungünstigen Standortverhältnisse heute forstwirtschaftlich uninteressant. In absehbarer Zeit ist eine Veränderung der Bestandesstruktur (ohne menschlichen Einfluß) nicht zu erwarten. Allenfalls der stellenweise randlich in die Bestände eindringende Buchenjungwuchs ist $\mathrm{zu}$ beobachten und gegebenenfalls zu entfernen. Außerdem sollten Beeinträchtigungen der nahe der Wege wachsenden Flechten, nicht nur in diesem Waldtyp, in jedem Fall vermieden werden. Weitere besondere Schutzmaßnahmen für die dortigen Erdflechten sind zum jetzigen Zeitpunkt nicht erforderlich. 


\section{Zusammenfassung}

Im Untersuchungsgebiet konnten insgesamt 16 Erdflechtengesellschaften und 4 Dominanzbestände aus zwei Vegetationsklassen nachgewiesen werden. Ein Großteil dieser Gesellschaften ist als ziemlich bis sehr selten und daher als schützenswert einzustufen. Geeignete Schutzmaßnahmen werden angegeben.

Auf basenreichen Substraten sind Gesellschaften der Klasse Toninio-Psoretea decipientis anzutreffen. Sie werden in der Regel von Krustenflechten dominiert. Einzige Federation innerhalb dieser Klasse ist das Toninion sedifoliae. Die artenreichsten Bestände sind der Union Toninio-Psoretum decipientis („Bunte-ErdflechtenGesellschaft") zuzuordnen, welche durch Fulgensia bracteata und F. fulgens charakterisiert wird. F. fulgens ist gleichzeitig Trennart der Subunion fulgensietosum. Seltene Erdflechtenarten treten jedoch auch in den Toninion-Fragmentgesellschaften auf. Relativ artenarm, jedoch floristisch dennoch wertvoll, sind dagegen die diversen Dominanzbestände. Gesellschaften der Toninio-Psoretea siedeln v.a. innerhalb von lückigen Kalkmagerrasen (Gentiano-Koelerietum), auf Felsköpfen und in Sekundärbiotopen wie z.B. aufgelassenen Gips-Steinbrüchen.

Die Erdflechtenvegetation kalkarmer bzw. kalkfreier Standorte gehört der Klasse Ceratodonto-Polytrichetea piliferi an. Diese wird in 3 Federationen unterteilt. Die Federation Baeomycion rosei umfaßt Pioniergesellschaften wie das Dibaeetum baeomycetis, welches besonders gut auf abgeplaggten Heideflächen der niederschlagsreichen Berglagen gedeiht. Rentierflechtenbestände (Cladonietum mitis) innerhalb von Heiden und Eichenwäldern sind in die Federation Cladonion arbusculae einzuordnen, der als intermediäre Entwicklungsstadien auch die von stiftförmigen, sorediösen Cladonien geprägten Gesellschaften ( $C l$. bacillaris-Ges.) angehören. Das ebenfalls hierzu zählende Cladonietum foliaceae siedelt auf vulkanischen Felsen und stellt eine Besonderheit in Nordhessen dar. Gesellschaften der Federation Cladonion rei sind bevorzugt auf Ruderalstandorten wie z.B. Bergbauflächen zu finden.

Cl. furcata ssp. furcata zeigt ihren Verbreitungsschwerpunkt im Cladonion arbusculae. Mit hoher Stetigkeit tritt sie dort in der Typischen Subunion des Cladonietum mitis, in der von ihr dominierten Cladonia furcata-Cladonion arbusculae-Fragmentgesellschaft und im Cladonietum foliaceae auf. Fruchtende Podetien zeigen sich jedoch nur in den beiden erstgenannten Einheiten. Darüber hinaus kommt diese Unterart auch im Baeomycion und im Cladonion rei vor, jedoch mit deutlich geringerer Stetigkeit. Nur ausnahmsweise siedelt Cl. furcata ssp. furcata in Beständen des Toninion. Dagegen bleibt $\boldsymbol{C l}$. furcata ssp. subrangiformis in ihrem Auftreten auf die Klasse ToninioPsoretea beschränkt. Sie ist dort hauptsächlich in den Cladonien-dominierten Stadien des Toninio-Psoretum und in den Toninion-Fragmentgesellschaften anzutreffen. Bisweilen bildet sie Dominanzbestände. Fruchtende Thalli wurden nur sehr selten beobachtet. 


\section{F Chemismus ausgewählter Cladonia-Arten}

Etliche Cladonien lassen sich aufgrund eines bestimmten Inhaltsstoffes bzw. einer bestimmten Stoffkombination eindeutig auf Art- bzw. Unterartebene bestimmen (s. dazu z.B. HuOvinen et al. 1990, PAus 1993/1997, WIRTH 1995). Bei einigen Cladonia-Arten treten jedoch innerhalb einer Unterart verschiedene Chemosippen (Chemotypen) auf, so beispielsweise bei Cladonia pyxidata ssp. grayi (s. Tab. 29). Diese Fälle sowie weitere interessante Ergebnisse der Dünnschichtchromatographie (DC) sollen nachfolgend erläutert und unter anderem mit den Ergebnissen von KÜMMERLING (1991) verglichen werden, deren Studie Proben aus einem Teil des Untersuchungsgebietes beinhaltet. Tab. 30 gibt Auskunft über Inhaltsstoffe und Probenzahl der im Rahmen dieser Arbeit untersuchten Flechtenarten.

Die Ergebnisse der DC wurden auch in Kap. E eingearbeitet, da sich beispielsweise die Chemosippen von Cl. pyxidata ssp. grayi teilweise als „Diagnostische Sippen“ bestimmter Vegetationseinheiten eignen. Darüber hinaus sind diese Resultate für die in Kap. I2 diskutierte Fragestellung über die Artberechtigung von Cl. subrangiformis sowie für die allgemeine Kenntnis der Verbreitung von Chemotypen innerhalb der Gattung Cladonia von Bedeutung (vgl. FISCHER \& GÜNZL 2004).

\section{Methodik zur Identifizierung der Flechtenstoffe}

Zur genauen Bestimmung und zum Nachweis etwaiger Chemotypen, wurden kritische Flechten-Exemplare aus den Vegetationsaufnahmeflächen sowie alle Cladonia furcataProben dünnschichtchromatographisch auf charakteristische Inhaltsstoffe hin untersucht (Methode nach CULBERSON \& AMMANN 1979). Bei den bestimmungskritischen Arten handelte es sich dabei in erster Linie um Exemplare von Cl. pyxidata sowie um die Artengruppe Cl. subulata/rei/glauca.

In den Podetienspitzen ist die Konzentration der Flechtensäuren am höchsten (CULBERSON 1969). Für die Untersuchungen wurden daher wenige Millimeter große Thallusbruchstücke an den Podetienenden bzw. im Bereich der Becher entnommen. Jedes Cl. furcata-Polster wurde an mindestens 3 Stellen beprobt. Die Thallusbruchstücke für die rDNA-Sequenzierung und die Proben für die DC entstammen bei Cladonia furcata unmittelbar benachbarten Podetienspitzen (wenn möglich vom selben Podetium). Die Thallusproben wurden in Eppendorf-Gefäßen mit einigen Tropfen Aceton extrahiert und der Extrakt mit Glaskapillaren (HaematokritKapillaren, $74 \mathrm{~mm} / 60 \mu \mathrm{l}$, Fa. Hirschmann Laborgeräte) auf die nicht weiter vorbehandelten DC-Platten aufgetragen. Zum Einsatz kamen $20 \times 20 \mathrm{~cm}$ große Kieselgelplatten der Fa. Merck (Merck Silica Gel 60 F 254, Schichtdicke 0,25 mm) bzw. der Fa. Macherey-Nagel (ALUGRAM SIL G/UV 254 , Schichtdicke 0,20 mm). Als interne Kontrollsubstanz diente ein Extrakt aus Cladonia symphycarpa, der Atranorin und Norstictinsäure enthält und an zwei Startpunkten aufgetragen wurde. Beim 
Laufmittel handelte es sich um das sogenannte Standardlaufmittel A (s. KÜMMERLING 1991), einem Gemisch aus Toluol, Dioxan und Eisessig (90:22,5:2,5 ml). Die Platten wurden bis zu einer Laufhöhe von $12 \mathrm{~cm}$ entwickelt. Auf den lufttrockenen Platten wurden zunächst unter Bestrahlung mit kurzwelligem UV-Licht (254 nm) alle Substanzen markiert, die in diesem Wellenbereich absorbieren und als dunkle, nicht fluoreszierende Banden erkennbar sind. Anschließendes Besprühen der Platten mit Wasser und vorsichtiges Fönen machte während des Trocknens lipophile Stoffe (z.B. die Fettsäure Rangiformsäure) als weißliche Flecken sichtbar. Zur Entwicklung des Chromatogramms wurden die Platten mit $10 \% \mathrm{H}_{2} \mathrm{SO}_{4}$ besprüht und bei $110{ }^{\circ} \mathrm{C}$ ca. 20 min. lang getrocknet.

Die Einteilung in Rf-Klassen richtete sich nach CULBERSON \& KRISTINSSON (1970). Die Identifikation der Flechtenstoffe erfolgte mit Hilfe bisher publizierter Inhaltsstoffanalysen (z.B. WALKER \& JAMES 1980, HoliEN \& TøNSBERG 1985, PAUS 1997). Insgesamt wurden 680 Cladonia-Proben analysiert.

\section{Ergebnisse der Dünnschichtchromatographie}

\section{Cladonia arbuscula:}

Anhand ihrer Flechtensäuren können die Proben in die ssp. squarrosa (Wallr.) Ruoss (mit Usninsäure und Fumarprotocetrarsäure) und die ssp. mitis (Sandst.) Ruoss (mit Usninsäure und Rangiformsäure) unterteilt werden. KÜMMERLING (1991) wies in ihren Cladonia arbuscula ssp. mitis zugeordneten Proben auch Fumarprotocetrarsäure nach, weist jedoch darauf hin, daß diese dem in RUOSS (1987) aufgeführten, sog. „,intermediären“ Chemotyp angehören, der sowohl den Fumarprotocetrarsäure-Komplex als auch den Rangiformsäure-Komplex enthält.

\section{Cladonia furcata:}

Die Unterteilung von Cladonia furcata in die zwei Unterarten furcata und subrangiformis erfolgt bei WIRTH (1995) in erster Linie anhand des Vorhandenseins oder Fehlens von Atranorin. Zusätzlich treten bei der Subspezies subrangiformis häufig weiß aufbrechende Beulen (Markaufbrüche) auf.

\section{Cladonia furcata ssp. furcata:}

Unter den von mir untersuchten nordhessischen Proben finden sich lediglich zwei, die außer Fumarprotocetrarsäure einen weiteren, als Bande sichtbaren, allerdings mittels DC nicht näher identifizierbaren Inhaltsstoff enthalten (Chemotyp II in Tab. 30). Die bisher bekannten, nachfolgend beschriebenen Chemotypen von Cl. furcata ssp. furcata kommen nicht vor.

Nach PAUS (1997) treten in der Unterart furcata als Hauptinhaltsstoff Fumarprotocetrarsäure sowie als Nebeninhaltsstoffe Confumarprotocetrar- und Protocetrarsäure auf. CULBERSON (1969) nennt für Cl. furcata ssp. furcata die Inhaltsstoffe \pm Atranorin, Fumarprotocetrarsäure, Ascorbinsäure und Zink. In Europa 
werden die Atranorin-haltigen Exemplare in der Regel Cl. furcata ssp. subrangiformis zugeordnet, Atranorin kann jedoch auch von der Cl. furcata ssp. furcata produziert werden. HUOVINEN et al. (1990) beispielsweise bezeichnen Atranorin als akzessorischen Inhaltsstoff der Subspezies furcata. Weiterhin ist von Cl. furcata ssp. furcata ein Chemotyp mit Psoromsäure bekannt. BARENDREGT et al. (1982) beschreiben ihn erstmals aus Portugal. Die schlanken, regelmäßig verzweigten Podetien dieses Chemotyps sollen der var. racemosa (=var. furcata, s. Kap. G2.1.1) entsprechen und wurden in offenen Heideflächen gefunden. Die Psoromsäure tritt in diesen Thalli anstelle von Fumarprotocetrarsäure oder Atranorin auf. FISCHER (2003) konnte im Rahmen ihrer vegetationsökologischen Untersuchungen an der Mittelelbe 3 verschiedene Psoromsäure-haltige Chemotypen nachweisen. Einer entspricht dem Chemotyp III von PAUs (1997) und enthält Atranorin und Psoromsäure, zwei weitere Chemotypen zeichnen sich durch die Inhaltsstoffe Psoromsäure und Fumarprotocetrarsäure bzw. ausschließlich Psoromsäure aus. Die Psoromsäure-haltigen Sippen wachsen in Trockenrasen des Spergulo-Corynephoretum sowie des DianthoArmerietum (vgl. FISCHER \& GüNZL 2004). Während BARENDREGT et al. (1982) und HuOvinEN et al. (1990) die Psoromsäure-haltigen Exemplare als der Cl. furcata zugehörig ansehen (und die ,subrangiformis“-Proben bei HUOVINEN et al. als Cl. subrangiformis abgetrennt werden), stellt PAUS (1997) den sogenannten Chemotyp III (mit Atranorin und Psoromsäure) als dritte Gruppe neben die Unterarten furcata und subrangiformis. Sie führt Belegexemplare aus einem Bestand der Cladonia bacillaris(Mikro-)Gesellschaft an.

\section{Cladonia furcata ssp. subrangiformis:}

Im Material des Untersuchungsgebietes treten 7 Proben auf, bei denen außer Atranorin und Fumarprotocetrarsäure eine dritte, jedoch mittels DC nicht näher bestimmbare Bande vorhanden ist (Chemotyp II in Tab. 30).

Hauptinhaltsstoffe sind Atranorin und Fumarprotocetrarsäure, als Nebeninhaltsstoffe treten Confumarprotocetrar- und Protocetrarsäure auf (PAUS 1997). KÜMMERLING (1991) führt als weiteren gelegentlich vorhandenen Inhaltsstoff Bourgeansäure an und trennt die Bourgeansäure-haltigen Sippen als „Chemorasse b“ gegenüber den lediglich Fumarprotocetrarsäure und Atranorin aufweisenden Proben der „Chemorasse a“ ab. In Burgaz \& AHTI (1992) findet sich der Hinweis auf einen Psoromsäure-haltigen Chemotyp bei Cl. (furcata ssp.) subrangiformis.

\section{Cladonia macilenta:}

Nach neueren taxonomischen Vorschlägen werden Cl. bacillaris, Cl. floerkeana und Cl. macilenta unter $\mathrm{Cl}$. macilenta zusammengefaßt. Cl. bacillaris wird als Thamnolsäure-freie Chemosippe in die ssp. macilenta integriert (CHRISTENSEN 1987, WIRTH 1994). Innerhalb der eigenen nordhessischen Proben lassen sich die beiden Unterarten von Cl. macilenta im allgemeinen anhand morphologischer Merkmale (Sorediengröße, Berindung etc.) gut trennen (s. dazu WIRTH 1995). Durch die stichprobenhafte Analyse einiger Cl. macilenta-Proben können zusätzlich Aussagen 
über die im Untersuchungsgebiet vorhandenen Chemotypen innerhalb der Unterarten gemacht werden:

\section{Cladonia macilenta ssp. floerkeana:}

Bei der Unterart floerkeana wurde im Untersuchungsgebiet nur ein Chemotyp festgestellt. Er entspricht der bei KÜMMERLING (1991) aufgeführten „Chemorasse a“ mit ausschließlich Barbatsäure als Hauptinhaltsstoff. Die sog. „Chemorasse b“ mit Barbatsäure und Didymsäure (KÜMMERLING 1991) tritt unter den untersuchten Exemplaren nicht auf. Auch KÜMMERLING belegt die „Chemorasse b“ nur durch eine Probe.

PAUS (1997) weist darauf hin, daß alle von ihr aufgrund morphologischer Merkmale als ssp. floerkeana eingeordneten Exemplare keine Thamnolsäure enthielten und sich dadurch vom Chemotyp I der Unterart macilenta (s.u.) abgrenzten. Nach WIRTH (1995) kann allerdings auch die ssp. floerkeana \pm Thamnolsäure enthalten. Thamnolsäurehaltige Thalli finden sich unter den von mir untersuchten floerkeana-Proben jedoch nicht.

\section{Cladonia macilenta ssp. macilenta:}

Die analysierten Proben lassen sich in zwei Chemotypen unterteilen: Chemotyp I beinhaltet außer Barbatsäure zusätzlich Thamnolsäure und entspricht der „Chemorasse a“ von KüMmerling (1991) bzw. dem Chemotyp I von PAUS (1997). Zwei dieser Proben zeigen einen dritte, allerdings nur schwach entwickelte Bande, die möglicherweise durch Didymsäure verursacht wird. Didymsäure kann als akzessorischer Inhaltsstoff in beiden Unterarten vorkommen (PAUS 1997). Nach KÜMMERLING (1991) wären Didymsäure-haltige Proben von Cl. macilenta s.str. als „Chemorasse b“ einzustufen. Chemotyp II (Syn. Cl. bacillaris) zeichnet sich durch das Fehlen von Thamnolsäure aus (s. PAUS 1997) und ist daher nur aufgrund der feinstaubigen Podetien von der ssp. floerkeana zu trennen.

\section{Cladonia portentosa:}

Folgende Inhaltsstoffe treten nach KÜMMERLING (1991) in Cl. portentosa auf: Usninsäure, Perlatolsäure sowie eine unbekannte, nicht mit Ursolsäure identische Substanz. In zwei Proben des Untersuchungsgebietes konnte jedoch nur Perlatolsäure nachgewiesen werden. Diese Tatsache bestätigt wiederum die Aussage von PAUS (1997), welche Usninsäure lediglich als akzessorischen Inhaltsstoff bezeichnet.

\section{Cladonia pyxidata:}

Es wurden insgesamt 222 Proben von Cl. pyxidata analysiert, darunter die 3 Unterarten ssp. chlorophaea, ssp. grayi und ssp. pyxidata. Innerhalb der Subspezies grayi traten alle 4 bei WIRTH (1995) genannten Chemosippen auf: Cl. cryptochlorophaea, Cl. grayi s.str., Cl. merochlorophaea und Cl. novochlorophaea (s. Tab. 29). Da von jedem Cl. pyxidata-Fundort Proben zur Analyse eingesammelt wurden, gibt die Verteilung der Probenzahl auf die unterschiedlichen Unterarten bzw. Chemosippen zugleich auch Auskunft über deren Häufigkeit im Untersuchungsgebiet. 
Cl. pyxidata ssp. chlorophaea weist als einzigen mittels DC nachweisbaren Inhaltstoff Fumarprotocetrarsäure auf. Ebenso Cl. pyxidata s.str., die jedoch im Gebiet wesentlich seltener auftritt und nur durch 6 Proben nachgewiesen wurde, im Gegensatz zu 53 Proben von Cl. pyxidata ssp. chlorophaea. Im Chemismus zwar übereinstimmend, lassen sich beide jedoch anhand morphologischer Merkmale unterscheiden (s. WIRTH 1995). Die häufigste Chemosippe der Subspezies grayi ist der Chemotyp I von Cl. merochlorophaea mit Fumarprotocetrarsäure, Merochlorophaeasäure und 4-0Methylcryptochlorophaeasäure. Dem Chemotyp II fehlt dagegen die Fumarprotocetrarsäure. Dieser Chemotyp tritt im Untersuchungsgebiet extrem selten auf, er wurde nur einmal nachgewiesen, im Gegensatz zu 103 Nachweisen des Chemotyps I.

Bei Cl. novochlorophaea lassen sich ebenfalls zwei Chemotypen unterscheiden. Chemotyp I zeichnet sich durch Fumarprotocetrarsäure, Homosekikasäure und Sekikasäure aus. Dem Chemotyp II fehlt, vergleichbar dem Chemotyp II von Cl. merochlorophaea, die Fumarprotocetrarsäure. Auch bei Cl. novochlorophaea ist der Chemotyp II wesentlich seltener, nur eine von insgesamt 23 Proben ist ihm zuzuordnen.

Cl. cryptochlorophaea und $\boldsymbol{C l}$. grayi s.str. treten etwa gleich häufig auf und stellen $9 \%$ bzw. $7 \%$ aller untersuchten Cl. pyxidata-Proben. Cl. cryptochlorophaea enthält außer Fumarprotocetrarsäure zusätzlich Cryptochlorophaeasäure, Cl. grayi s.str. zusätzlich Grayansäure.

Tab. 29: Flechtensäuren der Unterarten und Chemosippen von Cladonia pyxidata

\begin{tabular}{|c|c|c|c|c|c|c|c|}
\hline & 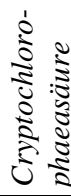 & 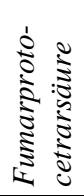 & 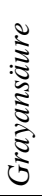 & 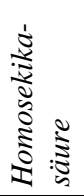 & 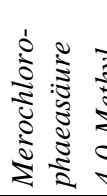 & 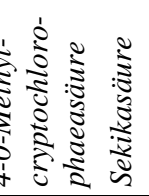 & 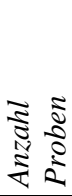 \\
\hline Cl. pyxidata ssp. chlorophaea & \multirow{8}{*}{$\mathrm{x}$} & $\bar{x}$ & & & & & 53 \\
\hline Cl. pyxidata ssp. pyxidata & & $\mathrm{x}$ & & & & & 6 \\
\hline Cl. cryptochlorophaea & & $\mathrm{x}$ & & & & & 20 \\
\hline Cl. grayi s.str. & & $\mathrm{x}$ & $\mathrm{x}$ & & & & 16 \\
\hline Cl. merochlorophaea, Chemotyp I & & $\mathrm{x}$ & & & $\mathrm{x}$ & $\mathrm{x}$ & 103 \\
\hline Cl. merochlorophaea, Chemotyp II & & \multirow{3}{*}{\multicolumn{2}{|c|}{$\mathrm{x}$}} & & $\mathrm{x}$ & $\mathrm{x}$ & 1 \\
\hline Cl. novochlorophaea, Chemotyp I & & & & $\mathrm{x}$ & & $\mathrm{x}$ & 22 \\
\hline Cl. novochlorophaea, Chemotyp II & & & & $\mathrm{x}$ & & $\mathrm{x}$ & 1 \\
\hline
\end{tabular}

\section{Cladonia rei:}

Da die Art in den erfaßten Flächen nur selten auftritt, liegen aus dem Untersuchungsgebiet lediglich Analyse-Ergebnisse einiger weniger Proben vor. Diese enthalten alle Homosekikasäure, Sekikasäure und Fumarprotocetrarsäure. Die SekikaBanden waren deutlich ausgebildet, obwohl diese Substanz nur in Spuren vorhanden sein soll (KüMMERLING 1991). Die nordhessischen Proben entsprechen somit alle dem Chemotyp I (PAUS 1997), bei dem das Fumarprotocetrarsäure-Chemosyndrom vorhanden ist. Dem Chemotyp II würde dieses Chemosyndrom fehlen. Nach KÜMMERLING (1991) ist bei Cladonia rei - ebenso wie bei anderen Cladonia-Arten im Gebiet, die Chemotypen mit und ohne Fumarprotocetrarsäure bilden (z.B. Cladonia merochlorophaea) - der Fumarprotocetrarsäure-haltige Chemotyp wesentlich häufiger 
vertreten. Die Angaben von PAUS et al. (1993) lauten ähnlich: 94 \% der Proben gehören dem Chemotyp I, lediglich $6 \%$ dem Chemotyp II an.

Tab. 30: Die Sekundärstoffe der untersuchten Cladonia-Arten

Cl. arbuscula ssp. mitis

Cl. arbuscula ssp. squarrosa

Cl. cervicornis ssp. verticillata et cervicornis

Cl. ciliata

Cl. coccifera et pleurota et deformis s.l.

Cl. coniocraea

Cl. crispata

Cl. cryptochlorophaea

Cl. digitata

Cl. fimbriata

Cl. furcata ssp. furcata, Chemotyp I

Cl. furcata ssp. furcata, Chemotyp II

Cl. furcata ssp. subrangiformis, Chemotyp I

Cl. furcata ssp. subrangiformis, Chemotyp II

Cl. glauca

Cl. gracilis

Cl. grayi s.str.

Cl. humilis

Cl. macilenta ssp. floerkeana

Cl. macilenta ssp. macilenta, Chemotyp I

Cl. macilenta ssp. macilenta, Chemotyp II

Cl. merochlorophaea, Chemotyp I

Cl. merochlorophaea, Chemotyp II

Cl. novochlorophaea, Chemotyp I

Cl. novochlorophaea, Chemotyp II

Cl. phyllophora

Cl. portentosa, Chemotyp I

Cl. portentosa, Chemotyp II

Cl. pyxidata ssp. chlorophaea

Cl. pyxidata ssp. pyxidata

Cl. ramulosa

Cl. rangiferina

Cl. rangiformis

Cl. rei

Cl. squamosa

Cl. subulata

Cl. uncialis
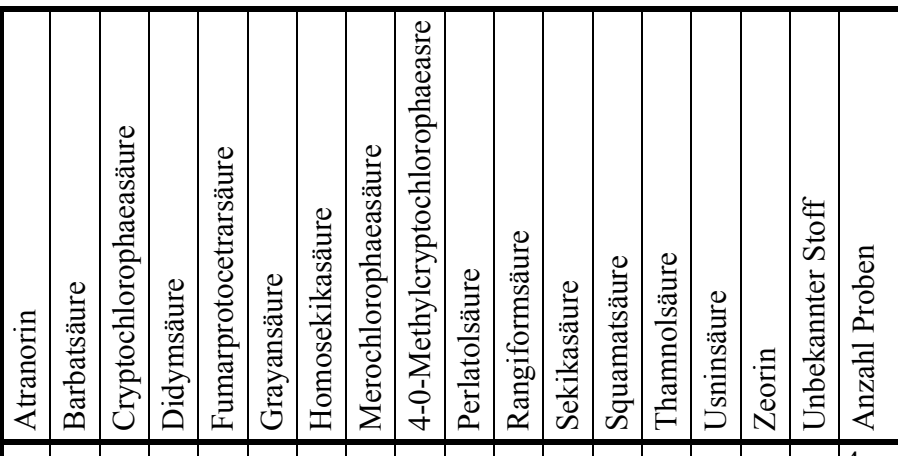

\section{Cladonia rangiformis:}

Innerhalb von Cl. rangiformis werden nach heutigem Wissensstand zwei Chemotypen unterschieden. PAUS (1997) konnte in ihrem Untersuchungsgebiet ausschließlich den Chemotyp I (Fumarprotocetrarsäure-Chemosyndrom fehlend) belegen, während KÜMMERLING (1991) in allen ihren Proben Fumarprotocetrarsäure zumindest in Spuren nachwies und bemerkt, die Fumarprotocetrarsäure-haltige „Chemorasse“ (Chemotyp II, 
PAUS 1997) sei in ihrem Gebiet wesentlich häufiger bzw. ausschließlich vertreten. Im Rahmen der vorliegenden Untersuchungen wurden zwar nur zwei Cl.rangiformisProben mittels DC untersucht, diese sind jedoch, in Übereinstimmung mit KÜMMERLING (1991), dem Chemotyp II (mit Fumarprotocetrarsäure) zuzuordnen.

\section{Zusammenfassung}

Es wurden 680 Proben der Gattung Cladonia untersucht. Innerhalb der Art Cl. pyxidata konnten alle 4 bei WIRTH (1995) aufgeführten Chemosippen (Cl. cryptochlorophaea, Cl. grayi s.str., Cl. merochlorophaea und Cl. novochlorophaea) festgestellt werden. Cl. merochlorophaea und Cl. novochlorophaea lassen sich jeweils in zwei Chemotypen weiter unterteilen: den Chemotyp I mit Fumarprotocetrarsäure und den Chemotyp II ohne Fumarprotocetrarsäure. Der Chemotyp I von Cl. merochlorophaea ist der häufigste Cl. pyxidata-Chemotyp im Untersuchungsgebiet.

Ein Chemotyp I mit Fumarprotocetrarsäure tritt auch bei $\mathbf{C l}$. rei auf, die darüber hinaus durch Sekika- und Homosekikasäure gekennzeichnet ist. Ein entsprechender Chemotyp II ohne Fumarprotocetrarsäure wurde dagegen in den nordhessischen Proben nicht nachgewiesen, obwohl dieser in anderen Gebieten vorkommt (s. PAUS et al. 1993).

Bei Cl. macilenta existieren ebenfalls Chemotypen. Alle Cl. floerkeana-Proben entsprechen dem Chemotyp I mit Barbatsäure als einzigem erkennbaren Inhaltsstoff. Ein Chemotyp mit Didymsäure (s. KÜMMERLING 1991) wurde im Untersuchungsgebiet nicht gefunden. Cl. macilenta teilt sich auf in einen Chemotyp I mit und einen Chemotyp II ohne Thamnolsäure. Chemotyp II ist nur aufgrund der feinstaubigen Podetien von der ssp. floerkeana zu trennen.

Innerhalb der Sippe $\boldsymbol{C l}$. furcata ssp. furcata tritt in der Regel nur Fumarprotocetrarsäure auf. Die aus der Literatur bekannten Chemotypen mit Psoromsäure und Atranorin (BARENDREgT et al. 1982, HuOVInEN et al. 1990, PAus 1997, Fischer \& GüNZL 2003) kommen im Untersuchungsgebiet nicht vor. Lediglich zwei Proben enthalten außer Fumarprotocetrarsäure noch eine weitere, mittels DC nicht näher identifizierbare Substanz.

Die Proben der Cl. furcata ssp. subrangiformis enthalten außer Fumarprotocetrarsäure noch Atranorin. In 7 Thalli der ssp. subrangiformis tritt ein weiterer, jedoch mit der DC ebenfalls nicht näher bestimmbarer Stoff auf. 


\title{
G Morphologische und anatomische Variabilität von Cladonia furcata
}

\begin{abstract}
„Wer eine Art genau auch in der Natur studiert, findet sie gewöhnlich so plastisch, dass es sinnlos oder geradezu unrichtig wäre, systematisch kleine Einheiten innerhalb derselben aufzustellen. Aber vielfach bedenkt man nicht, dass die Flechten lebende Organismen sind, sondern behandelt sie wie Briefmarken oder Knöpfe, denen man wegen zufällig entstandener Schäden verschiedene Namen beilegt. Hiermit will ich keineswegs behaupten, dass es sich nicht lohne, die Variationsamplitude einer Art zu studieren. Im Gegenteil! Solche Untersuchungen sind oft sehr interessant und fruchtbringend, allerdings mehr vom ökologischen als vom systematischen Gesichtspunkt aus. “(DEGELIUS 1939:103)
\end{abstract}

\section{Methoden}

\subsection{Wuchsformen-Untersuchungen}

\subsubsection{Probenahme und untersuchte Merkmale}

Das Ziel der morphologischen Untersuchungen ist, die Variabilität von Cladonia furcata ssp. furcata anhand möglichst vieler Merkmale zu erfassen und Abhängigkeiten der Merkmale untereinander bzw. von Standortfaktoren aufzuzeigen. Zu diesem Zweck wurden stichprobenhaft über den gesamten Untersuchungsraum verteilt, an verschiedenen Flechtenwuchsorten insgesamt 65 Polster gesammelt. Die Polster wurden zufällig aus der Flechtenvegetation herausgegriffen. Innerhalb jedes Polsters wurden maximal 10 zufällig ausgewählte Podetien, von der Basis an herausgetrennt (insgesamt 623 Podetien) und bei diesen 16 Merkmale protokolliert. Die Messungen und Merkmalsbestimmungen erfolgten an den Podetien, soweit nicht anders angegeben, in feuchtem Zustand unter dem Binokular. Die Beschreibung der Podetien umfaßt folgende Merkmale (s. Abb. 27):

1. Lagerfarbe und Farbe der Apothecien in trockenem Zustand bei Tageslicht

2. Vorhandensein oder Fehlen von Pyknidien (Py)

3. Anzahl Apothecien (AA)

4. Maximale Größe der Apothecien (GA)

5. Podetienlänge in $\mathrm{mm}(\mathrm{PL})$ : gemessen in gestrecktem Zustand.

6. Maximaler Podetiendurchmesser in $\mathrm{mm}(\mathrm{PD})$ : gemessen an der dicksten Stelle, jedoch nicht unmittelbar unter Verzweigungen, da sich die Podetien dort in der Regel trompetenartig erweitern.

7. Anzahl Schuppen (=Phylloide; vgl. UllRich 1954, SEMBDNER 1957/58) pro Podetium (AS). Es zählen nur deutlich flächige, nicht aber warzenähnliche Gebilde.

8. Maximale Größe der Schuppen (GS) in mm: längster Durchmesser der größten Schuppe. 
9. Anzahl der Verzweigungen (ZV): Gehen auf einer „Ebene“ mehrere Äste ab, werden sie als eine Verzweigung gezählt. Als Verzweigung zählen nur Äste, die annähernd halb so dick bis so dick wie der Hauptast sind oder dünner, dann jedoch länger als $5 \mathrm{~mm}$.

10. Fibrillen (Fi): Kategorien: fehlend bis max. 5; wenige; zahlreiche. Die Bezeichnung Fibrille ist im allgemeinen für kurze, meist waagrecht abstehende Seitenäste von Bartflechten gebräuchlich (WIRTH 1995), wird wegen der Ähnlichkeit der im Vergleich zu den Seitenzweigen kurzen und dünnen Auswüchse hier jedoch auch bei der Gattung Cladonia verwendet.

11. Zweige am Podetienende (ZP): Anzahl Verzweigungen im Bereich der letzten $5 \mathrm{~mm}$ einer Podetienspitze. Bei fruchtenden Exemplaren gilt als Ästchen, wenn ein Apothecium deutlich gestielt ist.

12. Weitere Podetienmerkmale: geschlitzt; offen (rinnig); rund (Mehrfachnennungen möglich).

13. Pigmentierung (Pi) (an der dunkelsten Stelle), eingeteilt in 3 Kategorien: ohne sichtbare Pigmentierung; leichte Bräunung; stark gebräunt.

14. Deckung der Algenschicht (Al): Ausdehnung der Algenschicht in \% der Podetienoberfläche. 4 Kategorien: - $25 \%$; - $50 \%$; - $75 \%$; > $75 \%$.

15. Anzahl Grundschuppen: fehlend/rar oder reichlich.

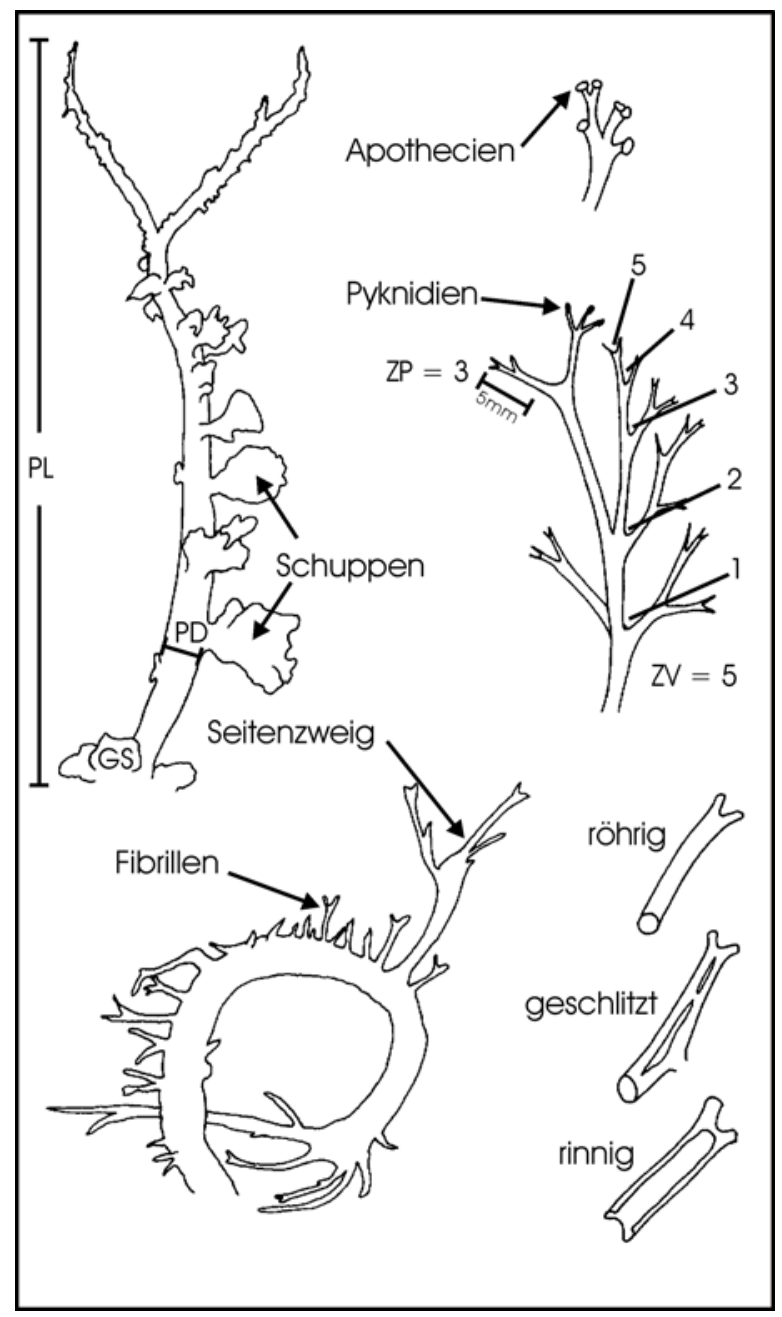

Abb. 27: Skizzen der untersuchten morphologischen Merkmale (Abkürzungen s. Text). 
Abb. 28 zeigt, welchen Meßtischblattquadranten und welchen Erdflechtengesellschaften die untersuchten Cladonia furcata-Proben entnommen wurden. Etwas außerhalb des eigentlichen Untersuchungsgebietes liegen die ebenfalls beprobten Heideflächen im Gladenbacher Bergland (MTB 5316).

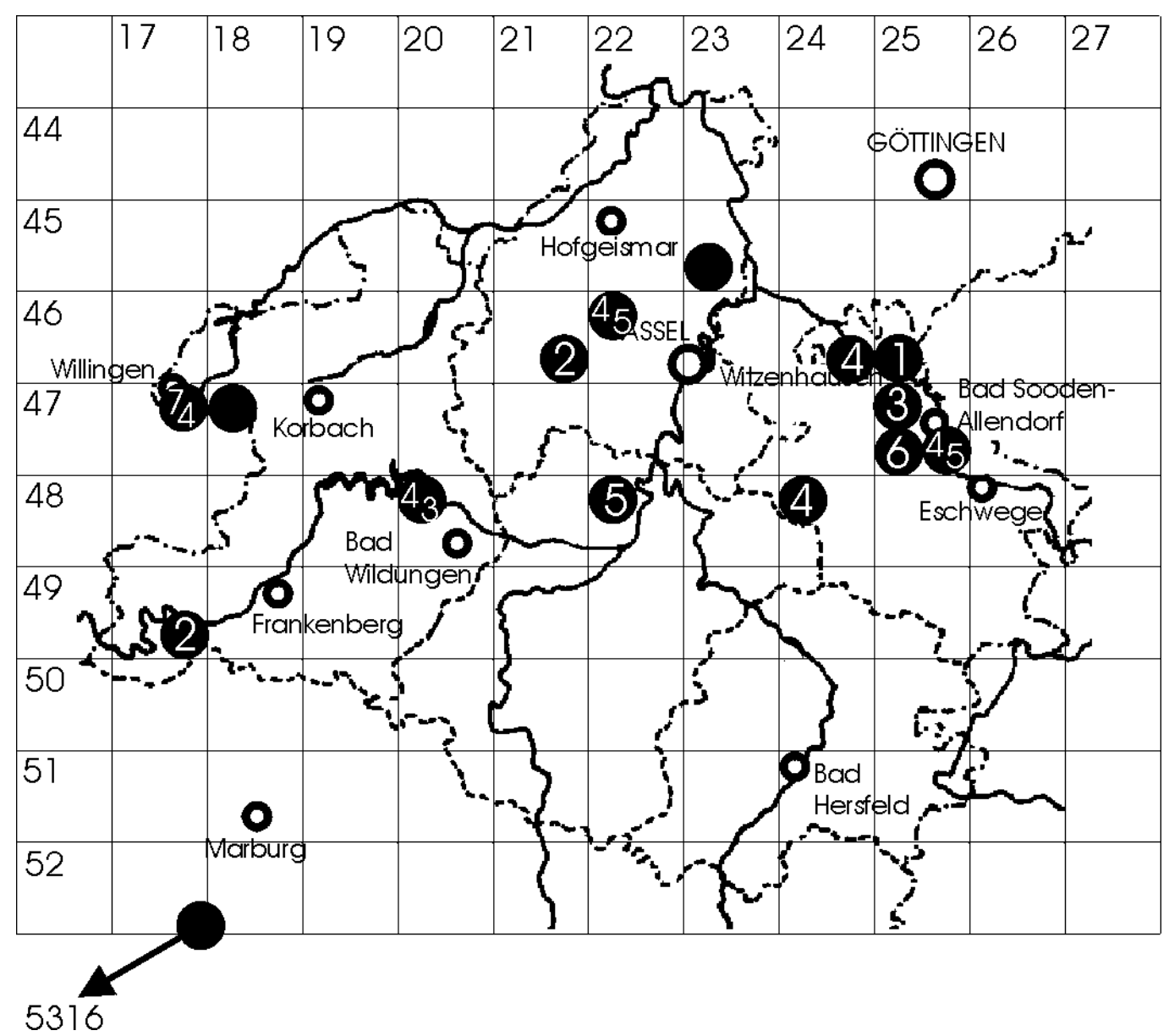

Abb. 28: Herkunftsorte der morphologisch untersuchten Cladonia furcata-Proben, angegeben auf Quadrantenbasis der MTB (TK 25). Die Zahlen bezeichnen die Gesellschaften, denen die Proben entstammen.

Es bedeuten:

1 Cladonia cervicornis-Baeomycion-Fragmentgesellschaft

2 Cladonietum mitis cladonietosum merochlorophaeae

3 Cladonietum mitis typicum, Cladonia rangiferina-Variante

4 Cladonia furcata-Cladonion arbusculae-Fragmentgesellschaft

5 Cladonietum foliaceae

6 Cladonia floerkeana-Cladonion rei-Fragmentgesellschaft

7 Cladonietum rei, Cladonia floerkeana-Ausbildung

Ohne Nummer: nicht vegetationskundlich bearbeitete Cladonia furcata-Bestände.

$\mathrm{Zu}$ jedem Polster wurde zudem eine Kurzbeschreibung des Wuchs- und Standortes angefertigt, die neben geographischen Daten auch Angaben zu Exposition, Inklination, Begleitvegetation, Lichtverhältnissen, Feuchtigkeitsverhältnissen, Windverhältnissen, Bodenmerkmalen sowie Nutzungs- und Störeinflüssen beinhaltet. Anhand dieser Angaben erfolgte eine Zuordnung der Standorte zu 5 Standorttypen (s. Belegeliste im Anhang 1). Die Standorttypen werden hier in der Reihenfolge zunehmender Feuchte und Beschattung beschrieben: 
- „Felsen“ (F): sonnige, exponierte, sehr trockene Felskuppen oder felsige Hänge (z.B. Maderstein, Dörnberg, Michelskopf).

- „Heiden“ (H): trockene, sonnige, offene Stellen in Zwergstrauchheiden in niederschlagsärmeren Regionen der tieferen Lagen (z.B. Battenfelder Driescher).

- „Waldränder“ (R): leicht beschattete, trockene Standorte in Waldrandlagen, etwa Wegböschungen in lichten Eichenwäldern (z.B. Roßkopf, Zeche Glimmerode).

- „Berglagen“ (B): exponierte Berglagen über $700 \mathrm{~m}$. ü. NN, geprägt durch hohe mittlere Jahresniederschläge. Leichte Beschattung der Flechten z.B. durch Calluna vulgaris (z.B. Ettelsberg bei Willingen, Meißner).

- „Wälder“ (W): stärker beschattete Stellen innerhalb der Eichenwälder (Roßkopf, Mühlecke am Edersee).

Darüber hinaus erfolgte eine Einstufung in 3 Kategorien nach der Schwere der mechanischen Einwirkungen auf die Flechten am Standort:

- $\quad$ sehr geringe mechanische Einwirkung

- mäßig starke mechanische Einwirkung

- sehr starke mechanische Einwirkung

Es werden bei dieser Einstufung nur die längerfristigen oder stets wiederkehrenden Störungen, wie Beweidung oder regelmäßiger Tritt durch Spaziergänger berücksichtigt. In der Vergangenheit aufgetretene, einmalige oder seltene Störereignisse können dabei jedoch unerkannt bleiben. Dies ist bei der Diskussion der Ergebnisse zu beachten.

\subsubsection{Auswertung und Darstellung der Ergebnisse}

Die statistische Auswertung der Daten erfolgte mit dem Statistik-Computerprogramm (Add-In) WinSTAT für Excel, Version 2001.1.

\section{a) Abhängigkeiten der Podetienmerkmale von den Standortbedingungen}

Als Untersuchungseinheiten wurden die Flechtenpolster bestimmt (die aus demselben Polster entnommenen Podetien sind als abhängig $\mathrm{zu}$ betrachten). Die Werte der einzelnen Podetien eines Polsters mußten deshalb gemittelt werden. Da die Polster zufällig an den Flechtenstandorten entnommen wurden, sind die daran ermittelten Daten als statistisch unabhängig aufzufassen. Die Überprüfung der Daten auf signifikante Unterschiede erfolgte mit dem U-Test (Mann-Whitney), der ein nicht-parametrisches Verfahren darstellt. Damit läßt sich der U-Test unabhängig von der Verteilungsart anwenden (im vorliegenden Fall sind nicht alle Daten normalverteilt). Für jedes Podetienmerkmal wurden die Standorttypen jeweils paarweise mit dem U-Test verglichen. Die Ergebnisse werden als signifikant betrachtet, wenn die Irrtumswahrscheinlichkeit $\mathrm{p} \leq 0,05$ beträgt.

Bei der graphischen Darstellung dieser Ergebnisse werden der arithmetische Durchschnitt sowie die Standardabweichung (zur Charakterisierung der Variabilität der Daten) in Form zweier Fehlerbalken angegeben. Mittelwerte und 
Standardabweichungen sollen lediglich der Beschreibung der Daten dienen, sie wurden nicht für die statistischen Berechnungen verwendet. Unter den Bezeichnungen für die Standorttypen (F, H, R, B, W) sind mit Buchstaben jeweils die Ergebnisse des U-Tests angegeben; verschiedene Buchstaben bedeuten signifikante Unterschiede. In den Tabellen wird bei den mit dem Exponent „a“ versehenen Werten das Signifikanzniveau für den jeweiligen Paarvergleich angegeben.

\section{b) Abhängigkeiten bzw. Korrelationen der Podetienmerkmale untereinander}

Die Überprüfung der Daten auf signifikante Unterschiede erfolgte immer dann mit dem U-Test, wenn metrische Daten von Merkmalen mit den in Kategorien eingeteilten Merkmalen (Nominal- und Ordinalskalen) verglichen wurden. Letzteres sind die Merkmale „Apothecien bzw. Pyknidien vorhanden oder fehlend“, „Grad der Fibrillenbildung“, „Grad der Pigmentierung“ sowie „Ausdehnung der Algenschicht". Die Ergebnisse werden wiederum als signifikant betrachtet, wenn die Irrtumswahrscheinlichkeit $\mathrm{p} \leq 0,05$ beträgt. Hierbei, wie auch bei der Korrelationsanalyse, konnten die Werte der einzelnen Podetien in die Berechnung eingehen, da die Fragestellung in diesem Fall lautet, in welchem Maße an einem Podetium Änderungen zweier Variablen (morphologischer Merkmale) miteinander verknüpft sind bzw. voneinander abhängen.

Eine Spearman Korrelationsanalyse wurde beim Vergleich ausschließlich der metrischen Daten durchgeführt. Bei dem verwendeten Spearmanschen Rangkorrelationskoeffizienten $\left(\mathrm{r}_{\mathrm{S}}\right)$ müssen die Daten in eine logische Rangfolge eingeordnet werden können und die Beziehung zwischen den Variablen muß monoton zu- oder abnehmen. Eine Normalverteilung der Daten ist nicht Voraussetzung. Der Wert von $r_{S}$ kann zwischen -1 (perfekte negative Korrelation) und +1 (perfekte positive Korrelation) variieren (BÄRLOCHER 1999, BORTZ et al. 1990). Neben der Berechnung der Korrelationskoeffizienten wird als Resultat von WinSTAT auch ein Wert für deren einseitige Signifikanz (p) ausgegeben. Dieser Wert gibt die Wahrscheinlichkeit dafür an, daß der beobachtete Korrelationskoeffizient zustande kommt, auch wenn die Grundgesamtheiten der Zufallsvariablen unabhängig sind, also nicht korrelieren, was der Nullhypothese entspricht. Eine Signifikanz kleiner als die festgelegte Irrtumswahrscheinlichkeit von $\mathrm{p}=0,05$ führt zur Ablehnung dieser Nullhypothese. 


\subsection{Verpflanzungsversuche}

\subsubsection{Standortwahl}

Die Verpflanzungsversuche erforderten mindestens zwei Standorte, die sich nur bezüglich ihrer mikroklimatischen Verhältnisse, nicht jedoch in ihrer Geologie, ihrer Phanerogamenvegetation und ihrem Makroklima unterscheiden. Diese Bedingungen erfüllen die 3 Flächen am Roßkopf (MTB 4725), die in unmittelbarer Nachbarschaft zueinander angelegt wurden, also dem gleichen Makroklima unterliegen. Das Ausgangsgestein am Roßkopf ist Grauwacke, bei der Phanerogamenvegetation handelt es sich um ein Luzulo-Quercetum. Der Ah-Horizont an den Standorten ist nur wenige $\mathrm{cm}$ mächtig. Anfallende Streu wird aufgrund der exponierten Lage vom Wind weg geweht. Alle Versuchsflächen weisen etwa die gleiche Inklination $\left(\sim 10^{\circ}\right)$ und Exposition (WSW) auf und liegen in 330 bis $335 \mathrm{~m}$ ü. NN. Die Versuchsflächen R1 und $\mathbf{R} 2$ befinden sich innerhalb des Luzulo-Quercetum. Kennzeichnend für beide Flächen ist eine mäßige bis deutliche Beschattung durch die Eichenkronen. In der Kryptogamenvegetation dominieren Moose (v.a. Dicranum scoparium) und Cladonia furcata ssp. furcata. Die Versuchsfläche R3 liegt etwas oberhalb von R1 und R2, an einem Waldrand des Luzulo-Quercetum, rund einen Meter neben einem Weg gelegen (s. Taf. 5 im Anhang 2 und Abb. 20 auf S. 59). Gegen Norden wird sie durch einige Eichen abgeschirmt, nach Süden hin ist sie jedoch über Mittag der direkten Sonneneinstrahlung ausgesetzt. Eine Beschattung durch die gegenüber stehenden Bäume setzt gegen Nachmittag ein. Die Kryptogamenvegetation wird von diversen Cladonien (u.a. Cl. portentosa und Cl. gracilis) dominiert. Der Standort sollte für Cl. furcata ssp. furcata geeignet sein, auch wenn die Art dort nur in Form eines kümmerlich entwickelten Polsters nachgewiesen werden konnte.

Ergänzend zu den Flächen am Roßkopf wurde eine weitere Fläche im Niestetal (MTB 4724) angelegt. Beide Gebiete liegen in Luftlinie nur wenige Kilometer auseinander und sind bezüglich des Makroklimas recht ähnlich. Die Versuchsfläche N1 weist eine Inklination von $5^{\circ}$ auf, ist südwest-exponiert, liegt an einem Waldrand und wird im Norden durch Fichten abgeschirmt. Durch den im Süden angrenzenden breiten Weg bedingt, ist sie mitunter starker Sonneneinstrahlung ausgesetzt und damit bezüglich der mikroklimatischen Verhältnisse der Versuchsfläche R3 ähnlich. Ausgangsgestein ist der Mittlere Buntsandstein. Der Boden wird von einer dicken Streuschicht bedeckt, die überwiegend aus Fichtennadeln besteht. Auffälligste Kryptogamenart am Standort ist Cladonia subulata, umgeben von Calluna vulgaris.

Bei der Auswertung werden in erster Linie die Daten der beiden Standorte am Roßkopf berücksichtigt, da dort auch Mikroklima-Messungen (s. Kap. G1.2.3) durchgeführt wurden. Die Daten der Fläche N1 werden zum Vergleich herangezogen. 


\subsubsection{Auswahl der Flechten-Polster und Bearbeitung der Podetien}

Der Verpflanzungsversuch wurde mit der Strauchflechte Cladonia furcata ssp. furcata durchgeführt. Die Art ist ungeschützt und im Gebiet häufig, so daß ausreichend Ausgangsmaterial für den Versuch zur Verfügung stand. Die verwendeten Polster wurden nach 3 Kriterien ausgewählt (s. auch Abb. 29):

1. Wenig bis nicht pigmentierte Polster eines beschatteten Standortes: Luzulo-Quercetum, Mühlecke am Edersee. Jede Versuchsfläche enthielt eines dieser Polster (Probe-Nummern I bis III). Die Proben Ia und Ib entstammen einem gemeinsamen großen Polster.

2. Stärker pigmentierte Polster eines besonnten Standortes: Festuca ovina-Bestand, Waldrand, ehemalige Zeche Glimmerode. In die Versuchsflächen R1 und N1 wurde je eine, in die Versuchsflächen R2 und R3 je zwei Proben aus Glimmerode gepflanzt. Diese Proben tragen die Nummern VI bis XI.

3. Innerhalb der Versuchsflächen R1 und R2 kommt von Natur aus Cladonia furcata ssp. furcata vor. Um zu überprüfen, wie diese Polster an dem direkt benachbarten Standort R3, unter stärkerer Sonneneinstrahlung gedeihen, wurde ein Polster in R1 belassen und ein weiteres in R3 umgesetzt (Nr. IV und V).

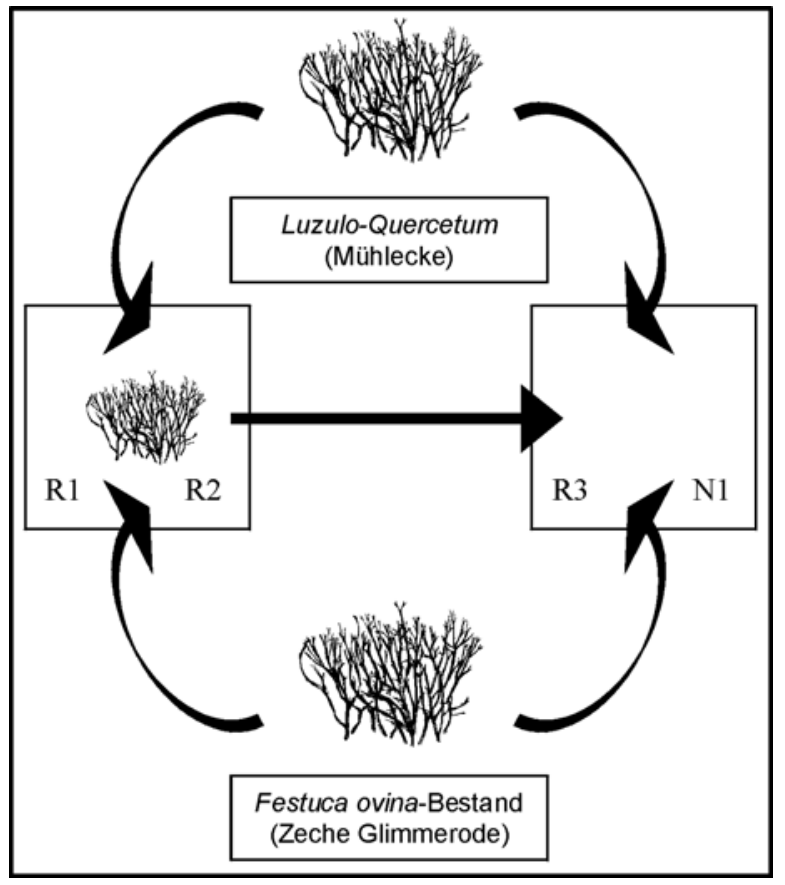

Abb. 29: Schema des Verpflanzungsversuches. R1 bis R3 und N1 bezeichnen die Versuchsflächen.

Alle Podetien, an denen die Zuwachsmessungen durchgeführt wurden, waren vital, zeigten keine Anzeichen von Fruchtkörper- oder Pyknidienbildung und wiesen zudem eine dichotome Verzweigung auf. Innerhalb der Polster wurden je nach Polstergröße zwischen 5 und 20 Podetien ausgewählt und mit rotem Polyester-Faden markiert:

Polster I-III, VI, IX:

Polster IV, V:

Polster VII, X:

Polster VIII, XI:
20 Podetien

10 Podetien

15 Podetien

5 Podetien 
Die Messungen erfolgten an den dichotom verzweigten Podetienspitzen, nach Besprühen mit Wasser und fünfminütigem Warten, in feuchtem Zustand. Gemessen wurden mit Hilfe eines Reißzirkels bzw. Binokulars (mit Meßokular) jeweils die Längen der Spitzen von der obersten Verzweigung ab (s. VAGTS et al. 1994). Jedes dieser Podetien erhielt eine Nummer. Der Polsterumriß sowie die Anordnung der Podetien innerhalb der Polster wurden auf Folie abgezeichnet, um die ausgemessenen Podetien nach Versuchsende wieder lokalisieren und ihren Zuwachs bestimmen zu können. Zusätzlich wurden Podetienbündel aus je 10 mit Polyesterfaden zusammengebundenen Podetien angefertigt (s. VAGTS et al. 1994) und in den Flächen ausgelegt, um die Regeneration der Podetien $\mathrm{zu}$ beobachten. Das Ausgangsmaterial hierfür stammte von der Mühlecke/Edersee. Die Bündel erhielten die Bezeichnung p1-4. Die ausgemessenen Polster und Podetienbündel wurden mit Polyethylen-Haken im Boden verankert und durch Holzgatter (s. Taf. 5.1 im Anhang 2) vor Wildtieren geschützt. Die Gatter wurden so konstruiert und verankert, daß sie allenfalls eine geringe Beschattung der Proben verursachten und die Polster in ihrer Entwicklung nicht beeinflußten. Im Einzelnen ergab sich für die Versuchsflächen folgende Proben-Belegung:

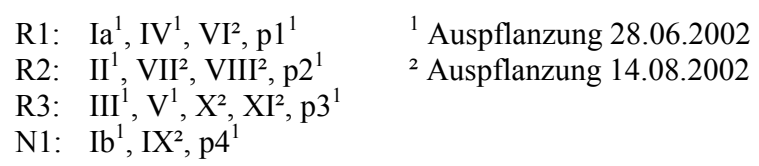

Bei der Verteilung der Polster auf die Versuchsflächen wurde darauf geachtet, daß sich in jedem der 0,25 $\mathrm{m}^{2}$ großen Gatter von jedem Ausgangsgebiet (Mühlecke, Glimmerode, Roßkopf) etwa gleich viele markierte Podetien befanden.

\subsubsection{Mikroklima-Messungen}

Um die beiden Roßkopf-Standorte etwas genauer charakterisieren zu können, wurden exemplarisch an einem Sommertag Mikroklima-Messungen durchgeführt. Die Messungen im Hochsommer zeigen einige Extreme des Mikroklimas, denen die Flechten an beiden Standorten ausgesetzt sind und erlauben Aussagen darüber, inwiefern die Thalli auch im Wald ungünstige Bedingungen bezüglich Temperatur und Feuchtigkeit ertragen müssen. Die Faktoren Licht und Luftemperatur am Flechtenstandort sowie Thallustemperatur und Thallusfeuchte der Cladonien (angegeben als Wassergehalt in \% des Trockengewichtes) wurden mit einem eigens für Flechten konstruierten tragbaren Meßgerät ermittelt. Eine genaue Beschreibung der Meßapparatur findet sich bei SCHUSTER et al. (1982/2002). Die Lichtintensität wurde nicht in der heute meist gebräuchlichen Einheit $\mu \mathrm{E} / \mathrm{m}^{2} / \mathrm{s}$ oder als relativer Lichtgenuß in \%, sondern in Lux gemessen, um die Daten mit denen anderer Arbeiten vergleichen zu können, bei denen die gleichen Meßgeräte verwendet wurden (z.B. SCHÖLLER 1991). Die Bestimmung der Evaporation in $2 \mathrm{~cm}$ Höhe erfolgte mit Piche-Evaporimetern mit grünen Filterpapierscheiben von $3 \mathrm{~cm}$ Durchmesser. Die Windgeschwindigkeit in $10 \mathrm{~cm}$ über der Bodenoberfläche konnte jeweils an einem Schalenkreuzanemometer abgelesen werden. Die relative Luftfeuchte wurde mit Aspirationspsychrometern (nach Assmann) bestimmt. Das Ablesen der Werte erfolgte von 6.15 Uhr bis 20.00 Uhr, mit Ausnahme der ersten Messung in 30-minütigem-Rhythmus. 


\section{Einführung: Varietäten und Formen}

\section{Die Untergliederung von Cladonia furcata in der Literatur des 20. Jahrhunderts}

Bei kaum einer anderen Flechtenart finden sich in der Literatur derartig viele Untereinheiten innerhalb einer Spezies wie bei Cladonia furcata ssp. furcata. Die zahlreichen Varietäten und Formen werden aufgrund morphologischer Merkmale aufgestellt. Bevor in Kap. G3 die Ergebnisse der eigenen Untersuchungen dargestellt werden, gibt dieses Kapitel einen Überblick über die verschiedenen Varietäten und Formen sowie deren kennzeichnende Merkmale. Die Frage nach dem Sinn solcher Untereinheiten wird in Kap. I1 diskutiert.

Bei der Zusammenstellung der Varietäten und Formen von Cladonia furcata wurden die furcata-ähnlichen, aber sorediösen Formen von Cl. scabriuscula nicht berücksichtigt. Es soll an dieser Stelle nur erwähnt werden, daß sie teilweise als var. scabriuscula zu Cl. furcata gestellt wurden (siehe z.B. LETTAU 1955, HENNIPMANN 1978). Jedoch haben schon ANDERS (1928) und SANDSTEDE (1931) Cl. scabriuscula als eigene Art aufgeführt. In die nachfolgenden Auswertungen wurden zahlreiche Literaturangaben überwiegend aus dem 20. Jahrhundert sowie einige Angaben aus dem Internet einbezogen (z.B. FEUERER 2003). Obwohl auf diese Weise eine lange Liste an Varietäten und Formen von Cl. furcata zusammengetragen wurde, erhebt diese keinen Anspruch auf Vollständigkeit, da sie in erster Linie Literatur des deutschsprachigen Raumes berücksichtigt.

\subsection{Cladonia furcata ssp. furcata}

In der aufgeführten Literatur finden sich zahlreiche Varietäten, von denen jedoch nur 3 für das Untersuchungsgebiet von Bedeutung sind:

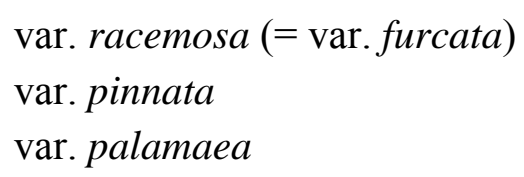

Nachfolgend sollen die Aussagen der verschiedenen Autoren über diese 3 Varietäten zusammenfassend dargestellt werden. Namen und Literaturquellen der anderen Varietäten sind Tab. $31 \mathrm{zu}$ entnehmen.

\subsubsection{Die Varietät racemosa (= furcata)}

Die Angaben über den Grad der Beschuppung (= Beblätterung) weichen bei den verschiedenen Autoren teilweise deutlich voneinander ab. Während HILLMANN \& GRUMMANN (1957) sie als nicht beblättert bezeichnen, gestehen ihr BRITZELMAYR (1904), ANDERs (1928) und ERICHSEN (1957) wenige Schuppen zu. KLEMENT (1952) zählt dagegen sowohl fast schuppenfreie als auch reich beschuppte Formen zur var. racemosa. ScHADE (1964) bemerkt zum Thema Beschuppung, daß die Fähigkeit zur Blättchenbildung zum Wesen der gesamten var. furcata (wie der Cladonia furcata überhaupt) gehöre, das Fehlen oder Vorhandensein von Podetiumblättchen daher kein 
taxonomisch wirksames Merkmal sein könne. In seiner Schilderung der charakteristischen Merkmale von Cl. furcata var. racemosa fehlen daher Angaben zur Beschuppung. Er beschreibt sie wie folgt: Podetien 10 bis $50 \mathrm{~mm}$ lang, 0,5 bis $2 \mathrm{~mm}$ dick, zusammenhängend grünlichgrau berindet, gegen das Ende der Zweige hin aber meist \pm fein weißscheckig, an sonnigen Orten \pm braun, meist spärlich bis dicht beblättert, an oft betretenen Stellen mit \pm zahlreichen dornig-hakigen Sprossen oder schon längeren Nachschüssen. Die Flanken der Podetien und ihrer Verzweigungen sind meist mehr oder weniger feinrissig. Besonders die fertilen sind oft breitklaffend aufgerissen.

\subsubsection{Die Varietät pinnata}

Die Varietät pinnata soll im Gegensatz zur var. racemosa bis oben \pm dicht beschuppt sein (FINK 1904, ANDERS 1928). Eine meist starke Beblätterung gehört auch bei SANDSTEDE (1931) und ERICHSEN (1957) zu ihren wichtigsten Erkennungsmerkmalen. SCHADE (1964) schreibt, die Beblätterung könne schwanken, sei aber offensichtlich auffälliger und etwas reichlicher als bei der var. furcata. Besonders fertile Zustände könnten aber auch fast blattlos sein. Weiterhin kennzeichnet er die Varietät folgendermaßen: die Podetien sind größer und kräftiger als bei der var. furcata, ca. 20 bis $120 \mathrm{~mm}$ lang und 0,9 bis $4 \mathrm{~mm}$ dick. Sie sind zusammenhängend dick und glatt berindet (wenigstens an den unteren Teilen der Podetien), glanzlos, grünlichgrau bis weißgrau, an den oberen Teilen oft ebenfalls weißscheckig, etwas gröber als bei der var. furcata, so besonders auf der Unterseite liegender Stämmchen. Dornig-hakige Sprosse treten weniger häufig auf, dagegen kommen aber zahlreiche kleinere bis klaffend breite Risse besonders in den Fruchtständen vor. Seitliche Risse fehlen kaum einem Podetium. An den ältesten Stücken ist die Varietät unten häufig grob querrissig oder faltig bis netzartig runzelig. Doch durchdringen die Risse nicht die ganze Podetienwand wie bei der var. furcata. Die Rißränder rollen sich nicht nach innen, sondern krempeln sich ab und zu nach außen schwach empor. Die Rindenfelder können sich ganz ablösen oder auf dem Mark wie Inseln sitzen bleiben.

BRITZELMAYR (1904) ordnet die var. pinnata nicht als eigenständige Varietät ein, sondern als beschuppte Form der var. racemosa (,var. racemosa f. squamulosa“).

\subsubsection{Die Varietät palamaea}

Die Varietät palamaea ist bei den meisten Autoren nur unbefriedigend gegen die anderen Varietäten abgegrenzt. Meist wird auf die braune bis dunkelbraune Färbung verwiesen. So schreiben HILLMANN \& GRUMMANN (1957) die var. palamaea sei von der var. racemosa f. furcatosubulata nur durch die kräftig braune Farbe unterschieden. Auch ERICHSEN (1957) berichtet, daß umgekehrt die var. racemosa im Gegensatz zur var. palamaea allenfalls in der Sonne hellbräunlich ist. Er nennt, ebenso wie ANDERS (1928), als weiteres Merkmal der var. palamaea das glänzende Aussehen der Podetien. SANDSTEDE $(1922,1931)$ bezeichnet sie zudem als meist sparrig gewachsen und häufig mit vielen dornig-hakigen Nebenästen versehen. SCHADE (1964) spricht sich gegen eine Varietät palamaea aus. Die dornig-pfriemlichen Nebenäste (von ihm als teras spinosum 
bezeichnet) seien nicht als Forma oder Varietät, sondern lediglich als eine Modifikation anzusehen. Zudem könnten weder die braune Farbe noch die vielen dornig-hakigen Nebenäste als der var. palamaea allein zukommende Merkmale gelten. In SCHADES Beschreibung der var. racemosa (s.o.) taucht das Merkmal „dornig-hakige Sprosse“ ebenfalls auf. Alle als var. palamaea beschriebenen Thalli sind demnach lediglich Modifikationen der var. furcata oder var. pinnata. GRUMMANN stuft in seinem Catalogus Lichenum Germaniae von 1963 solche Exemplare nur als Forma ein (,var. furcata f. palamaea“").

Zweifel an der Eigenständigkeit der Varietät palamaea lassen auch die Aussagen von SANDSTEDE (1931) aufkommen: „Die v. palamaea hält mit racemosa enge Fühlung, sie ist die sonnenständige Pflanze, daher ihre braune Färbung, ihre f. subulata Floerk. (...) ist die heller gefärbte Schattenpflanze. Man weiß wirklich nicht, wo die Grenzen liegen“ (SANDSTEDE 1931: 199) und „Es [die var. palamaea] ist die Form, wie sie von der furcato-subulata Hoffm., Wain. 327 bzw. subulata Floerk. 143 angenommen wird, wenn sie in einzelnen Rasen frei wächst" (SANDSTEDE 1922: 163). Auch dies rechtfertigt wohl eher die Einstufung als Modifkation (s. Kap. I1), denn als Varietät, scheint sie doch den Angaben von Anders (1928), KLEMENT (1952) und Hillmann \& GRUMMANN (1957) zufolge tatsächlich stets an sonnigen Standorten vorzukommen.

\subsubsection{Die Formen der Varietäten}

\section{a) Fruchtende Formen}

Der fertile Zustand der var. racemosa, mit schirmförmigen Fruchtständen, wird von BRITZELMAYR (1904), SANDSTEDE (1931) und ERICHSEN (1957) als f. corymbosa beschrieben. Doch scheint sich SANDSTEDE (1931) über die Benennung im Unklaren gewesen zu sein, da er in der selben Arbeit an anderer Stelle von der m. corymbosa spricht. Auch in SANDSTEDE (1922) und ANDERS (1928) werden diese fruchtenden Exemplare lediglich als „Modificatio“ eingeordnet und von ANDERS zur Forma furcatosubulata gestellt („f. furcato-subulata m. corymbosa“). Fruchtende Podetien können innerhalb der Varietät racemosa auch bei anderen Formen auftreten, so bei der f. racemosella (SANDSTEDE 1931) mit einseitswendig übergebogenen Lagerstielen, bei der f. crispatella (SANDSTEDE 1931) mit dornig-pfriemlichen Nebenästen und kleinen, trugdoldigen Fruchtständen und bei der f. arbuscula (SANDSTEDE 1931, ERICHSEN 1957) mit ausgebreiteten, fruchtenden Podetien. Bei ANDERS (1928) wird letztere als „f. furcatosubulata m. arbuscula“ eingeordnet.

Innerhalb der var. pinnata stellt die f. truncata den fertilen Zustand dar (FINK 1904, ANDERS 1928, SANDSTEDE 1931). Sie entspricht nach SANDSTEDE (1931) der f. corymbosa der var. racemosa. Apothecien treten aber auch bei der f. abbreviata (SANDSTEDE 1931) mit kurzen, derben Lagerstielen, bei der beblätterten f. foliolosa (ANDERS 1928), bei der hochwüchsigen f. regalis (SANDSTEDE 1931) und bei der truncata-ähnlichen f. intermedia auf. Letztere wird lediglich von SANDSTEDE (1931) erwähnt und nur aufgrund der Fruchtkörper von der f. foliolosa der var. pinnata unterschieden. 
VERSEGHY (1987/88) nennt als fruchtende Formen bei der Varietät pinnata die f. foliolosa, f. intermedia und f. truncata sowie bei der Varietät racemosa die f. arbuscula und f. corymbosa. Sie wertet alle diese Formen als phänologische Stadien, deren systematischer Wert umstritten sei. Dies trifft auch für die f. regalis der Varietät pinnata zu, die VERSEGHY als ökologische Modifikation einordnet, ohne Angaben über Auftreten oder Fehlen von Fruchtkörpern zu machen.

SCHADE (1964) hält dagegen ganz allgemein ein besonderes Taxon für den fertilen Zustand für überflüssig und schlägt statt dessen vor, den fertilen Zustand mit dem Kürzel „fert.“ zu kennzeichnen.

\section{b) Auffallend beschuppte Formen}

Formen, deren einziges kennzeichnendes Merkmal die auffallende Beschuppung ist, tauchen in allen 3 Varietäten auf. Innerhalb der var. racemosa ist dies die f. squamulifera (SANDSTEDE 1931), die allerdings von SCHADE (1964) als modificatio squamulifera herabgestuft wird, da die Beblätterung auf keine Varietät oder Form beschränkt sei. Die f. foliolosa der var. pinnata (z.B. SANDSTEDE 1931) und die f. rigidula der var. palamaea (ANDERS 1928; SANDSTEDE 1931, ERICHSEN 1957), stellen ebenfalls lediglich den beblätterten Zustand der jeweiligen Varietät dar.

Die beschuppte var. racemosa f. squamulosa von BRITZELMAYR (1904) ist, wie oben bereits erwähnt, mit der Varietät pinnata gleichzusetzen.

\section{c) Formen mit dornig-pfriemlichen Nebenästen}

In allen 3 Varietäten treten Formen mit dornig-pfriemlichen Nebenästen auf. SCHADE (1964) bezeichnet solche als teras spinosum und hält z.B. die von SANDSTEDE (1931) beschriebene var. racemosa f. crispatella lediglich für eine Modifikation. Eine entsprechende Form bei der Varietät pinnata ist die f. recurva (ANDERS 1928, SANDSTEDE 1931, ERICHSEN 1957), nach SCHADE (1964) ebenfalls lediglich ein teras spinosum. Sie wird von SANDSTEDE (1922) als Modificatio zur var. racemosa gestellt (,var. racemosa m. recurva“). KLEMENT (1952) hält sie für eine durch Trittwirkung geschädigte Form, ordnet sie allerdings als Form und nicht als Modifikation der var. racemosa $\mathrm{zu}$.

Die var. palamaea an sich gilt bei SANDSTEDE (1922/1931) als meist sparrig gewachsen und häufig mit vielen dornig-hakigen Nebenästen versehen (s.o.). Bei einigen ihrer Formen wird jedoch dieses Merkmal nochmals explizit in den Vordergrund gestellt. Als hakenförmig gekrümmt bezeichnen beispielsweise ANDERS (1928) und ERICHSEN (1957) die f. spadicea, welche SCHADE (1964) lediglich als eine var. pinnata von betretenem Boden wertet. Ebenso wie die f. simplex (ERICHSEN 1957) soll die f. implexa wirr verzweigt sein (ANDERS 1928, SANDSTEDE 1931), sie wird von zwei Autoren nur als Modifikation eingestuft (,m. implexa“; SCHADE 1964, SANDSTEDE 1922). Die f. recurva der var. palamaea (SANDSTEDE 1931) stellt nach SCHADE (1964) ein der gleichnamigen Forma der var. pinnata entsprechendes teras spinosum dar. 
Legende zu Tab. 31 (folgende Seite):
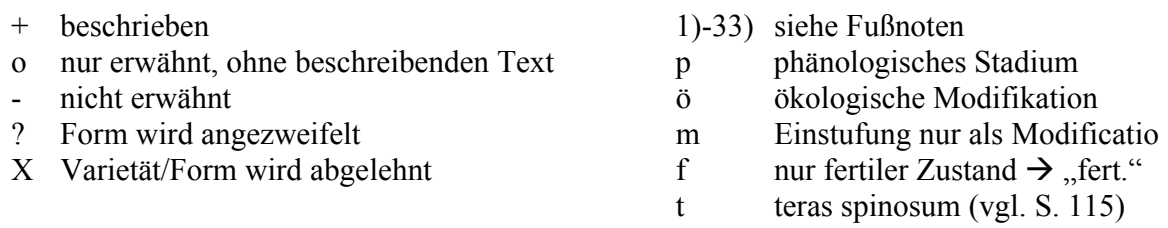

1) a) = var. furcata; b) HENNIPMANN (1978), LANGE et al. (1998): var. furcata

2) ssp. furcata var. racemosa

3) Checklisten Argentinien, Venezuela, Mexiko, Marokko oder Tunesien in Feuerer (2003)

4) Alterszustand, der zur f. fissa gehört

5) Verweis auf fissa Flörke, platystelis Wallr. und dilacerata-fissa Coëm.

6) zu var. racemosa? Erwähnt: dilacerato-fissa Coem. = platystelis Wallr.; ventricosa Aigr.

7) zu var. racemosa?

8) = subulata Floerk.

9) = f. furcato-subulata m. corymbosa (Ach.) Nyl.

10) = m. corymbosa (Ach.) Nyl., cymosa Flörke; Cl. furcata var. racemosa f. corymbosa = corymbosa Ach., Vain.

11) als Cl. furcata var. corymbosa aufgelistet (Checklisten Tunesien, Marokko, Mexiko in FeUERER 2003)

12) = f. furcatosubulata m. arbuscula Flörke

13) Evans (1950): Cladonia furcata var. racemosa f. prolifera f. nov.

14) = racemosa squamulosa Schär.

15) ssp. furcata var. pinnata

16) = var. racemosa m. foliosa Del.?

17) PUCCINI et al. (2003): Cl. furcata varieta foliosa aus Italien

18) = var. racemosa m. recurva Floerk.

19) zu var. racemosa

20) syn. furcata cymosa fissa

21) = var. racemosa m. regalis Floerk.

22) erwähnt wird Cl. furcata subulata L.

23) = var. racemosa m. subulata Floerk. Erwähnt ohne genaue Zuordnung: m. tenuior, f. truncata und f. gracilis

$24)=$ f. palamaea (Ach.) Nyl. der var. furcata

25) MÁzSA (1994): ssp. furcata var. palamaea (Ach.) Nyl.

26) aber auch: m. implexa Floerk. der var. racemosa?

27) furcata spadicea Fl. genannt

28) = zu var. furcata gehörend

29) Cl. furcata syrtica wird erwähnt

30) 2 Formen erwähnt: f. abortiva und f. monstrosa

31) f. finkii s. unter var. paradoxa

32) Checkliste Australien (in FeUERER 2003): var. cancellata Müll. Arg., Flora 65: 296 (1882), var. gracillima Müll. Arg., Flora 65: 296 (1882), var. hians Müll. Arg., Flora 65: 295 (1882), var. notabilis Müll. Arg., Flora 66: 18 (1883), var. virgulata Müll. Arg., Flora 66: 18 1883). Cl. furcata f. grandescens Nyl. in Crombie (Checkliste Argentinien in FEUERER 2003)

33) Checkliste Bayern, darin folgende Angaben: var. racemosa; var. racemosa f. squamulosa; var. subulata; var. subulata f. palamacea; $C l$. furcata f. corymbosa; Cl. furcata f. subulata ist Cl. subulata; Cl. furcata alpha racemosa * spinulosa; Cl. furcata alpha racemosa ** squamulosa; $\mathrm{Cl}$. furcata beta subulata * furcata; Cl. furcata beta subulata ** cymosa; $\mathrm{Cl}$. furcata delta stricta; $\mathrm{Cl}$. furcata gamma recurva; $\mathrm{Cl}$. furcata 1 . racemosa; $\mathrm{Cl}$. furcata 1 . racemosa $\mathrm{f}$. squamulosa; Cl. furcata 2. subulata.

\footnotetext{
* es wurden aus FLÖRKE (1828) lediglich die Bezeichnungen der Gestalttypen übernommen, ohne die systematische Einstufung zu beachten! Weitere in FlOERKE aufgeführte Bezeichnungen sind: tenuissima, cymosa, blastica, surrecta und polyphylla.
} 
Tab. 31: Übersicht der Varietäten und Formen von Cladonia furcata ssp. furcata

\begin{tabular}{|c|c|c|c|c|c|c|c|c|c|c|c|c|c|c|c|c|}
\hline & 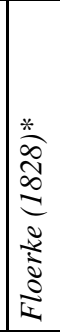 & 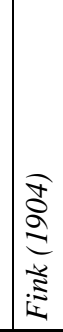 & 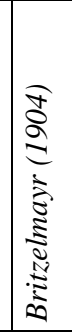 & 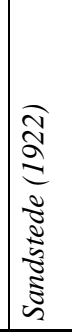 & 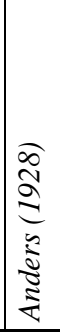 & 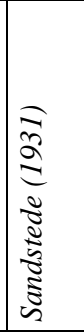 & 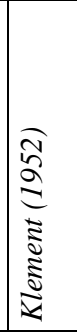 & 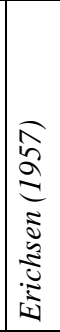 & 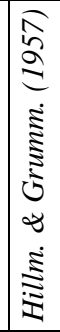 & 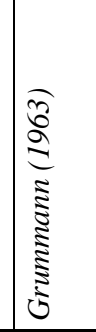 & 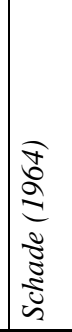 & 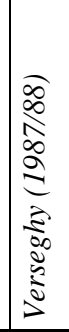 & 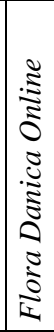 & 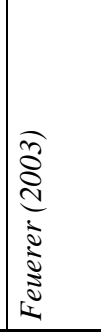 & 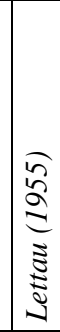 & 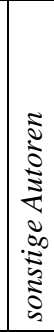 \\
\hline var. racemosa (Hoffm.) Flörke & + & + & + & + & + & + & + & + & + & 1a) & + & 2) & $\mathrm{O}$ & 3) & o & 1b) \\
\hline f. divulsa Klem. & - & - & - & - & - & - & + & - & - & 0 & 4) & - & - & - & - & - \\
\hline f. fissa Flörke & + & - & + & 5) & + & $+, 6)$ & $\mathrm{o}$ & + & + & - & $\mathrm{X}$ & - & - & - & $\mathrm{o}$ & - \\
\hline f. stricta Ach. & - & - & - & - & + & $+, 7)$ & $\mathrm{o}$ & - & - & - & - & $\mathrm{p}$ & - & - & - & - \\
\hline f. racemosella Flörke & - & - & - & $\mathrm{m}$ & - & + & - & + & - & - & $\mathrm{X}$ & - & - & - & - & - \\
\hline f. furcatosubulata (Hoffm.) Vain. & - & - & - & $\mathrm{m}$ & + & $8)$ & $\mathrm{o}$ & + & + & - & - & $\mathrm{p}$ & - & - & $\mathrm{o}$ & - \\
\hline f. squamulifera Sandst. & - & - & - & - & - & + & - & - & - & - & $\mathrm{m}$ & - & - & - & 0 & - \\
\hline f. flaccida Sandst. & - & - & - & - & - & + & - & + & - & - & - & - & - & - & - & - \\
\hline f. corymbosa (Ach.) Nyl. & - & - & + & $\mathrm{m}$ & 9) & 10) & o & + & + & - & $\mathrm{f}$ & $\mathrm{p}$ & - & 11) & o & - \\
\hline f. crispatella Flörke & + & - & - & - & - & + & - & - & - & - & $\mathrm{t}$ & - & - & - & - & - \\
\hline f. arbuscula Flörke & - & - & - & - & 12) & $+, 7)$ & 0 & + & - & - & - & $\mathrm{p}$ & - & - & - & - \\
\hline f. prolifera fo. nov. & - & - & - & - & - & - & - & - & - & - & - & - & - & - & - & 13) \\
\hline var. pinnata (Flörke) Vain. & - & + & 14) & - & + & + & + & + & + & $\mathrm{o}$ & + & 15) & - & 3) & & - \\
\hline f. abbreviata Scriba & - & - & - & - & - & + & - & - & - & - & $\mathrm{X}$ & - & - & - & $\mathrm{o}$ & - \\
\hline f. foliolosa Del. & - & - & - & 16) & + & + & - & + & - & $\mathrm{o}$ & $\mathrm{m}$ & $\mathrm{p}$ & - & & - & 17) \\
\hline f. truncata Flörke & + & + & + & - & + & + & - & + & - & - & $\mathrm{f}$ & $\mathrm{p}$ & - & - & $\mathrm{o}$ & - \\
\hline f. turgida Scriba & - & - & - & - & - & + & - & - & - & - & $\mathrm{X}$ & - & - & - & - & - \\
\hline f. recurva (Hoffm.) Flörke & + & - & - & 18) & + & + & $t, 19)$ & + & - & - & $\mathrm{t}$ & - & - & - & - & - \\
\hline f. regalis Flörke & + & - & 20) & 21) & + & + & - & - & - & $\mathrm{o}$ & $\mathrm{f}$ & ö & - & - & - & - \\
\hline f. intermedia Kusan & - & - & - & - & - & + & - & - & - & - & $\mathrm{f}$ & $\mathrm{p}$ & - & - & - & - \\
\hline f. robusta Kusan & - & - & - & - & - & + & - & - & - & - & $\mathrm{X}$ & ö & - & - & - & - \\
\hline f. subulata Flörke & + & - & 22) & 23) & + & - & - & - & - & - & - & - & - & - & - & - \\
\hline var. palamaea (Ach.) Nyl. & - & + & - & + & + & + & 0 & + & + & 24) & $\mathrm{t}$ & - & - & 3) & $\mathrm{o}$ & $25)$ \\
\hline f. implexa Flörke & + & - & 0 & $\mathrm{~m}$ & + & $+, 26)$ & - & - & - & - & $\mathrm{m}, \mathrm{t}$ & - & - & - & - & - \\
\hline f. ? subdecumbens & - & - & 0 & - & - & - & - & - & - & - & - & - & - & - & - & - \\
\hline f. simplex Flörke & - & - & - & - & - & - & - & + & - & - & - & - & - & - & - & - \\
\hline f. recurva Flörke & + & - & - & - & - & + & - & - & - & - & $\mathrm{t}$ & - & - & - & - & - \\
\hline f. spadicea (Ach.) Aigr. & + & - & 27) & - & + & $\mathrm{o}$ & - & + & - & - & $\mathrm{X}$ & - & - & - & - & - \\
\hline f. rigidula Mass. & - & - & - & - & + & + & - & + & - & $+, 28)$ & $\mathrm{m}$ & - & - & - & - & - \\
\hline f. syrtica Ohlert. & - & - & - & 29) & + & - & - & - & - & - & - & - & - & 3) & - & - \\
\hline f. conspersa Vain. & - & - & - & - & + & - & - & - & - & - & - & - & - & - & - & - \\
\hline var. paradoxa Vain. & - & + & - & + & - & + & - & - & - & - & $?$ & - & - & - & - & - \\
\hline var. tecta Savicz. & - & - & - & - & - & $+, 30)$ & - & - & - & - & - & - & - & - & - & - \\
\hline var. finckii Vain. & - & + & - & - & - & $31)$ & - & - & - & - & - & - & - & - & - & - \\
\hline var. crispata & + & - & - & - & - & - & - & - & - & - & - & - & 0 & - & - & - \\
\hline weitere Varietäten/Formen & + & - & - & - & - & - & - & - & - & - & - & - & - & $32,33)$ & - & - \\
\hline
\end{tabular}




\section{d) Alters- und Kümmerzustände}

Alters- und Kümmerzustände von Cladonia furcata ssp. furcata können abweichende Thallusmerkmale aufweisen, aufgrund derer einige möglicherweise als eigene Formen eingestuft wurden. Alters- oder ökologisch bedingt kann beispielsweise die Bildung von seitlichen Rissen sein (SCHADE 1964), wie sie für die var. racemosa f. fissa (ANDERS 1928, SANDSTEDE 1931, ERICHSEN 1957) typisch sind. Die var. racemosa f. divulsa (KLEMENT 1952) sowie die var. pinnata f. robusta (SANDSTEDE 1931) hält ScHADE (1964) aufgrund ihres Erscheinungsbildes für Alters-, die var. pinnata f. turgida und f. abbreviata (SANDSTEDE 1931) für Kümmerzustände von Cl. furcata.

VERSEGHY (1987/88) stuft viele der von anderen Autoren aufgeführten Varietäten und Formen als phänologische Stadien oder ökologische Modifikation ein und zweifelt damit deren systematischen Wert an. So ist ihrer Meinung nach die f. regalis (var. pinnata) lediglich eine sehr gut entwickelte Flechte an optimalen Standorten, ebenso die f. robusta. Im Gegensatz zu den Beobachtungen von SCHADE (1964) führt VERSEGHY (1987/88: 44) ein Beispiel für die var. pinnata f. robusta an, welches eine im „optimalen Verhältnisse“ gut entwickelte Flechte, also eine ökologische Modifikation darstellt. Weitere Beispiele für ökologische Modifikationen wären nach VERSEGHY die f. stricta (Desorganisationsstadium), die f. furcatosubulata (steriles Jugendstadium) und die f. corymbosa (Jugendstadium mit Apothecien), alle 3 der var. racemosa zugehörig, sowie die f. intermedia der var. pinnata (altes, gut entwickeltes, fertiles Stadium).

Weiterhin ist in den Beschreibungen der Formen immer wieder vom Einfluß der Standortfaktoren auf die Gestalt zu lesen. Einige Autoren geben explizit Hinweise auf Schattenformen und Lichtformen. Die f. flaccida der Varietät racemosa beispielsweise wird bei SANDSTEDE (1931) und ERICHSEN (1957) aufgeführt und von beiden Autoren als „Schattenpflanze“ bezeichnet. Auf die Auswirkungen der Standortfaktoren Licht und Tritt (mechanische Störungen) wird in Kap. G3.2 ausführlicher eingegangen.

\subsection{Cladonia furcata ssp. subrangiformis}

Nach WiRTH (1995) und SchOlZ (2000) wird die Atranorin-haltige Sippe basenreicher Standorte als Subspezies subrangiformis innerhalb der Art Cladonia furcata gewertet. Diese Einstufung soll in der vorliegenden Arbeit zunächst übernommen, anhand der in den nachfolgenden Kapiteln aufgeführten Untersuchungsergebnisse jedoch in Kap. I2 diskutiert werden. Andere Einstufungen der Sippe subrangiformis sind denkbar. In der Literatur finden sich die folgenden:

Cladonia subrangiformis Sandst.: z.B. SANDSTEDE (1922), PURVIS et al. (1992), AHTI (1993), SANTESSON (1993), COPPINS (2002); unveröffentlichtes Synonym: Cl. rangiformis var. hercynica Scriba (s. SANDSTEDE 1931, UlLRICH 1956, HILLMANN \& GRUMMANN 1957, SCHADE 1966).

Cladonia furcata var. subrangiformis (Sandst.) Hennipm.: HeNNIPMAN (1967/1978), FEUERER (2003, Checkliste Slowenien). 
Cladonia furcata m. subrangiformis (Sandst.) Schade: SCHADE (1966)

Die Anzahl an Varietäten und Formen, die für Cladonia furcata ssp. subrangiformis beschrieben wurden ist gering. In der ausgewerteten Literatur fanden sich lediglich Angaben zu einer Varietät sowie zu 5 Formen (s. Tab. 32).

Lettau (1955) liefert die Erstbeschreibung der Varietät praestigiosa. Das Aussehen dieser sterilen Flechte soll an Stereocaulon erinnern: zierlich, niedrigwüchsig, dicht „horstig“. Eventuell liege hierbei ein pathologischer Zustand vor. Der Lichenologe v. ZWACKH bezeichnete sie als „Cladonia pungens var. sorediophora“ (LETTAU 1955: 40). SANDSTEDE (1931) rechnete das betreffende Exemplar zu Cl. subrangiformis.

SANDSTEDE (1931), der die Sippe subrangiformis wie LETTAU (1955) als eigene Art ansieht, beschreibt die Forma vagans (= „Cladonia rangiformis f. vagans“, SANDSTEDE 1931:234) als eine Wanderflechte mit unförmig dicken, waagerecht ausgebreiteten Lagerstielen und kurzen, dicken, aufrechten Sprossungen. Weitere Merkmale seien die starke Berindung, die runzelige, eingerissene Podetienoberfläche und die dicht beieinander stehenden weißen Markaufbrüche. Eine f. vagans, allerdings innerhalb Cl. furcata var. subrangiformis, nennt auch VERSEGHY (1975). Als weitere Form der var. subrangiformis taucht in dieser Arbeit die f. pustarum auf. In VERSEGHYs Veröffentlichung aus dem Jahre 1987/88 findet sich diese Form ebenfalls wieder, nun jedoch innerhalb von Cl. furcata ssp. subrangiformis. SzATALA (1938) hatte sie als Cl. subrangiformis f. pustarum beschrieben. 3 weitere Formen werden in SzATALA (1938) aufgeführt.

Tab. 32: Übersicht der Varietäten und Formen von Cladonia furcata ssp. subrangiformis (Legende s. Tab. 31)

\begin{tabular}{|c|c|c|c|c|c|c|}
\hline & 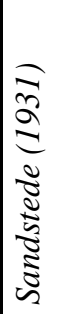 & 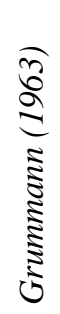 & 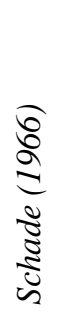 & 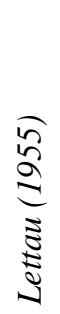 & 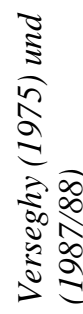 & 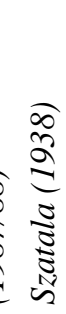 \\
\hline var. praestigiosa Lett. nova var. & - & $\mathrm{o}$ & - & + & - & - \\
\hline f. pustarum (Szat.) comb. nov. & - & - & - & - & o & + \\
\hline f. vagans Tomin n.f. & + & o & o & - & o & - \\
\hline f. foliofera Szatala 1938 & - & - & - & - & - & + \\
\hline f. subuliformis Szatala 1938 & - & - & - & - & - & + \\
\hline f. truncatula Szatala 1938 & I & - & - & - & - & + \\
\hline
\end{tabular}

\subsection{Ein kurzer Vergleich mit der Variabilität anderer Cladonia-Arten}

Neben Cladonia furcata sind zahlreiche weitere Arten der Gattung für eine große morphologische Variabilität bekannt. Als Beispiele seien hier Cl. gracilis, Cl. squamosa und Cl. crispata genannt. Eine Vielzahl von Varietäten und Formen innerhalb dieser Sippen beschreibt SANDSTEDE (1931). Der von ihm verfaßte Band über die Gattung Cladonia soll als Grundlage des nachfolgenden Vergleichs dienen. SANDSTEDE (1931) unterteilt Cl. crispata in 5 Varietäten (mit insgesamt 15 Formen), Cl. gracilis in ebenfalls 5 Varietäten (mit insgesamt 27 Formen, inklusive 4 bei ANDERS (1928) 
genannter Formen der „Cl. elongata“) und Cl. squamosa in 3 Varietäten (mit insgesamt 25 Formen).

Die Merkmale aufgrund derer bei Cladonia gracilis, Cl. crispata und Cl. squamosa eine weitere Unterteilung vollzogen wurde, entsprechen in vielen Fällen denen, die auch bei Cl. furcata ssp. furcata eine Rolle spielen. So treten sowohl bei Cl. gracilis als auch bei Cl. crispata innerhalb der Varietäten Formen auf, die durch eine auffallende Beschuppung gekennzeichnet sind. Auch bei Cl. squamosa wird häufig auf die Beblätterung (bzw. Beschuppung) eingegangen, jedoch spielen bei dieser Art nicht nur das Auftreten bzw. Fehlen von Blättchen, sondern z.B. auch deren Form und Größe eine Rolle. Die Fruchtkörperbildung bzw. die Anordnung der Apothecien kann bei einzelnen Formen ein wesentliches oder auch nur ergänzendes Merkmal sein. Analog zu Cl. furcata ssp. furcata treten Formen auf, die durch dornige, eingekrümmte Sprosse gekennzeichnet sind, z.B. innerhalb der Cl. crispata var. cetrariaeformis oder der Cl. gracilis var. chordalis. Da sowohl Cl. gracilis als auch Cl. crispata und Cl. squamosa Becher ausbilden können, spielt das Vorhandensein oder Fehlen, neben der Gestalt der Becher, bei der Unterteilung dieser Arten eine wesentliche Rolle. Solche Merkmale fehlen bei Cl. furcata naturgemäß. Daneben werden bei allen diesen Arten weitere Merkmale, wie die Wuchsform der Podetien (kümmerlich oder hochwüchsig) und die Pigmentierung als Kennzeichen zur Unterteilung verwendet. Die Möglichkeit des Übergehens von einer Form/Varietät in eine andere im Rahmen einer fortschreitenden Entwicklung der Thalli oder aufgrund von Standortveränderungen wird bei SANDSTEDE ebenfalls angesprochen.

SANDSTEDE (1931) revidiert Exsiccate, die ursprünglich fälschlicherweise zu Cladonia furcata gestellt wurden, eigentlich aber der Cl. gracilis bzw. Cl. crispata zugehörig sind und weist wiederholt auf die starke Ähnlichkeit von Formen der Sippen Cl. gracilis und Cl. crispata mit Cl. furcata hin: „In Moospolster eingeschlossene, schlanke Lagerstiele der Cl. crispata var. gracilescens, Cl. furcata f. furcato-subulata, Cl. gracilis f. chordalis m. leucochlora können sich derart ähneln, daß man nicht hin und her weiß (...)“(SANDSTEDE 1931:3).

\section{Morphologie und morphologische Variabilität in Nordhessen}

\subsection{Allgemeine Beschreibung der Proben}

Bei Cladonia furcata ssp. furcata handelt es sich um eine Strauchflechte, die lockere bis dichte Polster bilden, jedoch auch in Form von einzelnen miteinander verflochtenen und dem Boden anliegenden Podetien auftreten kann. Die Farbe des Lagers variiert im Untersuchungsgebiet, je nach Grad der Pigmentierung, von grüngrau bis braungrau. Einfluß auf die Lagerfarbe hat zudem die Ausdehnung der Algenschicht. Sie kann nur stellenweise ausgebildet sein, die Podetien erscheinen dann gefleckt, oder fast den gesamten Podetienkörper durchziehen, was zu einer grünlichen Färbung führt (vgl. auch Abb. 32, S. 146). Grundschuppen sind bei der Mehrzahl der Polster nicht oder kaum 
mehr erkennbar. Proben mit zahlreichen Grundschuppen sind eher die Ausnahme. Die Podetien sind stielrund bis leicht abgeflacht oder auch von Rissen durchzogen bis röhrig geöffnet. Die Länge der ausgemessenen Podetien liegt bei gut entwickelten Polstern zwischen 15 und $92 \mathrm{~mm}$, der Durchmesser zwischen 0,4 und 3,2 $\mathrm{mm}$. Thalli, bei denen die Grundschuppen dominieren, weisen dagegen in der Regel nur wenige Millimeter lange Podetien auf. Die Lagerstiele verzweigen sich zur Spitze hin ein- bis 14 mal. Am Podetienende treten 1 bis 30 Zweige auf. Die Podetienoberfläche ist glatt oder zeigt wenige bis zahlreiche (max. 85) schuppenartige Auswüchse. Diese können bis zu 4,2 mm groß werden. Fibrillen kommen ebenfalls gelegentlich in großer Zahl vor. Als Fruchtkörper treten Apothecien auf, deren Farbe von hellem Braun bis dunkelbraun reicht. Maximal wurden 280 Apothecien pro Podetium gezählt. Die Fruchtkörper erreichen einen Durchmesser von bis zu $2 \mathrm{~mm}$. Als Organe, in denen asexuelle Keime gebildet werden, weist $C l$. furcata ssp. furcata relativ häufig Pyknidien auf. Diese sitzen an den Enden der Podetien, sind meist schwarz, gelegentlich jedoch auch mittelbraun gefärbt und bei guter Entwicklung urnenförmig mit deutlicher Öffnung (= Ostiolum).

Eine Probe der Subspezies furcata, vom Typ her einer palamaea entsprechend, zeigt an der Basis einiger Podetien weiße Markaufbrüche, wie sie eigentlich für die Unterart subrangiformis typisch sind (z.B. WIRTH 1995). In den betreffenden Lagerstielen ließ sich aber mittels Dünnschichtchromatographie kein Atranorin nachweisen, weswegen sie Cl. furcata ssp. furcata zugeordnet wurden. Die betreffende Probe (s. Taf. 2.12 im Anhang 2) stammt von einem Basaltstandort (Maderstein, MTB 4822).

Zum Vergleich werden nachfolgend die ebenfalls im Untersuchungsgebiet erhobenen Daten von 47 Podetien der Subspezies subrangiformis aufgeführt. Die Wuchsform dieser Unterart unterscheidet sich insofern von der Subspezies furcata, als daß viel seltener aufrecht wachsende Polster auftreten. In der Regel sind die Thalli niedergedrückt, dem Boden anliegend oder sie erheben sich nur wenig über die Bodenoberfläche. Die Mehrzahl der Podetien ist deutlich pigmentiert und daher von bräunlicher Farbe. Gelegentlich treten fast schwarze Lagerstiele oder aber, im Schatten der Phanerogamenvegetation, auch nahezu unpigmentierte Podetien auf. Die Algenschicht durchzieht das Podetium meist großflächig. Grundschuppen wurden bei diesen Proben nicht beobachtet. Die Podetien sind stielrund, selten etwas flachgedrückt und nur ausnahmsweise aufgerissen. Sie erreichen eine Länge von maximal $81 \mathrm{~mm}$ und einen Durchmesser von bis zu $2 \mathrm{~mm}$, durchschnittlich sind sie aber nur etwa $46 \mathrm{~mm}$ lang und 1,3 mm dick. Der geringste gemessene Durchmesser betrug 0,7 mm. Die Lagerstiele verzweigen sich zwischen einem- und achtmal. Am Podetienende treten meist 2 bis 3, maximal 6 Zweige auf. Eine Beschuppung ist bei Cladonia furcata ssp. subrangiformis viel seltener und in viel geringerem Maße zu beobachten als bei der ssp. furcata. Gezählt wurden maximal 8 Schuppen mit höchstens 1,7 mm Größe. Meistens treten jedoch gar keine Schuppen auf. Fibrillen sind ebenfalls die Ausnahme und nie in großer Zahl vorhanden. Eine Apothecienbildung zeigten einige der untersuchten Exemplare, allerdings waren die Fruchtkörper noch nicht vollständig entwickelt. Nur ein einziges Podetium trug ein Pyknidium. Die für Cl. furcata ssp. subrangiformis als 
charakteristisches Merkmal geltenden Markaufbrüche (s.o.) ließen sich im Rahmen dieser stichprobenhaften Untersuchung bei gut der Hälfte der Podetien nachweisen.

\subsection{Abhängigkeiten der morphologischen Merkmale von den Standortbedingungen}

\section{a) Podetienlänge, Podetiendurchmesser und Verzweigungsgrad}

(Abb. 30; Tab. 61 bis Tab. 64 im Anhang 1)

Die im Mittel längsten Podetien $(51,7 \mathrm{~mm})$ haben die Polster der Heidestandorte der tieferen Lagen. Die mit durchschnittlich 36,6 mm Länge kürzesten Podetien kommen auf Felsstandorten vor. Sie sind signifikant kürzer als an den anderen Standorttypen. Den größten mittleren Podetiendurchmesser von 1,4 mm haben die Proben der Waldränder und Wälder. Die Mittelwerte der anderen Standorttypen liegen etwas darunter. Signifikant ist nur der Unterschied zwischen den Werten der Wälder und der Felsen. Polster mit relativ stark verzweigten Podetien (durchschnittlich 6,3 bis 7,1 Verzweigungen) kommen in den Wäldern, an Waldrändern und in den Heiden vor. Weniger verzweigt (mit im Mittel nur 5,0 bzw. 5,6 Verzweigungen) sind die Podetien auf den Felsen bzw. in den Berglagen. Diese Unterschiede sind signifikant zwischen den Standorttypen Felsen und Waldrändern, zwischen Heiden und Berglagen und zwischen Wäldern und Berglagen. Bei der Anzahl Zweige an den Podetienspitzen zeigen sich kaum nennenswerte Unterschiede. Die Mittelwerte liegen hier zwischen 4,6 und 5,7 Zweigen.

b) Beschuppung (Abb. 30; Tab. 65 und Tab. 66 im Anhang 1)

Die am stärksten beschuppten Exemplare sind in den schattigen Wäldern zu finden. Der Mittelwert für diesen Standorttyp liegt mit 17,0 deutlich über den anderen Werten, allerdings streuen die Werte innerhalb des Standorttyps Wälder ziemlich stark: der kleinste Durchschnittswert eines Polsters beträgt 0,3, der größte 48,7 Schuppen (s. Tab. $66 \mathrm{im}$ Anhang 1). Signifikant ist der Unterschied zwischen Wäldern einerseits sowie Felsen, Heiden und Waldrändern andererseits. Die Polster der Felsstandorte beispielsweise tragen im Mittel lediglich maximal 4,0 Schuppen pro Podetium, die der Heiden sogar nur 0,5. Auch werden die Schuppen in den Wäldern größer. Im Mittel sogar signifikant größer als an den Heide-Standorten. Die zugehörigen Mittelwerte dieser beiden Typen betragen 1,4 bzw. 0,7 mm.

\section{c) Apothecien und Pyknidien (Abb. 31; Tab. 67 bis Tab. 69 im Anhang 1)}

Die Polster, deren Podetien durchschnittlich am zahlreichsten Apothecien ausbilden, stammen von Standorten des Typs Heide (MW 38,8) oder des Typs Waldrand (MW 36,4). In beiden Fällen streuen die Werte jedoch ziemlich stark: die einzelnen PolsterMittelwerte liegen zwischen 0 und 78,2 in den Heiden bzw. 0 und 115,7 Fruchtkörpern an den Waldrändern (s. Tab. 67 im Anhang 1). Die wenigsten Apothecien, durchschnittlich nur 6,7 Stück, zeigen sich an Polstern aus den Wäldern. Der Unterschied zwischen Wäldern und Heiden ist signifikant. Auch an den trockensten 
Stellen, den Felsstandorten, bilden sich relativ wenige Fruchtkörper aus. Die größten Durchmesser von im Mittel 0,5 mm erreichen Apothecien an Heide-Standorten. Mit nur 0,2 $\mathrm{mm}$ ziemlich geringe Werte ergeben sich dagegen bei den Standorttypen Felsen, Berglagen und Wäldern. Statistisch absichern läßt sich der Unterschied zwischen Felsen und Heiden. Pyknidientragende Podetien nehmen in Polstern der schattigen Wälder mit $73,1 \%$ einen signifikant höheren Anteil ein, als in Polstern von Felsstandorten (47,8 \%). Die Werte der 3 anderen Standorttypen liegen etwa im Bereich des Wertes der Felsen.

d) Weitere Podetienmerkmale (Abb. 31; Tab. 70 bis Tab. 72 im Anhang 1)

Polster, deren Podetien zahlreiche Fibrillen aufweisen, sind für die Standorttypen Felsen und Heiden charakteristisch. Sie haben dort einen Anteil von durchschnittlich 7,8 bzw. 10,0 \%. Mit Mittelwerten von 0 bis 3,6 \% ist der Anteil derartiger Lagerstiele an den anderen Standorten deutlich geringer. Statistisch signifikant ist der Unterschied zwischen Felsen und Waldrändern. Die Polster der hellsten Standorte (Felsen und Heiden) haben erwartungsgemäß den höchsten Anteil stark pigmentierter Podetien. Der Wert der Felsen (34,8\%) ist signifikant höher als die Werte der 3 zumindest gelegentlich beschatteten Standorte, an deren Polstern dunkelbraune Podetien lediglich einen Anteil von durchschnittlich 1,1 bis 5,7 \% haben. Bei beiden Merkmalen Fibrillenzahl und Pigmentierung variieren allerdings die einzelnen Werte der Heideund Felsstandorte stark, erkennbar an der relativ hohen Standardabweichung. Die geringste Ausdehnung der Algenschicht, d.h. das fleckigste Aussehen zeigen die Podetien der Heiden, während an den anderen Standorten die Algenschicht in den Lagerstielen viel häufiger eine Ausdehnung von mehr als $75 \%$ erreicht. Die Unterschiede zwischen den Werten der Heiden (MW 5,0\%) auf der einen und den Werten der Felsen, Berglagen und Wälder (MW 23,3 bis 41,7 \%) auf der anderen Seite sind signifikant. 

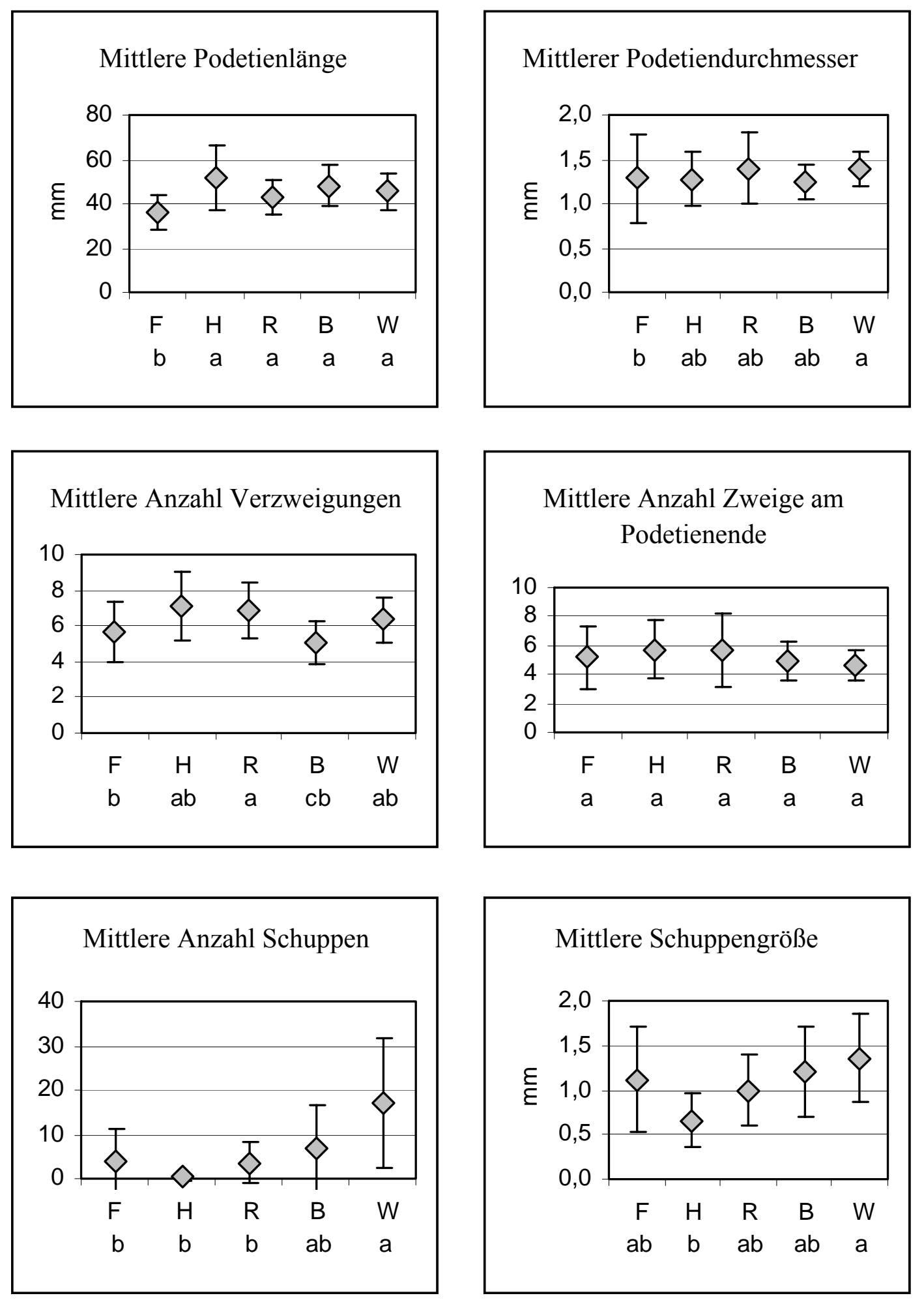

Abb. 30: Mittelwerte und Standardabweichungen der Podetienmerkmale Podetienlänge,

Podetiendurchmesser, Verzweigungen und Beschuppung. (Abkürzungen s. S. 113). 


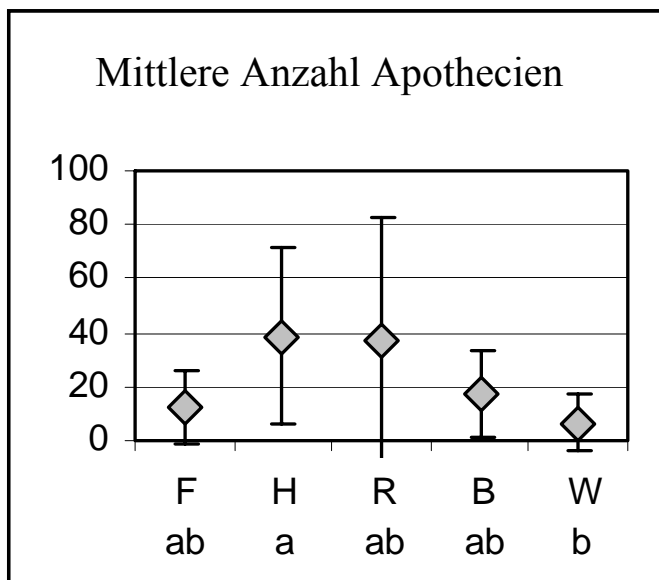

Mittlere Größe der Apothecien

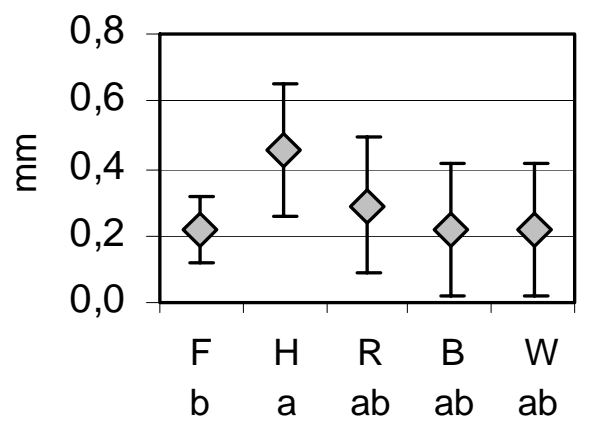

Mittlerer Anteil Podetien mit Pyknidien

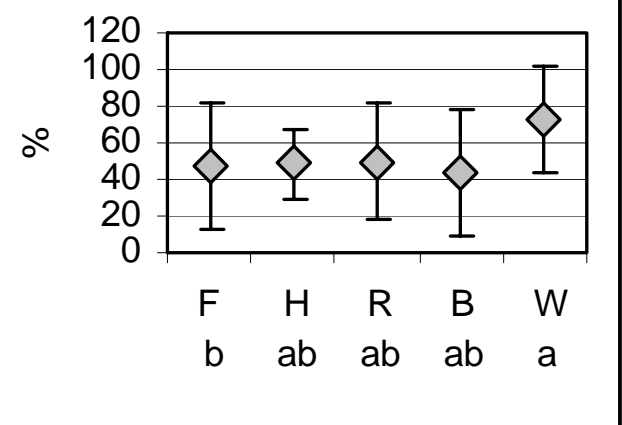

Mittl. Anteil stark pigmentierter Podetien

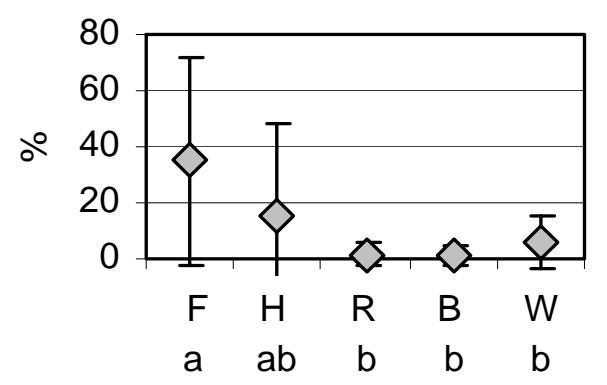

Mittl. Anteil Podetien mit zahlreichen Fibrillen

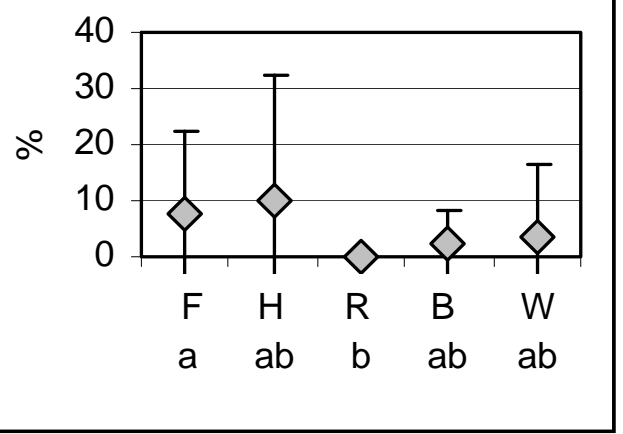

Mittl. Anteil Podetien mit $>75 \%$ bedeckender Algenschicht

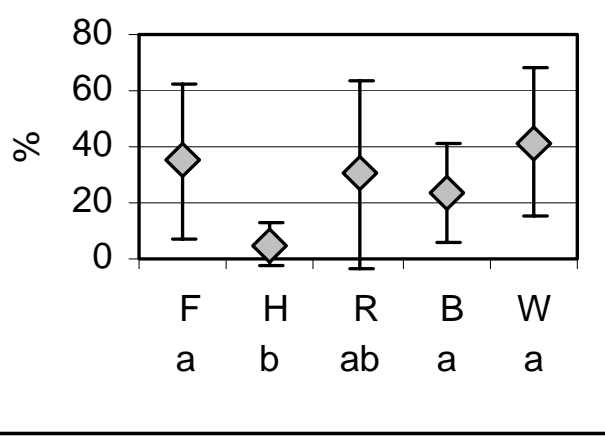

Abb. 31: Mittelwerte und Standardabweichungen der Podetienmerkmale Apothecien, Pyknidien, Fibrillen und Algenschicht. (Abkürzungen s. S. 113). 


\section{e) Auswirkung mechanischer Störungen}

Die Unterteilung erfolgt in 3 Kategorien von sehr geringer bis sehr starker mechanischer Einwirkung (vgl. Kap. G1.1.1). Tab. 33 ist zu entnehmen, daß keiner der Standorttypen nur einer Kategorie angehört. Die meisten Fels- und Heidestandorte weisen jedoch einen mäßigen bis starken Tritteinfluß (bzw. deutliche Substratinstabilität) auf, während die Waldstandorte eher weniger trittbelastet sind.

Tab. 33: Anteile der 3 Kategorien mechanischer Störung an den Standorttypen [\%]

\begin{tabular}{l|lll}
\hline mechanische Störung & sehr gering & mäßig stark & sehr stark \\
\hline Felsen $(\mathrm{F}) \mathrm{n}=23$ & 4 & 48 & 48 \\
Heiden $(\mathrm{H}) \mathrm{n}=6$ & 0 & 83 & 17 \\
Waldränder (R) $\mathrm{n}=13$ & 23 & 62 & 15 \\
Berglagen (B) $\mathrm{n}=9$ & 22 & 33 & 44 \\
Wälder (W) $\mathrm{n}=14$ & 57 & 29 & 14 \\
\hline
\end{tabular}

Die Auswertung der Daten mit Hilfe des U-Tests ergab nur in Ausnahmefällen signifikante Unterschiede (s. Tab. 34): Der Wert der Podetienlänge beträgt an den wenig trittbeeinflußten Standorten $48,4 \mathrm{~mm}$ und ist damit signifikant größer als an den trittbelasteten Stellen, wo im Mittel bei starker Störung nur 38,7 mm erreicht werden. Der Podetiendurchmesser ist mit durchschnittlich $1,1 \mathrm{~mm}$ an den am stärksten beeinträchtigten Stellen signifikant geringer als an den weniger belasteten (1,3 bzw. 1,5 mm). Die Größe der Schuppen unterscheidet sich signifikant zwischen den sehr wenig und den sehr stark gestörten Standorten (1,4 mm gegenüber 1,0 mm), ebenso der Anteil pyknidientragender Podetien (71,4\% gegenüber 38,7\%). Bei allen anderen Podetienmerkmalen wurden keine signifikanten Unterschiede festgestellt.

Tab. 34: Mittelwerte der Podetienmerkmale in den 3 Kategorien mechanischer Störung

\begin{tabular}{l|lll}
\hline mechanische Störung & sehr gering & mäßig stark & sehr stark \\
\hline max. Podetienlänge [mm] & $48,4^{\mathrm{a}^{* * *}}$ & $43,1^{\mathrm{b}}$ & $38,7^{\mathrm{b}}$ \\
max. Podetiendurchmesser [mm] & $1,3^{\mathrm{a}^{* * *}}$ & $1,5^{\mathrm{a}^{* * *}}$ & $1,1^{\mathrm{b}}$ \\
Anzahl Verzweigungen & $6,0^{\mathrm{a}}$ & $6,4^{\mathrm{a}}$ & $5,6^{\mathrm{a}}$ \\
Anzahl Zweige am Podetienende & $4,7^{\mathrm{a}}$ & $5,7^{\mathrm{a}}$ & $4,8^{\mathrm{a}}$ \\
max. Anzahl Schuppen & $9,5^{\mathrm{a}}$ & $5,8^{\mathrm{a}}$ & $6,4^{\mathrm{a}}$ \\
max. Größe Schuppen [mm] & $1,4^{\mathrm{a}^{*}}$ & $1,1^{\mathrm{ab}}$ & $1,0^{\mathrm{b}}$ \\
Anzahl Apothecien & $14,7^{\mathrm{a}}$ & $23,3^{\mathrm{a}}$ & $15,8^{\mathrm{a}}$ \\
max. Größe Apothecien [mm] & $0,2^{\mathrm{a}}$ & $0,3^{\mathrm{a}}$ & $0,3^{\mathrm{a}}$ \\
Anteil pyknidientragender Podetien [\%] & $71,4^{\mathrm{a}^{* *}}$ & $54,4^{\mathrm{ab}}$ & $38,7^{\mathrm{b}}$ \\
Anteil Podetien mit zahlreichen Fibrillen [\%] & $1,4^{\mathrm{a}}$ & $4,8^{\mathrm{a}}$ & $7,0^{\mathrm{a}}$ \\
Anteil stark pigmentierter Podetien [\%] & $9,3^{\mathrm{a}}$ & $12,0^{\mathrm{a}}$ & $25,0^{\mathrm{a}}$ \\
Anteil Podetien mit > 75 \% deckender Algenschicht [\%] & $34,3^{\mathrm{a}}$ & $33,3^{\mathrm{a}}$ & $25,2^{\mathrm{a}}$ \\
\hline
\end{tabular}

Signifikanzniveau: $* \mathrm{p} \leq 0,05 ; * * \mathrm{p} \leq 0,01 ; * * * \mathrm{p} \leq 0,001$;

Stichprobenumfang $\mathrm{n}$ siehe Tab. 61 bis Tab. 72 im Anhang 1 


\section{f) Podetienform}

Tab. 35 listet auf, in wieviel Prozent der Polster geschlitzte bzw. offen-rinnige Podetien auftreten. Bei beiden Merkmalsausprägungen lassen sich keine Tendenzen von sonnigtrockenen zu schattig-frischen Standorten erkennen. Auffallend häufig treten allerdings in den Polstern der Heiden offen-rinnige Podetien auf. Geschlitzte Podetien sind öfter an wenig trittbelasteten als an häufig gestörten Standorten anzutreffen.

Tab. 35: Häufigkeit geschlitzter bzw. offen-rinniger Podetien in Abhängigkeit von Standorttyp und mechanischer Störung

\begin{tabular}{l|ll}
\hline Standorttyp & geschlitzte Podetien & offen-rinnige Podetien \\
\hline Felsen $(\mathrm{n}=23)$ & $61 \%$ & $17 \%$ \\
Heiden $(\mathrm{n}=6)$ & $50 \%$ & $83 \%$ \\
Waldränder $(\mathrm{n}=13)$ & $31 \%$ & $46 \%$ \\
Berglagen $(\mathrm{n}=9)$ & $78 \%$ & $33 \%$ \\
Wälder $(\mathrm{n}=14)$ & $64 \%$ & $21 \%$ \\
\hline mechan. Störung & geschlitzte Podetien & offen-rinnige Podetien \\
\hline sehr gering $(\mathrm{n}=14)$ & $79 \%$ & $36 \%$ \\
mäßig stark $(\mathrm{n}=31)$ & $61 \%$ & $39 \%$ \\
sehr stark $(\mathrm{n}=20)$ & $35 \%$ & $20 \%$ \\
\hline
\end{tabular}

\subsection{Abhängigkeiten und Korrelationen der morphologischen Merkmale untereinander}

\section{a) Vergleich fertiler und steriler sowie pyknidientragender und pyknidienloser} Podetien (Tab. 36; Tab. 73 und Tab. 74 im Anhang 1)

Signifikante Unterschiede zwischen fruchtenden und nicht fruchtenden Podetien zeigen sich bei der Podetienlänge, der Anzahl Verzweigungen, der Anzahl Zweige am Podetienende sowie der Anzahl und Größe der Schuppen. Apothecientragende Lagerstiele sind im Durchschnitt länger, stärker verzweigt und weisen am Podetienende deutlich mehr Äste auf als sterile Podetien. Dagegen ist die Anzahl und Größe der Schuppen bei den nicht fruchtenden Lagerstielen im Mittel höher.

Tab. 36: Mittelwerte der Podetienmerkmale bei fertilen und sterilen bzw. bei pyknidientragenden und pyknidienlosen Podetien

\begin{tabular}{l|ll|ll}
\hline & fruchtend & nicht fruchtend & mit Pyknidien & ohne Pyknidien \\
\hline max. Podetienlänge [mm] & $44,9^{\mathrm{a}^{* * *}}$ & $41,2^{\mathrm{b}}$ & $43,4^{\mathrm{a}}$ & $42,5^{\mathrm{a}}$ \\
max. Podetiendurchmesser [mm] & $1,3^{\mathrm{a}}$ & $1,3^{\mathrm{a}}$ & $1,4^{\mathrm{a}^{* * *}}$ & $1,3^{\mathrm{b}}$ \\
Anzahl Verzweigungen & $6,5^{\mathrm{a}^{* * *}}$ & $5,5^{\mathrm{b}}$ & $6,1^{\mathrm{a}}$ & $5,9^{\mathrm{a}}$ \\
Anzahl Zweige am Podetienende & $6,7^{\mathrm{a}^{* * *}}$ & $3,7^{\mathrm{b}}$ & $5,2^{\mathrm{a}}$ & $5,2^{\mathrm{a}}$ \\
max. Anzahl Schuppen & $4,8^{\mathrm{b}}$ & $9,0^{\mathrm{a}^{* * *}}$ & $7,9^{\mathrm{a}}$ & $5,8^{\mathrm{a}}$ \\
max. Größe Schuppen [mm] & $1,1^{\mathrm{b}}$ & $1,4^{\mathrm{a}^{*}}$ & $1,5^{\mathrm{a}^{* * *}}$ & $1,1^{\mathrm{b}}$ \\
Anzahl Apothecien & - & - & $15,1^{\mathrm{b}}$ & $25,5^{\mathrm{a}^{* *}}$ \\
max. Größe Apothecien [mm] & - & - & $0,3^{\mathrm{a}}$ & $0,3^{\mathrm{a}}$ \\
\hline
\end{tabular}

Signifikanzniveau: $* \mathrm{p} \leq 0,05 ; * * \mathrm{p} \leq 0,01 ; * * * \mathrm{p} \leq 0,001$;

Stichprobenumfang n siehe Tab. 73 und Tab. 74 im Anhang 1 
Pyknidientragende Podetien haben durchschnittlich einen signifikant größeren Durchmesser und signifikant größere Schuppen als pyknidienlose Lagerstiele. Letztere weisen dagegen im Mittel eine signifikant höhere Anzahl Apothecien auf.

\section{b) Vergleich unterschiedlich stark mit Fibrillen besetzter und pigmentierter} Podetien (Tab. 37; Tab. 75 und Tab. 77 im Anhang 1)

Stark mit Fibrillen besetzte Podetien zeichnen sich durch einen im Mittel größeren Durchmesser und eine stärkere Verzweigung aus. Die Unterschiede sind jeweils signifikant. Lagerstiele ohne Fibrillen haben signifikant höhere Durchschnittswerte der Zweige am Podetienende, der Anzahl Apothecien und der Größe der Apothecien als Podetien mit wenigen Fibrillen. Auch zwischen den Podetien mit zahlreichen Fibrillen und denen mit wenigen Fibrillen zeigt sich ein signifikanter Unterschied beim Mittelwert der Apothecienzahl. Dieser ist bei den stark mit Fibrillen besetzten Lagerstielen höher. Der Mittelwert der Schuppenzahl ist bei ihnen dagegen signifikant geringer als bei den Podetien der beiden anderen Kategorien.

Einige signifikante Unterschiede zeigen sich auch beim Vergleich unterschiedlich pigmentierter Lagerstiele: Weniger gebräunte Podetien sind im Mittel länger und dicker als stärker gebräunte und haben durchschnittlich größere Schuppen. Die unpigmentierten weisen darüber hinaus mehr Schuppen auf als die wenig bis stark pigmentierten, auch dieser Unterschied ist signifikant. Die mittlere Anzahl Apothecien ist bei den schwach pigmentierten signifikant höher als bei den stark gebräunten Exemplaren.

Tab. 37: Mittelwerte der Podetienmerkmale unterschiedlich stark mit Fibrillen besetzter bzw. pigmentierter Podetien

\begin{tabular}{|c|c|c|c|c|c|c|}
\hline & $\begin{array}{l}\text { Fibrillen } \pm \\
\text { fehlend }\end{array}$ & $\begin{array}{l}\text { wenige } \\
\text { Fibrillen }\end{array}$ & $\begin{array}{l}\text { zahlreiche } \\
\text { Fibrillen }\end{array}$ & $\begin{array}{l}\text { nicht } \\
\text { gebräunt }\end{array}$ & $\begin{array}{l}\text { wenig } \\
\text { gebräunt }\end{array}$ & $\begin{array}{l}\text { stark } \\
\text { gebräunt }\end{array}$ \\
\hline max. Podetienlänge [mm] & $42,9^{\mathrm{a}}$ & $43,2^{\mathrm{a}}$ & $43,3^{\mathrm{a}}$ & $43,8^{\mathrm{a}^{* * *}}$ & $43,9^{\mathrm{a}^{* * *}}$ & $37,7^{b}$ \\
\hline max. Podetiendurchmesser [mm] & $1,3^{b}$ & $1,3^{b}$ & $1,5^{\mathrm{a}^{* * *}}$ & $1,4^{\mathrm{a}^{* * *}}$ & $1,3^{\mathrm{a}^{* * *}}$ & $1,2^{b}$ \\
\hline Anzahl Verzweigungen & $5,8^{\mathrm{c}}$ & $6,4^{\mathrm{b}^{* *}}$ & $7,8^{\mathrm{a}^{* / * * *}}$ & $6,0^{\mathrm{a}}$ & $6,0^{\mathrm{a}}$ & $5,9^{\mathrm{a}}$ \\
\hline Anzahl Zweige am Podetienende & $5,4^{\mathrm{a}^{* * *}}$ & $4,4^{b}$ & $5,1^{\mathrm{ab}}$ & $4,7^{\mathrm{b}}$ & $5,4^{\mathrm{a}^{*}}$ & $5,6^{\mathrm{ab}}$ \\
\hline max. Anzahl Schuppen & $6,1^{\mathrm{a}^{*}}$ & $11,5^{\mathrm{a}^{* *}}$ & $2,8^{\mathrm{b}}$ & $14,0^{\mathrm{a}^{* * *}}$ & $3,7^{\mathrm{b}}$ & $3,5^{\mathrm{b}}$ \\
\hline max. Größe Schuppen [mm] & $1,3^{\mathrm{a}}$ & $1,3^{\mathrm{a}}$ & $1,1^{\mathrm{a}}$ & $1,4^{a^{* *}}$ & $1,3^{\mathrm{a}^{*}}$ & $0,9^{\mathrm{b}}$ \\
\hline Anzahl Apothecien & $22,7^{\mathrm{a}^{* * *}}$ & $6,3^{\mathrm{b}}$ & $12,8^{\mathrm{a}^{*}}$ & $16,3^{\mathrm{ab}}$ & $24,2^{\mathrm{a}^{* *}}$ & $8,5^{\mathrm{b}}$ \\
\hline max. Größe Apothecien [mm] & $0,3^{\mathrm{a}^{\mathrm{***}}}$ & $0,1^{\mathrm{b}}$ & $0,2^{\mathrm{ab}}$ & $0,3^{\mathrm{a}}$ & $0,3^{\mathrm{a}}$ & $0,2^{\mathrm{a}}$ \\
\hline
\end{tabular}

Signifikanzniveau: $* \mathrm{p} \leq 0,05 ; * * \mathrm{p} \leq 0,01 ; * * * \mathrm{p} \leq 0,001$;

Stichprobenumfang $\mathrm{n}$ siehe Tab. 75 und Tab. 77 im Anhang 1

\section{c) Vergleich von Podetien mit unterschiedlich stark ausgedehnter Algenschicht} (Tab. 38 und Tab. 78 im Anhang 1)

Es sind nur in zwei Fällen signifikante Unterschiede erkennbar. Podetien, die sich durch eine mehr als $75 \%$ der Oberfläche einnehmende Algenschicht auszeichnen, haben im Mittel die größte Länge und einen relativ großen Durchmesser. Signifikant sind hierbei nur die Unterschiede zu den Podetien der nächst niedrigeren Kategorie, also denen mit 50 bis $75 \%$ Ausdehnung. 
Tab. 38: Mittelwerte der Podetienmerkmale von Podetien mit unterschiedlich ausgedehnter Algenschicht

\begin{tabular}{l|llll}
\hline Ausdehnung der Algenschicht & bis 25\% & bis 50\% & bis 75\% & $>75 \%$ \\
\hline max. Podetienlänge [mm] & $34,5^{\mathrm{ab}}$ & $41,5^{\mathrm{ab}}$ & $42,4^{\mathrm{b}}$ & $44,5^{\mathrm{a}^{*}}$ \\
max. Podetiendurchmesser [mm] & $2,0^{\mathrm{ab}}$ & $1,3^{\mathrm{ab}}$ & $1,3^{\mathrm{b}}$ & $1,4^{\mathrm{a}^{* *}}$ \\
Anzahl Verzweigungen & $3,5^{\mathrm{a}}$ & $5,7^{\mathrm{a}}$ & $6,0^{\mathrm{a}}$ & $6,2^{\mathrm{a}}$ \\
Anzahl Zweige am Podetienende & $6,5^{\mathrm{a}}$ & $5,5^{\mathrm{a}}$ & $5,0^{\mathrm{a}}$ & $5,5^{\mathrm{a}}$ \\
max. Anzahl Schuppen & $0,0^{\mathrm{a}}$ & $3,9^{\mathrm{a}}$ & $7,3^{\mathrm{a}}$ & $7,1^{\mathrm{a}}$ \\
max. Größe Schuppen [mm] & - & $1,1^{\mathrm{a}}$ & $1,3^{\mathrm{a}}$ & $1,4^{\mathrm{a}}$ \\
Anzahl Apothecien & $0,0^{\mathrm{a}}$ & $17,2^{\mathrm{a}}$ & $19,5^{\mathrm{a}}$ & $20,3^{\mathrm{a}}$ \\
max. Größe Apothecien [mm] & - & $0,3^{\mathrm{a}}$ & $0,3^{\mathrm{a}}$ & $0,3^{\mathrm{a}}$ \\
\hline
\end{tabular}

Signifikanzniveau: $* \mathrm{p} \leq 0,05 ; * * \mathrm{p} \leq 0,01$;

Stichprobenumfang n siehe Tab. 78 im Anhang 1

\section{d) Podetienform}

Die Podetienform zeigt eine Abhängigkeit von der Fruchtkörperbildung. In Polstern mit Apothecien treten weitaus häufiger geschlitzte und offen-rinnige Podetien auf als in den sterilen Polstern. Naheliegend wäre auch ein Zusammenhang zwischen Podetienform und Alter der Flechte. Das Merkmal Podetienlänge sagt etwas über das Thallusalter aus, allerdings mit der Einschränkung, daß Standortfaktoren auf die Podetienlänge ebenfalls Einfluß nehmen und das Wachstum fördern bzw. hemmen können. Eine Auswertung mit unterschiedlichen Längenklassen ergibt, daß in Polstern mit relativ langen Podetien die beiden Merkmalsausprägungen geschlitzt und offen-rinnige Lagerstiele öfter zu beobachten sind als in kleinwüchsigeren Polstern (s. Tab. 39).

Tab. 39: Häufigkeit geschlitzter bzw. offen-rinniger Podetien in Abhängigkeit von der Fruchtkörperbildung und der Podetienlänge

\begin{tabular}{l|ll}
\hline Fruchtkörperbildung & geschlitzte Podetien & offen-rinnige Podetien \\
\hline nicht fruchtend $(\mathrm{n}=17)$ & $35 \%$ & $6 \%$ \\
fruchtend $(\mathrm{n}=48)$ & $65 \%$ & $38 \%$ \\
\hline Podetienlänge & geschlitzte Podetien & offen-rinnige Podetien \\
\hline bis $30 \mathrm{~mm}(\mathrm{n}=7)$ & $43 \%$ & $14 \%$ \\
bis $40 \mathrm{~mm}(\mathrm{n}=22)$ & $50 \%$ & $18 \%$ \\
bis $50 \mathrm{~mm}(\mathrm{n}=22)$ & $68 \%$ & $41 \%$ \\
$>50 \mathrm{~mm}(\mathrm{n}=14)$ & $57 \%$ & $50 \%$ \\
\hline
\end{tabular}

\section{e) Korrelationen der morphologischen Merkmale}

Die Spearman Korrelationsanalyse zeigt lediglich in einem Fall eine deutliche Korrelation zweier morphologischer Merkmale (s. Tab. 40): das Merkmal Apothecienzahl korreliert positiv $(\mathrm{r}=0,65)$ mit der Anzahl Zweige am Podetienende. Bei allen weiteren Merkmalskombinationen sind keine bzw. nur schwache Korrelationen erkennbar, so z.B. zwischen Anzahl und Größe der Schuppen $(r=0,51)$. 
Tab. 40: Ergebnisse der Korrelationsanalyse nach Spearman.

\begin{tabular}{|c|c|c|c|c|c|c|c|}
\hline & $P D$ & $Z V$ & $Z P$ & $A S$ & $G S$ & $A A$ & $G A$ \\
\hline$\overline{P L}$ & $\begin{array}{l}0,22 * * * \\
\mathrm{n}=622\end{array}$ & $\begin{array}{l}0,41 * * * \\
\mathrm{n}=622\end{array}$ & $\begin{array}{l}0,08^{*} \\
\mathrm{n}=619\end{array}$ & $\begin{array}{l}0,06 \\
n=622\end{array}$ & $\begin{array}{l}0,05 \\
\mathrm{n}=287\end{array}$ & $\begin{array}{l}0,18 * * * \\
\mathrm{n}=538\end{array}$ & $\begin{array}{l}-0,02 \\
\mathrm{n}=295\end{array}$ \\
\hline$P D$ & & $\begin{array}{l}0,29 * * * \\
\mathrm{n}=623\end{array}$ & $\begin{array}{l}0,12 * * \\
\mathrm{n}=620\end{array}$ & $\begin{array}{l}0,21 * * * \\
\mathrm{n}=623\end{array}$ & $\begin{array}{l}0,44 * * * \\
\mathrm{n}=288\end{array}$ & $\begin{array}{l}0,18 * * * \\
\mathrm{n}=539\end{array}$ & $\begin{array}{l}0,34 * * * \\
\mathrm{n}=295\end{array}$ \\
\hline$Z V$ & & & $\begin{array}{l}0,36 * * * \\
\mathrm{n}=620\end{array}$ & $\begin{array}{l}0,00 \\
\mathrm{n}=623\end{array}$ & $\begin{array}{l}-0,13^{* *} \\
\mathrm{n}=288\end{array}$ & $\begin{array}{l}0,34 * * * \\
\mathrm{n}=539\end{array}$ & $\begin{array}{l}0,18 * * * \\
\mathrm{n}=295\end{array}$ \\
\hline$Z P$ & & & & $\begin{array}{l}-0,23 * * * \\
\mathrm{n}=620\end{array}$ & $\begin{array}{l}-0,13^{* *} \\
\mathrm{n}=287\end{array}$ & $\begin{array}{l}0,65^{* * *} \\
\mathrm{n}=536\end{array}$ & $\begin{array}{l}0,40 * * * \\
\mathrm{n}=295\end{array}$ \\
\hline AS & & & & & $\begin{array}{l}0,51^{* * * *} \\
\mathrm{n}=288\end{array}$ & $\begin{array}{l}-0,31 * * * \\
\mathrm{n}=539\end{array}$ & $\begin{array}{l}-0,17 * * \\
\mathrm{n}=295\end{array}$ \\
\hline GS & & & & & & $\begin{array}{l}-0,16^{* *} \\
\mathrm{n}=247\end{array}$ & $\begin{array}{l}-0,05 \\
n=103\end{array}$ \\
\hline$A A$ & & & & & & & $\begin{array}{l}0,39 * * * \\
\mathrm{n}=222\end{array}$ \\
\hline
\end{tabular}

einseitige Signifikanz: ${ }^{*} \mathrm{p} \leq 0,05 ; * * \mathrm{p} \leq 0,01 ; * * * \mathrm{p} \leq 0,001$

angegeben sind die Rangkorrelationskoeffizienten $\left(\mathrm{r}_{\mathrm{s}}\right)$ für die Korrelationen

der morphologischen Merkmale untereinander (Abkürzungen s. Kap. G1.1.1, S. 106)

\subsection{Rolle äußerer und innerer Faktoren bei der Merkmalsausbildung}

Trotz der bei derartigen Untersuchungen bestehenden Schwierigkeit aus einer Vielzahl sich gegenseitig beeinflussender, auf die Thalli einwirkender Faktoren, die prägenden herauszufinden, soll der Versuch einer Interpretation der in Kap. G3.2 und G3.3 dargestellten Ergebnisse unternommen werden. Gleichzeitig wird die Rolle bzw. die Stärke des Einflusses von Standortfaktoren bzw. „inneren“ Faktoren auf die Gestalt herausgearbeitet. Die Schwierigkeit der Interpretation beruht beispielsweise darauf, daß der U-Test bei der Frage nach der Abhängigkeit der Merkmale untereinander einige signifikante Unterschiede aufzeigen, also auf einen Einfluß innerer Faktoren hinweisen kann; dies muß aber trotzdem nicht bedeuten, daß die entsprechenden Merkmale einander beeinflussen. Beide können in ähnlicher Deutlichkeit auf bestimmte Standortbedingungen reagieren, so daß beim Vergleich zweier solcher morphologischer Merkmale ein nur scheinbarer Zusammenhang entsteht. Eine ausführliche Diskussion der Rolle der Standortfaktoren unter Einbeziehung von weiteren, als den hier aufgeführten Angaben aus der Literatur sowie von molekularbiologischen Daten findet sich in an späterer Stelle in Kap. I1.

\section{a) Podetienlänge und Podetiendurchmesser}

Die Podetien wachsen an Standorten mit häufigen Trockenperioden weniger gut als unter den etwas günstigeren Feuchtigkeitsverhältnissen in Heiden, Berglagen, Wäldern oder an Waldrändern. Da sich die Polster dieser Standorte zudem durch eine geringere bis fehlende Pigmentierung und eine oft großflächig deckende Algenschicht auszeichnen, ergibt sich auch zwischen wenig und stark pigmentierten sowie zwischen Podetien mit unterschiedlich ausgedehnter Algenschicht ein signifikanter Unterschied bei den Mittelwerten der Podetienlänge. Wenig Tritteinfluß (wie z.B. in Wäldern) ist ebenfalls positiv für die ungestörte Entwicklung hochwüchsiger Podetien. Fruchtende 
Podetien sind im Mittel länger als sterile, da die Fruchtkörperbildung das Endstadium der Entwicklung darstellt (JAHNS et al. 1978), die Podetien also im fruchtenden Zustand quasi ausgewachsen sind.

Fazit: Günstige Standortbedingungen, vor allem eine gute Feuchtigkeitsversorgung aber auch ausreichend Licht (s. Wert der Heiden), führen zu hochwüchsigen Podetien, sofern nicht mechanische Störungen die Entwicklung beeinträchtigen. Aber auch das Alter (also der vorangegangene Entwicklungszeitraum) spielt eine Rolle.

Podetien sind im Wald und an Waldrändern dicker. Insgesamt sind die Unterschiede beim Podetiendurchmesser jedoch gering. Polster aus Wäldern sind, wie oben bereits erwähnt, durch geringe Trittbeeinflussung, wenig Pigmentierung und eine ausgedehnte Algenschicht gekennzeichnet, zudem tragen sie häufiger Pyknidien. Deshalb ergeben sich auch signifikante Unterschiede bei den Mittelwerten des Podetiendurchmessers zwischen Podetien unterschiedlich stark trittbeeinflusster Standorte, zwischen pyknidientragenden und pyknidienlosen, zwischen wenig und stark pigmentierten und zwischen solchen mit verschieden ausgedehnter Algenschicht. $\mathrm{Zu}$ erwarten wäre auch ein Unterschied zwischen fruchtenden und sterilen Lagerstielen, da fertile in Wäldern am seltensten auftreten. Diese Vermutung läßt sich aber anhand des Datenmaterials nicht bestätigen.

Auf den ersten Blick mag es erstaunen, daß stark mit Fibrillen besetzte Podetien im Mittel einen signifikant größeren Durchmesser aufweisen als Exemplare mit weniger Fibrillen, obwohl erstere häufiger in Heiden und an Felsstandorten auftreten, also in den Standorttypen, deren Mittelwerte der Podetiendurchmesser verhältnismäßig gering sind. Erklären könnte dies die Tatsache, daß viele der durch zahlreiche Fibrillen gekennzeichneten Podetien eine ungewöhnliche Wuchsform mit teilweise ziemlich dicken Abschnitten aufweisen (s. Taf. 2.11 und Taf. 2.12 im Anhang 2) und in dieser Hinsicht nicht unbedingt der Mehrzahl der Podetien an entsprechenden Standorten entsprechen.

Fazit: Günstige Standortbedingungen (v.a. Feuchtigkeitsverhältnisse) fördern das Entstehen relativ dicker Podetien. Dicke Podetienabschnitte können aber auch an zu Boden gedrückten, z.B. trittbeeinflussten Lagerstielen auftreten.

\section{b) Anzahl der Verzweigungen und der Zweige am Podetienende, Fibrillenbildung}

Die Zahl der Verzweigungen ist bei Exemplaren der Heiden besonders hoch. Dort treten aber auch besonders lange (weil besonders alte?) sowie viele fruchtende Lagerstiele auf. Für den signifikanten Unterschied bei den Mittelwerten der Anzahl Verzweigungen zwischen Podetien mäßig trittbelasteter Standorte auf der einen sowie wenig bzw. stark trittbelasteter Stellen auf der anderen Seite spielt wahrscheinlich auch eine Rolle, daß die untersuchten Heiden meist mäßig trittbeeinflusste Standorte sind.

Fruchtende Exemplare weisen durchschnittlich mehr Verzweigungen auf als sterile, sicherlich auch deshalb, weil sie ein fortgeschrittenes Entwicklungsstadium (s.o.) darstellen und somit für eine bereits verhältnismäßig lange andauernde Entwicklungszeit stehen. 
Fazit: Günstige Standortbedingungen, die ein langes, ungestörtes Wachstum ermöglichen, fördern die Entwicklung von Podetien, die sich zur Spitze hin mehrfach verzweigen. Aber auch ein Einfluß der Fruchtkörperbildung kann nicht ausgeschlossen werden, da sich diese, wie nachfolgend beschrieben, auf die Wuchsform auswirkt. Starker Tritt fragmentiert die Thalli, gelegentliche Tritteinwirkung kann jedoch zu stärker verzweigten Exemplaren führen, da sich an umgekippten Lagerstielen neue Seitensprosse ausbilden.

Die Anzahl Zweige am Podetienende korreliert positiv mit der Anzahl Apothecien. Fruchtende Exemplare sind im Mittel signifikant stärker verzweigt als sterile. Dagegen bestehen keine signifikanten Unterschiede zwischen den Standorttypen bei der Zahl der Zweige am Podetienende. Der Wert der Heiden ist etwas höher, wohl weil dort mehr fruchtende Podetien anzutreffen sind. Daher ist auch der Wert mäßig gestörter Standorte, wie den Heiden, etwas höher als die Werte der wenig bzw. stark gestörten Stellen.

Podetien ohne bzw. mit vielen Fibrillen sind im Mittel stärker verzweigt als solche mit wenigen Fibrillen. Ersteres wohl deshalb, weil sie ungestörter wachsen, also sich besser entwickeln (und fruchten) können. Fibrillen und Seitenzweige unterscheiden sich laut Definition (s. Kap. G1.1.1) nur aufgrund ihrer Länge und Dicke. Beide stellen Verzweigungen des Hauptastes dar, weshalb sich ein höherer Mittelwert auch bei stark mit Fibrillen besetzten Lagerstielen ergibt. Fibrillen treten vermehrt an trittbeeinflussten Stellen auf. Teilweise zeigen sich daher signifikante Unterschiede zwischen den durch Tritt geprägten Standorttypen auf der einen und den ungestörteren auf der anderen Seite.

Fazit: Apothecienbildung führt zu einer stärkeren Verzweigung am Podetienende. Der Standort hat nur insofern Einfluß, als daß die dort herrschenden Bedingungen eine Fruchtkörperbildung fördern oder bremsen (s.u.). Auch mechanische Störungen können eine vermehrte Zweig- und Fibrillenbildung auslösen. Fibrillen entstehen v.a. an trittbelasteten Standorten.

\section{c) Anzahl und Größe der Schuppen}

Im Schatten (Standorttyp Wald) werden mehr Schuppen gebildet, um die Oberfläche zu vergrößern (vgl. BRITZELMAYR 1904). An Waldstandorten werden die Flechten wenig gestört, bilden häufiger Pyknidien, tragen weniger Fibrillen, zeigen kaum Pigmentierung aber eine ausgedehnte Algenschicht und fruchten weniger oft. Daher treten (teilweise signifikante) Unterschiede bei den Mittelwerten zwischen Podetien unterschiedlich gestörter Standorte, zwischen pyknidientragenden und pyknidienlosen, zwischen unterschiedlich mit Fibrillen besetzten bzw. pigmentierten und zwischen fruchtenden bzw. nicht fruchtenden Lagerstielen auf.

Fazit: Die Anzahl Schuppen ist in erster Linie von den Lichtverhältnissen abhängig. Alle weiteren scheinbaren Zusammenhänge zwischen diesem und anderen Podetienmerkmalen, z.B. zwischen Beschuppung und Apothecienbildung, beruhen wohl eher darauf, daß jeweils beide Merkmale deutlich aber unabhängig voneinander auf die Bedingungen am Standort reagieren. 
Die größten Schuppen wachsen an Waldstandorten. Daher zeigen sich auch hier, analog $\mathrm{zu}$ den Ausführungen im vorigen Abschnitt, teilweise signifikante Unterschiede zwischen Podetien unterschiedlich stark gestörter Standorte, zwischen solchen mit und ohne Pyknidien, zwischen unterschiedlich pigmentierten bzw. durch eine unterschiedliche Ausdehnung der Algenschicht gekennzeichneten sowie zwischen fruchtenden und nicht fruchtenden Lagerstielen. Die Anzahl der Schuppen korreliert nur schwach mit der Schuppengröße.

Fazit: Die Schuppengröße ist weniger deutlich vom Standort abhängig als die Schuppenzahl. Es können auch unter anderen, als den in Wäldern herrschenden Bedingungen größere Schuppen auftreten. „Viele Schuppen“ muß nicht immer „größere Schuppen“ bedeuten und umgekehrt. Exemplare mit wenigen Schuppen können ebenfalls große Schuppen haben.

\section{d) Apothecien und Pyknidien}

Die für die Apothecienbildung günstigsten Standortverhältnisse finden sich in Heiden und an Waldrändern, daher auch der etwas höhere Mittelwert bei der Apothecienzahl für die mäßig trittbeeinflussten Standorte, die größtenteils Heiden oder Waldrändern zuzuordnen sind. Ebenso verhält es sich mit dem Mittelwert der schwach pigmentierten Podetien. Dieser ist höher, weil sich derartige Exemplare häufig in Heiden und an Waldrändern finden. Starker Tritt verhindert eine ungestörte Podetien-Entwicklung, mäßige Tritteinwirkung scheint aber die günstigen mikroklimatischen Bedingungen an beiden Standorttypen bezüglich der Apothecienbildung nicht aufheben zu können. Exemplare mit wenigen Fibrillen weisen besonders viele Apothecien auf. Die höchsten Mittelwerte der Apothecienzahl erhält man für die Heiden und Waldränder, den höchsten Mittelwert für den Anteil an Pyknidien weist dagegen der Standorttyp Wald (also die schwach gestörten Stellen) auf.

Fazit: Apothecien bilden sich besonders an sonnigen, jedoch nicht zu extrem sonnigtrockenen Standorten (wie sie beispielsweise die Felsen darstellen). Dies wird durch die Feststellung bestätigt, daß Cladonia furcata ssp. furcata im Cladonietum foliaceae, einer Gesellschaft der Basalt- und Diabasfelsen, nur sehr selten fruchtet (s. Kap. E5). Pyknidien entstehen dagegen vermehrt unter schattigen Bedingungen mit besseren Feuchtigkeitsverhältnissen.

Apothecien werden in Heiden und an Exemplaren ohne Fibrillen am größten.

Fazit: Günstige Standortbedingungen ohne mechanische Störeinflüsse können die Ausbildung relativ großer Fruchtkörper fördern. 


\section{e) Pigmentierung und Algenschicht}

Die Algenschicht dehnt sich unter weniger strahlungsintensiven Bedingungen weiter über das Podetium aus als bei stärkerer Sonneneinstrahlung. Daher ist ist die Algenschicht in den Podetien der Wälder, Waldrändern und Berglagen großflächiger ausgebildet als in den Podetien der Heiden. Allerdings ist der Anteil Podetien mit ausgedehnter Algenschicht auch an den sonnigen Felsstandorten im Mittel wesentlich höher als in den Heiden. Durch die starke Pigmentierung zum Schutz vor schädlicher Strahlung könnte auch weniger photosynthetisch nutzbare Strahlung zu den Algen gelangen. Hinzu kommt, daß Phasen photosythetischer Aktivität nur bei ausreichender Feuchtigkeit und somit in der Regel bei geringerer Lichtintensität möglich sind. Es herrschen dann aus Sicht der Algen ähnliche Lichtverhältnisse wie an schattigen Standorten (vgl. Kap. I1.1).

Fazit: Die Pigmentierung hängt ausschließlich von den Lichtverhältnissen ab, die Ausdehnung der Algenschicht vermutlich auch.

\section{f) Podetienform}

Fazit: Anhand der in Kap. G3.2 und G3.3 dargestellten Ergebnisse läßt sich festhalten, daß die Podetienform hauptsächlich eine Frage des Alters ist, daher die Abhängigkeit von der Fruchtkörperbildung (fruchtende Thalli stellen die Endstadien der Flechtenentwicklung dar). Dies erklärt auch, warum hochwüchsige Polster häufiger geschlitzte bzw. offen-rinnige Podetien aufweisen.

\section{g) Zusammenfassung der Rolle innerer und äußerer Faktoren}

Tab. 41 faßt die Bedeutung der Standortverhältnisse bzw. des Entwicklungszustandes für die Ausprägung der einzelnen Podetienmerkmale zusammen. Zwei Kreuze bedeuten eine starken, ein Kreuz einen schwächeren Einfluß. Ein Fragezeichen steht in Fällen, in denen die Untersuchung keine eindeutigen Aussagen ermöglichen, ein Einfluß aber denkbar ist. Da die Thalli nach der Pyknidienbildung ihr Wachstum einstellen (VoBIS 1980), ist beispielsweise bei diesem Merkmal, wie auch bei der Apothecienbildung, ein „,innerer“ Einfluß wahrscheinlich. Zusammenfassend läßt sich festhalten, daß offensichtlich der Großteil der morphologischen Merkmale eine deutliche Abhängigkeit von den Standortbedingungen zeigt. Weniger vom Standort, dagegen hauptsächlich vom Entwicklungszustand abhängig ist das Merkmal Anzahl Zweige am Podetienende. 
Tab. 41: Zusammenfassung der Bedeutung von Standortfaktoren und Entwicklungszustand auf die einzelnen Podetienmerkmale bei Cladonia furcata ssp. furcata (Erläuterungen im Text)

\begin{tabular}{l|ll}
\hline Podetienmerkmale & Standortfaktoren & $\begin{array}{l}\text { Entwicklungszustand } \\
\text { (z.B. Alter) }\end{array}$ \\
\hline max. Podetienlänge & \multicolumn{2}{l}{} \\
max. Podetiendurchmesser & ++ & + \\
Anzahl Verzweigungen & ++ & ++ \\
Anzahl Zweige am Podetienende & + & ++ \\
max. Anzahl Schuppen & ++ & \\
max. Größe Schuppen & ++ & + \\
Anzahl Apothecien & ++ & + \\
max. Größe Apothecien & + & $?$ \\
Anteil pyknidientragender Podetien & ++ & + \\
Anteil Podetien mit zahlreichen Fibrillen & ++ & + (s. Kap. I1.1) \\
Pigmentierung & ++ & \\
Deckung der Algenschicht & ++ &
\end{tabular}

\subsection{Modifikationen}

Besonders die Subspezies furcata tritt in Nordhessen in einer bemerkenswerten morphologischen Vielfalt auf. Aber auch bei der Subspezies subrangiformis lassen sich verschiedene Modifikationen erkennen. Um die Fülle verschiedenster morphologischer Erscheinungsformen zu bündeln, werden sie in der folgenden Besprechung nach dem dominierenden Merkmal zusammengefaßt. Die ganze Bandbreite an Variabilität darzustellen, würde den Rahmen dieses Kapitels sprengen. Text und Bildtafeln (Taf. 2 bis 4 im Anhang 2) sollen dennoch einen Eindruck von der Vielfalt unterschiedlichster Gestalttypen im Untersuchungsgebiet vermitteln. Weiterhin wird ein Vergleich mit den in Kap. G2 besprochenen Varietäten und Formen durchgeführt und sofern dabei Übereinstimmungen erkennbar sind, auf diese jeweils hingewiesen.

\subsubsection{Cladonia furcata ssp. furcata}

Das Merkmal „Fruchtkörper vorhanden“ vereint im Untersuchungsgebiet Thalli, die sich in anderen Merkmalen deutlich voneinander unterscheiden. Es kommen Exemplare mit stark längsrissigen und daher rinnigen Podetien vor (s. Taf. 2.1 und Taf. 2.2 im Anhang 2), die der var. racemosa f. fissa entsprechen (Beschreibung z.B. bei ANDERS 1928, SANDSTEDE 1931 und ERICHSEN 1957). Fertile Stadien mit schlanken, bis zu $70 \mathrm{~mm}$ hohen, entfernt beblätterten Lagerstielen zeigen dagegen Ähnlichkeit mit der f. foliolosa (var. pinnata) von SANDSTEDE (1931). Auch schlanke, aufrechte Podetien mit schirmförmigen Fruchtständen (s. Taf. 2.3 im Anhang 2), wie sie für die var. racemosa f. corymbosa (bzw. modificatio corymbosa) als charakteristisch angegeben werden (z.B. Britzelmayr 1904, SANDSTEDE 1922/1931, HiLlmann \& GRUMmanN 1957) und Polster mit gleich hohen, auf einer Ebene fruchtenden Podetien, wie sie die var. pinnata f. truncata auszeichnen, wurden beobachtet. Darüber hinaus treten weitere, nicht eindeutig zuzuordnende fertile Thalli auf (s. Taf. 2.4 im Anhang 2). Ein eindeutiger Typus „fruchtender Thallus“ ist nicht erkennbar, lediglich die etwas stärkere Verzweigung ist allen fertilen Polstern gemein. Eine Auflistung aller 
Erdflechtengesellschaften, in denen fruchtende Podetien auftraten, findet sich in Kap. E5.

Beschuppte, jedoch oft sterile Thalli kommen im Untersuchungsgebiet weit verbreitet vor. Der Grad der Beschuppung reicht von wenigen kleinen Schuppen bis hin zu Podetien, die fast vollständig mit Schuppen bedeckt sind (s. Taf. 2.5 bis Taf. $2.8 \mathrm{im}$ Anhang 2). Ein Vergleich mit Literaturangaben gestaltet sich schwierig, eine eindeutige Zuordnung ist kaum möglich. Schlanke, gutwüchsige, stark beschuppte Exemplare (s. Taf. $2.7 \mathrm{im}$ Anhang 2) zeigen Übereinstimmungen mit der var. pinnata f. foliolosa. Etwas niedrigwüchsigere gleichen der var. racemosa f. squamulifera (SANDSTEDE 1931) und weniger aufrecht wachsende, gebräunte Thalli mit kleinen Schuppen ähneln der var. palamaea f. rigidula (s. ANDERS 1928, SANDSTEDE 1931). Reich beschuppte Thalli, mit durchschnittlich mehr als 20 Schuppen pro Podetium, sind besonders in der Cladonia rangiferina-Variante des Cladonietum mitis typicum an Waldstandorten anzutreffen, z.B. in den Eichenwäldern des Ederseegebietes oder am Roßkopf.

Modifikationen mit zahlreichen dornig-pfriemlichen Nebenästen (Fibrillen) sind in Nordhessen nur gelegentlich zu beobachten (s. Taf. 2.11 und Taf. 2.12 im Anhang 2). Sie kommen z.B. im Bereich des Segelflugplatzes am Dörnberg, am Michelskopf (Edersee) und auf dem Maderstein vor, also sehr sonnigen Felsstandorten, an denen zumindest zeitweise mechanische Störungen in Form von Tritt (durch Weidevieh oder Mensch) bzw. durch herabrutschendes Gestein auf die Flechten einwirken und eine verstärkte Fibrillenbildung bewirken können. Dies führt zur Ausbildung teilweise bizarr anmutender Gestalten oder zahlreicher Auswüchse (s. Taf. 2.9 und 2.10 im Anhang 2). Nach SCHADE (1964) wäre solch eine Erscheinungsform als „teras spinosum“ zu bezeichnen. Ein Vergleich der nordhessischen Proben mit Abbildungen in der Literatur ergibt deutliche Übereinstimmungen in Bezug auf Wuchsform, Pigmentierung und Verzweigung mit der var. palamaea bei ANDERS (1928) bzw. der f. palamaea bei SANDSTEDE (1931). Die Flechtenbestände der ausgewählten nordhessischen Fundorte, an denen diese Modifikation auftritt, sind dem Cladonietum foliaceae und der Cladonia furcata-Cladonion arbusculae-Fragmentgesellschaft zuzuordnen.

Glatte schlanke Podetien mit zugespitzten Astenden, der var. racemosa f. furcatosubulata (s. z.B. SANDSTEDE 1931, ERICHSEN 1957, HiLlmANN \& GRUMMANN 1957) entsprechend, treten im Untersuchungsgebiet vielerorts auf. Beispiele für Wuchsorte sind die Zeche Glimmerode, der Orenberg bei Willingen, der Michelskopf/Edersee, der Battenfelder Driescher und der Burghasunger Burgberg. Erdflechtengesellschaften des Untersuchungsgebietes, in denen diese Modifikation gefunden wurde, sind neben der Cladonia furcata-Cladonion arbusculaeFragmentgesellschaft auch die Cladonia floerkeana-Variante des Cladonietum rei und die Cladonia bacillaris-Gesellschaft, also zwei Gesellschaften, die als intermediäre Sukzessionsstadien gelten (s. Kap. E2.1.4). Geht man davon aus, daß Thalli auf derartigen Standorten noch keine sehr lange Wachstumszeit hatten, so würde dies die Aussage von VERSEGHY (1987/88) bestätigen, wonach die f. furcatosubulata lediglich ein Jugendstadium der Flechte darstellt (s. Kap. G2.1.4). 


\section{Verwechslungsmöglichkeiten mit Cladonia gracilis und $\mathrm{Cl}$. crispata:}

Eine Verwechslungsmöglichkeit von Cladonia furcata ssp. furcata und Cl. gracilis besteht besonders bei kümmerlich entwickelten Exemplaren mit deutlichen Grundschuppen und spärlich ausgebildeten Podetien (s. Taf. 3.2 im Anhang 2). Allenfalls der etwas schlankere Wuchs der unverzweigten oder wenig verzweigten Podetien deutet dann auf Cl. gracilis hin, sofern Lagerstiele deutlich genug erkennbar sind. Auch die im Untersuchungsgebiet gelegentlich auftretenden höherwüchsigen aber becherlosen Formen von Cl. gracilis lassen sich oft nur aufgrund der geschlossenen Achseln und des geringeren Verzweigungsgrades exakt bestimmen (s. Taf. 3.1 und Taf. $3.3 \mathrm{im}$ Anhang). Beide Arten stimmen bezüglich der Inhaltsstoffe überein. Eine typisch ausgebildete Cl.gracilis, wie sie im Untersuchungsgebiet ebenfalls auftritt, ist u.a. durch schmale Becher gekennzeichnet (s. Taf. 3.4 im Anhang 2).

Cladonia crispata kann mit Cl. furcata ssp. furcata verwechselt werden, wenn ihre Polster untypisch ausgebildet, d.h. ihre Podetien nach oben hin kaum (becherförmig) erweitert und am Rand wenig gezähnt sind (s. Taf. 3.5 bis Taf. 3.7 im Anhang 2). Sie sehen dann zierlichen Formen von Cl. furcata ssp. furcata ähnlich, die nur kurze Zweige an der Podetienspitze aufweisen (s. Taf. 3.8 im Anhang 2). Beide Arten können jedoch aufgrund verschiedener Inhaltsstoffe eindeutig unterschieden werden: Cl. furcata enthält Fumarprotocetrarsäure, Cl. crispata dagegen Squamatsäure (s. Kap. F2). Dies läßt sich bereits mit dem K-Test erkennen, da die furcata-Exemplare aufgrund ihres Fumarprotocetrarsäure-Gehaltes $\mathrm{K}+$ braun, die crispata-Exemplare dagegen $\mathrm{K}$ reagieren.

\subsubsection{Cladonia furcata ssp. subrangiformis}

Fruchtende Exemplare kommen bei der Subspezies subrangiformis im Untersuchungsgebiet viel seltener vor als bei der Subspezies furcata. Parallelen in der Gestalt lassen sich dennoch erkennen, da auch bei Cladonia furcata ssp. subrangiformis die fruchtenden Podetien rinnig aufreißen können (s. Taf. 4.6 im Anhang 2). Beschuppte Formen spielen bei dieser Unterart eine wesentlich geringere Rolle als bei der Cl. furcata ssp. furcata; Schuppen treten nur sporadisch und in geringer Anzahl auf (s. Taf. 4.1 im Anhang 2, oberes Podetium). Da Cl. furcata ssp. subrangiformis häufig auf beweideten Standorten siedelt, sind durch Tritteinwirkung in ihrer Wuchsform beeinflußte Podetien nicht selten. Unregelmäßig verzweigte, dem Boden anliegende Lagerstiele und zahlreiche Nachschüsse können die Folge sein (s. Taf. 4.3 und Taf. 4.4 im Anhang 2). Oft sind die Podetien von Cl. furcata ssp. subrangiformis glatt und relativ schlank (s. Taf. 4.1 und Taf. 4.2 im Anhang 2), bisweilen treten jedoch Modifikationen mit aufgedunsenen, zum Teil auch längsrissigen Podetien auf (s. Taf. 4.5 und Taf. 4.6 im Anhang 2). Bei dem in Taf. 4.5 abgebildeten Exemplar sind die für die Unterart charakteristischen punktförmigen, weißen Markaufbrüche besonders gut erkennbar. Übereinstimmungen zeigen sich zwischen diesen aufgedunsenen Exemplaren und der bei SANDSTEDE (1931) beschriebenen sowie von weiteren Autoren (z.B. VeRSEGHY 1975) erwähnten Forma vagans. 
Die enormen Unterschiede in der Pigmentierung (manche Exemplare sind oberseits fast schwarz) werden bei einem Vergleich der in Taf. 4 (im Anhang 2) zusammengestellten Thalli ersichtlich.

\section{Anatomische Merkmale}

Das vorhergehende Kapitel beschreibt die Zusammenhänge zwischen den äußerlich sichtbaren Merkmalen und dem Standort. Ergänzend dazu stellt sich die Frage, ob auch anatomische Unterschiede zwischen Thalli bestehen, die während ihres Wachstums unterschiedlichen Standortbedingungen ausgesetzt waren. Hierfür wurden PodetienQuerschnitte einiger exemplarisch ausgewählter morphologischer Erscheinungsformen von Cladonia furcata ssp. furcata sowie einer gut entwickelten Cl. furcata ssp. subrangiformis-Probe angefertigt. Darüber hinaus werden auch die anatomischen Verhältnisse dreier untypisch ausgebildeter, von Cl.furcata nur schwer zu unterscheidender Podetien von Cl. gracilis beschrieben, welche auch für die ITS rDNASequenzierung verwendet wurden (s. Kap. H). Herkunft sowie einige morphologische Merkmale der Cl. furcata- und Cl. gracilis-Proben sind Tab. 42 zu entnehmen.

Tab. 42: Daten zu den lichtmikroskopisch untersuchten Proben von Cladonia furcata und Cl. gracilis

\begin{tabular}{|c|c|c|c|c|c|c|c|}
\hline Probe & $\begin{array}{l}\text { Her- } \\
\text { kunft }\end{array}$ & $\begin{array}{l}\text { Stand- } \\
\text { orttyp }\end{array}$ & $\begin{array}{l}\text { Verteilung der Algenschicht } \\
\text { (= Gonidienzone) } \\
\text { über die Podetien }\end{array}$ & $\begin{array}{l}\text { Pigmen- } \\
\text { tierung }\end{array}$ & $\begin{array}{l}\text { Schup- } \\
\text { pen }\end{array}$ & Fibrillen & Sonstige Merkmale \\
\hline furcata-1 & DT & $\mathrm{W}$ & $\begin{array}{l}\text { fleckig, aber meist nur } \\
\text { kleinflächig ohne Algen }\end{array}$ & - & ++ & - & - \\
\hline furcata-2 & ME & $\mathrm{W}$ & $\begin{array}{l}\text { fleckig bis dicht, jedoch } \\
\text { stellenweise auch deutlich } \\
\text { algenfreies Mark sichtbar }\end{array}$ & - & o & - & $\begin{array}{l}\text { fruchtend; fast schon } \\
\text { Degenerationsstadium }\end{array}$ \\
\hline furcata-3 & RK & W & $\begin{array}{l}\text { fleckig bis großflächig } \\
\text { deckend, aber auch deutlich } \\
\text { algenfreies Mark sichtbar }\end{array}$ & - & ++ & o & - \\
\hline furcata-4 & ZG & $\mathrm{R}$ & $\begin{array}{l}\text { dicht fleckig bis gepunktet; } \\
\text { zur Spitze hin punktförmige } \\
\text { Areale mit Algen, zur Basis } \\
\text { hin größere Flecken ohne } \\
\text { Algen. }\end{array}$ & + & - & - & $\begin{array}{l}\text { sehr glatt, schlank und } \\
\text { regelmäßig aussehend }\end{array}$ \\
\hline furcata-5 & MI & $\mathrm{F}$ & fleckig-locker bis dicht & + & - & o & - \\
\hline furcata-6 & KM & B & $\begin{array}{l}\text { fleckig/gepunktet bis (zur } \\
\text { Basis hin) fast geschlossene } \\
\text { Algenschicht }\end{array}$ & - & - & o & - \\
\hline furcata-7 & DB & $\mathrm{F}$ & $\begin{array}{l}\text { dicht gepunktet, zur Basis hin } \\
\text { eher fleckig bis großflächig } \\
\text { deckend }\end{array}$ & + & o & o & $\begin{array}{l}\text { Trittbelastung; } \\
\text { Oberfläche } \\
\text { stellenweise warzig }\end{array}$ \\
\hline furcata-8 & MK & B & $\begin{array}{l}\text { locker fleckig bis dicht; zur } \\
\text { Spitze hin gepunktet }\end{array}$ & - & o & o & - \\
\hline furcata-9 & MK & B & $\begin{array}{l}\text { zur Spitze hin dicht gepunktet, } \\
\text { zur Basis hin flächig deckend, } \\
\text { jedoch mit größeren Flecken } \\
\text { ohne Algenschicht }\end{array}$ & - & ++ & + & - \\
\hline gracilis-1 & $\overline{\mathrm{SZ}}$ & $\mathrm{F}$ & $\begin{array}{l}\text { gefleckt (größere rundliche } \\
\text { Flecken) }\end{array}$ & ++ & - & - & $\begin{array}{l}\text { Kümmerwuchs } \\
\text { (Trittbelastung?), } \\
\text { Oberfläche warzig }\end{array}$ \\
\hline gracilis-2 & NT & $\mathrm{R}$ & $\begin{array}{l}\text { gepunktet, locker bis, gegen } \\
\text { die Basis hin, dichter }\end{array}$ & + & o & o & $\begin{array}{l}\text { zierlich, glatt } \\
\text { aussehend }\end{array}$ \\
\hline gracilis-3 & MM & B & $\begin{array}{l}\text { fleckig, zur Spitze hin } \\
\text { gepunktet, aber oft lückig }\end{array}$ & + & + & o & $\begin{array}{l}\text { zierlich, aufrecht und } \\
\text { wenig verzweigt }\end{array}$ \\
\hline
\end{tabular}

Merkmalsausprägung: - nicht/keine, o wenig, + stark/viele, ++ sehr stark/sehr viele

Abkürzungen der Standorttypen s. Kap. G1.1.1, S. 109. Zur Herkunft siehe Fundortverzeichnis im Anhang 1. 


\subsection{Podetien}

Die Podetien wurden jeweils in der oberen, gut entwickelten Hälfte geschnitten. Im Querschnitt der Podetien sind ein zentraler Hohlraum und 3 ihn umgebende Schichten abgrenzbar. Der zentrale Hohlraum bildet sich sehr früh. Er tritt bereits in nur etwa 0,5 mm dicken Seitenästen auf. Nach HENSSEN \& JAHNS (1974) wird allgemein bei Flechten eine Rinde (= Cortex), eine Algenschicht und eine Markschicht (= Medulla) unterschieden. Passender für die Cladonien erscheint allerdings die Einteilung von SANDSTEDE (1931), die im weiteren verwendet wird. Von außen nach innen, kommen folgende Schichten vor:

- Rindenschicht (stratum corticale)

- äußeres Mark (stratum myelohyphicum), mit Gonidienzone und gonidienfreier Zone

- inneres Mark (stratum chondroideum)

Ehe auf die Besonderheiten der einzelnen Querschnitte eingegangen wird, sollen zunächst einige allgemeine, unter dem Lichtmikroskop sichtbare Merkmale der Schichten angesprochen werden.

Die Rindenschicht ist kompakt, durchscheinend und gelatinös. Die Wände der Rindenzellen sind gallertig verquollen (vgl. HENSSEN \& JAHNS 1974). Die Hyphenoberfläche erscheint glatt bis stellenweise rauh. Bei dunkel gefärbten Proben ist eine deutliche Pigmentierung (Braunton) erkennbar. Stellenweise löst sich die Rinde auf, so daß das Mark nach außen hin nur durch eine körnige Struktur abgeschlossen wird. Die Hyphen sind rund $1 \mu \mathrm{m}$ dick.

Die äußere Markschicht ist mehr oder weniger durchgängig entwickelt, kann jedoch bezüglich der Schichtdicke innerhalb desselben Querschnitts deutlich variieren. Es lassen sich zwei Zonen unterscheiden: Zur Rinde hin liegt die Gonidienzone, ein Geflecht aus Pilzhyphen und Algenzellen. Die kokkalen Algen (= Gonidien) liegen darin meist dicht beieinander. Sie variieren wenig in der Größe und haben einen Durchmesser von maximal 10 bis $11 \mu \mathrm{m}$ (bei der Probe „subrangiformis“ bis $14 \mu \mathrm{m}$ ), meist jedoch um $7 \mu \mathrm{m}$. Darunter findet sich die gonidienfreie Zone als dunkel erscheinendes, da luftgefülltes, relativ lockeres Hyphengeflecht, welches ein Luftreservoir für den Gasaustausch bildet. Die Hyphen sind mit $4 \mu \mathrm{m}$ deutlich dicker als die Hyphen der Rinde bzw. des inneren Marks und weisen eine unter dem Lichtmikroskop undeutlich erkennbare rauhe Oberflächenstruktur auf. Eventuell handelt es sich dabei um Flechtenstoffe, welche die Zellwände mit einer dicken Kristallschicht überziehen können (HENSSEN \& JAHNS 1974). Die innere Markschicht stellt einen kompakten Zylinder aus verleimten Längshyphen dar, der im Querschnitt eine sehr unterschiedliche Dicke aufweist. Die Hyphen selbst sind mit $1 \mu \mathrm{m}$ Durchmesser relativ dünn.

Die Querschnitte sowie die einzelnen Schichten wurden unter dem Lichtmikroskop ausgemessen und aus den Werten anschließend der maximale prozentuale Anteil der Schichten am Radius des Querschnitts ausgerechnet (s. Tab. 43). Auf diese Weise können die verschiedenen Querschnitte miteinander verglichen werden. Da beim 
inneren Mark, über den gesamten Querschnitt betrachtet, dicke und dünne Stellen meist etwa gleichen Anteil haben, konnte hier zur Orientierung auch der mittlere Anteil ausgerechnet werden. Beim äußeren Mark war dies aufgrund der zu großen Unregelmäßigkeiten nicht möglich.

Tab. 43: Maximaler prozentualer Anteil der einzelnen Schichten am Radius des Podetiums (Standorttypen s. Kap. G1.1.1, S. 109)

\begin{tabular}{|c|c|c|c|c|c|c|c|c|c|c|c|c|c|}
\hline & 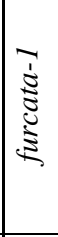 & $\begin{array}{l}n \\
1 \\
0 \\
0 \\
0 \\
\vdots \\
S t a\end{array}$ & 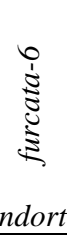 & 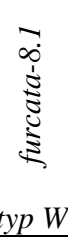 & 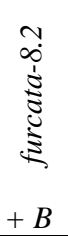 & 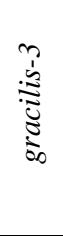 & 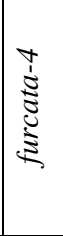 & 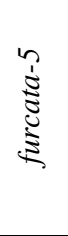 & 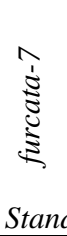 & 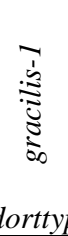 & 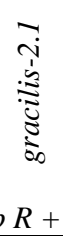 & 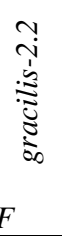 & $\begin{array}{l}\frac{1}{0} \\
\frac{1}{5} \\
\vdots \\
\frac{1}{\Xi} \cdot \cong \\
\omega\end{array}$ \\
\hline Radius Podetium $[\mu \mathrm{m}]$ & 400 & 625 & 325 & 575 & 900 & 300 & 375 & 525 & 375 & 325 & 275 & 375 & 500 \\
\hline Rinde maximal [\%] & 6 & 4 & 8 & 4 & 2 & 8 & 5 & 4 & 5 & 10 & 7 & 6 & 4 \\
\hline Gonidienzone maximal [\%] & 8 & 4 & 10 & 6 & 5 & 12 & 11 & 7 & 11 & 24 & 13 & 10 & 15 \\
\hline gonidienlose Zone maximal [\%] & 2 & 10 & 7 & 6 & 5 & 31 & 5 & 19 & 5 & 11 & 7 & 26 & 13 \\
\hline inneres Mark maximal [\%] & 27 & 25 & 26 & 39 & 20 & 20 & 31 & 24 & 38 & 17 & 22 & 15 & 23 \\
\hline inneres Mark MW [\%] & 21 & 21 & 22 & 25 & 15 & 14 & 25 & 13 & 29 & 14 & 18 & 10 & - \\
\hline
\end{tabular}

Es zeigen sich folgende Tendenzen (s. Tab. 43):

Bei Podetien mit dünner Rindenschicht ist auch die Gonidienschicht eher dünn und umgekehrt. Die Exemplare mit dünner Rinde und dünner Algenschicht entstammen verhältnismäßig beschatteten Standorten (Standorttyp Wälder und Berglagen). Um das geringere Lichtangebot nutzen zu können, liegen die Algen tendenziell wohl weniger über- als nebeneinander. Der mittlere maximale Anteil der Gonidienzone dieser Proben beträgt 7,9\% (Cl. furcata-Proben: 6,9\%). Einen mittleren maximalen Anteil der Algenschicht von 13,3\% (Cl. furcata-Proben: 11,0\%) haben dagegen die Thalli der besonnten Standorte (Standorttypen Felsen, Waldränder). Bei ihnen ist die Rinde dicker und die Algenschicht mächtiger ausgebildet.

Podetien mit dickerer gonidienloser Zone haben ein eher dünnes inneres Mark und umgekehrt. Bei Betrachtung der Herkunft der einzelnen Proben zeigt sich hierbei kein klarer Zusammenhang zwischen den Standortbedingungen und der Schichtdicke. 


\subsection{Beschreibung der einzelnen Podetienquerschnitte}

\section{a) Podetien von Standorten des Typs Wälder oder Berglagen}

Probe furcata-1 aus einem Eichenwald zeigt ein Podetium mit ziemlich regelmäßigem Querschnitt (Abb. 32a). Das innere Mark ist relativ dick, die äußere Markschicht dagegen verhältnismäßig dünn. Sie wird fast vollständig von der Gonidienzone eingenommen. Wie in Kap. G3.2 gezeigt, ist eine derart ausgedehnte Algenschicht typisch für Podetien von Waldstandorten. Die Rindenschicht wird von zahlreichen Spalten durchbrochen. Es treten am Podetium zudem Stellen auf, an denen Rindenschicht und Gonidienzone auf größerer Fläche komplett fehlen.

Ähnlich ist die Anatomie bei Probe furcata-8 von einer Basaltblockhalde am Meißner. Die Algen liegen locker, aber ziemlich gleichmäßig um den gesamten Querschnitt verteilt unter der Rinde. Darunter folgt eine schmale, gonidienlose Zone. Genau wie bei Probe furcata-1 durchbrechen im Querschnitt immer wieder Spalten die Rinde, so daß die Hyphen der gonidienlosen Zone in direktem Kontakt zur Außenwelt stehen. In den weiter zur Podetienbasis hin entnommenen, sehr großen Querschnitten (Probe furcata8.2, s. Tab. 43), lassen sich Gonidienzone und gonidienlose Zone nur noch schwer voneinander trennen, da die Algen dort locker verteilt im gesamten äußeren Mark liegen. Der Querschnitt der dickeren unteren Podetienhälfte ist gleichmäßiger als der Querschnitt aus der Podetienspitze. Probe furcata-6 wuchs an einem ähnlichen Standort und zeigt auch einen im wesentlichen dem der Probe furcata- 8 vergleichbar aufgebauten Querschnitt. Ebenso die Probe furcata-3 aus einem Eichentrockenwald auf Grauwacke, jedoch liegen dort die Algen weniger durchgängig, sondern etwas unregelmäßiger verteilt unter der Rinde und bilden nur an einigen Stellen eine über größere Abschnitte reichende Schicht.

Die Probe gracilis-3 (Abb. 32f) stellt ein zierliches Exemplar von einer Basaltblockhalde dar. Im Querschnitt zeigt sich, daß die pustelförmigen Aufwölbungen am Podetium im Inneren ein sehr dickes, aber extrem lockeres äußeres Mark (gonidienlose Zone) mit großen Zwischenräumen aufweisen. Die Rinde ist relativ betrachtet dicker als bei den Cl. furcata-Proben des selben Standorttyps und, zumindest oberseits, deutlich braun pigmentiert. Die Gonidienzone ist gut entwickelt, allerdings konzentrieren sich die Algen vermehrt auf die pigmentierte, dem Licht zugewandte Podetienoberseite. Den zentralen Hohlraum umgibt ein verhältnismäßig dünner, aber regelmäßiger Zylinder der inneren Markschicht.

\section{b) Podetien von Standorten des Typs Waldränder oder Felsen}

Die Podetien der Probe furcata-4 eines Waldrand-Standortes (Abb. 32b) haben eine Rinde, die an einigen Stellen körnig aufgelöst ist. Bis ans innere Mark heranreichende Risse treten ebenfalls auf. Die äußere Markschicht ist schmal und besteht in den Bereichen, in denen die Algenzellen vorkommen nur aus einer Gonidienzone. Gonidienlose Bereiche kommen lediglich stellenweise, quasi zwischen der Gonidienzone gelegen vor und liegen dann dementsprechend unmittelbar unter der 
Rinde. Das Podetium ist an den Bereichen mit Flechtenalgen stellenweise etwas nach außen gewölbt. Der Zylinder aus innerem Mark ist kräftig entwickelt.

Probe furcata-5 (Abb. 32c) stammt von einem sonnigen Tonschiefer-Steilhang. Das im Umriss unregelmäßige Podetium weist eine für derart sonnige Felsstandorte typische braun pigmentierte Rindenschicht auf (vgl. Kap. G3.2). Diese ist jedoch stellenweise sehr dünn oder fehlt gänzlich. Die Algen finden sich überwiegend in Bereichen, an denen sich die Podetienoberfläche leicht nach außen wölbt. Dazwischen liegen nur einzelne Algenzellen ins äußere Mark eingestreut. Die Algenschicht ist nicht so durchgängig, wie es nach den Ergebnissen der morphologischen Untersuchungen (s. Kap. G3.2) bei Podetien des Standorttyps Felsen zu erwarten gewesen wäre. Eine typische Probe dieser Standorte ist daher wohl die Probe furcata-7, bei der die Algen im gesamten äußeren Mark liegen. Äußeres und inneres Mark sind bei Probe furcata-7 bezüglich der Schichtdicke ziemlich gleichmäßig entwickelt.

Der Querschnitt von Cladonia furcata ssp. subrangiformis (Abb. 32d) entspricht im wesentlichen denen der Cl. furcata ssp. furcata-Proben. In der Gonidienzone liegen die relativ großen Algenzellen verhältnismäßig locker verteilt. Die Gonidienzone ist ausgedehnt, ähnlich der Cl. furcata-Proben von Felsstandorten, jedoch nicht durchgängig entwickelt. Sie wird immer wieder von Hyphen der gut entwickelten gonidienlosen Zone unterbrochen. Die Rinde ist an solchen Stellen dünn oder körnig aufgelöst.

Probe gracilis-1 repräsentiert eine Kümmerform, im Erscheinungsbild geprägt durch Trittbelastung an ihrem Wuchsort, einer von Pfaden durchzogenen stark besonnten Basaltblockhalde. Die Podetien sind kurz und von geringem Durchmesser. Im Querschnitt (Abb. 32e) ist wie bei Probe furcata-5 eine dicke, braun pigmentierte Rindenschicht erkennbar. Im Vergleich zu den Cl. furcata-Proben ist die Rinde ziemlich mächtig ausgebildet (vgl. auch Probe gracilis-3). Die innere Markschicht ist dagegen relativ dünn und im gesamten Querschnitt bezüglich der Schichtdicke wenig variabel. Bei dem deutlich dickeren äußeren Mark fällt auf, daß die Zone mit Algen nicht ringförmig den ganzen Querschnitt durchzieht, sondern in einer Hälfte des Querschnitts und auch dort nur fleckweise ausgebildet ist. Hierin unterscheidet sich diese Probe also von typischen $\mathrm{Cl}$. furcata-Podetien der Felsstandorte.

Dem Querschnitt in Abb. 32f ähnlich ist der von Probe gracilis-2 aus dem Niestetal. Er weist ebenfalls ein relativ dünnes inneres Mark auf und die Schnitte aus der Podetienmitte zeigen auch pustelförmige bzw. beulenartige Auswölbungen, die mit locker verflochtenen Hyphen der gonidienlosen Zone ausgefüllt sind. Zwischen den Auswölbungen ist die gonidienlose Zone lediglich dünn oder fehlt vollständig, die Gonidienzone nimmt dann fast das gesamte äußere Mark ein. In Übereinstimmung mit Cl. furcata-Proben vom selben Standorttyp, fehlt die Gonidienzone nur an wenigen Stellen, die Hyphen der gonidienlosen Zone liegen an solchen Stellen locker geknäuelt direkt unter der Rinde, die dort dünner bzw. körnig aussieht. Querschnitte, die weiter basal am Podetium entnommen wurden (Probe gracilis-2.2) zeigen, daß die Algen dort weniger dicht gedrängt zwischen den locker verflochtenen Hyphen des äußeren Marks 
liegen und die Gonidienzone relativ gesehen weniger Raum einnimmt. Die Rinde weist Lücken auf, die bis zum inneren Mark reichen.

\section{c) Längsschnitt Podetium}

Von den Proben furcata-5 und furcata-3 wurden Podetien-Längsschnitte angefertigt (Abb. 33a). In diesen zeigen sich folgende Verhältnisse: Die Rindenschicht sieht kompakt, fast zellulär aus. Die Rinde fehlt stellenweise bzw. erscheint körnig aufgelöst. In einigen Schnitten ist die Gonidienzone sehr unregelmäßig dick. Während sie an manchen Stellen des Schnittes fast vollständig fehlt, bildet sie an anderen deutliche, knollige Verdickungen aus. In der gonidienfreien Zone sind kleine Hohlräume zwischen den Hyphen erkennbar. Die innere Markschicht ist im gesamten Längsschnitt annähernd gleich dick.

\subsection{Schuppen am Podetium (= Phylloide) und Grundschuppen}

Ergänzend zu den Podetienquerschnitten wird nachfolgend der Aufbau der Phylloide ebenfalls kurz erläutert. Die untersuchten Schuppen sind den Proben furcata-3 und furcata-9 entnommen. Die Oberseite der Phylloide ist ebenfalls nicht durchgängig, sondern fleckig grün, da Bereiche mit Algen durch Bereiche ohne Algen abgegrenzt sind. Die Oberfläche sieht glatt, zum Rand hin etwas glänzend aus. Die Unterseite ist weiß und filzig. Weiße Strahlen ziehen von der Schuppenbasis zum Rand, sind jedoch nur stellenweise sichtbar (s. Abb. 33d).

Im Querschnitt ist zu erkennen, daß die Schichten in den Schuppen, denen in den Podetien gleichen (Abb. 33e+f). Die Rindenschicht ist durchscheinend trüb und ungefärbt, wie es für die Standorttypen Wald bzw. Berglagen typisch ist. Ihre Oberfläche erscheint im Querschnittbild ziemlich rauh. Die Gonidienzone weist dicht gepackte Algenzellen auf. Die gonidienlose Zone umschließt stellenweise Areolen des inneren Marks und bildet am Rand sowie an der Spitze der Schuppen das Abschlußgewebe nach unten, welches unter dem Binokular als weißer Filz zu erkennen ist. Die innere Markschicht ist nicht durchgängig entwickelt. Sie ist dort, wo sie vorkommt an den dünnen, verklebten Hyphen erkennbar. Äußeres und inneres Mark liegen also in den Schuppen nicht in Schichten übereinander. Das innere Mark bildet nur in der Mitte der Schuppen durchgängige Bereiche. An der Spitze und am Rand der Schuppen ist eine innere Markschicht fast nicht vorhanden. Je weiter die Schnitte zur Schuppenmitte hin angefertigt wurden, desto ausgedehnter sind die Bereiche mit innerem Mark. Jedoch liegen diesem oft noch filzige Reste der gonidienlosen Zone auf, so daß nur an vereinzelten Stellen das innere Mark das untere Abschlußgewebe bildet. Die Areolen mit innerer Markschicht entsprechen Querschnitten der im Binokular erkennbaren Strahlen, die von diesen Hyphen gebildet werden und ähnlich den Blattadern höherer Pflanzen die Blättchenfläche durchziehen. 

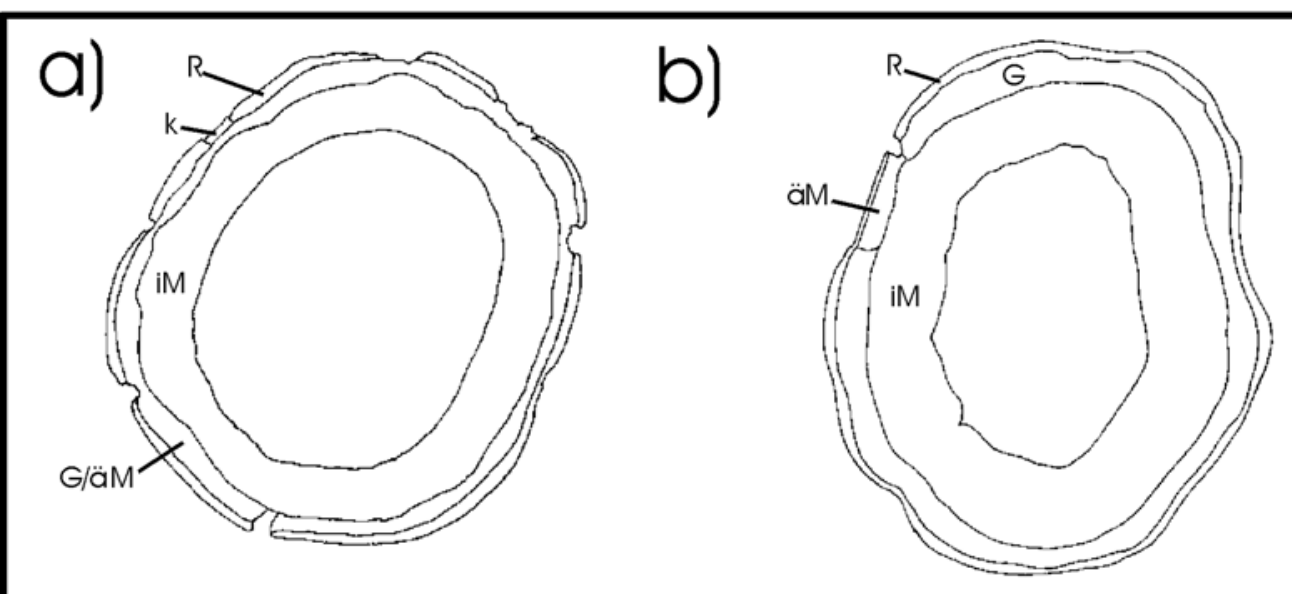

C)

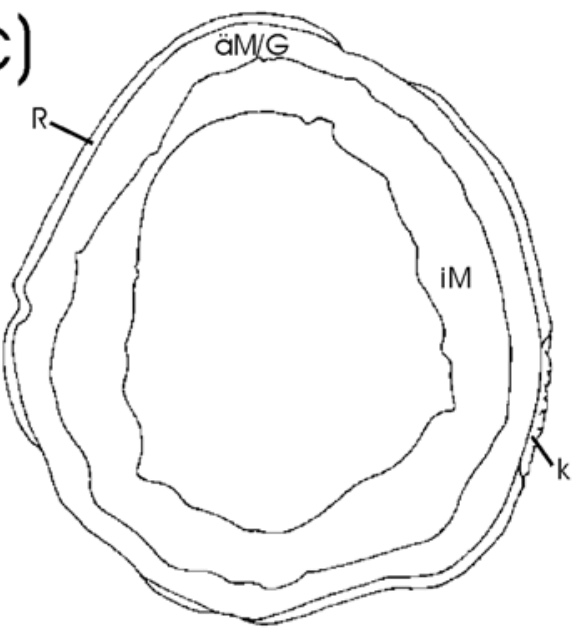

d)

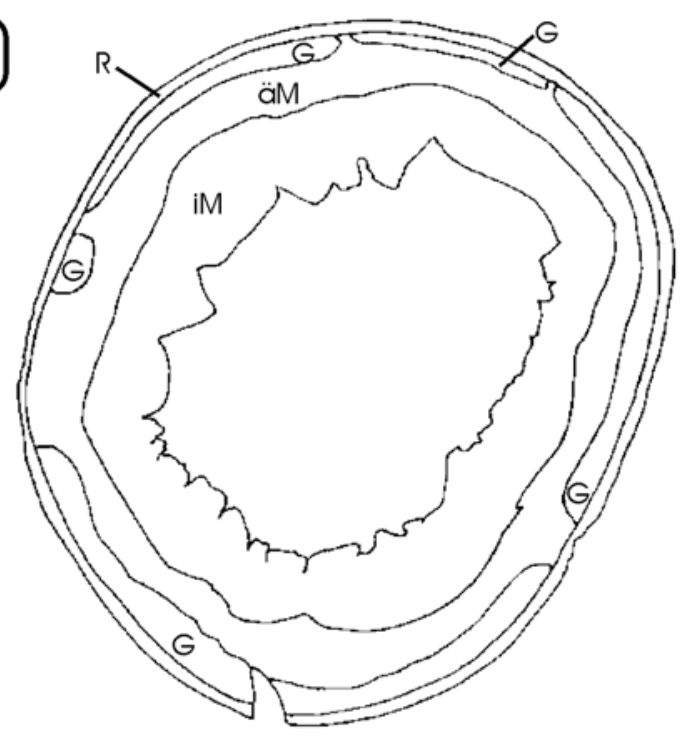

e)

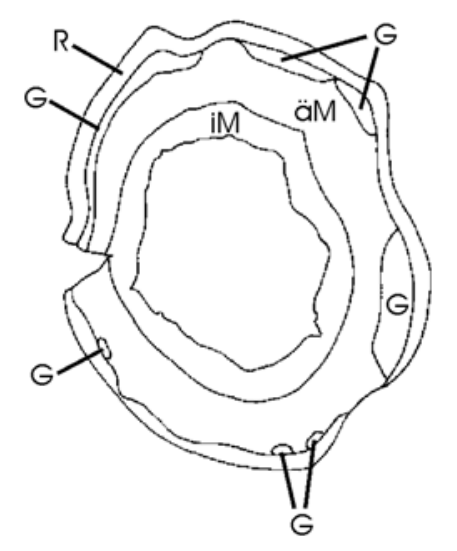

f)

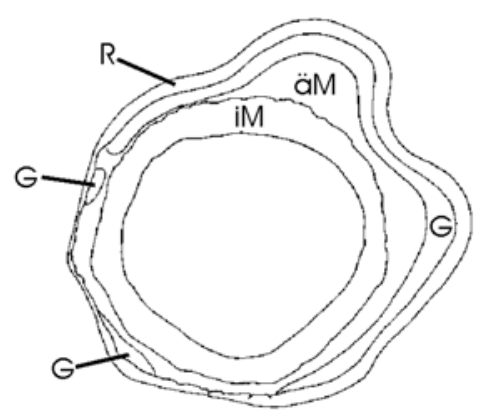

$0,5 \mathrm{~mm}$

Abb. 32: Podetienquerschnitte. a) Probe furcata-1; b) Probe furcata-4; c) Probe furcata-5;

d) Probe subrangiformis; e) Probe gracilis-1; f) Probe gracilis-3.

$R=$ Rinde $; G=$ Gonidienzone äußeres Mark; $\ddot{a} M=$ gonidienlose Zone äußeres Mark; $i M=$ inneres Mark; $k=$ nicht weiter definierte körnige Struktur. 


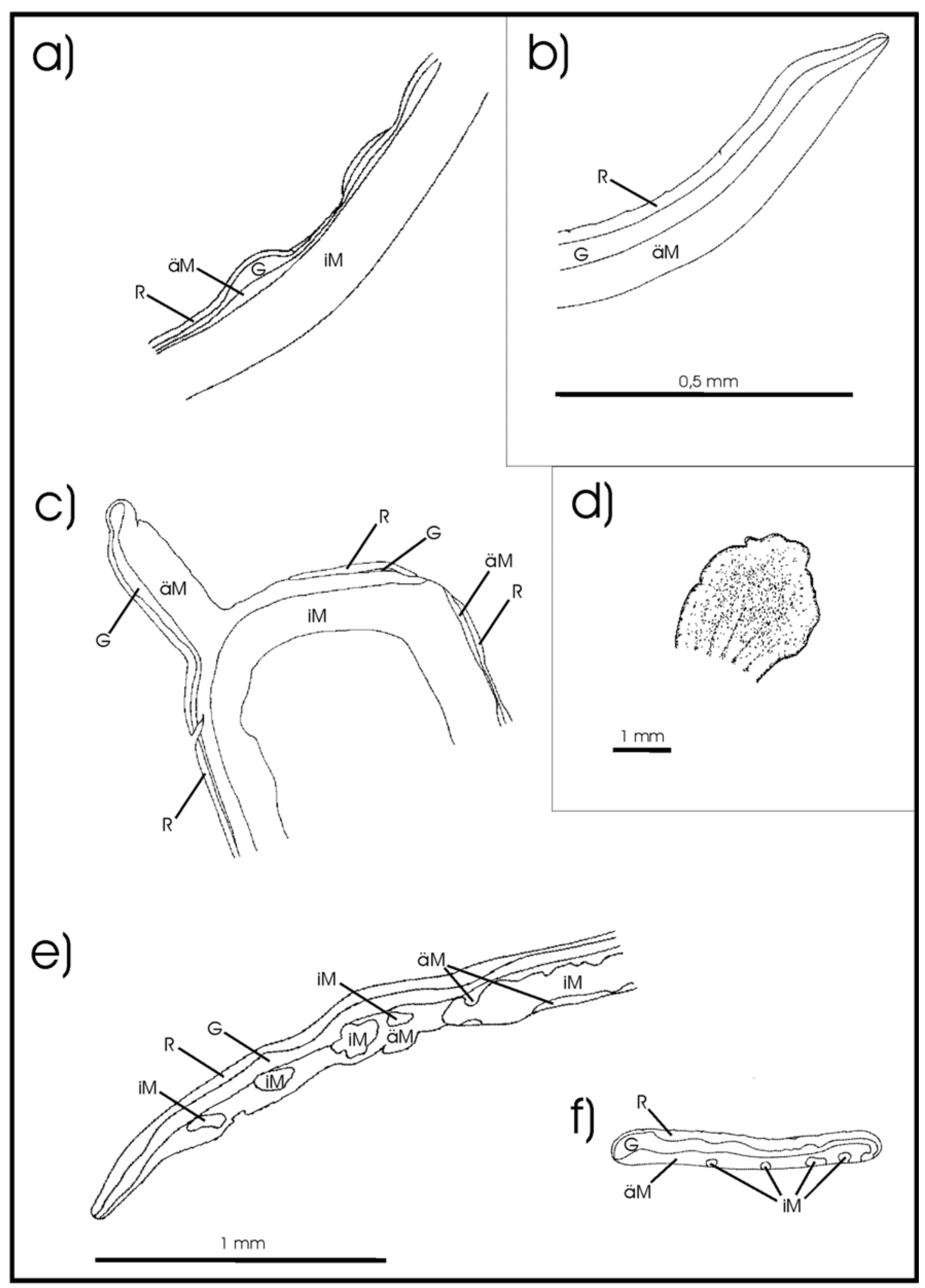

Abb. 33: Weitere lichtmikroskopische Schnitte durch Podetien, Phylloide und Grundschuppen. a) Längschnitt, Probe furcata-3; b) Querschnitt Grundschuppe Probe gracilis-1; c) Schuppenansatzstelle quer, Probe furcata-3; d) Gesamtansicht Schuppenunterseite, Probe furcata-9; e) Phylloid (Schuppe), Querschnitt Schuppenmitte, Probe furcata-3; f) Phylloid (Schuppe), Querschnitt Schuppenspitze, Probe furcata-3. Legende s. Abb. 32. 
Phylloide entstehen durch eine Gonidienvermehrung verbunden mit einer Hyphenneubildung (ULLRICH 1954). Rinde und äußere Markschicht heben sich in der weiteren Entwicklung zunehmend vom inneren Mark ab, die warzigen Erhebungen entwickeln sich zu kleinen Schüppchen. Abb. 33c zeigt einen Schnitt durch die Ansatzstelle einer jungen Schuppe. Eine Verbindung von innerem Mark und Schuppen ist in dieser Schnittebene nicht zu erkennen.

\section{d) Grundschuppen}

Bei den Proben gracilis-1 und gracilis-3 waren die Grundschuppen noch erkennbar, so daß auch von den Grundschuppen Querschnitte angefertigt werden konnten (Abb. 33b).

Die Rindenoberfläche der Grundschuppe von Probe gracilis-1 ist unregelmäßig und stellenweise eingekerbt. Das äußere Mark bildet das Abschlußgewebe an der Schuppenunterseite. Dort ragen zahlreiche Hyphen aus der Markschicht heraus. Eine innere Markschicht ist nicht vorhanden. Algenzellen scheinen reichlicher vorhanden zu sein als in den zugehörigen Podetien!

\section{Verpflanzungsversuche}

Wie in Kap. G3 gezeigt werden konnte, spielen Standortfaktoren bei der Entstehung der zahlreichen Modifikationen von Cladonia furcata eine wesentliche Rolle. Einzelne Modifikationen können bei Veränderung der Standortbedingungen in andere übergehen. So schreibt SANDSTEDE (1931) über Cl. crispata var. gracilescens, sie sei die Schattenform der var. cetrariaeformis und daher graugrün gefärbt. Sobald jedoch Sonnenstrahlen auf die var. gracilescens treffen, beginne die Bräunung. Gleiches ist auch bei Cl. furcata beobachtet worden: „Die v. palamaea hält mit racemosa enge Fühlung, sie ist die sonnenständige Pflanze, daher die braune Färbung, ihre f. subulata (...) ist die heller gefärbte Schattenpflanze.“ (SANDSTEDE 1931:199).

Der nachfolgend beschriebenen Verpflanzungsversuch zeigt, wie gut Cladonia furcata ssp. furcata auf sich ändernde Bedingungen an ihrem Wuchsort reagieren kann und welche Merkmale sich dabei in welcher Weise und innerhalb welchen Zeitraumes verändern. Interessant ist auch, ob das gesamte Polster oder lediglich die neu wachsenden Abschnitte von den Veränderungen betroffen sind.

\subsection{Das Makroklima im Versuchszeitraum (Tab. 44)}

Der Untersuchungszeitraum umfaßt die Monate Juni 2002 bis Oktober 2003. In den Sommermonaten zu Beginn der Untersuchungen wurden Temperaturen von maximal 30,0 bis $33,4{ }^{\circ} \mathrm{C}$ erreicht. Die Monatsmittel lagen in diesen Monaten bis zu $2,2{ }^{\circ} \mathrm{C}$ über den Durchschnittswerten der Jahre 1961 bis 1990. Der Juni 2002 war insgesamt sehr warm. Der Juli 2002 zeigte sich sonnenscheinarm und regenreich. Die Sonne schien nur 132,6 Stunden verglichen mit einer durchschnittlichen Sonnenscheindauer von 196 Stunden während der Referenzperiode. Leider liegen von der Station Kassel für Juni 2002 und Juli 2002 keine Werte der Niederschlagssummen vor. An der nur wenig weiter südwestlich liegenden Station Fritzlar wurde aber im Juli 2002 die beachtliche 
Niederschlagssumme von 165,3 mm gemessen. Der August 2002 war in ganz Deutschland sehr warm und sehr nass bei unterdurchschnittlichem Sonnenschein. Ein Großteil der Niederschläge fiel innerhalb zweier Extremsituationen in der ersten Monatshälfte (MÜLLER-WeSTERMEIER \& RIECKE 2003). Im September 2002 stieg die Tagestemperatur tagsüber noch bis auf $27,9^{\circ} \mathrm{C}$ an. Es fiel weniger Niederschlag als im Referenzzeitraum. Oktober und November 2002 waren hingegen relativ niederschlagsreich. Ende Oktober zog ein Sturmtief mit orkanartigen Böen über Deutschland hinweg (MÜLLER-WESTERMEIER \& RIECKE 2003). Während der November mild war (MÜLLER-WESTERMEIER \& RIECKE 2003), kamen im Dezember 2002 Tage mit deutlichem Frost von bis zu $-11,3{ }^{\circ} \mathrm{C}$ vor. Leider fehlen die entsprechenden Angaben für die Monate Januar und Februar 2003. Der Februar 2003 war im Mittel deutlich kälter als in der Referenzperiode. Bis in den April hinein trat deutlicher Frost auf. Großen Temperaturunterschieden waren die Flechten im April und Mai 2003 ausgesetzt. Im Mai kletterte das Thermometer schon auf sommerliche $28,4{ }^{\circ} \mathrm{C}$. Im Mittel war dieser Monat wärmer als in der Referenzperiode. Das Frühjahr 2003 kann insgesamt als relativ trocken, März und April 2003 können als ungewöhnlich sonnig bezeichnet werden.

Tab. 44: Klimadaten des Untersuchungszeitraumes, Klimastation Kassel (Daten aus: DEUTSCHER WETTERDIENST 2003a)

\begin{tabular}{|c|c|c|c|c|c|c|c|c|c|c|c|}
\hline & 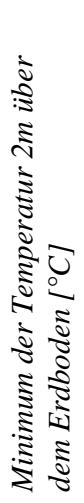 & 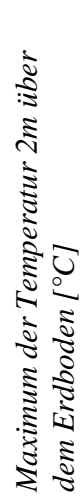 & 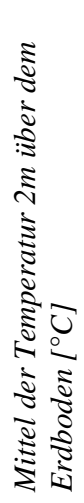 & 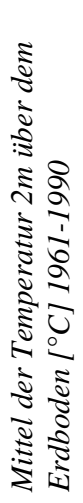 & 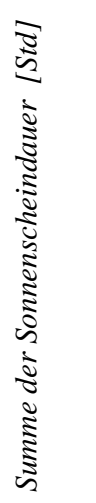 & 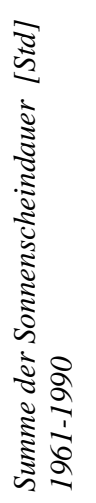 & 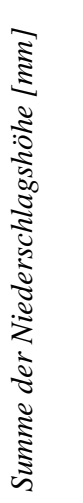 & 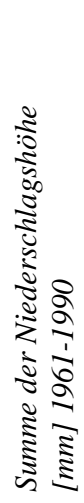 & 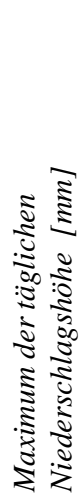 & 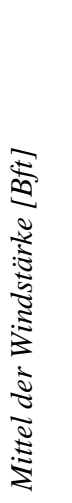 & 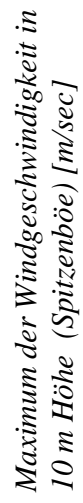 \\
\hline Sept & 2,5 & 29,2 & 13,8 & 13,5 & 198,3 & 135,0 & $\overline{46,4}$ & 54,0 & 18,6 & 20 & 18,6 \\
\hline Aug. 2003 & 8, & 36,7 & 21,2 & 16,9 & 2717 & 188,0 & 13 & 63 & 3,9 & & 13,6 \\
\hline Juli & 10,4 & 33,8 & 19,1 & 17 & 20 & 196,0 & 51 & 6 & 9,3 & & 17,8 \\
\hline Jun & 8,8 & 30,6 & 19 & 15 & 29 & 19 & 97 & 7 & 25,7 & & 18,8 \\
\hline $\mathrm{Ma}$ & 1,9 & 28,4 & 14 , & 12 & 1 & & 36 & 67 & 6 & & 14,3 \\
\hline $\mathrm{Ap}$ & $-6,4$ & 22,9 & 8,9 & 7,9 & 21 & 150,0 & 22,9 & 50 & 5 , & 2 , & 16,5 \\
\hline $\mathrm{M}^{\prime}$ & $-3,7$ & 19,2 & 6,2 & 3,9 & 161,9 & 110,0 & 30,4 & 51,0 & 9,0 & 2 , & 13,2 \\
\hline $\mathrm{Fel}$ & - & 12,3 & $-1,5$ & 0,8 & - & 72,0 & - & 43 & 7,6 & 2 , & 13,5 \\
\hline Jan. 2 & - & 9,5 & 0,2 & $-0,2$ & - & 39,0 & - & 55,0 & 19,3 & 2,5 & 17,9 \\
\hline Dez. 2002 & $-11,3$ & 12,4 & 0,7 & 1,1 & 37,1 & 31,0 & 86,4 & 67,0 & 23,1 & 2,0 & 13,2 \\
\hline Nov. 2002 & - & 13,7 & 6,4 & 4,1 & 25,4 & 45,0 & 79,1 & 59,0 & 14,3 & 2,1 & 17,2 \\
\hline Okt. 2002 & - & 18,4 & 8,3 & 9,3 & - & 99,0 & 84,7 & 46,0 & 14,0 & 2,3 & 24,8 \\
\hline Sept. 2002 & 0,9 & 27,9 & 13,2 & 13,5 & 136,1 & 135,0 & 33,8 & 54,0 & 15,7 & - & 14,3 \\
\hline Aug. 2002 & 11,5 & 30,0 & 19,1 & 16,9 & 170,7 & 188,0 & 35,7 & 63,0 & 19,0 & 1,9 & 14,5 \\
\hline Juli 2002 & 10,2 & 31,0 & 17,3 & 17,2 & 132,6 & 196,0 & - & 64,0 & 54,2 & 2,1 & 25,5 \\
\hline Juni 2002 & 6,2 & 33,4 & 17,1 & 15,6 & 181,0 & 190,0 & - & 79,0 & 21,4 & 2,0 & 15,6 \\
\hline
\end{tabular}

Die Sommermonate Juni, Juli und August 2003 waren erheblich zu warm. Juni und August waren im Gebietsmittel von Deutschland jeweils die wärmsten seit 1901. Anhaltende Hochdruckwetterlagen führten zu deutlich überdurchschnittlicher Sonnenscheindauer und $\mathrm{zu}$ einem erheblichen Niederschlagsdefizit. Mit Ausnahme einiger 
Stationen in Nord- und Nordwestdeutschland war dies der heißeste Sommer seit Beginn der Messreihen 1901 (Deutscher WetTerdienst 2003b). Die höchste Temperatur trat im August auf, sie betrug fast $37^{\circ} \mathrm{C}$ ! Auch die Sonnenscheindauer lag während dieser Monate deutlich über dem Durchschnitt. Dagegen fielen im Juli und vor allem auch im August deutlich weniger Niederschläge als in den vergleichbaren Monaten der Jahre 1961 bis 1990. Der Sommer 2003 war also nicht nur ungewöhnlich warm, sondern auch sehr trocken. Der September 2003 war ebenfalls überdurchschnittlich sonnig, es fiel mehr Regen als im August, jedoch weniger als im Mittel der Jahre 1961 bis 1990. Weitere Angaben zu Temperatur, Sonnenscheindauer, Niederschlagshöhen und zur Windstärke während des Untersuchungszeitraumes sind Tab. 44 zu entnehmen.

\section{Das Wetter am 16. Juli 2003:}

Es war ein zunächst sonniger, sehr heißer Hochsommertag, dem mehrere trockene und ebenfalls heiße Tage vorausgegangen waren. Ab 14:00 Uhr zogen erste Wolken einer herannahenden Gewitterfront herauf. Bewölkte und sonnige Phasen wechselten sich ab. Erst am frühen Abend hatte sich eine geschlossene Wolkendecke gebildet, aus der um 20:00 Uhr die ersten Regentropfen fielen.

\subsection{Das Mikroklima an den Erdflechtenstandorten}

Die folgenden Ausführungen stellen die extremen mikroklimatischen Verhältnisse während der Sommerperiode an den Standorten zweier verschiedener Erdflechtengesellschaften dar, in denen Cladonia furcata ssp. furcata auftritt. Die Vegetation an den Standorten R1 und R2 ist der Cl. furcata-Cladonion arbusculae-FG, die Vegetation am Standort R3 dem Cladonietum mitis cladonietosum merochlorophaeae zuzuordnen.

\section{a) Licht (Abb. 34)}

In den ersten Tagesstunden unterscheiden sich beide Flächen bezüglich der Lichtintensitäten kaum voneinander. Bis $13.00 \mathrm{Uhr}$ werden beide Versuchsflächen durch die umgebenden Gehölze abgeschirmt. Lediglich einzelne Sonnenflecken führen zu kleinen Peaks. Ab 13.00 Uhr ist der Standort R3 der vollen Besonnung ausgesetzt, die Lichtintensität steigt steil an. Es herrschen nun Lichtverhältnisse wie im Freiland. Auch Fläche R2 wird jetzt stärker besonnt, allerdings im Gegensatz zu R3 nicht flächendeckend. Das Blätterdach mindert nach wie vor die Einstrahlung. Die Lichtintensität steigt dort zwar ebenfalls an, erreicht jedoch, verglichen mit R3, einen wesentlich geringeren Maximalwert. Leider tritt gegen 14.00 Uhr eine Wetteränderung ein, der Himmel bewölkte sich zunehmend, so daß die Lichtintensitäten an beiden Standorten schon kurze Zeit nach Erreichen der hohen Werte wieder deutlich absinken. Nachmittags wäre Fläche R3 eigentlich besonnt, jedoch gelangen nur gelegentlich durch Lücken in der Wolkendecke einige Sonnenstrahlen auf die Fläche. Dies führt jeweils zu einem kurzzeitigen Anstieg der Lichtintensität. Fläche R2 liegt auch während des Nachmittags weiterhin im Schatten der Eichen, dementsprechend niedriger sind die Lichtintensitäten auch in der zweiten Tageshälfte. 
Die maximalen Lichtintensitäten können an Cladonien-Wuchsorten deutlich höher ausfallen, als am 16. Juli am Roßkopf. Die Lichtmessungen von JAHNS \& FRITZLER (1982) auf einer Blockhalde im Taunus ergaben an einem Sommertag, bei voller Besonnung, Werte von über 90000 Lux. Leider wurde an keinem Wuchsort von Cladonia furcata gemessen, welche auf der betreffenden Blockhalde in randlichen Bereichen ebenfalls vorkommt. ScHÖLLER (1991) vergleicht Schattenzone, Übergangszone und Vollichtzone in flechtenreichen Traubeneichenwäldern. Während der Sommerperiode liegen die maximalen Lichtintensitäten in der Schattenzone bei unter 20000 Lux, in der Übergangszone bei fast 80000 Lux und in der Vollichtzone bei etwa 120000 Lux. Cladonia furcata ssp. furcata tritt in allen 3 Zonen auf.

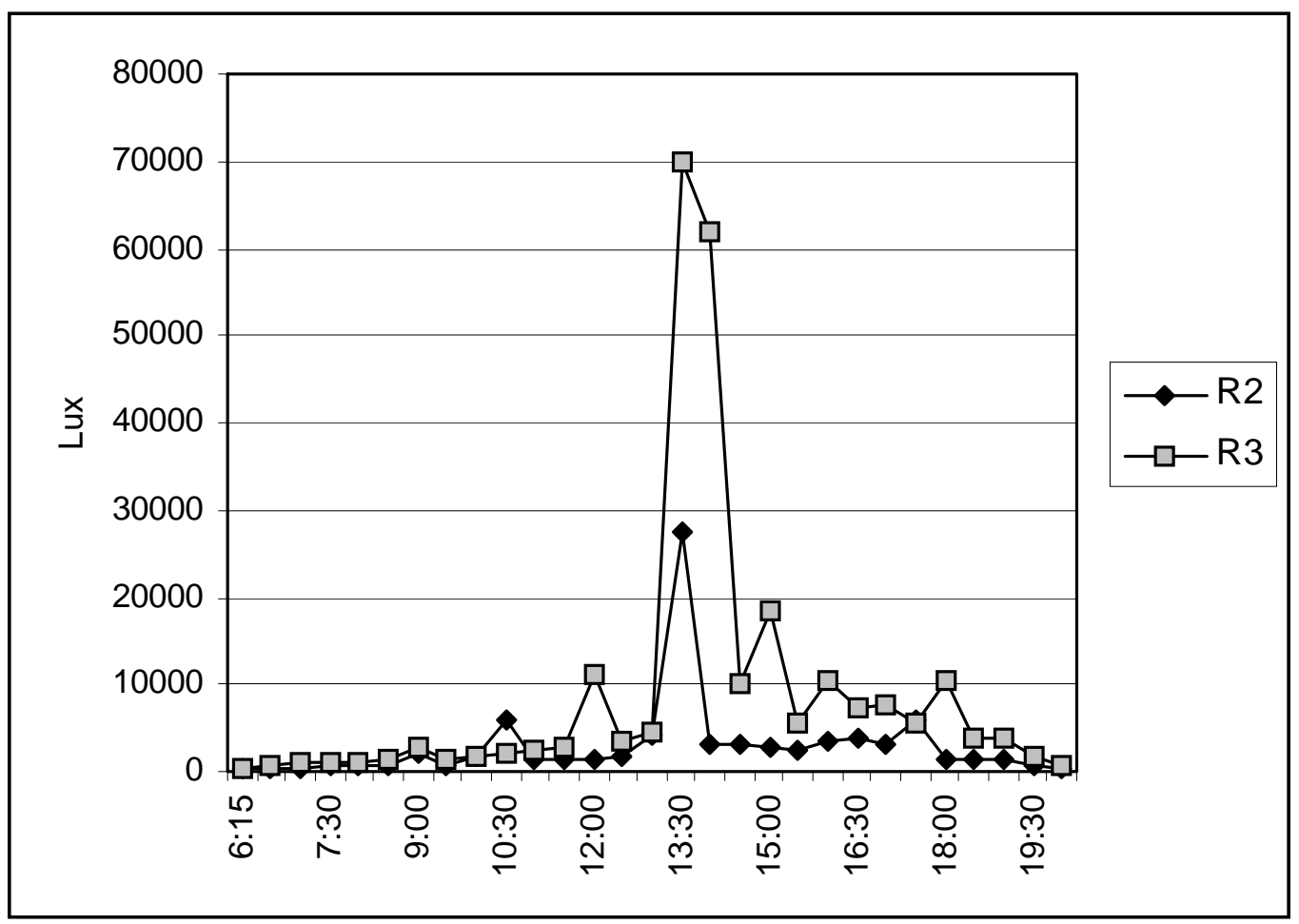

Abb. 34: Tagesverlauf der Lichtintensität an den Standorten R2 und R3 am 16.07.2003.

b) Lufttemperatur und Thallustemperatur (Abb. 35 bis Abb. 37)

Da die Temperaturen von Luft und Thallus in erster Linie von der Einstrahlung und Ausstrahlung beeinflußt werden (s. JAHNS \& OTT 1983), zeigt sich eine Korrelation zwischen den Temperaturkurven und den entsprechenden Kurven der Lichtintensitäten. Im Laufe des Vormittags steigen die Temperaturen an beiden Standorten etwa in gleichem Maße an. Als gegen 13.00 Uhr die Mittagssonne die Standorte erreicht, steigt die Lufttemperatur auf der direkt besonnten Fläche R3 deutlich, auf der etwas beschatteten Fläche R2 leicht an. Mit Einsetzen der Bewölkung geht sie jedoch an beiden Meßstellen wieder zurück. In gleicher Weise reagieren auch die Thallustemperaturen. Der direkten Sonnenstrahlung ausgesetzt, erwärmen sich die Thalli am Standort R3 rasch auf bis zu $47^{\circ} \mathrm{C}$ bei einer Lufttemperatur von $35,5^{\circ} \mathrm{C}$. Möglicherweise hätten sie sich noch weiter aufgeheizt, wäre nicht der Wetterwechsel eingetreten. Deutlich kühler bleiben die Thalli in R2; sie erreichen nur maximal $34,6{ }^{\circ} \mathrm{C}$ bei einer Luftemperatur von $32{ }^{\circ} \mathrm{C}$. 
c) Luft- und Thallusfeuchte (Abb. 38 und Abb. 39)

Die Luftfeuchtigkeitswerte sind an beiden Standorten stärkeren Schwankungen unterlegen (Schwankungen beobachteten auch JAHNS \& OTT (1983) bei ihren Mikroklimamessungen). Tendenziell sinkt aber auf beiden Flächen zum Mittag hin die relative Luftfeuchtigkeit ab und erreicht auf R2 einen Minimumwert von knapp unter $40 \%$, auf R3 von etwa $30 \%$. Das Einsetzen der Bewölkung ist auch in diesen Kurven an einem leichten Anstieg der Werte erkennbar. Bis zum frühen Abend bleiben die Werte jedoch auf relativ niedrigem Niveau und steigen erst gegen 19.30 Uhr wieder deutlicher an.

Der Wassergehalt der Flechten steht in enger Beziehung zur relativen Luftfeuchtigkeit (JAHNS 1984). Bis zur Mittagszeit nimmt der Wassergehalt der Flechten einhergehend mit den Luftfeuchtigkeitswerten an beiden Standorten stetig ab. Gegen $12.00 \mathrm{Uhr}$ fallen die Werte des Wassergehalts unter $15 \%$ und somit unter die Schwelle ab, für die die verwendete Messapparatur zuverlässige Daten liefert (s. SCHUSTER et al. 1982). Die Messwerte schwanken in der Mittagshitze vielleicht auch deshalb relativ stark. Für den Standort R3 ergibt sich in einem Falle sogar ein negativer Wert (in Abb. 39 nicht dargestellt). Ab 18.00 Uhr und mit leicht zunehmender Luftfeuchtigkeit fangen auch die Thalli beider Standorte wieder an Feuchtigkeit aufzunehmen. Jedoch ist gegen 20.00 Uhr noch nicht einmal die $15 \%$-Grenze überschritten. Der Wassergehalt der Flechten ist insgesamt am Standort R3 geringfügig niedriger als am Standort R2. Nach SCHUSTER et al. (1982) ist ab einem Wassergehalt von unter $15 \%$ der Stoffwechsel der Flechten nur noch extrem gering und sie befinden sich in einer Trockenstarre. D.h. an beiden Standorten ist an einem solchen Sommertag, wie dem 16.07.2003, den ganzen Tag über die Stoffwechselaktivität von Cladonia furcata ssp. furcata äußerst gering. Die Polster weisen selbst am frühen Morgen kaum mehr als $20 \%$ Wassergehalt auf. Der für die Photosynthese optimale Wassergehalt liegt bei dieser Art bei $100 \%$ der Trockenmasse (TUBA et al. 1996)!

d) Wind und Evaporation (Abb. 40 bis Abb. 42)

Während es am Morgen und am frühen Vormittag fast windstill war, frischte der Wind gegen Mittag deutlich auf, wurde dann wiederum etwas schwächer, um gegen 17.00 Uhr ein zweites Maximum zu erreichen. Insgesamt war es jedoch ein nur schwach windiger Tag. Die Kurven beider Standorte zeigen einen ähnlichen Verlauf, Fläche R3 ist allerdings etwas mehr dem Wind ausgesetzt als Fläche R2. Nach JAHNS \& OTT (1983) kann der Wind die Verdunstung und damit die Temperatur beeinflußen. Da aber an diesem Sommertag die Thalli bereits morgens ausgetrocknet waren, dürfte dies für den Juli-Meßtag ohne Bedeutung sein. Auch die Evaporation ist auf der Fläche R3 höher als an dem im Wald gelegenen Standort. Um die Mittagszeit sind die Unterschiede zwischen beiden Flächen besonders deutlich. Über den gesamten Tag betrachtet ist die Evaporationssumme auf der Fläche R3 deutlich höher als auf der Fläche R2. 


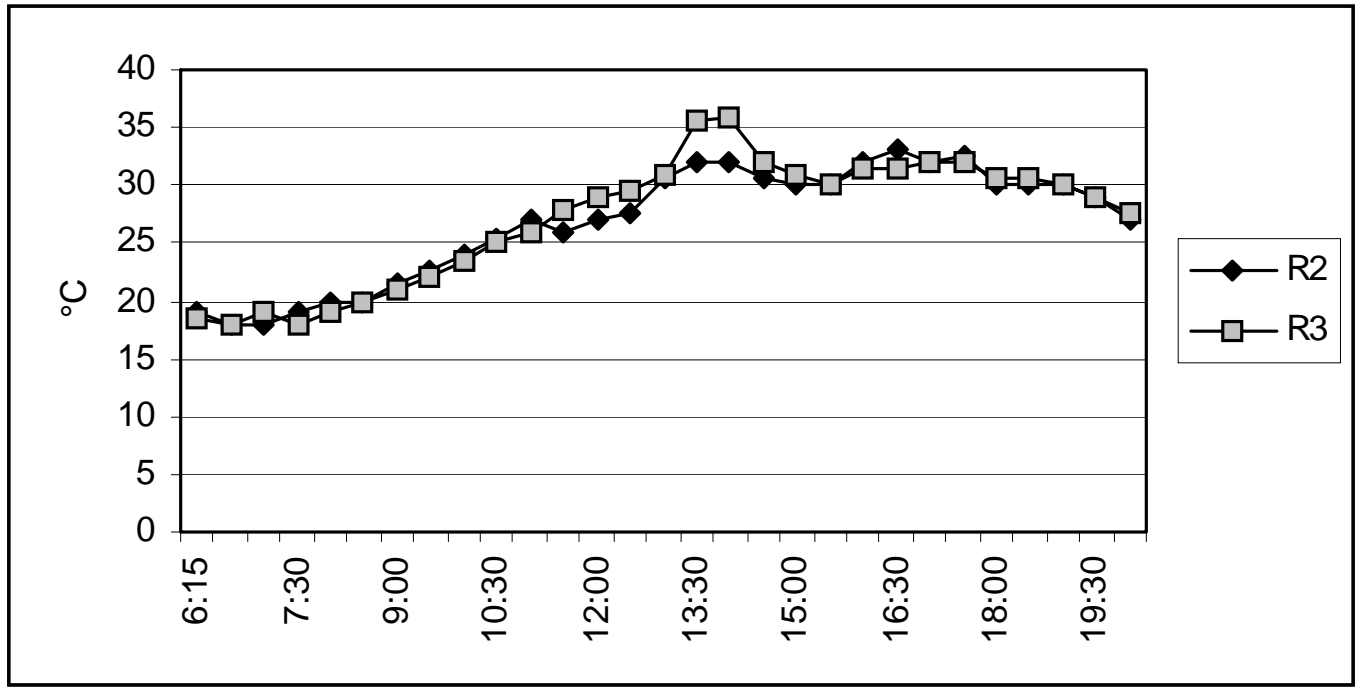

Abb. 35: Tagesverlauf der Lufttemperatur an den Standorten R2 und R3 am 16.07.2003.

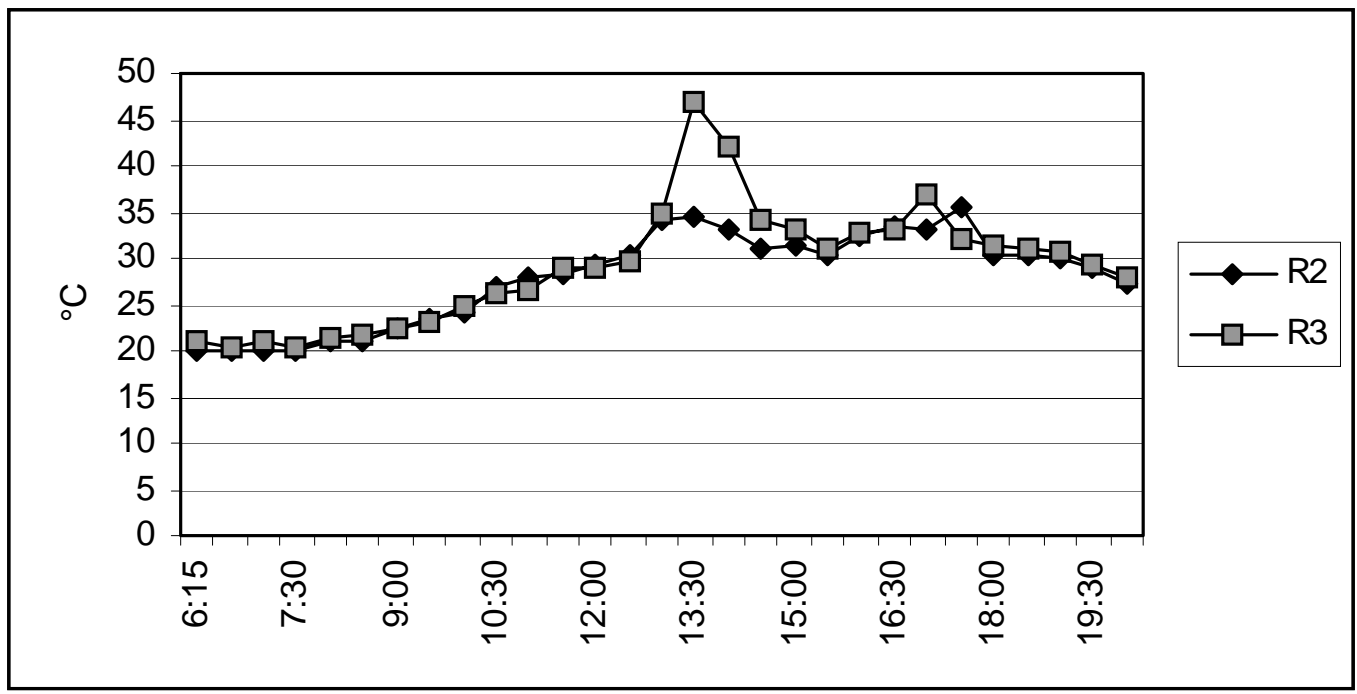

Abb. 36: Tagesverlauf der Thallustemperatur an den Standorten R2 und R3 am 16.07.2003.

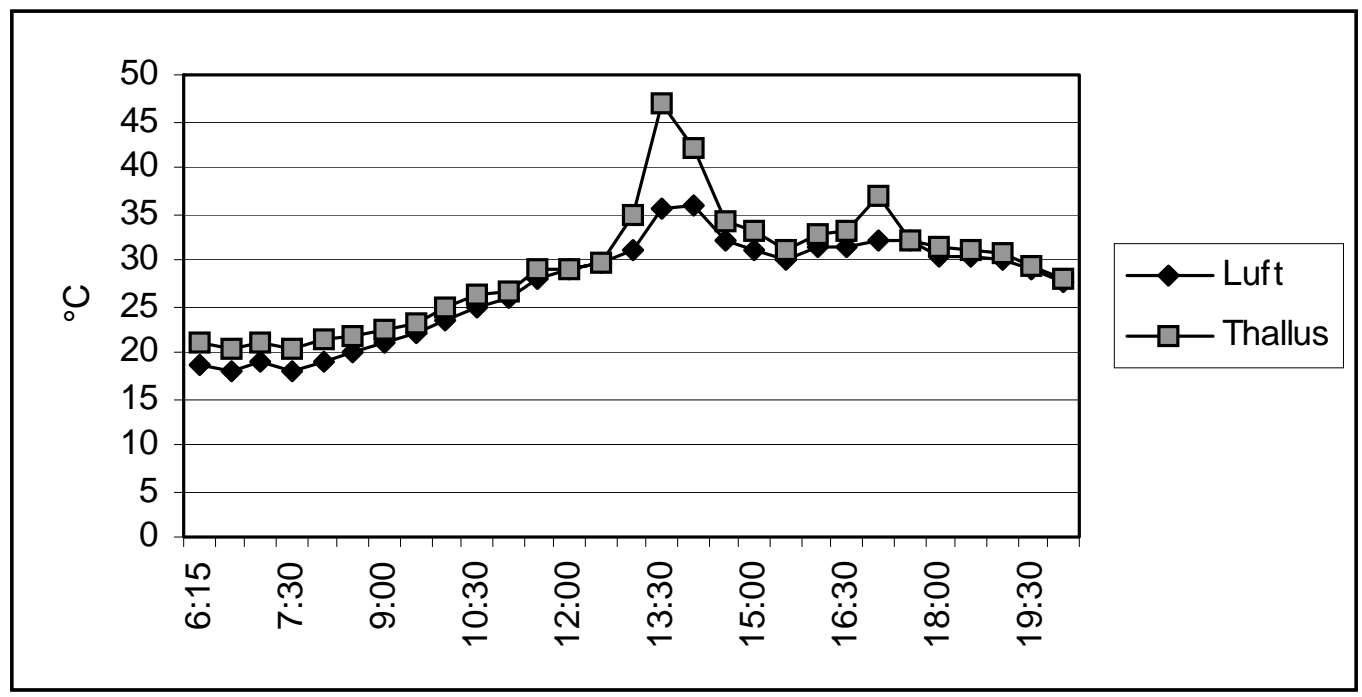

Abb. 37: Vergleich von Luft- und Thallustemperatur am Standort R3 am 16.07.2003. 


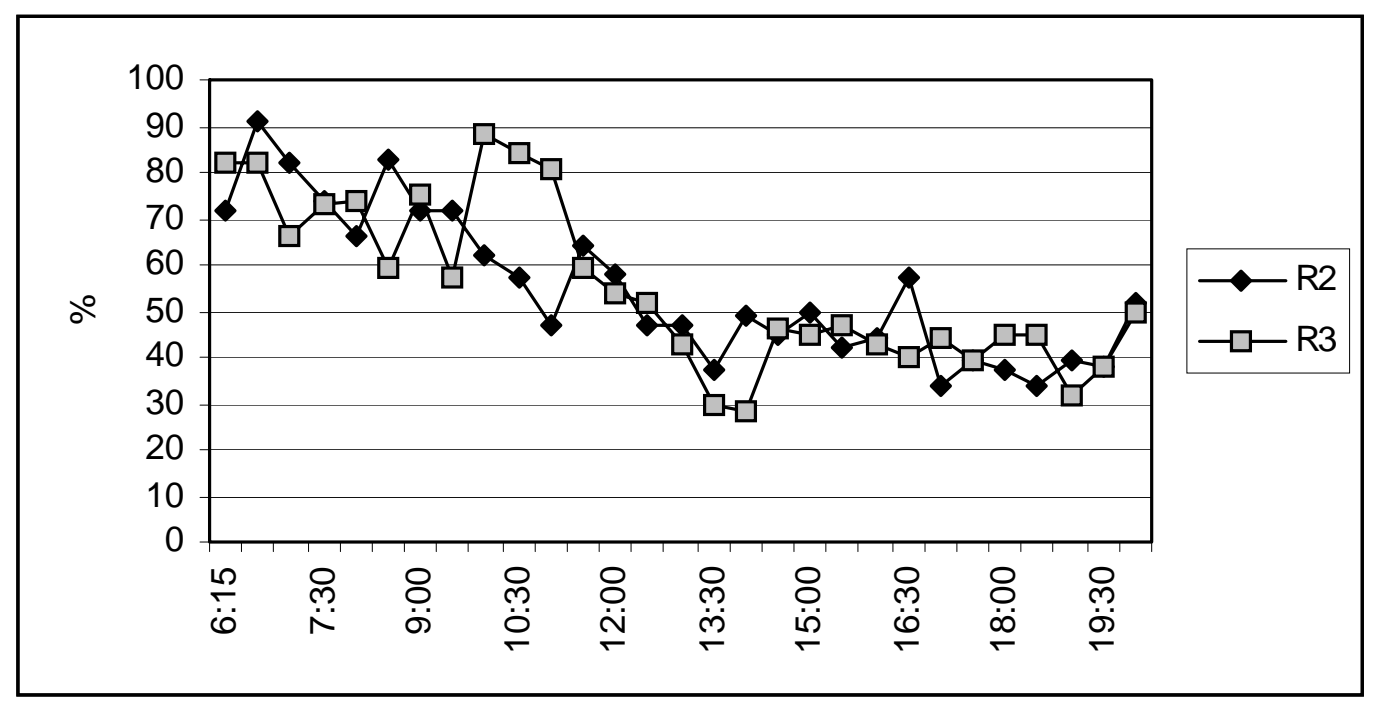

Abb. 38: Tagesverlauf der relativen Luftfeuchte an den Standorten R2 und R3 am 16.07.2003.

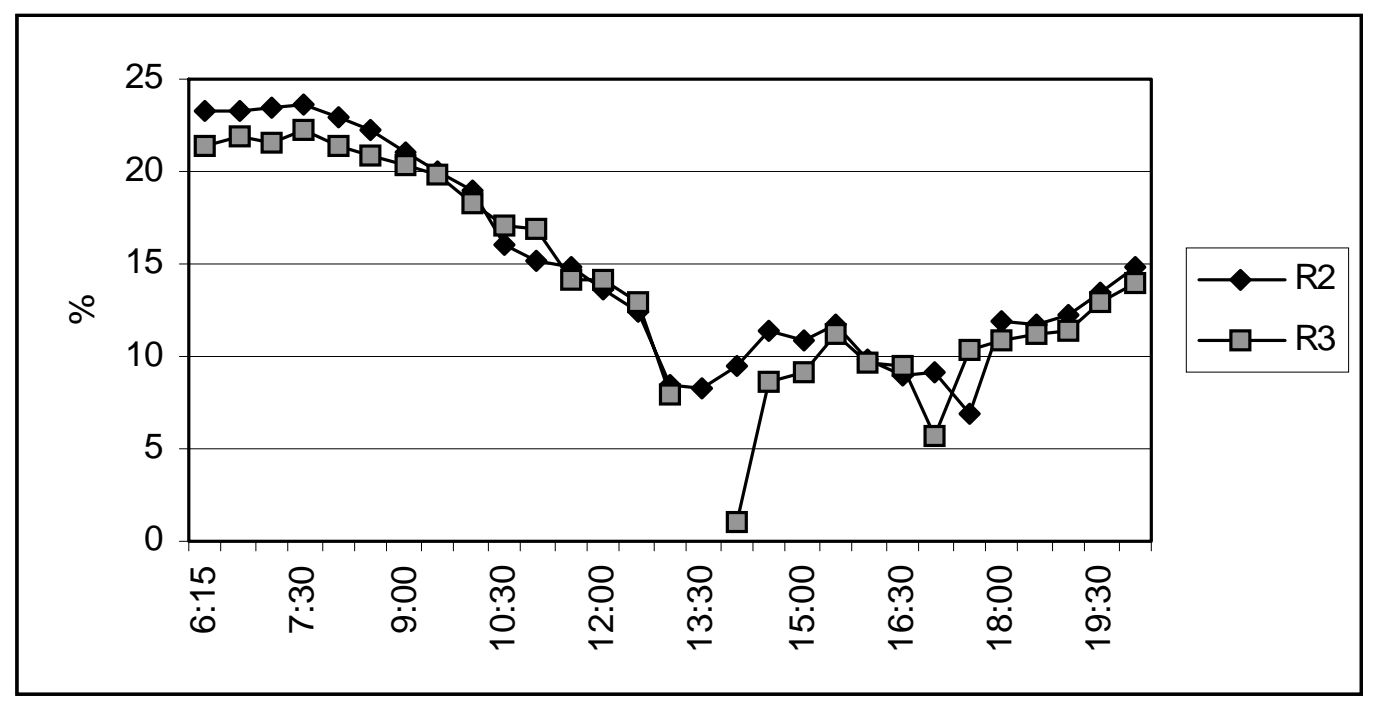

Abb. 39: Tagesverlauf derThallusfeuchtigkeit an den Standorten R2 und R3 am 16.07.2003. 


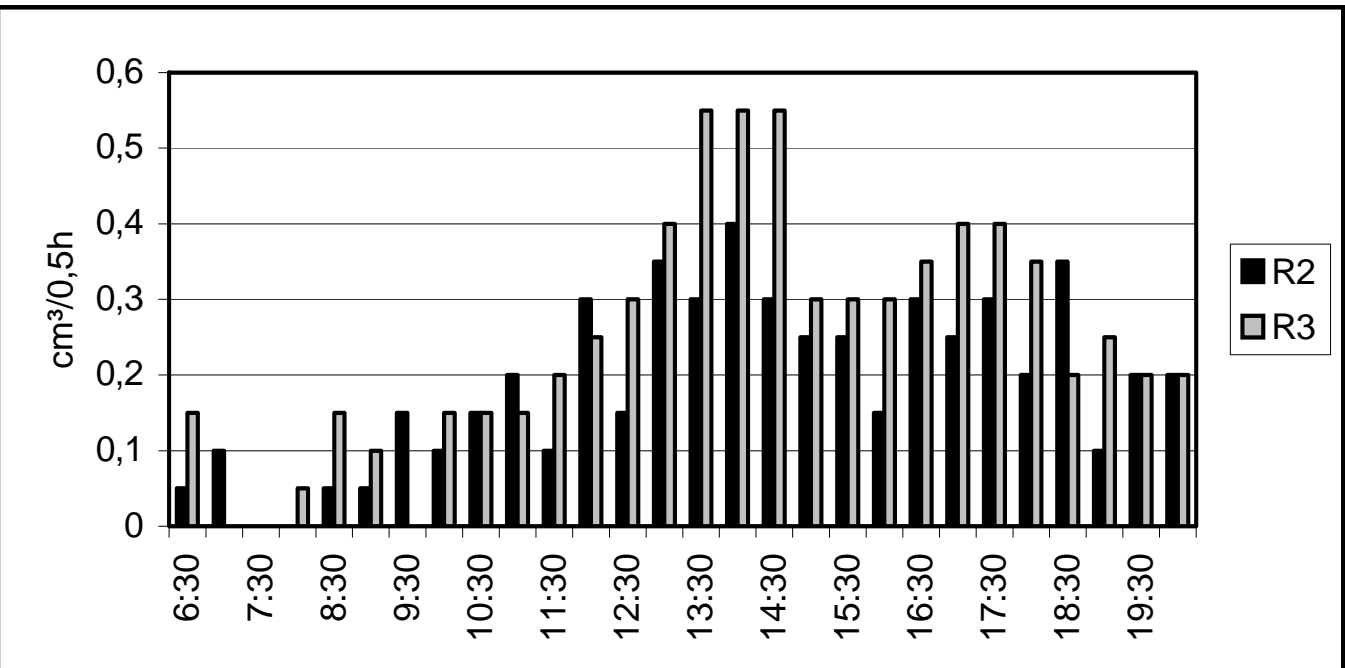

Abb. 40: Tagesverlauf der Evaporation an den Standorten R2 und R3 am 16.07.2003.

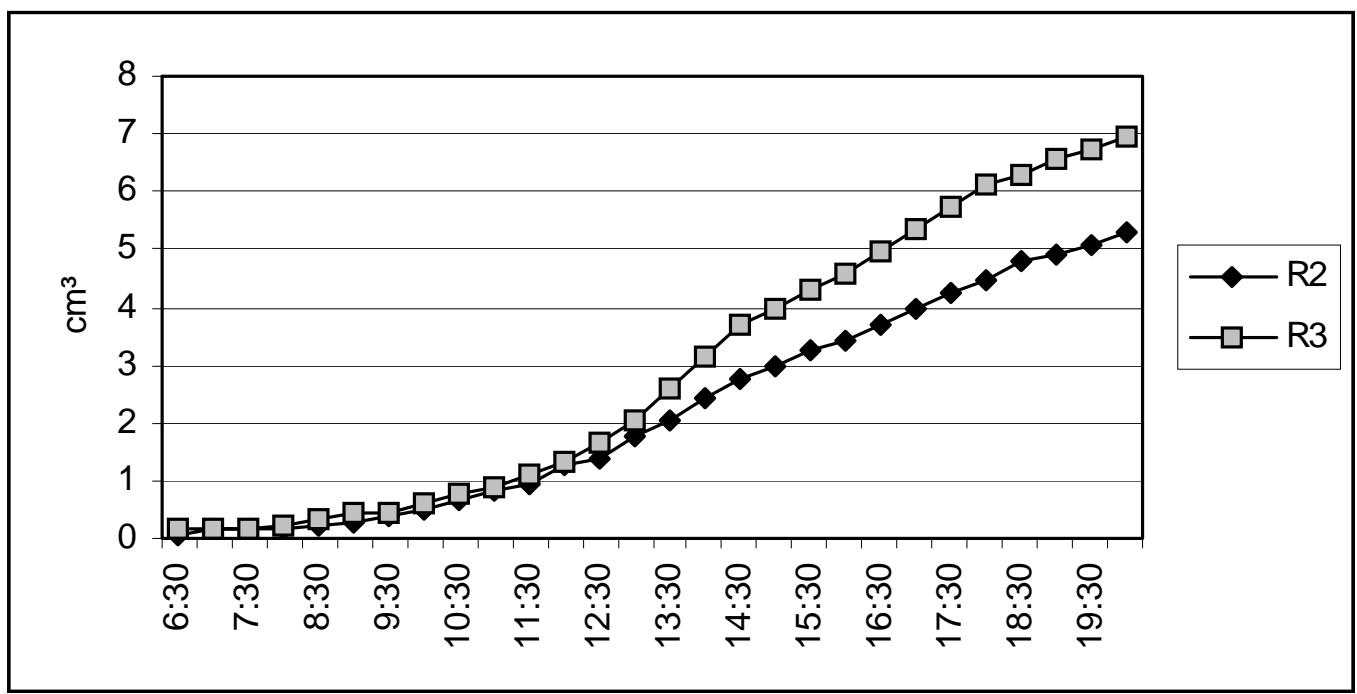

Abb. 41: Evaporationssummen an den Standorten R2 und R3 am 16.07.2003.

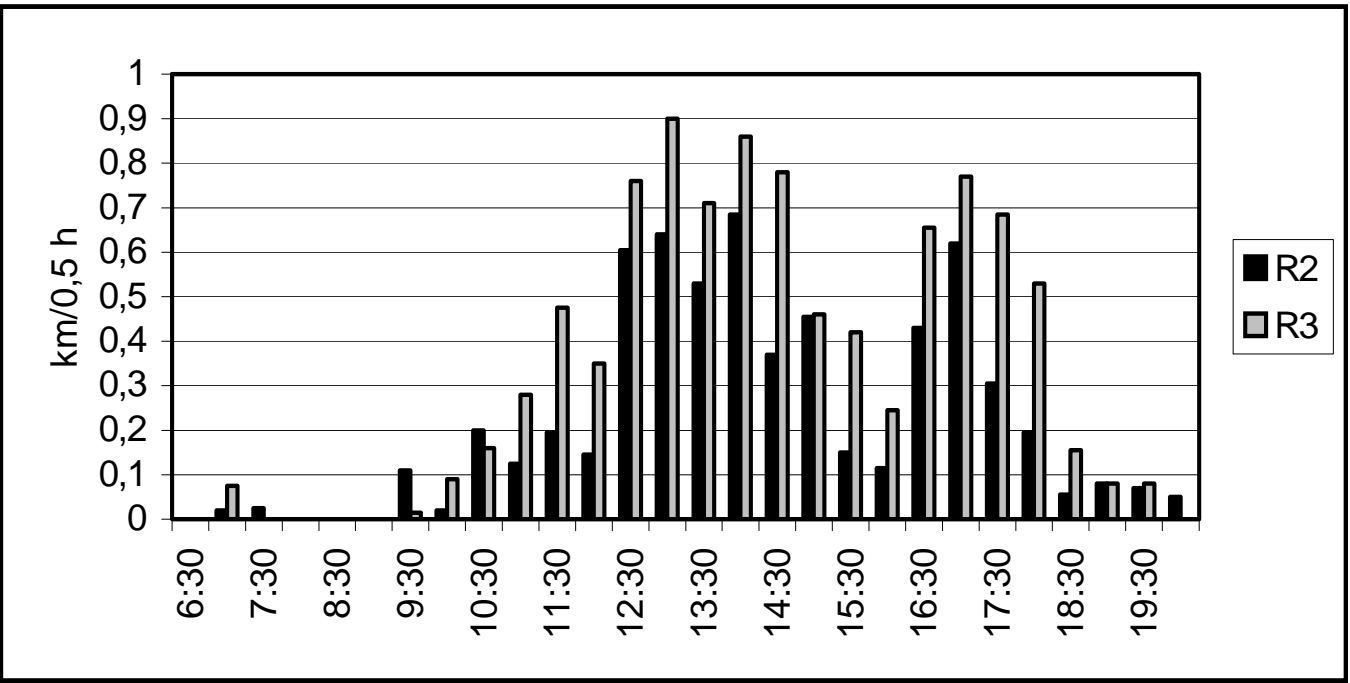

Abb. 42: Tagesverlauf der Windmessungen an den Standorten R2 und R3 am 16.07.2003. 


\section{e) Einstufung und abschließender Vergleich der beiden Erdflechtenstandorte}

Wie ein Vergleich der Kurvenverläufe der Lichtintensitäten und des Arteninventars mit den Angaben von SchÖLlER (1991) zeigt, ist die Fläche R2, mit der darauf siedelnden Cladonia furcata-Cladonion arbusculae-FG, der sog. Schattenzone zuzuordnen. Diese wird dominiert von Dicranum scoparium. Die gemessenen Lichtintensitäten deuten allerdings einen gewissen Übergangscharakter an, da der Standort nicht den ganzen Tag gleichmäßig beschattet ist. Die Schattenzone bei SCHÖLLER entspricht dem Standorttyp „Wald“ der vorliegenden Untersuchung. Fläche R3 zählt zur sogenannten Übergangszone jedoch mit deutlicher Tendenz zur Vollichtzone, erkennbar an den für die hellsten Standorte typischen Flechtenarten Dibaeis baeomyces und Trapeliopsis granulosa (s. ScHÖLLER 1991). Diese Übergangszone ist dem Standorttyp „Waldrand“ gleichzusetzen und wird hier vom Cladonietum mitis cladonietosum merochlorophaeae besiedelt.

Nach ScHÖLLER (1991) kann Cladonia furcata ssp. furcata von der Schatten- bis in die Vollichtzone auftreten. In Nordhessen kommt sie auch auf sonnig-trockenen Felsstandorten vor (Cladonietum foliaceae, s. Kap. E2.1.2.4). Die Fläche R2 steht somit für die schattigsten Standorte, an denen Cl. furcata ssp. furcata gedeihen kann, während R3 einen intermediären Wuchsort repräsentiert, der zwar relativ sonnig und trocken ist, aber noch nicht die extremsten Bedingungen aufweist, die Cl. furcata ssp. furcata zu ertragen in der Lage ist.

Wie der mikroklimatische Vergleich der Versuchsflächen zeigt, sind beide Standorte verhältnismäßig trocken. Die Fläche R3 am Waldrand ist jedoch insgesamt sonniger und wärmer als die Fläche R2, so daß die Thalli dort etwas schneller austrocknen, stärkerer Einstrahlung ausgesetzt sind und sich bei Sonneneinstrahlung deutlich mehr erwärmen.

\subsection{Morphologische Veränderungen der Thalli und Zuwachsraten}

\subsubsection{Die Morphologie der Polster am Versuchsende}

Alle ausgepflanzten Polster machen zum Zeitpunkt des Versuchsendes einen vitalen Eindruck. Bei den Polstern Nr. III und Nr. IX der Waldrand-Standorte R3 bzw. N1 ist deutlicher als bei den anderen Polstern der selben Flächen zu erkennen, daß sich die Podetien im Verlaufe des Auspflanzungszeitraumes in Richtung Mittagssonne ausgerichtet haben (s. Taf. 5.2 im Anhang 2). Dies ist bei den Polstern der WaldStandorte R1 und R2 nicht der Fall.

Die Polster der Fläche R3 zeigen nach Versuchsende eine deutlich stärkere Pigmentierung als die der Flächen im Wald (s. Taf. 5.2 im Anhang 2). Die Polster des Niestetals (N1) nehmen eine Zwischenstellung ein - sie sind dunkler als die der Flächen R1 und R2, aber nicht so stark gebräunt wie die der Fläche R3. Jedoch ist auch der Grad der Pigmentierung, wie er sich am Standort R3 entwickelt hat, noch nicht das Maximum an Bräunung, welches ganz allgemein im Untersuchungsgebiet beobachtet wurde. Die neu entstandene verstärkte Pigmentierung betrifft nicht nur die Podetienspitzen, sondern 
alle Teile der Podetien, die der Sonnenstrahlung ausgesetzt waren, auch die wenigen Schuppen an den Lagerstielen.

Eine nennenswerte Neubildung von Schuppen an den wachsenden Podetienspitzen konnte an keinem der Standorte beobachtet werden. Keines der markierten Podetien hat im Versuchszeitraum Apothecien ausgebildet. Lediglich 4 Zweigspitzen wiesen neu entstandene Pyknidien auf.

\subsubsection{Zuwachsraten und Neubildung von Verzweigungen}

\section{a) Vergleich der Zuwachsraten an den verschiedenen Standorten (Tab. 45)}

Einige der markierten Zweige haben den Zeitraum der Auspflanzung leider nicht unbeschadet überstanden und mußten, meist aufgrund von Bruchschäden, bei der Auswertung unberücksichtigt bleiben. Zwei Polster (Nr. VII und VIII) waren derart stark beschädigt, daß keine einzige intakte, markierte Zweigspitze mehr übrig war. Bei einem Polster (Nr. IX) haben sich die Podetien so stark in eine Richtung verbogen, daß eine eindeutige Zuordnung der nummerierten Zweige nicht mehr möglich war. Insgesamt hatten jedoch die meisten Podetien die Zeit in den Gattern ohne sichtbare Schäden überdauert und sich gut entwickelt.

Die Auswertung der Zuwachsraten erbrachte folgendes Ergebnis: Die Podetien der von Ende Juni 2002 bis Mitte Juli 2003 ausgepflanzten Polster sind an den Standorten R1 und R2 (im Wald) etwas weniger gut gewachsen als am Standort R3 (am Waldrand). Nicht so dagegen die Podetien der Proben, die von Mitte August 2002 bis Mitte Oktober 2003 in den Gattern belassen wurden. Bei diesen Thalli aus der Zeche Glimmerode zeigen sich keine Unterschiede zwischen den Standorten.

Tab. 45: Mittlere Zuwachsraten der Podetien

\begin{tabular}{l|lllll}
\hline Fläche & Zeitraum & Herkunft & Polster-Nr. & $\begin{array}{l}\text { Anzahl } \\
\text { Ausgemessener Zweige }\end{array}$ & $\begin{array}{l}\text { Mittlerer } \\
\text { Zuwachs der } \\
\text { Podetien [mm] }\end{array}$ \\
\hline R1 & J & ME & Ia & 25 & 1,3 \\
& J & RK & IV & 13 & 1,3 \\
& A & ZG & VI & 31 & 2,9 \\
\hline$R 2$ & J & ME & II & 26 & 0,9 \\
& A & ZG & VII & Polster defekt & - \\
& A & ZG & VIII & Polster defekt & - \\
\hline R3 & J & ME & III & 28 & 2,0 \\
& J & RK & V & 20 & 1,5 \\
& A & ZG & X & 22 & 3,0 \\
& A & ZG & XI & 9 & 1,6 \\
\hline$N 1$ & J + A & ME & Ib & 24 & 5,0 \\
& A & ZG & IX & Podetien verschoben & - \\
\hline
\end{tabular}

Auspflanzungszeiträume:

$\mathrm{J}=$ Ende Juni 2002 bis Mitte Juli 2003; A = Mitte August 2002 bis Mitte Oktober 2003

Zur Herkunft der Proben s. Kap. G1.2.2 und Tab. 57 im Anhang

Die zwei größeren Polster aus der Zeche Glimmerode (Nr. VI und X) sind wesentlich mehr gewachsen als die im Juni ausgepflanzten Polster von der Mühlecke und vom Roßkopf. Ursache hierfür können zum einen (genetisch bedingte) Unterschiede der 
Polster selbst sein, zum anderen jedoch auch die Tatsache, daß die Polster aus Glimmerode nach dem extrem trockenen Sommer 2003 noch einige Herbst-Wochen länger im Gelände waren, an denen gelegentlicher Regen, tagsüber relativ milde Temperaturen und ausreichend Sonne günstige Wachstumsbedingungen geschaffen haben. Von den im Herbst günstigen Wachstumsbedingungen für Flechten berichten auch JAhns \& Fritzler (1982) sowie MÁzSA (1994). An Sommertagen weisen die Thalli dagegen, wie unter Kap. G5.2 beschrieben, aufgrund der Trockenheit kaum nennenswerte Stoffwechselaktivität auf. Bei einem Vergleich der ursprünglich zusammenhängenden Polster Nr. Ia und Ib ist ersichtlich, daß Polster Nr. Ib, welches den gesamten Auspflanzungszeitraum (bis Mitte Oktober 2003) im Gelände verbracht hat, wesentlich mehr gewachsen ist, als Polster Ia, das nur bis Juli 2003 im Gatter ausgepflanzt war. Beide wuchsen auf Flächen am Waldrand. Da das zweite Polster des Standorts N1 (Polster Nr. IX) aus den oben erwähnten Gründen nicht ausgewertet werden konnte, bleibt unklar, ob die Polster am Standort N1 generell mehr gewachsen sind. Evtl. sind für den größeren Zuwachs des Polsters Nr. Ib gegenüber Ia ausschließlich die günstigen Witterungsbedingungen im Herbst 2003 verantwortlich.

\section{b) Bildung neuer Verzweigungen}

Ein Teil der markierten Podetienspitzen hat sich im Laufe des Untersuchungszeitraumes verzweigt. Ein Vergleich beider Standorte zeigt, daß auf der sonnigeren Fläche R3 der Anteil an Zweigen, welche neue Verzweigungen gebildet haben, mit $42 \%$ deutlich höher ist, als an den schattigen Standorten R1 und R2. Dort treten nur an $23 \%$ der markierten Äste neue Verzweigungen auf (Tab. 46).

Tab. 46: Anteil sich neu verzweigender Podetienspitzen an den Standorten R1/R2 und R3 in Abhängigkeit von der Ausgangslänge der Zweige

\begin{tabular}{l|ll|ll}
\hline $\begin{array}{l}\text { Länge } \\
\text { der Zweige zu } \\
\text { Versuchsbeginn }\end{array}$ & $\begin{array}{l}\text { Gesamtzahl } \\
\text { Zweige } \\
R 1+2\end{array}$ & $\begin{array}{l}\text { Gesamtzahl } \\
\text { Zweige } \\
R 3\end{array}$ & $\begin{array}{l}\text { Anteil neu verzweigter Podetienspitzen } \\
\text { am Versuchsende }\end{array}$ \\
\hline bis $0,5 \mathrm{~mm}$ & 18 & 24 & $39 \%$ & Flächen R1 und R2 \\
bis $1,0 \mathrm{~mm}$ & 26 & 25 & $19 \%$ & $25 \%$ \\
bis $1,5 \mathrm{~mm}$ & 32 & 19 & $16 \%$ & $44 \%$ \\
bis $2,0 \mathrm{~mm}$ & 14 & 9 & $29 \%$ & $47 \%$ \\
über $2,0 \mathrm{~mm}$ & 5 & 2 & $20 \%$ & $56 \%$ \\
\hline Gesamt & $\mathbf{9 5}$ & $\mathbf{7 9}$ & $\mathbf{2 3 \%}$ & $100 \%$ \\
\hline
\end{tabular}

Ähnliche Werte zeigen sich auch bei getrennter Betrachtung der Polster, aufgeteilt in die beiden Auspflanzungszeiträume (Tab. 47). Die Ergebnisse stehen im Einklang mit den Erkenntnissen aus Kap. G3.2, wonach sich die Polster in den Standorttypen Heiden und Waldränder stärker verzweigen als beispielsweise in den relativ schattigen Wäldern.

Bei den Podetien der Fläche R3 zeigt sich, daß die Häufigkeit neuer Verzweigungen mit der Länge der Zweige zu Versuchsbeginn zunimmt (Tab. 46). An den schattigen Standorten R1 und R2 ist dies nicht zu beobachten. 
Tab. 47: Anteil sich neu verzweigender Podetienspitzen an den Standorten R1/R2 und R3 in Abhängigkeit vom Auspflanzungszeitraum

\begin{tabular}{l|ll|ll}
\hline $\begin{array}{l}\text { Länge } \\
\text { der Zweige zu } \\
\text { Versuchsbeginn }\end{array}$ & $\begin{array}{l}\text { Gesamtzahl } \\
\text { Zweige }\end{array}$ & $\begin{array}{l}\text { Gesamtzahl } \\
\text { Zweige }\end{array}$ & \multicolumn{2}{|c}{$\begin{array}{c}\text { Anteil neu verzweigter Podetienspitzen } \\
\text { am Versuchsende }\end{array}$} \\
\hline Zeitraum J: & & $R 3$ & Flächen R1 und R2 & Fläche R3 \\
bis $0,5 \mathrm{~mm}$ & 12 & 16 & $50 \%$ & $25 \%$ \\
bis $1,0 \mathrm{~mm}$ & 15 & 16 & $33 \%$ & $44 \%$ \\
bis $1,5 \mathrm{~mm}$ & 20 & 8 & $25 \%$ & $38 \%$ \\
bis $2,0 \mathrm{~mm}$ & 12 & 6 & $17 \%$ & $50 \%$ \\
über $2,0 \mathrm{~mm}$ & 5 & 2 & $20 \%$ & $100 \%$ \\
\hline Gesamt & $\mathbf{6 4}$ & $\mathbf{4 8}$ & $\mathbf{2 8} \%$ & $\mathbf{4 0} \%$ \\
\hline Zeitraum A: & & & & \\
bis $0,5 \mathrm{~mm}$ & 6 & 8 & $17 \%$ & $25 \%$ \\
bis $1,0 \mathrm{~mm}$ & 11 & 9 & $0 \%$ & $44 \%$ \\
bis $1,5 \mathrm{~mm}$ & 12 & 11 & $8 \%$ & $55 \%$ \\
bis $2,0 \mathrm{~mm}$ & 2 & 3 & $100 \%$ & $66 \%$ \\
über $2,0 \mathrm{~mm}$ & - & - & - & - \\
\hline Gesamt & $\mathbf{3 1}$ & $\mathbf{3 1}$ & $\mathbf{1 3} \%$ & $\mathbf{4 5 \%}$ \\
\hline
\end{tabular}

Auspflanzungszeiträume:

J = Ende Juni 2002 bis Mitte Juli 2003; A = Mitte August 2002 bis Mitte Oktober 2003

Die sich neu verzweigenden Podetienspitzen sind unter beiden Standortbedingungen wesentlich stärker gewachsen als die auch am Versuchsende noch unverzweigten, und zwar um 2,7 mm im Vergleich zu 1,7 mm am Standort R3 bzw. 1,4 mm auf den Flächen R1 und R2. Der Unterschied bei den Zuwachsraten zwischen sich verzweigenden und unverzweigt bleibenden Podetienspitzen ist hochsignifikant (U-Test nach MannWhitney; s. Tab. 48).

Tab. 48: Mittlerer Zuwachs sich verzweigender sowie unverzweigt bleibender Podetienspitzen

\begin{tabular}{l|ll}
\hline Fläche & $\begin{array}{l}\text { Mitterer Zuwachs } \\
\text { der sich verzweigenden } \\
\text { Podetien [mm] }\end{array}$ & $\begin{array}{l}\text { Mittlerer Zuwachs } \\
\text { der unverzweigt bleibenden } \\
\text { Podetien [mm] }\end{array}$ \\
\hline R1/R2 & $2,7 \quad(\mathrm{n}=22)$ & $1,4 \quad(\mathrm{n}=73)$ \\
$\mathrm{R} 3$ & $2,7 \quad(\mathrm{n}=33)$ & $1,7 \quad(\mathrm{n}=46)$ \\
\hline Gesamt & $2,7 * * * \quad(\mathrm{n}=55)$ & $1,5 \quad(\mathrm{n}=119)$ \\
\hline
\end{tabular}

Signifikanzniveau: $* * * \mathrm{p} \leq 0,001$

\section{c) Vergleich der Zuwachsraten von randlich stehenden Podetien mit solchen aus der Polstermitte}

Anhand der Polster, die als Ganzes verpflanzt wurden (also nicht wie Polster I halbiert), läßt sich feststellen, ob randlich stehende Podetien besser wachsen als Podetien der Polstermitte. Als randlich werden dabei Podetien angesehen, die weniger als $1 \mathrm{~cm}$ vom Umriß des Polsters entfernt stehen. Als aus der Polstermitte gelten alle, die mehr als $1 \mathrm{~cm}$ vom Rand entfernt wachsen. Hintergrund dieser Fragestellung ist die Vermutung, daß ein Polster an seinen Rändern wächst, die randlich stehenden Podetien also jünger und wuchskräftiger sind als die der Polstermitte. Tatsächlich zeigt sich bei Auswertung des Datenmaterials ein Unterschied bei den Zuwachsraten: die am Rande stehenden Lagerstiele sind durchschnittlich 2,3 $\mathrm{mm}$ gewachsen, die aus der Polstermitte nur 
1,8 mm. Dieser Unterschied ist allerdings, wie eine Überprüfung mit dem U-Test (nach Mann \& Whitney) zeigte, nicht signifikant (Tab. 49).

Tab. 49: Vergleich der Zuwachsraten von randlich stehenden Podetien und solchen aus der Polstermitte

\begin{tabular}{l|ll}
\hline Fläche & $\begin{array}{l}\text { Mitterer Zuwachs } \\
\text { der randlich stehenden } \\
\text { Podetien }[\mathrm{mm}]\end{array}$ & $\begin{array}{l}\text { Mittlerer Zuwachs } \\
\text { der in der Polstermitte } \\
\text { stehenden Podetien }[\mathrm{mm}]\end{array}$ \\
\hline R1/R2 & $2,6 \quad(\mathrm{n}=23)$ & $1,5 \quad(\mathrm{n}=47)$ \\
R3 & $2,1 \quad(\mathrm{n}=33)$ & $2,1 \quad(\mathrm{n}=46)$ \\
\hline Gesamt & 2,3 n.s. $(\mathrm{n}=56)$ & $1,8 \quad(\mathrm{n}=93)$ \\
\hline
\end{tabular}

n.s. $=$ Unterschied nicht signifikant

d) Abhängigkeit der Zuwachsraten von der Länge der Verzweigungen zu Versuchsbeginn (Tab. 50)

Es besteht keine Abhängigkeit der Zuwachsrate von der Länge der Zweige am Beginn des Versuches. Bei Betrachtung des gesamten Datenmaterials zeigen lediglich die zu Versuchsbeginn zwischen 1 und $2 \mathrm{~mm}$ langen Zweige einen etwas größeren Zuwachs als die kürzeren bzw. als die mehr als $2 \mathrm{~mm}$ langen.

Tab. 50: Jahreszuwachs in Abhängigkeit von der Zweiglänge bei Versuchsbeginn

\begin{tabular}{|c|c|c|c|c|c|}
\hline $\begin{array}{l}\text { Länge } \\
\text { der Zweige zu } \\
\text { Versuchsbeginn }\end{array}$ & Gesamt & Zeitraum J & Zeitraum A & $\begin{array}{l}\text { Standorte } \\
R 1 / R 2\end{array}$ & $\begin{array}{l}\text { Standort } \\
\text { R3 }\end{array}$ \\
\hline bis $0,5 \mathrm{~mm}$ & $1,8 \quad(n=42)$ & $1,3 \quad(n=28)$ & $2,9 \quad(n=14)$ & $2,1 \quad(n=18)$ & $1,7 \quad(n=24)$ \\
\hline bis $1,0 \mathrm{~mm}$ & $1,8 \quad(n=51)$ & $1,7 \quad(n=31)$ & $2,1 \quad(n=20)$ & $1,5 \quad(n=26)$ & $2,2 \quad(n=25)$ \\
\hline bis $1,5 \mathrm{~mm}$ & $2,0 \quad(n=51)$ & $1,2 \quad(n=28)$ & $2,9 \quad(n=23)$ & $1,8 \quad(n=32)$ & $2,3 \quad(n=19)$ \\
\hline bis $2,0 \mathrm{~mm}$ & $2,2 \quad(n=23)$ & $1,5 \quad(n=18)$ & $4,4 \quad(n=5)$ & $1,9 \quad(n=14)$ & $2,6 \quad(n=9)$ \\
\hline über $2,0 \mathrm{~mm}$ & $1,5 \quad(n=7)$ & $1,5 \quad(n=7)$ & - & $0,9 \quad(n=5)$ & $3,2 \quad(n=2)$ \\
\hline
\end{tabular}

Auspflanzungszeiträume:

$\mathrm{J}=$ Ende Juni 2002 bis Mitte Juli 2003;

A = Mitte August 2002 bis Mitte Oktober 2003

\subsubsection{Veränderungen der Podetienbündel}

An beiden sonnigen Standorten (R3 und N1) haben die liegenden Podetien kräftige senkrechte Auswüchse gebildet. Während am Standort N1 auch die waagerechten Teile der Podetien einen vitalen Eindruck machen, wurden diese auf der Fläche R3 von Moosen überwachsen und sind wohl daher gebleicht, d. h. die Algen offensichtlich abgestorben. Die senkrechten Auswüchse weisen an beiden Waldrand-Standorten zahlreiche Verzweigungen auf, ihre Oberfläche ist stellenweise leicht pigmentiert, überall glatt und schuppenlos. Weit weniger gut haben sich die Podetienbündel an den beschatteten Standorten R1 und R2 entwickelt. Die Lagerstiele sehen vergleichsweise kümmerlich aus und sind kaum gewachsen. An einem der senkrechten Podetienzweige treten kleine Schuppen auf. 


\section{Zusammenfassung}

$\mathrm{Zu}$ Beginn des Kapitels wird ein Überblick gegeben über die in der meist deutschsprachigen Literatur des 20. Jahrhunderts aufgeführten Varietäten und Formen von Cladonia furcata sowie ein kurzer Vergleich mit der morphologischen Variabilität anderer Cladonien angestellt. Es folgt eine allgemeine Beschreibung der untersuchten Cl. furcata-Proben, in der für beide Unterarten Wuchsform, Färbung, Fruchtkörperbildung etc., aber auch Minimum- und Maximumwerte der Thallusmerkmale angegeben sind. Daran anschließend werden die weiteren Ergebnisse der morphologischen Untersuchungen dargestellt und interpretiert. Es zeigt sich, daß einige Thallusmerkmale von den Bedingungen am Standort abhängen. Günstige Lichtverhältnisse bei nicht $\mathrm{zu}$ starker Trockenheit sind wichtig für das Podetienwachstum und die Fruchtkörperbildung. Stärkere Sonneneinstrahlung führt zu einer deutlichen Pigmentierung der Thalli. Im Schatten treten dagegen an den Podetien vermehrt Schuppen und Pyknidien auf und die Algenschicht dehnt sich weiter aus. Letzteres ist auch an den sonnig-trockensten Standorten der Fall. Vermutlich hält dort die starke Pigmentierung auch photosynthetisch wirksame Strahlung zurück, so daß die Bedingungen für die Algenzellen, denen im Schatten entsprechen. Stark verzweigte Podetien treten bevorzugt an Standorten auf, an denen die mikroklimatischen Verhältnisse günstig sind und weitgehend ungestörtes Wachstum möglich ist. Die Apothecienbildung führt zu verstärkter Verzweigung am Podetienende. Weitere deutliche Korrelationen zwischen den Podetienmerkmalen bestehen nicht.

Anhand einiger exemplarisch ausgewählter Proben von Cladonia furcata und Cl. gracilis wird auf die anatomischen Verhältnisse eingegangen. Der maximale prozentuale Anteil der Dicke der Schichten am Podetienradius wird angegeben und verglichen. Es zeigt sich, daß die Gonidienzone tendenziell in Podetien lichtreicher Standorte etwas dicker ist als in Podetien, die an stärker beschatteten Stellen gewachsen waren. Bei Podetien mit dünner Gonidienschicht ist auch die Rindenschicht eher dünn, und umgekehrt. Einzelne Podetienquerschnitte werden detailliert beschrieben.

Die Ergebnisse des Verpflanzungsversuches bestätigen den Zusammenhang zwischen Podetienwachstum bzw. Grad der Verzweigung und günstigen Lichtverhältnissen, da die Podetien der lichtreicheren Standorte tendenziell etwas mehr gewachsen sind und sich mehr verzweigt haben als die der Vergleichsflächen im Wald. Dabei sind die randlich stehenden, vermutlich jüngeren Podetien zwar etwas, aber nicht signifikant mehr gewachsen als die Lagerstiele der Polstermitte. Das Mikroklima beider Versuchsflächen wird exemplarisch anhand eines Sommertages dargestellt. Es zeigt sich, daß die Thalli am Waldrand etwas schneller austrocknen, sich mehr aufheizen und stärkere Sonneneinstrahlung ertragen müssen. An beiden Standorten sind sie unter den sommerlichen Witterungsverhältnissen jedoch bereits morgens so stark ausgetrocknet, daß kaum Stoffwechselaktivität stattfinden kann. 


\section{H Genetische Variabilität der Bionten von Cladonia furcata und Cladonia gracilis}

Zur Untersuchung der intraspezifischen Variabilität bei Cladonia furcata werden neben den bereits beschriebenen phänotypischen Merkmalen im Folgenden auch DNASequenzdaten herangezogen. Diese werden darüber hinaus mit Sequenzen der morphologisch ähnlichen Becherflechte $C l$. gracilis verglichen.

Um bei den nah verwandten Arten und Unterarten eine informative Menge an Sequenzunterschieden zu erhalten, wurde ein Sequenzbereich mit hoher Variabilität gewählt. Hierfür erschien der im Kern kodierte ITS-Bereich geeignet, der bereits in einer Reihe ähnlicher Studien erfolgreich analysiert wurde (ARUP \& GRUBE 1998, Lohtander et al. 1998, Mattsson \& Wedin 1998, Piercey-Normore \& DePriest 2001, Stenroos et al. 2002). Dieser Bereich setzt sich zusammen aus den hochvariablen, nicht-kodierenden Abschnitten ITS1 und ITS2 (ITS: internal transcribed spacer) und der dazwischenliegenden 5.8S rDNA. Dieser ITS-Bereich befindet sich zwischen den Genen der kleinen (18S) und großen (26S) Untereinheit der ribosomalen RNA (s. Abb. 43).

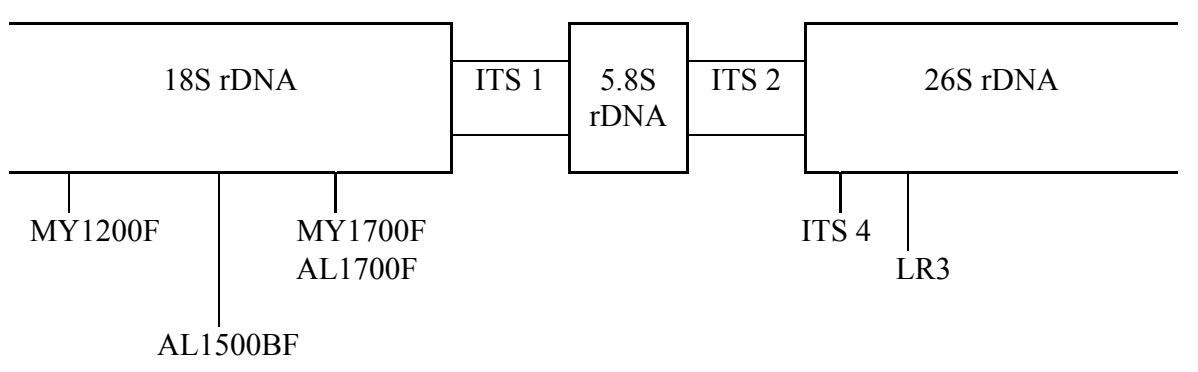

Abb. 43: ITS-Region mit Ansatzstellen der in Kap. H1.2 aufgeführten PCR-Primer (verändert nach BALDWIN et al. 1995).

\section{Molekularbiologische Methoden}

\subsection{Auswahl und Herkunft der Proben, DNA-Extraktion}

Für die Sequenzierung der rDNA wurden die Proben nach folgenden Kriterien ausgewählt (s. auch Tab. 51 und Tab. 52):

\section{a) Cladonia furcata ssp. furcata}

Die untersuchten Podetien stammen von Standorten mit saurem, mäßig saurem oder basenreichem Ausgangsgestein sowie von schattigen (in Wäldern) bis stark besonnten Standorten (auf Felsen etc.). Es wurden morphologisch unterschiedliche Lagerstiele ausgewählt, d.h. niederliegende und aufrecht gewachsene, zierliche und kräftige, 
unterschiedlich stark verzweigte, unterschiedlich beschuppte, unterschiedlich stark pigmentierte, fruchtende und sterile sowie geschlossen röhrige und offen rinnige.

\section{b) Cladonia furcata ssp. subrangiformis}

Die Thallus-Proben entstammen basenreichen Standorten mit unterschiedlichem Ausgangsgestein und unterscheiden sich zudem hinsichtlich Wuchsform, Beschuppung, Häufigkeit von Markaufbrüchen, Fruchtkörperbildung sowie weiteren Podetienmerkmalen wie Pigmentierung, Podetienform etc.

Tab. 51: Bezeichnung, Herkunft und Charakterisierung der Proben für die rDNA-Sequenzierung

\begin{tabular}{|c|c|c|c|c|c|c|c|c|c|c|c|c|c|c|c|c|c|c|}
\hline 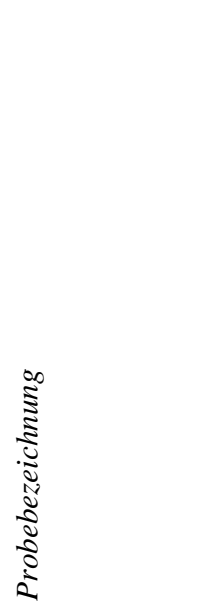 & 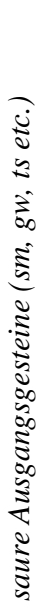 & $\begin{array}{l}\vec{\Xi} \\
\tilde{E} \\
\tilde{D}\end{array}$ & 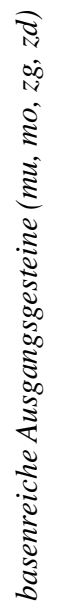 & 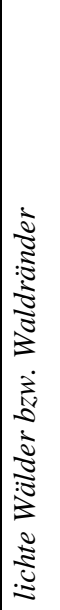 & 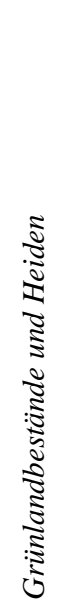 & 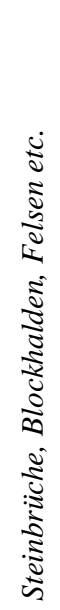 & 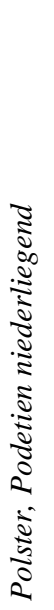 & 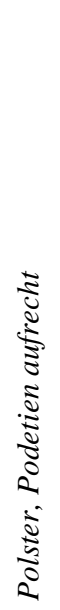 & 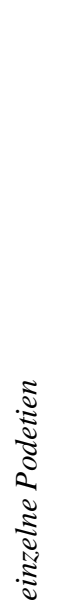 & 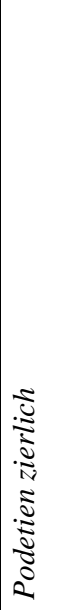 & 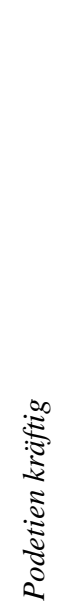 & 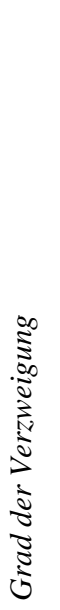 & 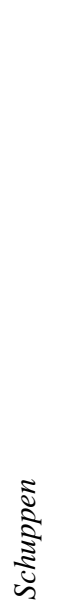 & 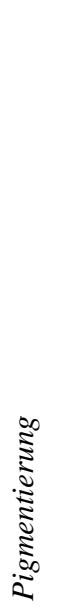 & $\begin{array}{l}\tilde{\Xi} \\
\tilde{\Xi} \\
\tilde{\Xi} \\
\tilde{z}\end{array}$ & 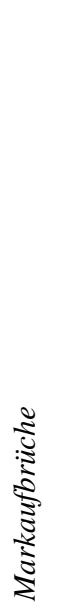 & 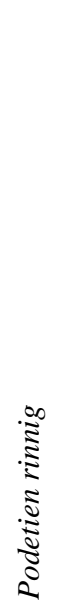 & 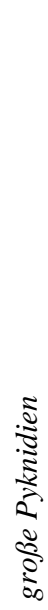 \\
\hline clfufu@2MI & * & & & & & * & * & & & & + & ++ & - & ++ & - & - & - & - \\
\hline Clfufu@3RK & * & & & * & & & * & * & & & + & + & ++ & 0 & - & - & - & - \\
\hline Clfufu@4KB & * & & & * & & & * & & & & 0 & + & 0 & 0 & - & - & - & + \\
\hline Clfufu05DT & * & & & * & & & & * & & & + & ++ & ++ & 0 & ++ & - & ++ & - \\
\hline clfufu@9ZG & * & & & & ћ & & & * & & & + & ++ & - & + & + & - & + & - \\
\hline Clfufu10ZG & * & & & & * & & & * & & & 0 & ++ & + & 0 & 0 & - & - & - \\
\hline clfufu@7DB & & * & & & & * & & * & & & + & ++ & - & + & 0 & - & - & - \\
\hline Clfufu13GM & & * & & & & * & * & & & & + & ++ & 0 & ++ & - & - & - & - \\
\hline Clfufu16DH & & & * & & & * & * & & * & & 0 & 0 & 0 & ++ & - & - & - & - \\
\hline clfusue2SI & & & $\star$ & & $\star$ & & & * & & & + & + & 0 & ++ & - & + & - & + \\
\hline Clfusu03KL & & & * & & * & & * & & & & + & 0 & 0 & ++ & - & + & - & - \\
\hline clfusu@4KA & & & * & & * & & * & & $\star$ & & + & + & - & ++ & - & 0 & - & - \\
\hline Clfusu05IG & & & * & & & * & * & & * & & + & 0 & - & + & - & - & - & - \\
\hline clfusu@7R0 & & & * & & ћ & & * & * & & & + & + & - & 0 & 0 & + & + & - \\
\hline Clfusu@৪HE & & & * & & * & & * & & & & + & + & - & + & - & + & - & - \\
\hline Clfusu10SF & & & * & & * & & * & & * & & + & + & - & + & - & + & - & - \\
\hline Clfusu12HO & & & $\star$ & & $\star$ & & * & & & & + & + & - & ++ & - & + & - & - \\
\hline Clfusu14WB & & & * & & & * & * & & $\star$ & & 0 & - & - & 0 & - & 0 & - & - \\
\hline clfusu11BK & & * & & & * & & * & & * & + & & + & - & 0 & - & + & - & - \\
\hline Clgrac06MM & & * & & & & * & & * & & + & & 0 & ++ & + & - & - & - & - \\
\hline Clgrac12SZ & & * & & & & * & * & & & + & & - & + & + & - & - & - & - \\
\hline Clgrac14BP & & * & & & & * & * & * & & + & & + & ++ & + & - & - & - & - \\
\hline Clgrac11NT & * & & & * & & & & * & & + & & 0 & 0 & + & - & - & - & - \\
\hline
\end{tabular}

Die Probebezeichnung setzt sich wie folgt zusammen: erste sechs Buchstaben $=$ Art bzw. Unterart (Clfufu = Cladonia furcata ssp. furcata; Clfusu = Cladonia furcata ssp. subrangiformis; Clgrac = Cladonia gracilis), Ziffer $7+8=$ Nummern für die Sequenzierung der zwei Gruppen „,ssp. furcata/gracilis“ und „ssp. subrangiformis“; die letzten beiden Buchstaben geben den Fundort an, weitere Angaben dazu siehe Tab. 57 im Anhang 1; Abkürzungen zur Geologie s. Anhang 1. Merkmalsausprägung: - nicht/keine, o wenig, + stark/viele, ++ sehr stark/sehr viele. 


\section{c) Cladonia gracilis}

Für die Sequenzierung wurden morphologisch der Cl. furcata ähnlich sehende, anhand der Podetienmerkmale nur schwer bestimmbare Exemplare ausgewählt. Die Thalli wiesen keine Becher auf!

Tab. 52: Vegetationseinheiten, denen die Proben für die rDNA-Sequenzierung entnommen wurden

\begin{tabular}{l|l}
\hline Probe & Vegetationseinheit (s. Kapitel E) \\
\hline Clfufu02MI & Cladonia furcata-Cladonion arbusculae-Fragmentgesellschaft \\
Clfufu03RK & Cladonia furcata-Einzelpolster \\
Clfufu04KB & Cladonia cervicornis-Baeomycion-Fragmentgesellschaft \\
Clfufu05DT & Cladonia furcata-Cladonion arbusculae-Fragmentgesellschaft \\
Clfufu07DB & Cladonietum foliaceae \\
Clfufu09ZG & Cladonia furcata-Cladonion arbusculae-Fragmentgesellschaft \\
Clfufu10ZG & Cladonia furcata-Cladonion arbusculae-Fragmentgesellschaft \\
Clfufu13GM & Cladonietum foliaceae \\
Clfufu16DH & Toninio-Psoretum fulgensietosum, Cl. symphycarpa-Variante \\
\hline Clfusu02SI & Toninio-Psoretum typicum + Peltigera rufescens-Toninion-FG \\
Clfusu03KL & Toninio-Psoretum fulgensietosum, Cl. symphycarpa-Variante \\
Clfusu04KA & Peltigera rufescens-Toninion-Fragmentgesellschaft \\
Clfusu05IG & Cetraria aculeata-Dominanzbestand \\
Clfusu07R0 & Cladonia furcata-Einzelpolster \\
Clfusu08HE & Peltigera rufescens-Toninion-Fragmentgesellschaft \\
Clfusu10SF & Cladonia furcata-Einzelpolster \\
Clfusu11BK & Cladonia rangiformis-Dominanzbestand \\
Clfusu12H0 & Peltigera rufescens-Toninion-Fragmentgesellschaft \\
Clfusu14WB & Toninio-Psoretum fulgensietosum, Cl. symphycarpa-Variante \\
\hline Clgrac06MM & Cladonia gracilis-Einzelpolster \\
Clgrac11NT & Cladonia bacillaris-Gesellschaft \\
Clgrac12SZ & Cladonia gracilis-Cladonion arbusculae-Fragmentgesellschaft \\
Clgrac14BP & Cladonia gracilis-Cladonion arbusculae-Fragmentgesellschaft \\
\hline
\end{tabular}

Alle Podetien wurden erst kurze Zeit vor Beginn der Laborarbeiten dem Standort im Gelände entnommen, um möglichst frische Thalli für die Sequenzierung zu haben. Die verwendeten Proben dienten als Ausgangsmaterial sowohl für die molekularbiologischen Untersuchungen als auch für die Dünnschichtchromatographie (s. Kap. F). In beide Untersuchungen gingen dabei jeweils benachbarte Podetienabschnitte ein. Für die Sequenzierung der rDNA wurden unter dem Binokular gesund erscheinende Podetienspitzen, ohne Parasitenbefall, epiphytischen Algenbewuchs oder Degenerationserscheinungen ausgewählt. Die wenige Millimeter langen Podetienspitzen wurden in 1,5 $\mathrm{ml}$ Eppendorf-Tubes in einen mit flüssigem Stickstoff vorgekühlten Aluminiumblock gestellt und fein zermörsert. Die Extraktion der DNA aus dem pulverisierten Thallus erfolgte mit dem DNeasy Plant Mini Kit (Quiagen), dem Invisorb Spin Plant Kit (Invitek) bzw. dem REDExtract-N-AMP Plant PCR Kit (Sigma) jeweils nach Protokoll des Herstellers. Die Flechten-DNA wurde in 50 bis $100 \mu 1$ TE Puffer gelöst (mit Ausnahme der mit dem Sigma-Kit extrahierten DNA).

\subsection{Polymerase-Chain-Reaktion (PCR), Gelelektrophorese und DNA- Aufreinigung}

Durch die Auswahl des Primerpaares (Vorwärts- und Rückwärts-Primer) wird der zu vervielfältigende rDNA-Abschnitt festgelegt und mit Hilfe der Taq-Polymerase in einer PCR amplifiziert. Die eingesetzten Primer tragen die Bezeichnungen MY1200F, MY1700F (HELMS et al. 2003) und ITS4 (WhiTE et al. 1990) für die Mycobionten sowie AL1500BF, AL1700F (HELMS et al. 2001), LR3 (FrIEDL \& RoKITTA 1997) und 
ITS4 (WhITE et al. 1990) für die Photobionten. Die PCR wurde durchgeführt mit 1 bis $2 \mu 1$ DNA-Extrakt in $50 \mu 1$ Reaktionsvolumen. Der Reaktionsansatz beinhaltet $0,2 \mathrm{mM}$ von jeder der 4 dNTPs (dNTP-Mix, Hybaid-AGS), 2 mM MgCl $2,4 \%$ DMSO, 0,2 $\mu \mathrm{M}$ von jedem PCR-Primer und 1 Einheit SilverStar Taq-Polymerase (Eurogentech). Einer anfänglichen Denaturierungszeit von 5 min bei $95^{\circ} \mathrm{C}$ schließen sich 35 Zyklen an. Ein Zyklus beginnt mit einer Denaturierung bei $94{ }^{\circ} \mathrm{C}$ (Dauer $40 \mathrm{sec}$.), gefolgt von einer Primer-Anlagerung (Annealing) bei $51{ }^{\circ} \mathrm{C}$ (Dauer $40 \mathrm{sec}$.) und einer Elongation bei $72{ }^{\circ} \mathrm{C}$ (Dauer $2 \mathrm{~min} .+5 \mathrm{sec}$.). Nach Beendigung der 35 Zyklen wurde der Reaktionsansatz für $10 \mathrm{~min}$. bei $72^{\circ} \mathrm{C}$ inkubiert.

Die Überprüfung der Amplifikate erfolgte mit Hilfe einer Gelelektrophorese. Zu diesem Zweck wurden $2 \mu 1$ der Reaktionsansätze mit $3 \mu$ Loading Dye (1,5fach) auf 1\%ige Agarose-Gele aufgetragen. Als Laufpuffer diente 0,5-facher TBE-Puffer. Zur Anfärbung der DNA wurde eine 0,1\%ige Ethidiumbromid-Lösung verwendet. Unter UV-Licht (312 nm) erscheint der entstandene DNA-Ethidiumbromid-Komplex als hell leuchtende Bande. Das Ergebnis wurde fotographisch dokumentiert. Die Längen der amplifizierten Fragmente konnten anhand eines Markers (Lambda, EcoRI/Hind III) bestimmt werden. Sofern die Proben schon nach der ersten PCR und anschließender Elektrophorese eine deutliche Bande zeigten, wurden sie direkt sequenziert. Proben mit ebenfalls nur einer, aber etwas schwächeren Bande wurden reamplifiziert, d.h. einer erneuten PCR unterzogen, um genügend Ausgangsmaterial für die Sequenzierung zu erhalten.

Da überschüssige dNTP`s und PCR-Primer die Sequenzierreaktion beeinträchtigen, müssen die PCR-Produkte zunächst gereinigt werden. Diese Aufreinigung erfolgte mit dem High Pure ${ }^{\mathrm{TM}}$ PCR Product Purifikation Kit (Roche) nach Protokoll, bzw. mit NaAcetat und Isopropanol nach folgendem Schema: $50 \mu 1$ PCR-Ansatz werden mit $5 \mu 1$ $3 \mathrm{M} \mathrm{Na}$-Acetat ( $\mathrm{pH} 5,5)$ und $50 \mu 1$ Isopropanol versetzt und anschließend über Nacht bei $-20^{\circ} \mathrm{C}$ gekühlt (s. SAMBROOK et al. 1989). Nach 30minütigem Zentrifugieren bei maximaler Umdrehung wird der Überstand verworfen und $150 \mu 170 \%$ Ethanol zugegeben. Das Gemisch bleibt weitere $2 \mathrm{~min}$ stehen, wird danach $10 \mathrm{~min}$ bei maximaler Umdrehung zentrifugiert und der Überstand wiederum verworfen. $\mathrm{Zu}$ der verbleibenden DNA wird $50 \mu \mathrm{l}$ TE-Puffer zugegeben. Mit einem Biophotometer (Eppendorf) wurde anschließend die Konzentration des aufgereinigten Produkts bestimmt, mit Hilfe derer die für die Sequenzier-Ansätze benötigte Template-Menge ermittelt werden konnte.

\subsection{DNA-Sequenzierung}

Die Sequenzierung erfolgte mit verschiedenen Sequenziergeräten. Für den ALFexpress II automated sequencer (Pharmacia) wurden CY-5-markierte Primer in Kombination mit dem Thermo Sequenase Sequencing Kit mit 7-deaza-dGTP (Amersham) verwendet. Derselbe Sequencing Kit in Kombination mit IR-700 und IR800 markierten Primern kam beim LI-COR IR ${ }^{2}$ DNA Sequencer zum Einsatz. Das Reaktionsvolumen betrug $15 \mu \mathrm{l}$. Das zugehörige PCR-Temperaturprogramm umfaßt, neben einer anfänglichen zweiminütigen Denaturierung bei $95^{\circ} \mathrm{C}, 30$ Zyklen. Diese 
beginnen jeweils mit einer Denaturierung bei $94{ }^{\circ} \mathrm{C}$ (Dauer $30 \mathrm{sec}$.), darauf folgen die Anlagerung der Primer bei $50{ }^{\circ} \mathrm{C}$ (Dauer $30 \mathrm{sec}$.) und die Elongation bei $72{ }^{\circ} \mathrm{C}$ (Dauer $1 \mathrm{~min}$.). Die finale Elongation erfolgt ebenfalls bei $72^{\circ} \mathrm{C}$ und dauert $7 \mathrm{~min}$. Die Sequenzieransätze für den ABI PRISM 3100 Genetic Analyzer wurden mit dem Big Dye Terminator V2.0 Cycle Sequencing Kit (ABI) sowie unmarkierten Primern und markierten ddNTPs angesetzt. Das Reaktionsvolumen betrug $5 \mu 1$, bei geringer DNAKonzentration $10 \mu \mathrm{l}$. Das PCR-Temperaturprogramm für die Sequenzierung mit dem ABI beinhaltet lediglich 25 Zyklen. Einer 10 sec. dauernden Denaturierung bei $96^{\circ} \mathrm{C}$ folgt bei diesen das Annealing bei $45^{\circ} \mathrm{C}$ (Dauer $5 \mathrm{sec}$.) und die Extension bei $60{ }^{\circ} \mathrm{C}$ (Dauer 4 min.). Die Sequenzen wurden mit der ALF-Software bzw. der AlignIR V1.2 Software (LI-COR) zusammengestellt.

\subsection{Auswertung der Daten}

Die ermittelten ITS rDNA-Sequenzen wurden mit dem Computerprogramm BioEdit (http://www.mbio.ncsu.edu/RnaseP/info/programms/BIOEDIT/bioedit.html; s. HALL 1999) manuell angeordnet und mit den in Tab. 53 aufgelisteten Sequenzen aus der Datenbank des NCBI (GenBank; http://www.ncbi.nlm.nih.gov/) verglichen. Die phylogenetischen Analysen wurden mit dem Programm PAUP* V4.0b10 (SwOFFORD 2000) durchgeführt. Die dargestellten Stammbäume basieren auf dem Maximum Likelihood-Verfahren (ML), welches eine Berechnung der Wahrscheinlichkeit von Ereignissen auf der Grundlage statistischer Modellvorstellungen und Bevorzugung der danach wahrscheinlichsten Erklärung durchführt. Die Wahl eines geeigneten Evolutionsmodells erfolgte mit dem Programm MODELTEST 3.0 (PosADA \& Crandall 1998). Bei den Photobionten wurde das TrNef+G-Modell (TAMURA \& NeI 1993), bei den Mycobionten das K80-Modell (KIMURA 1980) als das passenste ermittelt. Zur Überprüfung der Zuverlässigkeit der Stammbäume wurden BootstrapTests durchgeführt. In den Stammbaum-Darstellungen werden Bootsstrap-Werte aus dem ML-, Maximium Parsimony- (MP) und Neighbor-Joining-Verfahren (NJ) angegeben. Die Distanztabellen wurden mit der Software MEGA 2.1 erstellt (KUMAR et al. 2001; http://www.megasoftware.net).

Tab. 53: Vergleichssequenzen aus GenBank

\begin{tabular}{|c|c|c|c|}
\hline \multicolumn{2}{|c|}{ Accession-Nr./Bezeichnung } & Autoren & zugehörige Publikationen \\
\hline AF184752 & $\begin{array}{l}\text { Cl. gracilis ssp. turbinata } \\
\text { [Herkunft ?] }\end{array}$ & Wedin, M., Doring, H., Ekman, S. & Lichenologist 32(2), 171-187 (2000) \\
\hline AF345414 & $\begin{array}{l}\text { Algal symbiont of } \\
\text { Cl. gracilis ssp. gracilis IO50 } \\
\text { (Russia) }\end{array}$ & $\begin{array}{l}\text { Piercey-Normore, M.D., } \\
\text { DePriest, P.T. }\end{array}$ & Am. J. Bot. 88, 1490-1498 (2001) \\
\hline AF345429 & $\begin{array}{l}\text { Algal symbiont of } \\
\text { Cl. furcata IO24 (USA) }\end{array}$ & $\begin{array}{l}\text { Piercey-Normore, M.D., } \\
\text { DePriest, P.T. }\end{array}$ & Am. J. Bot. 88, 1490-1498 (2001) \\
\hline AF455193 & $\begin{array}{l}\text { Cl. gracilis ssp. turbinata } \\
\text { (Finland) }\end{array}$ & $\begin{array}{l}\text { Stenroos, S., Hyvoenen, J., } \\
\text { Myllys, L., Thell, A., Ahti, T. }\end{array}$ & Cladistics 18, 237-278 (2002) \\
\hline AF455194 & $\begin{array}{l}\text { Cl. gracilis ssp. gracilis } \\
\text { (Sweden) }\end{array}$ & $\begin{array}{l}\text { Stenroos, S., Hyvoenen, J., } \\
\text { Myllys, L., Thell, A., Ahti, T. }\end{array}$ & Cladistics 18, 237-278 (2002) \\
\hline AF455198 & $\begin{array}{l}\text { Cl. gracilis ssp. elongata } \\
\text { (Chile) }\end{array}$ & $\begin{array}{l}\text { Stenroos, S., Hyvoenen, J., } \\
\text { Myllys, L., Thell, A., Ahti, T. }\end{array}$ & Cladistics 18, 237-278 (2002) \\
\hline AF455200 & $\begin{array}{l}\text { Cl. macroceras } \\
\text { (Island) }\end{array}$ & $\begin{array}{l}\text { Stenroos, S., Hyvoenen, J., } \\
\text { Myllys, L., Thell, A., Ahti, T. }\end{array}$ & Cladistics 18, 237-278 (2002) \\
\hline AF455220 & Cl. furcata (USA) & $\begin{array}{l}\text { Stenroos, S., Hyvoenen, J., } \\
\text { Myllys, L., Thell, A., Ahti, T. }\end{array}$ & Cladistics 18, 237-278 (2002) \\
\hline
\end{tabular}




\section{Ergebnisse der ITS rDNA-Sequenzierung}

\subsection{ITS rDNA-Sequenzen der Mycobionten}

\subsubsection{Polymorphismen der sequenzierten ITS-Bereiche}

a) Cladonia furcata (Abb. 44 und Abb. 45)

Es lassen sich innerhalb von $\mathrm{Cl}$. furcata 4 ITS-Varianten abgrenzen:

ITS-Variante 1: Cl. furcata ssp. subrangiformis Proben 2, 5, 7, 10, 14, Cl. furcata ssp. furcata Proben 4, 5, 10, 13

ITS-Variante 2: Cl. furcata ssp. subrangiformis Probe 11, Cl. furcata ssp. furcata Probe 16

ITS-Variante 3: Cl. furcata ssp. furcata Proben 2, 3, 7

ITS-Variante 4: Cl. furcata ssp. subrangiformis Probe 8

Die intraspezifische Variabilität von Cladonia furcata ist gering - die Sequenzen aller 4 ITS-Varianten weichen nur in maximal 10 Basen voneinander ab (s. Tab. 55). Die Abgrenzung der ITS-Variante 1 von den anderen Varianten wird durch Bootstrap-Werte von 81 (ML), 57 (MP) bzw. 78 (NJ) unterstützt (s. Abb. 44). Die Sequenzen der zweiten ITS-Variante stehen der ersten nahe und werden mit ihr gemeinsam von den Varianten 3 und 4 abgetrennt (Bootstrap-Werte 60 (ML), 40 (MP) und 76 (NJ)). Unterstützt durch relativ hohe Bootstrap-Werte von 94 (ML), $92(\mathrm{MP})$ und 90 (NJ) bilden die beiden Sequenzen Clfufu03RKmy und Clfufu02MImy die ITS-Variante 3. (Zu dieser zählt auch die Sequenz Clfufu07DBmy, welche aufgrund der unzureichenden Länge nicht in die phylogenetischen Analysen einbezogen wurde. Dies gilt auch für die Sequenz Clfufu13GMmy der Variante 1). Variante 4 umfaßt nur eine Sequenz von Cl. furcata ssp. subrangiformis, die verhältnismäßig deutlich, d.h. in 8 bis 10 Positionen von den anderen Sequenzen abweicht. Die Cl. furcata-Sequenz aus GenBank (AF455220) läßt sich keiner der 4 ITS-Varianten eindeutig zuordnen. Von den Sequenzen der Variante 1 unterscheidet sie sich im dargestellten Sequenzabschnitt beispielsweise in 11 bis 13 Positionen (Abb. 45).

\section{b) Cladonia gracilis (Abb. 46)}

Die 4 Sequenzen der Mycobionten von Cl. gracilis zeigen eine deutliche Variabilität in den ITS-Bereichen. Sie weichen in bis zu 17 Positionen voneinander ab. Die Sequenz Clgrac11NTmy unterscheidet sich lediglich in 3 Positionen von der GenBank-Sequenz von Cladonia macroceras (AF455200). Probe Clgrac12SZmy weist die größte Ähnlichkeit mit Cl. gracilis ssp. turbinata (AF 455193) auf (5 verschiedene Positionen). Die Sequenzen Clgrac06MMmy und Clgrac14BPmy stehen der GenBank-Sequenz von Cl. gracilis ssp. gracilis (AF455194) nahe (3 bzw. 4 variierende Positionen). Die Anzahl unterschiedlicher Positionen bezieht sich jeweils auf den in Abb. 46 dargestellten Sequenzbereich, der jedoch nicht immer den gesamten ITS-Abschnitt umfaßt. 


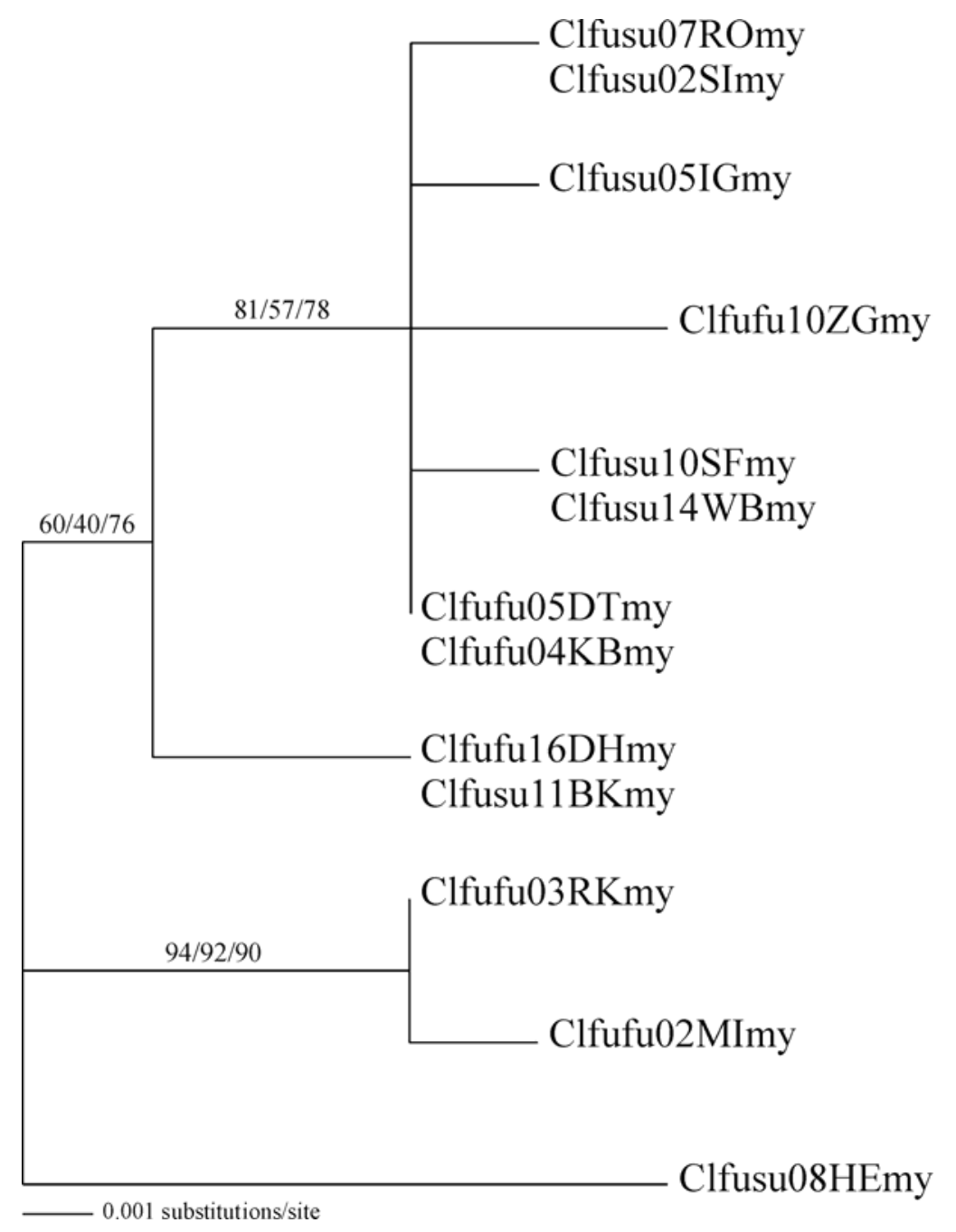

Abb. 44: rDNA-Stammbaum der Mycobionten von Cladonia furcata berechnet nach der Maximum Likelihood-Methode (Midpoint rooting) mit zusätzlichen Bootstrap-Werten aus dem Maximum Parsimony- und Neighbor-Joining-Verfahren (angegeben in dieser Reihenfolge).

Tab. 54: Distanztabelle der Mycobionten von Cladonia furcata (fett gedruckt: Distanzen innerhalb einer ITS-Variante)

\begin{tabular}{|c|c|c|c|c|c|c|c|c|c|c|c|c|}
\hline & 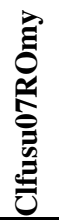 & 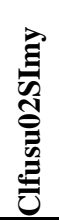 & 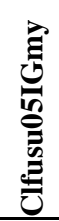 & 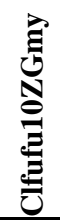 & 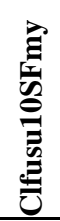 & 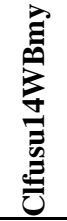 & 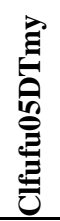 & 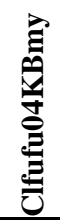 & 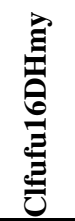 & 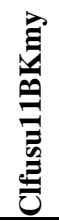 & 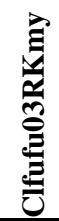 & 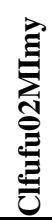 \\
\hline Clfusu02SImy & $\mathbf{0}$ & & & & & & & & & & & \\
\hline Clfusu05IGmy & 2 & 2 & & & & & & & & & & \\
\hline Clfufu10ZGmy & 3 & 3 & 3 & & & & & & & & & \\
\hline Clfusu10SFmy & 2 & 2 & 2 & 3 & & & & & & & & \\
\hline Clfusu14WBmy & 2 & 2 & 2 & 3 & 0 & & & & & & & \\
\hline Clfufu05DTmy & 1 & 1 & 1 & 2 & 1 & 1 & & & & & & \\
\hline Clfufu04KBmy & 1 & 1 & 1 & 2 & 1 & 1 & 0 & & & & & \\
\hline Clfufu16DHmy & 5 & 5 & -5 & 6 & 5 & 5 & 4 & 4 & & & & \\
\hline Clfusu11BKmy & 5 & 5 & 5 & 6 & 5 & 5 & 4 & 4 & 0 & & & \\
\hline Clfufu03RKmy & 7 & 7 & 7 & 6 & 7 & 7 & 6 & 6 & 6 & 6 & & \\
\hline Clfufu02MImy & 8 & 8 & 8 & 7 & 8 & 8 & 7 & 7 & 7 & 7 & 1 & \\
\hline Clfusu08HEmy & 9 & 9 & 9 & 10 & 9 & 9 & 8 & 8 & 8 & 8 & 8 & 8 \\
\hline
\end{tabular}




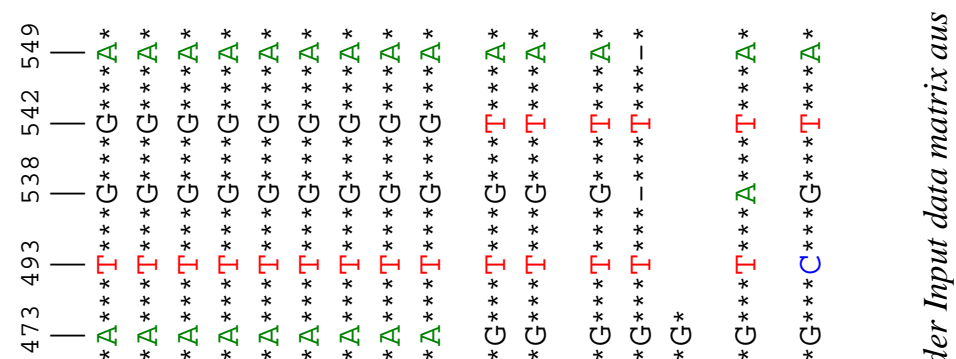

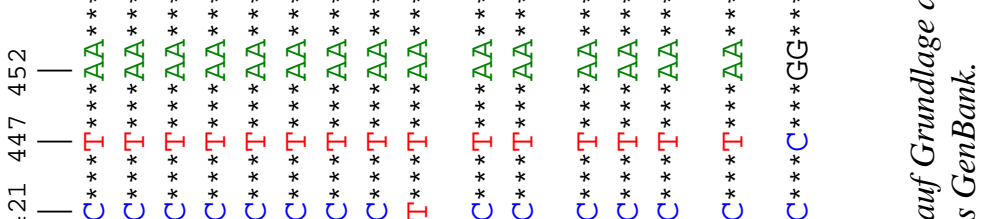

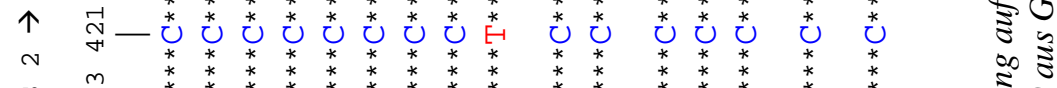

点

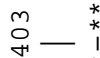

in

$$
\text { ind }
$$

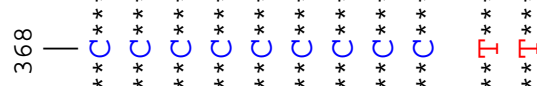

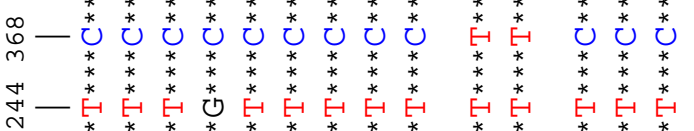

点

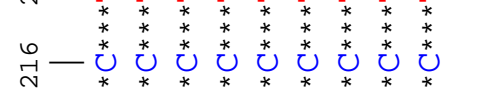

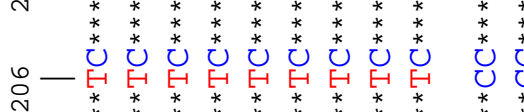

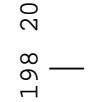

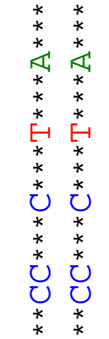

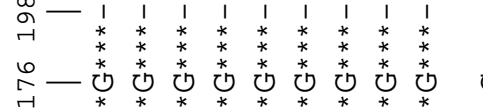

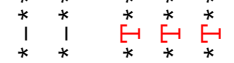

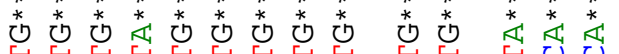

م-ب

怘袋

U.

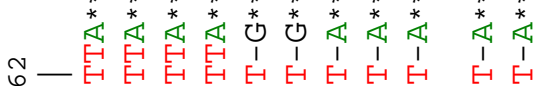

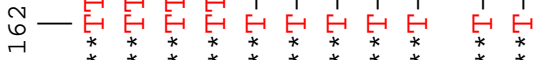

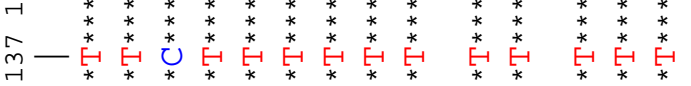

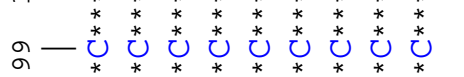

$\bigcup_{*}^{*} \bigcup_{*}^{*}$

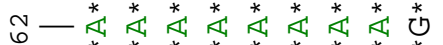

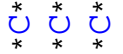

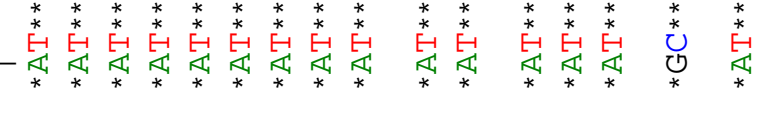

is

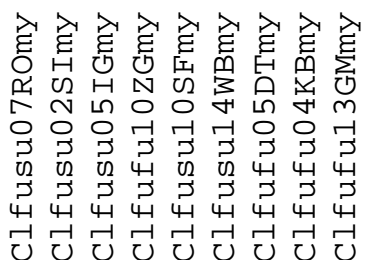

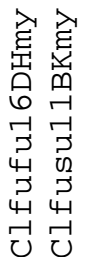

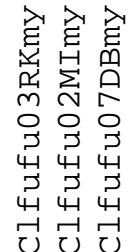

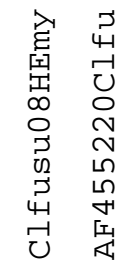

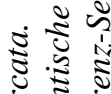

这造

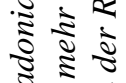

บิ

$\stackrel{\frac{1}{2}}{\frac{2}{2}} \cdot \frac{\tilde{n}}{4}$

ฐ

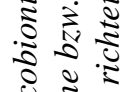

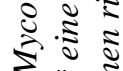

ชิ

ปิ

है

के

है है है 


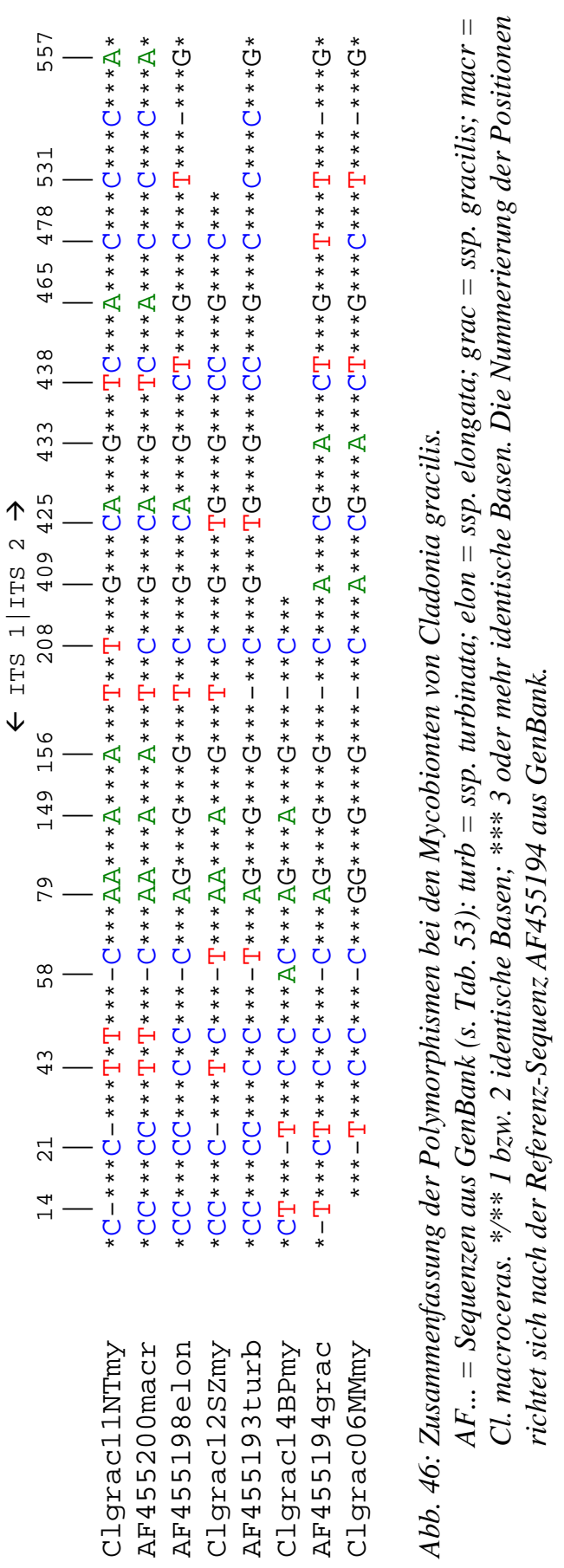




\subsubsection{Korrelation der ITS-Varianten mit systematischer Einstufung, Chemismus, Morphologie und Standortbedingungen}

Die Cladonia gracilis-Proben unterscheiden sich in ihren ITS rDNA-Sequenzen deutlich von den Cl.furcata-Proben, obwohl sie eine starke morphologische Ähnlichkeit aufweisen. In den betrachteten ITS-Regionen (528 Positionen) treten zwischen 29 und 35 Positionen auf, in denen gracilis-Proben und furcata-Proben variieren (s. Tab. 55). Die stellvertretend für die 4 ITS-Varianten aufgeführten Sequenzen von Cl. furcata unterscheiden sich in diesem Bereich lediglich in 5 bis 9 Positionen. Die in Tab. 55 angegebenen Distanz-Werte beziehen sich auf den Bereich zwischen Position 27 (ITS1) und 553 (ITS2) entsprechend der Nummerierung der Referenz-Sequenz AF455220 (Cl. furcata) aus GenBank.

Tab. 55: Distanztabelle zum Vergleich von Cladonia furcata und Cl. gracilis

\begin{tabular}{|c|c|c|c|c|c|}
\hline & 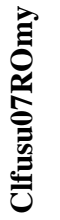 & 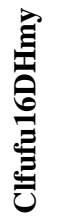 & 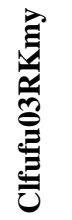 & 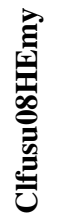 & 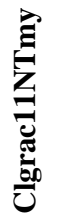 \\
\hline Clfufu16DHmy & 5 & & & & \\
\hline Clfufu03RKmy & 7 & 6 & & & \\
\hline Clfusu08HEmy. & 9 & 8 & 8 & & \\
\hline Clgrac11NTmy & 29 & 30 & 29 & 29 & \\
\hline Clgrac06MMmy & 35 & 34 & 35 & 33 & 13 \\
\hline
\end{tabular}

Die zwei Unterarten von Cladonia furcata lassen sich aufgrund ihrer ITS rDNASequenzen nicht trennen. Sie treten in den ITS-Varianten 1 und 2 sogar gemeinsam auf. Eine Korrelation zwischen ITS-Varianten und dem Chemismus (d.h. dem Merkmal „Atranorin vorhanden bzw. fehlend“) bzw. dem morphologischen Merkmal „Markaufbrüche“ (bei der Sippe subrangiformis) ist daher nicht gegeben. Die Zusammensetzung der Variante 1 deutet darauf hin, daß ein Zusammenhang zwischen Morphologie und ITS-Varianten auch innerhalb der ssp. furcata nicht besteht. Eine deutlich unterschiedliche äußere Gestalt weisen innerhalb dieser Gruppierung beispielsweise die Proben Clfufu05DTmy und Clfufu13GMmy auf. Bei der zuerst genannten handelt es sich um aufrecht wachsende, stark verzweigte und beschuppte, kaum pigmentierte Podetien aus einem Waldgebiet auf Grauwacke, bei der zweiten um zwar ebenfalls stark verzweigte, jedoch niederliegende, kaum beschuppte, stark pigmentierte Exemplare eines Basalt-Felsstandortes. Die ITS rDNA-Sequenzen morphologisch unähnlicher Cl. furcata-Exemplare können demnach bis auf 3 (oder weniger) Positionen identisch sein.

Anhand der Variante 1 wird deutlich, daß nicht immer ein Zusammenhang zwischen Standorten und molekularen Daten besteht, da in ihr Proben sowohl von Wuchsorten mit basenreichem als auch mit basenarmem Ausgangsgestein vereinigt sind. Die Variante 2 setzt sich aus je einer Probe von einem Dolomit- bzw. Basaltstandort, die Variante 3 nur aus Proben von Standorten mit basenreichem Gestein zusammen. 
Entsprechend unterschiedlich wie die Standorte sind auch die Gesellschaften, denen die Thalli entnommen wurden (s. dazu Tab. 52).

\subsection{ITS rDNA-Sequenzen der Photobionten}

\subsubsection{Polymorphismen der sequenzierten ITS-Bereiche}

Bei den Photobionten können 4 ITS-Varianten unterschieden werden (Abb. 47 und Abb. 49). Sie umfassen folgende Sequenzen:

ITS-Variante 1: Cl. furcata ssp. subrangiformis Proben Nr. 3, 4, 11, 12, Cl. furcata ssp. furcata Proben 9, 16

ITS-Variante 2: Cl. furcata ssp. subrangiformis Proben Nr. 5, 8, 10, 14

ITS-Variante 3: Cl. furcata ssp. furcata Probe Nr. 7

ITS-Variante 4: Cl. gracilis Probe Nr. 11

Aus Abb. 47 wird ersichtlich, daß die ITS-Varianten 1 und 2 durch hohe BootstrapWerte von 92 (ML), 94 (MP) und 100 (NJ) bzw. 72 (ML), 81 (MP) und 100 (NJ) unterstützt werden. Die Sequenzen der Varianten 1 und 2 werden gemeinsam den ITSVarianten 3 und 4 gegenübergestellt (Bootstrap-Werte 96 (ML), 91 (MP), 100 (NJ)). Die Abtrennung der ITS-Varianten 3 und 4 wird durch Bootstrap-Werte von 96 (ML), 91 (MP) und $100(\mathrm{NJ})$ unterstützt.

Abb. 48 zeigt die Polymorphismen der sequenzierten ITS-Bereiche. In Tab. 56 sind die Distanzen der Sequenzen untereinander dargestellt. Die ersten 5 Sequenzen der ITSVariante 1 stimmen untereinander überein (4 davon sind in Tab. 56 dargestellt). Die 6. Sequenz (Clfufu09ZGal) weicht in 2 Positionen von den anderen Sequenzen ab. Innerhalb der ITS-Variante 2 sind die ersten 3 Sequenzen identisch, die vierte unterscheidet sich an 3 Positionen von den anderen Sequenzen der selben Variante. Die beiden Sequenzen der Varianten 3 und 4 zeigen im Vergleich an 9 Positionen Unterschiede. Insgesamt betrachtet können die Sequenzen der verschiedenen ITSVarianten in bis zu 17 der 567 bei der Berechnung berücksichtigten Positionen voneinander abweichen. D.h. sie variieren in bis zu $3 \%$ der betrachteten Positionen. Die auffälligsten Unterschiede finden sich zwischen Position 55 und 58 (ITS 1) sowie ab Position 380 ff (ITS 2).

\subsubsection{Vergleich mit Photobionten-Sequenzen aus GenBank}

Die ITS-Variante 1 ähnelt Trebouxia erici (AF345441), deren Sequenz jedoch eine vermittelnde Stellung zwischen den Varianten einnimmt. Die Sequenz des Photobionten aus Cladonia rangiformis (AF345435) stimmt im betrachteten Sequenzbereich bis auf eine Position mit 3 der 4 Sequenzen der Variante 2 überein. Ebenfalls zu dieser Gruppe gehört die Sequenz des Photobionten aus Cl. furcata (AF345429), die sich allerdings in mindestens 11 Positionen von den anderen Sequenzen der Variante 2 unterscheidet. Die Sequenz des Photobionten aus Cl. gracilis (AF345414) läßt sich ebenso wie die von Trebouxia irregularis (AF345411) in die Variante 3 einordnen. 


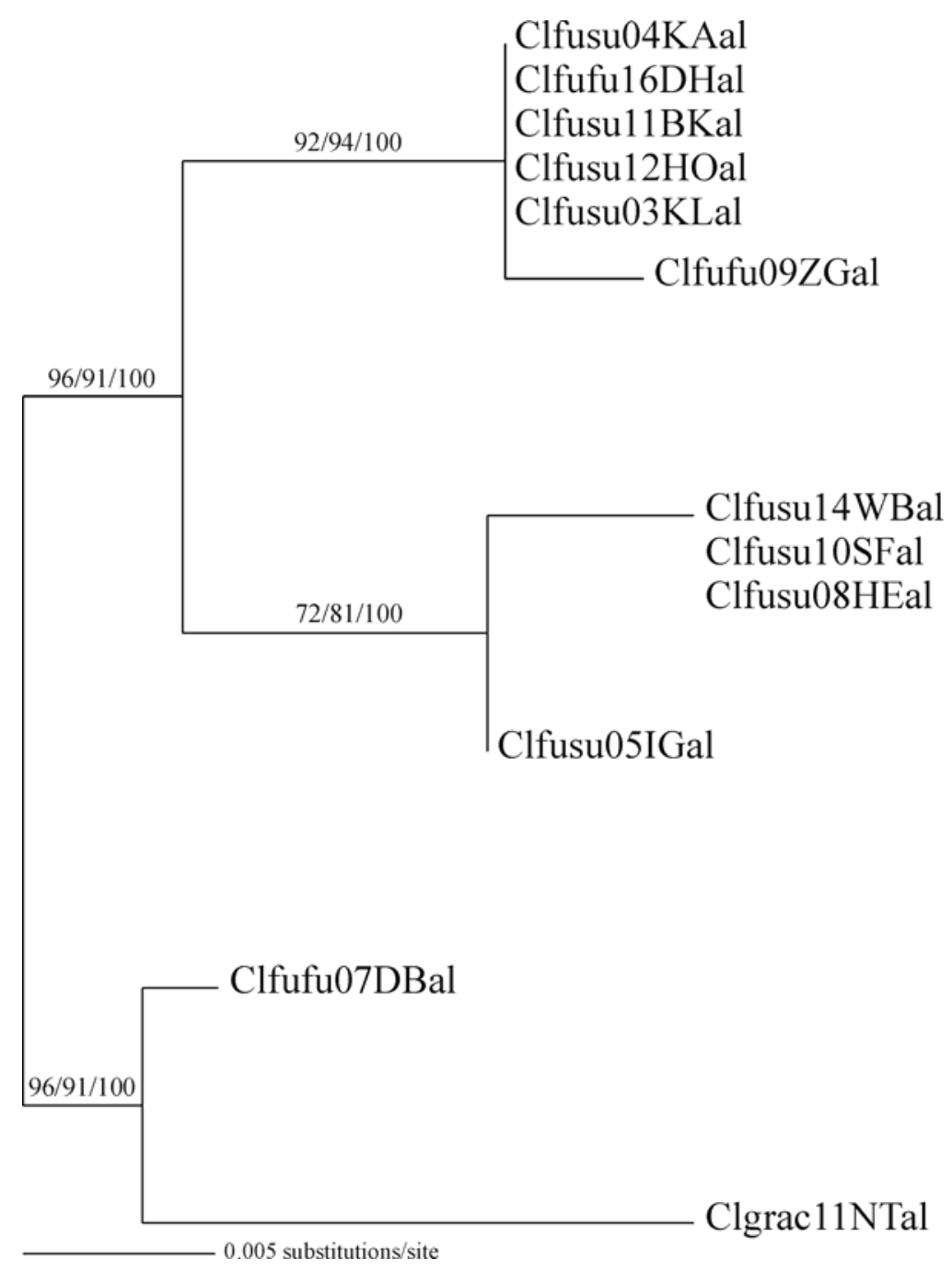

Abb. 47: rDNA-Stammbaum der Photobionten aus Cladonia furcata und Cl. gracilis berechnet nach der Maximum Likelihood-Methode (Midpoint rooting) mit zusätzlichen Bootstrap-Werten aus dem Maximum Parsimony- und Neighbor-Joining-Verfahren (angegeben in dieser Reihenfolge).

Tab. 56: Distanztabelle der Photobionten (fett gedruckt: Distanzen innerhalb einer ITS-Variante)

\begin{tabular}{|c|c|c|c|c|c|c|c|c|c|c|c|}
\hline & 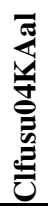 & 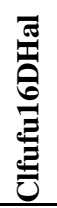 & 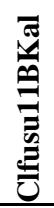 & 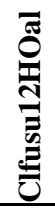 & 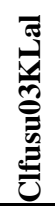 & 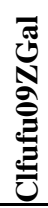 & 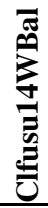 & 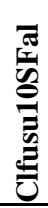 & 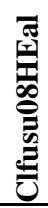 & $\begin{array}{l}\bar{J} \\
0 \\
0 \\
0 \\
0 \\
0 \\
0 \\
0 \\
0\end{array}$ & 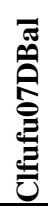 \\
\hline Clfufu16DHal & $\mathbf{0}$ & & & & & & & & & & \\
\hline Clfusu11BKal & 0 & 0 & & & & & & & & & \\
\hline Clfusu12HOal & $\mathbf{0}$ & 0 & $\mathbf{0}$ & & & & & & & & \\
\hline Clfusu03KLal & $\mathbf{0}$ & $\mathbf{0}$ & $\mathbf{0}$ & $\mathbf{0}$ & & & & & & & \\
\hline Clfufu09ZGal & 2 & 2 & 2 & 2 & 2 & & & & & & \\
\hline Clfusu14WBal & 9 & 9 & 9 & 9 & 9 & 10 & & & & & \\
\hline Clfusu10SFal & 9 & 9 & 9 & 9 & 9 & 10 & $\mathbf{0}$ & & & & \\
\hline Clfusu08HEal & 9 & 9 & 9 & 9 & 9 & 10 & $\mathbf{0}$ & $\mathbf{0}$ & & & \\
\hline Clfusu05IGal & 9 & 9 & 9 & 9 & 9 & 11 & 3 & 3 & 3 & & \\
\hline CIfufu07DBal & 9 & 9 & 9 & 9 & 9 & 10 & 12 & 12 & 12 & 9 & \\
\hline Clgrac11NTal & 16 & 16 & 16 & 16 & 16 & 17 & 16 & 16 & 16 & 14 & 9 \\
\hline
\end{tabular}




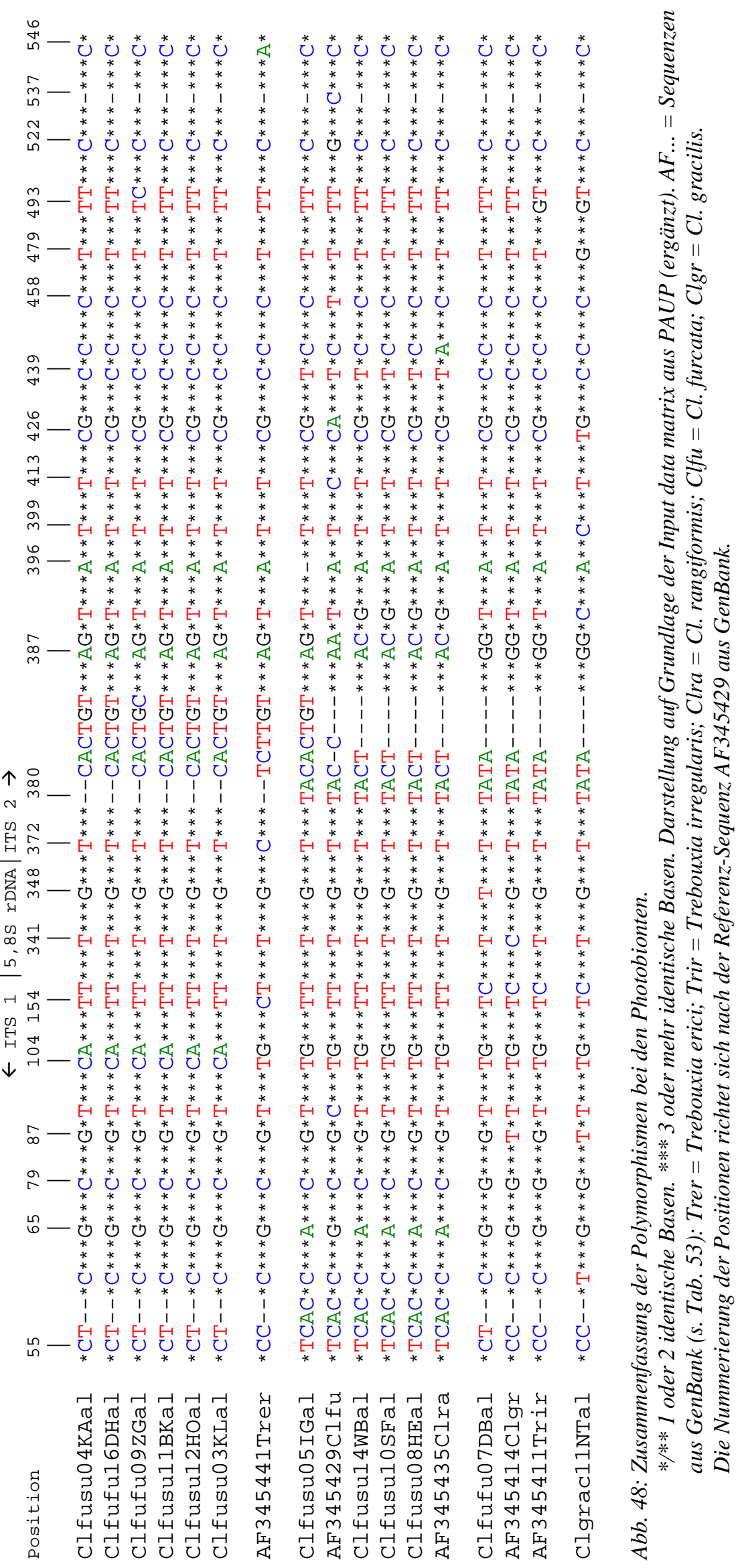




\subsubsection{Korrelation der ITS-Varianten mit systematischer Einstufung, Chemismus, Morphologie und Standortbedingungen}

ITS-Variante 1 umfaßt Photobionten beider Unterarten von Cladonia furcata, d.h. aus Thalli, die sich in ihrem Chemismus voneinander unterscheiden. Variante 2 beinhaltet nur Photobionten-Sequenzen aus Cl. furcata ssp. subrangiformis. Variante 3 besteht lediglich aus einer Photobiontensequenz von Cl. furcata ssp. furcata. Das bedeutet, jede Unterart von Cl. furcata geht mit kokkalen Grünalgen mindestens zweier verschiedener ITS-Varianten eine Lebensgemeinschaft ein. Von den 8 Photobionten-Sequenzen aus Cl. furcata ssp. subrangiformis gehören je 4 der ITS-Variante 1 bzw. der ITS-Variante 2 an. Die 3 Sequenzen aus der Subspezies furcata verteilen sich wie folgt: 2 sind der Variante 1, eine der Variante 3 zuzuordnen. Cl. gracilis unterscheidet sich in ihrem Photobionten (ITS-Variante 4) von Cl. furcata.

Es lassen sich keine Korrelationen zwischen ITS-Varianten und morphologischen Merkmalen der Cladonien, etwa dem Grad der Verzweigung oder der Beschuppung, erkennen. Leider stehen jedoch keine ausreichend guten Sequenzen beschuppter Exemplare zur Verfügung. Ein Zusammenhang zwischen Standortfaktoren und molekularen Daten deutet sich bei Betrachtung der Varianten 1 und 2 an. Deren AlgenSequenzen stammen (mit Ausnahme der Sequenz Clfufu09ZGal) alle aus Flechten, die auf basenreichem Ausgangsgestein wuchsen. Interessanterweise fügt sich in die Variante 2 auch der Cl. rangiformis-Photobiont (AF345435) ein, über dessen Herkunft leider keine Details bekannt sind. Cl. rangiformis gilt jedoch als eine Flechtenart, die gewöhnlich auf basenreichen Böden wächst (WIRTH 1995).

Mit Ausnahme der Probe aus der Zeche Glimmerode beinhalten die ITS-Varianten 1 und 2 nur Photobionten aus Cladonien, die innerhalb von Pflanzen- bzw. Flechtengesellschaften wuchsen, die für derartige basenreiche Standorte typisch sind.

\subsection{Kombinationen der Bionten (Abb. 49)}

Es wurde versucht, die Sequenzen beider Bionten zu ermitteln, jedoch liegen nur bei einem Teil der Proben beide Sequenzen vor. Ein Vergleich der ITS-Varianten von Pilz und Alge führt zu einigen interessanten Erkenntnissen, die allerdings aufgrund der geringen Anzahl an Sequenzen lediglich Hinweise auf einen möglichen Zusammenhang zwischen Mycobionten und Photobionten-Varianten geben können:

1. Die Mycobionten der Variante 1 weisen Photobionten der ITS-Variante 2 auf (Clfusu 5, 10, 14).

2. Die Mycobionten der Proben ClfufuDH16 und ClfusuBK11 bilden die ITSVariante 2 und unterscheiden sich auch bezüglich der Photobionten von den Proben der Mycobionten-Variante 1. Ihre Photobionten-Sequenzen zählen zur ITS-Variante 1.

3. Bei der Probe Clfufu7 gehören beide Bionten jeweils einer anderen als den unter Punkt 1 und 2 genannten ITS-Varianten an.

4. Bei Cl. gracilis bilden ebenfalls beide Bionten eigene Varianten. 


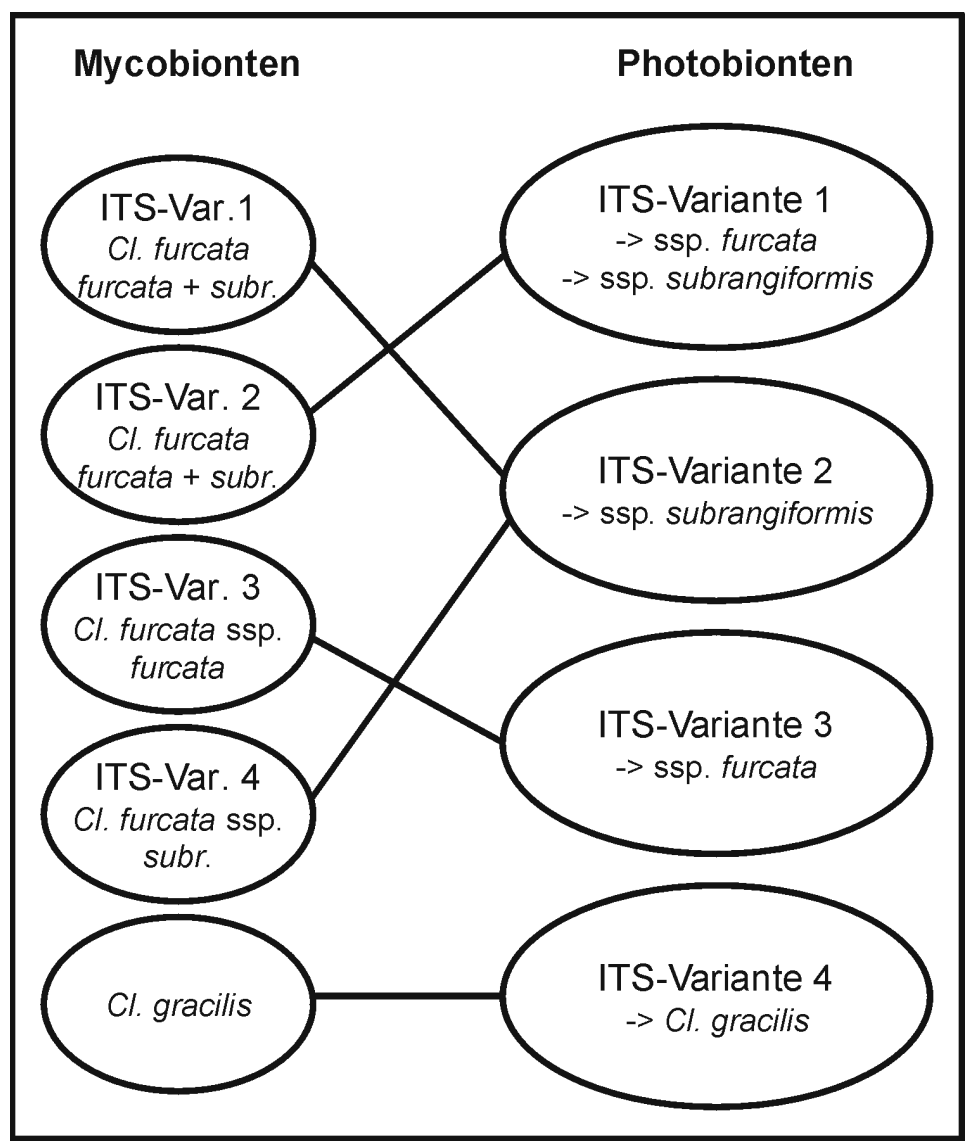

Abb. 49: Kombinationen der Bionten innerhalb der Arten Cladonia furcata und Cl. gracilis.

\section{Diskussion}

\section{a) Asterochloris spec. - Photobiont von Cladonia furcata und Cl. gracilis}

Als einzige Art ihrer Gattung wurde Asterochloris phycobiontica aus der Flechte Anzina carneonivea (Anzi) Scheid. (Syn. Varicellaria carneonivea (Anzi) Erichs.) erstmals von TSCHERMAK-WOESS (1980) isoliert und beschrieben. TSCHERMAK-WOESS (1989) unterteilte die Gattung Trebouxia in die zwei Subgenera Trebouxia und Eleutherococcus und erkannte aufgrund phänotypischer Merkmale die Übereinstimmung von Asterochloris phycobiontica mit den Eleutherococcus-Arten. Ein von PIERCEY-NORMORE \& DEPRIEST (2001) vorgenommener Vergleich von Sequenzdaten ergab ebenfalls die nahe Verwandtschaft von Asterochloris phycobiontica mit den Eleutherococcus-Arten von TSCHERMAK-WOESS (1989).

Die von AHMADJIAN \& JAKOBS (1981) durchgeführten Resynthese-Experimente belegen die Abgrenzung von Asterochloris und Trebouxia s. str. auf eindrucksvolle Weise. Der Mycobiont Cladonia cristatella Tuck. bildet mit allen Algenstämmen der AsterochlorisGruppe Thallusschuppen, während er die anderen getesteten Trebouxia-Stämme parasitiert und abtötet. RAMBOLD et al. (1998) nehmen an, daß alle Asterochlorisähnlichen Trebouxia-Arten die einzigen kompatiblen Algen für die Mehrheit der 
Cladoniaceae sind. Diese Annahme wird von Piercey-Normore \& DePriest (2001) unterstützt. Ein Vergleich mit den von PIERCEY-NORMORE \& DEPRIEST (2001) sequenzierten Asterochloris-Stämmen führte zu dem Ergebnis, daß die im Rahmen der vorliegenden Untersuchung erstellten Photobionten-Sequenzen aus Cladonia furcata und Cl. gracilis ebenfalls Asterochloris zuzuordnen sind.

Helms (2003) greift die Ergebnisse von Ahmadjan \& JaKobs (1981), PierceyNORMORE \& DEPRIEST (2001) und FrIEDL (1989) sowie weiterer Studien auf und publiziert erstmals einen tabellarischen Vergleich der bis zum heutigen Zeitpunkt bekannten molekularen und anatomischen Merkmale, aufgrund derer Trebouxia s.str. und Asterochloris voneinander getrennt werden können. Zur Asterochloris-Gruppe zählen demnach u.a. die Arten Trebouxia irregularis, T. pyriformis, T. glomerata und T. erici (s. PierceY-Normore \& DePriest 2001). Der Photobiont T. impressa ist dagegen beispielsweise ein Vertreter der Trebouxia s.str.

Bislang sind nur von wenigen Flechten die Algenpartner bekannt. In der Literatur finden sich zu den Photobionten von Cladonia furcata und Cl. gracilis lediglich die nachfolgend aufgeführten Informationen. WANG-YANG (1970 in NAKANO \& IGUCHI 1994) nennt Trebouxia impressa, MEISCH (1981) T. pyriformis als Photobionten von Cl. furcata. Die Angabe von WANG-YANG bezüglich T. impressa ist nach dem oben beschriebenen heutigen Kenntnisstand der Photobiontenwahl bei Cladonien allerdings zweifelhaft. Als Photobionten in Cl. gracilis werden T. erici (NAGANO \& IGUCHI 1994) und T. glomerata (WARÉN 1918/19 in NAGANO \& IGUCHI 1994) genannt. BECK (2002) führt den von ihm aus Cl. furcata isolierten Photobionten als Asterochloris irregularis (Chodat) T. Friedl (ined.) auf. Er synonymisiert zudem die Arten Asterochloris (= Trebouxia) pyriformis und Asterochloris (= Trebouxia) glomerata mit A. irregularis. Diese Synonymisierung wird durch die molekularen Daten von PIERCEY-NORMORE \& DEPRIEST (2001) bestätigt, da alle sequenzierten Klone dieser Arten fast identische ITSSequenzen haben und zusammen die als „Clade I“ (s.u.) bezeichnete Gruppe bilden.

\section{b) ITS-Varianten der Photobionten}

PiERCEY-Normore \& DePriest (2001) untersuchten anhand der ITS 1 und 2 die verwandtschaftlichen Beziehungen der Photobionten von 33 Cladoniaceen. Die Übereinstimmung der Photobionten-Sequenzen ist dabei, wie auch bei den nordhessischen Sequenzen, mit mehr als $90 \%$ ziemlich hoch. Es ergeben sich zwei Clades. Clade I beeinhaltet u.a. den Photobionten aus Cladonia gracilis ssp. gracilis (GenBank AF345414). Die ITS-Varianten 3 und 4 der vorliegenden Untersuchung entsprechen diesem Clade I, wie ein Vergleich der Sequenzen zeigt. Die Sequenz Clfufu07DBal (ITS-Variante 3) unterscheidet sich in 4, die Sequenz Clgrac11NTal (ITS-Variante 4) in 7 Positionen von der oben genannten GenBank-Sequenz. Der Photobiont aus Cl.furcata gehört dagegen Clade II (nach PIERCEY-NORMORE \& DEPRIEST 2001) an. Diesem entspricht die ITS-Variante 2 der vorliegenden Untersuchung, die ausschließlich in der Subspezies subrangiformis nachgewiesen wurde. Darüber hinaus tritt bei den nordhessischen $\mathrm{Cl}$. furcata-Proben noch eine weitere ITS-Variante (Nr. 1) auf, die Clade II näher steht als Clade I. 
Nach PierCeY-Normore \& DePriest (2001) können die Clade I-Genotypen (die möglicherweise alle T. irregularis angehören) mit Pilzen verschiedener Gattungen, Familien und sogar Ordnungen assoziiert sein. Generell sind die Algen wohl wenig spezifisch, so daß eine Algenart, bzw. ein Algen-Genotyp, mit verschiedenen, verwandtschaftlich sich nicht nahestehenden Flechtenpilzen zusammenleben kann (s. HiLdRETH \& AHMADJIAN 1981, Helms 2003). In der vorliegenden Untersuchung bleiben die Algen der ITS-Varianten jedoch auf jeweils eine der beiden Cladonia-Arten beschränkt. Umgekehrt zeigen die Ergebnisse aus Nordhessen aber auch, daß Cl. furcata weniger selektiv gegenüber dem Algenpartner ist, als nach der Studie von Piercey-Normore \& DePriest (2001) zu erwarten wäre. Da im Gegensatz zu den vorangegangenen Untersuchungen nicht nur eine, sondern mehrere Sequenzen aus derselben Cladonia-Art zur Verfügung standen, konnte belegt werden, daß Cl. furcata mindestens 3 verschiedene ITS-Varianten (bzw. zwei Algen-Genotypen) als Photobionten aufweist. Mycobionten mit mehreren kompatiblen Algen-ITS-Varianten lassen sich beispielsweise auch bei Arten innerhalb der Physciaceae beobachten (HELMS 2003).

Die Asterochloris-ITS-Varianten der nordhessischen Cladonien unterscheiden sich, wie in Kap. G2.2.1 aufgeführt, in bis zu $3 \%$ aller betrachteten Positionen voneinander. Dieses Maß an Variation ist vergleichsweise gering und entspricht etwa der Variabilität innerhalb von Trebouxia-Subclades (Helms 2003). Subclades werden als dem taxonomischen Rang einer Art entsprechend angesehen (HELMS 2003). Innerhalb einer Trebouxia-Art können deutlich größere Unterschiede auftreten. BECK et al. (1998) stellten bis zu 40 variable Positionen zwischen den Stämmen einer Trebouxia-Art fest. Die Sequenzen der in RoMEIKE et al. (2002) beschriebenen „Antarctic variant A“ (=Trebouxia spec.) weisen bis zu 11 verschiedene Positionen auf. Die paarweisen Differenzen unter den insgesamt 5 ,Antarctic“-Varianten betragen 52 bis 119 Positionen. Die Varianten A und E, die sich in bis zu 119 Positionen unterscheiden, gehören allerdings verschiedenen Trebouxia-Arten an.

\section{c) Genetische Variabilität der Mycobionten}

ZOLLER et al. (1999) fanden 3 variable Positionen innerhalb der ITS 1 bei Lobaria pulmonaria. Die Mycobionten-Sequenzen der von ROMEIKE (2002) untersuchten, morphologisch ebenfalls variablen Umbilicaria-Arten zeigen fast keine Variationen in der ITS rDNA-Region: Von den 11 Proben des Mycobionten von Umbilicaria antarctica weisen 2 Proben identische Sequenzen mit der Vergleichs-Sequenz aus GenBank (AF096213) auf, 9 unterscheiden sich von dieser in lediglich einer Position (vgl. auch RoMEIKE et al. 2002). Die Mycobionten von Cladonia furcata sind in ihrer ITS rDNA dagegen verhältnismäßig variabel. Sie unterscheiden sich in immerhin bis zu 14 Positionen von der Vergleichs-Sequenz aus GenBank (AF455220; s. Abb. 45) und in bis zu 10 Positionen untereinander (s. Tab. 54).

Leider liegen keine Vergleichssequenzen von Cladonia subrangiformis vor. OKSANEN et al. (2000) haben diese Flechte offensichtlich in ihren Untersuchungen mitbearbeitet, 
da sie sie als zur Sektion Ascyphiferae gehörig nennen. Es wurden bislang jedoch keine entsprechenden Sequenzen in GenBank publiziert.

\section{d) Korrelation von molekularen mit morphologischen Merkmalen?}

In der vorliegenden Untersuchung zeigte sich kein Zusammenhang zwischen ITSVarianten der Mycobionten und den verschiedenen Gestalttypen der Flechte. LOHTANDER et al. (1998) verglichen ITS-Sequenzen innerhalb von Roccella capensis und stellten fest, daß sich die sexuell ausbreitenden Proben bezüglich ihrer ITS nicht von denen mit asexueller Verbreitungsstrategie unterscheiden.

Wie sich in anderen Studien herausstellte, lassen sich auf Ebene der Arten und höherer taxonomischer Einheiten unter Umständen Übereinstimmungen zwischen molekularen und morphologischen Merkmalen feststellen. Beispielsweise korreliert nach den Untersuchungen von HeLMS et al. (2003) bei den Physciaceae die ITS Phylogenie mit Merkmalen der Frucktkörper (Apothecien-, Ascus- und Sporenmerkmalen).

Ein Einfluß der Alge auf die Gestalt der Flechte ist ebenfalls denkbar, auch wenn sich dies im Fall der Cladonia furcata-Proben nicht bestätigen ließ. FRIEDL (1989) konnte bei Flechten mit verschiedenen Algenkombinationen keine Unterschiede in der Morphologie der Thalli in Abhängigkeit von einem bestimmten Algenpartner feststellen. Nach HeLmS (2003) treten bei Trebouxia Arten auf, die aufgrund morphologischer Merkmale getrennt werden, jedoch ein und dieselbe ITS-Variante darstellen. Umgekehrt können aber auch verschiedene Subclades phänotypisch eine Art repräsentieren. Darüber hinaus konnte Helms (2003), anders als im Rahmen der vorliegenden Untersuchung, Zusammenhänge zwischen pH-Kategorien des Substrates und Algen-Phylogenie, also zwischen Standortfaktoren und molekularen Merkmalen feststellen. BECK (2002) erkannte, daß alle von ihm untersuchten Flechten eine ausgeprägte Selektivität bezüglich ihres Algenpartners haben, der in einer Flechte gefundene Photobiont jedoch nicht von der Flechtenart, sondern von deren Standort, also von klimatischen und geographischen Faktoren abhängt.

\section{$4 \quad$ Zusammenfassung}

Anhand der ITS rDNA-Sequenzen beider Bionten von Cladonia furcata und Cl. gracilis werden verschiedene Fragestellungen untersucht. Im Vordergrund steht die Frage, ob sich beide Unterarten von Cl. furcata anhand der Sequenzdaten unterscheiden lassen. Weiterhin wird nach Zusammenhängen zwischen morphologischen und molekularen Merkmalen sowie zwischen Sequenzdaten und Standortbedingungen gesucht. Zudem werden Proben von Cl. furcata und Cl. gracilis verglichen, die eine starke morphologische Ähnlichkeit aufweisen.

Die Sequenzen der Mycobionten von Cl. furcata können in 4 ITS-Varianten unterteilt werden. Ein nach dem Maximum Likelihood-Verfahren errechneter Stammbaum wird angegeben. Die ITS-Bereiche der Mycobionten weisen eine relativ geringe Variabilität auf, da sie sich in maximal 10 Positionen voneinander unterscheiden. Beide Unterarten von Cl. furcata können der selben Mycobionten-ITS-Variante angehören. Die 
Sequenzdaten unterstützen daher eine Wertung der Subspezies subrangiformis als eigene Art nicht. Es besteht kein Zusammenhang zwischen dem Auftreten bzw. Fehlen von Atranorin in den Cl. furcata-Proben und den ITS-Varianten.

Das äußere Erscheinungsbild der Subspezies furcata korreliert nicht mit den ITSVarianten der Mycobionten bzw. Photobionten. Dagegen unterscheiden sich die morphologisch ähnlichen Thalli von Cl. furcata und Cl. gracilis deutlich in ihren Mycobionten-Sequenzen. Ein Zusammenhang zwischen Standortverhältnissen und ITSVariante deutet sich nur bei einem Teil der ITS-Varianten von Pilz und Alge an.

Die Grünalgen sind zu den Trebouxia-Arten zu stellen, die BECK (2002) und HeLMS (2003) als Asterochloris bezeichnen. Sie lassen sich in 4 ITS-Varianten unterteilen, welche ebenfalls in einer Maximum Likelihood-Phylogenie dargestellt werden. Cladonia furcata ist nicht ganz so selektiv bei der Wahl des Algenpartners wie bisherige Studien vermuten ließen, sondern kann mit mindestens 3 verschiedenen Photobionten-ITS-Varianten zusammenleben, welche sich allerdings nur in maximal 12 Positionen unterscheiden. Der Photobiont von Cl. gracilis gehört einer anderen ITSVariante an, als die Photobionten aus Cl. furcata, von denen er in immerhin 9 bis 17 Positionen abweicht. 


\section{Zusammenfassende Diskussion zu Cladonia furcata}

\section{Ursachen der morphologischen Erscheinungsformen der Subspezies furcata}

\subsection{Auswirkungen des Mikroklimas}

\section{a) Anpassungen an starke Sonneneinstrahlung}

Viele Flechten sind in der Lage sich an hohe Einstrahlung durch SonnenschutzPigmente (RIKKINEN 1995) oder durch eine Verdickung der Rinde (z.B. ERTL 1951, HYVÄRINEN 1992, PANNEWITZ et al. 2003) anzupassen. Daher zeigt die Pigmentierung eine besonders deutliche Abhängigkeit von der Lichtintensität am Standort (vgl. auch WAINIO 1897, SANDSTEDE 1931 und SEMBDNER 1957/58). Allerdings ist zu beachten, daß eine verstärkte Pigmentierung auch eine Alterserscheinung sein kann (SEMBDNER 1957/58) oder die Folge eines Befalls mit lichenicolen Pilzen (z.B. Arthrorhaphis aeruginosa, FAHSELT et al. 2000).

DIETZ et al. (2000) weisen am Beispiel von Peltigera praetextata und P. rufescens nach, daß Flechten bei verstärkter Einstrahlung bereits innerhalb weniger Tage eine stärkere Pigmentierung aufweisen. Im Rahmen des von mir durchgeführten Verpflanzungsversuches reagierten alle der höheren Sonneneinstrahlung ausgesetzten Podetienbereiche mit vermehrter Pigmentierung, so daß sich an den sonnigen Standorten nach wenigen Wochen eine schwache, innerhalb eines Jahres eine deutlichen Verfärbung der Thalli beobachten ließ.

Anhand der anatomischen Untersuchungen (Kap. G4) wurde deutlich, daß Thalli von Cladonia furcata an helleren Standorten eine dickere Rinde aufweisen, als an beschatteten Wuchsorten. HYVÄRINEN (1992) stellte bei der Blattflechte Hypogymnia physodes teilweise signifikante Unterschiede zwischen Proben von lichten (trockenen) Waldstandorten mit dickerer Rinde und solchen von beschatteten Flächen, mit dünnerer Rinde fest. Die dickere Rinde an stärker besonnten Standorten könnte den Photobionten einen gewissen Schutz vor schädlicher UV-Strahlung bieten. Tatsächlich lassen die Rindenschichten von Schattenexemplaren mehr Licht durch, da sie dünner sind als die von Flechten sonniger Standorte (ERTL 1951). Eine dicke Rinde verringert v.a. in trockenem Zustand die Lichtdurchlässigkeit (ERTL 1951, PANNEWITZ et al. 2003). Allerdings folgern DIETZ et al. (2000) aus ihren Untersuchungen, daß weniger die Dicke der Rinde als vielmehr die Pigmentierung die Lichtdurchlässigkeit des Cortex beeinflußt. Die im Rahmen der Studie von SANCHO et al. (2003) erhobenen morphologischen Daten für Umbilicaria aprina weisen darauf hin, daß bei stark pigmentierten Thalli, bedingt durch die dunkle Schicht in der Rinde, so viel Licht verloren geht, daß der Photosynthetische Apparat sich wie ein schattenadaptiertes System verhält. Dies würde die in Kap. G3.4 aufgestellte Vermutung unterstützen, 
wonach die ausgedehnte Algenschicht der von sonnigen Felsstandorten stammenden Cl. furcata-Podetien eine Folge der starken Pigmentierung dieser Thalli ist. Bezüglich der Ausdehnung der Algenschicht und der Lichtverhältnisse für die Photobionten sind sich also Exemplare stark besonnter und beschatteter Standorte ähnlich.

Bei den nordhessischen Cladonia-Proben ist die Gonidienzone in Podetien von schattigen Standorten dünner als in denen sonniger Wuchsorte (vgl. hierzu auch SEMBDNER 1957/58 und SANCHO et al. 2003). Laut ERTL (1951) wird das physiologische Manko der geringen Lichtzufuhr zu den Algen bei den Sonnenformen durch die größere Dicke der Assimilationsschicht ungefähr wieder ausgeglichen. Somit wäre die Dicke der Algenschicht also keine Folge von besonders guten Lichtverhältnissen in den Thalli besonnter Standorte, die es auch weiter im Inneren des Hyphengeflechts liegenden Photobionten ermöglicht Photosynthese $\mathrm{zu}$ betreiben, sondern, im Gegenteil, eine Reaktion auf das ungünstige Lichtangebot.

Ergänzend ist hier anzuführen, daß die oben beschriebenen Verhältnisse offensichtlich nicht auf alle Flechten zutreffen. In Hypogymnia physodes ist die Algenschicht nicht an den sonnig-trockenen, sondern an den feuchten Standorten signifikant dicker (HyVÄRINEN 1992). SEMBDNER (1957/58) berichtet, daß die Rinde bei den von ihm bearbeiteten Cladonien unter feucht-schattigen Verhältnissen dicker sei.

\section{b) Vergrößerung der assimilierenden Oberfläche}

Wie am Beispiel von Cladonia furcata ssp. furcata gezeigt werden konnte, ist die Anzahl der Schuppen am Podetium ebenfalls vom Sonnenlicht abhängig. Die Podetien sind im Schatten oft deutlich beschuppt, der Sonne ausgesetzt dagegen mehr oder weniger schuppenlos. Entsprechende Beschreibungen über Cl. furcata bzw. Cl. coniocraea finden sich auch bei BRITZELMAYR (1904), ANDERS (1928), SANDSTEDE (1931), ErichSEN (1957), SEMBdNER (1957/58) und Schade (1964). Britzelmayr (1904) bemerkt hierzu, die in der Schattenlage reichlichere Beschuppung bei Cl. furcata diene wohl dem Zwecke, das Lichtbedürfnis solcher Formen durch Darbietung größerer Flächen zu befriedigen. WAINIO (1897) führt die beblätterten Formen vieler CladoniaArten auf die Wirkung übermäßiger Feuchtigkeit zurück. Die Aussage BRITZELMAYRs erscheint mir für $\mathrm{Cl}$. furcata am zutreffensten. Eine derartige Oberflächenvergrößerung ist für die Flechte jedoch nur unter günstigen Feuchtigkeitsverhältnissen, wie beispielsweise an den Waldstandorten, akzeptabel.

\section{c) Podetienwachstum und Fibrillenbildung}

In gewissem Maße wird das Podetienwachstum ebenfalls von der Lichtintensität beeinflußt, wie VAGTS et al. (1994) und die eigenen Verpflanzungsversuche belegen: Die Thalli der beschatteten Versuchsflächen zeigen häufig ein geringeres Podetienwachstum als die Polster der stärker, jedoch nicht extrem besonnten Flächen. VAGTS et al. (1994) maßen im Mittel einen Zuwachs von 2,9 mm/a an Cl. furcata-Thalli im Corynephoretum. Bei geeigneter Düngung lassen sich Zuwachsraten von bis zu 6,9 mm/a erzielen (VAGTS \& KINDER 1999). Im Vergleich dazu beträgt der Jahreszuwachs der am Roßkopf ausgepflanzten Thalli je nach Standort nur 0,9 bis 2,0 mm (betrachtet 
man die etwa ein Jahr lang ausgesetzten Polster des Verpflanzungszeitraumes Juni 2002 bis Juli 2003). Unter günstigen Bedingungen im Labor sollen die Podetien von Cl. furcata ssp. furcata 2-3 mm innerhalb von 3-4 Wochen wachsen (STOCKERWÖRGÖTTER \& TÜRK 1993). Diesen hohen Wachstumsraten im Labor widersprechen allerdings nicht nur die eigenen Beobachtungen aus dem Freiland, sondern auch die von FINK (1917), VAGTS et al. (1994) sowie VAGTS \& KINDER (1999).

Unter welchen Bedingungen das stärkste Podetienwachstum auftritt, ist offensichtlich von den Ansprüchen der Flechtenart abhängig, wie ein Vergleich mit den Ergebnissen von SEMBDNER (1957/58) zeigt: Cladonia [pyxidata ssp.] chlorophaea weist, vergleichbar mit $\mathrm{Cl}$. furcata ssp. furcata, auf normalen, d.h. weder besonders sonnigtrockenen noch besonders schattig-feuchten Standorten, im Mittel die größte Podetienlänge und den größten Podetiendurchmesser auf. Bei stärkerer Trockenheit entwickeln sich lediglich kleinere Podetien. Cl. coniocraea erreicht ihre größte Podetienlänge dagegen im Schatten unter vermehrter Feuchtigkeit. An den schattigeren Standorten dürfte für einige Arten das Licht den begrenzenden Faktor für das Podetienwachstum darstellen (vgl. SEMBDNER 1957/58).

Die vorliegende Untersuchung kommt zu dem Resultat, daß Fibrillen an den beiden hellsten Standorttypen häufiger gebildet werden, als unter weniger günstigen Lichtverhältnissen. Dies würde sich mit der Aussage von SEMBDNER (1957/58) decken, wonach dornige und hakige Nachsprossungen der Podetien von Cladonia coniocraea hauptsächlich auf starke Lichtreize zurückzuführen seien. Er hält jedoch auch Lageveränderungen, wie mechanisches Umstoßen, als Ursache für möglich. Letzteres ist meiner Meinung nach der Hauptgrund für die Fibrillenbildung. Auch ScHADE (1964) bestätigt dies. Er schreibt über das Entstehen der für viele Formen und Varietäten (besonders der var. palamaea) typischen dornig-hakigen Auswüchse, daß sie kein Organisationsmerkmal darstellten, sondern Reaktionen des Flechtenkörpers auf eine plötzlich erfolgte und bleibende Veränderung der Lage seien. Mechanischer Druck habe aufrechte Podetien längere Zeit zu Boden gedrückt oder gar umgebrochen und die nun nach oben gewendete Flanke treibe dann hakige, spitze Sprosse hervor. Im Extremfall können Tritt und ähnliche mechanische Störungen die Gestalt von Cladonien so stark beeinflussen, daß nur ein dauerhafter Primärthallus, aber keine Podetien ausgebildet werden, wie LANGE et al. (1998) an einem Thallus von Cl. furcata var. furcata (= ssp. furcata) beobachteten, der an einer stark trittbelasteten Stelle wuchs.

In der vorliegenden Arbeit wird versucht, den Einfluß mechanischer Störungen auf die Flechtengestalt herauszuarbeiten. Allerdings haben die Ergebnisse nur begrenzte Aussagekraft, da die Einstufung in verschiedene Grade mechanischer Störung anhand der zum Aufnahmezeitpunkt erkennbaren Einflüsse erfolgte. Einmalige oder länger zurückliegende Ereignisse können dabei unerkannt bleiben, so daß u.U. einige Zusammenhänge von mechanischen Störungen und Thallusmerkmalen, so auch der Fibrillenbildung unerkannt bleiben. Darüber hinaus überlagern sich stets mikroklimatische und mechanische Einflüsse. Die beiden hellsten Standorttypen, Felsen und Heiden, sind beispielsweise zugleich auch am stärksten Tritteinwirkungen 
ausgesetzt; Faktor Licht und Faktor Tritt daher oftmals bei der Ergebnisinterpretation schwer zu trennen.

\section{d) Fruchtkörperbildung - eine Frage des Mikroklimas?}

Bereits die geringe Apothecienbildung von Cladonia furcata ssp. furcata im Cladonietum foliaceae weist auf einen möglichen Einfluß des Mikroklimas hin: Die Art tritt in dieser Union zwar hochstet auf, wurde jedoch an den zugehörigen trockenwarmen Standorten nur in einem Fall fertil angetroffen (s. Kap. E5). SCHÖLLER (1991) bemerkt, daß fruchtende Exemplare von $\mathrm{Cl}$. furcata ssp. furcata meist an feuchten Standorten auftreten. Bei meinen Untersuchungen stellte sich heraus, daß günstige Lichtverhältnisse wohl ebenfalls von Bedeutung sind, da diese Flechte unter den lichtreichen, nur mäßig trockenen Bedingungen in Heiden häufiger Fruchtkörper bildet als an anderen, z.T. auch frischeren Standorten. In der Literatur finden sich einige weitere Angaben, die den Einfluß des Mikroklimas, besonders der Feuchtigkeitsverhältnisse, auf die Apothecienbildung bestätigen. VERSEGHY (1983) beobachtete beispielsweise die Entwicklung von $\mathrm{Cl}$. furcata ssp. furcata innerhalb verschiedener Vegetationstypen der Ungarischen Tiefebene und stellte fest, daß sich die Art unter trockenen, ungünstigen Umständen hauptsächlich auf vegetative Weise verbreitet und allenfalls spärliche Apothecienbildung zeigt, entsprechend den Gegebenheiten im Cladonietum foliaceae in Nordhessen. Stärkere Apothecienbildung tritt dagegen in Gesellschaften mit günstigeren Feuchtigkeitsverhältnissen auf. Auch diese Beobachtungen lassen sich auf das nordhessische Untersuchungsgebiet übertragen, da $\mathrm{Cl}$. furcata ssp. furcata hier ebenfalls in Flechtengesellschaften etwas schattigerer aber dafür frischer Standorte (z.B. in der Cl. rangiferina-Variante des Cladonietum mitis typicum) häufiger zur Fruchtbildung kommt als auf den bereits erwähnten Felsstandorten.

JAHNS et al. (1978) erforschten die Fruchtkörperbildung von Cladonia furcata ssp. furcata auf der Nordseeinsel Schiermonnigkoog und kamen zu dem Ergebnis, daß Sexualstadien dieser Flechte dort vorzugsweise im Winter gebildet werden. Es ist ein deutlicher Zusammenhang mit der Wasserversorgung erkennbar. Dabei besteht keine positive Korrelation mit dem Niederschlag, sondern mit der Anzahl Tagen pro Monat, an denen die Luftfeuchtigkeitswerte den ganzen Tag bei einem sehr hohen Wert liegen. Dies würde für den im Rahmen der vorliegenden Untersuchungen bearbeiteten Standorttyp Berglagen sprechen. Da fertile Lagerstiele dort jedoch nicht am häufigsten sind, ist die geringere Fruchtkörperbildung wohl mit dem unzureichenden Lichtangebot (Beschattung durch Calluna vulgaris etc.) zu erklären. Entscheidend für eine reichliche Fruchtkörperbildung sind demnach bei Cl. furcata nicht zu trockene Bedingungen am Standort sowie günstige Lichtverhältnisse.

Bei der Untersuchung der Fruchtkörperbildung in der vorliegenden Arbeit, dürfte die bei JAHNS et al. (1978) beschriebene Tatsache, daß Cladonia furcata ssp. furcata unter Umständen nur zu einer bestimmten Jahreszeit Fruchtkörper bildet, ohne Bedeutung sein, da die Apothecien eigenen Beobachtungen und JAHNS \& OTT (1982) zufolge 
längere Zeit (1-2 Jahre) erhalten bleiben und so, unabhängig vom Aufnahmezeitraum, erfaßt werden konnten.

Durch die Untersuchung des Standorteinflusses auf verschiedene Merkmale von Cladonia furcata ssp. furcata wird weiterhin belegt, daß Pyknidien, im Gegensatz zu den Apothecien, mehr an schattigen Standorten gebildet werden. Die Beobachtungen von FISCHER (2003), wonach Cl. coccifera im Mittelelbegebiet in sehr dichten Trockenrasen nur Pyknidien und kaum Apothecien aufweist, bestätigen diese Erkenntnis. Im Gegensatz dazu schreibt SEMBDNER (1957/58), die Pyknidienentwicklung sei bei Cl. [pyxidata ssp.] chlorophaea gerade auf den extrem sonnigen Standorten am stärksten. Dies erscheint nur dann nachvollziehbar, wenn man davon ausgeht, daß sie dort gelegentlich vom Regen durchnässt werden. Das Aufreißen der Pyknidien kann nämlich nur in feuchtem Zustand erfolgen. Nur dann quillt der Schleim in der Höhlung der Pyknidien und erhöht den Innendruck derart, daß durch die Öffnung die Conidienmasse heraustritt und die als Spermatien (oder zur vegetativen Verbreitung des Mycobionten?) dienenden Conidien freigesetzt werden können (VOBIS 1980, vgl. auch POELT 1986).

Eine exogene Steuerung der Fruchtkörperbildung besteht nicht nur bei Cladonia furcata ssp. furcata, wie auch Отт (1987) am Beispiel der epiphytischen Blattflechte Physcia tenella zeigt. Die im Rahmen meiner Arbeit erhobenen Daten zur Fruchtkörperbildung (Kap. E5) zeigen bei Peltigera rufescens ebenfalls eine Abhängigkeit von den Standortbedingungen. Als typische Art der Toninio-Psoretea decipientis bildet diese Blattflechte wohl aufgrund der relativen Trockenheit der entsprechenden Standorte im Untersuchungsgebiet keine Fruchtkörper aus, die im allgemeinen sonst bei ihr nicht selten sind.

Eine interessante Abhängigkeit von Mikroklima und Reproduktionsstrategie beobachtete MONTE (1993) an epilithischen Flechten: Mikroklimatische Bedingungen bestimmen hier die Reproduktionsstrategien der Arten. Die sexuelle Fortpflanzung durch Sporen tritt besonders häufig unter intermediären Mikroklima-Bedingungen auf. Die vegetative Reproduktion durch Soredien erreicht ein Maximum unter extremen Bedingungen wie minimale Lichtintensität und maximale Feuchtigkeit oder maximale Lichtintensität und minimale Feuchtigkeit.

\section{e) Der zeitliche Rahmen der Fruchtkörperbildung}

Die Fruchtkörperbildung stellt das Endstadium der Thallusentwicklung dar. Die Individuen sterben nach dem Fruchten ab, wie auch die von Cladonia pyxidata ssp. chlorophaea, Baeomyces rufus und Dibaeis baeomyces (JAHNS et al. 1978, JAHNS \& OTT 1982, JAHNS 1982). Die Gesamtlebensdauer von Cl. furcata ssp. furcata geben JAHNS et al. (1978) mit maximal 10 Jahren an. Voll entwickelte Cladonia-Thalli, einschließlich Stadien mit Fruchtkörpern, brauchen nach FINK (1917) eine Entwicklungszeit von bis zu 8 Jahren. Einigen anderen Autoren erscheint diese Zeitspanne allerdings zu lang (STOCKER-WÖRGÖTTER \& TÜRK 1993, JAHNS et al. 1978). Ähnliche Verhältnisse liegen auch bei den Pyknidien vor: Mit der Pyknidienbildung kommt das Wachstum des Thallus ebenfalls zum Stillstand (VoBIS 1980). 
Interessant ist daher die Frage, ob sich Cladonia furcata ssp. furcata, die offensichtlich eine Entwicklungszeit von mehreren Jahren braucht, in allen Gesellschaften lange genug entwickeln kann, um zur Fruchtreife zu gelangen. Intermediäre Stadien der Sukzession, z.B. die Cladonia bacillaris-Gesellschaft, sind häufig geprägt durch sorediöse, stiftförmige Arten. Die Bildung von Soredien, in denen Pilz- und Algenpartner gemeinsam verbreitet werden, ermöglicht die rasche Besiedlung neu entstandener, auch weiter entfernt liegender Standorte. Die eigenen Beobachtungen lassen darauf schließen, daß derartige Bestände aber oft schon nach wenigen Jahren von Phanerogamen oder Folgegesellschaften der Erdflechten verdrängt werden. Thalli von Cl. furcata ssp. furcata entstehen in der Natur nicht nur aus Ascosporen, die auf den geeigneten Algenpartner treffen, sondern auch durch Fragmente bereits vorhandener Thalli. Die Art hat eine sehr hohe und effektive Regenerationsfähigkeit aus Thallusfragmenten (STOCKER-WÖRGÖTTER \& TÜRK 1993). Allerdings sind Thallusfragmente gegenüber Soredien weniger gut für eine Ausbreitung über größere Distanzen geeignet (vgl. HEINKEN 1999). Cl. furcata kann sich auf derartigen Standorten also zum einen schlechter ansiedeln, zum anderen könnte die Entwicklung bis zur Apothecienbildung auf solchen, nur begrenzte Zeit zur Verfügung stehenden Standorten durch konkurrenzstärkere Organismen gestoppt werden. Dies würde die geringen Deckungsgrade und die geringe Ausdehnung der Lager von Cl. furcata ssp. furcata in der Cl. bacillaris-Gesellschaft erklären (s. Tab. 8 in der Einbandtasche).

\subsection{Gestaltveränderungen bei fortschreitender Podetienentwicklung}

Einen Einfluß der ,äußeren“ Faktoren auf die Gestalt der Lagerstiele vermutet SEMBDNER (1957/58) aufgrund der Tatsache, daß Risse und Löcher in den von ihm untersuchten Cladonien häufiger unter schattig-feuchteren Verhältnissen auftreten. Er erklärt dies damit, daß durch die langanhaltende Wassersättigung die Elastizität des Hyphenzylinders, dessen Struktur an einen mehr oder weniger raschen Wechsel von Quellung und Entquellung angepaßt scheint, zu stark beansprucht wird, so daß die Podetienwände allmählich erschlaffen und einreißen. Einen Einfluß der Standortfaktoren auf die Rissbildung bzw. das Aufklaffen der Podetien läßt sich anhand der vorliegenden Untersuchungen allerdings nicht bestätigen. Unter den lichten Bedingungen der Tieflagen-Heiden treten auffallend viele offen-rinnige Podetien auf, und dies wohl nur indirekt der Standortbedingungen wegen. Der Standort ermöglicht die Entwicklung der Podetien bis zur Fruchtkörperbildung, welche dort besonders oft zu beobachten ist (s.o.). Aufgrund dieses fortgeschrittenen Entwicklungsstadiums (also des relativ hohen Podetienalters) treten in diesem Standorttyp häufig rissige Podetien auf. Risse sind demnach als Alterserscheinung anzusehen.

Wie anhand des nordhessischen Materials gezeigt werden konnte, ist die Ausbildung der frühen Sexualstadien von Cladonia furcata ssp. furcata mit einer Änderung der äußeren Wuchsform verbunden, und zwar in der Weise, daß die Zahl der Verzweigungen deutlich zunimmt (s. auch JAHNS et al. 1978). Solche Veränderungen treten bei anderen Flechtenarten ebenfalls auf. Die Arbeiten von JAHNS \& SCHUSTER (1981) und JAHNS \& FREY (1982) beschäftigen sich beispielsweise mit demselben Phänomen bei der 
Strauchflechte Cetraria islandica und der Blattflechte Peltigera canina. Bei beiden Arten bewirkt die Entwicklung der Apothecien eine Förderung des Breitenwachstums bei gleichzeitiger Hemmung des Längenwachstums.

\subsection{Modifikatorische Varianten oder phänologische Stadien}

Bevor die Frage der Benennung der unterschiedlichen morphologischen Erscheinungsformen von Cladonia furcata ssp. furcata diskutiert wird, ist festzuhalten, daß sie alle derselben Art angehören. Bei den molekularbiologischen Untersuchungen (Kap. H) zeigten sich keine Unterschiede in den Mycobionten-Sequenzen, die für eine Einordnung als getrennte Arten sprechen würden. Geringe Sequenzunterschiede sind zwar vorhanden und führen zur Aufstellung verschiedener ITS-Varianten. Es besteht aber kein Zusammenhang zwischen ITS-Varianten und äußerer Gestalt. (Dagegen können, wie am Beispiel von Cl.gracilis und Cl. furcata gezeigt, genetisch deutlich unterschiedliche Flechtenarten bei entsprechenden Standortbedingungen ähnliche Gestalt annehmen).

Eine Einordung der zahlreichen morphologischen Erscheinungsformen in ein System, wie dies beispielsweise SANDSTEDE (1931) probiert hat, ist in jedem Falle fraglich, beachtet man die zahllosen Übergänge, die selbst SANDSTEDE (1931:199) eingestehen mußte: „man weiß wirklich nicht, wo die Grenzen liegen“ [zwischen der sonnenständigen v. palamaea und der heller gefärbten Schattenpflanze f. subulata]. Selbst innerhalb eines Polsters von Cladonia furcata können die Podetien unterschiedliche Gestalt aufweisen, die randlich stehenden beispielsweise anders geformt sein als die inneren und somit eigentlich unterschiedlichen Formen angehören (vgl. SANDSTEDE 1931). Wie vielfach ausgeführt und anhand der eigenen Verpflanzungsversuche belegt, können sich zudem die Formen ineinander umwandeln. Viele der bei SANDSTEDE und anderen Autoren als Varietäten oder Formen beschriebenen Morphotypen stellen lediglich besonders extreme oder leicht wiedererkennbare Erscheinungsformen dar.

GRUMMANN (1954) kritisiert in seinem Beitrag über eine einheitliche Benennung von Bildungsabweichung bei Flechten, daß einige Lichenologen dazu übergegangen waren, für die kurze Heraushebung einer Bildungsabweichung eine Kategorie der Nomenklatur $\mathrm{zu}$ verwenden (meist die Varietät oder Form). Er schlägt für die Benennung der Bildungsabweichungen den Begriff Terata (kurz ter.) vor und definiert ihn wie folgt: „Terata sind morphologisch-anatomische Wachstumserscheinungen, die nicht erblich sind und also nur das einzelne Individuum betreffen (...) und nicht zum normalen Entwicklungsgang gehören, und deren Merkmale nicht zu denen gehören, die das Taxon umschreiben, zu dem das Individuum gehört“ (GRUMMANN 1954: 61). In diesem Begriff sind pathologische und nicht pathologische Bildungsabweichungen enthalten. Die bei Cladonia furcata ssp. furcata untersuchten „Merkmalsausbildungen“ treten jedoch in GRUMMANNs Liste der Bildungsabweichungen nicht auf, sondern sind eher als Merkmale des Taxon zu sehen (z.B. Beschuppung, vgl. SchAdE 1966) bzw. Teil des normalen Entwicklungsganges (z.B. Apothecienbildung). Aus diesem Grunde sind zumindest für die meisten der Erscheinungsformen von Cl. furcata ssp. furcata die 
Begriffe „phänologisches Stadium“ und „modifikatorische Variante“ passender. Es konnte gezeigt werden, daß die Standortfaktoren für die Ausprägung der Gestalt ganz wesentlich verantwortlich sind und die Flechte sich in ihrer Gestalt den jeweiligen Standortbedingungen anpaßt. Häufig trifft daher auf die Gestalttypen der Begriff Modifikation zu, d.h. die Umwelteinflüsse bedingen eine Änderung des Phänotypus, die adaptiven Charakter besitzt (s. WAGENITZ 1996: 242). In geringerem Maße spielt auch der jeweilige Entwicklungszustand der Flechte eine Rolle. Bei jeder Erscheinungsform ist daher getrennt zu beurteilen, ob sie als modifikatorische Variante, phänologisches Stadium oder auch als „teras“ im Sinne GRUMMANNs anzusprechen ist. Oft wird eine eindeutige Zuordnung allerdings nicht möglich sein.

Fazit:

Cladonia furcata ssp. furcata ändert im Laufe ihrer Entwicklung deutlich ihre Gestalt und hat zudem die Fähigkeit sich erstaunlich gut an die jeweiligen Umwelteinflüsse anzupassen. Beides führt zu einer enormen Vielfalt an morphologischen Erscheinungsformen, welche je nach ihrer Ursache als modifikatorische Varianten oder phänologische Stadien betrachtet werden sollten.

\section{Zur Artberechtigung von Cladonia subrangiformis}

Cladonia subrangiformis Sandst. wird erstmals 1922 von SANDSTEDE als eigene Art beschrieben. Wie aus SANDSTEDE (1931: 231) hervorgeht, hat SCRIBA sie zwar zuerst erkannt, jedoch im Herbar als Cl. rangiformis v. hercynica bezeichnet. Der taxonomischen Wertung als eigener Art schließen sich in neuerer Zeit beispielsweise PURVIS et al. (1992) an. PURVIS et al. (1992: 208) beenden die Beschreibung von Cl. subrangiformis allerdings mit dem Hinweis: „Possibly a morph of Cl. furcata deformed by excessive production of calcium oxalate crystals." Andere Autoren halten diese Sippe, wie in Kap. G2.2 dargelegt, nicht für eine eigene Spezies, sondern werten sie lediglich als Unterart, Varietät oder Modifikation der Cl. furcata. Während in früheren Zeiten die wesentlichen Gesichtspunkte der Flechtensystematik die Gestalt, der Stoffwechsel, die Vermehrungsweise und die Flechtensäuren waren (s. TOBLER 1929, SEMBDNER 1957/58), gewinnen heute molekularbiologische Methoden, darunter auch die Sequenzierung der rDNA, zunehmend an Bedeutung.

In der vorliegenden Arbeit wurden Erkenntnisse über die Vergesellschaftung, die Morphologie und den Chemismus von Cl. furcata gewonnen sowie zusätzlich Informationen über die ITS rDNA-Sequenzen von Mycobionten und Photobionten beider Unterarten. Anhand dieser Ergebnisse soll die in den Arbeiten von UlLRICH (1956) und SCHADE (1966) geführte Diskussion um die Artberechtigung von Cl. subrangiformis wieder aufgenommen und unter neuen Gesichtspunkten fortgeführt werden.

\subsection{Morphologische und chemische Merkmale}

Kap. G3.1 ist zu entnehmen, daß beide Unterarten von Cladonia furcata im Untersuchungsgebiet morphologisch schlecht $\mathrm{zu}$ unterscheiden sind. Zwar tritt die 
Subspezies subrangiformis fast nie in Polsterform auf, jedoch gehören auch zur Cl. furcata ssp. furcata Exemplare, die als einzelne, unregelmäßig verzweigte Podetien dem Boden aufliegen. Nachfolgend soll anhand der eigenen Ergebnisse und anhand von Angaben aus der Literatur, der Wert der beiden die Subspezies subrangiformis kennzeichnenden Merkmale „Markaufbrüche“ und „Atranoringehalt“ diskutiert werden (vgl. Tab. 1, S. 17).

\section{a) Markaufbrüche}

Markaufbrüche gelten innerhalb der Sippe Cladonia furcata als charakteristisches Merkmal der Unterart subrangiformis bzw. je nach Einstufung des Autors als trennendes Merkmal von Cl. subrangiformis und Cl. furcata (s. z.B. SANDSTEDE 1922, UllRICH 1956, WIRTH 1995). Sie stellen eine feinkrümelige, mit Hyphen durchsetzte Masse von Calciumoxalatkristallen dar (UlLRICH 1956). BACHMANN (zitiert in SANDSTEDE 1922) deutete die Markwucherungen als Wasserspeicher, die bei den Flechten aufgrund sehr trockener Standortbedingungen aufträten. Dann müßten sie aber an trockenen Standorten auch an den Thalli der Cl. furcata ssp. furcata zu sehen sein, das ist jedoch nur ausnahmsweise der Fall (vgl. Kap. G3.1). UlLRICH (1956) schreibt dagegen über die Ursache dieser Wucherungen, daß durch die übermäßige Aufnahme von Calciumsalzen bei der acidophilen Cl. furcata offensichtlich eine starke Exkretion von Calciumoxalat stattfindet, die zu so starken Anhäufungen im Podetium führen kann, daß schließlich die Rinde aufreißt und das Mark mit dem gespeicherten Oxalat nach außen tritt.

Markaufbrüche sind also die Folgen eines physiologischen Prozesses und kommen, wie sich auch anhand der eigenen Proben bestätigen ließ, nur an den untersten Podetienteilen vor, die in engem Kontakt mit der Unterlage stehen. Der Satz SANDSTEDES (1931:232) „Sie liebt Kalkboden“ trifft damit wohl nicht zu. Die Flechte kann auf solchen Substraten gedeihen, aber nicht weil, sondern obwohl diese aus Kalk bestehen, da sie diesen unschädlich macht, wie SCHADE (1966) bemerkt. PINTARIC \& TÜRK (1995) stellten fest, daß Cl. furcata ssp. furcata je nach besiedeltem Substrat sehr stark variierende Ca-Gehalte im Thallus aufweist. Die Autoren meinen, die große Differenz sei nicht nur durch morphologische, sondern durch physiologische Besonderheiten erklärbar. Die Flechte kann $\mathrm{Ca}$ als Oxalat binden und daher als physiologisch adaptierte Form betrachtet werden. Sie ist aber nicht als calciphil zu bezeichnen, da sie das $\mathrm{Ca}$ nicht braucht, um etwa überschüssige Oxalat-Anionen zu binden, sondern es auf diesem Wege vom zellbiologischen Geschehen fern hält.

Calciumoxalat-Exkrete wurden auch bei anderen, auf kalkhaltiger Unterlage wachsenden Flechten beobachtet: z.B. bei Cladonia rangiformis (z.B. MATTICK 1932, SANDSTEde 1931, Schade 1966), Cl. pyxidata [ssp.] pocillum (MATTICK 1932, SCHADE 1966), Cl. symphicarpia (Syn. Cl. symphycarpa) (SCHADE 1966), Cl. foliacea (SCHADE 1966), Cetraria islandica (SCHADE 1966), Cornicularia (Syn. Cetraria) aculeata (SCHAdE 1966) sowie bei Fulgensia-Arten (MATtick 1932, Schade 1966). SANDSTEdE (1931) beschreibt darüber hinaus eine Cl. gracilis ssp. elongata (ohne Fundortangaben), eine Cl. crispata f. punctata (ebenfalls ohne Fundortangaben) und eine Cl. furcata var. 
palamaea (von einer Kalksteinheide in Westergotland), die alle Markaufbrüche wie Cl. subrangiformis aufweisen. Letzteres deckt sich wiederum mit der in Kap. G3.1 beschriebenen Beobachtung, wonach im Rahmen der vorliegenden Untersuchung ebenfalls eine der palamaea entsprechende Probe von Cl. furcata ssp. furcata mit weißen Markaufbrüchen entdeckt wurde (s. Taf. 2.12 im Anhang 2). Bereits DES ABBAYES (1937) hat ausgeführt, daß sich Cl. subrangiformis und Cl. furcata var. palamaea nahestehen. LETTAU (1955:39) meint jedoch im Widerspruch zu SANDSTEDE und zu meinen eigenen Ergebnissen, die Cl. subrangiformis sei u.a. aufgrund dieser charakteristischen Markaufbrüche „oft von Cl. furcata var. palamaea usw. gut zu unterscheiden“. WIRTH (1995: 296) bezeichnet die „weiß aufbrechenden Beulen“ als häufig, also nicht stets vorhanden. HILLMANN \& GRUMMANN (1957) schreiben, Cl.furcata habe keine, Cl.subrangiformis oft Markwucherungen, diese Anschwellungen der Rinde platzten jedoch nur ab und $\mathrm{zu}$ auf, wodurch dann die weißliche Markschicht zutage träte (vgl. auch SANDSTEDE 1922).

Von den untersuchten nordhessischen subrangiformis-Proben wiesen nur rund die Hälfte derartige Bildungen auf. Für Nordhessen bleibt daher festzuhalten: Markaufbrüche sind ein morphologisches Merkmal, daß bei der Unterart subrangiformis auftreten kann, ausnahmsweise jedoch auch bei Cl. furcata ssp. furcata vorkommt. PAUS (1997) hat im Übrigen an dem von ihr untersuchten nordwestdeutschen Material der ssp. subrangiformis keine Markaufbrüche festgestellt.

\section{b) Atranoringehalt}

In der vorliegenden Untersuchung wurden in Anlehnung an WIRTH (1995) die Atranorin-haltigen Thalli als Subspezies subrangiformis eingestuft. Alle derartigen Proben entstammen Gebieten mit basenreichem Ausgangsgestein. In Nordhessen findet sich in Cladonia furcata ssp. furcata von Standorten mit mehr oder weniger saurem Ausgangsgestein nur Fumarprotocetrarsäure; es tritt also keiner der unten bzw. in Kap. F2 erwähnten Chemotypen auf.

Der Abtrennung der Atranorin-haltigen Sippen als Cladonia subrangiformis (bzw. Cl. furcata ssp. subrangiformis) steht eine Beobachtung entgegen, die schon DES AbBayes (1937) und Hillmann \& Grummann (1957) machten. Demnach kann Atranorin auch in Cl. furcata [ssp. furcata] auftreten. HUOVINEN et al. (1990) bestätigen dies. Darüber hinaus sind weitere Flechtensäuren aus Cl. furcata ssp. furcata bekannt. BARENDREGT et al. (1982) publizierten Funde eines neu entdeckten Chemotyps von Cl. furcata var. furcata (= ssp. furcata), der Psoromsäure anstelle von Fumarprotocetrarsäure oder Atranorin enthält. PAUS (1997) wies in Nordwestdeutschland einen Cl.furcata-Chemotyp mit Psoromsäure und Atranorin nach, ohne ihn einer der beiden Unterarten zuzuordnen. In Trockenrasen des MittelelbeGebietes tritt neben den Chemotypen mit Atranorin und Psoromsäure bzw. nur mit Psoromsäure noch ein weiterer Chemotyp mit Psoromsäure und Fumarprotocetrarsäure auf (FISCHER \& GÜNZL 2004).

HAWKSWORTH (1976) schlägt vor, Exemplare lediglich als Varietäten innerhalb einer Art einzustufen, sofern eine oder mehrere Substanzen auftreten, die keine andere(n) 
Substanzen ersetzen, und dieses Auftreten korreliert ist mit Unterschieden in der ökologischen Amplitude bzw. mit Unterschieden in der lokalen Verbreitung. Auf den zuletzt genannten Punkt wird an späterer Stelle eingegangen.

\section{c) Korrelation der morphologischen und chemischen Merkmale?}

Die Atranorin-haltigen Thalli von Cladonia furcata zeigen nicht immer, jedoch wesentlich häufiger Markaufbrüche als die Thalli ohne Atranorin. Beide Merkmale korrelieren daher schwach miteinander. $\mathrm{Ob}$ der Atranoringehalt eine Folge der Standortbedingungen ist, so wie die Markaufbrüche, bleibt unklar. Da im Untersuchungsgebiet neben den Fumarprotocetrarsäure-haltigen Sippen von Cl. furcata ssp. furcata keine weiteren Chemotypen eindeutig nachzuweisen waren, konnten bei dieser Unterart keine Zusammenhänge zwischen den morphologischen Merkmalen und dem Chemismus untersucht werden. Bislang sind bei Cl. furcata ssp. furcata keine die jeweiligen Chemotypen kennzeichnenden morphologischen Merkmale bekannt (vgl. BARENDREGT et al. 1982, PAUS 1997).

\section{$2.2 \quad$ Vergesellschaftung}

Häufig werden als Argument für die Unterteilung von Cladonia furcata in Unterarten deren unterschiedliche Standortpräferenzen und Vergesellschaftung angeführt (vgl. PIŠUT 1961, PAUS 1997). In einigen Gegenden, so auch in Nordhessen, ist dies tatsächlich der Fall. Wie in Kap. E2 dargestellt, ist Cl. furcata ssp. subrangiformis Federationskennart des Toninion sedifoliae und tritt nie außerhalb dieser Federation auf. Die Einstufung als Federationskennart erfolgt in Anlehnung an WIRTH (1995). Cl. furcata ssp. furcata hat ihren Verbreitungsschwerpunkt in Nordhessen im Cladonion arbusculae, greift jedoch in Gesellschaften des Baeomycion rosei und des Cladonion rei über (vgl. auch PAUs 1997). Nur selten kommt sie darüber hinaus auch in Beständen des Toninion sedifoliae vor. Die Vergesellschaftung beider Subspezies überschneidet sich also im Untersuchungsgebiet nur marginal. SCHADE (1966) bemerkt hierzu, daß auch die ,normale“ Cl. furcata in Kalkgebieten dort aufzutreten vermag, wo schwacher Kalkgehalt bereits verlorengegangen ist oder dicke Humusschichten sie von der Gesteinsunterlage isolieren oder sich eine Insel sauren Gesteins eingeschaltet hat, so daß sie an diesen Stellen keine Sekrete bilden muß.

In dem von FiSCHER (2003) untersuchten Mittelelbe-Gebiet treten beide Unterarten gemeinsam in Koelerio-Corynephoretea-Beständen auf. Das soziologische Kriterium ist somit zumindest fragwürdig.

\subsection{ITS rDNA-Sequenzen der Mycobionten}

Anhand der ITS rDNA-Sequenzen lassen sich die beiden Unterarten von Cladonia furcata nicht voneinander unterscheiden, während im Vergleich hierzu, Cl. furcata und Cl. gracilis deutliche Sequenzunterschiede zeigen. Von Cl. furcata ssp. subrangiformis liegt bislang gar keine, von $\mathrm{Cl}$. furcata ssp. furcata lediglich eine publizierte und über GenBank zugängliche Sequenz vor. Die Ergebnisse meiner Untersuchungen stellen 
daher vorerst den einzigen Beleg für übereinstimmende Sequenzen beider Unterarten dar und sprechen gegen eine Einstufung als getrennte Arten.

\section{4 „subrangiformis“ - eine edaphisch bedingte Modifikation innerhalb der Cladonia furcata?}

Anhand der obigen Ausführungen wird deutlich: Markaufbrüche sind nicht auf die Sippe subrangiformis beschränkt, bei ihr aber deutlich häufiger als bei Cladonia furcata ssp. furcata. Als Unterscheidungsmerkmal sind sie daher nur eingeschränkt zu gebrauchen. In anderen Gebieten treten sie gar nicht auf (vgl. PAUS 1997) und fallen somit als Kennzeichen der ssp. subrangiformis weg. Sie stellen eine ökologische Erscheinung bei Exemplaren kalkhaltiger Substrate dar. PIŠUT (1961) betrachtet die Markaufbrüche ebenfalls als wertlos für die Taxonomie und meint der einzig verläßliche Unterschied zu Cl. furcata ssp. furcata sei das Vorkommen von Atranorin sowie die deutlich veränderliche Verbreitung und spricht sich für eine Subspezies subrangiformis aus. Da jedoch auch der Atranoringehalt, wie oben ausgeführt, kein auf die Sippe subrangiformis beschränktes Merkmal darstellt, würde demnach nur die unterschiedliche Verbreitung für eine Einstufung als Unterart sprechen. Auch dieses Kriterium trifft jedoch, wie gezeigt, nicht überall zu. PAUS (1997) kommt trotzdem aufgrund ihrer Untersuchungen zu dem Schluß, die Sippen furcata und subrangiformis als eigenständige Taxa im Rang einer Subspezies zu belassen, obwohl morphologische Merkmale nicht zur Differenzierung beitragen. Entscheidend sei die (in Nordwestdeutschland) unterschiedliche Einbindung der chemisch definierten Sippen in Vegetationseinheiten. Daneben beständen offensichtlich auch chorologische Unterschiede. Anderer Meinung ist dagegen Des ABBAYES (1937). Er erkannte, daß zwischen Cl. subrangiformis und Cl. furcata eine ganze Reihe von Zwischenformen in morphologischer und chemischer Hinsicht bestehen und akzeptiert subrangiformis auch nicht als gute Varietät von Cl. furcata sondern nennt sie einen ,écotype“. Auch SCHADE (1966) spricht sich gegen eine Einstufung als Subspezies aus. Er schlägt eine Benennung als m. subrangiformis vor.

Anhand eigener Ergebnisse und den Angaben aus der Literatur, wird die These aufgestellt, daß es sich bei der Sippe subrangiformis nicht um eine eigene Art handelt, sondern sie statt dessen in Cladonia furcata zu integrieren ist. Ein Argument ist hierbei das Resultat der ITS rDNA-Analysen, wonach bei den Mycobionten der Sippen subrangiformis und furcata keine Unterschiede in den Sequenzen bestehen. Aufgrund des relativ häufigen Auftretens von Markaufbrüchen als Reaktion auf ein vermehrtes Ca-Angebot, kann sie als edaphisch bedingte Modifikation der Cl. furcata angesehen werden. Um dies zu bestätigen wären Verpflanzungsversuche von Interesse, bei denen Thalli von $C l$. furcata ssp. furcata (ohne Markaufbrüche) auf Kalkstandorte verpflanzt würden und umgekehrt solche von $\mathrm{Cl}$. furcata ssp. subrangiformis auf basenarmes Substrat. 


\section{J Gesamtzusammenfassung}

Es werden für Nordhessen 16 Erdflechtengesellschaften sowie 4 Dominanzbestände aus zwei Vegetationsklassen belegt. Die Gesellschaften der Klasse Toninio-Psoretea decipientis, $\mathrm{zu}$ denen auch die Bunte-Erdflechten-Gesellschaft (Toninio-Psoretum decipientis) zählt, sind häufig durch Krustenflechten gekennzeichnet und gedeihen auf basenreichen Substraten. Sie siedeln v.a. innerhalb von lückigen Kalkmagerrasen (Gentiano-Koelerietum), auf Felsköpfen und in Sekundärbiotopen wie z.B. aufgelassenen Gips-Steinbrüchen. Die Erdflechtenvegetation kalkarmer bzw. kalkfreier Standorte gehört der Klasse Ceratodonto-Polytrichetea piliferi an, welche sich in 3 Federationen unterteilen läßt. Die Federation Baeomycion rosei umfaßt Gesellschaften mit Pioniercharakter. Dem Cladonion arbusculae zugehörig sind sowohl intermediäre Entwicklungsstadien, die von stiftförmigen, sorediösen Cladonien geprägt werden als auch Strauchflechten-Bestände wie die Rentierflechten-Teppiche in Heiden. Gesellschaften der Federation Cladonion rei sind bevorzugt auf Ruderalstandorten wie z.B. Bergbauflächen zu finden, auch sie werden von stiftförmigen, sorediösen Arten dominiert. Ein Großteil der im Untersuchungsgebiet auftretenden Erdflechtengesellschaften ist als ziemlich bis sehr selten und daher als schützenswert einzustufen. Geeignete Schutzmaßnahmen werden angegeben.

Die Vergesellschaftung von Cladonia furcata wird detailliert dargestellt. Die Unterart furcata zeigt ihren Verbreitungsschwerpunkt im Cladonion arbusculae, greift jedoch auch in Gesellschaften der anderen Federationen über. Nur gelegentlich tritt sie in Beständen der Klasse Toninio-Psoretea decipientis auf. Nicht in allen Gesellschaften werden jedoch fertile Podetien ausgebildet. Eingehende Untersuchungen der morphologischen Merkmale und ihrer Abhängigkeiten von Standortfaktoren zeigen, daß die Apothecienbildung bei $\mathrm{Cl}$. furcata ssp. furcata von den Lichtverhältnissen beeinflußt wird. Auch die meisten anderen Merkmale werden von den Bedingungen am Standort geprägt, wobei allgemein besonders den Licht- und Feuchtigkeitsverhältnissen, aber auch den mechanischen Störungen große Bedeutung zukommt. In unterschiedlichem Maße hat auch der Entwicklungszustand der Flechte einen Einfluß auf die äußere Gestalt. So wird beispielsweise die Zahl der Zweige am Podetienende von der Fruchtkörperbildung beeinflußt. Die Ursachen für die unterschiedliche Ausprägung der einzelnen Merkmale werden aufgelistet und diskutiert.

Cladonia furcata ssp. subrangiformis bleibt in ihrem Auftreten auf die Klasse ToninioPsoretea beschränkt. Sie bildet gelegentlich Dominanzbestände. Auch ihre Morphologie wird anhand einiger stichprobenhaft ausgemessener Podetien skizziert.

Die Zuordnung der Cladonia furcata-Proben zu den Unterarten erfolgte anhand des Auftretens bzw. Fehlens von Atranorin. Neben Fumarprotocetrarsäure tritt im Untersuchungsgebiet in den Thalli von Cl. furcata ssp. furcata kein weiterer, mittels Dünnschichtchromatographie eindeutig nachweisbarer Stoff auf. Außer Atranorin und Fumarprotocetrarsäure konnte in der Unterart subrangiformis ebenfalls nur 
ausnahmsweise ein weiterer Inhaltsstoff nachgewiesen, jedoch nicht identifiziert werden.

Die Sequenzierung der ITS rDNA der Bionten beider Unterarten von Cladonia furcata zeigt, daß bei den Mycobionten verschiedene ITS-Varianten auftreten, diese jedoch offensichtlich von der Subspezies unabhängig sind. Ein Zusammenhang zwischen Morphologie und ITS-Varianten ist ebenfalls nicht erkennbar. Dagegen zeigen Mycobionten-Sequenzen von Cl. gracilis, trotz hoher morphologischer Ähnlichkeit der untersuchten Proben beider Arten, deutliche Unterschiede zu Cl. furcata. ITS-Varianten treten auch bei den Photobionten auf, welche anhand der Sequenzen der Gattung Asterochloris zugeordnet werden. Beide Unterarten von $\mathrm{Cl}$. furcata können Photobionten derselben ITS-Variante als Partner nutzen.

Anhand der Ergebnisse der vegetationskundlichen und morphologischen Untersuchungen sowie der Dünnschichtchromatographie und der rDNA-Sequenzierung wird die systematische Unterteilung von Cladonia furcata sowie die Artberechtigung von Cl. subrangiformis eingehend diskutiert. Es wird vorgeschlagen, sowohl die morphologischen Erscheinungsformen von Cl. furcata ssp. furcata als auch die ssp. subrangiformis als modifikatorische Varianten bzw. phänologische Stadien zu bezeichnen. 


\section{K Summary}

\section{Terricolous lichens and their communities in North Hessen (Germany) with special emphasis on the morphological and genetical variability of Cladonia furcata (Hudson) Schrader}

Sixteen terricolous lichen communities assignable to two vegetation classes are described from the northern part of the German state of Hessen. The class ToninioPsoretea decipientis, including the association Toninio-Psoretum decipientis, is characterized by crustose species and grows on base-rich soils. Communities of this class colonize soils over gypsum, dolomite and limestone and can mostly be found in oligotrophic grasslands (Gentiano-Koelerietum) or abandoned quarries. All these communities at present occur only rarely in this area. They need open sites with poorly developed vegetation. In the course of succession, the lichens are displaced by phanerogames.

The lichen vegetation on more or less acid soils, deficient in lime, belongs to the class Ceratodonto-Polytrichetea piliferi. Three alliances of this class can be differentiated. The alliance Baeomycion rosei includes pioneer communities. Some communities of the second alliance Cladonion arbusculae are dominated by subulate Cladonias with soredia. This type of vegetation represents successional stages of intermediate character. Communities of reindeer lichens (e.g. Cladonia arbuscula) also belong to this alliance. They are terminal stages of the lichen succession. The alliance Cladonion rei is typical for ruderal sites, for example mining areas. Its communities are also dominated by subulate and sorediate Cladonias. In the course of succession, phanerogames will displace the lichens on these sites.

Most of the lichen communities in North Hessen at present occur very rarely in this area. The same holds true for the whole of Germany. This justifies the necessity to afford special environmental protection to terricolous lichen vegetation. Suitable protective measures are specified.

The most important genus in terricolous lichen vegetation is the genus Cladonia. Another part of this study deals with the variability of the common species Cladonia furcata. The subspecies furcata mainly occurs in the Cladonion arbusculae, but can also be found in communities of the other alliances in this class. Very seldom the subspecies furcata grows in communities of the class Toninio-Psoretea decipientis as well. However, Cl. furcata ssp. furcata does not develop fertile podetias in all communities. Detailed investigations of the morphological characters point out that the development of apothecia is influenced by light. Most of the morphological characters show a dependence on light and moisture. But mechanical influences are also important. There are also internal factors which affect the morphology of this lichen.

The number of branches at the top of the podetia belongs to the presence or absence of apothecia, for example. The reasons for the variability of all the morphological characters are described and discussed in this study. 
Cladonia furcata ssp. subrangiformis can be found only in lichen communities of the class Toninio-Psoretea decipientis. Sometimes this lichen can be the dominant species in the lichen vegetation. The morphology of this subspecies in North Hessen is also described.

One reason for the division of Cladonia furcata into two subspecies is the presence or absence of atranorin. Usually, in addition to fumarprotocetraric acid there is no other acid in the analyzed thalli of $\mathrm{Cl}$. furcata ssp. furcata which could be demonstrated using thin-layer chromatography. The investigated thalli of the subspecies subrangiformis contain atranorin and fumarprotocetraric acid. As an exception, there is also another substance in the thalli of both subspecies. This substance could however not be identified using thin-layer chromatography.

The last part of this study is concerned with the sequencing of ITS rDNA. Both bionts (photobionts and mycobionts) of Cladonia furcata and Cl. gracilis were analysed. There are different ITS-variants of the photobiont and mycobiont. These ITS-variants do not depend on the two subspecies of Cl. furcata. The mycobiont ITS rDNA-sequences of the subspecies furcata and the subspecies subrangiformis are identical. A correlation between morphology and ITS-variants could not be found. The sequences of Cl. furcata and $\mathrm{Cl}$. gracilis show clear differences, despite the fact that the analyzed thalli are very similar in morphology.

The photobionts belong to the genus Asterochloris. Both subspecies of Cladonia furcata can live in symbiosis with photobionts of the same ITS-variant.

The question whether Cladonia subrangiformis might best be classified as a subspecies of $C l$. furcata or as a species for its own is discussed on the basis of morphological and chemical datas as well as on the basis of datas of phytosociology and molecular biology (sequences of ITS rDNA). The division of Cl. furcata in varieties and forms is also discussed. It is proposed, that the morphs of Cl. furcata ssp. furcata as well as the subspecies subrangiformis should be classified as modificatory variants and phenological stages respectively. 


\section{Literaturverzeichnis}

AHMAdJIAN, V. \& JAKOBS, J.B. (1981): Relationship between fungus and alga in the lichen Cladonia cristatella Tuck. - Nature 289: 169-172.

AHTI, T. (1962): Notes on the lichen Cladonia pseudorangiformis Asah. - Arch. Soc. Zool. Bot. Fenn. Vanamo 17(1): 38-41.

Ahti, T. (1966): Correlation of the chemical and morphological characters in Cladonia chlorophaea and allied lichens. - Ann. Bot. Fenn. 3: 380-390.

AHTI, T. (1993): Names in current use in the Cladoniaceae (lichen-forming Ascomycetes) in the ranks of genus to variety. - In: GREUTER, W. (ed.): NCU-2. Names in Current Use in the Families Trichocomaceae, Cladoniaceae, Pinaceae and Lemnaceae. Regnum Vegetabile 128: 58-106. Koeltz Scientific Books. Königstein.

AHTI, T. (2000): Cladoniaceae. - Flora Neotropica Monogr. 78: 1-362.

Ahti, T. \& LAI, M.-J. (1979): The lichen genera Cladonia, Cladina and Cladia in Taiwan. Ann. Bot. Fenn. 16: 228-236.

ANDERS, J. (1928): Die Strauch- und Laubflechten Mitteleuropas. I-IV. - Jena. 217 S.

ANONYMUS (1994): Naturschutzgebiete im Regierungsbezirk Kassel. - Naturschutz Nordhessen 14: $73-81$.

ANONYMUS (2001): Exkursionsbericht Gladenbacher Bergland 6.-8.4.2001. - Aktuelle Lichenol. Mitt. N.F. 6: 9-17.

ARCHER, A.W. (1992): Cladoniaceae. - In: Flora Australia 54: 107-143. Australian Government Publishing Service Press. Canberra.

ARUP, U. \& GRUBE, M. (1998): Molecular systematics of Lecanora subgenus Placodium. Lichenologist 30(4-5): 415-425.

AUgE, K. (2000): Auswirkungen von Kiefernanflugwäldern auf Kalkmagerrasen im Unteren Werraland. - Unveröff. Diplomarb. Univ. Göttingen. 100 S.

Baldwin, B.G., SANDerson, M.J., Porter, J.M., Wojciechowski, M.F., CAMPBell, C.S. \& DONOGHUE, M.J. (1995): The ITS region of nuclear ribosomal DNA: a valuable source of evidence an angiosperm phylogeny. - Ann. Missouri Bot. Gard. 82: 247-277.

BARENDREgT, A., VAN DEN DRIES, P.L.V. \& SipMAN, H.J.M. (1982): A new chemical strain of Cladonia furcata (Huds.) Schrad. (Lichenes). - Acta Bot. Neerl. 31: 491-494.

BARKMAN, J.J. (1958): Phytosociology and ecology of cryptogamic epiphytes. - Van Gorkum, Hak, Brakke. Assen. $628 \mathrm{~S}$.

BARKMAN, J.J. (1968): Das synsystematische Problem der Mikrogesellschaften innerhalb der Biozönosen. - In: TÜXEN, R. (ed.): Pflanzensoziologische Systematik. Ber. Int. Symp. IVV Stolzenau 1964: 21-53. Junk. Den Haag. 
BARKMAN, J.J. (1973): Synusial approaches to classification. - In: WHITTAKER, R.H. (ed.): Ordination and classification of vegetation. Handbook Veg. Sci. 5: 435-491. Junk. The Hague.

BÄRLOCHER, F. (1999): Biostatistik - Praktische Einführung in Konzepte und Methoden. Thieme. Stuttgart, New York. 206 S.

BAUFELD, R. (1991): Laubwald-Gesellschaften im Unteren Werraland. - Unveröff. Diplomarb. Univ. Göttingen. $120 \mathrm{~S}$.

BAUMANN, K. (2000): Vegetation und Ökologie der Kleinseggenriede des Harzes: Wissenschaftliche Grundlagen und Anwendungen im Naturschutz. - Cuvillier-Verlag. Göttingen. $219 \mathrm{~S}$.

BECK, A. (2002): Selektivität der Symbionten schwermetalltoleranter Flechten. - Diss. Univ. München. 194 S.

Beck, A., Friedl, T. \& Rambold, G. (1998): Selectivity of photobiont choice in a defined lichen community: inferences from cultural and molecular studies. - New Phytol. 139: 709-720.

BERGMEIER, E. (1987): Magerrasen und Therophytenfluren im NSG „Wacholderheiden bei Niederlemp“(Lahn-Dill-Kreis, Hessen). - Tuexenia 7: 267-293.

Bergmeier, E., Härdtle, W., Mierwald, U., NowaK, B. \& Peppler, C. (1990): Vorschläge zur syntaxonomischen Arbeitsweise in der Pflanzensoziologie. - Kieler Not. Pflanzenk. Schleswig-Holst. Hamburg 20(4): 92-103.

BERND, R. (1998): Flora und Vegetation der Gudensberger Basaltkuppen (Nordhessen). Unveröff. Diplomarb. Univ. Göttingen.155 S.

BEUG, H.-J. (1963-1989): Moos- und Flechtenexkursionen zum Meißner und seinem Vorland. Unveröff. Manuskripte. Univ. Göttingen.

BEYER, H. (1968): Versuche zur Erhaltung von Heideflächen durch Heidschnucken im Naturschutzgebiet „Heiliges Meer“. - Natur \& Heimat 28(4): 145-149.

Biermann, R., Breder, Ch., Daniels, F., Kniffe, K. \& Paus, S. (1994): Heideflächen im Raum Munster, Lüneburger Heide: Eine floristisch-pflanzensoziologische Erfassung als Grundlage für Pflege- und Optimierungsmaßnahmen. - Ber. Naturhist. Ges. Hannover 135: 105-161.

BöHLE, U.-R. \& HaLfmanN, J. (1992): Die Vegetation der Halbtrockenrasen im südlichen Ringgau (Nordhessen) im Hinblick auf ihre Schutzwürdigkeit sowie Anregungen zu ihrer Erhaltung und Pflege. - Naturschutz Nordhessen 12: 81-104.

Bonnemann, A. (1984): Der Reinhardswald. - Weser-Buchhandlung. Hann. Münden. 451 S.

BorNKAMM, R. (1958): Die Bunte-Erdflechten-Gesellschaft im südwestlichen Harzvorland. Ber. Deutsch. Bot. Ges. 71(7): 253-270.

Bortz, J., Lienert, G. \& BoeHnKe, K. (1990): Verteilungsfreie Methoden in der Biostatistik. Springer. Berlin, Heidelberg, New York. 939 S. 
Braun-Blanquet, J. (1964): Pflanzensoziologie. Grundzüge der Vegetationskunde. 3., neu bearb. Aufl. - Springer. Berlin, Wien, New York. 865 S.

BritzelmaYR, M. (1904): Cladonia furcata Huds. und squamosa L. im Gebiet der süddeutschen Hochebene von 450-600 m über der Nordsee. - Hedwigia 43: 126-131.

BRosius, M. (1959): Die Tertiärmulde von Glimmerode. - Hess. Lagerstättenarchiv 4: 1-48.

BRÜCKNER, A.F. (1803): Florae Neobrandenburgensis Prodronum. - Jena.

BRUELHeIDE, H. (1989): Die Vegetation der Kalkmagerrasen im östlichen und westlichen Meißner-Vorland. - Unveröff. Diplomarb. Univ. Göttingen. 174 S.

BRUELHEIDE, H. (1991): Kalkmagerrasen im östlichen und westlichen Meißner-Vorland. Tuexenia 11: 205-233.

BRUELHEIDE, H. (1992): Kartierung der Kalkmagerrasen im östlichen und westlichen MeißnerVorland. - Bot. Naturschutz Hessen, Beih. 4: 85-100.

BRÜHL, H. \& HEGER, B. (1989): Zur Hydrogeologie des Hohen Meißners. - Berliner Geowiss. Abh. (A) 114: 77-90.

BRUN-HOOL, J. (1966): Ackerunkraut-Fragmentgesellschaften. - In: TÜXEN, R. (ed.): Anthropogene Vegetation. Ber. Internat. Sympos. IVV Stolzenau 1961: 38-50. Junk. Den Haag.

BultmanN, M. (1993): Flora und Vegetation der Kalkmagerrasen an der unteren Diemel. Philippia VI/4: 331-380.

Burgaz, A.R. \& AhTI, T. (1992): Contribution to the study of the genera Cladina and Cladonia in Spain. I. - Nova Hedwigia 55(1-2): 37-53.

CANTERS, K.J. (1988): Ökologische Untersuchungen in einem kleinräumig gegliederten Waldgebiet. - Diss. Univ. Frankfurt/M. 285 S.

CERnohorsky, Z. (1950): Cladonia subrangiformis Scriba v Cechach. [C.s. new to Bohemia.]. - Ces. Bot. Listy 2: 107-108.

Christensen, S.N. (1987): Morphological and chemical variation in the Cladonia macilenta/bacillaris aggregate in Denmark. - Lichenologist 19: 61-69.

COPPINS, B.J. (2002): Checklist of Lichens of Great Britain and Ireland London. - British Lichen Society. London. $95 \mathrm{~S}$.

Culberson, C.F. (1969): Chemical and Botanical Guide to Lichen Products. - Chapel Hill, N.C. University of North Carolina Press. 628 S.

Culberson, C.F. \& AmmanN, K. (1979): Standardmethode zur Dünnschichtchromatographie von Flechtensubstanzen. - Herzogia 5: 1-24.

CUlberson, C.F. \& Kristinsson, H. (1970): A standardized method for the identification of lichen products. - Jour. Chromatogr. 46: 85-93. 
DANiels, F.J.A., BIERMANN, R. \& BREDER, C. (1993): Über Kryptogamen-Synusien in Vegetationskomplexen binnenländischer Heidelandschaften. - Ber. Reinhold-TüxenGes. 5. 199-219.

DANiEls, F.J.A., SloOF, F.J.E. \& VAN DE WeTERING, H.T.J. (1987): Veränderungen in der Vegetation der Binnendünen in den Niederlanden. - In: SCHUBERT, R. \& HILBIG, W. (eds.): Erfassung und Bewertung anthropogener Vegetationsveränderungen. Martin Luther Univ. Halle Wittemberg. Wiss. Beitrag Halle/Saale 1987/46 (P31): 24-44.

Degelius, G. (1939): Die Flechten von Norra Skaftön. Ein Beitrag zur Kenntnis der Flechtenflora und Flechtenvegetation im äusseren Teil der schwedischen Westküste. Uppsala Universitets Arsskrift 11: 1-206.

DEPRIEST, P.T. (1993): Variation in the Cladonia chlorophaea complex I: morphological and chemical variation in Southern Appalachian populations. - Bryologist 96(4): 555-563.

DES ABBAYES, H. (1937): Contributions nouvelles à la connaissance des lichens armoricains III. Cladonia subrangiformis Sandst. emend. H. des Abb. - Bull. Soc. Sci. Bretagne 14: 154-164.

DEUTSCHER WETTERDIENST (2003a): Klimadaten ausgewählter deutscher Stationen. Abruf der Monatswerte. - URL: http://www.dwd.de/de/FundE/Klima/KLIS/daten/online/nat/ ausgabe_monatswerte.htm.

DEUTSCHER WETTERDIENST (2003b): Der Rekordsommer 2003. - URL: http://www.dwd.de/ de/FundE/Klima/KLIS/prod/spezial/temp/Rekordsommer_2003.pdf.

DeUtSChER WeTtERDIENST IN DER US-Zone (ed.) (1950): Klima-Atlas von Hessen. Selbstverlag. Bad Kissingen. 20 S. Erläuterungen, 75 Karten, 9 Diagramme.

DiEDERICH, G. (1991): Hydrogeologisches Kartenwerk Hessen 1: 300 000. - Geologische Abhandlungen Hessen 95. Wiesbaden. 83 S. +5 Karten.

DierschKe, H. (1994): Pflanzensoziologie: Grundlagen und Methoden. - Ulmer. Stuttgart (Hohenheim). $683 \mathrm{~S}$.

DietZ, S., BÜDEL, B., LANGe, O.L. \& BILGER, W. (2000): Transmittance of light through the cortex of lichens from contrasting habitats. - Biblioth. Lichenol. 75: 171-182.

DoIgDE, E.M. (1950): The lichens of South Africa to the end of 1945. - Bothalia 5: 1-1094.

DoLL, R. (1996): Lichenologische Beiträge aus Nordostdeutschland I. - Gleditschia 24(1/2): 179-188.

DrehWald, U. (1993): Die Pflanzengesellschaften Niedersachsens - Bestandsentwicklung, Gefährdung, Schutzprobleme - Flechtengesellschaften. - Naturschutz Landschaftspfl. Niedersachs. 20/10: 1-122.

EGEA, J.M. (1996): Catalogue of lichenized and lichenicolous fungi of Morocco. - Bocconea 6: $19-114$.

EhrENDORFER, F. (1973): Liste der Gefäßpflanzen Mitteleuropas. 2. Aufl. - Gustav Fischer. Stuttgart. $318 \mathrm{~S}$. 
EIGNER, J. (1991): Hochmoor und Heide. - In: MuES, D. (ed.): Biotoppflege, Biotopentwicklung/FLL Forschungsgesellschaft Landschaftsentwicklung Landschaftsbau e.V.; eine Initiative des Arbeitskreises Biotopentwicklung der Forschungsgesellschaft Landschaftsentwicklung Landschaftsbau. Teil I: 17-31. Bonn.

Engelhard, K. (1971): Entwicklung der Kulturlandschaft. - In: MARTin, B. \& WeTEKAM, R. (eds.): Waldeckische Landeskunde: 99-170. Bing. Korbach.

ERICHSEN, C.F.E. (1957): Flechtenflora von Nordwestdeutschland. - Gustav Fischer. Stuttgart. $411 \mathrm{~S}$.

ERTL, L. (1951): Über die Lichtverhältnisse in Laubflechten. - Planta 39: 245-270.

Evans, A.W. (1950): Notes on the Cladoniae of Connecticut IV. - Rhodora 52(617): 77-123.

Evans, A.W. (1954): Cladonia subrangiformis in North America. - Rhodora 56(672): 266-272.

FAhSElt, D., Krol, M., HÜNER, N. \& Tønsberg, T. (2000): Pigmentation of Cladonia infected by the lichenicolous fungus Arthrorhaphis aeruginosa. - Lichenologist 32(3): 300-303.

FEUERER, T. (ed.) (2003): Checklists of lichens and lichenicolous fungi. Version 1 June 2003. URL: http://www.checklists.de.

FINK, B. (1904): Further notes on Cladonias III. - Bryologist 7: 53-58.

FINK, B. (1917): The rate of growth and ecesis in lichens. - Mycologia 9: 138-158.

FISCHER, P. (2003): Trockenrasen des Biosphärenreservates „Flußlandschaft Elbe“. Vegetation, Ökologie und Naturschutz. - Arch. naturwiss. Diss. 15: 1-286.

FISCHER, P. \& GÜNZL, B. (2004): Ein Beitrag zur Verbreitung der Chemotypen ausgewählter Cladonia-Arten. - Aktuelle Lichenol. Mitt. N.F. 13 (im Druck).

Floerke, H.G. (1828): Festos Jesu Christi Nati anniversarios (...) indicit. Universitatis Literariae Rostochiensis Rector Henricus Gustavus Floerke (...) celebranda civibus Academicis commendat. De Cladoniis, difficillimo Lichenum genere; Commentatio 1.Adler. Rostock. 94 S.

Flora Danica Online: Issue no. 37, Tables 2209 Fig. 2, 2210 Fig 1. - URL: http://www. pictures.dnlb.dk/Homepage/Hefter/22012220.html.

FollmanN, G. (1974): Nordhessische Flechtengesellschaften I. Das Fulgensietum fulgentis GAMS. - Hess. Florist. Briefe 23: 18-25.

FollmanN, G. (1979): Neue Flechtenfunde aus dem Hessischen Bergland. - Hess. Florist. Briefe 28: 10-16.

FollmanN, G. (1981): Das Vorkommen der Strauchflechte Cladonia rei Schaer. (Cladoniaceae) im Hessischen Bergland. - Hess. Florist. Briefe 30: 14-16.

FollmanN, G. (1983): Das Vorkommen der Scharlachflechte Cladonia floerkeana (E. M. Fires) Floerke (Cladoniaceae) im Hessischen Bergland. - Hess. Florist. Briefe 32: 26-32.

FollmanN, G. \& MALECKI, B. (1973): Fulgensia bracteata (Hoffm.) Raes. subspec. deformis (Erichs.) Poelt in Hessen. - Hess. Florist. Briefe 22: 38-41. 
FrAHM, J.-P. \& FREY, W. (1992): Moosflora. 3. überarb. Aufl. - Ulmer. Stuttgart (Hohenheim). $528 \mathrm{~S}$.

FRIEDL, T. (1989): Systematik und Biologie von Trebouxia (Microthamniales, Chlorophyta) als Phycobiont der Parmeliaceae (lichenisierte Ascomyceten). - Diss. Univ. Bayreuth. $218 \mathrm{~S}$.

FrIEDL, T. \& RokitTA, C. (1997): Species relationships in the lichen alga Trebouxia (Chlorophyta, Trebouxiophyceae): Molecular phylogenetic analyses of nuclear-encoded large subunit rRNA gene sequence. - Symbiosis 23: 125-148.

GALLOWAY, D.J. (1985): Flora of New Zealand Lichens. - Hasselberg. Wellington. 662 S.

Glavac, V., Schlage, A. \& Schlage, R. (1979): Das Gentiano-Koelerietum am Kleinen Dörnberg bei Zierenberg (Kreis Kassel). - Mitt. Florist.-Soziol. Arbeitsgem. 21: 105109.

Goward, T. (1999): The Lichens of British Columbia. Illustrated Keys. Part 2, Fruticose Species. - British Columbia Ministry of Forests. Victoria. 319 S.

GRUMMANN, V.J. (1954): Über eine einheitliche Benennung von Bildungsabweichungen bei den Flechten. - Ber. Deutsch. Bot. Ges. 67: 59-68.

GrummanN, V.J. (1963): Catalogus Lichenum Germaniae. - Gustav Fischer. Stuttgart. 208 S.

GÜNZL, B. (2001a): Die Bunte-Erdflechten-Gesellschaft (Toninio-Psoretum decipientis Stodiek 1937) in Nordhessen - aktuelle Erfassung und Gliederung. - Tuexenia 21: 179-191.

GÜNZL, B. (2001b): Die Bunte-Erdflechten-Gesellschaft in Nordhessen - Bestandssituation, Gefährdungsursachen und Schutzmöglichkeiten. - Jahrb. Naturschutz Hessen 6: 115124.

HAKES, W. (1987): Einfluß von Wiederbewaldungsvorgängen in Kalkmagerrasen auf die floristische Artenvielfalt und Möglichkeiten der Steuerung durch Pflegemaßnahmen. Diss. Bot. 109: 1-151.

HALL, T.A. (1999): BioEdit: a user-friendly biological sequence alignment editor and analyses program for Windows 95/98/NT. - Nucl. Acids Symp. Ser. 41: 95-98.

HAWKSWORTH, D.L. (1969): The lichen flora of Derbyshire. - Lichenologist 4: 105-193.

HAWKSWORTH, D.L. (1976): Lichen chemotaxonomy. - In: BROWN, D.H., HAWKSWORTH, D.L. \& BAILEY, R.H. (eds.): Lichenology: Progress and Problems: 139-184. Academic Press. London.

HEINKEN, T. (1999): Dispersal patterns of terricolous lichens by thallus fragments. Lichenologist 31(6): 603-612.

HeINTZE, G. (1966): Landschaftsrahmenplan Naturpark Meißner-Kaufunger Wald. - Schriftenr. Inst. Natursch. Darmstadt 8(4): 1-115.

Helms, G. (2003): Taxonomy and symbiosis in associations of Physciaceae and Trebouxia. Diss. Univ. Göttingen. URL: http://webdoc.sub.gwdg.de/diss/2003/helms/index.html. $156 \mathrm{~S}$. 
Helms, G., FriedL, T. \& RAMBOLd G. (2003): Phylogenetic relationships of the Physciaceae inferred from rDNA sequence data and selected phenotypic characters. - Mycologia 95(6) im Druck.

Helms, G., Friedl, T., RAmbold, G. \& MAYRhOFER, H. (2001): Identification of photobionts from the lichen family Physciaceae using algal-specific ITS rDNA sequencing. Lichenologist 33(1): 73-87.

Henningsen, D. \& Katzung, G. (1998): Einführung in die Geologie Deutschlands. 5., neu bearb. Auflage. - Enke. Stuttgart. 244 S. + Farbanhang.

HenNiPMAN, E. (1967): Notes on some Dutch Cladoniae (Lichenes). - Persoonia 4: 427-428.

Hennipman, E. (1978): De nederlandse Cladonia`s (Lichenes). - Wet. Mededel. K. Nederl. Natuurh. Veren. 124: 1-80.

Henssen, A. \& JAHNS, H.M. (1974): Lichenes. - Georg Thieme. Stuttgart. 467 S.

HESSISCHES MINISTERIUM FÜR LANDESENTWICKLUNG, WOHNEN, LANDWIRTSCHAFT, FORSTEN UND NATURSCHUTZ (1994): Verzeichnis der Naturschutzgebiete in Hessen. Wiesbaden. $53 \mathrm{~S}$.

HiLDRETH, K.C. \& AHMAdJIAN, V. (1981): A study of Trebouxia and Pseudotrebouxia isolates from different lichens. - Lichenologist 13: 65-86.

HillmanN, J. \& GRUMmanN, V.J. (1957): Flechten. - Kryptogamenflora der Mark Brandenburg und angrenzender Gebiete. 8: I-X, 1-898. Berlin.

HLUG (Hessisches LANDESAMt FÜR UMwelt Und GeOlogie) (2001): Geologische Entwicklung in Hessen. - URL: http://www.hlug.de/medien/boden/natraum.html. Stand August 2001.

HöLzER, A. (1977): Vegetationskundliche und ökologische Untersuchungen im BlindenseeMoor bei Schonach (Mittlerer Schwarzwald) unter besonderer Berücksichtigung des Kationengehaltes. - Diss. Bot. 36: 1-196.

HöLzer, A. (1984): Chemische Analyse in Moorwasser und ihre Problematik. - Libellula $3(1 / 2): 1-9$.

Holien, H. \& Tønsberg, T. (1985): Notes on Cladonia asahinae, C. conista and the C. grayigroup in Norway. - Gunneria 51: 1-26.

Huovinen, K., Ahti, T. \& Stenroos, S. (1990): The composition and contents of aromatic lichen substances in Cladonia section Cladonia and group Furcatae. - In: JAHNS, H.M. (ed.): Contributions to Lichenology in Honour of A. Henssen. Biblioth. Lichenol. 38: 209-241.

HYVÄRINEN, M. (1992): Adaptivity of the thallus structure of Hypogymnia physodes to microclimatic conditions. - Lichenologist 24(3): 267-279.

JAHNS, H.M. (1982): The cyclic development of the lichen Baeomyces rufus and mosses in an ecosystem. - Lichenologist 14: 261-265. 
JAHNS, H.M. (1984): Morphology, reproduction and water relations - a system of morphogenetic interactions in Parmelia saxatilis. - In: HERTEL, H. \& OBERWINKLER, F.: Festschrift J. Poelt. Nova Hedwigia Beih. 79: 715-789.

JAHNS, H.M. \& FREY, P. (1982): Thallus growth and the development of fruit bodies in Peltigera canina. - Nova Hedwigia 36: 485-498.

JAHNS, H.M. \& FRITZLER, E. (1982): Flechtenstandorte auf einer Blockhalde. - Herzogia 6: 243-270.

JAHNS, H.M., Herold, K. \& BeltMAN, H.A. (1978): Chronological sequence, synchronization and induction of development of fruit bodies in Cladonia furcata var. furcata (Huds.) Schrad. - Nova Hedwigia 30: 469-526.

JAHNS, H.M. \& OTT, S. (1982): Flechtenentwicklung an dicht benachbarten Standorten. Herzogia 6: 201-241.

JAHNS, H.M. \& OTT, S. (1983): Das Mikroklima dicht benachbarter Flechtenstandorte. - Flora 173: $183-222$.

JAHNS, H.M. \& SCHUSTER, G. (1981): Morphogenetische Untersuchungen an Cetraria islandica. - Beitr. Biol. Pflanzen 55: 427-473.

KAISER, E. (1926): Die Pflanzenwelt des Hennebergisch-Fränkischen Muschelkalkgebietes. Beih. Feddes Repert. 44: 1-152.

KALB, M. \& Vent-SchmidT, V. (1981): Das Klima von Hessen: Standortkarte im Rahmen der agrarstrukturellen Vorplanung. - Hess. Landesamt für Ernährung, Landwirtschaft u. Landentwicklung, Abt. Landentwicklung. Wiesbaden.

KIMURA, M. (1980): A simple method for estimating evolutionary rate of base substitutions through comparative studies of nucleotide sequences. - J. Molec. Evol. 16: 111-120.

Klausing, O. (Bearb.) (1988): Die Naturräume Hessens: mit einer Karte der naturräumlichen Gliederung 1:200 000. - Hessische Landesanstalt für Umwelt. Wiesbaden. 43 S., 1 Kt.

KLEMENT, O. (1952): Zur Flechtenflora Schwabens. - Ber. Naturf. Ges. Augsburg 5: 43-91.

KLEMENT, O. (1955): Prodromus der mitteleuropäischen Flechtengesellschaften. - Beih. Feddes Repert. 135: 5-194.

Kollmann, K. (ed.) (1993): Geschichte der Stadt Eschwege. - Selbstverlag Kreisstadt Eschwege. $530 \mathrm{~S}$.

Koperski, M., SaUer, M., Braun, W. \& Gradstein, S. R. (2000): Referenzliste der Moose Deutschlands. - Schriftenr. Vegetationsk. 34: 1-519.

KrausSe, H.-F. (1971): Geologie des Waldecker Landes. - In: MARTIN, B. \& WeteKAM, R. (eds.): Waldeckische Landeskunde: 1-48. Bing. Korbach.

Kremer, B.P. \& Muhle, H. (1991): Steinbachs Naturführer (Hrsg. G. SteInBACH), Flechten, Moose, Farne. - Mosaik. München. 288 S.

KRIEGER, H. (1937): Die flechtenreichen Pflanzengesellschaften der Mark Brandenburg. - Beih. Bot. Centralbl. 57, Abt. B: 1-76. 
Kumar, S., TAMURA, K., JAKobSEn, I.B. \& NeI, M. (2001): MEGA2: Molecular Evolutionary Genetics Analysis software. - Bioinformatics 17(12): 1244-1245.

KÜMMERLING, H. (1991): Zur Kenntnis der Flechtenflora am Hohen Meißner und in seinem Vorland (Hessen) unter besonderer Berücksichtigung chemischer Merkmale. - Biblioth. Lichenol. 41: 1-315.

Kuntze, H., Roeschmann, G. \& SChWERdTFEger, G. (1994): Bodenkunde. 5., neubearb. u. erweit. Aufl. - Ulmer. Stuttgart (Hohenheim). 424 S.

LANGE, O., GREEN, T.G.A. \& TÜRK, R. (1998): An unusual growth form of Cladonia furcata: the trampling-resistant primary thallus colonising a paved pathway. - Lichenologist 30(6): 583-588.

LANGERFELDT, J. (1939): Die Flechten-Gesellschaften der Kieskuppen und Sandheiden zwischen Jade und Ems. - Repert. Spec. Nov. Regni Veg., Beih. 66: 1-48.

LEICHT, E. (1988): Zum Stand der Naturschutzgebietsausweisung in Nordhessen. - Naturschutz Nordhessen 10: 13-24.

LEICHT, E. (1990): Die neuen Naturschutzgebiete im Regierungsbezirk Kassel (Stand 31.12.1989). - Naturschutz Nordhessen 11: 81-97.

LENARDUZZI, M. (1999a): Naturschutzfachliche Bewirtschaftung eines Biotopverbundes in stationärer Hütehaltung im Werra-Meißner-Kreis - Ein Naturschutzkonzept schlägt Wurzeln. - Jahrb. Naturschutz Hessen 4: 43-47.

LENARDUZZI, M. (1999b): Ein Naturschutzkonzept schlägt Wurzeln. - Zeitschr. Deutsche Schafzucht 20: 496-499.

LetTAU, G. (1955): Flechten aus Mitteleuropa X. - Feddes Repert. 57: 1-94.

LiTTERSKI, B. (1999): Pflanzengeographische und ökologische Bewertung der Flechtenflora Mecklenburg-Vorpommerns. - Diss. Bot. 307: 1-391.

LohtAnder, K., KÄLlersjö, M. \& Tehler, A. (1998): Dispersal strategies in Roccellina capensis (Arthoniales). - Lichenologist 30(4-5): 341-350.

LOTZ, K. (1995): Einführung in die Geologie des Landes Hessen. - Hitzeroth. Marburg. 267 S.

MAGNUSSON, M. (1982): Composition and succession of lichen communities in an inner coastal dune area in southern Sweden. - Lichenologist 14: 153-163.

MANZ, E. (1993): Vegetation und standörtliche Differenzierung der Niederwälder im Nahe- und Moselraum. - Pollichia-Buch 28: 1-413. Bad Dürkheim.

MarstalleR, R. (1968): Die Xerothermflora der Gipshänge bei Jena (Ostthüringen) unter besonderer Berücksichtigung der Bunten-Erdflechten-Gesellschaft. - Hercynia 5: 352372.

MARSTALLER, R. (1971): Zur Kenntnis der Gesellschaften des Toninion-Verbandes im Unstruttal zwischen Nebra und Artern sowie im Kyffhäusergebirge. - Hercynia N.F. 8: $34-51$. 
MAтTick, F. (1932): Bodenreaktion und Flechtenverbreitung. - Beih. Bot. Centralbl. 49: 241271.

Mattsson, J-E. \& Wedin, M. (1998): Phylogeny of the Parmeliaceae - DNA data versus morphological data. - Lichenologist 30(4-5): 463-472.

MÁzSA, K. (1994): Field studies on $\mathrm{CO}_{2}$ fixation of Cladonia furcata and Cladonia convoluta. Cryp. Bot. 4: 207-211.

MeISCH, J.P. (1981): Beiträge zur Isolation, Kultur und Systematik von Flechtenalgen. - Diss. Univ. Innsbruck. $160 \mathrm{~S}$.

MÖLLER, K. (1989): Die geomorphologischen Verhältnisse am Hohen Meißner und ihre Interpretation. - Berliner Geowiss. Abh. (A) 114: 103-114.

MONTE, M. (1993): The influence of environmental conditions on the reproduction and distribution of epilithic lichens. - Aerobiologia 9: 169-179.

MÜHR, B. (2002/2003): Klimadiagramme weltweit. - URL: http://www.klimadiagramme.de.

Müller, J., VAGTS, I. \& Frese, E. (1997): Pflanzliche Regenerationsstrategien und Besiedlungsdynamik in nordwestdeutschen Calluna-Heiden nach Brand. - NNA-Ber. 5: 87-104.

Müller-Westermeier, G. \& Riecke, W. (2003): Die Witterung in Deutschland. - In: DEUTSCHER WETTERDIENST: Klimastatusbericht 2002. URL: http://www.dwd.de/de/FundE/Klima/KLIS/prod/KSB/ksb02/Witterung.pdf.

NAKANO, T. \& IGUCHI, K. (1994): Photobionts isolated from some Japanese species of Cladonia (Lichens). - Symbiosis 17(1): 65-73.

Nebel, M. \& PhILIPPI, G. (eds.) (2000): Die Moose Baden-Württembergs. Band 1: Allgemeiner Teil; Spezieller Teil (Bryophytina I, Andreaeales bis Funariales). - Ulmer. Stuttgart (Hohenheim). $512 \mathrm{~S}$.

Nieschalk, A. \& NieschalK, C. (1983a): Der Osterkopf bei Usseln eine Hochheide des Waldecker Uplandes. - Naturschutz Nordhessen 6: 49-55.

NieschalK, A. \& NieschalK, C. (1983b): Hochheiden im Waldecker Upland und angrenzenden westfälischen Sauerland. - Philippia 5/2: 127-150.

NIMIS, P.L. (1993): The Lichens of Italy. - Museo Regionale di Scienze Naturali, Monographie XII. Torino. $897 \mathrm{~S}$.

Nitsche, L. (1995): Heideflächen in Nordhessen und ihre Pflege. - Naturschutz Nordhessen 15: 78-91.

NiTSCHE, L. \& NiTSCHE S. (2003): Naturschutzgebiete in Hessen schützen - erleben - pflegen. Bd. 2. Stadt Kassel, Landkreis Kassel und Schwalm-Eder-Kreis. - Cognito. Niedenstein. $256 \mathrm{~S}$.

Nitsche, S. \& Bultmann, M. (1995): Magerrasen und Heiden im Raum Kassel. - Naturschutz Hessen, Sonderheft 1: 1-108. 
Oksanen, I., DePriest, P.T., KärkkÄinen, K., Piercey-Normore, M., Sikaroodi, M. \& Aht, T. (2000): Phylogenetic analyses of Cladonia sect. Ascyphiferae (lichen-forming Ascomycota). - In: „Progress and Problems in Lichenology at the Turn of the Millenium: The Fourth IAL Symposium“, Addendum zum Abstract Book. Univ. Barcelona. Barcelona.

OTT, S. (1987): Exogene und endogene Einflüsse auf die Entwicklung von Ascocarpien bei Physcia tenella. - Nova Hedwigia 45(3-4): 327-346.

PAnnewitz, S., Schroeter, B., ScheidegGer, C. \& Kappen, L. (2003): Habitat selection and light conditions: a field study with Lobaria pulmonaria. - Biblioth. Lichenol. 86: 281297.

PAUS, S. (1993): Die Inhaltsstoffe der in Deutschland vorkommenden Cladonia-Arten. Aktuelle Lichenol. Mitt. 4: 7-11.

PAUS, S. (1997): Die Erdflechtenvegetation Nordwestdeutschlands und einiger Randgebiete. Biblioth. Lichenol. 66: 1-222.

Paus, S., DANiels, F.J.A. \& LumBSCH, H.T. (1993): Chemical and ecological studies in the Cladonia subulata complex in northern Germany (Cladoniaceae, lichenised Ascomycotina). - In: FeIGe, G.B. \& LumBSCH, H.T. (eds.): Phytochemistry and Chemotaxonomy of Lichenized Ascomycetes - A Festschrift in Honour of Siegfried Huneck. Biblioth. Lichenol. 53: 191-200.

PePPleR, C. (1999): Anleitung zur Benutzung des Programmes „Tab für Windows” zum Sortieren und Bearbeiten pflanzensoziologischer Tabellen, Version 3.3. - Unveröff. Manuskript, Universität Oldenburg.

PfuHL, E. (1935): Östliches Westfalen und Lippe. - In: ScHUCHT, F.: Die Muschelkalkböden Mitteldeutschlands und ihre land- und forstwirtschaftliche Nutzung: 357-392. Reichsnährstand-Verl. Berlin.

PiERCEY-Normore, M.D. \& DePriest, P.T. (2001): Algal switching among lichen symbioses. - Amer. J. Bot. 88(8): 1490-1498.

PINTARIC, M. \& TÜRK, R. (1995): Vergleichende Untersuchungen über den Ca-, Mg- und KGehalt von Flechten und ihrem Substrat von Kalk- und Silikatstandorten. - Biblioth. Lichenol. 57: 363-385.

PIŠUT, I. (1961): Bemerkungen über einige Arten der Flechtengattung Cladonia in der Slowakei. - Acta Fac. rer. nat. Univ. Comen. VI, Fasc. VIII-X. Botanica: 513-531.

PIŠUT, I. \& WAGNER, B. (1973): Doplnek k rozsireni lisejniku Cladonia furcata (Huds.) Schrad. subsp. subrangiformis (Sandst.) des Abb. a Collema crispum (Huds.) Web. var. crispum v Cechach. [Nachtrag zur Verbreitung der Flechten Cladonia furcata (Huds.) Schrad. subsp. subrangiformis (Sandst.) des Abb. und Collema crispum (Huds.) Web. var. crispum in Böhmen.]. - Zpravy Ceskoslov. Bot. Spolecn. 8: 27-29.

POELT, J. (1969): Bestimmungsschlüssel europäischer Flechten. - Cramer. Lehre. 757 S. 
Poelt, J. (1986): Morphologie der Flechten - Fortschritte und Probleme. - Ber. Deutsch. Bot. Ges. 99: 3-29.

POSADA, D. \& CRANDALL, K.A. (1998): MODELTEST: testing the model of DNA substitution. - Bioinformatics 14: 817-818.

POTT, R. (1981): Der Einfluß der Niederwaldwirtschaft auf die Physiognomie und die floristische Struktur von Kalkbuchenwäldern. - Tuexenia 1: 233-242.

PотT, R. (1985): Vegetationsgeschichtliche und pflanzensoziologische Untersuchungen zur Niederwaldwirtschaft in Westfalen. - Abh. Westfäl. Mus. Naturk. 47(4): 1-75.

PuCCINI, F., PASQuinelli, J. \& PASQuinelli, P. (2003): Cladonia furcata, varietà foliosa. URL: http://www.jep.it/furcata.htm.

Purvis, O.W. \& JAmeS, P.W. (1992): Cladonia Hill ex Browne (1756). - In: PURVIS, O.W., Coppins, B.J., Hawksworth, D.L., James, P.W. \& MoOre, D.M. (eds): The lichen flora of Great Britain and Ireland: 188-210. Nat. Hist. Mus. Publs \& Br. Lichen Soc. London.

RAMBOLD, G., FriedL, T. \& BECK, A. (1998): Photobionts in lichens: possible indicators of phylogenetic relationships? - Bryologist 101(3): 392-397.

REIMERS, H. (1940a): Bemerkenswerte Moos- und Flechtengesellschaften auf Zechsteingips am Südrande des Kyffhäusers und Harzes. - Hedwigia 79: 81- 174.

REIMERS, H. (1951): Beiträge zur Kenntnis der Bunten-Erdflechten-Gesellschaft. I. Zur Systematik und Verbreitung der Charakterflechten der Gesellschaft besonders im Harzvorland. - Ber. Deutsch. Bot. Ges. 63(5): 147-156.

Richter-BERnBURG, G. (1955): Stratigraphische Gliederung des deutschen Zechsteins. - Z. Dt. Geol. Ges. 105: 843-854.

RIKKINEN, L. (1995): What's behind the pretty colours? A study on the photobiology of lichens. - Bryobrothera 4: 1-239.

RITZKOWSKI, S. (1978): Geologie des Unterwerrasattels und seiner Randstrukturen zwischen Eschwege und Witzenhausen (Nordhessen). - Der Aufschluß, Sonderband 28: 187-204.

ROMEIKE, J. (2002): Mikroklima und Musterbildungsprozesse: Komplexe Interaktionen in der Flechtenvegetation entlang eines Transekts der maritimen Antarktis. - Diss., Univ. Düsseldorf. URL: Archivserver der Deutschen Bibliothek http://www.ulb.uniduesseldorf.de/diss/mathnat/2002/romeike.pdf. $196 \mathrm{~S}$.

Romeike, J., Friedl, T., Helms, G. \& OTt, S. (2002): Genetic diversity of algal and fungal partners in four species of Umbilicaria (lichenized Ascomycetes) along a transekt in the Antarctic Peninsula. - Molec. Biol. Evol. 19(8): 1209-1217.

Ruoss, E. (1987): Chemotaxonomische und morphologische Untersuchungen an den Rentierflechten Cladonia arbuscula und C. mitis. - Botanica Helvetica 97(2): 239-263.

SAMBroOK, J., Fritsch, E.F. \& MANiATIS, T. (1989): Molecular Cloning - a laboratory manual. - Cold Spring Harbor Laboratory Press. Cold Spring Harbor. 
Sancho, L.G., Pintado, A., Green, T.G.A., Pannewitz, S. \& Schroeter, B. (2003): Photosynthetic and morphological variation within and among populations of the antarctic lichen Umbilicaria aprina: implications of the thallus size. - Biblioth. Lichenol. 86: 299-311.

SAndstede, H. (1922): Die Cladonien des nordwestdeutschen Tieflandes und der deutschen Nordseeinseln. III. - Abh. Naturwiss. Verein Bremen 25: 89-243.

SANDSTEDE, H. (1931): Die Gattung Cladonia. Rabenhorst Kryptogamenflora. 2. Aufl. Bd. 9. Akademische Verlagsgesellschaft. Leipzig.

SANTESSON, R. (1993): The lichens and lichenicolous fungi of Sweden and Norway. - SBTförlaget. Lund.

SCHADE, A. (1964): Cladonia furcata (Huds.) Schrad. und die Ursachen ihrer schwierigen Taxonomie. Die Flechten Sachsens VIII. - Abh. Ber. Naturkundemus. Görlitz ; 39/14: $1-39$.

SCHADE, A. (1966): Über die Artberechtigung der Cladonia subrangiformis Sandst. sowie das Auftreten von Calciumoxalat-Exkreten bei ihr und einigen anderen Flechten. - Nova Hedwigia 11: 285-308.

SCHÄFER, M. (1998): Laubwald-Gesellschaften trockener Standorte der Edersee-Region. Unveröff. Diplomarb. Univ. Göttingen. 139 S.

SCHMIDT, M. (2000): Die Blaugras-Rasen des nördlichen deutschen Mittelgebirgsraumes und ihre Kontaktgesellschaften. - Diss. Bot. 328: 1-294.

SCHÖLLER, H. (1991): Flechtenverbreitung und Klima. Vegetationsökologische Untersuchungen zur Rolle der Flechten in naturnahen Traubeneichenwäldern des Taunus. - Biblioth. Lichenol. 42:1-250.

SchOLZ, P. (2000): Katalog der Flechten und flechtenbewohnenden Pilze Deutschlands. Schriftenr. Vegetationsk. 31: 1-298.

SCHUHWERK, F. (1986): Kryptogamengemeinschaften in Waldassoziationen - ein methodischer Vorschlag zur Synthese. - Phytocoenologia 14(1): 79-108.

Schuster, G., Herold, K. \& JAHnS, H.M. (1982): Mikroklimatische Messungen an Flechtenstandorten. Neue Meßapparaturen. - Herzogia 6: 183-200.

Schuster, G., OTt, S., GASSMANN, A. \& RoMeIKe, J. (2002): In situ measurement of the water content of lichens. - In: KRANNER, I., BECKETT, R.P. \& VARMA, A.K. (eds.): Protocols in Lichenology. Culturing, Biochemistry, Ecophysiology and Use in Biomonitoring: 224-235. Springer. Berlin, Heidelberg.

SEMBDNER, G. (1957/58): Standortseinflüsse auf die anatomische und morphologische Ausgestaltung bei einigen Cladonia-Arten. - Flora 145: 589-611.

SJÖRS, H. (1950): On the relation between vegetation and electrolytes in North Swedish mire waters. - Oikos 2: 241-258. 
SloOf, J., DANiEls, F.J.A. \& VAN DE Wetering, H.T.J. (1986): Typology en elementeninhoud van Cladonia portentosa (Duf.) Zahlbr. - The Utrecht Plant Ecology News Report 6: 6177.

SOMMER, W.-H. (1972): Die Flechtenvegetation des Naturschutzgebietes „Heiliger Hain“ nördlich Gifhorn. - Herzogia 2: 291-304.

Stenroos, S. \& Ahti, T. (1992): The lichen family Cladoniaceae in the Falkland Islands. Ann. Bot. Fenn. 29: 67-73.

Stenroos, S., Hyvoenen, J., Myllys, L., Thell, A. \& Ahti, T. (2002): Phylogeny of the genus Cladonia s.lat. (Cladoniaceae, Ascomycetes) inferred from molecular, morphological, and chemical data. - Cladistics 18: 237-278.

STOCKER-WÖRGÖTTER, E. \& TÜRK, R. (1993): Redifferentiation of the lichen Cladonia furcata ssp. furcata from cultivated lichen tissue. - Crypt. Bot. 3: 283-289.

STODIEK, E. (1937): Soziologische und ökologische Untersuchungen an den xerotopen Moosen und Flechten des Muschelkalkes in der Umgebung Jenas. - Repert. Spec. Nov. Regni Veg., Beih. 99: 1-46.

STOKER, T. \& DE SMIDT, J.T. (1985): Het effect van begrazing op de struktuur en de soortensamenstelling van droge heidevegetaties. - The Utrecht Plant Ecology News Report 1: 65-70.

Suppan, U., Prügger, J. \& Mayrhofer, H. (2000): Catalogue of the lichenized and lichenicolous fungi of Slovenia. - Biblioth. Lichenol. 76: 1-215.

SUZA, J. (1937): Les lichens remarquables de la région xérotherme de la Tchècoslovaquie. Cas. nár. Muz. Praha 111: 136-156.

SWOFFORD, D.L. (2000): PAUP*: Phylogenetic Analysis Using Parsimony (*and other methods). Version 4. - Sinauer Associates. Sunderland.

SzAtAlA, O. (1938): Cladoniae novae e Hungaria. - Borbasia 1: 5-6.

TAMURA, K. \& NEI, M. (1993): Estimation of the number of nucleotide substitutions in the control region of mitochondrial DNA in humans and chimpanzees. - Molec. Biol. Evol. 10: $512-526$.

Thomson, J.W. (1967): The Lichen Genus Cladonia in North America. - University of Toronto Press. Toronto. $172 \mathrm{~S}$.

TOBLER, F. (1929): Erfolg und Ziel der Flechtensymbiose. - Scientia halensis 46(23): 237-244.

TobleR, F. \& MAtTick, F. (1938): Die Flechtenbestände der Heiden und der Reetdächer Nordwestdeutschlands. - Biblioth. Botanica 117: 1-71.

TOEPFER, A. (1971): Die Birkenplage und ihre Bekämpfung. - Naturschutz und Naturparke 61: 56-57.

TSCHERMAK-WOESS, E. (1980): Asterochloris phycobionta, gen. et spec. nov., der Phycobiont der Flechte Varicellaria carneonivea (Anzi) Erichs. - Pl. Syst. Evol. 135: 279-294. 
TSCHERMAK-WOESS, E. (1989): Developmental studies in trebouxioid algae and taxonomical consequences. - Pl. Syst. Evol. 164: 161-195.

Tuba, Z., Csintalan, Z. \& Proctor, M.C.F. (1996): Photosynthetic responses of a moss, Tortula ruralis, ssp. ruralis, and the lichens Cladonia convoluta and C. furcata to water deficit and short periods of desiccation, and their ecophysiological significance: a baseline study at present-day $\mathrm{CO}_{2}$ concentration. - New Phytol. 133: 353-361.

UlLRICH, J. (1954): Beobachtungen über die vegetative Verbreitung der Cladonien durch Thallusfragmente. - Ber. Deutsch. Bot. Ges. 67(10): 391-394.

UllRiCH, J. (1956): Beobachtungen an Cladonien. 1. Partiell isolaterale Primarthalli der Cladonien. 2. Zur Artberechtigung von Cladonia subrangiformis Scriba. - Ber. Deutsch. Bot. Ges. 69(5): 239-244.

VAGTS, I., KindeR, M. \& MÜLLER, J. (1994): The effect of agrochemicals on the growth of Cladonia furcata. - Lichenologist 26(1): 73-82.

VAGTS, I. \& KINDER, M. (1999): The response of different Cladonia species after treatment with fertilizer or lime in heathland. - Lichenologist 31(1): 75-83.

VERSEGHY, K. (1975): Talajlako xerofiton zuzmofajok okologiaja es elterjedese Magyarorszagon (II.) s nehany taxon revisioja. [Ecology and distribution of terricolous xerophytous lichen species in Hungary. II]. - Studia Bot. Hung. 10: 41-61.

Verseghy, K. (1983): Phänologische Untersuchungen der Art Cladonia furcata (Hds.) Schrad. (Lichenes). - Ann. Hist.-Nat. Mus. Natl. Hung. 75: 55-60.

VERSEGHY, K. (1987/88): Magyarorszagi zuzmoflora reviziojanak eredmenyei. Ergebnisse der Revision der Flechtenflora von Ungarn. - Botanikai Kozlemenyek 74-75: 31-46.

Vitikainen, O. (1994): Taxonomic revision of Peltigera (lichenized Ascomycotina) in Europe. - Acta Bot. Fenn. 152: 1-96.

VoBIS, G. (1980): Bau und Entwicklung der Flechten-Pycnidien und ihrer Conidien. - Biblioth. Lichenol. 14: 1-141 + Anhang.

VON DER HEIDE, K. (1984): Kalkmagerrasen bei Witzenhausen. - Unveröff. Diplomarb. Univ. Göttingen. $91 \mathrm{~S}$.

WAgENITZ, G. (1996): Wörterbuch der Botanik: Morphologie, Anatomie, Taxanomie, Evolution; die Termini in ihrem historischen Zusammenhang. - Gustav Fischer. Jena, Stuttgart, Lübeck, Ulm. 532 S.

WAINIO, E. (1897): Monographia Cladoniarum universalis. Pars tertia. - Acta Soc. Fauna Fl. Fenn. 14(1): 1-268. Reprint 1978. Koeltz. Königstein.

WALKER, F.J. \& JAMES, P.W. (1980): A revised guide to microchemical techniques for the identification of lichen products. - Bull. Brit. Lichen Soc. 46: 13-29.

Wedin, M., DÖRING, H. \& EKMAN, S. (2000): Molecular phylogeny of the lichen families Cladoniaceae, Sphaerophoraceae and Stereocaulaceae (Lecanorales, Ascomycotina). Lichenologist 32(2): 171-187. 
White, T.J., BuRns, T., LEE, S. \& TAYLOR, J. (1990): Amplification and direct sequencing of fungal ribosomal RNA gene for phylogenetics. - In: INNES, M.A., GELFAND, D.H., SNINSKY, J.J. \& WHITE, T.J. (eds.): PCR protocols: a guide to methods and applications. Academic Press. San Diego: 315-322.

WiLKE, E. (1996): Entwicklung der Schäferei in Hessen und Aspekte der Beweidung. - Jahrb. Naturschutz Hessen 1: 86-89.

WiLMANNS, O. (1959): Zur Kenntnis des Toninion coeruleonigricantis Reimers 1951 in Südwestdeutschland. - Bot. Jahrb. 78(4): 481-488.

WilmanNS, O. (1962): Rindenbewohnende Epiphytengemeinschaften in Südwestdeutschland. Beitr. Naturk. Forsch. Südwestdeutschl. 21: 87-164.

WiLMANNS, O. (1966): Kryptogamen-Gesellschaften oder Kryptogamen-Synusien? - In: TUEXEN, R. (ed.): Gesellschaftsmorphologie. Ber. Int. Symp. IVV Stolzenau: 1-7. Junk. The Hague.

WilmanNs, O. (1998): Ökologische Pflanzensoziologie. 6. Aufl. - Quelle \& Meyer. Wiesbaden. $405 \mathrm{~S}$.

WIRTH, V. (1972): Die Silikatflechten-Gemeinschaften im außeralpinen Zentraleuropa. - Diss. Bot. 17:1-306.

Wirth, V. (1991): Zeigerwerte von Flechten. - In: EllenberG, H., WeBER, H.E., DÜLl, R., Wirth, V., Werner, W., Paulissen, D. (eds.): Zeigerwerte von Pflanzen in Mitteleuropa. Scripta Geobot. 18: 215-237.

WIRTH, V. (1994): Checkliste der Flechten und flechtenbewohnenden Pilze Deutschlands - eine Arbeitshilfe. - Stuttgarter Beitr. Naturk., Serie A 517: 1-63.

WirTH, V. (1995): Die Flechten Baden-Württembergs, Teil 1 + 2, 2. Aufl. - Ulmer. Stuttgart (Hohenheim). $1006 \mathrm{~S}$.

WIRTH, V. (2002): Indikator Flechte: Naturschutz aus der Flechtenperspektive. - Stuttgarter Beitr. Naturk., Serie C - Wissen für alle, 50: 1-96.

WIRTH, V. \& DÜLL, R. (2000): Farbatlas Flechten und Moose. - Ulmer. Stuttgart (Hohenheim). $320 \mathrm{~S}$.

Wirth, V., SchÖller, H., Scholz, P., ERnst, G., Feuerer, T., GNÜChtel, A., HAUCK, M., JaCOBSEn, P., John, V. \& LitTerski, B. (1996): Rote Liste der Flechten (Lichenes) in der Bundesrepublik Deutschland. - Schriftenr. Vegetationsk. 28: 307-368.

Wisskirchen, R. \& HAEuPleR, H. (1998): Standardliste der Farn- und Blütenpflanzen Deutschlands. - Ulmer. Stuttgart (Hohenheim). 765 S.

Zoller, S., Lutzoni, F. \& SCHEIDEGGER, C. (1999): Genetic variation within and among populations of the threatened lichen Lobaria pulmonaria in Switzerland and implications for its conservation. - Molecular Ecology 8: 2049-2059. 


\section{Tabellen- und Abbildungsverzeichnis}

\section{Tabellen:}

Tab. 1: Merkmale der beiden Unterarten von Cladonia furcata................................................ 17

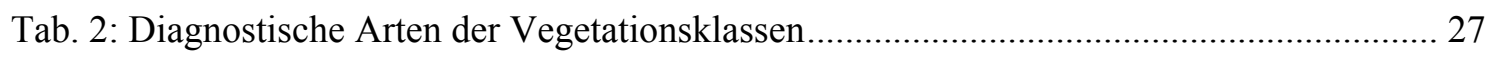

Tab. 3: Syntaxonomische Übersicht der Gesellschaften innerhalb der Klasse

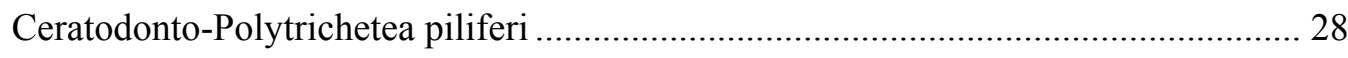

Tab. 4: Übersichtstabelle Ceratodonto-Polytrichetea piliferi.................................................... 29

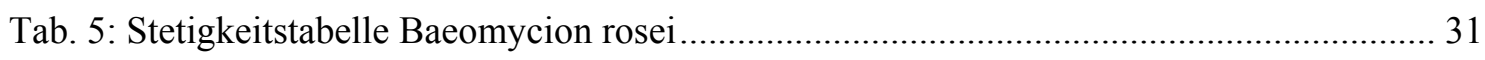

Tab. 6: Baeomycion rosei (Anhang 3)

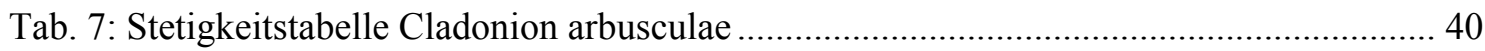

Tab. 8: Cladonion arbusculae (Anhang 3)

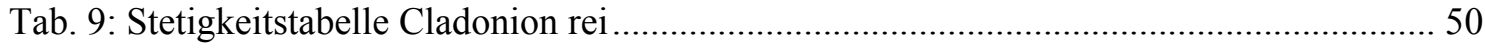

Tab. 10: Cladonion rei (Anhang 3)

Tab. 11: Ceratodonto-Polytrichetea piliferi: $\mathrm{pH}-$ Werte und elektrolytische Leitfähigkeiten

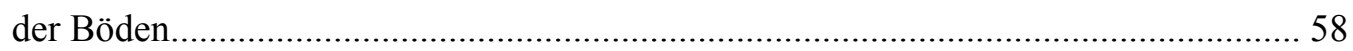

Tab. 12: Syntaxonomische Übersicht der Gesellschaften innerhalb der Klasse

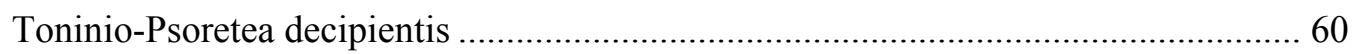

Tab. 13: Autorenangaben zu Kennarten innerhalb der Klasse Toninio-Psoretea decipientis..... 61

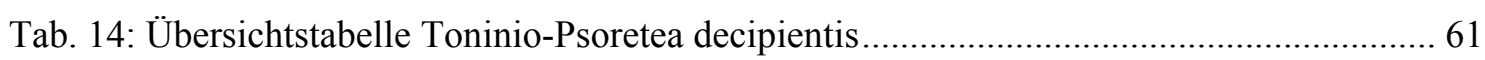

Tab. 15: Toninion-Initialgesellschaft und Toninion-Fragmentgesellschaften und Dominanzbestände (Anhang 3)

Tab. 16: Stetigkeitstabelle Toninio-Psoretum decipientis

Tab. 17: Toninio-Psoretum decipientis (Anhang 3)

Tab. 18: Medianwerte von $\mathrm{pH}$ und elektrolytischer Leitfähigkeit in Abhängigkeit vom Ausgangsgestein 75

Tab. 19: Toninio-Psoretea decipientis: pH-Werte und elektrolytische Leitfähigkeiten der Böden..

Tab. 20: Stetigkeiten, Ausdehnung der Lager und Deckungsgrade von Cladonia furcata ssp.

furcata 77

Tab. 21: Stetigkeiten von Cladonia furcata ssp. furcata: Vergleich der Daten aus

Nordhessen und Nordwestdeutschland. 78

Tab. 22: Stetigkeiten, Ausdehnung der Lager und Deckungsgrade von Cladonia furcata ssp. subrangiformis.... 
Tab. 23: Stetigkeiten von Cladonia furcata ssp. subrangiformis: Vergleich der Daten aus Nordhessen und Nordwestdeutschland 80

Tab. 24: Stetigkeiten fruchtender Thalli und Gesamtstetigkeiten ausgewählter Arten 87

Tab. 25: Prozentualer Anteil der fruchtenden Thalli ausgewählter Arten 88

Tab. 26: Anteil fruchtender Thalli an den absoluten Stetigkeiten ausgewählter Arten 90

Tab. 27: Rote Liste-Arten der Flechten 91

Tab. 27: Bestandssituation der Erdflechtengesellschaften in Nordhessen .92

Tab. 29: Flechtensäuren der Unterarten und Chemosippen von Cladonia pyxidata 103

Tab. 30: Die Sekundärstoffe der untersuchten Cladonia-Arten. 104

Tab. 31: Übersicht über die Varietäten und Formen von Cladonia furcata ssp. furcata.

Tab. 32: Übersicht über die Varietäten und Formen von Cladonia furcata ssp. subrangiformis

Tab. 33: Anteile der 3 Kategorien mechanischer Störung an den Standorttypen 128

Tab. 34: Mittelwerte der Podetienmerkmale in den 3 Kategorien mechanischer Störung

Tab. 35: Häufigkeit geschlitzter bzw. offen-rinniger Podetien in Abhängigkeit von Standorttyp und mechanischer Störung

Tab. 36: Mittelwerte der Podetienmerkmale bei fertilen und sterilen bzw. bei pyknidientragenden und pyknidienlosen Podetien

Tab. 37: Mittelwerte der Podetienmerkmale unterschiedlich stark mit Fibrillen besetzter bzw. pigmentierter Podetien

Tab. 38: Mittelwerte der Podetienmerkmale von Podetien mit unterschiedlich ausgedehnter Algenschicht.

Tab. 39: Häufigkeit geschlitzter bzw. offen-rinniger Podetien in Abhängigkeit von der Fruchtkörperbildung und der Podetienlänge

Tab. 40: Ergebnisse der Korrelationsanalyse nach Spearman.

Tab. 41: Zusammenfassung der Bedeutung von Standortfaktoren und Entwicklungszustand auf die einzelnen Podetienmerkmale bei Cladonia furcata ssp. furcata

Tab. 42: Daten zu den lichtmikroskopisch untersuchten Proben von Cladonia furcata und $\mathrm{Cl}$. gracilis

Tab. 43: Maximaler prozentualer Anteil der einzelnen Schichten am Radius des Podetiums

Tab. 44: Klimadaten des Untersuchungszeitraumes, Klimastation Kassel

Tab. 45: Mittlere Zuwachsraten der Podetien

Tab. 46: Anteil sich neu verzweigender Podetienspitzen an den Standorten

R1/R2 und R3 in Abhängigkeit von der Ausgangslänge der Zweige 
Tab. 47: Anteil sich neu verzweigender Podetienspitzen an den Standorten R1/R2 und R3 in Abhängigkeit vom Auspflanzungszeitraum 158

Tab. 48: Mittlerer Zuwachs sich verzweigender sowie unverzweigt bleibender Podetienspitzen 159

Tab. 49: Vergleich der Zuwachsraten von randlich stehenden Podetien und solchen aus der Polstermitte 160

Tab. 50: Jahreszuwachs in Abhängigkeit von der Zweiglänge bei Versuchsbeginn 160

Tab. 51: Bezeichnung, Herkunft und Charakterisierung der Proben für die rDNA-Sequenzierung

Tab. 52: Vegetationseinheiten, denen die Proben für die rDNA-Sequenzierung entnommen wurden. 164

Tab. 53: Vergleichssequenzen aus GenBank 166

Tab. 54: Distanztabelle der Mycobionten von Cladonia furcata............................................. 170

Tab. 55: Distanztabelle zum Vergleich von Cladonia furcata und $\mathrm{Cl}$. gracilis . 173

Tab. 56: Distanztabelle der Photobionten 175

Tab. 57: Fundortverzeichnis 219

Tab. 58: Abkürzungen der Ausgangsgesteine. 221

Tab. 59: Stetigkeiten fruchtender Thalli und Gesamtstetigkeiten. 225

Tab. 60: Prozentualer Anteil der fruchtenden Thalli 226

Tab. 61: Mittlere maximale Podetienlänge 227

Tab. 62: Mittlerer maximaler Podetiendurchmesser. 227

Tab. 63: Mittlere Anzahl Verzweigungen. 227

Tab. 64: Mittlere Anzahl Zweige am Podetienende. 228

Tab. 65: Mittlere maximale Anzahl Schuppen an den Podetien 228

Tab. 66: Mittlere maximale Größe der Schuppen an den Podetien 228

Tab. 67: Mittlere maximale Anzahl Apothecien 228

Tab. 68: Mittlere maximale Größe der Apothecien 228

Tab. 69: Mittlerer Anteil pyknidientragender Podetien 229

Tab. 70: Mittlerer Anteil mit zahlreichen Fibrillen besetzter Podetien. 229

Tab. 71: Mittlerer Anteil stark pigmentierter Podetien 229

Tab. 72: Mittlerer Anteil Podetien mit mehr als $75 \%$ deckender Algenschicht 229

Tab. 73: Wertetabelle für fruchtende bzw. sterile Podetien 230

Tab. 74: Wertetabelle für Podetien mit bzw. ohne Pyknidien 230 
Tab. 75: Wertetabelle für Podetien ohne, mit wenigen bzw. mit zahlreichen Fibrillen .............223

Tab. 76: Wertetabelle für nicht, wenig und stark pigmentierte Podetien ..................................22

Tab. 77: Wertetabelle für Podetien mit unterschiedlicher Ausdehnung der Algenschicht........232

\section{Abbildungen:}

Abb. 1: Schematische Darstellung der Gliederung der Arbeit ................................................. 4

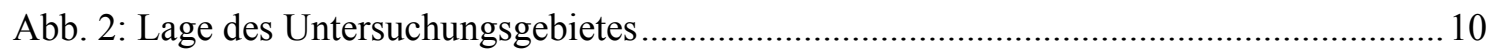

Abb. 3: Übersichtskarte des Untersuchungsgebietes mit den bearbeiteten Landkreisen.............. 10

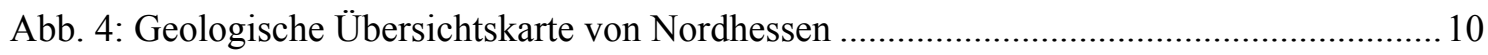

Abb. 5: Klimadiagramme der Städte Eschwege und Kassel sowie des Kahlen Asten

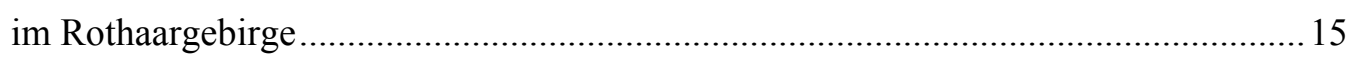

Abb. 6: Mittlere Niederschlagshöhen im Regierungsbezirk Kassel (1931-1960) ........................ 12

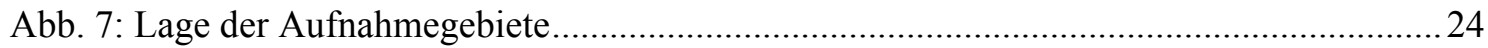

Abb. 8: Verbreitung des Dibaeetum baeomycetis im Untersuchungsgebiet ............................... 38

Abb. 9: Verbreitung des Lecideetum uliginosae im Untersuchungsgebiet .................................40

Abb. 10: Verbreitung des Pycnothelio-Cladonietum cervicornis und der Cladonia cervicornis-Baeomycion-FG im Untersuchungsgebiet ............................................... 41

Abb. 11: Verbreitung der Cladonia bacillaris-Gesellschaft im Untersuchungsgebiet ...................46

Abb. 12: Verbreitung des Cladonietum mitis im Untersuchungsgebiet .......................................48

Abb. 13: Verbreitung der Cladonia furcata- und der Cladonia gracilis-Cladonion

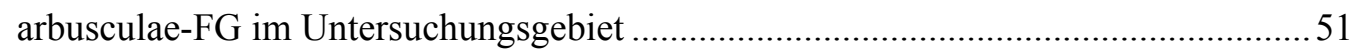

Abb. 14: Verbreitung des Cladonietum foliaceae im Untersuchungsgebiet...............................52

Abb. 15: Verbreitung der Cladonia floerkeana-Cladonion rei-FG im Untersuchungsgebiet .......56

Abb. 16: Verbreitung des Cladonietum rei im Untersuchungsgebiet........................................57

Abb. 17: Verbreitung der Cladonia subulata-Cladonion rei-FG im Untersuchungsgebiet...........59

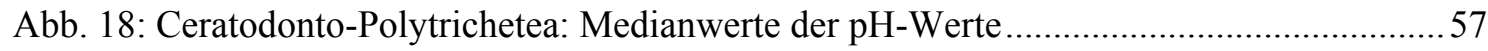

Abb. 19: Ceratodonto-Polytrichetea: Medianwerte der elektrolytischen Leitfähigkeit................57

Abb. 20: Transekt an einem Wegrand innerhalb der Eichenwälder am Roßkopf ........................59

Abb. 21: Verbreitung der Toninia sedifolia-Toninion-Initialgesellschaft im

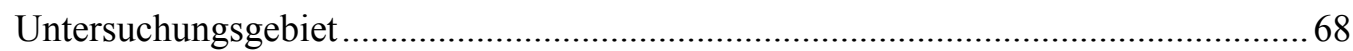

Abb. 22: Verbreitung des Toninio-Psoretum decipientis im Untersuchungsgebiet ..................... 73

Abb. 23: Verbreitung der Cladonia symphycarpa-Toninion-FG im Untersuchungsgebiet.......... 75

Abb. 24: Verbreitung der Peltigera rufescens-Toninion-FG im Untersuchungsgebiet ................. 77 
Abb. 25: Lage der bearbeiteten Dominanzbestände im Untersuchungsgebiet 78

Abb. 26: Gesellschaftsdiversität im Untersuchungsgebiet. 97

Abb. 27: Skizzen der untersuchten morphologischen Merkmale 107

Abb. 28: Herkunftsorte der morphologisch untersuchten Cladonia furcata-Proben 108

Abb. 29: Schema des Verpflanzungsversuches 112

Abb. 30: Mittelwerte und Standardabweichungen der Podetienmerkmale Podetienlänge, Podetiendurchmesser, Verzweigungen und Beschuppung. 130

Abb. 31: Mittelwerte und Standardabweichungen der Podetienmerkmale Apothecien, Pyknidien, Fibrillen und Algenschicht 131

Abb. 32: Podetienquerschnitte. 146

Abb. 33: Weitere lichtmikroskopische Schnitte durch Podetien, Phylloide und Grundschuppen 147

Abb. 34: Tagesverlauf der Lichtintensität an den Standorten R2 und R3 151

Abb. 35: Tagesverlauf der Lufttemperatur an den Standorten R2 und R3 153

Abb. 36: Tagesverlauf der Thallustemperatur an den Standorten R2 und R3 153

Abb. 37: Vergleich von Luft- und Thallustemperatur am Standort R3 153

Abb. 38: Tagesverlauf der relativen Luftfeuchte an den Standorten R2 und R3 154

Abb. 39: Tagesverlauf derThallusfeuchtigkeit an den Standorten R2 und R3. 156

Abb. 40: Tagesverlauf der Evaporation an den Standorten R2 und R3 155

Abb. 41: Evaporationssummen an den Standorten R2 und R3 ..... 155

Abb. 42: Tagesverlauf der Windmessungen an den Standorten R2 und R3 155

Abb. 43: ITS-Region mit Ansatzstellen der PCR-Primer ... 162

Abb. 44: rDNA-Stammbaum der Mycobionten von Cladonia furcata .... 168

Abb. 45: Zusammenfassung der Polymorphismen bei den Mycobionten von

Cladonia furcata.

Abb. 46: Zusammenfassung der Polymorphismen bei den Mycobionten von

Cladonia gracilis. 174

Abb. 47: rDNA-Stammbaum der Photobionten aus Cladonia furcata und $\mathrm{Cl}$. gracilis 173

Abb. 48: Zusammenfassung der Polymorphismen bei den Photobionten 178

Abb. 49: Kombinationen der Bionten innerhalb der Arten Cladonia furcata und Cl. gracilis. 176 


\section{Bildtafeln:}

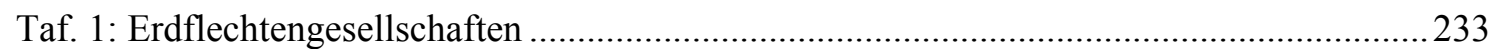

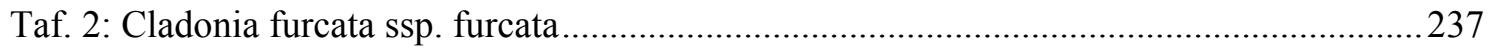

Taf. 3: Cladonia gracilis, Cl. crispata, Cladonia furcata ssp. furcata........................................239

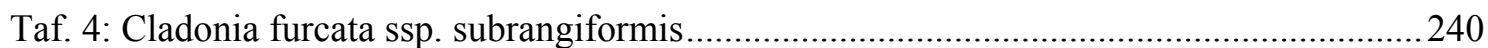

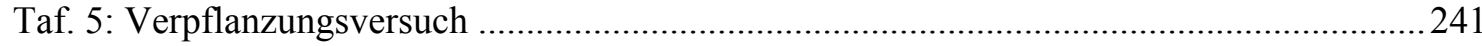




\section{N Anhang 1: Abkürzungen, Arten- und Belegeliste, Tabellen}

\section{a) Abkürzungen der Fundorte}

Tab. 57: Fundortverzeichnis

\begin{tabular}{|c|c|c|c|c|c|c|}
\hline Kürzel & Fundort & Beschreibung & Geologie & $\begin{array}{l}\text { Höhe ü. } \\
\text { NN [m] }\end{array}$ & MTB/Q & NSG \\
\hline$\overline{\mathrm{AS}}$ & Alte Stadt, Frankershausen & $\begin{array}{l}\text { Wegböschung mit } \\
\text { Kalkmagerrasenfragmenten }\end{array}$ & $\mathrm{zg}+\mathrm{zd}$ & 265 & $4725 / 3$ & \\
\hline BA & Bilstein bei Albungen & Felswand & $\mathrm{d}$ & 240 & $4725 / 4$ & $\mathrm{x}$ \\
\hline BB & Burghasunger Berg & Felsköpfe, -kuppen & $\mathrm{b}$ & 480 & $4621 / 4$ & $\mathrm{x}$ \\
\hline $\mathrm{BD}$ & Battenfelder Driescher & Zwergstrauchheide & ts & 380 & $4917 / 4$ & $\mathrm{x}$ \\
\hline BK & Blaue Kuppe, Eschwege & $\begin{array}{l}\text { Ehem. Steinbruch mit Magerrasen, } \\
\text { Rand eines Trampelpfads }\end{array}$ & $\mathrm{b}$ & 320 & $4826 / 1$ & $\mathrm{x}$ \\
\hline $\mathrm{BP}$ & $\begin{array}{l}\text { Blockhalde bei Petersruh, } \\
\text { Meißner }\end{array}$ & Blockhalde umgeben von Wald & $\mathrm{b}$ & 642 & $4725 / 3$ & $\mathrm{x}$ \\
\hline BT & $\begin{array}{l}\text { Breites Tal, Kaufunger } \\
\text { Wald }\end{array}$ & Schneise im Luzulo-Fagetum & sm & 420 & $4724 / 1$ & \\
\hline BÜ & Bühlchen bei Weißenbach & Kalkmagerrasen & $\mathrm{mu}$ & 515 & $4725 / 3$ & $\mathrm{x}$ \\
\hline $\mathrm{CM}$ & Carlsstollen, Meißner & Erdanrisse an einer Wegböschung & $\mathrm{sm}$ & $591-595$ & $4725 / 3$ & \\
\hline DB & $\begin{array}{l}\text { Dörnberggebiet } \\
\text { (incl. Helfensteine) }\end{array}$ & $\begin{array}{l}\text { Kuppen im Segelflugplatz mit } \\
\text { Felsfluren und Mesobromion-Ges. }\end{array}$ & $\mathrm{b}$ & $452-475$ & $4622 / 1$ & \\
\hline DH & $\begin{array}{l}\text { Dolomitfelsen bei } \\
\text { Hergershausen }\end{array}$ & $\begin{array}{l}\text { Felsen, umgeben und z.T. } \\
\text { bewachsen mit Kalkmagerrasen }\end{array}$ & $\mathrm{zd}$ & 240 & $4924 / 1$ & \\
\hline DÖ & Dörnberg & Kalkmagerrasen & $\mathrm{mu}$ & 403 & $4621 / 2$ & $\mathrm{x}$ \\
\hline DP & $\begin{array}{l}\text { Diemeltal, } \\
\text { Papenbreite bei Sielen }\end{array}$ & Kalkmagerrasen, Wegrand & $\mathrm{mu}$ & 220 & $4422 / 1$ & \\
\hline DS & $\begin{array}{l}\text { Diemeltal, } \\
\text { Sommerberg bei Sielen }\end{array}$ & $\begin{array}{l}\text { Kalkmagerrasen, Pfade und } \\
\text { Wegränder }\end{array}$ & $\mathrm{mu}$ & 200 & $4422 / 1$ & \\
\hline DT & Dohlsbachtal & Traubeneichenwald & gw & $310-330$ & $4725 / 4$ & \\
\hline EI & Eisenberg bei Walburg & Kalkmagerrasen & mo & 420 & $4724 / 4$ & $\mathrm{x}$ \\
\hline EW & Ettelsberg bei Willingen & Zwergstrauchheide, Wegränder & ts & $740-780$ & $4717 / 2$ & \\
\hline FT & Fahrenbachtal & altes Stallgebäude & - & 180 & $4625 / 3$ & \\
\hline GB & $\begin{array}{l}\text { Böddiger Dorfberg } \\
\text { bei Gudensberg }\end{array}$ & Basaltfelsen im Ort & $\mathrm{b}$ & 180 & $4822 / 1$ & \\
\hline $\mathrm{GH}$ & $\begin{array}{l}\text { Grenzstreifen } \\
\text { bei der Burg Hanstein }\end{array}$ & $\begin{array}{l}\text { Wegränder in degenerierender } \\
\text { Zwergstrauchheide }\end{array}$ & $\mathrm{sm}$ & $390-400$ & $4625 / 4$ & \\
\hline GM & $\begin{array}{l}\text { Gudensberger } \\
\text { Basaltkuppen, Maderstein }\end{array}$ & $\begin{array}{l}\text { Kuppe mit Berg-Lauch- } \\
\text { Felsgesellschaften }\end{array}$ & $\mathrm{b}$ & 263 & $4822 / 1$ & \\
\hline GO & $\begin{array}{l}\text { Gottesberg } \\
\text { bei Hundelshausen }\end{array}$ & $\begin{array}{l}\text { Felskuppe, } \sim 0,5 \mathrm{~km} \text { östl. } \\
\text { Hundelshausen }\end{array}$ & $\mathrm{zd}$ & 280 & $4725 / 1$ & \\
\hline GS & $\begin{array}{l}\text { Scharfenstein } \\
\text { bei Gudensberg }\end{array}$ & Felskuppe mit Felsfluren & $\mathrm{b}$ & $\sim 270$ & $4822 / 1$ & \\
\hline $\mathrm{HE}$ & Heiligenberg & $\begin{array}{l}\text { Kalkmagerrasen und Felsflur am } \\
\text { Sendemast }\end{array}$ & mo & $570-580$ & $4725 / 3$ & z.T. \\
\hline HG & Heinebach & $\begin{array}{l}\text { aufgelassener Steinbruch, z.T. } \\
\text { Kalkmagerrasenfragmente }\end{array}$ & $\mathrm{zg}$ & $180-210$ & $4923 / 2$ & \\
\hline $\mathrm{HH}$ & $\begin{array}{l}\text { Heide „Steinbülz“ } \\
\text { bei Hombressen }\end{array}$ & Erdanrisse in Zwergstrauchheide & sm & 200 & $4422 / 4$ & \\
\hline HK & $\begin{array}{l}\text { zwischen Hie- und } \\
\text { Kripplöchern }\end{array}$ & $\begin{array}{l}\text { Kalkmagerrasenfragmente am } \\
\text { Wegrand }\end{array}$ & $\mathrm{zd}$ & 280 & $4725 / 2$ & \\
\hline HL & Hielöcher Frankershausen & Kalkmagerrasen, Pfade & $z d+z g$ & $280-317$ & $4725 / 3$ & $\mathrm{x}$ \\
\hline $\mathrm{HO}$ & Hohekopf bei Epterode & $\begin{array}{l}\text { Kalkmagerrasen und Wegränder an } \\
\text { der Grenze zum NSG }\end{array}$ & $\mathrm{mu}$ & 485 & $4724 / 4$ & z.T. \\
\hline HS & Hang oberhalb Seulenbach & Traubeneichenwald & gw & 280 & $4725 / 1$ & \\
\hline IG & Iberg bei der Grube Gustav & $\begin{array}{l}\text { Felskopf und Trampelpfad in } \\
\text { Kalkmagerrasen }\end{array}$ & $\mathrm{zd}$ & 245 & $4725 / 4$ & \\
\hline JW & Jestädter Weinberg & Kalkmagerrasen, Felskopf & $\mathrm{zg}$ & 180 & $4725 / 4$ & $\mathrm{x}$ \\
\hline KA & Krösselberg bei Abterode & Kalkmagerrasen & $z d+z g$ & 260 & $4725 / 4$ & \\
\hline
\end{tabular}


Forts. Tab. 57: Fundortverzeichnis

\begin{tabular}{|c|c|c|c|c|c|c|}
\hline Kürzel & Fundort & Beschreibung & Geologie & $\begin{array}{l}\text { Höhe ü. } \\
\text { NN [m] }\end{array}$ & $\mathbf{M T B} / \mathbf{Q}$ & NSG \\
\hline$\overline{\mathrm{KB}}$ & Klepperberg, Flachsbachtal & $\begin{array}{l}\text { schmaler Bergrücken mit krüppel- } \\
\text { wüchsigen Eichen und Buchen }\end{array}$ & $\overline{g w}$ & 260 & $4625 / 3$ & \\
\hline $\mathrm{KL}$ & Kripplöcher Frankershausen & Kalkmagerrasen, Pfade & $\mathrm{zd}$ & $324-329$ & $4725 / 2$ & \\
\hline KM & Kitzkammer, Meißner & Basaltschuttfläche & $\mathrm{b}$ & 620 & $4725 / 3$ & \\
\hline $\mathrm{KN}$ & $\begin{array}{l}\text { Straße zw. Kleinalmerode } \\
\text { und Nieste }\end{array}$ & moosbewachsene Straßenböschung & $\mathrm{su}$ & 365 & $4624 / 4$ & \\
\hline KP & Kahle Pön & $\begin{array}{l}\text { Zwergstrauchheide, mit vor } \sim 26 \mathrm{~J} \text {. } \\
\text { abgeplaggter Fläche }\end{array}$ & ts & 750 & $4718 / 1$ & $\mathrm{x}$ \\
\hline KR & $\begin{array}{l}\text { Kalkmagerrasen } \\
\text { bei Roßbach }\end{array}$ & $\begin{array}{l}\text { Schafweide, Erdanriss an einem } \\
\text { Trampelpfad }\end{array}$ & $\mathrm{zd}$ & 280 & $4624 / 4$ & $\mathrm{x}$ \\
\hline $\mathrm{ME}$ & Mühlecke, Edersee & $\begin{array}{l}\text { Traubeneichenwald, z.T. } \\
\text { Wegböschungen und Schutthalden }\end{array}$ & $\mathrm{ts}+\mathrm{gw}$ & $330-380$ & $4820 / 1$ & \\
\hline MI & Michelskopf, Edersee & $\begin{array}{l}\text { Schutthalde und Felsen unterhalb } \\
\text { eines Traubeneichenwaldes }\end{array}$ & ts & $280-285$ & $4820 / 1$ & \\
\hline MK & Meißner, Kalbe & $\begin{array}{l}\text { Schutthalde von ehem. } \\
\text { Braunkohlegrube und Blockhalde }\end{array}$ & $\mathrm{b}$ & $705-710$ & $4725 / 3$ & z.T. \\
\hline MM & Meißner bei Meißnerhaus & Blockhalde & $\mathrm{b}$ & 610 & $4725 / 3$ & $\mathrm{x}$ \\
\hline NT & Niestetal, Kaufunger Wald & $\begin{array}{l}\text { Waldrand, Fichtenforst und } \\
\text { Wegböschungen }\end{array}$ & sm & $377-520$ & $4724 / 1$ & \\
\hline $\mathrm{OL}$ & Oberlemp, Lempetal & $\begin{array}{l}\text { kleine Zwergstrauchheide im Wald } \\
\text { und aufgelassener Steinbruch }\end{array}$ & ts & 300 & $5316 / 4$ & \\
\hline $\mathrm{OU}$ & Osterkopf bei Usseln & $\begin{array}{l}\text { Zwergstrauchheide, Felsköpfe und } \\
\text { abgeplaggte Flächen }\end{array}$ & ts & $690-709$ & $4718 / 1$ & $\mathrm{x}$ \\
\hline OW & Orenberg bei Willingen & $\begin{array}{l}\text { kleine Zwergstrauchheide, } \\
\text { Erdanrisse an Pfaden }\end{array}$ & ts & $655-660$ & $4717 / 2$ & \\
\hline PT & Festberg bei Philippinenthal & Kalkmagerrasen & $\mathrm{mu}$ & 330 & $4621 / 3$ & $\mathrm{x}$ \\
\hline RK & Roßkopf, Riedbachtal & Traubeneichenwald & gw & $335-360$ & $4725 / 1$ & \\
\hline $\mathrm{RO}$ & Rohrberg bei Reichenbach & verbrachter Kalkmagerrasen & $\mathrm{mu}$ & 460 & $4824 / 2$ & \\
\hline SA & $\begin{array}{l}\text { Steinbruch bei der } \\
\text { Kirchenruine Abterode }\end{array}$ & Kalkmagerrasen & $\mathrm{zd}$ & 260 & $4725 / 4$ & \\
\hline SB & Schmachteberg & kleine Schutthalde im Wald & gw & $\sim 270$ & $4625 / 3$ & \\
\hline $\mathrm{SC}$ & Steinbruch Carmshausen & aufgelassener Steinbruch & gw & 200 & $4625 / 3$ & \\
\hline SE & Sengelharth & Traubeneichenwald & gw & 300 & $4725 / 1$ & \\
\hline SF & $\begin{array}{l}\text { Schenklengsfeld } \\
\text { bei Bad Hersfeld }\end{array}$ & Kalkmagerrasen & $\mathrm{mu}$ & $300-380$ & $5125 / 3$ & \\
\hline SG & Schäferburg, Graburg & Felsen/Felsspalten & $\mathrm{mu}$ & $\sim 480$ & $4826 / 4$ & \\
\hline SI & Steinküppel bei Iba & $\begin{array}{l}\text { ehem. Steinbruch, Kalkmagerrasen, } \\
\text { Felsköpfe und Wegränder }\end{array}$ & $\mathrm{zd}$ & 355 & $5025 / 1$ & $\mathrm{x}$ \\
\hline SK & Steinbruch Kupferbachtal & $\begin{array}{l}\text { aufgelassener Steinbruch, } \sim 0,75 \mathrm{~km} \\
\text { oberhalb der Grube Gustav }\end{array}$ & $z d$ & 230 & $4725 / 4$ & \\
\hline $\mathrm{SN}$ & Schnellerskopf, Riedbachtal & Traubeneichenwald & gw & $\sim 250$ & $4725 / 1$ & \\
\hline SR & Steinröllchen, Oberhone & aufgelassener Steinbruch & $\mathrm{zg}$ & 200 & $4826 / 1$ & \\
\hline SS & Steinbruch Schafhof & $\begin{array}{l}\text { Oberkante eines aufgelassenen } \\
\text { Steinbruchs }\end{array}$ & gw & 240 & $4725 / 4$ & \\
\hline ST & $\begin{array}{l}\text { Schweinsbachtal, } \\
\text { Eltmannshausen }\end{array}$ & aufgelassener Steinbruch & $\mathrm{zd}$ & 200 & $4825 / 2$ & \\
\hline $\mathrm{SZ}$ & $\begin{array}{l}\text { Gr. Schreckenberg } \\
\text { bei Zierenberg }\end{array}$ & $\begin{array}{l}\text { Blockhalde und angrenzende } \\
\text { Flächen }\end{array}$ & b & 415 & $4621 / 2$ & \\
\hline $\mathrm{TE}$ & $\begin{array}{l}\text { Termenei } \\
\text { bei Wilhelmshausen }\end{array}$ & $\begin{array}{l}\text { Zwergstrauchheide mit feuchten } \\
\text { Senken und ehem. Sportplatz }\end{array}$ & $\mathrm{sm}$ & 260 & $4523 / 3$ & $\mathrm{x}$ \\
\hline WB & $\begin{array}{l}\text { Wenkeberg } \\
\text { bei Frankershausen }\end{array}$ & Kalkmagerrasen, Gipsfelsen & $\mathrm{zg}$ & 280 & $4725 / 3$ & \\
\hline WH & $\begin{array}{l}\text { Hügel zwischen Wenkeberg } \\
\text { und Hielöcher }\end{array}$ & Kalkmagerrasen & $\mathrm{zg}$ & 270 & $4725 / 3$ & \\
\hline WN & $\begin{array}{l}\text { Wachholderheide } \\
\text { bei Niederlemp }\end{array}$ & Zwergstrauchheide & ts & 300 & $5316 / 1+2$ & $\mathrm{x}$ \\
\hline WZ & Warmberg bei Zwergen & Kalkmagerrasen, unter einer Kiefer & $\mathrm{mu}$ & 210 & $4521 / 2$ & $\mathrm{x}$ \\
\hline $\mathrm{ZG}$ & $\begin{array}{l}\text { Ehemalige Zeche } \\
\text { Glimmerode }\end{array}$ & $\begin{array}{l}\text { Seeufer, Erdanrisse, z.T. mit } \\
\text { Pioniervegetation }\end{array}$ & st, $\mathrm{tt}, \mathrm{bt}$ & $410-425$ & $4824 / 1$ & \\
\hline
\end{tabular}




\section{b) Abkürzungen zur Geologie}

Tab. 58: Abkürzungen der Ausgangsgesteine

\begin{tabular}{ll|ll}
\hline $\mathrm{b}$ & Basalt & su & Unterer Buntsandstein \\
$\mathrm{bt}$ & tertiäre Braunkohle & sug & Gips, Unterer Buntsandstein \\
$\mathrm{st}$ & tertiäre Sande & $\mathrm{zd}$ & Zechstein-Dolomit \\
$\mathrm{tt}$ & tertiärer Ton & $\mathrm{zg}$ & Zechstein-Gips \\
$\mathrm{mo}$ & Oberer Muschelkalk & $\mathrm{gw}$ & Grauwacke (Karbon) \\
$\mathrm{mm}$ & Mittlerer Muschelkalk & $\mathrm{ts}$ & Tonschiefer (Devon, Karbon) \\
$\mathrm{mu}$ & Unterer Muschelkalk & $\mathrm{d}$ & Diabas (Devon) \\
$\mathrm{so}$ & Oberer Buntsandstein & $\mathrm{dp}$ & Dachpappe \\
$\mathrm{sm}$ & Mittlerer Buntsandstein & & \\
\hline
\end{tabular}

\section{c) Gesamtartenliste der Flechten}

In der folgenden Artenliste sind alle Erdflechtenfunde der Jahre 1999 bis 2003 aufgeführt. Alle mit einem * gekennzeichneten Arten wurden nicht in den Vegetationsaufnahmen erfaßt. Ein * hinter den Fundortangaben bedeutet, daß die betreffende Art an diesem Fundort nicht in einer der Aufnahmeflächen zu finden war. Die Nomenklatur richtet sich nach SCHOLZ (2000) und WIRTH (1995)

Artname

Arthrorhaphis citrinella

Bacidia bagliettoana

Baeomyces rufus

Buellia epigaea

Cetraria aculeata

Cetraria islandica

Cetraria muricata

Cladonia arbuscula

Cladonia arbuscula ssp. mitis

Cladonia arbuscula ssp. squarrosa

Cladonia cf. caespiticia

Cladonia cervicornis ssp. cervicornis

Cladonia cervicornis

Cladonia cervicornis ssp. verticillata

Cladonia ciliata

Cladonia coccifera

Cladonia coccifera/deformis s.1.

Cladonia coniocraea

Cladonia cf. cornuta

Cladonia crispata

Cladonia digitata

Cladonia fimbriata

Cladonia foliacea

Cladonia furcata ssp. furcata

Cladonia furcata ssp. subrangiformis

Cladonia glauca

Cladonia gracilis

Cladonia humilis

Cladonia macilenta ssp. floerkeana

Cladonia macilenta ssp. macilenta

Cladonia phyllophora

Cladonia pleurota

Cladonia polydactyla

Cladonia portentosa
Fundorte (s. Tab. 57)

OW

BÜ; AS*

RK; KB; MK; ZG; SC*; SE*; HS*; DT*; MI*; ME*; DB; CM; BD; OU; $\mathrm{KP}$; OW

$\mathrm{KR}$

SC; IG; HE; MI; DÖ; KL*; DB; BD; DS; BB; PT; OU

$\mathrm{BD}$; OU; EW

$\mathrm{MK}^{*}$

$\mathrm{MI}$; MK; BD; ME; BB

$\mathrm{BD}$

MK; BB; ME; MI; OW*

$\mathrm{SZ} * \mathrm{CM} ; \mathrm{GH}$

$\mathrm{KB}$; DT; $\mathrm{SE}^{*}$; DB*

RK; KB; DB; ME; MI; BB

RK; SE; HS*; DT*; BD

$\mathrm{GO}$; $\mathrm{DT}^{*} ; \mathrm{BD} ; \mathrm{MK} ; \mathrm{ME} ; \mathrm{KP}^{*} ; \mathrm{EW}^{*}$; OU

RK; HS; SE; KB; TE; BP; DB; MK; BA; BD; ME; MI; BB; KP; OU

DB; HS; SE; ME; OU

RK; SS; ZG; HS*; SE*; SN*; BK*; NT; BT

ZG; OW; KP

RK; HS*; BD*; OU

$\mathrm{RK}^{*} ; \mathrm{SE}^{*} ; \mathrm{CM}$

FT; GH; TE; ZG; SC*; KM*; BT; CM; NT; HG*; DB; GB*; MK*; BD; $\mathrm{EW}^{*} ; \mathrm{KP}$

$\mathrm{GM}$; DB; BA; BD*

RK; SC; KB; DT; HE; ZG; HS*; SE*; SB*; ME; MI; GM; DB; NT;

$\mathrm{MK}$; BD; SZ; DH*; BB; HH; KP; EW; OU; BA; TE; GB*

KA; IG; WB; WH; HO; SR; HE; BÜ; DÖ; KL; BK; HL; HK; SI; DH; DS;

DP; EI; RO*; WZ; PT

RK; HS; HH; SE*; ME*; NT; DB; BP; SZ; BD; BA; BB; MI

$\mathrm{RK} ; \mathrm{KB} ; \mathrm{BB} ; \mathrm{SC}^{*} ; \mathrm{ME}$; MI; DB; MK; BD; OU

$\mathrm{SS} ; \mathrm{ZG} ; \mathrm{BK}^{*} ; \mathrm{GH} ; \mathrm{NT}^{*}$; BD*

RK; HS; SE; KB; GH; TE; HH; ZG; ME; DT*; DB; NT; KM; BD; MK;

MI; BB; OU; KP; OW

HS; KB; GH; SS; TE; HH; ZG; SE*; BT; NT; MK; BD; ME; MI; BA; EW*; $\mathrm{KP}$; OW

$\mathrm{ME} ; \mathrm{DB} ; \mathrm{BD}^{*}$; MI; BB; MK

$\mathrm{HH}$

NT

RK; HH; SE*; DT*; MI*; KL*; MK; BD; ME; EW* 


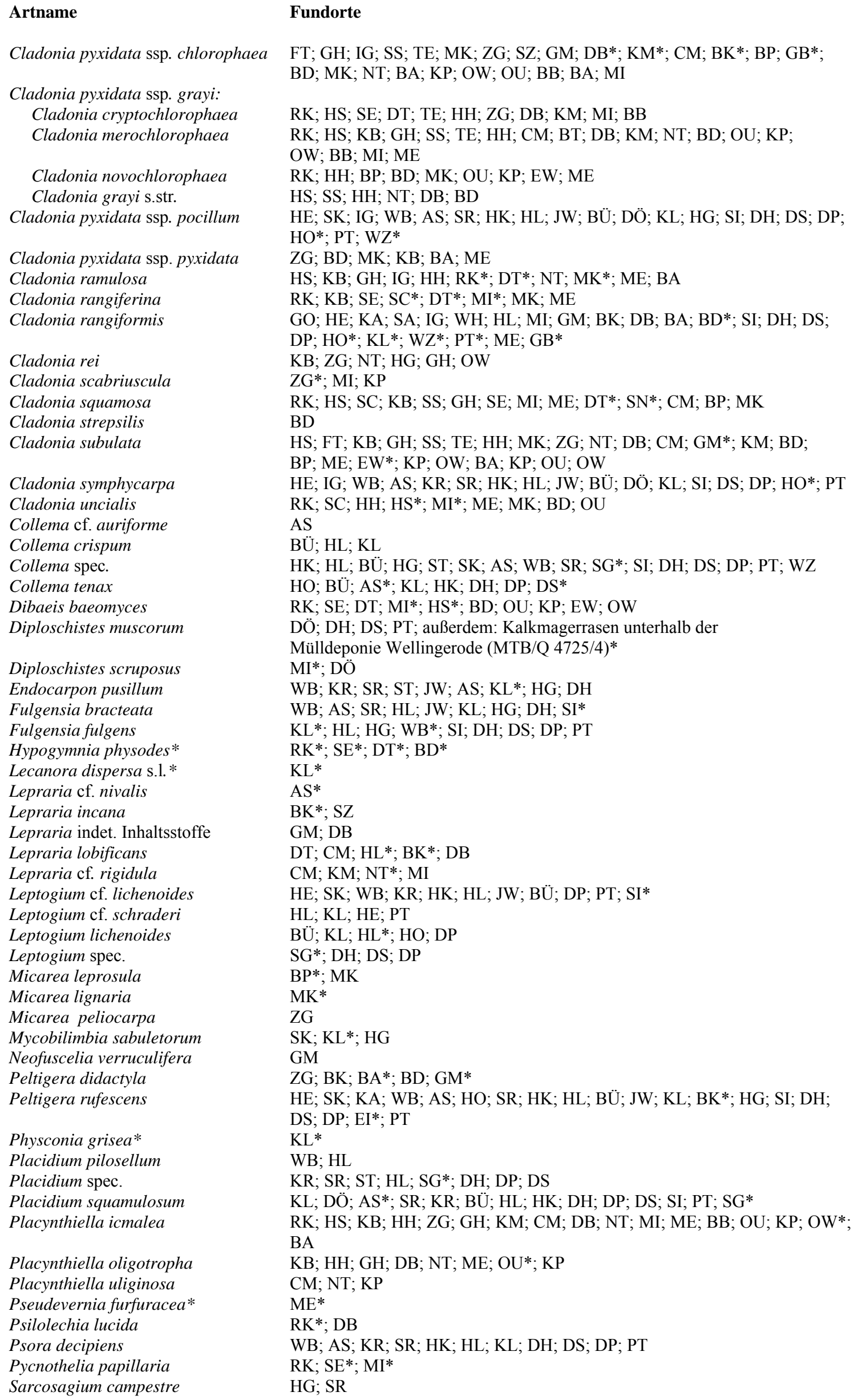




\section{Artname}

Solorina saccata*

Squamarina cartilaginea

Squamarina lentigera

Toninia sedifolia

Trapeliopsis flexuosa

Trapeliopsis gelatinosa

Trapeliopsis granulosa

Trapeliopsis pseudogranulosa

\section{Fundorte}

WB; KL

WB; AS; SR; HL; KL; DH; DP

DH

HE; SK; WB; AS; KR; SR; ST; HK; JW; BÜ; DÖ; KL; HG; HL; SG*; SI;

DH; DS; PT

$\mathrm{HS}^{*} ; \mathrm{SE}^{*} ; \mathrm{RK}^{*}$

$\mathrm{HS}^{*} ; \mathrm{RK}^{*} ; \mathrm{CM}$

RK; HS; KB; GH; SE*; DB; KM; BD; ME; MI; BB; EW*; KP; OU; OW*

RK; HS; SE*; KB*; DT*; DB; SZ; ME*

Eine Flechten-Artenliste der Wachholderheide bei Niederlemp findet sich in den Aktuellen Lichenologischen Mitteilungen NF 6 (ANONYMUS 2001).

\section{d) Belegeliste}

Die nachfolgend aufgeführten Belege wurden im Herbar Göttingen (GOET) bzw. im Herbar der Verfasserin (Günzl) hinterlegt. Darüber hinaus befinden sich im Herbar der Verfasserin einige weitere, meist für die Dünnschichtchromatographie verwendete Flechten-Proben. Da diese Proben jedoch nur aus Thallusbruchstücken, einzelnen Bechern oder Bruchstücken derselben bestehen, werden sie hier nicht explizit aufgelistet.

Arthrorhaphis citrinella: OW, 03.09.02 (GOET/Günzl)

Bacidia bagliettoana: AS, 15.06.00 (Günzl)

Baeomyces rufus: ZG, 06.04.99 (GOET/Günzl); RK, 19.05.99 (Günzl)

Cetraria aculeata: MI, 24.06.02 (GOET); IG, 04.08.99 (GOET)

Cladonia arbuscula ssp. mitis: BD, 08.04.02 (GOET)

Cladonia arbuscula ssp. squarrosa: ME, 24.06.02 (GOET)

Cladonia caespiticia: SZ, 27.04.00 (Günzl); CM, 28.04.00 (Günzl)

Cladonia cervicornis ssp. verticillata: HS, 20.05.99 (GOET)

Cladonia ciliata: EW, 03.09.02 (GOET); ME, 24.06.02 (Günzl)

Cladonia crispata: RK, 26.06 .98 (Günzl); HS, 1998 (Günzl)

Cladonia fimbriata: ZG, 06.09.99 (Günzl); FT, 13.08.99 (Günzl); RK, 19.05.99 (GOET)

Cladonia furcata ssp. furcata (für die morphologischen Untersuchungen (s. Kap. G) verwendete Thalli. In Klammern die Anzahl der Proben, sofern bei ausgedehnten Vorkommen mehrere Proben in einem Gebiet genommen wurden): Felsstandorte: MI (2+8), 07.03.1999+24.06.02; GM, 09.05.00; GS, 07.03.99; DB (4), 15.05.00; BA, 13.03.01; BB (5), 20.08.02; SC, 31.08.98; Heiden der Tieflagen: OW, 02.09.02; WN (3), 07.04.01; BD (2), 08.04.01; Waldränder: RK, 24.06.98+01.05.99; HS, 19.06.98; ZG (3+1), 06.09.99+07.09.99; OL (3), 07.04.01; TE, 14.06.98; KB, 21.07.99; KM, 28.04.00; Berglagen: EW (2), 03.09.02; KP (3), 02.09.02; MK (2), 21.11.00; OU, 02.09.02+03.09.02; Waldstandorte: ME (8), 24.06.02; RK (3), 26.06.98; DT, 22.07.99, 18.08.98; SB 1998; (alle Günzl)

Cladonia furcata ssp. subrangiformis: SF, 02.07 .01 (leg. C. Becker; Günzl)

Cladonia glauca: RK, 19.05.99 (Günzl); NT, 30.09.00 (GOET/Günzl); MI, 24.06.02 (Günzl)

Cladonia gracilis: DB, 15.05.00 (Günzl)

Cladonia humilis: OL, 07.04.01 (Günzl); ZG, 06.09 .99 (Günzl); GH, 21.03.00 (GOET)

Cladonia macilenta ssp. macilenta: HS, 20.05.99 (Chemotyp 1; Günzl); HH, 17.08.99 (Günzl)

Cladonia phyllophora: ME, 24.06.02 (Günzl)

Cladonia portentosa: EW, 03.09 .02 (GOET)

Cladonia pyxidata ssp. chlorophaea: NT, 30.09 .00 (GOET); GH, 21.03.00 (GOET)

Cladonia pyxidata ssp. grayi:

Cladonia cryptochlorophaea: TE, 17.08.99 (GOET)

Cladonia grayi s.str.: HS, 20.05.99 (GOET); NT, 30.09.00 (GOET)

Cladonia merochlorophaea: NT, 30.09.00 (GOET); BT, 18.05.00 (GOET)

Cladonia novochlorophaea: MK, 21.11.00 (GOET); BP, 28.04.00 (GOET)

Cladonia pyxidata ssp. pocillum: IG, 04.08.99 (GOET)

Cladonia pyxidata ssp. pyxidata: BD, 02.07.01 (GOET)

Cladonia ramulosa: KB, 21.07.99 (Günzl); OL, 07.04.01 (Günzl)

Cladonia rangiformis: IG, 04.08.99 (GOET); HK, 06.08.99 (GOET)

Cladonia rei: HG, 14.09.00 (Günzl); NT, 30.09 .00 (GOET) 
Cladonia scabriuscula: ZG, 06.09.99 (Günzl)

Cladonia subulata: FT, 13.08.99 (Günzl); GH, 21.07.99 (Günzl); MK, 01.09.99 (Günzl); HS, 01.05.99 (GOET) Cladonia symphycarpa: IG, 04.08 .99 (GOET)

Collema tenax: HK, 02.09.99 (Günzl); AS, 15.06.00 (Günzl); DH, 25.07.01 (Günzl); DS, 01.08.01 (GOET)

Dibaeis baeomyces: RK, 19.05.99 (GOET); DT, 22.07.99 (Günzl)

Diploschistes muscorum: Kalkmagerrasen unterhalb Mülldeponie Wellingerode (MTB/Q 4725/4) 24.08.99 (Günzl)

Endocarpon pusillum: WB, 06.08.99 (Günzl); GH, 14.09.00 (GOET)

Lepraria cf. nivalis: AS, 15.06.00 (Günzl)

Lepraria incana: SZ, 27.04.00 (Günzl)

Lepraria lobificans: DB, 26.04.00 (Günzl)

Lepraria rigidula: CM, 02.06.00 (GOET/Günzl); NT, 30.09.00 (Günzl); KM, 28.04.00 (Günzl)

Leptogium cf. schraderi: KR, 13.08.99 (Günzl)

Leptogium cf. lichenoides: HK, 02.09 .99 (Günzl)

Micarea leprosula: MK, 21.11.00 (Günzl)

Micarea lignaria: MK, 21.11.00 (Günzl)

Mycobilimbia sabuletorum: HG, 14.09 .00 (Günzl)

Neofuscelia verruculifera: GM, 09.05.00 (Günzl)

Peltigera didactyla: ZG, 03.09.99 (Günzl)

Peltigera rufescens: HE, 28.07.99 (Günzl); SR, 23.08.99 (Günzl); SK, 02.08.99 (GOET)

Placidium pilosellum: HL, 20.09.99 (GOET/Günzl); WB, 06.08.99 (Günzl)

Placidium squamulosum: AS, 15.06.00 (Günzl); DP, 01.08.01 (Günzl); SG, Feb.01 (Günzl)

Placynthiella icmalea: GH, 21.03.00 (Günzl); CM, 02.06.00 (Günzl); KP, 02.09.02 (GOET); OU, 02.09.02 (GOET)

Placynthiella oligotropha: NT, 30.09.00 (GOET); HH, 17.08.99 (GOET); GH, 21.03.00 (Günzl); DB, 15.05.00 (Günzl)

Placynthiella uliginosa: CM, 28.04.00 (Günzl)

Sarcosagium campestre: SR, 23.08.99 (GOET)

Squamarina cartilaginea: AS, 06.08 .99 (GOET)

Toninia sedifolia: HK, 02.09.99 (GOET/Günzl); AS, 06.08.99 (Günzl)

Trapeliopsis flexuosa: NT, 14.09.00 (GOET/Günzl)

Trapeliopsis gelatinosa: CM, 02.07.01 (GOET)

Trapeliopsis granulosa: GH, 21.03.00 (Günzl); CM, 28.04.00 (GOET)

Trapeliopsis pseudogranulosa: OL, 07.04.01 (GOET); RK, 19.05.99 (Günzl); DB, 07.04 .00 (Günzl) 


\section{f) Tabellen zur Fruchtkörperbildung}

Tab. 59: Stetigkeiten fruchtender Thalli (obere Hälfte) und Gesamtstetigkeiten (untere Hälfte, s. Tab. 24)

\begin{tabular}{|c|c|c|c|c|c|c|c|c|c|c|c|c|c|c|c|c|c|c|c|c|c|c|}
\hline & 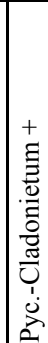 & 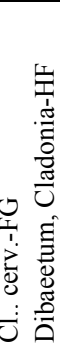 & 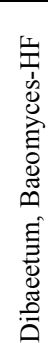 & 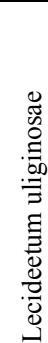 & 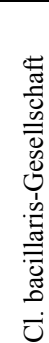 & 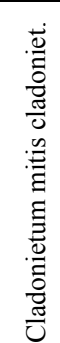 & 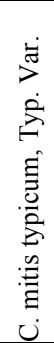 & 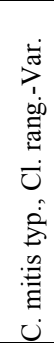 & 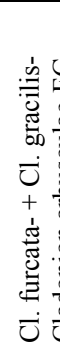 & & 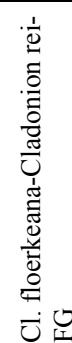 & 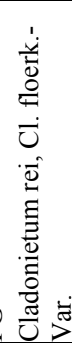 & 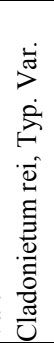 & 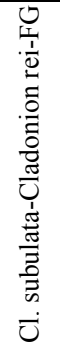 & 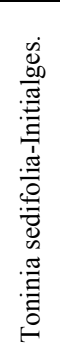 & 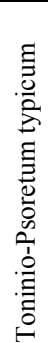 & 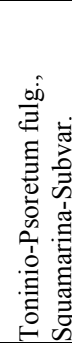 & 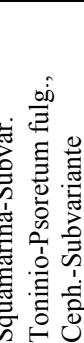 & 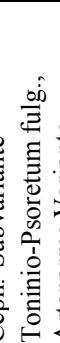 & 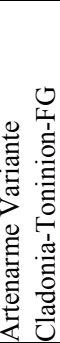 & 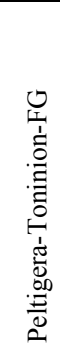 & 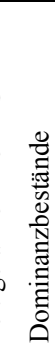 \\
\hline Cl. cervicornis & 2 & $\overline{\text { III }}$ & & & 0 & $\mathrm{I}$ & 4 & + & 0 & 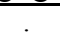 & & I & . & . & . & . & . & & . & . & . & \\
\hline Dibaeis baeomyces & 1 & III & II & 0 & . & 0 & . & . & . & ${ }^{\circ}$ & . & 0 & r & . & . & . & . & & $\cdot$ & . & r. & \\
\hline Baeomyces rufus & 0 & . & IV & I & 0 & 0 & . & . & . & 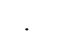 & 0 & $\mathrm{I}$ & & II & . & & . & & . & & r. & . \\
\hline Placynthiella icmalea & 0 & . & . & 0 & 0 & I & 0 & 0 & 0 & 0 & 0 & 0 & 0 & 0 & - & . & . & & . & & . & - \\
\hline $\mathrm{Cl}$. gracilis & 0 & . & - & . & 0 & III & III & III & II & I & . & 0 & . & . & . & . & . & 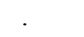 & . & . & . & \\
\hline $\mathrm{Cl}$. furcata ssp. furcata & 0 & 0 & . & . & 0 & + & 0 & III & II & I & . & 0 & . & . & . & . & . & . & . & 0 & . & - \\
\hline Cl. rangiformis & $v^{\circ}$ & . & - & . & . & . & . & 0 & 0 & I & & . & 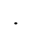 & . & . & 0 & 0 & . & . & 0 & 0 & + \\
\hline Cl. fimbriata & & . & 0 & 0 & 0 & & . & . & 0 & 0 & III & II & II & II & . & . & . & 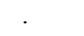 & . & . & . & 0 \\
\hline Cl. pyxidata ssp. chloroph. & 0 & . & + & I & . & 0 & 0 & . & + & I & . & IV & IV & II & . & . & . & . & . & 0 & . & 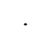 \\
\hline Trapeliopsis granulosa & 0 & . & . & . & 0 & II & . & 0 & 0 & . & I & 0 & . & . & . & . & . & . & . & . & . & . \\
\hline Cl. merochlorophaea & 0 & 0 & . & I & IV & II & 0 & + & 0 & 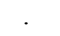 & II & III & . & . & . & . & . & . & . & . & $\cdot$ & . \\
\hline Cl. macil. ssp. floerkeana & 3 & II & 0 & I & $\mathrm{V}$ & III & I & I & I & 0 & $\mathrm{~V}$ & IV & . & . & . & . & . & . & . & . & $\cdot$ & \\
\hline Cl. macil. ssp. macilenta & 0 & . & . & II & IV & III & . & . & . & 0 & III & $\mathrm{V}$ & . & . & . & . & . & . & . & . & . & . \\
\hline ulata & 0 & 0 & 0 & . & II & 0 & - & . & 0 & 0 & I & II & 0 & II & . & . & . & . & . & ${ }^{\circ}$ & r & . \\
\hline Cl. $\operatorname{cocc}$ & 1 & II & . & . & II & III & . & + & II & I & . & II & - & . & . & 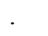 & . & . & $\cdot$ & r. & $\cdot$ & \\
\hline Cl. cryptochlorophaea & 0 & I & 0 & II & I & 0 & . & . & & . & . & I & I & . & . & 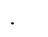 & . & . & . & . & . & \\
\hline Cl. glauca & 0 & . & . & . & 0 & 0 & . & 0 & 0 & 0 & . & . & . & . & . & & . & & . & . & . & \\
\hline Fulgensia fulgens & . & . & . & . & . & . & . & . & . & . & . & . & . & . & . & IV & I & I & III & . & . & . \\
\hline Fulgens & . & . & . & . & . & . & . & . & . & . & . & . & . & . & . & & $\mathrm{V}$ & $\mathrm{V}$ & $\mathrm{V}$ & . & . & \\
\hline Cl. sy & . & . & . & . & . & . & . & . & . & . & . & . & . & . & . & 0 & 0 & 0 & . & + & 0 & \\
\hline Toninia s & 相 & . & . & . & . & . & . & . & . & . & . & . & . & . & 2 & II & II & 0 & II & II & . & \\
\hline Psora decipiens & . & . & . & . & . & . & . & . & . & . & . & . & ${ }^{\circ}$ & . & . & III & III & I & . & I & . & \\
\hline Placidium squamulosum & & . & . & . & . & . & . & . & . & . & . & . & . & . & . & II & II & II & . & I & . & \\
\hline Peltigera rufescens & & . & . & . & . & . & . & . & . & . & . & . & . & . & . & 0 & 0 & 0 & 0 & 0 & 0 & \\
\hline Cl. pyxidata ssp. pocillum & . & . & . & . & . & . & . & . & . & . & . & . & . & . & . & 0 & + & 0 & 0 & + & I & 0 \\
\hline $\mathrm{Cl}$. furcata ssp. subrangif. & . & . & . & . & . & . & . & . & . & . & . & . & . & . & . & 0 & 0 & 0 & . & 0 & 0 & 0 \\
\hline Cl. cervicornis & 4 & $\mathrm{~V}$ & 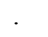 & . & I & II & . & + & + & . & . & I & . & . & . & . & . & . & . & . & . & . \\
\hline Dibaeis baeomyces & 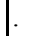 & V & $\mathrm{V}$ & I & . & + & . & . & . & . & . & I & . & . & . & . & . & . & . & . & . & \\
\hline Baeomy & 3 & . & $\mathrm{V}$ & III & + & + & . & . & . & . & + & II & . & II & . & . & . & . & . & . & . & r \\
\hline Placynthiella icmalea & 2 & . & . & III & II & II & I & + & + & I & I & II & I & I & . & . & . & . & . & . & . & . \\
\hline Cl. gracilis & 1 & . & . & . & + & IV & IV & IV & II & I & . & I & . & . & . & . & . & . & . & . & . & \\
\hline $\mathrm{Cl}$. furcata ssp. furcata & 2 & II & . & . & I & II & I & IV & $\mathrm{V}$ & IV & . & I & . & . & . & & 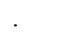 & . & . & + & . & \\
\hline Cl. rangiformis & . & . & . & . & . & . & . & I & + & $\mathrm{V}$ & . & . & . & . & . & II & I & . & . & II & III & III \\
\hline Cl. fimbriata & . & . & + & I & + & . & . & . & I & I & $\mathrm{V}$ & II & II & IV & . & . & . & . & . & . & . & + \\
\hline Cl. pyxidata ssp. chloroph. & 1 & . & I & II & . & I & I & . & II & II & . & $\mathrm{V}$ & $\mathrm{V}$ & IV & . & . & . & . & . & + & . & . \\
\hline Trapeliopsis granulosa & 3 & . & . & . & II & III & & + & II & . & IV & II & . & . & . & . & . & . & . & . & . & \\
\hline Cl. merochlorophaea & 1 & I & . & II & $\mathrm{V}$ & IV & II & II & II & . & IV & IV & . & . & . & 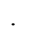 & . & . & . & . & . & \\
\hline Cl. macil. ssp. floerkeana & 4 & II & II & I & $\mathrm{V}$ & IV & II & II & I & I & $\mathrm{V}$ & IV & . & . & . & . & . & 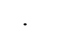 & . & . & . & \\
\hline Cl. macil. ssp. macilenta & 1 & . & . & II & $\mathrm{V}$ & III & & . & . & I & III & $\mathrm{V}$ & & . & . & 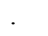 & . & 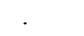 & . & . & . & \\
\hline Cl. subulata & 1 & I & I & 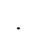 & IV & II & & . & I & I & IV & $\mathrm{V}$ & I & $\mathrm{V}$ & . & 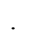 & 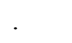 & . & . & 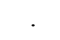 & . & \\
\hline Cl. coccifera s.str. & 4 & II & . & . & II & IV & & I & II & II & . & II & . & . & . & . & . & . & . & . & . & \\
\hline Cl. cryptochlorophaea & 1 & II & + & II & II & I & 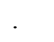 & . & . & . & . & I & I & . & . & . & . & . & . & . & . & \\
\hline Cl. glauca & 1 & . & . & . & II & III & & I & II & II & . & . & . & . & . & & . & . & . & . & . & \\
\hline Fulgensia fulgens & . & . & . & . & . & . & . & . & . & . & . & . & . & . & . & $\mathrm{V}$ & I & I & III & . & . & \\
\hline Fulgensia bracteata & . & . & . & . & . & . & . & . & . & 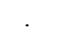 & . & . & . & . & . & . & $\mathrm{V}$ & $\mathrm{V}$ & $\mathrm{V}$ & . & 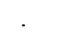 & \\
\hline Cl. symphycarpa & . & . & . & . & . & . & . & . & . & . & . & . & . & . & - & III & IV & IV & . & V & I & \\
\hline Toninia sedifolia & . & . & . & . & . & . & . & . & . & . & . & . & . & . & 3 & IV & III & III & II & IV & . & \\
\hline Psora decipiens & & . & . & . & . & 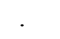 & . & . & . & . & . & . & . & . & . & III & IV & $\mathrm{V}$ & . & II & . & \\
\hline Placidium squamulosum & & . & . & . & . & 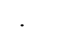 & . & . & . & 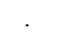 & . & . & . & . & . & $\mathrm{V}$ & III & $\mathrm{V}$ & . & II & . & \\
\hline Peltigera rufescens & . & . & . & . & . & 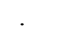 & . & . & . & . & . & . & . & . & . & $\mathrm{V}$ & $\mathrm{V}$ & I & I & IV & V & \\
\hline Cl. pyxidata ssp. pocillum & & . & . & & . & . & & . & . & . & . & . & 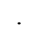 & . & . & III & IV & $\mathrm{V}$ & III & IV & II & + \\
\hline $\mathrm{Cl}$. furcata ssp. subrangif. & & . & & & & & & & & & & & & & & III & I & I & & IV & III & III \\
\hline
\end{tabular}


Tab. 60: Prozentualer Anteil der fruchtenden Thalli (s. Tab. 25)

\begin{tabular}{|c|c|c|c|c|c|c|c|c|c|c|c|c|c|c|c|c|c|c|c|c|c|c|c|}
\hline & 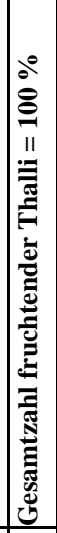 & 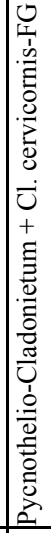 & 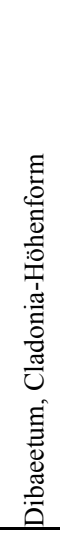 & 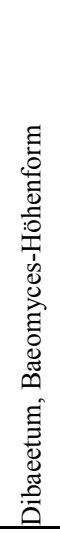 & 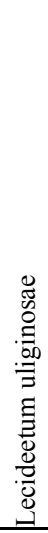 & 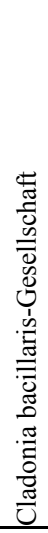 & 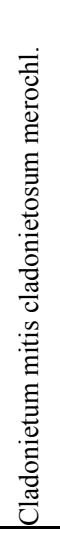 & 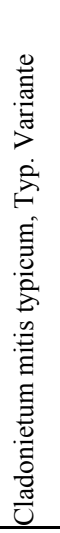 & 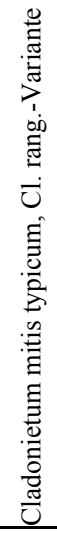 & 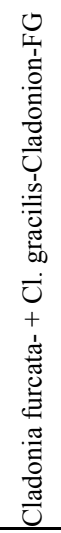 & 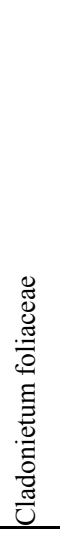 & 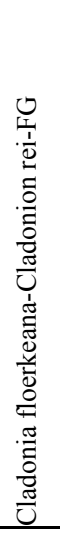 & 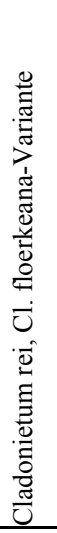 & 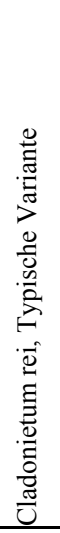 & 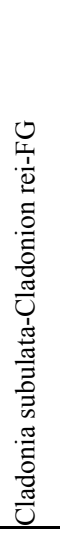 & 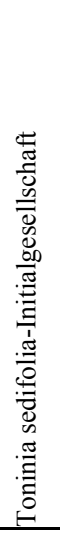 & 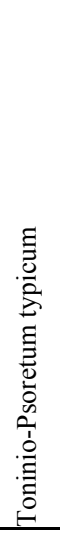 & 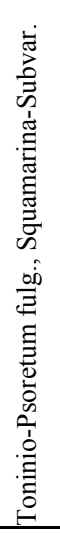 & 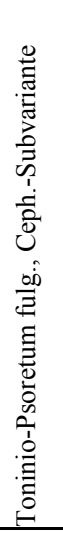 & 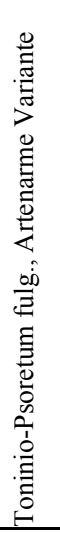 & 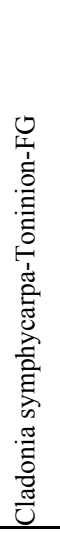 & 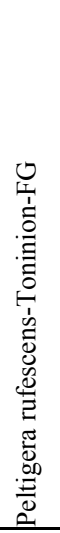 & 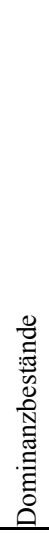 \\
\hline Cl. cervicornis & 9 & 22 & 33 & & & 0 & 22 & . & 11 & 0 & . & . & 11 &. & & & . & . & . & . & . & . &. \\
\hline Dibaeis & 7 & & 43 & 57 & 0 & & 0 & . & 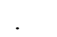 & . & . & - & 0 & - & & & . & 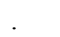 & . & . & . & $\cdot$ & \\
\hline Baeomyces rufus & 13 & 0 & . & 69 & 8 & 0 & 0 & . & - & & . & 0 & 8 & - & 15 & & . & . & . & . & . & . & . \\
\hline Placynthiella icmalea & 2 & 0 & . & . & 0 & 0 & 100 & 0 & 0 & 0 & 0 & 0 & 0 & 0 & 0 & & . & . & . & . & . & . & . \\
\hline Cl. gracilis & 18 & 0 & & 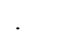 & 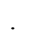 & 0 & 33 & 17 & 28 & 17 & 6 & 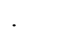 & 0 & 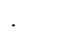 & & & 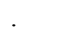 & . & . & & & & . \\
\hline $\mathrm{Cl}$. furcata ssp. furcata & 13 & 0 & 0 & . & . & 0 & 8 & 0 & 46 & 38 & 8 & . & 0 & - & . & & & & - & 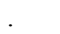 & 0 & & \\
\hline Cl. ra & 2 & & . & & & . & $\cdot$ & . & 0 & 0 & 50 & . & & . & & & 0 & 0 & . & . & 0 & 0 & 50 \\
\hline Cl. fimbriata & 11 & & . & 0 & 0 & 0 & & . & . & 0 & 0 & 45 & 18 & 18 & 18 & & . & . & . & . & . & . & 0 \\
\hline Cl. pyxidata ssp. chlor. & 17 & 0 & . & 6 & 6 & . & 0 & 0 & 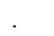 & 6 & 6 & 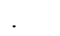 & 35 & 24 & 18 & & . & - & . & . & 0 & . & . \\
\hline Trapeliopsis granulosa & 5 & 0 & - & . & - & 0 & 60 & & 0 & 0 & . & 40 & 0 & . & . & & . & . & . & . & . & . & . \\
\hline Cl. merochlorophaea & 26 & 0 & 0 & & 4 & 46 & 15 & 0 & 4 & 0 & & 15 & 15 & - & 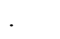 & & - & - & . & 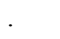 & . & . & . \\
\hline Cl. macilenta ssp. floerk. & 49 & 6 & 4 & 0 & 2 & 33 & 14 & 2 & 4 & 4 & 0 & 18 & 12 & . & & & . & . & . & . & . & . & . \\
\hline Cl. macilenta ssp. macil. & 36 & 0 & . & . & 6 & 39 & 17 & . & . & & 0 & 14 & 25 & & & & . & . & . & r. & . & & . \\
\hline Cl. subulata & 13 & 0 & 0 & 0 & . & 38 & 0 & . & . & 0 & 0 & 15 & 23 & 0 & 23 & & . & - & & 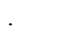 & . & . & . \\
\hline Cl. cocciferas & 24 & 4 & 8 & & . & 21 & 29 & . & 4 & 21 & 4 & . & 8 & . & . & 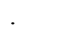 & $\cdot$ & $\cdot$ & 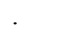 & . & . & $\cdot$ & . \\
\hline Cl. cryptochlorophaea & 7 & 0 & 14 & 0 & 29 & 29 & 0 & . & . & & . & . & 14 & 14 & . & & 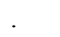 & . & . & . & . & . & . \\
\hline Cl. glauca & 0 & 0 & . & . & . & 0 & 0 & . & 0 & 0 & 0 & . & & . & & & & & & & . & & . \\
\hline Fulgensia $\mathrm{f}$ & 15 & & . & . & 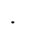 & . & . & . & . & . & . & . & & . & & & 47 & 20 & 7 & 27 & & & . \\
\hline Fulgensia bracteata & 29 & & . & - & 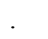 & . & & . & . & & . & . & & . & r & & . & 55 & 17 & 28 & & & 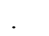 \\
\hline Cl. symphycarpa & 1 & & . & . & . & . & & . & . & & . & . & & . & . & & 0 & 0 & 0 & . & 100 & 0 & . \\
\hline Toninia sedifolia & 16 & & . & . & & . & & 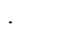 & . & & . & 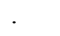 & & . & & 13 & 19 & 38 & 0 & 13 & 19 & & . \\
\hline Psor & 15 & & . & . & & 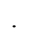 & & ${ }^{\circ}$ & . & & $\cdot$ & 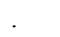 & & . & & . & 33 & 47 & 7 & 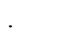 & 13 & & . \\
\hline Placidium squamulosum & 11 & & . & . & . & 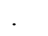 & & ${ }^{\circ}$ & . & & . & 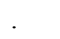 & & . & . & & 27 & 36 & 18 & & 18 & & . \\
\hline Peltigera rufescens & 0 & & . & . & & . & & 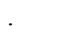 & . & & . & & & . & & & 0 & 0 & 0 & 0 & 0 & 0 & \\
\hline Cl. pyxidata ssp. poc. & 3 & & . & & & . & & $\cdot$ & $v^{\circ}$ & & . & . & & . & . & & 0 & 33 & 0 & 0 & 33 & 33 & 0 \\
\hline Cl. furcata ssp. subrang. & 0 & & & & & & & & & & & & & & & & 0 & 0 & 0 & & 0 & 0 & 0 \\
\hline
\end{tabular}




\section{g) Tabellen zu den Abhängigkeiten der morphologischen Merkmale von den Standortbedingungen}

\section{Anmerkung zu Tab. 61 bis Tab. 72:}

Es sind jeweils die Mittelwerte für die Standorttypen angegeben. Sie errechnen sich aus den Mittelwerten der einzelnen Polster, die wiederum aus den Werten aller untersuchter Podetien eines Polsters ermittelt wurden. Die Maximal- bzw. Minimalwerte, die an einzelnen Podetien gemessen wurden, sind nicht diesen Tabellen, sondern Kap. G3.1 zu entnehmen.

Erläuterungen der Abkürzungen in Tab. 61 bis Tab. 72:

$\begin{array}{lllll}\text { Standorttypen: } & \text { F } & \text { Felsen } & \text { Tritteinfluß: } & \text { sehr geringe mechanische Störung } \\ & \mathrm{H} & \text { Heiden tieferer Lagen } & & \text { mäßig starke mechanische Störung } \\ \mathrm{R} & \text { Waldränder } & & & \\ \mathrm{B} & \text { Berglagen } & & & \\ \mathrm{W} & \text { Wälder } & & \end{array}$

Quartile: $\mathrm{Q}_{1}$ (bei $25 \%$ aller Daten), $\mathrm{Q}_{2}$ (bei $75 \%$ aller Daten)

Tab. 61: Mittlere maximale Podetienlänge [mm]

\begin{tabular}{l|lllll|lll}
\hline & $F$ & $H$ & $R$ & $B$ & $W$ & $\bullet$ & $\bullet$ & $\bullet$ \\
& $n=23$ & $n=6$ & $n=13$ & $n=9$ & $n=14$ & $n=14$ & $n=31$ & $n=20$ \\
\hline MW & 36,6 & 51,7 & 43,2 & 48,3 & 45,7 & 48,4 & 43,1 & 38,7 \\
Median & 35,9 & 49,0 & 43,4 & 48,8 & 44,3 & 46,1 & 41,0 & 36,4 \\
Q $_{1}$ & 31,0 & 45,3 & 36,9 & 42,5 & 40,8 & 44,2 & 35,1 & 31,8 \\
Q $_{3}$ & 38,3 & 61,5 & 45,5 & 54,3 & 51,4 & 53,0 & 47,8 & 42,0 \\
Min. & 27,1 & 27,8 & 31,8 & 31,8 & 32,7 & 34,6 & 29,0 & 27,1 \\
Max. & 64,0 & 74,4 & 58,2 & 62,4 & 60,5 & 64,0 & 74,4 & 62,4 \\
Stabw. & 7,8 & 14,9 & 7,7 & 9,5 & 8,3 & 7,4 & 10,9 & 9,4 \\
\hline
\end{tabular}

Tab. 62: Mittlerer maximaler Podetiendurchmesser [mm]

\begin{tabular}{l|lllll|lll}
\hline & $F$ & $H$ & $R$ & $B$ & $W$ & $\bullet$ & $\bullet$ & $\bullet$ \\
& $n=23$ & $n=6$ & $n=13$ & $n=9$ & $n=14$ & $n=14$ & $n=31$ & $n=20$ \\
\hline MW & 1,3 & 1,3 & 1,4 & 1,2 & 1,4 & 1,3 & 1,5 & 1,1 \\
Median & 1,1 & 1,2 & 1,3 & 1,1 & 1,4 & 1,3 & 1,4 & 1,0 \\
Q $_{1}$ & 1,0 & 1,1 & 1,2 & 1,1 & 1,2 & 1,2 & 1,2 & 1,0 \\
Q $_{3}$ & 1,2 & 1,4 & 1,5 & 1,4 & 1,5 & 1,5 & 1,6 & 1,1 \\
Min. & 0,8 & 0,9 & 1,0 & 1,0 & 1,1 & 1,1 & 1,0 & 0,8 \\
Max. & 2,4 & 1,8 & 2,3 & 1,5 & 1,9 & 1,6 & 2,4 & 1,9 \\
Stabw. & 0,5 & 0,3 & 0,4 & 0,2 & 0,2 & 0,2 & 0,4 & 0,3 \\
\hline
\end{tabular}

Tab. 63: Mittlere Anzahl Verzweigungen der Podetien

\begin{tabular}{l|lllll|lll}
\hline & $F$ & $H$ & $R$ & $B$ & $W$ & $\bullet$ & $\bullet$ & $\bullet \bullet$ \\
& $n=23$ & $n=6$ & $n=13$ & $n=9$ & $n=14$ & $n=14$ & $n=31$ & $n=20$ \\
\hline MW & 5,6 & 7,1 & 6,9 & 5,0 & 6,3 & 6,0 & 6,4 & 5,6 \\
Median & 5,1 & 6,8 & 6,6 & 4,8 & 5,9 & 5,8 & 6,2 & 5,1 \\
$\mathrm{Q}_{1}$ & 4,5 & 5,7 & 6,2 & 4,6 & 5,4 & 5,2 & 5,0 & 4,7 \\
$\mathrm{Q}_{3}$ & 6,2 & 8,1 & 7,8 & 5,1 & 7,1 & 6,6 & 7,6 & 6,2 \\
Min. & 3,6 & 4,7 & 4,7 & 3,0 & 4,8 & 3,0 & 4,0 & 3,6 \\
Max. & 9,5 & 10,4 & 10,5 & 7,6 & 9,6 & 8,7 & 10,5 & 9,5 \\
Stabw. & 1,7 & 1,9 & 1,6 & 1,2 & 1,3 & 1,4 & 1,8 & 1,6 \\
\hline
\end{tabular}


Tab. 64: Mittlere Anzahl Zweige am Podetienende

\begin{tabular}{l|lllll|lll}
\hline & $F$ & $H$ & $R$ & $B$ & $W$ & $\bullet$ & $\bullet$ & $\bullet$ \\
& $n=23$ & $n=6$ & $n=13$ & $n=9$ & $n=14$ & $n=14$ & $n=31$ & $n=20$ \\
\hline MW & 5,2 & 5,7 & 5,7 & 4,9 & 4,6 & 4,7 & 5,7 & 4,8 \\
Median & 5,3 & 5,6 & 5,4 & 5,4 & 4,9 & 4,8 & 5,4 & 4,9 \\
Q $_{1}$ & 3,4 & 4,2 & 3,9 & 4,9 & 3,7 & 3,3 & 4,3 & 3,5 \\
Q $_{3}$ & 6,3 & 7,0 & 7,1 & 6,0 & 5,2 & 6,0 & 6,6 & 5,9 \\
Min. & 2,3 & 3,1 & 2,8 & 2,2 & 2,7 & 2,2 & 2,6 & 2,3 \\
Max. & 12,4 & 8,8 & 12,4 & 6,5 & 6,7 & 7,1 & 12,4 & 7,8 \\
Stabw. & 2,2 & 2,0 & 2,5 & 1,4 & 1,1 & 1,5 & 2,3 & 1,5 \\
\hline
\end{tabular}

Tab. 65: Mittlere maximale Anzahl Schuppen an den Podetien

\begin{tabular}{l|lllll|lll}
\hline & $F$ & $H$ & $R$ & $B$ & $W$ & $\bullet$ & $\bullet$ & $\bullet$ \\
& $n=23$ & $n=6$ & $n=13$ & $n=9$ & $n=14$ & $n=14$ & $n=31$ & $n=20$ \\
\hline MW & 4,0 & 0,5 & 3,6 & 6,8 & 17,0 & 9,5 & 5,8 & 6,4 \\
Median & 1,2 & 0,1 & 1,3 & 0,6 & 10,8 & 7,7 & 1,3 & 1,0 \\
$\mathrm{Q}_{1}$ & 0,4 & 0,0 & 0,0 & 0,0 & 6,7 & 0,2 & 0,4 & 0,0 \\
$\mathrm{Q}_{3}$ & 3,8 & 0,5 & 5,6 & 12,6 & 27,1 & 12,4 & 8,3 & 4,0 \\
Min. & 0,0 & 0,0 & 0,0 & 0,0 & 0,3 & 0,0 & 0,0 & 0,0 \\
Max. & 33,8 & 1,9 & 15,5 & 29,8 & 48,7 & 29,8 & 48,7 & 41,5 \\
Stabw. & 7,2 & 0,7 & 4,8 & 9,6 & 14,8 & 10,3 & 10,0 & 11,6 \\
\hline
\end{tabular}

Tab. 66: Mittlere maximale Größe der Schuppen an den Podetien [mm]

\begin{tabular}{l|lllll|lll}
\hline & $F$ & $H$ & $R$ & $B$ & $W$ & $\bullet$ & $\bullet$ & $\bullet$ \\
& $n=18$ & $n=3$ & $n=9$ & $n=6$ & $n=14$ & $n=10$ & $n=26$ & $n=14$ \\
\hline MW & 1,1 & 0,7 & 1,0 & 1,2 & 1,4 & 1,4 & 1,1 & 1,0 \\
Median & 1,0 & 0,7 & 1,0 & 1,1 & 1,2 & 1,4 & 1,0 & 0,9 \\
$\mathrm{Q}_{1}$ & 0,7 & 0,5 & 0,7 & 1,0 & 1,0 & 1,1 & 0,7 & 0,7 \\
$\mathrm{Q}_{3}$ & 1,3 & 0,8 & 1,3 & 1,1 & 1,8 & 1,8 & 1,4 & 1,1 \\
Min. & 0,2 & 0,3 & 0,5 & 0,6 & 0,6 & 0,6 & 0,3 & 0,2 \\
Max. & 2,7 & 1,0 & 1,6 & 2,3 & 2,4 & 2,3 & 2,7 & 2,4 \\
Stabw. & 0,6 & 0,3 & 0,4 & 0,5 & 0,5 & 0,5 & 0,6 & 0,5 \\
\hline
\end{tabular}

Tab. 67: Mittlere maximale Anzahl Apothecien

\begin{tabular}{l|lllll|lll}
\hline & $F$ & $H$ & $R$ & $B$ & $W$ & $\bullet$ & $\bullet$ & $\bullet$ \\
& $n=22$ & $n=6$ & $n=12$ & $n=9$ & $n=13$ & $n=14$ & $n=28$ & $n=20$ \\
\hline MW & 12,1 & 38,8 & 36,4 & 16,8 & 6,7 & 14,7 & 23,3 & 15,8 \\
Median & 7,2 & 36,8 & 5,2 & 16,3 & 1,9 & 2,0 & 8,9 & 7,8 \\
Q $_{1}$ & 0,1 & 8,8 & 0,0 & 0,9 & 0,0 & 0,3 & 0,0 & 0,0 \\
Q $_{3}$ & 20,7 & 70,3 & 88,4 & 28,8 & 4,2 & 18,0 & 34,3 & 24,6 \\
Min. & 0,0 & 0,0 & 0,0 & 0,0 & 0,0 & 0,0 & 0,0 & 0,0 \\
Max. & 43,8 & 78,2 & 115,7 & 45,8 & 33,9 & 103,3 & 115,7 & 84,0 \\
Stabw. & 13,8 & 33,2 & 46,6 & 16,0 & 10,4 & 27,5 & 32,4 & 20,7 \\
\hline
\end{tabular}

Tab. 68: Mittlere maximale Größe der Apothecien [mm]

\begin{tabular}{l|lllll|lll}
\hline & $F$ & $H$ & $R$ & $B$ & $W$ & $\bullet$ & $\bullet$ & $\bullet$ \\
& $n=16$ & $n=5$ & $n=8$ & $n=8$ & $n=11$ & $n=12$ & $n=22$ & $n=14$ \\
\hline MW & 0,2 & 0,5 & 0,3 & 0,2 & 0,2 & 0,2 & 0,3 & 0,3 \\
Median & 0,2 & 0,5 & 0,3 & 0,1 & 0,1 & 0,2 & 0,2 & 0,2 \\
$\mathrm{Q}_{1}$ & 0,1 & 0,4 & 0,2 & 0,1 & 0,1 & 0,1 & 0,1 & 0,1 \\
$\mathrm{Q}_{3}$ & 0,3 & 0,6 & 0,4 & 0,3 & 0,2 & 0,3 & 0,4 & 0,3 \\
Min. & 0,1 & 0,1 & 0,1 & 0,1 & 0,1 & 0,1 & 0,1 & 0,1 \\
Max. & 0,4 & 0,6 & 0,5 & 0,5 & 0,8 & 0,5 & 0,6 & 0,8 \\
Stabw. & 0,1 & 0,2 & 0,2 & 0,2 & 0,2 & 0,1 & 0,2 & 0,2 \\
\hline
\end{tabular}


Tab. 69: Mittlerer Anteil pyknidientragender Podetien [\%]

\begin{tabular}{l|lllll|lll}
\hline & $F$ & $H$ & $R$ & $B$ & $W$ & $\bullet$ & $\bullet$ & $\bullet$ \\
& $n=23$ & $n=6$ & $n=13$ & $n=9$ & $n=14$ & $n=14$ & $n=31$ & $n=20$ \\
\hline MW & 47,8 & 48,3 & 49,7 & 44,4 & 73,1 & 71,4 & 54,4 & 38,7 \\
Median & 50,0 & 40,0 & 40,0 & 50,0 & 80,0 & 80,0 & 50,0 & 36,7 \\
Q $_{1}$ & 15,0 & 40,0 & 30,0 & 10,0 & 60,0 & 60,0 & 30,0 & 17,5 \\
Q $_{3}$ & 80,0 & 47,5 & 80,0 & 60,0 & 100,0 & 90,0 & 90,0 & 60,0 \\
Min. & 0,0 & 30,0 & 0,0 & 0,0 & 10,0 & 20,0 & 0,0 & 0,0 \\
Max. & 100,0 & 90,0 & 100,0 & 100,0 & 100,0 & 100,0 & 100,0 & 100,0 \\
Stabw. & 34,4 & 19,5 & 32,3 & 34,4 & 29,0 & 24,7 & 34,2 & 30,7 \\
\hline
\end{tabular}

Tab. 70: Mittlerer Anteil mit zahlreichen Fibrillen besetzter Podetien [\%]

\begin{tabular}{l|lllll|lll}
\hline & $F$ & $H$ & $R$ & $B$ & $W$ & $\bullet$ & $\bullet$ & $\bullet$ \\
& $n=23$ & $n=6$ & $n=13$ & $n=9$ & $n=14$ & $n=14$ & $n=31$ & $n=20$ \\
\hline MW & 7,8 & 10,0 & 0,0 & 2,2 & 3,6 & 1,4 & 4,8 & 7,0 \\
Median & 0,0 & 0,0 & 0,0 & 0,0 & 0,0 & 0,0 & 0,0 & 0,0 \\
$\mathrm{Q}_{1}$ & 0,0 & 0,0 & 0,0 & 0,0 & 0,0 & 0,0 & 0,0 & 0,0 \\
$\mathrm{Q}_{3}$ & 10,0 & 0,0 & 0,0 & 0,0 & 0,0 & 0,0 & 0,0 & 0,0 \\
Min. & 0,0 & 0,0 & 0,0 & 0,0 & 0,0 & 0,0 & 0,0 & 0,0 \\
Max. & 50,0 & 60,0 & 0,0 & 20,0 & 50,0 & 20,0 & 60,0 & 50,0 \\
Stabw. & 14,7 & 22,4 & 0,0 & 6,3 & 12,9 & 5,2 & 13,9 & 15,5 \\
\hline
\end{tabular}

Tab. 71: Mittlerer Anteil stark pigmentierter Podetien [\%]

\begin{tabular}{l|lllll|lll}
\hline & $F$ & $H$ & $R$ & $B$ & $W$ & $\bullet$ & $\bullet$ & $\bullet$ \\
& $n=23$ & $n=6$ & $n=13$ & $n=9$ & $n=14$ & $n=14$ & $n=31$ & $n=20$ \\
\hline MW & 34,8 & 15,0 & 1,6 & 1,1 & 5,7 & 9,3 & 12,0 & 25,0 \\
Median & 30,0 & 0,0 & 0,0 & 0,0 & 0,0 & 0,0 & 0,0 & 0,0 \\
Q $_{1}$ & 0,0 & 0,0 & 0,0 & 0,0 & 0,0 & 0,0 & 0,0 & 0,0 \\
Q $_{3}$ & 65,0 & 0,0 & 0,0 & 0,0 & 7,5 & 17,5 & 0,0 & 40,0 \\
Min. & 0,0 & 0,0 & 0,0 & 0,0 & 0,0 & 0,0 & 0,0 & 0,0 \\
Max. & 100,0 & 90,0 & 11,0 & 10,0 & 30,0 & 40,0 & 100,0 & 100,0 \\
Stabw. & 36,9 & 33,5 & 3,8 & 3,1 & 9,8 & 12,8 & 27,4 & 35,8 \\
\hline
\end{tabular}

Tab. 72: Mittlerer Anteil Podetien mit mehr als $75 \%$ deckender Algenschicht [\%]

\begin{tabular}{l|lllll|lll}
\hline & $F$ & $H$ & $R$ & $B$ & $W$ & $\bullet$ & $\bullet$ & $\bullet$ \\
& $n=23$ & $n=6$ & $n=13$ & $n=9$ & $n=14$ & $n=14$ & $n=31$ & $n=20$ \\
\hline MW & 34,8 & 5,0 & 30,2 & 23,3 & 41,7 & 34,3 & 33,3 & 25,2 \\
Median & 30,0 & 0,0 & 13,0 & 20,0 & 47,0 & 20,0 & 30,0 & 15,0 \\
$\mathrm{Q}_{1}$ & 10,0 & 0,0 & 0,0 & 10,0 & 12,5 & 10,0 & 10,0 & 0,0 \\
$\mathrm{Q}_{3}$ & 50,0 & 7,5 & 50,0 & 30,0 & 60,0 & 60,0 & 50,0 & 41,0 \\
Min. & 0,0 & 0,0 & 0,0 & 0,0 & 10,0 & 0,0 & 0,0 & 0,0 \\
Max. & 100,0 & 20,0 & 100,0 & 50,0 & 80,0 & 80,0 & 100,0 & 100,0 \\
Stabw. & 27,6 & 7,6 & 33,2 & 17,6 & 26,4 & 29,7 & 27,2 & 27,4 \\
\hline
\end{tabular}




\section{h) Tabellen zu den Abhängigkeiten und Korrelationen der morphologischen Merkmale untereinander}

Abkürzungen in Tab. 73 bis

Tab. 77:

$\begin{array}{llll}\text { PL } & \text { Podetienlänge } & \text { AS } & \text { Anzahl Schuppen } \\ \text { PD } & \text { Podetiendurchmesser } & \text { GS } & \text { Größe der Schuppen } \\ \text { ZV } & \text { Anzahl Verzweigungen am Podetienende } & \text { AA } & \text { Anzahl Apothecien } \\ \text { ZP } & \text { Anzahl Zweige am Podetienende } & \text { GA } & \text { Größe Apothecien }\end{array}$

Quartile: $\mathrm{Q}_{1}$ (bei $25 \%$ aller Daten), $\mathrm{Q}_{2}$ (bei $75 \%$ aller Daten)

Tab. 73: Wertetabelle für fruchtende bzw. sterile Podetien

\begin{tabular}{|c|c|c|c|c|c|c|c|}
\hline fertil & & $\begin{array}{l}P L \\
n=306\end{array}$ & $\begin{array}{l}P D \\
n=306\end{array}$ & $\begin{array}{l}Z V \\
n=306\end{array}$ & $\begin{array}{l}Z P \\
n=306\end{array}$ & $\begin{array}{l}A S \\
n=306\end{array}$ & $\begin{array}{l}G S \\
n=104\end{array}$ \\
\hline & MW & 44,9 & 1,3 & 6,5 & 6,7 & 4,8 & 1,1 \\
\hline & Median & 43,0 & 1,2 & 6,0 & 6,0 & 0,0 & 1,0 \\
\hline & $\mathrm{Q}_{1}$ & 35,0 & 1,0 & 5,0 & 4,0 & 0,0 & 0,7 \\
\hline & $\mathrm{Q}_{3}$ & 52,0 & 1,6 & 8,0 & 8,0 & 3,0 & 1,4 \\
\hline & Min. & 15,0 & 0,6 & 2,0 & 1,0 & 0,0 & 0,1 \\
\hline & Max. & 92,0 & 3,2 & 14,0 & 30,0 & 67,0 & 4,2 \\
\hline & Stabw. & 13,7 & 0,5 & 2,3 & 3,4 & 10,7 & 0,6 \\
\hline steril & & $\begin{array}{l}P L \\
n=316\end{array}$ & $\begin{array}{l}P D \\
n=317\end{array}$ & $\begin{array}{l}Z V \\
n=317\end{array}$ & $\begin{array}{l}Z P \\
n=314\end{array}$ & $\begin{array}{l}\text { AS } \\
n=317\end{array}$ & $\begin{array}{l}G S \\
n=184\end{array}$ \\
\hline & MW & 41,2 & 1,3 & 5,5 & 3,7 & 9,0 & 1,4 \\
\hline & Median & 40,0 & 1,2 & 5,0 & 3,0 & 2,0 & 1,2 \\
\hline & $\mathrm{Q}_{1}$ & 32,0 & 1,0 & 4,0 & 2,0 & 0,0 & 0,8 \\
\hline & $\mathrm{Q}_{3}$ & 49,0 & 1,5 & 7,0 & 5,0 & 13,0 & 1,9 \\
\hline & Min. & 16,0 & 0,4 & 1,0 & 1,0 & 0,0 & 0,1 \\
\hline & Max. & 79,0 & 3,1 & 13,0 & 12,0 & 85,0 & 4,1 \\
\hline & Stabw. & 11,7 & 0,4 & 1,9 & 1,8 & 14,8 & 0,9 \\
\hline
\end{tabular}

Tab. 74: Wertetabelle für Podetien mit bzw. ohne Pyknidien

\begin{tabular}{ll|llllllll}
\hline Pyknidien vorhanden & & $P L$ & $P D$ & $Z V$ & $Z P$ & $A S$ & $G S$ & $A A$ & $G A$ \\
& & $n=337$ & $n=337$ & $n=337$ & $n=336$ & $n=337$ & $n=165$ & $n=310$ & $n=137$ \\
\hline & MW & 43,4 & 1,4 & 6,1 & 5,2 & 7,9 & 1,5 & 15,1 & 0,3 \\
& Median & 41,0 & 1,3 & 6,0 & 5,0 & 0,0 & 1,3 & 0,0 & 0,2 \\
& $Q_{1}$ & 33,0 & 1,0 & 5,0 & 3,0 & 0,0 & 0,9 & 0,0 & 0,1 \\
& $Q_{3}$ & 51,0 & 1,6 & 7,0 & 7,0 & 9,0 & 2,0 & 14,5 & 0,3 \\
& Min. & 18,0 & 0,6 & 1,0 & 1,0 & 0,0 & 0,1 & 0,0 & 0,1 \\
& Max. & 92,0 & 3,1 & 14,0 & 18,0 & 85,0 & 4,2 & 229,0 & 2,0 \\
& Stabw. & 13,6 & 0,5 & 2,1 & 2,7 & 14,5 & 0,9 & 32,4 & 0,3 \\
\hline Pyknidien fehlend & $P L$ & $P D$ & $Z V$ & $Z P$ & $A S$ & $G S$ & $A A$ & $G A$ \\
& & $n=285$ & $n=286$ & $n=286$ & $n=284$ & $n=286$ & $n=123$ & $n=229$ & $n=159$ \\
\hline & MW & 42,5 & 1,3 & 5,9 & 5,2 & 5,8 & 1,1 & 25,5 & 0,3 \\
& Median & 42,0 & 1,2 & 5,0 & 4,0 & 0,0 & 1,0 & 0,0 & 0,2 \\
& Q & 34,0 & 1,0 & 4,0 & 3,0 & 0,0 & 0,7 & 0,0 & 0,1 \\
& $Q_{3}$ & 50,0 & 1,5 & 7,0 & 6,0 & 7,0 & 1,4 & 35,0 & 0,5 \\
& Min. & 15,0 & 0,4 & 1,0 & 1,0 & 0,0 & 0,1 & 0,0 & 0,1 \\
& Max. & 85,0 & 3,2 & 13,0 & 30,0 & 68,0 & 3,0 & 280,0 & 1,4 \\
& Stabw. & 11,9 & 0,4 & 2,3 & 3,4 & 11,3 & 0,6 & 44,8 & 0,3 \\
\hline
\end{tabular}


Tab. 75: Wertetabelle für Podetien ohne, mit wenigen bzw. mit zahlreichen Fibrillen

\begin{tabular}{|c|c|c|c|c|c|c|c|c|c|}
\hline fehlend (bis max. 5) & & $\begin{array}{l}P L \\
n=485\end{array}$ & $\begin{array}{l}P D \\
n=486\end{array}$ & $\begin{array}{l}Z V \\
n=486\end{array}$ & $\begin{array}{l}Z P \\
n=484\end{array}$ & $\begin{array}{l}A S \\
n=486\end{array}$ & $\begin{array}{l}G S \\
n=230\end{array}$ & $\begin{array}{l}A A \\
n=423\end{array}$ & $\begin{array}{l}G A \\
n=248\end{array}$ \\
\hline & MW & 42,9 & 1,3 & 5,8 & 5,4 & 6,1 & 1,3 & 22,7 & 0,3 \\
\hline & Median & 41,0 & 1,2 & 5,0 & 5,0 & 0,0 & 1,1 & 0,0 & 0,3 \\
\hline & $\mathrm{Q}_{1}$ & 33,0 & 1,0 & 4,0 & 3,0 & 0,0 & 0,7 & 0,0 & 0,1 \\
\hline & $\mathrm{Q}_{3}$ & 51,0 & 1,5 & 7,0 & 7,0 & 8,0 & 1,7 & 27,0 & 0,4 \\
\hline & Min. & 15,0 & 0,4 & 1,0 & 1,0 & 0,0 & 0,1 & 0,0 & 0,1 \\
\hline & Max. & 92,0 & 3,2 & 12,0 & 30,0 & 85,0 & 4,2 & 280,0 & 2,0 \\
\hline & Stabw. & 13,1 & 0,5 & 2,1 & 3,1 & 11,3 & 0,8 & 41,8 & 0,3 \\
\hline \multirow[t]{9}{*}{ wenige } & & $P L$ & $P D$ & $Z V$ & $Z P$ & $A S$ & $G S$ & $A A$ & $G A$ \\
\hline & & $n=110$ & $n=110$ & $n=110$ & $n=109$ & $n=110$ & $n=52$ & $n=89$ & $n=37$ \\
\hline & $\mathrm{MW}$ & 43,2 & 1,3 & 6,4 & 4,4 & 11,5 & 1,3 & 6,3 & 0,1 \\
\hline & Median & 42,0 & 1,2 & 6,0 & 4,0 & 0,0 & 1,1 & 0,0 & 0,1 \\
\hline & $\mathrm{Q}_{1}$ & 36,0 & 1,0 & 5,0 & 2,0 & 0,0 & 0,8 & 0,0 & 0,1 \\
\hline & $\mathrm{Q}_{3}$ & 49,0 & 1,5 & 8,0 & 5,0 & 14,0 & 1,8 & 0,0 & 0,1 \\
\hline & Min. & 18,0 & 0,6 & 1,0 & 1,0 & 0,0 & 0,2 & 0,0 & 0,1 \\
\hline & Max. & 88,0 & 2,5 & 14,0 & 18,0 & 77,0 & 3,5 & 107,0 & 0,6 \\
\hline & Stabw. & 12,0 & 0,4 & 2,3 & 2,9 & 19,3 & 0,9 & 17,6 & 0,1 \\
\hline \multirow[t]{8}{*}{ zahlreich } & & $\begin{array}{l}P L \\
n=27\end{array}$ & $\begin{array}{l}P D \\
n=27\end{array}$ & $\begin{array}{l}Z V \\
n=27\end{array}$ & $\begin{array}{l}Z P \\
n=27\end{array}$ & $\begin{array}{l}A S \\
n=27\end{array}$ & $\begin{array}{l}G S \\
n=6\end{array}$ & $\begin{array}{l}A A \\
n=27\end{array}$ & $\begin{array}{l}G A \\
n=11\end{array}$ \\
\hline & MW & 43,3 & 1,5 & 7,8 & 5,1 & 2,8 & 1,1 & 12,8 & 0,2 \\
\hline & Median & 42,0 & 1,5 & 7,0 & 4,0 & 0,0 & 1,1 & 0,0 & 0,1 \\
\hline & $\mathrm{Q}_{1}$ & 34,5 & 1,3 & 6,0 & 3,0 & 0,0 & 0,8 & 0,0 & 0,1 \\
\hline & $\mathrm{Q}_{3}$ & 47,0 & 1,7 & 9,5 & 7,0 & 0,0 & 1,3 & 23,0 & 0,2 \\
\hline & Min. & 26,0 & 1,0 & 4,0 & 2,0 & 0,0 & 0,7 & 0,0 & 0,1 \\
\hline & Max. & 73,0 & 3,0 & 13,0 & 10,0 & 38,0 & 1,6 & 94,0 & 0,6 \\
\hline & Stabw. & 11,7 & 0,4 & 2,7 & 2,5 & 7,8 & 0,3 & 21,4 & 0,2 \\
\hline
\end{tabular}

Tab. 76: Wertetabelle für nicht, wenig und stark pigmentierte Podetien (Forts. S. 232)

\begin{tabular}{cl|llllllll}
\hline nicht gebräunt & & $P L$ & $P D$ & $Z V$ & $Z P$ & $A S$ & $G S$ & $A A$ & $G A$ \\
& & $n=198$ & $n=198$ & $n=198$ & $n=198$ & $n=198$ & $n=136$ & $n=169$ & $n=95$ \\
\hline MW & 43,8 & 1,4 & 6,0 & 4,7 & 14,0 & 1,4 & 16,3 & 0,3 \\
& Median & 42,0 & 1,3 & 6,0 & 4,0 & 6,0 & 1,2 & 0,0 & 0,1 \\
& $Q_{1}$ & 34,3 & 1,1 & 5,0 & 3,0 & 0,0 & 0,8 & 0,0 & 0,1 \\
& $Q_{3}$ & 51,0 & 1,6 & 7,0 & 6,0 & 19,8 & 1,8 & 19,0 & 0,4 \\
& Min. & 19,0 & 0,5 & 1,0 & 1,0 & 0,0 & 0,1 & 0,0 & 0,1 \\
& Max. & 85,0 & 3,0 & 14,0 & 15,0 & 85,0 & 4,2 & 145,0 & 1,5 \\
& Stabw. & 12,4 & 0,4 & 2,3 & 2,6 & 18,4 & 0,8 & 30,1 & 0,3 \\
\hline leicht gebräunt & $P L$ & $P D$ & $Z V$ & $Z P$ & $A S$ & $G S$ & $A A$ & $G A$ \\
& & $n=333$ & $n=333$ & $n=333$ & $n=331$ & $n=333$ & $n=123$ & $n=294$ & $n=166$ \\
\hline & MW & 43,9 & 1,3 & 6,0 & 5,4 & 3,7 & 1,3 & 24,2 & 0,3 \\
& Median & 43,0 & 1,2 & 6,0 & 5,0 & 0,0 & 1,1 & 0,0 & 0,2 \\
& $Q_{1}$ & 34,0 & 1,0 & 5,0 & 3,0 & 0,0 & 0,7 & 0,0 & 0,1 \\
& $Q_{3}$ & 51,0 & 1,6 & 7,0 & 7,0 & 4,0 & 1,7 & 27,0 & 0,4 \\
& Min. & 16,0 & 0,4 & 2,0 & 2,0 & 0,0 & 0,2 & 0,0 & 0,1 \\
& Max. & 92,0 & 3,2 & 12,0 & 24,0 & 53,0 & 4,0 & 280,0 & 2,0 \\
Stabw. & 13,3 & 0,5 & 2,1 & 3,0 & 7,8 & 0,8 & 45,3 & 0,3
\end{tabular}


Forts. Tab. 76: Wertetabelle für nicht, wenig und stark pigmentierte Podetien

\begin{tabular}{ll|llllllll}
\hline stark gebräunt & & $P L$ & $P D$ & $Z V$ & $Z P$ & $A S$ & $G S$ & $A A$ & $G A$ \\
& & $n=91$ & $n=92$ & $n=92$ & $n=91$ & $n=92$ & $n=29$ & $n=76$ & $n=35$ \\
\hline MW & 37,7 & 1,2 & 5,9 & 5,6 & 3,5 & 0,9 & 8,5 & 0,2 \\
& Median & 37,0 & 1,1 & 6,0 & 4,0 & 0,0 & 0,8 & 0,0 & 0,1 \\
& $\mathrm{Q}_{1}$ & 30,0 & 1,0 & 4,0 & 3,0 & 0,0 & 0,7 & 0,0 & 0,1 \\
& $\mathrm{Q}_{3}$ & 43,0 & 1,3 & 7,0 & 7,0 & 2,0 & 1,1 & 7,3 & 0,3 \\
& Min. & 15,0 & 0,6 & 2,0 & 2,0 & 0,0 & 0,1 & 0,0 & 0,1 \\
& Max. & 79,0 & 2,0 & 13,0 & 30,0 & 40,0 & 2,5 & 81,0 & 0,7 \\
& Stabw. & 10,9 & 0,3 & 2,2 & 4,1 & 7,8 & 0,6 & 18,0 & 0,2 \\
\hline
\end{tabular}

Tab. 77: Wertetabelle für Podetien mit unterschiedlicher Ausdehnung der Algenschicht

\begin{tabular}{|c|c|c|c|c|c|c|c|c|c|}
\hline bis $25 \%$ Deckung & & $\begin{array}{l}P L \\
n=2\end{array}$ & $\begin{array}{l}P D \\
n=2\end{array}$ & $\begin{array}{l}Z V \\
n=2\end{array}$ & $\begin{array}{l}Z P \\
n=2\end{array}$ & $\begin{array}{l}A S \\
n=2\end{array}$ & $G S$ & $\begin{array}{l}A A \\
n=2\end{array}$ & $G A$ \\
\hline & MW & 34,5 & 2,0 & 3,5 & 6,5 & 0,0 & - & 0,0 & - \\
\hline & Median & 34,5 & 2,0 & 3,5 & 6,5 & 0,0 & - & 0,0 & - \\
\hline & $\mathrm{Q}_{1}$ & 33,8 & 1,5 & 2,8 & 5,3 & 0,0 & - & 0,0 & - \\
\hline & $\mathrm{Q}_{3}$ & 35,3 & 2,5 & 4,3 & 7,8 & 0,0 & - & 0,0 & - \\
\hline & Min. & 33,0 & 1,0 & 2,0 & 4,0 & 0,0 & - & 0,0 & - \\
\hline & Max. & 36,0 & 3,0 & 5,0 & 9,0 & 0,0 & - & 0,0 & - \\
\hline & Stabw. & 1,5 & 1,0 & 1,5 & 2,5 & 0,0 & - & 0,0 & - \\
\hline \multirow[t]{8}{*}{ bis $50 \%$ Deckung } & & $\begin{array}{l}P L \\
n=58\end{array}$ & $\begin{array}{l}P D \\
n=58\end{array}$ & $\begin{array}{l}Z V \\
n=58\end{array}$ & $\begin{array}{l}Z P \\
n=57\end{array}$ & $\begin{array}{l}A S \\
n=58\end{array}$ & $\begin{array}{l}G S \\
n=25\end{array}$ & $\begin{array}{l}A A \\
n=46\end{array}$ & $\begin{array}{l}G A \\
n=30\end{array}$ \\
\hline & MW & 41,5 & 1,3 & 5,7 & 5,5 & 3,9 & 1,1 & 17,2 & 0,3 \\
\hline & Median & 41,0 & 1,2 & 5,5 & 5,0 & 0,0 & 1,0 & 0,0 & 0,2 \\
\hline & $\mathrm{Q}_{1}$ & 33,3 & 1,0 & 4,0 & 3,0 & 0,0 & 0,7 & 0,0 & 0,1 \\
\hline & $\mathrm{Q}_{3}$ & 49,8 & 1,5 & 6,0 & 7,0 & 6,0 & 1,5 & 19,3 & 0,4 \\
\hline & Min. & 15,0 & 0,6 & 2,0 & 2,0 & 0,0 & 0,2 & 0,0 & 0,1 \\
\hline & Max. & 65,0 & 3,1 & 12,0 & 11,0 & 44,0 & 2,3 & 112,0 & 1,0 \\
\hline & Stabw. & 10,6 & 0,5 & 2,0 & 2,5 & 7,7 & 0,6 & 29,4 & 0,2 \\
\hline \multirow[t]{8}{*}{ bis $75 \%$ Deckung } & & $\begin{array}{l}P L \\
n=367\end{array}$ & $\begin{array}{l}P D \\
n=368\end{array}$ & $\begin{array}{l}Z V \\
n=368\end{array}$ & $\begin{array}{l}Z P \\
n=367\end{array}$ & $\begin{array}{l}A S \\
n=368\end{array}$ & $\begin{array}{l}G S \\
n=165\end{array}$ & $\begin{array}{l}A A \\
n=317\end{array}$ & $\begin{array}{l}G A \\
n=186\end{array}$ \\
\hline & MW & 42,4 & 1,3 & 6,0 & 5,0 & 7,3 & 1,3 & 19,5 & 0,3 \\
\hline & Median & 41,0 & 1,2 & 6,0 & 4,0 & 0,0 & 1,0 & 0,0 & 0,2 \\
\hline & $\mathrm{Q}_{1}$ & 32,5 & 1,0 & 5,0 & 3,0 & 0,0 & 0,7 & 0,0 & 0,1 \\
\hline & $\mathrm{Q}_{3}$ & 50,0 & 1,5 & 7,0 & 6,0 & 9,3 & 1,7 & 27,0 & 0,4 \\
\hline & Min. & 16,0 & 0,4 & 1,0 & 1,0 & 0,0 & 0,1 & 0,0 & 0,1 \\
\hline & Max. & 92,0 & 2,9 & 13,0 & 30,0 & 85,0 & 4,2 & 240,0 & 2,0 \\
\hline & Stabw. & 13,5 & 0,4 & 2,1 & 2,9 & 13,8 & 0,8 & 35,1 & 0,3 \\
\hline \multirow[t]{8}{*}{ > $75 \%$ Deckung } & & \begin{tabular}{|l}
$P L$ \\
$n=195$
\end{tabular} & $\begin{array}{l}P D \\
n=195\end{array}$ & $\begin{array}{l}Z V \\
n=195\end{array}$ & $\begin{array}{l}Z P \\
n=194\end{array}$ & $\begin{array}{l}\text { AS } \\
n=195\end{array}$ & $\begin{array}{l}G S \\
n=98\end{array}$ & $\begin{array}{l}A A \\
n=175\end{array}$ & $\begin{array}{l}G A \\
n=79\end{array}$ \\
\hline & MW & 44,5 & 1,4 & 6,2 & 5,5 & 7,1 & 1,4 & 20,3 & 0,3 \\
\hline & Median & 42,0 & 1,3 & 6,0 & 5,0 & 1,0 & 1,3 & 0,0 & 0,3 \\
\hline & $\mathrm{Q}_{1}$ & 36,0 & 1,1 & 5,0 & 3,0 & 0,0 & 0,9 & 0,0 & 0,1 \\
\hline & $\mathrm{Q}_{3}$ & 51,0 & 1,6 & 8,0 & 7,0 & 9,0 & 1,8 & 18,0 & 0,4 \\
\hline & Min. & 22,0 & 0,7 & 2,0 & 1,0 & 0,0 & 0,1 & 0,0 & 0,1 \\
\hline & Max. & 88,0 & 3,2 & 14,0 & 24,0 & 77,0 & 4,1 & 280,0 & 1,5 \\
\hline & Stabw. & 12,1 & 0,5 & 2,3 & 3,5 & 13,1 & 0,8 & 45,9 & 0,3 \\
\hline
\end{tabular}




\section{O Anhang 2: Bildtafeln}

\section{Bildtafel 1: Erdflechtengesellschaften}

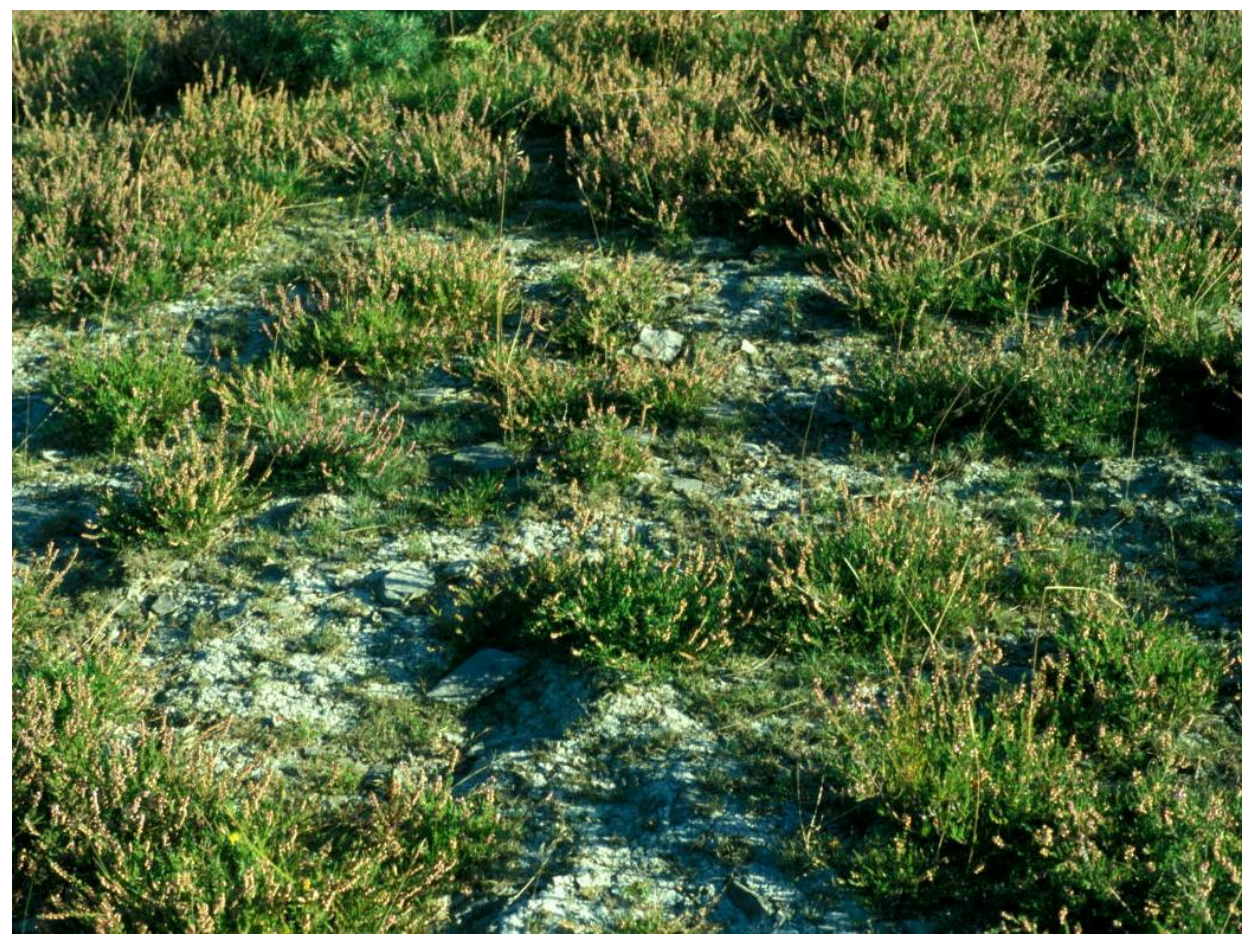

Taf. 1.1: Dibaeetum baeomycetis zwischen Calluna vulgaris am Osterkopf bei Usseln.

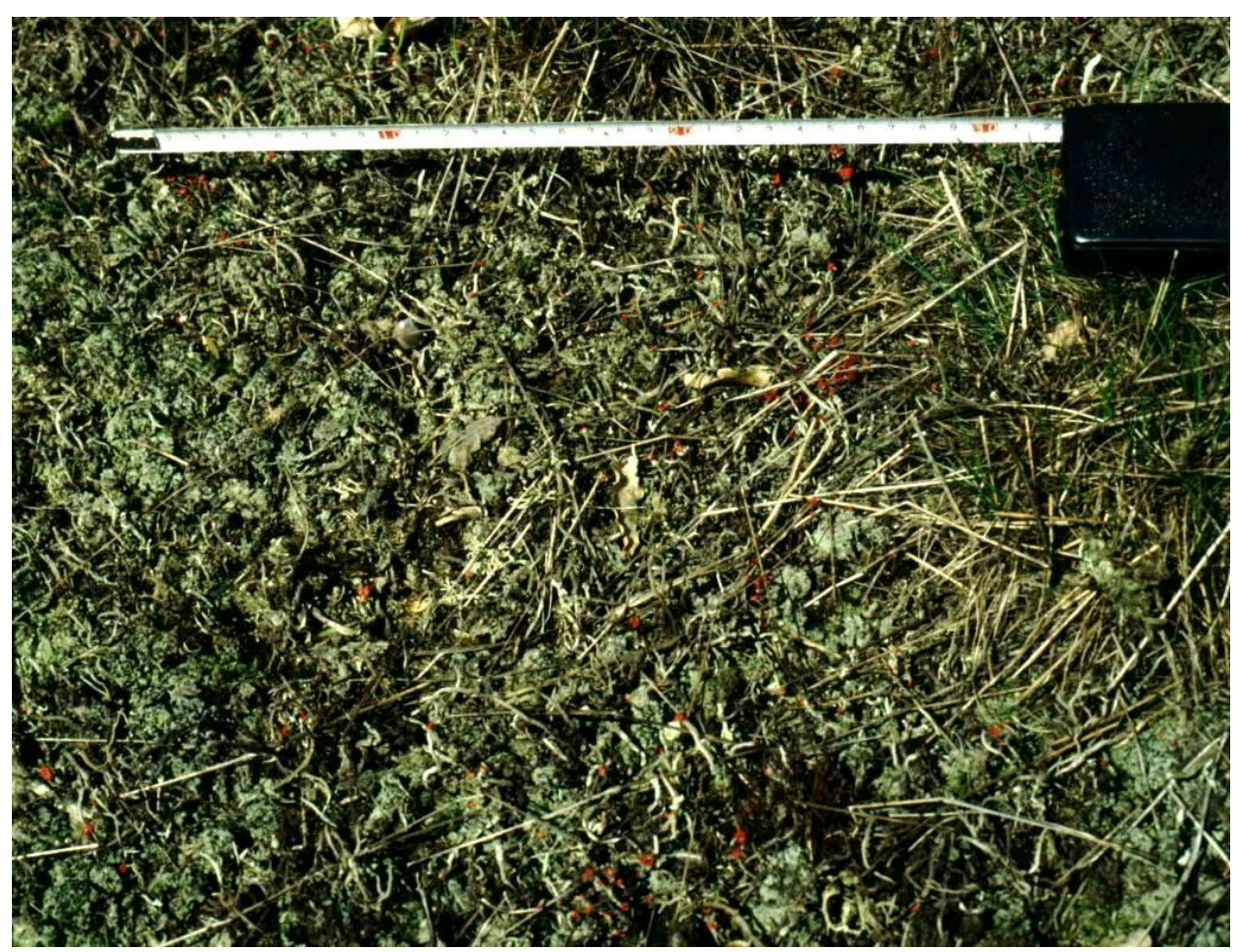

Taf. 1.2: Cladonia bacillaris-Gesellschaft im Battenfelder Driescher. Es dominieren in diesem Bestand Trapeliopsis granulosa (graue Kruste) und Cladonia macilenta (rote Fruchtkörper). 


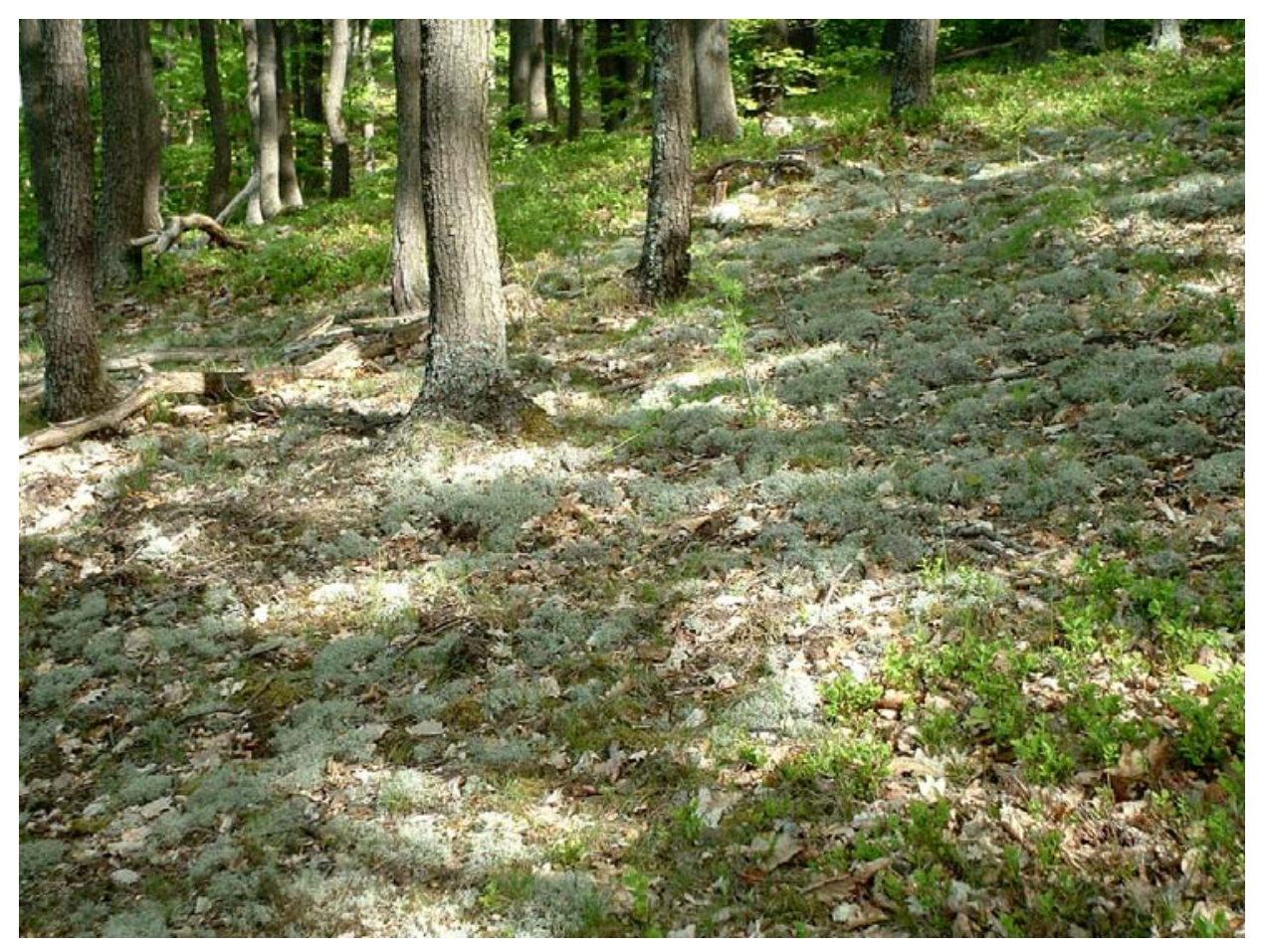

Taf. 1.3: Cladonietum mitis typicum am Roßkopf.

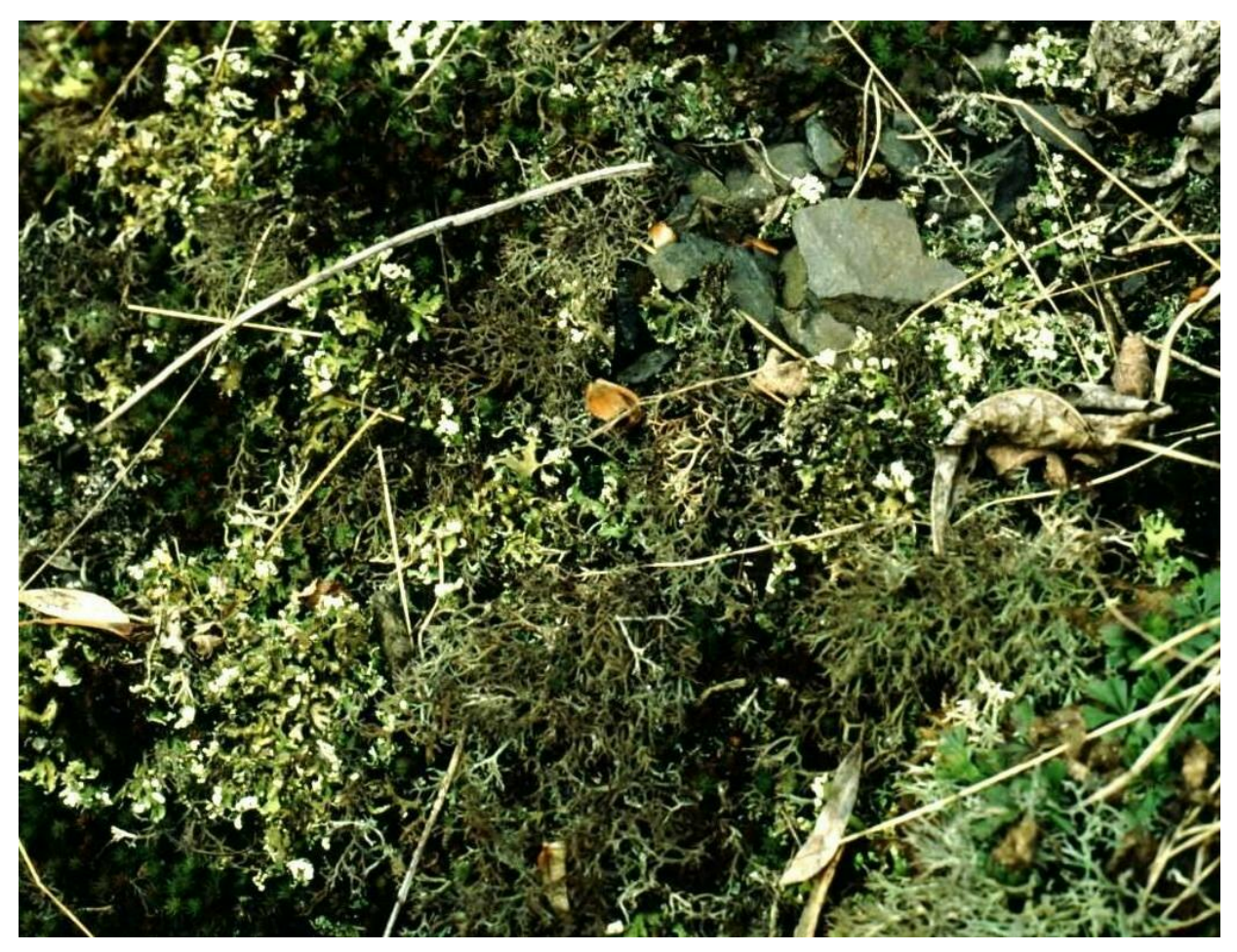

Taf. 1.4: Cladonietum foliaceae am Bilstein bei Albungen (Diabasfelswand). 


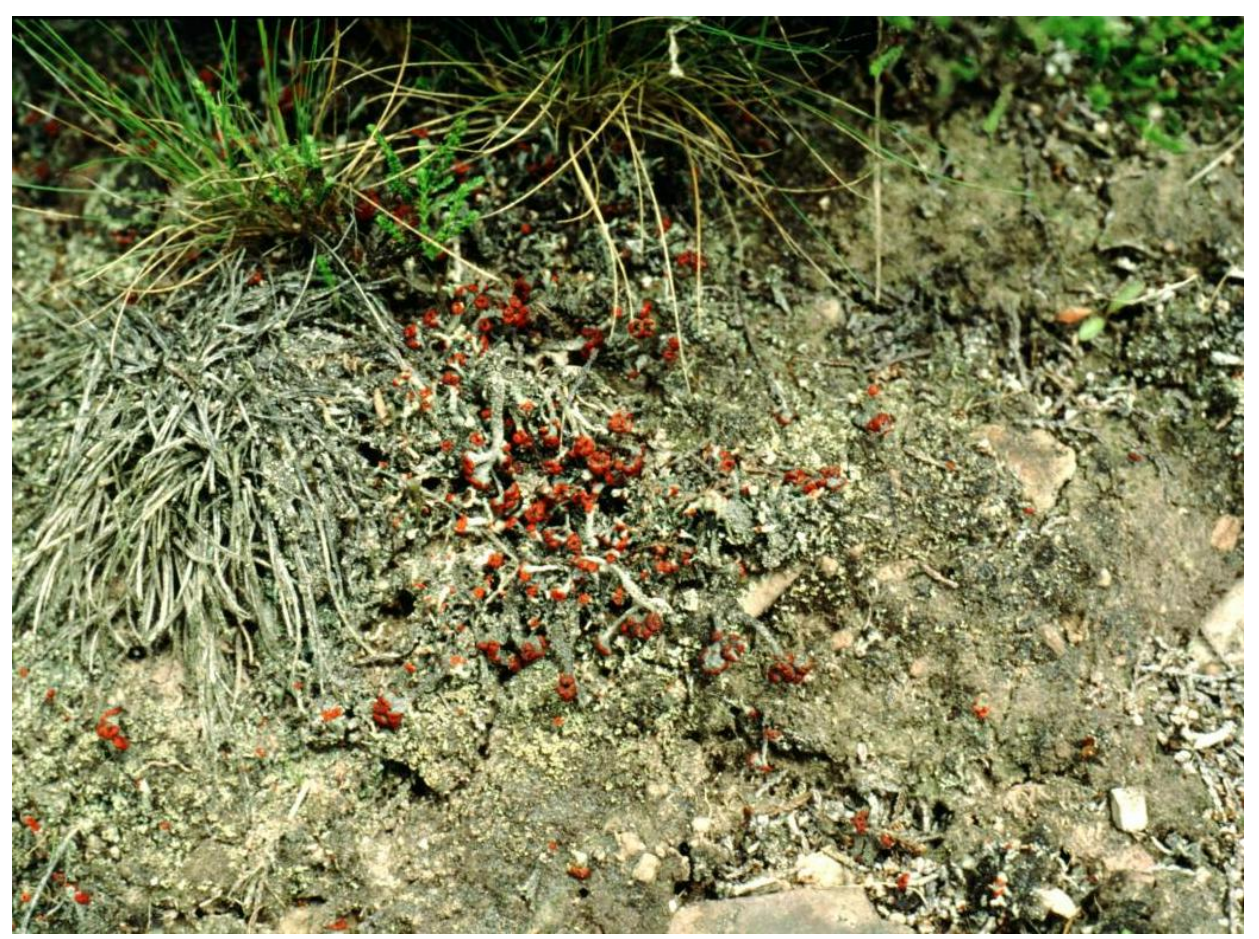

Taf. 1.5: Cladonia floerkeana-Cladonion rei-Fragmentgesellschaft auf dem ehemaligen Grenzstreifen bei der Burg Hanstein.

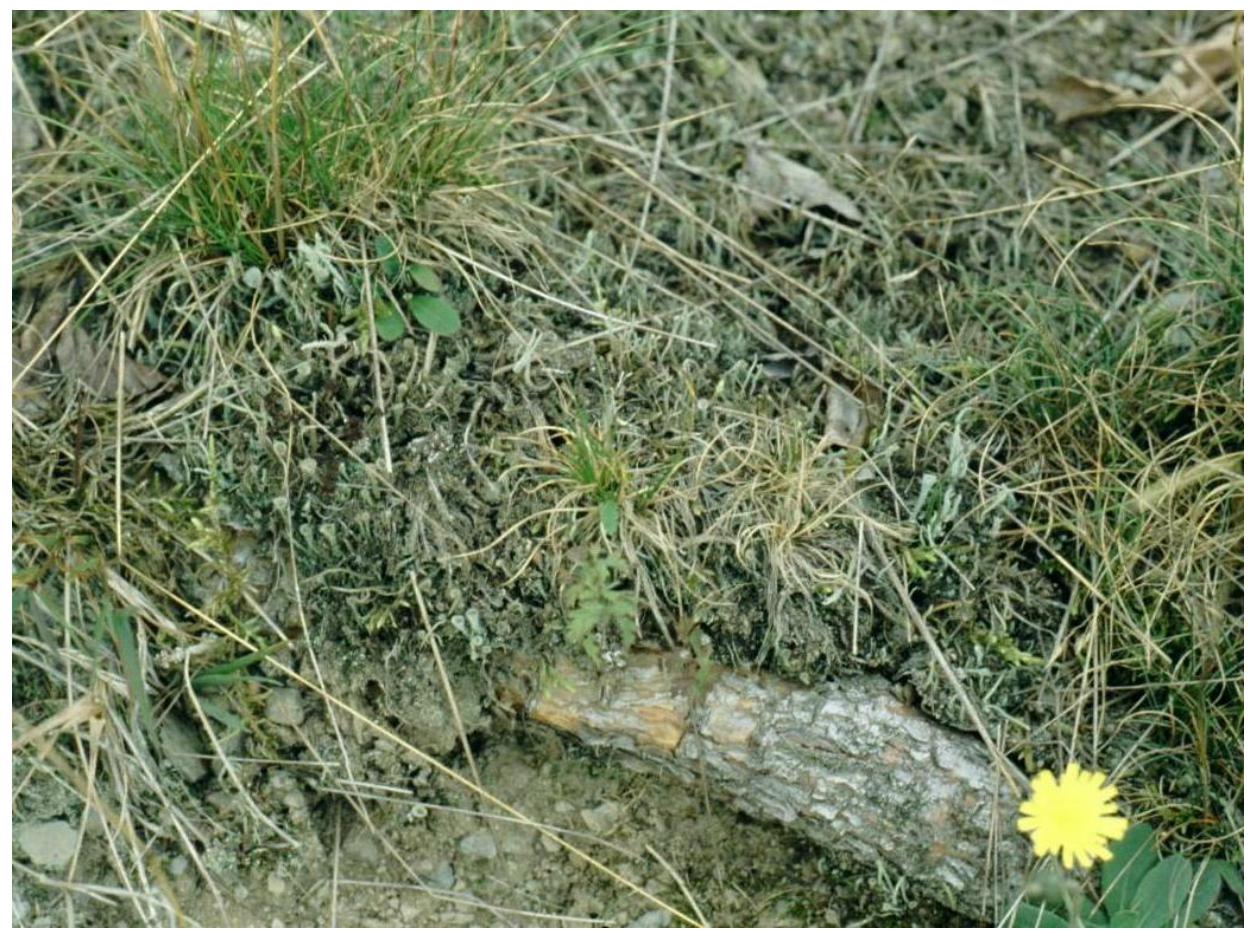

Taf. 1.6: Cladonietum rei am Orenberg bei Willingen. 


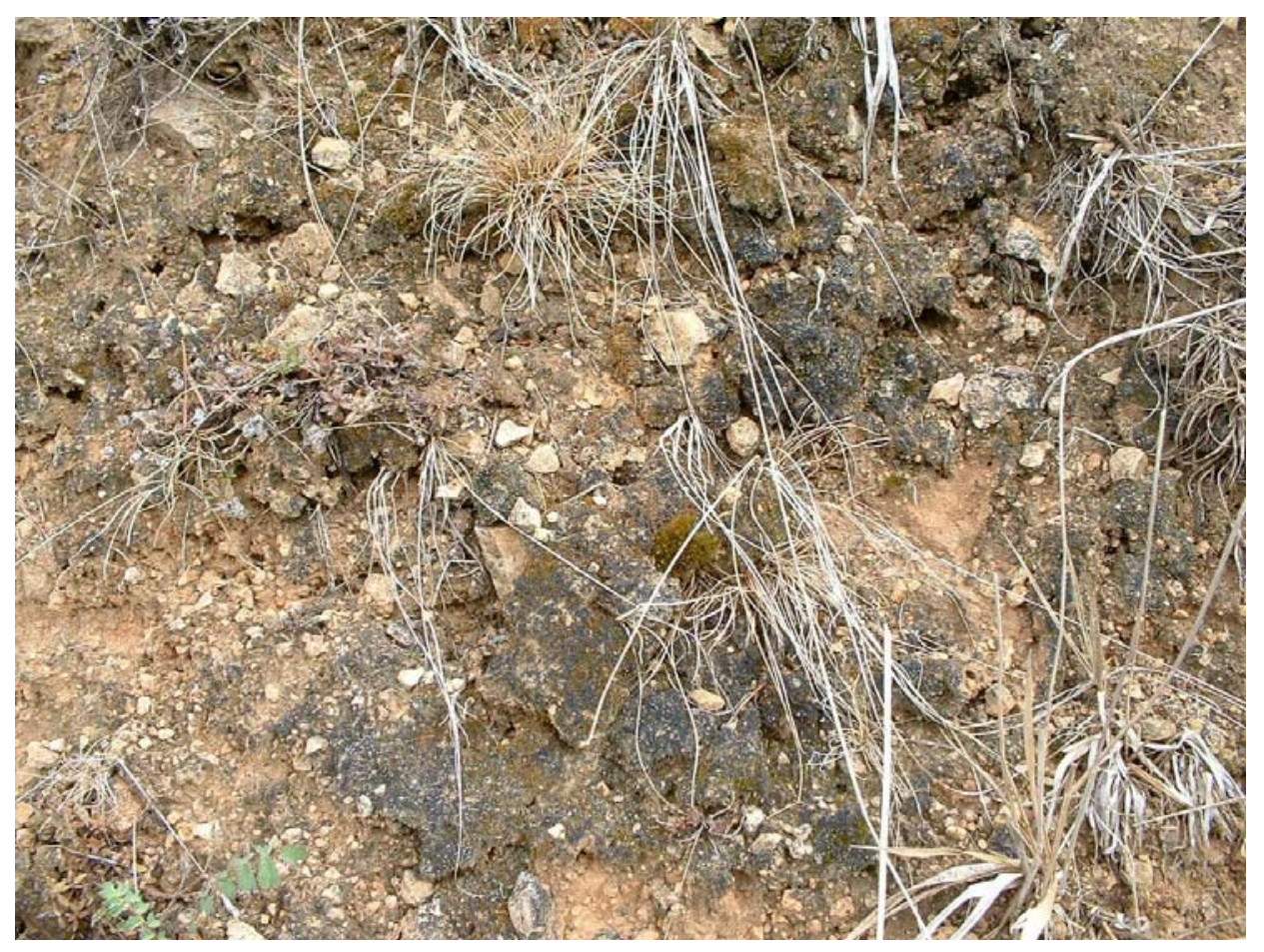

Taf. 1.7: Toninia sedifolia-Toninion-Initialgesellschaft in einem aufgelassenen Dolomit-Steinbruch bei Abterode.

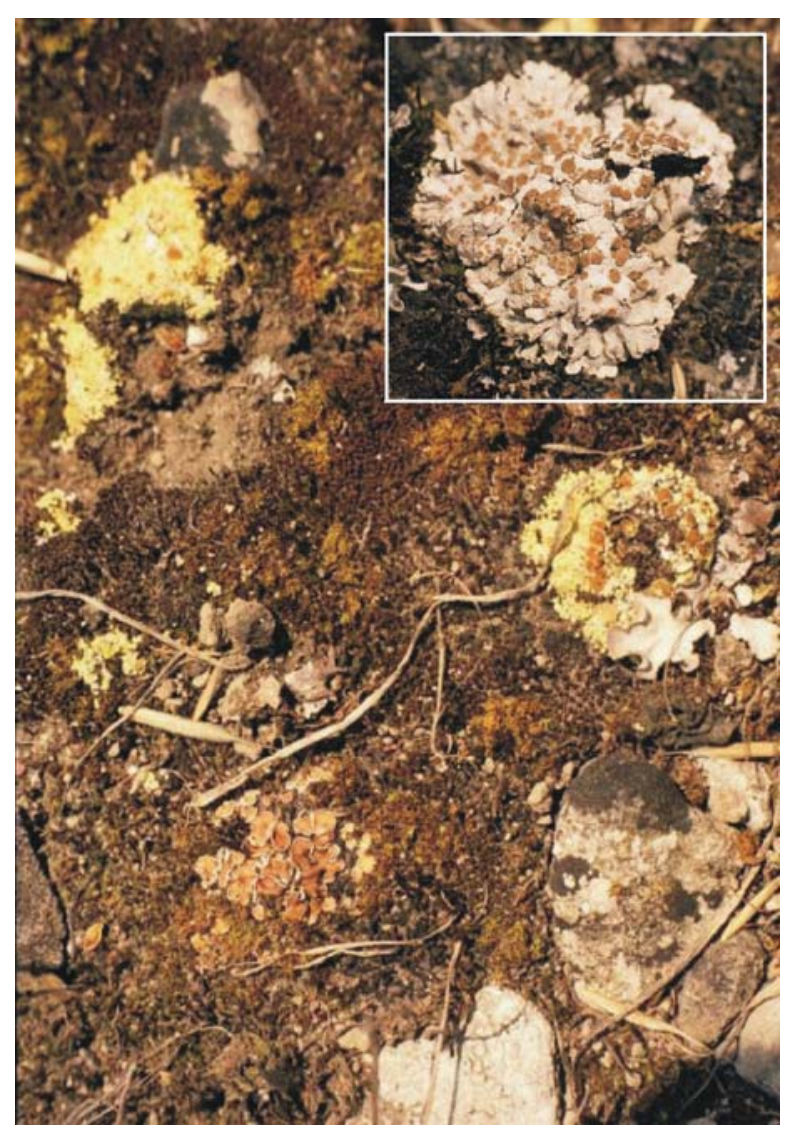

Taf. 1.8:

Arten der Bunten

Erdflechten-Gesellschaft:

Psora decipiens;

Fulgensia bracteata;

Peltigera rufescens,

Einschubbild:

Squamarina lentigera. 


\section{Bildtafel 2: Cladonia furcata ssp. furcata $(1,8 x)$}

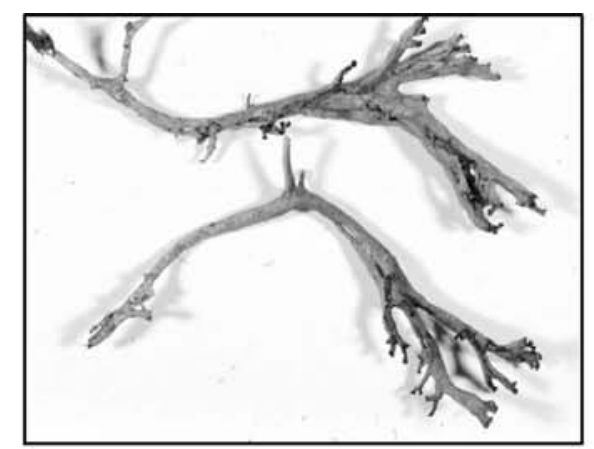

Taf. 2.1: Cl. furcata ssp. furcata. Fertile Podetien, nach oben hin rinnig aufgerissen.

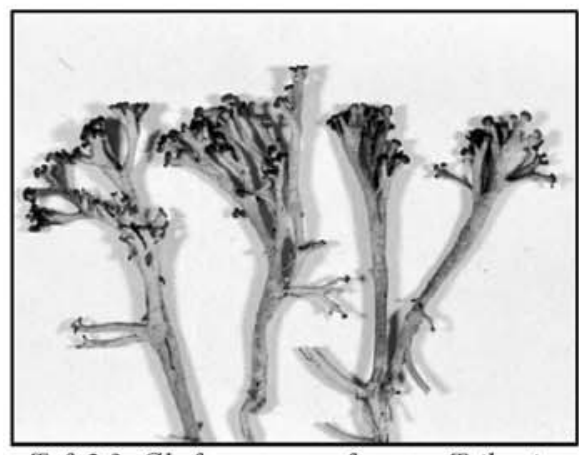

Taf. 2.3: Cl. furcata ssp. furcata. Teilweise doldenähnliche Fruchtstände.

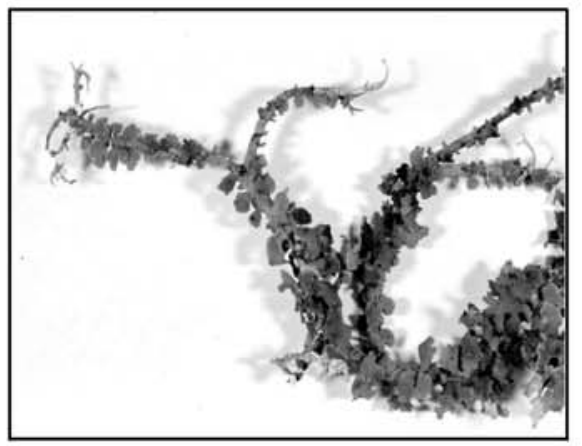

Taf. 2.5: Cl. furcata ssp. furcata. Stark beschupptes Podetium (Oberseite).

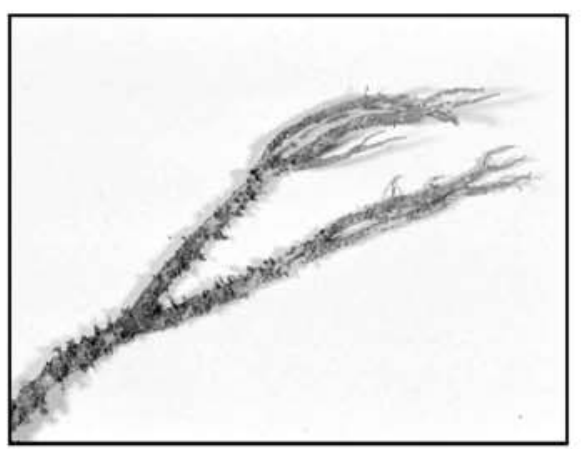

Taf. 2.7: Cl. furcata ssp. furcata. Stark beschupptes Podetium mit kleinen Schuppen. Verwechslungsgefahr mit der sörediösen $\mathrm{Cl}$. scabriuscula.

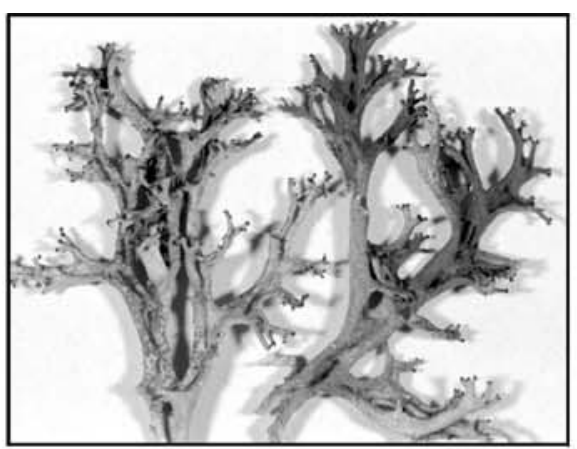

Taf. 2.2: Cl. furcata ssp. furcata. Fertile, stark längsrissige Podetien.

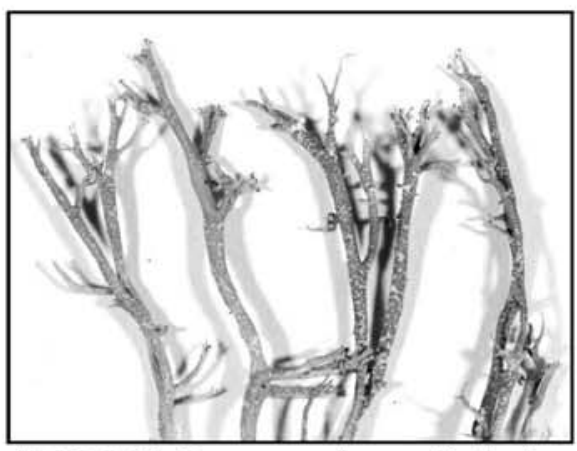

Taf. 2.4: Cl. furcata ssp. furcata. Schlanke Podetien mit sehr kleinen Apothecien.

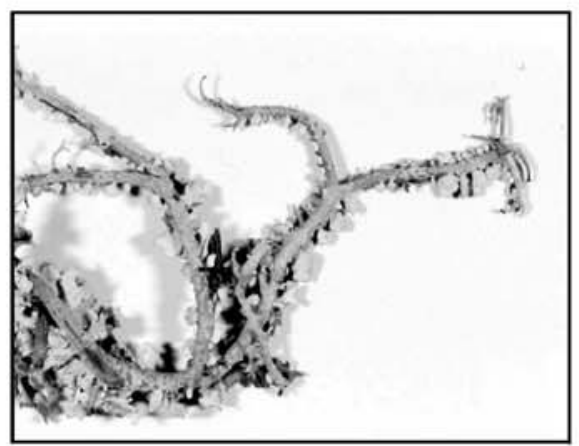

Taf. 2.6: Cl. furcata ssp. furcata. PodetienUnterseite, Probe wie bei Taf. 2.5.

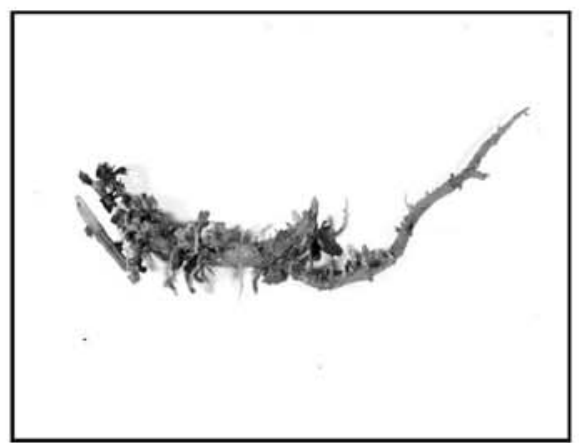

Taf. 2.8: Cl. furcata ssp. furcata. Podetium mit fast wirtelartig angeordneten Schuppen. 


\section{Forts. Bildtafel 2: Cladonia furcata ssp. furcata $(\mathbf{1 , 8 x})$}

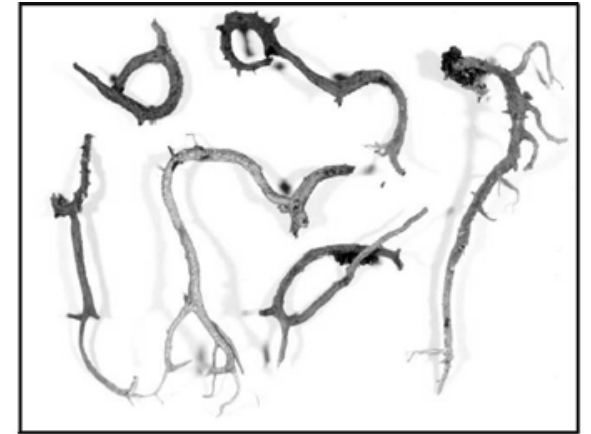

Taf. 2.9: Cl. furcata ssp. furcata. Durch mechanische Einwirkung geprägt.

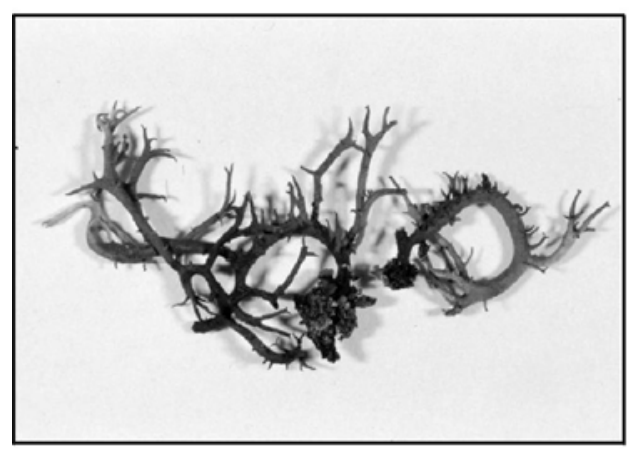

Taf. 2.11: Cl. furcata ssp. furcata. Mit zahlreichen dornigen Auswüchsen.

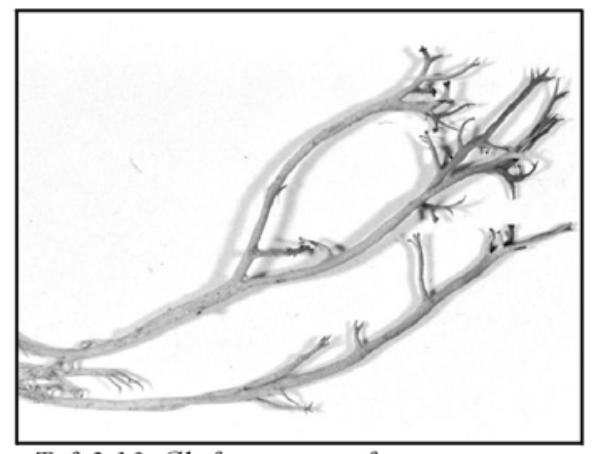

Taf. 2.13: Cl. furcata ssp. furcata. Zierliche, hochwüchsige Podetien.

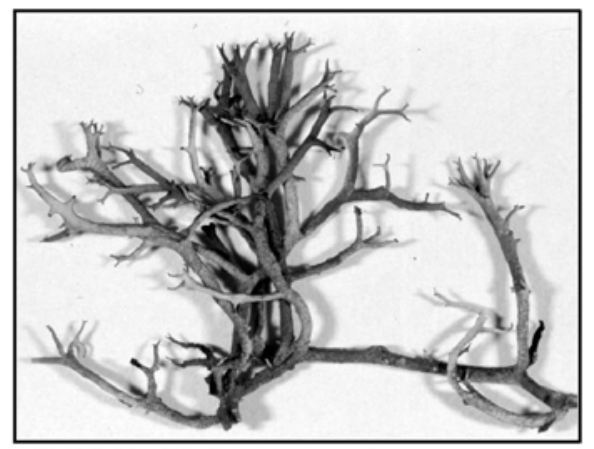

Taf. 2.15: Cl. furcata ssp. furcata.

Podetien einem gemeinsamen

basalen Strang entspringend.

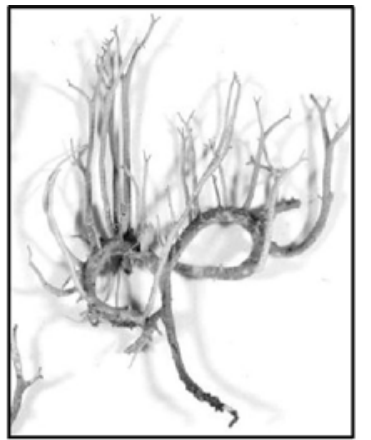

Taf. 2.10: Cl. furcata ssp. furcata.

Podetium mit neugebildeten Ästen.

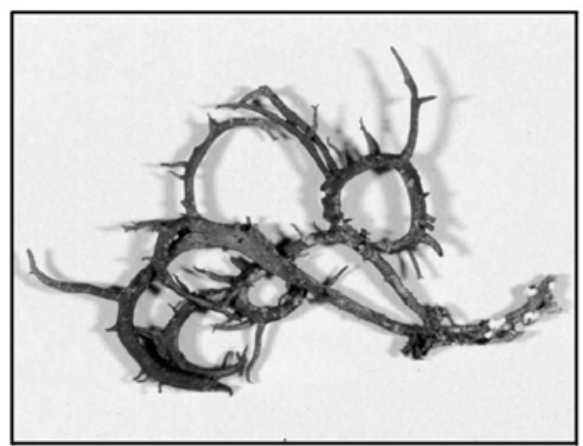

Taf. 2.12: Cl. furcata ssp. furcata. An der Basis mit weißen Markaufbrïchen.

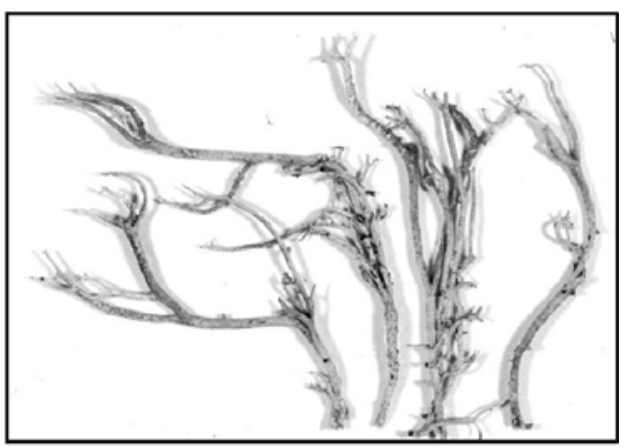

Taf. 2.14: Cl. furcata ssp. furcata.

Kümmerliche, dïnne Podetien.

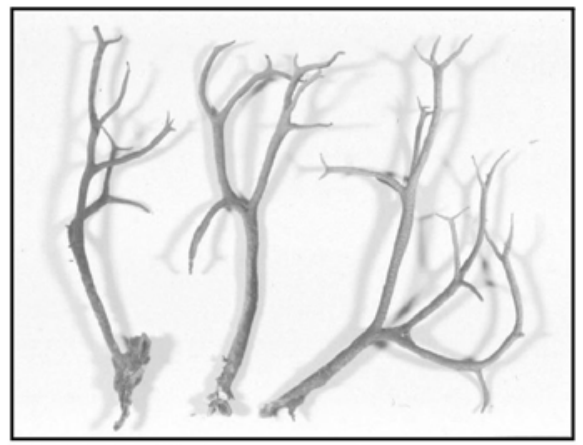

Taf. 2.16: Cl. furcata ssp. furcata.

Schlanke, schuppenlose Podetien. 


\section{Bildtafel 3: Cladonia gracilis, $\mathrm{Cl}$. crispata und $\mathrm{Cl}$. furcata ssp. furcata $(\mathbf{1 , 8 x})$}

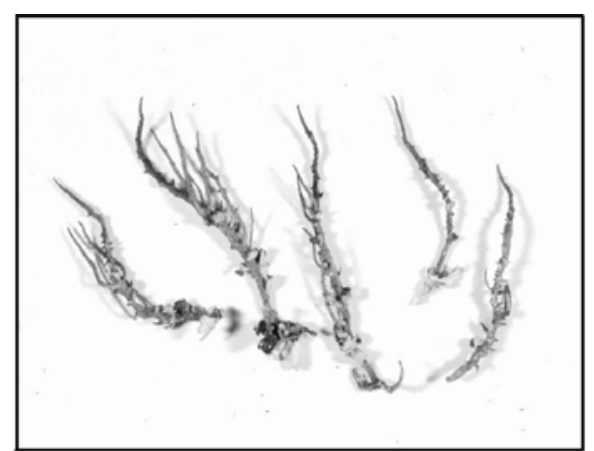

Taf. 3.1:Cl. gracilis. Becherlose Podetien.

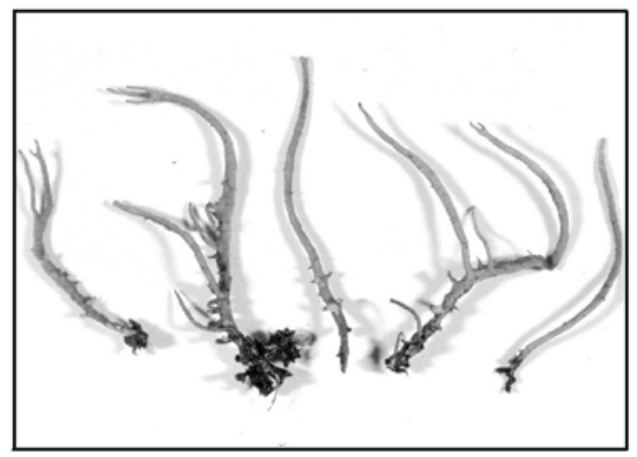

Taf. 3.3: Cl. gracilis. Podetien z.T. mit Bechern, z.T. pfriemförmig endend.

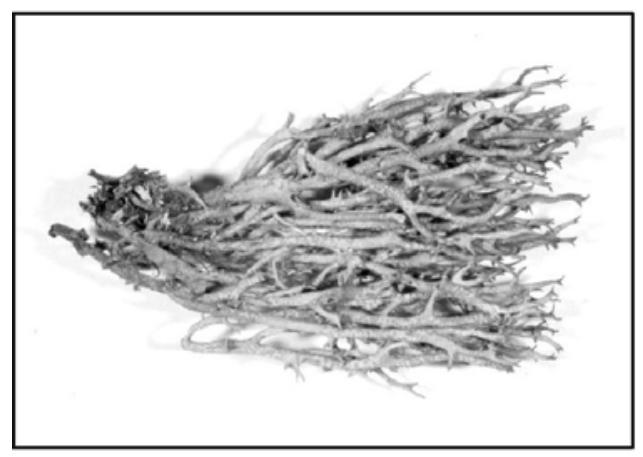

Taf. 3.5: Polster von Cl. crispata.

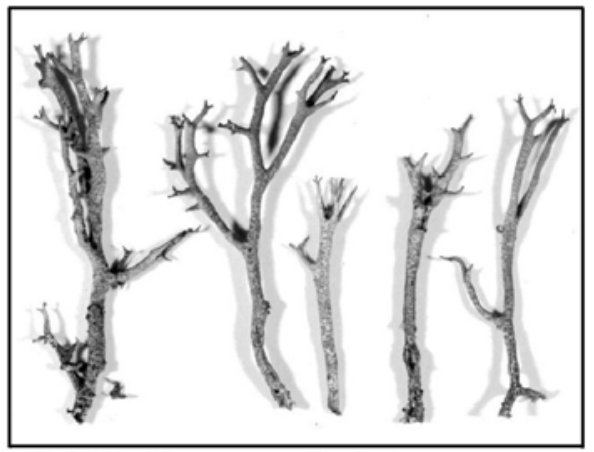

Taf. 3.7: Cl. crispata. Podetien mit undeutlich becheratig erweiterten Spitzen.

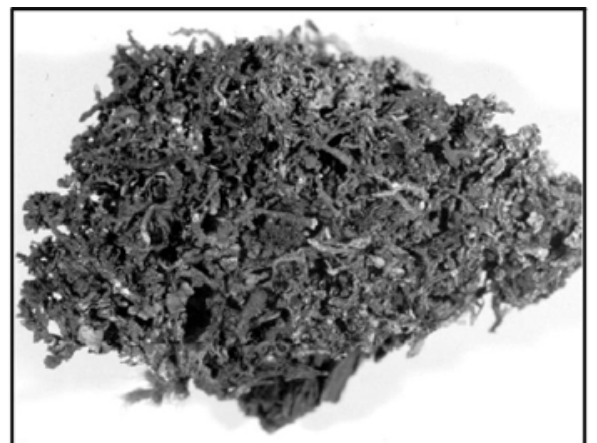

Taf. 3.2: Cl. gracilis. Kümmerexemplar von einer Basaltblockhalde.

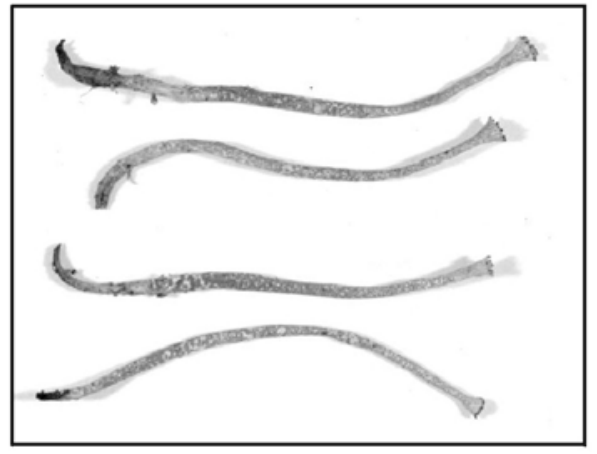

Taf. 3.4: Typische Cl. gracilis. Schlanke Podetien mit schmalen Bechern.

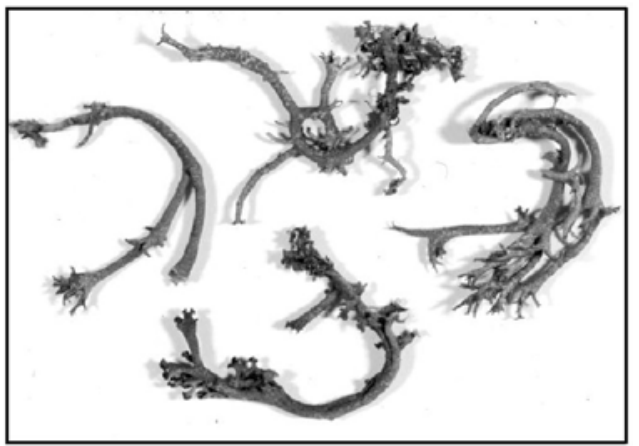

Taf. 3.6: Cl. crispata. Untypisch entwickelte Podetien.

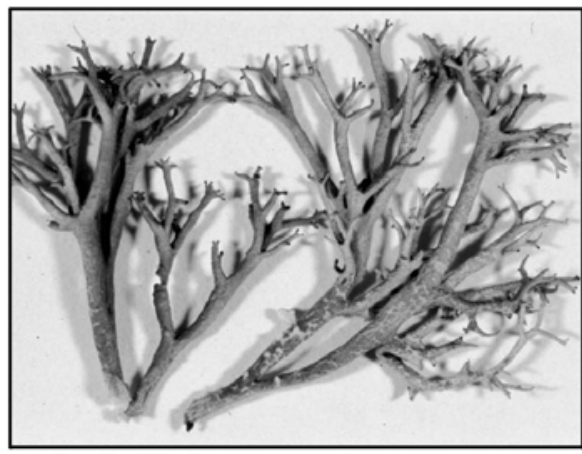

Taf. 3.8: Cl. furcata ssp. furcata.

Crispata-ähnliches Exemplar. 
Bildtafel 4: Cladonia furcata ssp. subrangiformis $(1,8 x)$

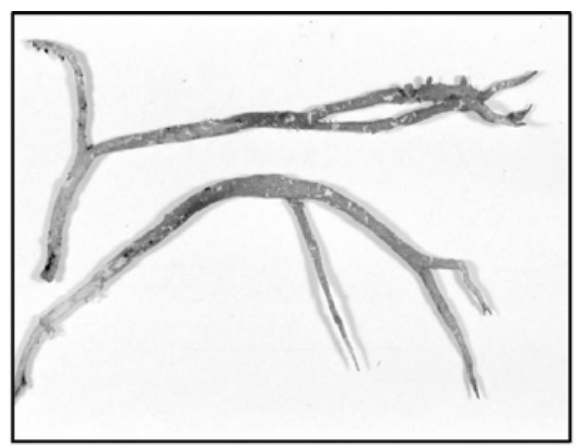

Taf. 4.1: Cl. furcata ssp. subrangiformis. Schwach pigmentiertes Exemplar.

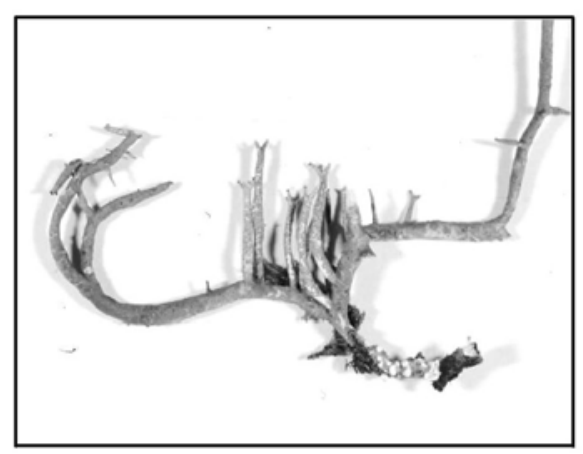

Taf. 4.3: Cl. furcata ssp. subrangiformis. Podetium mit zahlreichen Auswïchsen.

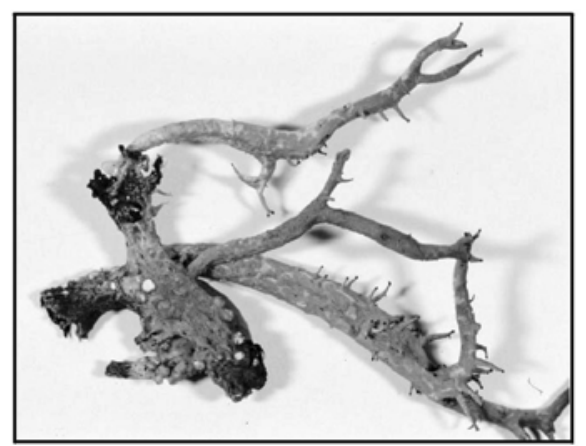

Taf. 4.5: Cl. furcata ssp. subrangiformis. Stark verbreiterte Podetienbasis mit punktförmigen Markaufbrüchen.

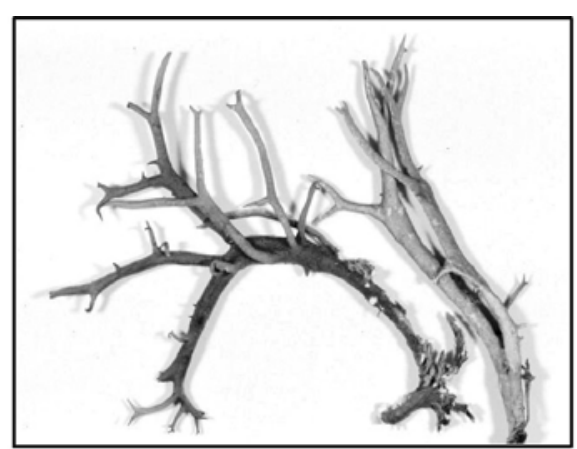

Taf. 4.2: Cl. furcata ssp. subrangiformis. Links die stark pigmentierte Ober-, rechts die helle Unterseite.

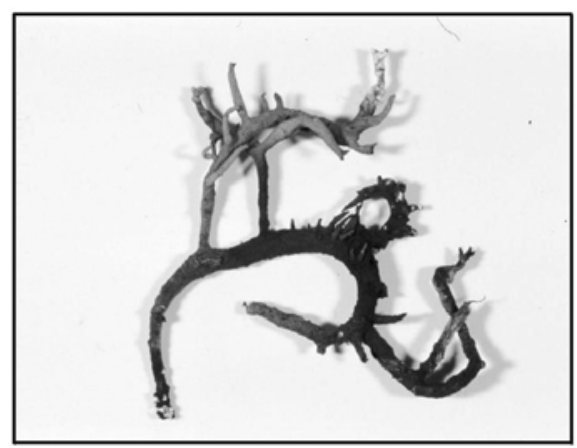

Taf. 4.4:Cl. furcata ssp. subrangiformis. Dunkel pigmentiertes, durch

Trittein wirkung deformiertes Podetium.

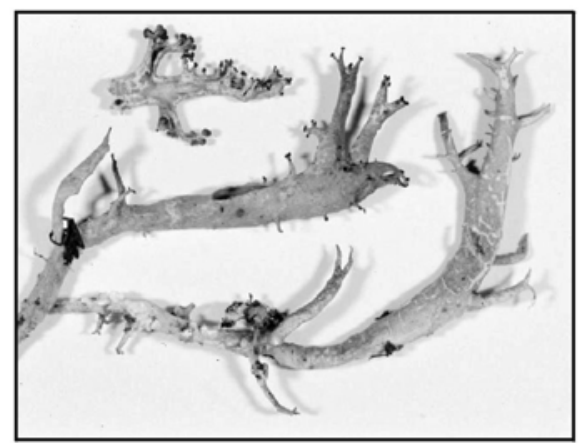

Taf. 4.6: Cl. furcata ssp. subrangiformis. Verbreiterte Podetien und rinnig aufgerissener Fruchtstand. 


\section{Bildtafel 5: Verpflanzungsversuch}

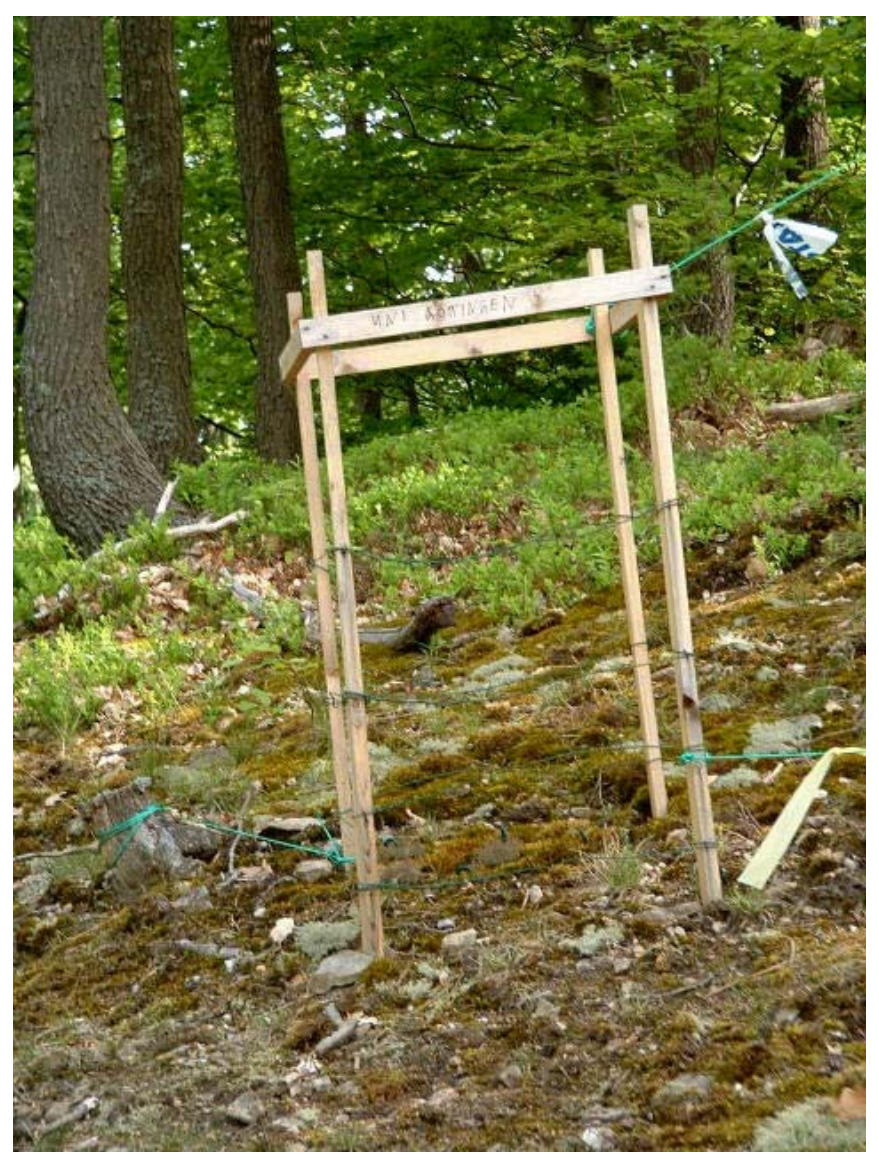

Taf. 5.1:

Versuchsfläche R3 am

Roßkopf

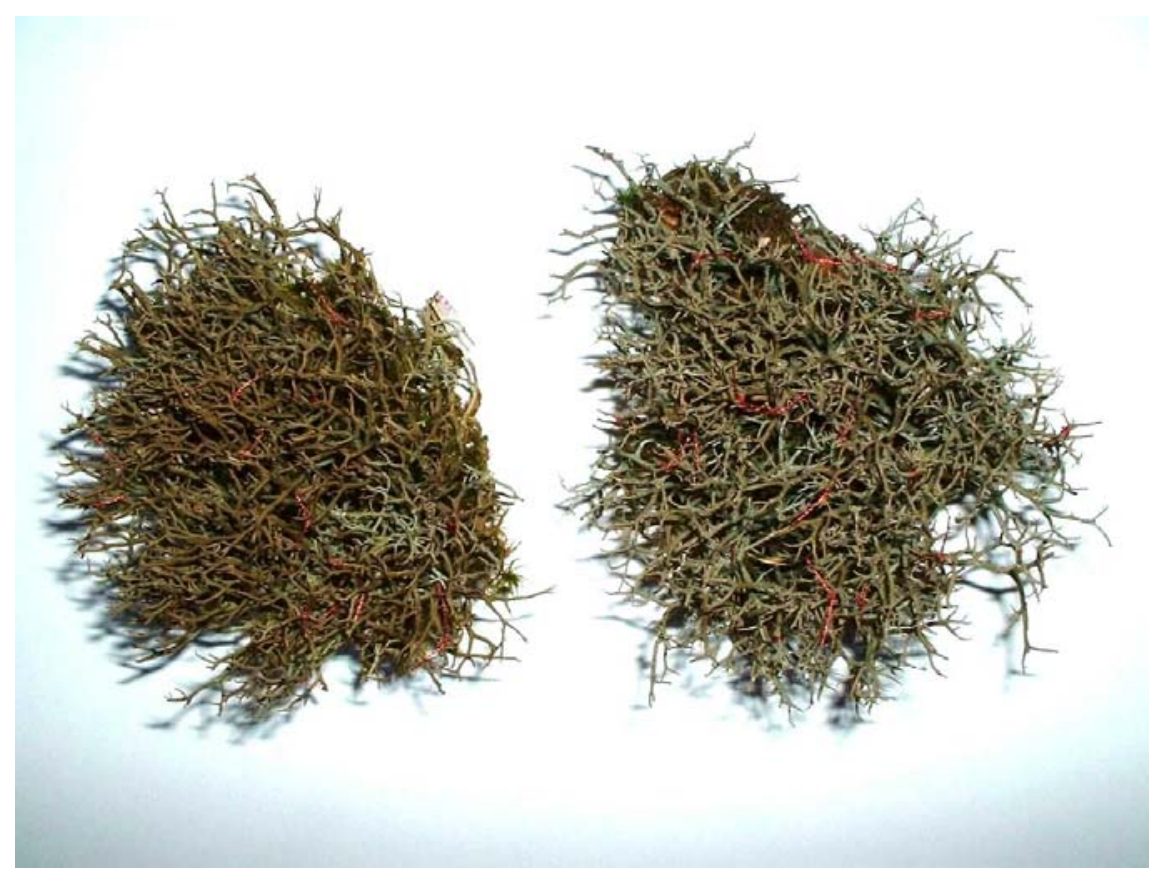

Taf. 5.2: Zwei Polster am Versuchsende: links ein stärker gebräuntes, einseitswendiges Polster von der Fläche R3, rechts ein wenig gebräuntes Polster von der Fläche R2. 
P Anhang 3: Vegetationstabellen 
Tab. 6: Baeomycion rosei

Einheit 1: Pycnothelio-Cladonietum cervicornis

Einheit 2: Cladonia cervicornis-Baeomycion-Fragmentgesellschaft

Einheit 3. Dibaeetum baeomycetis

3.1 Cladonia cervicornis-Höhenform

Einheit 4: Lecideetum uliginosae

\begin{tabular}{|c|c|c|c|c|c|c|c|c|c|c|c|c|c|c|c|c|c|c|c|c|c|c|c|c|c|c|c|}
\hline \multirow{2}{*}{ Einheit } & \multirow{2}{*}{\multicolumn{2}{|c|}{1}} & \multirow{2}{*}{\multicolumn{2}{|c|}{2}} & \multicolumn{18}{|c|}{3} & \multicolumn{5}{|c|}{4} \\
\hline & & & & & \multicolumn{6}{|c|}{3.1} & \multicolumn{12}{|c|}{3.2} & & & & & \\
\hline \begin{tabular}{|l} 
Laufende Nummer \\
\end{tabular} & 1 & 2 & 3 & 4 & 5 & 6 & 7 & 8 & 9 & 10 & 11 & 12 & 13 & 14 & 15 & 16 & 17 & 18 & 19 & 20 & 21 & 22 & 23 & 24 & 25 & 26 & 27 \\
\hline & RK & $\mathrm{MI}$ & KB & KB & $\mathrm{BD}$ & SE & DT & ME & RK & ME & EW & EW & OW & OW & OW & OU & OU & $\mathrm{KP}$ & $\mathrm{KP}$ & $\mathrm{KP}$ & OU & ZG & $\mathrm{HH}$ & $\mathrm{HH}$ & $\mathrm{KP}$ & $\mathrm{CM}$ & $\mathrm{CM}$ \\
\hline Höhe [müNN] & 340 & 285 & 260 & 260 & 400 & 300 & 330 & 330 & 360 & 330 & 780 & 775 & 660 & 660 & 660 & 690 & 690 & 750 & 750 & 750 & 690 & 410 & 200 & 200 & 750 & 595 & 595 \\
\hline Exposition & sw & w & so & so & - & ssw & $\mathrm{s}$ & $\mathrm{s}$ & sw & $\mathrm{s}$ & $\mathrm{n}$ & $\mathrm{n}$ & $\mathrm{s}$ & $s$ & $\mathrm{~s}$ & 0 & 0 & nnw & - & - & 0 & nw & so & w & - & so & 0 \\
\hline Inklination $\left[{ }^{\circ}\right]$ & 20 & 5 & 5 & 5 & - & 30 & 60 & 45 & 60 & 45 & 10 & 10 & 5 & 5 & 5 & 5 & $<5$ & 5 & 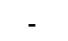 & - & 5 & 10 & 30 & $<5$ & 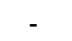 & -90 & 45 \\
\hline Ausgangsgestein & $g w$ & ts & gw & gw & ts & $g w$ & gw & tsgw & gw & tsgw & ts & ts & ts & ts & ts & ts & ts & ts & ts & ts & ts & st & sm & $\mathrm{sm}$ & ts & $\mathrm{sm}$ & $\mathrm{sm}$ \\
\hline $\mathrm{pH}$-Wert $\left(\mathrm{H}_{2} \mathrm{O}\right)$ & 3.9 & 4.2 & 4.1 & 4.0 & 4.4 & 4.2 & 4.3 & 4.7 & 4.2 & 4.7 & 4.5 & 4.3 & 4.8 & 4.8 & 4.8 & 4.3 & 4.3 & 4.4 & 4.6 & 4.4 & 4.1 & 4.1 & 3.8 & 3.8 & 3.8 & 3.8 & 4.0 \\
\hline $\mathrm{pH}$-Wert $(\mathrm{KCl})$ & 3.3 & 4.2 & 3.5 & 3.4 & 4.0 & 3.7 & 3.7 & 3.8 & 3.6 & 3.8 & 4.0 & 3.8 & 4.3 & 4.3 & 4.3 & 3.8 & 3.8 & 3.9 & 3.9 & 3.9 & 3.7 & 3.4 & 3.2 & 3.2 & 3.8 & 3.3 & 3.6 \\
\hline Leitfähigkeit [uS/cm] & 33 & 10 & 53 & 46 & 45 & 29 & 49 & 16 & 32 & 16 & 9 & 10 & 35 & 35 & 35 & 2 & 2 & 0 & 11 & 6 & 4 & 41 & 44 & 35 & 396 & 96 & 85 \\
\hline Skelettanteil im Oberboden & ++ & ++ & ++ & ++ & ++ & + & ++ & ++ & + & ++ & ++ & ++ & ++ & ++ & ++ & + & + & + & + & + & + & 0 & - & - & + & + & + \\
\hline Gesamtdeckung [\%] & 30 & 70 & 55 & 40 & 90 & 25 & 90 & 80 & 85 & 75 & 85 & 75 & 80 & 65 & 75 & 75 & 80 & 95 & 95 & 95 & 95 & 75 & 90 & 95 & 85 & 80 & 80 \\
\hline Deckung Phanerogamen [\%] & $<5$ & - & $<5$ & - & 10 & 10 & $<1$ & - & $<5$ & - & 15 & $<5$ & 10 & 5 & 30 & 5 & 15 & 10 & 20 & 15 & 20 & - & 10 & 20 & 20 & $<5$ & $<1$ \\
\hline Deckung Flechten [\%] & 10 & 70 & 50 & 40 & 75 & 20 & 70 & 50 & 80 & 70 & 75 & 55 & 70 & 60 & 50 & 75 & 60 & 90 & 85 & 90 & 80 & 55 & 80 & 75 & 45 & 60 & 70 \\
\hline Deckung Moose [\%] & 20 & $<5$ & $<5$ & $<1$ & 10 & $<1$ & 20 & $<1$ & $<5$ & 1 & 1 & $<5$ & $<1$ & $<1$ & $<1$ & - & 10 & $<1$ & $<5$ & $<1$ & $<1$ & 20 & $<1$ & $<5$ & 25 & 20 & 20 \\
\hline Deckung Algen [\%] & $<1$ & & & & & & $<5$ & 30 & $<1$ & $<5$ & 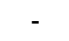 & 15 & 5 & $<1$ & $<1$ & $<5$ & & $<1$ & $<1$ & - & & 5 & $<1$ & - & 5 & 10 & $<5$ \\
\hline Deckung Steine & 15 & 30 & 25 & 55 & 10 & 10 & $<5$ & 10 & $<5$ & 15 & 15 & 20 & 20 & 30 & 15 & 15 & 15 & $<5$ & $<1$ & $<5$ & $<5$ & 10 & - & - & 5 & $<5$ & 5 \\
\hline Deckung offener Boden [\%] & 50 & $<5$ & 15 & 5 & - & 45 & 10 & 10 & 10 & 5 & $<1$ & 5 & $<1$ & 5 & 10 & 10 & 5 & $<5$ & $<1$ & $<1$ & $<1$ & 15 & 10 & - & 10 & 10 & 15 \\
\hline Deckung Streu [\%] & 5 & $<5$ & 10 & 5 & $<5$ & 20 & - & - & 1 & 5 & $<1$ & $<1$ & $<1$ & $<1$ & $<1$ & $<1$ & $<1$ & $<1$ & $<1$ & $<1$ & $<1$ & $<1$ & 5 & 10 & $<1$ & 10 & 10 \\
\hline Gesamtartenzah & 15 & 14 & 14 & 12 & 16 & 10 & 12 & 8 & 10 & 10 & 11 & 8 & 11 & 9 & 10 & 6 & 5 & 14 & 15 & 10 & 7 & 10 & 10 & 10 & 14 & 20 & 10 \\
\hline Artenzahl Phanerogamen & 1 & - & - & - & 4 & 1 & 1 & - & 1 & 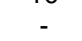 & 4 & 3 & 4 & 3 & 4 & 2 & 2 & 5 & 4 & 3 & 3 & - & 2 & 2 & 4 & 2 & 1 \\
\hline Artenzahl Flechten & 6 & 12 & 9 & 10 & 10 & 7 & 5 & 5 & 3 & 6 & 1 & 1 & 4 & 4 & 4 & 2 & 2 & 3 & 7 & 5 & 2 & 5 & 4 & 6 & 5 & 9 & 3 \\
\hline Artenzahl Moose & 5 & 2 & 4 & 2 & 2 & 2 & 5 & 1 & 5 & 2 & 5 & 3 & 2 & 1 & 1 & 1 & 1 & 5 & 4 & 2 & 2 & 4 & 3 & 2 & 4 & 8 & 5 \\
\hline $\begin{array}{l}\text { D 1: } \\
\text { UC } \quad \text { Pycnothelia papillaria }\end{array}$ & $r^{2}$ & $2 a^{2 f}$ & & & & & & $1^{2 f}$ & & & & & & & & & & & & & & & & & 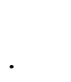 & & \\
\hline $\begin{array}{l}\text { D 1, } \mathbf{A} \text { H.1: } \\
\text { Sch Cladonia cervicornis s.l }\end{array}$ & $2 \mathrm{a}^{2}$ & $3^{2}$ & $3^{3+1}$ & $2 a^{2+}$ & $1^{3+}$ & $+2 T$ & +27 & & $1^{1}$ & $1^{1}$ & & & & . & & & & & & & & & & & . & & \\
\hline
\end{tabular}

KC Polytrichum piliferum

D 3:

UC Dibaeis baeomyces

$\Delta$ H 3.2:

FC Baeomyces rufus Arthrorhaphis citrinella

D 4:

Sch Placynthiella icmalea

C Placynthiella oligotropha Cladonia macilenta ssp. macilenta Trapeliopsis gelatinosa

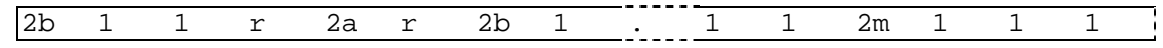

OC Peltigeretalia:

Cladonia furcata ssp. furcata

Cladonia coccifera

Cladonia macilenta ssp. floerkeana

Cladonia subulata

Trapeliopsis granulosa

Cetraria aculeata

Cladonia rei

Cladonia arbuscula s.I.

Cladonia portentosa

Cladonia humilis

Cetraria islandica

Cladonia uncialis

Begleiter Kryptogamen: Chlorophyta div. spec. Cephaloziella spec.

Pohlia nutans

Cladonia cryptochlorophaea

Cladonia pyxidata ssp. chlorophaea

Ditrichum heteromallum

Dicranum scoparium

Cladonia spec.
Dicranella heteromalla

Cyanophyta div. spec.

Cladonia merochlorophae

Hypnum cupressiforme s.

Cladonia coccifera et deformis agg.

Cladonia digitata

Diplophyllum albican

Ceratodon purpureus

Polytrichum formosum

Dicranella cf. heteromalla

Phanerogamen:

Deschampsia flexuosa

Festuca ovina agg

Calluna vulgaris

Agrostis capillaris

Nardus stricta

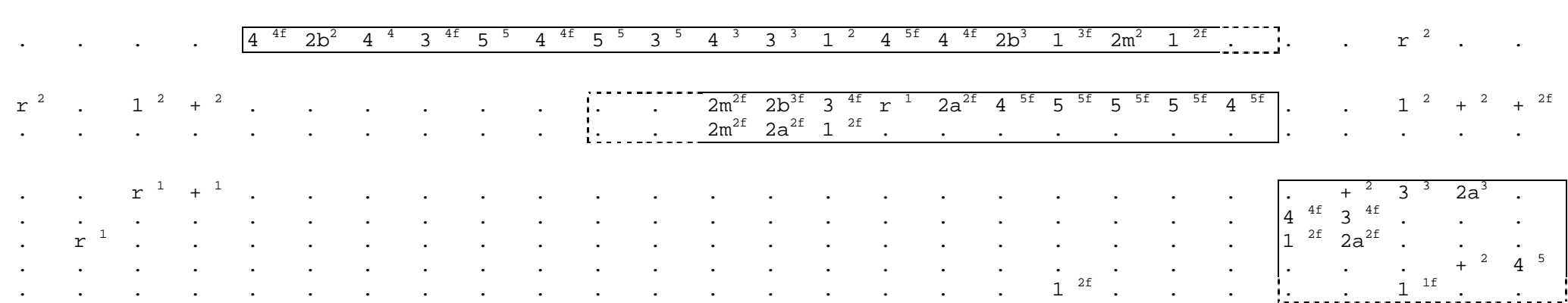

$r_{1+}+1^{3} \quad 2 a^{3}$

${ }_{1 f}+{ }^{1} r^{1} r^{1} r_{1} a_{1 f}+{ }_{1 f}$

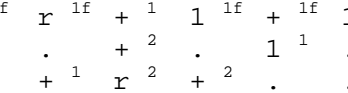

+1
$2 b^{3}$

${ }_{1}^{2 f} i^{2}$

$\begin{array}{ll}i_{1} & 1 \\ r^{2}\end{array}$

$\cdot$

ußerdem mit Stetigkeit < II und in weniger als drei Einheiten:

Brachythecium rutabulum 22:r; Bryophyta spec. 12:2m; Buxbaumia aphylla 1:r; Campanula rotundifolia 18:r; Campylopus introflexus 23:1; Carex spec. 15:+; Cladonia cf. cornuta 19: $+^{1}$; Cladonia coniocraea 22:1 ${ }^{2}$;

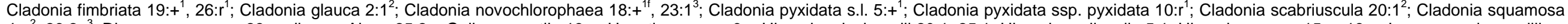
4: ${ }^{2}, 26: 2 a^{3}$; Dicranaceae spec. 23:+; diverse Algen 25:2a; Galium saxatile 13:+; Hepaticae spec. 9:r; Hieracium lachenalii 20:1, 25:1; Hieracium pilosella 5:1; Hieracium spec. 15:+, 18:r; Jungermannia gracillima 11:+, 12:1; Jungermannia spec. 11:+; Lepidozia reptans 26:+; Lepraria lobificans $7: r^{2}, 26: 2 a^{3}$; Lepraria rigidula 2:+1 ${ }^{1}, 26: 2 a^{3} ;$ Lophocolea heterophylla 26:+; Luzula campestris agg. 21:r; Musci spec 11:+, 16:+ Pinus sylvestris juv. 5:r; Plagiothecium laetum 26:+; Pleurozium schreberi 5:+; Polytrichum juniperinum 18:1; Potentilla erecta 18:+, 25:+; Rhytidiadelphus squarrosus 19:1; Tetraphis pellucida 26:2m; Trapeliopsis pseudogranulosa 9: $r^{2}$; Vaccinium myrtillus 11:r, 19:r; Veronica verna agg. 26: 


\section{ab. 8: Cladonion arbusculae}

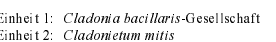

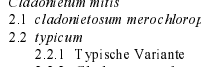

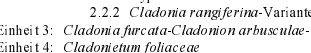

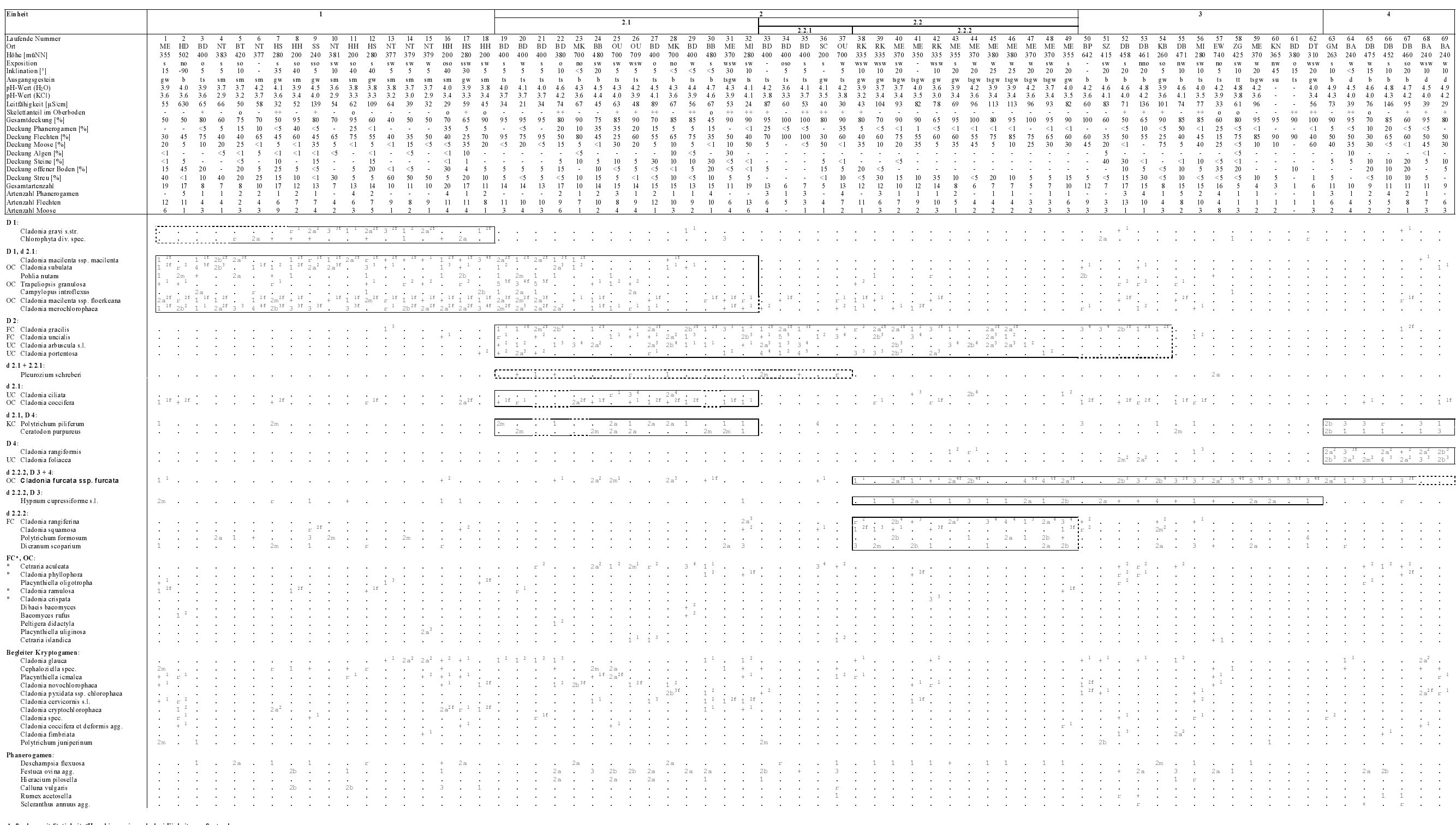

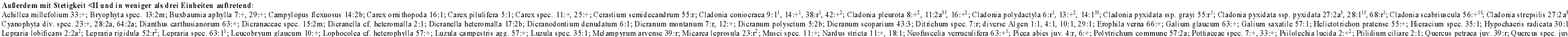


Tab. 10: Cladonion rei

Einheit 1: Cladonia floerkeana-Cladonion rei-Fragmentgesellschaft

Einheit 2: Cladonietum rei

2.1 Cladonia floerkeana-Variante
2.2 Typische Variante

Einheit 3: Cladonia subulata-Cladonion rei-Fragmentgesellschaft

\begin{tabular}{|c|c|c|c|c|c|c|c|c|c|c|c|c|c|c|c|c|c|c|c|c|c|c|c|c|c|c|c|c|}
\hline Einheit & & & & & & 1 & & & & & & & & & & 2.1 & & & $\underline{2}$ & & & & 2.2 & & & & & \\
\hline Laufende Nummer & 1 & 2 & 3 & 4 & 5 & 6 & 7 & 8 & 9 & 10 & 11 & 12 & 13 & 14 & 15 & 16 & 17 & 18 & 19 & 20 & 21 & 22 & 23 & 24 & 25 & 26 & 27 & 28 \\
\hline Ort & NT & $\mathrm{GH}$ & $\mathrm{KP}$ & BD & NT & BT & $\mathrm{KM}$ & $\mathrm{CM}$ & $\mathrm{GH}$ & $\mathrm{GH}$ & $\mathrm{GH}$ & $\mathrm{KB}$ & OU & TE & $\mathrm{KP}$ & ow & ZG & SS & $\mathrm{GH}$ & ZG & $\overline{Z G}$ & $\mathrm{GH}$ & ZG & NT & NT & NT & $\mathrm{MK}$ & $\mathrm{ZG}$ \\
\hline Höhe [müNN] & 379 & 400 & 755 & 400 & 377 & 420 & 620 & 591 & 400 & 400 & 390 & 260 & 690 & 270 & 750 & 660 & 410 & 240 & 400 & 420 & 410 & 380 & 410 & 520 & 517 & 520 & 710 & 4104 \\
\hline Exposition & w & - & nnw & - & sw & so & so & sso & ssw & sw & $\mathrm{s}$ & so & 0 & sw & - & $s$ & $s$ & sso & ssw & $\mathrm{n}$ & $\mathrm{nw}$ & oso & $\mathrm{n}$ & 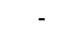 & - & sw & no & sw \\
\hline 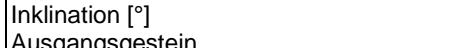 & 5 & - & 5 & - & 5 & 10 & 70 & 50 & 30 & 40 & 35 & 5 & 5 & 20 & - & 5 & 60 & 5 & 5 & $<5$ & 40 & 35 & 60 & - & - & 5 & 45 & 40 \\
\hline $\begin{array}{l}\text { Ausgangsgestein } \\
\text { pH-Wert }\left(\mathrm{H}_{2} \mathrm{O}\right)\end{array}$ & sm & sm & ts & ts & sm & sm & $\mathrm{b}$ & sm & sm & sm & sm & gw & ts & sm & ts & ts & st & gw & sm & stbt & st & sm & bt & sm & sm & sm & b & st \\
\hline $\mid \begin{array}{l}\mathrm{pH}-\text { Wert }\left(\mathrm{H}_{2} \mathrm{O}\right) \\
\mathrm{n} \text { n-Wert } \mathrm{KCll}\end{array}$ & 3.7 & & 3.9 & 3.9 & 4.7 & 3.8 & 4.5 & 3.9 & 4.2 & 4.6 & 4.8 & 3.9 & 4.8 & 4.0 & 4.5 & 4.5 & 4.0 & 4.6 & 4.0 & 3.3 & 6.0 & 4.7 & 4.9 & 6.5 & 6.2 & 6.0 & 4.5 & 5.9 \\
\hline $\mathrm{pH}$-Wert $(\mathrm{KCl})$ & 2.9 & 3.8 & 3.5 & 3.6 & 4.3 & 3.1 & 3.9 & 3.2 & 3.6 & 4.0 & 3.8 & 3.5 & 4.3 & 3.5 & 3.9 & 4.0 & 3.5 & 4.2 & 3.5 & 2.9 & 5.6 & 3.8 & 4.5 & 6.0 & 5.8 & 5.6 & 4.0 & 5.5 \\
\hline $\begin{array}{l}\text { Leitfähigkeit }[\mu \mathrm{\mu S} / \mathrm{cm}] \\
\text { Skelettanteil im Oberboden }\end{array}$ & 32 & 23 & -5 & 82 & 61 & 47 & 94 & 27 & 45 & 88 & 42 & 70 & 25 & 50 & 10 & 26 & 82 & 209 & 59 & 372 & 78 & 34 & 356 & 132 & 102 & 80 & 95 & 40 \\
\hline $\begin{array}{l}\text { Skelettanteil im Oberboden } \\
\text { Gesamtdeckung [\%] }\end{array}$ & & + & + & & 65 & $\overline{15}$ & + & م० & o & $\stackrel{+}{+}$ & + & ++ & + & 0 & + & ++ & OR & + & - & o & & + & + & مص & - & + & $\therefore$ & \\
\hline $\begin{array}{l}\text { Gesamtdeckung [\%] } \\
\text { Deckung Phanerogamen [\%] }\end{array}$ & 70 & 90 & 90 & 95 & 65 & 45 & $\begin{array}{l}50 \\
<1\end{array}$ & 90 & 95 & 75 & 60 & 80 & 85 & 75 & 100 & 75 & 60 & 80 & 85 & 40 & 75 & 85 & 60 & 80 & 80 & 90 & 90 & 60 \\
\hline $\begin{array}{l}\text { Deckung Phanerogamen [\%] } \\
\text { Deckung Flechten [\%] }\end{array}$ & - & 40 & 15 & $<5$ & - & & $<1$ & $<5$ & 1 & - & $<1$ & $<5$ & 45 & 25 & 30 & 10 & $<5$ & - & $<5$ & - & 20 & 5 & 10 & 10 & 10 & $<5$ & - & 15 \\
\hline Deckung Flechten [\%] & 70 & 60 & 70 & 90 & 65 & 45 & 35 & 85 & 90 & 75 & 50 & 75 & 50 & 50 & 70 & 70 & 55 & 40 & 85 & 40 & 35 & 75 & 50 & 70 & 70 & 90 & 40 & 10 \\
\hline Deckung Moose [\%] & $<5$ & - & 10 & 5 & $<5$ & $<5$ & 15 & 5 & 5 & 1 & 15 & $<1$ & 10 & 10 & 10 & $<5$ & $<5$ & 40 & $<1$ & & 10 & 10 & 5 & $<5$ & $<5$ & $<5$ & 50 & 40 \\
\hline Deckung Algen $[\%]$ & - & - & - & - & - & $<5$ & $<5$ & 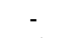 & 1 & 1 & & & - & 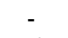 & & - & 10 & - & - & $<1$ & 15 & 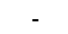 & - & 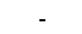 & - & 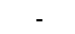 & $<5$ & - \\
\hline Deckung Steine [\%] & - & - & $<1$ & - & $<5$ & 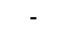 & 25 & $<1$ & $<1$ & $<1$ & 20 & $<5$ & 10 & $<1$ & $<1$ & 10 & & 15 & & & $<1$ & 10 & 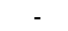 & $<5$ & 5 & 5 & 10 & $<1$ \\
\hline Deckung offener Boden [\%] & 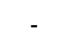 & 10 & $<1$ & 5 & 5 & $<5$ & 10 & 5 & 5 & 1 & 20 & $<5$ & 5 & 20 & 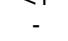 & 15 & 40 & 5 & 15 & 50 & 20 & 5 & 40 & 20 & 10 & 5 & - & 40 \\
\hline Deckung Streu [\%] & 30 & $<5$ & 15 & - & 30 & 55 & 15 & 5 & $<1$ & 25 & 5 & 15 & $<1$ & 10 & $<1$ & $<5$ & 5 & - & 5 & 10 & 5 & $<5$ & $<5$ & 5 & 10 & 10 & - & 1 \\
\hline Gesamtartenzahl & 8 & 9 & 13 & 11 & 7 & 6 & 18 & 13 & 9 & 6 & 7 & 18 & 18 & 10 & 13 & 13 & 9 & 9 & 11 & 9 & 14 & 7 & 15 & 11 & 11 & 10 & 11 & 14 \\
\hline Artenza & & 3 & 3 & 1 & $r$ & - & 1 & 1 & 2 & 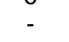 & 1 & 1 & 6 & 2 & 3 & 4 & 1 & - & 5 & & 9 & 1 & 3 & 2 & 3 & 2 & $\stackrel{1+}{-}$ & $\begin{array}{l}14 \\
5\end{array}$ \\
\hline Artenza & 5 & & 8 & 7 & 4 & 4 & 7 & 8 & 5 & 4 & 4 & 15 & 8 & 6 & 6 & 7 & 5 & 6 & 7 & 8 & 3 & 4 & 5 & 4 & 4 & 3 & 4 & 6 \\
\hline Artenzahl Moose & 3 & 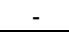 & 2 & 3 & 2 & 1 & 8 & 4 & 1 & 1 & 2 & 2 & 4 & 2 & 4 & 2 & 2 & 3 & 1 & 1 & 1 & 2 & 7 & 5 & 4 & 5 & 6 & 3 \\
\hline D 1, d 2.1: & & & & & & & & & & & & & & & & & & & & & & & & & & & & \\
\hline OC Cladonia macilenta s & $++^{17}$ & $2 \mathrm{~b}^{3+}$ & + & $2 a^{1+}$ & & & $r_{2}^{17}$ & & & & & $2 \mathrm{a}^{2+}$ & $2 b^{3 f} 1$ & & 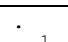 & +24 & $1^{3}$ & $3 f$ & 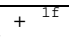 & & & & . & & . & & . & . \\
\hline Cladonia merochloro & $1^{1 \mathrm{ff}}$ & & & & & & & & & & & & & & & & & $=32 f$ & $2 h^{3 f}$ & $13 f$ & & & & & & & & \\
\hline $\begin{array}{ll} & \text { Cladonia } 1 \\
\text { oc } & \text { Trapeliops }\end{array}$ & & $\begin{array}{l}2 b^{3+} \\
+2\end{array}$ & & $3 \mathrm{~h}^{34}$ & & & & & & & & & & & & & & & & & & & . & & . & & " & 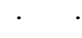 \\
\hline $\begin{array}{l}\text { OC Trapeliopss granulosa } \\
\text { OC Cladonia subulata }\end{array}$ & $2 \mathrm{a}^{2}$ & $2 b^{3}$ & $\begin{array}{ll}1 & 1 \\
+ & 1 \\
\end{array}$ & $1_{1}^{20}$ & $3_{3} 4$ & & $2 a^{3 f}$ & $2 b^{3}$ & & & $2 b^{4}$ & & & & $+1 f$ & $3^{4 f}$ & $3^{3 f}$ & $2 a^{3}$ & $2 b^{3}$ & $2 b^{3}$ & & & $i^{3}$ & & . & 2 & $\dot{1}^{3}$ & $2 \mathrm{~m}^{1}$ \\
\hline D 2+3: & & & & & & & & & & & & & & & & & & & & & & & & & & & & \\
\hline $\begin{array}{l}\text { UC Cladonia pyxidata ssp. chlorophaea } \\
\text { UC Cladonia humilis }\end{array}$ & : & & : & & . & & . & . & . & : & & : & $++^{1}$ & & & $1^{2}$ & $\begin{array}{l}r^{r} \\
1^{2 f}\end{array}$ & $\begin{array}{l}r 1 \\
+1\end{array}$ & & & & $\begin{array}{l}11^{17} \\
4^{5}\end{array}$ & $\begin{array}{l}+{ }^{16} \\
3^{5}\end{array}$ & $1^{2}$ & $r$ & 1 & $2 a^{3 f}$ & $\begin{array}{l}r^{2 t} \\
+{ }^{1}+\end{array}$ \\
\hline d 2.2: & & & & & & & & & & & & & & & & & & & & & & & & & & & & \\
\hline UC Cladonia rei &. & & . & · & . & & . &. & . & . & & $2 b^{3}$ & & ${ }^{\circ}$ & & +1 & . & & . & & $r^{3}$ & & $r 1$ & 1 & $\frac{12}{12}$ & & & . \\
\hline Atrichum undulatum & . & & . & & . & & . & 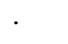 & . & . & & 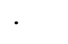 & & & & & . & & 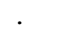 & & $2 a$ & & $2 \mathrm{~m}$ & & & & . & 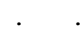 \\
\hline $\begin{array}{l}\text { D Cladonion rei: } \\
\text { Cladonia fimb }\end{array}$ & & & & & & & & & & & & & & & & & & & & & & & & & & & & \\
\hline Clad & $2 \mathrm{~b}^{2}$ & $r^{1}$ & $2 b^{4 \mathrm{f}}$ & $r^{2}$ & & $3^{2}$ & $2 b^{3 t}$ & $2 a^{2}$ & $1^{3 f}$ & +1 & $2 b^{3 f}$ & & & & & & & & & & & & & $4^{4 f}$ & $4^{4}$ & 5 & & $r^{1} 2$ \\
\hline Ceratodon purpureus & 1 & & $\cdot 1$ & 1 & . & & 1 & . & $2 \mathrm{a}$ & 1 & $2 \mathrm{~b}$ & . & 1 & . & $2 \mathrm{~m}$ & $2 \mathrm{~m}$ & . & . & + & & . & $2 a$ & . & + & r & + & . & $2 a$ \\
\hline oc, $\mathrm{KC}^{*}:$ & & & & & & & & & & & & & & & & & & & & & & & & & & & & \\
\hline $\mathrm{Be}$ & . & & . & · & 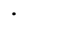 & . & . & $r^{1}$ & 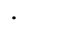 & . & & $2{ }_{2}^{2 f}$ & $1^{2 f}$ & & $+{ }^{3}$ & 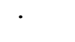 & . & . & $r_{1 f}$ & & . & & . & & & . & 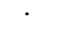 & . \\
\hline $\begin{array}{l}\text { Cladoni } \\
\text { Cladoni }\end{array}$ & : & & · & & . & · & . & . & . & . & & $2 a^{2 t}$ & & $\dot{r}_{1 f}$ & $\dot{r}_{1 f}$ & & . & . & $r^{17}$ & & · & & . & +27 & . & · & . & . \\
\hline Placynt & : & : & $i^{2}$ &. & : & . & : & $\therefore$ & : & $i^{3}$ & & +2 & & . & . & $:$ & $\therefore$ & $:$ & : & & r & 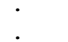 & : & & r & · & . & ' \\
\hline * Polytricl & . & . & . & . & . & . & . & . & . & . & & 1 & . & . & . & . & . & . & . & & . & & . & & . & . & 3 & . \\
\hline Cladonia furcata ssp. & & & & . & . & & . & . & . & . & & $1^{1}$ & & . & . & . & . & & . & & . & & . & & . & . & 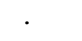 & . \\
\hline Dibaeis baeon & . & . & . & . & . & . & . & . & . & . & . & . & $1^{2}$ & . & . & . & . & . & . & & . & & . & & . & . & if & . \\
\hline Cladonia phyll & . & . & . & . & . & . & . & . & . & . & & . & & . & . & . & . & . & . & & . & & . & & . & . & $2 b^{4 f}$ & : \\
\hline Peltige & - & & 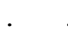 & & 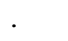 & & & & & 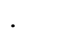 & & . & & & & & . & & . & & . & & 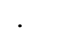 & & . & . & 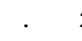 & $2 a^{2 f}$. \\
\hline Placynthiella uliginosa & . & & . & . & . & . & . & $2 a^{3}$ & . & . & . & . & . & . & . & . & . & . & . & & . & & . & & . & . & . & . \\
\hline Begleiter Krypto & & & & & & & & & & & & & & & & & & & & & & & & & & & & \\
\hline $\begin{array}{l}\text { Ceph } \\
\text { Hypn }\end{array}$ & $i$ & & : & & : & . & $\begin{array}{l}2 \mathrm{a} \\
1\end{array}$ & + & : & : & & $\dot{r}$ & $2 \mathrm{a}$ & i & $\begin{array}{l}2 \mathrm{a} \\
2 \mathrm{~m}\end{array}$ & : & 1 & $\begin{array}{l}r \\
1\end{array}$ & $:$ & & $\therefore$ & 1 & $\begin{array}{l}+ \\
+\end{array}$ & & 1 & : & 1 & 1. \\
\hline Chlore & & & 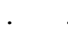 & & 1 & + & 1 & . & 1 & 1 & & & & . & & . & $2 a$ & & . & & & & & & . & . & . & 3 \\
\hline & 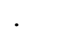 & & . & & & & $r$ & & $1^{2}$ & . & & +1 & +1 & 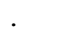 & & 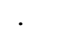 & & & . & & & & & & . & . & . & $r^{1}$ \\
\hline & $r^{\circ}$ & &. & & . & & 1 & $2 \mathrm{~m}$ & 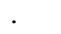 & . & $r$ & . & . & ${ }^{\circ}$ & . & . & + & & . & & . & & r. & & . & . & 1 & . \\
\hline $\mathrm{Pc}$ & . & 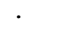 & $2 a$ & & . & $2 m$ & . & $r$ & . & . & & . & & . & . & & . & 3 & . & & . & & . & 1 & $r$ & . & . & . \\
\hline $\mathrm{PI}$ & . & . & 1 & 1 & . & $\cdot$ & i & . & . & . & . & . & + & $\cdot$ & . & 1 & . & ${ }^{\circ}$ & . & & . & & $\cdot$ & & . & · & . & . \\
\hline & 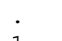 & & . & & . & . & 1 & . & . & . & & . & 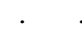 & . & . & . & . & . & . & & $2 b$ & & . & & . & · & 1 & . \\
\hline C & 1 & & . & 1 & + & 1 & $\cdot$ & . & 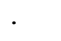 & . & . & . & 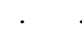 & . & . & . & . & . & . & & . & & . & & . & · & 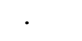 & . \\
\hline C & . & . & . & 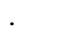 & . & +1 & . & . & . & . & . & . & . & . & . & . & . & . & . & & . & & 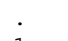 & & r 1 & & . & . \\
\hline S & . & . & . & . & . & . & . & . & . & . & . & . & . & . & . & . & . & . & . & & . & & 1 & + & . & + & . & . \\
\hline $\mathrm{Cl}$ & . & & . & . & . & . & . & . & . & . & . & . & . & . & . & +2 & . & . & . & +3 & . & & . & & . & & . & . \\
\hline & & & 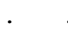 & . & & & & & & 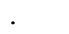 & & 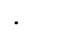 & 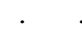 & . & & & . & & . & & 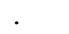 & & & + & . & + & . & . \\
\hline Dicranella cf. heteromalla & . & . & . & . & + & . & 1 & $2 \mathrm{~m}$ & . & . & . & . & & . & . & 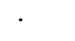 & . & . & 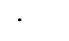 & & . & & . & & 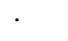 & . & . & . \\
\hline Phanero & & & & & & & & & & & & & & & & & & & & & & & & & & & & \\
\hline & . & + & . & 1 & . & . & $r$ & $r$ & + & . & + & . & 1 & & & . & . & . & + & & . & & 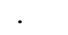 & + & + & + & . & . \\
\hline & & 3 & & & & & & . & 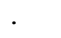 & & & + & 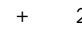 & $2 \mathrm{~b}$ & $2 \mathrm{~b}$ & & . & & $r$ & & & & 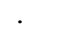 & & . & 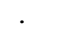 & . & 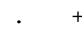 \\
\hline & . & & r & r. & . & $\cdot$ & . & . & . & & & · & & . & & & . & . & ' & & . & & 1 & & . & . & . & $r$ \\
\hline & . & & . & . & . & . & . & . & . & r & & . & 3 & $\dot{2}$ & & + & 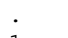 & & . & & . & $2 a$ & & & + & . & . & . \\
\hline & . & & 1 & . & . & . & . & . & . & . & & . & 2 & $2 \mathrm{a}$ & $2 a$ & 1 & 1 & . & r. & & . & & 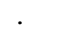 & & . & r. & . & 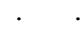 \\
\hline & . & & $\therefore$ & . & . & . & . & . & & . & . & . & 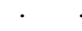 & . & . & . & . & . & . & & 1 & & $2 a$ & & . & . & . & $2 a$ \\
\hline & . & & . & . & . & . & 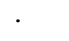 & 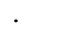 & $\mathrm{r}$ & . & & 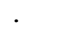 & . & 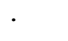 & 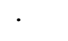 & 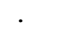 & . & 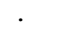 & . & & $2 a$ & & $\dot{r}$ & & . & · & . & . \\
\hline Betula pen & . & & 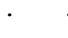 & . & . & & . & . & . & 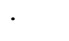 & & & 1 & 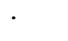 & & 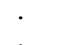 & . & & i & & + & & $r$ & & . & · & . & . \\
\hline Rumex acetosella & & & & & & & & & & & & & & & & & & & & & & & & & & & & \\
\hline
\end{tabular}

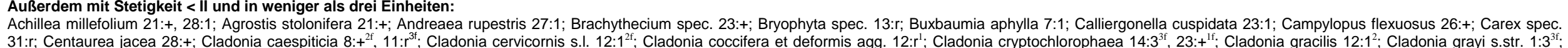

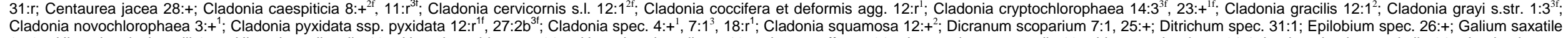
25:2a; Hieracium lachenalii 15:+; Hieracium pilosella 16:; Hypericum hirsutum 21:+; Hypochaeris radicata 13:+; Juncus effusus 31:r; Leontodon autumnalis 13:r; Linum catharticum 21:1; Lophocolea heterophylla 31:r; Lophoziaceae

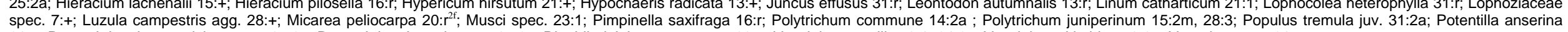
21:+; Racomitrium heterostichum agg. 27:2a; Racomitrium lanuginosum 27:2a; Rhytidiadelphus squarrosus 26:+; Vaccinium myrtillus 3:1; 24:2a; Vaccinium vitis-idaea 3:2a; Veronica spec. 21:r 
Einheit 1: Toninia sedifolia-Toninion-Initialgesellschaft

Einheit 2: Cladonia symphycarpa-Toninion-Fragmentgesellschaft

Einheit 3: Peltigera rufescens-Toninion-Fragmentgesellschaft

Einheit 4: Dominanzbestände

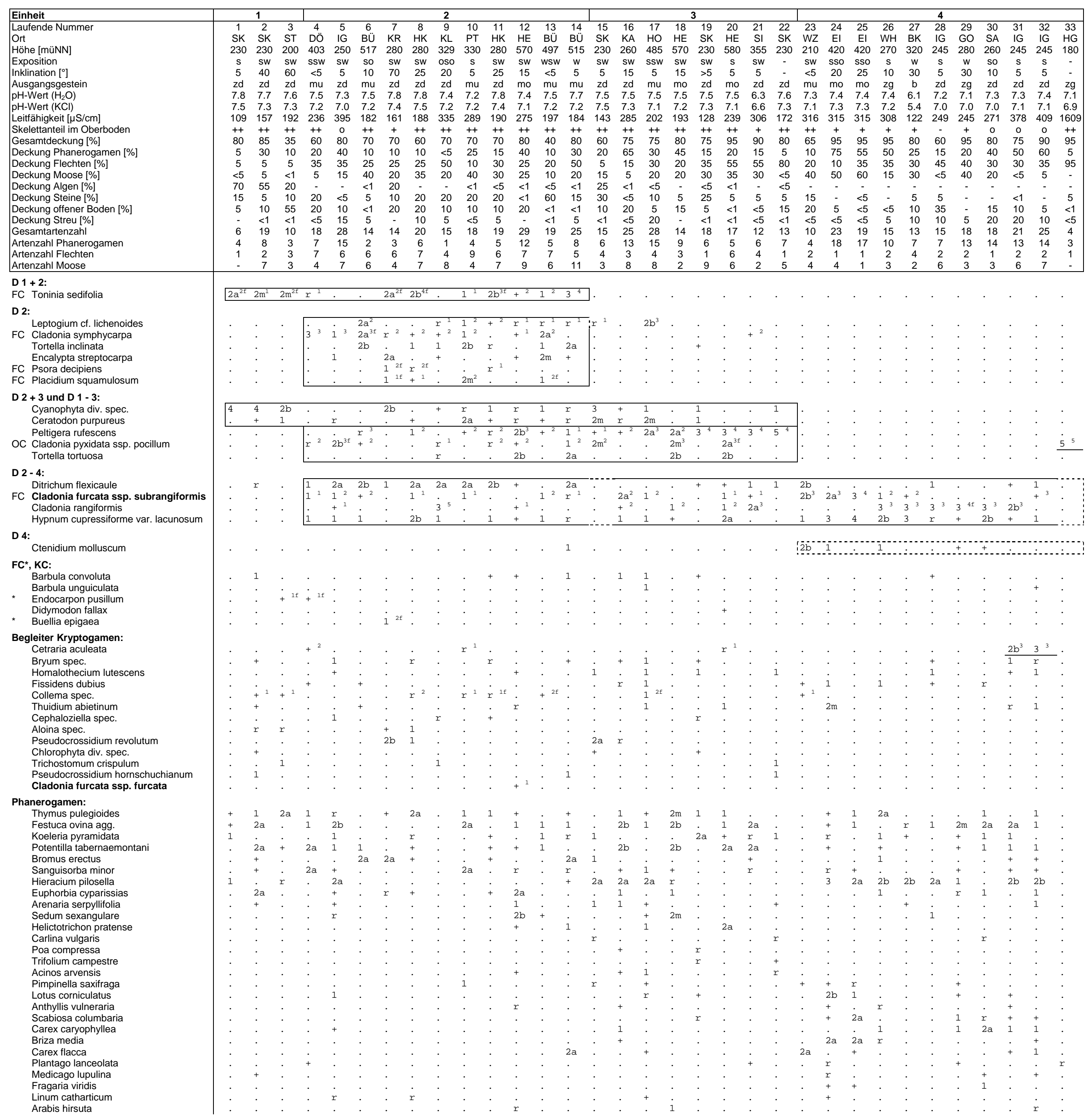

Außerdem mit Stetigkeit < II und in weniger als drei Einheiten:

Achillea millefolium 28:r 33:+ Asplenium ruta-muraria 9:1: Bacidia bagliettoana 6:rir; Barbula spec. 4:r; Brachypodium pinnatum 17:2a; Bryophyta spec. 9:r; Campanula rotundifolia 5:r, 22:r; Centaurea jacea 17:1; Centaurea scabiosa 31:+,

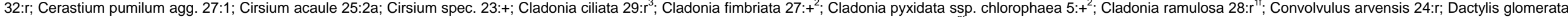
28:1, 30:1; Daucus carota 24:r; Dianthus carthusianorum 16:r; Dicranum scoparium 29:3; Didymodon rigidulus 14:r; Diploschistes muscorum 4:1'1, 10:+ 2; Encalypta vulgaris 16:+, 27:1; Erophila verna 12:1, 13:r, 27:1; Euphrasia officinalis 25.+, Eurhynchium hians 19.;, Festuca rubra agg. 32:1; Fissidens spec. 13.+; Fissidens taxifolius 32:1; Galium mollugo 30:1; Galium pumilum 5.+, 25:+, 26.1; Galium verum 25:r; Gentianella germanica 25:r; Gyroweisia tenuis 16:+;

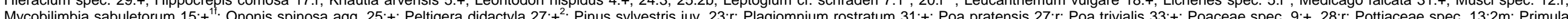

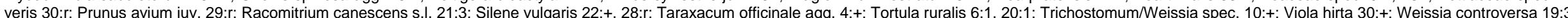


Tab. 17: Toninio-Psoretum decipientis

Einheit 1: Toninio-Psoretum decipientis typicum, Cladonia-Variante
Einheit 2: Toninio-Psoretum decipientis fulgensietosum bracteatae 2.1 Cladonia symphycarpa-Variante
2.1.1 Squamarina cartilaginea-Subvariante
2.1.2 Cephaloziella-Subvariante
2.2 Artenarme Variante

\begin{tabular}{|c|c|}
\hline Einhe & heit \\
\hline & 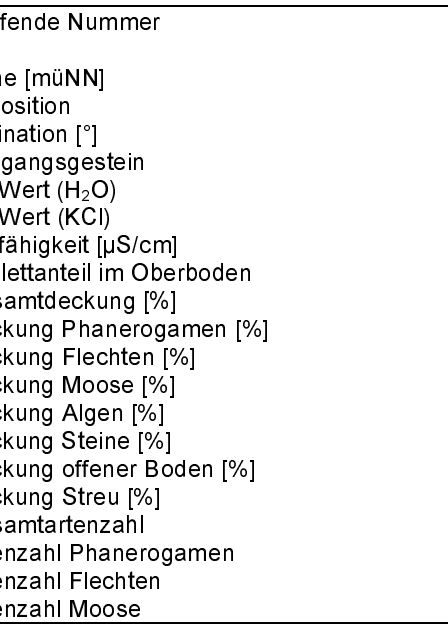 \\
\hline & $\begin{array}{l}\text { + d 1: } \\
\text { Fulgensia fulgens } \\
\text { Cladonia furcata ssp. subrangiformis } \\
\text { Leptogium cf. schraderi } \\
\text { Thuidium abietinum }\end{array}$ \\
\hline & $\begin{array}{l}\text { Fulgensia bracteata } \\
\text { Endocarpon pusillum }\end{array}$ \\
\hline & $\begin{array}{l}\text { +2.1: } \\
\text { Cladonia symphycarpa } \\
\text { Psora decipiens } \\
\text { Placidium squamulosum } \\
\text { Ditrichum flexicaule } \\
\text { Hypnum cupressiforme var. lacunosum }\end{array}$ \\
\hline & $\begin{array}{l}\text { + 2.1.1: } \\
\text { Peltigera rufescens } \\
\text { Tortella inclinata } \\
\text { Encalypta streptocarpa } \\
\text { Leptogium cf. lichenoides }\end{array}$ \\
\hline & $\begin{array}{l}\text { 1.1: } \\
\text { Encalypta vulgaris } \\
\text { Squamarina cartilaginea } \\
\text { Squamarina lentigera } \\
\text { Placidium pilosellum }\end{array}$ \\
\hline & $\begin{array}{l}\text { 1.2 + 2.2: } \\
\text { Cephaloziella spec. } \\
\text { Trichostomum et Weissia spec. }\end{array}$ \\
\hline & $\begin{array}{l}\text { 2: } \\
\text { Aloina spec. } \\
\text { Bryum spec. } \\
\text { Barbula unguiculata }\end{array}$ \\
\hline & $\begin{array}{l}\text { OC, KC: } \\
\text { Toninia sedifolia } \\
\text { Cladonia pyxidata ssp. pocillum } \\
\text { Barbula convoluta }\end{array}$ \\
\hline & $\begin{array}{l}\text { leiter Kryptogamen: } \\
\text { Ceratodon purpureus } \\
\text { Cyanophyta div. spec. } \\
\text { Collema spec. } \\
\text { Pseudocrossidium revolutum } \\
\text { Pseudocrossidium hornschuchianum } \\
\text { Didymodon rigidulus } \\
\text { Chlorophyta div. spec. } \\
\text { Cladonia rangiformis } \\
\text { Barbula spec. } \\
\text { Fissidens dubius } \\
\text { Pottiaceae spec. } \\
\text { Brachythecium albicans } \\
\text { Cetraria aculeata }\end{array}$ \\
\hline & $\begin{array}{l}\text { Inerogamen: } \\
\text { Thymus pulegioides } \\
\text { Festuca ovina agg. } \\
\text { Bromus erectus } \\
\text { Koeleria pyramidata } \\
\text { Potentilla tabernaemontani } \\
\text { Sanguisorba minor } \\
\text { Sedum sexangulare } \\
\text { Arenaria serpyllifolia } \\
\text { Scabiosa columbaria } \\
\text { Poa compressa } \\
\text { Carex ornithopoda } \\
\text { Plantago media } \\
\text { Plantago lanceolata } \\
\text { Pimpinella saxifraga } \\
\text { Lotus corniculatus } \\
\text { Helictotrichon pratense } \\
\text { Briza media }\end{array}$ \\
\hline
\end{tabular}

\begin{tabular}{|c|c|c|c|c|c|c|c|c|c|c|c|c|c|}
\hline \multicolumn{9}{|c|}{1} & \multirow[b]{2}{*}{10} & & & & \\
\hline 1 & 2 & 3 & 4 & 5 & 6 & 7 & 8 & 9 & & 11 & 12 & 13 & 14 \\
\hline DP & DS & $\begin{array}{l}\text { PT } \\
\text { f }\end{array}$ & PT & PT & DS & SI & $\mathrm{SI}$ & $\mathrm{SI}$ & $\mathrm{HL}$ & $\mathrm{DH}$ & WB & JW & SR \\
\hline 220 & 200 & 330 & 330 & 330 & 200 & 355 & 355 & 355 & 315 & 220 & 280 & 180 & 200 \\
\hline s & & 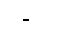 & - & & & & sw & & & w & & sw & 0 \\
\hline$<5$ & - & - & $<5$ & $<5$ & - & - & 5 & - & - & 20 & - & 5 & 5 \\
\hline $\mathrm{mu}$ & $\mathrm{mu}$ & $\mathrm{mu}$ & $\mathrm{mu}$ & $\mathrm{mu}$ & $\mathrm{mu}$ & $z d$ & $\mathrm{zd}$ & $z d$ & $z d$ & $\mathrm{zd}$ & $\mathrm{zg}$ & $\mathrm{zg}$ & sug \\
\hline 7.2 & 7.3 & 7.3 & 7.3 & 7.3 & 7.3 & 7.3 & 7.2 & 7.3 & 7.3 & 7.4 & 7.2 & 7.1 & ing \\
\hline 7.1 & 7.1 & 7.1 & 7.1 & 7.1 & 7.1 & 7.2 & 7.1 & 7.2 & 7.0 & 7.2 & 7.1 & 7.0 & 7.0 \\
\hline 362 & 309 & 301 & 301 & 301 & 309 & 258 & 326 & 261 & 406 & 279 & 1322 & & 15 \\
\hline & & ++ & ++ & ++ & ++ & o & & ++ & ++ & ++ & & ++ & 70 \\
\hline 80 & 60 & 70 & 80 & 50 & 60 & 60 & 95 & 65 & 80 & 40 & 60 & 75 & 85 \\
\hline & 25 & 30 & 35 & 20 & 30 & 15 & 35 & & 25 & 10 & $<5$ & $<1$ & $<1$ \\
\hline & & & 20 & 15 & 20 & 20 & 15 & 25 & 30 & 10 & 15 & 25 & 65 \\
\hline & 20 & 35 & 30 & 20 & 20 & 15 & 60 & 10 & 30 & 25 & 45 & 50 & ov \\
\hline & 5 & $<5$ & & & $<5$ & 15 & $<5$ & 30 & $<1$ & $\begin{array}{l}20 \\
<1\end{array}$ & $\begin{array}{l}<0 \\
<1\end{array}$ & $<5$ & 20 \\
\hline 15 & 35 & 15 & 10 & 25 & 30 & 35 & $<5$ & 25 & 15 & 40 & 40 & 25 & 10 \\
\hline & 5 & 15 & 10 & 25 & 10 & 5 & $<5$ & 10 & 5 & 20 & & $<1$ & 5 \\
\hline$<1$ & $<1$ & $<1$ & $<5$ & $<1$ & $<1$ & $<1$ & $<5$ & $<1$ & $<1$ & $<1$ & 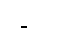 & & $<1$ \\
\hline 20 & 25 & 22 & 28 & 21 & 28 & 14 & 23 & 12 & 26 & 20 & 25 & 18 & 14 \\
\hline 20 & 9 & 6 & 10 & 9 & 7 & & 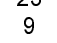 & & 12 & 3 & & & \\
\hline 6 & 10 & 9 & & & 12 & 3 & & - & & 10 & 11 & 5 & 5 \\
\hline & & & & & & & & & & & & & \\
\hline
\end{tabular}

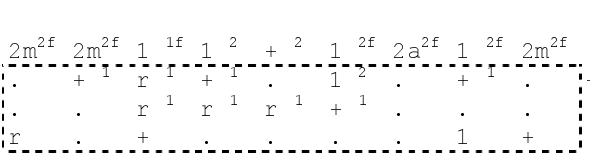

$\begin{array}{lllllllllllllllllllllllllllllll} & & & \end{array}$

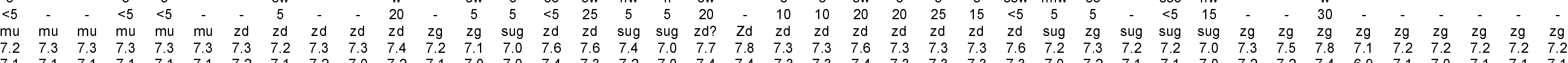

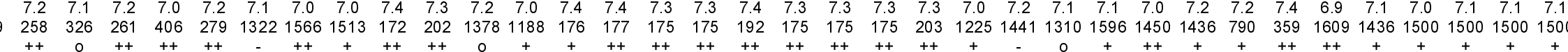

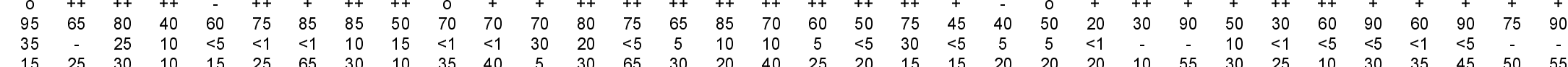

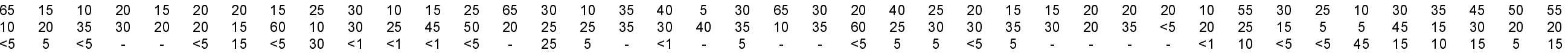

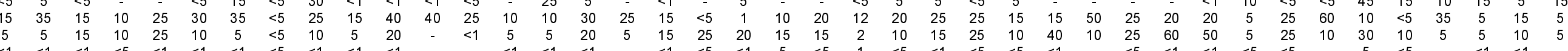
$\begin{array}{ccccccccccccccccccccccccccc}27 & 19 & 18 & 22 & 23 & 24 & 13 & 12 & 21 & 20 & 17 & 11 & 24 & 18 & 17 & 17 & 9 & 17 & 13 & 13 & 11 & 12 & 14 & 11 & 11 & 8 & 8 \\ 7 & 5 & 1 & 2 & 5 & 7 & 2 & 1 & 7 & 4 & 3 & 1 & 10 & 2 & 2 & 5 & 2 & 7 & 1 & 2 & 1 & 3 & 3 & 1 & 3 & - & - \\ & & & & & & & & & & & & & & & & & & & & \end{array}$

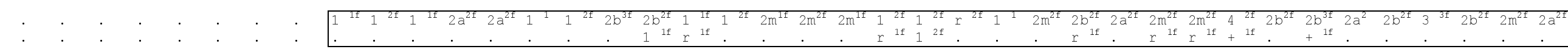

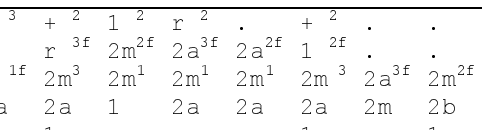

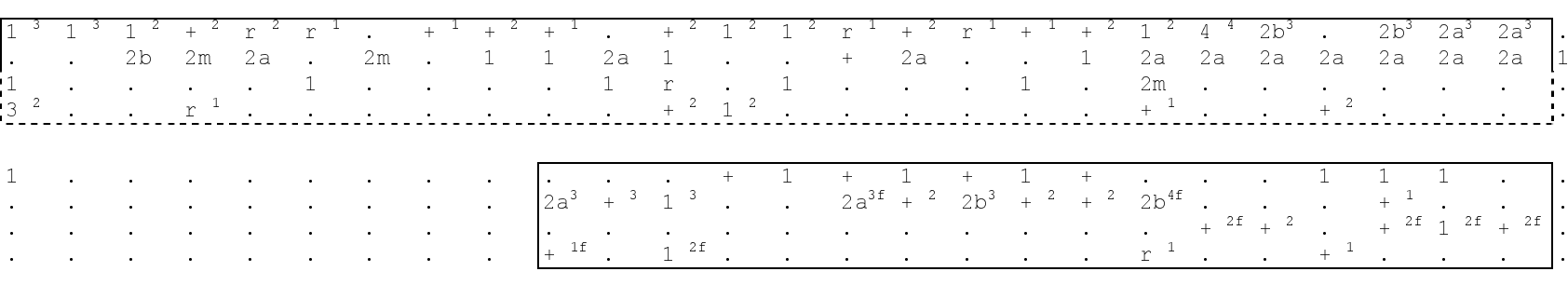

Außerdem mit Stetigkeit < 11 und in weniger als drei Einheiten:

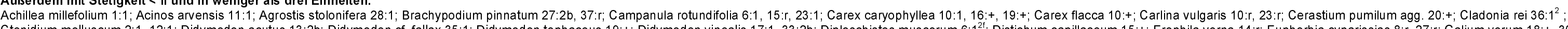

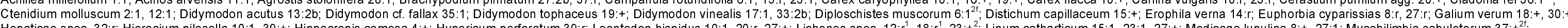

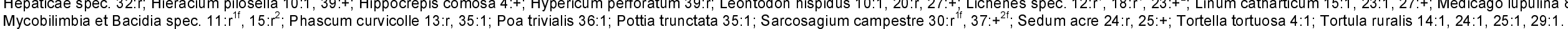




\section{Danksagung}

An erster Stelle möchte ich Herrn Prof. Dr. Hartmut Dierschke danken, der mir die Möglichkeit gab, meiner Begeisterung für Flechten nachzugehen und im Rahmen dieser Dissertation ein flechtenkundliches Thema zu bearbeiten. Er hatte stets ein offenes Ohr für Fragen und stand mir mit seinem Rat zur Seite. Herr Prof. Dr. Erwin Bergmeier übernahm freundlicherweise das Korreferat. Herrn Dr. Heribert Schöller (Schmitten) danke ich ganz besonders für die Hilfe beim Erarbeiten des Konzeptes und die gemeinsamen Flechten-Exkursionen im Untersuchungsgebiet.

Mit Petra Fischer arbeitete ich mich in die Methodik der Dünnschichtchromatographie ein, wir führten viele anregende Flechten-Diskussionen und sie stand mir immer mit Tips und Hilfestellungen zur Seite. In Zusammenarbeit mit Gunnar Waesch ließen sich zahlreiche mehr oder weniger hartnäckige Computerprobleme in den Griff bekommen. Danke für die gute Zusammenarbeit und die Hilfe in der Endphase der Arbeit! Brigitte Siegesmund hat mich bei den Laborarbeiten und beim Ausmessen der Cladonia-Podetien unterstützt - ohne sie hätten wohl nicht nur die morphologischen Untersuchungen wesentlich mehr Zeit in Anspruch genommen. Ute Wergen half ebenfalls bei der Dünnschichtchromatographie der unzähligen Flechten-Proben und bei der Dateneingabe. Auch den beiden Technischen Assistentinnen der Abteilung daher ein herzliches Dankeschön! Bei allen weiteren aktuellen und ehemaligen Mitgliedern der Abteilung für Vegetationsanalyse und Phytodiversität bedanke ich mich für die nette Arbeitsatmosphäre.

Ein besonderer Dank gilt meinem Vater Dr. Hans Günzl (Tübingen) für die Möglichkeit seine Ausrüstung zum Erstellen der Makrophotographien zu nutzen und die Hilfestellungen, die er mir hierzu gab. Bei Herrn Ernst Baier (Witzenhausen) bedanke ich mich für die zahlreichen Informationen zum Untersuchungsgebiet und die schönen gemeinsamen Exkursionen in Nordhessen, auf der Suche nach Buxbaumia aphylla und anderen interessanten Moosen sowie für die große Hilfe beim Bestimmen vor allem der kleinen Kalkmagerrasen-Moose. Dr. Marcus Schmidt (Göttingen) half in der Planungs- und Endphase der Doktorarbeit und zeigte mir einige interessante Gebiete in Nordhessen.

Herr Prof. Dr. Thomas Friedl (Abt. Experimentelle Phykologie, Univ. Göttingen) ermöglichte mir, die Einrichtungen und das Know-how seiner Abteilung für die rDNA-Sequenzierung zu nutzen, unterstützte mich bei der Datenauswertung und bei der Interpretation der Ergebnisse. Er führte zudem die phylogenetischen Analysen mit PAUP durch. Vielen Dank dafür! Gert Helms brachte mir die notwendigen Arbeitsschritte bei, half mir bei so manchem Labor- und Auswertungsproblem und war darüber hinaus bezüglich des Genetik-Kapitels ein wichtiger Ansprechpartner beim Zusammenschreiben der Arbeit. Bei ihm sowie bei den weiteren Mitarbeitern der Abteilung Phykologie Anke Behnke, Dominik Hepperle, Birgit Olberg, Ruth Pilot und Elke Zufall-Roth, bedanke ich mich für die Hilfe, die mir als Neuling in Sachen molekularer Methoden den Einstieg in diese Thematik erleichterte. Herr Prof. Dr. Eberhard Grüger (Abt. Palynologie und Quartärwissenschaften) gestattete mir die Nutzung eines Laborraumes seiner Abteilung. Dr. Michael Hoppert (Univ. Göttingen) bestimmte die Algen einiger Algenkrusten aus meinem Untersuchungsgebiet. Prof. Dr. H.-J. Beug (Abt. Palynologie und Quartärwissenschaften) stellte bislang unpublizierte Daten früherer Kryptogamenexkursionen ins Meißner-Vorland zur Verfügung.

Dr. Holger Thues (Kaiserslautern) und Dr. Günter Schuster (Düsseldorf) weihten mich in die Technik der Mikroklima-Messungen mit den eigens für Flechten konstruierten Geräten ein. Mark Eckhardt war zum frühen Aufstehen bereit und arbeitete bei den zeitaufwendigen Mikroklima-Messungen mit. Dr. Thilo Heinken (Berlin) half bei den statistischen Auswertungen. Bernd Raufeisen (Göttingen) fertigte die Karten zum Untersuchungsgebiet an. Carolin Held (Tübingen) und Toby Spribille (Göttingen) korrigierten den englischen Text.

Von den Forstämtern Bad Sooden-Allendorf und Witzenhausen bekam ich ebenfalls Informationen zum Untersuchungsgebiet. Besonders Herrn Marco Lenarduzzi (Germerode) möchte ich an dieser Stelle Dank sagen für die zahlreichen Informationen und interessanten Gespräche zu den bzw. über die Magerrasen des Meißnervorlandes. Hessen Forst sowie das RP Kassel unterstützten diese Arbeit durch eine Wegebenutzungserlaubnis bzw. durch eine Ausnahmegenehmigung zum Bearbeiten einiger nordhessischer NSGs.

Zum Abschluß gilt meinem Lebensgefährten Börries Schlimbach ein ganz besonderes Dankeschön für die vielfältige Hilfe und Unterstützung im Verlauf dieser Arbeit. 


\section{Lebenslauf}

1970

geboren in Tübingen

\section{Schulabschluß:}

1989

Abitur in Tübingen

\section{Studium:}

1989 - 1993 Biologiestudium (Diplom) an der Universität Tübingen

1991 Vordiplom

1992

DAAD-Stipendium: Auslandsaufenthalt in Finnland, Studium an den Universitäten Turku und Oulu

1993-1997 Weiterführung des Biologiestudiums an der Universität Göttingen

1996 Diplomprüfungen

1996 - 1997 Diplomarbeit mit dem Titel:

„Einfluß von Nutzung und Struktur der Fichtenwälder des

Hochharzes auf die epiphytische Flechtenflora und -vegetation”

\section{Studienabschluß:}

1997

Diplom-Biologin

\section{Promotion:}

1999 - 2003 Promotion am A.-V.-Haller-Institut für

Pflanzenwissenschaften, Universität Göttingen

\section{Berufstätigkeiten:}

1994/1995 Praktika bei der Akademie für Natur- und Umweltschutz Baden-Württemberg

1997/1998 Freiberufliche Biologin

1998/1999 Wissenschaftliche Hilfskraft an der Biologischen

Fakultät der Universität Göttingen

seit 1999 Wissenschaftliche Mitarbeiterin an der Abteilung für

Vegetationsanalyse und Phytodiversität (ehem. Vegetationskunde und Populationsbiologie) der Universität Göttingen 